JASA-CP-2383 19860013491
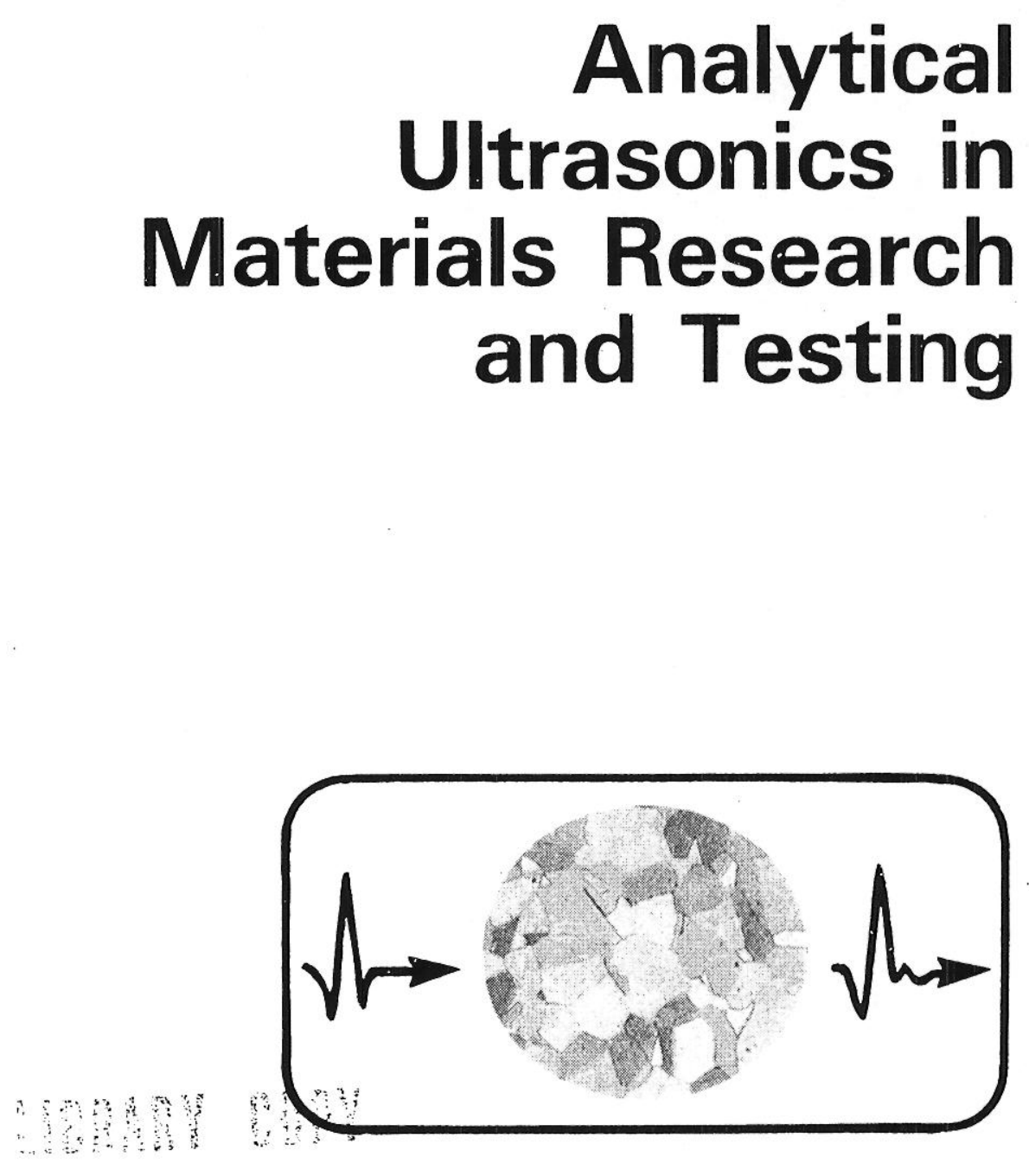

Proceedings of a conference held at the NASA Lewis Research Center Cleveland, Ohio

November 13-14, 1984 



\section{Analytical Ultrasonics in Materials Research and Testing}

Proceedings of a conference sponsored by the National Aeronautics and Space Administration, Washingtron, D.C., and held at the NASA Lewis Research Center Cleveland, Ohio November 13-14, 1984 



\section{PREFACE}

Analytical ultrasonics implies the measurement of material microstructure and associated factors that govern mechanical properties and dynamic response. It goes beyond flaw detection, flaw imaging, and defect characterization and includes assessing the inherent properties of material environments in which the flaws reside. Analytical ultrasonics can assess and verify material moduli, strength, toughness, and a host of other mechanical properties and morphological conditions. Analytical ultrasonics can provide a nondestructive approach to characterizing initial states of engineering solids and their degradation or modification on exposure to service environments. Also, analytical ultrasonics can provide data relevant to materials research concerned with understanding and tailoring material microstructures to enhance properties and dynamic response.

Realization of these potentials will demand the advancement of signal acquisition, processing, and analysis methods with emphas is on automated, digital techniques. Moreover, it will be necessary to establish theoretical foundations for the empirical correlations that can be found among ultrasonic measurements, microstructures, mechanical properties, dynamic response, microdamage and degradation effects, and microcrack nucleation mechanics.

This conference proceedings presents recent research results and is intended to stimulate further investigations and advancements in analytical ultrasonics for characterizing structural materials from metals and ceramics to composites. General topics covered by the conference included

(1) Status and advances in analytical ultrasonics for characterizing material microstructures and mechanical properties

(2) Status and prospects for ultrasonic measurements of microdamage, degradation, and underlying morphological factors

(3) Status and problems in precision measurements of frequency-dependent velocity and attenuation for materials analysis

(4) Procedures and requirements for automated, digital signal acquisition, processing, analysis, and interpretation

(5) Incentives for analytical ultrasonics in materials research and materials processing, testing, and inspection

(6) Examples of progress in ultrasonics for interrelating microstructure, mechanical properties, and dynamic response

Because of the diversity of topics covered, no attempt was made to arrange the papers into systematic categories. With two exceptions, the papers appear in the order in which they were presented at the conference.

The keynote paper was given by Professor Robert E. Green, Jr., Chairman of The Johns Hopkins University Materials Science and Engineering Department and Director of the Center for NDE at the University, who stressed the impor- 
tance of advancing the theoretical foundations of ultrasonics for fuller explanations of interactions of ultrasound with solid materials. The general theme of materials characterization through better understanding of interrelations among ultrasonic measurements, material microstructure and morphology, and mechanical properties was emphasized from diverse viewpoints by the speakers.

I express my gratitude to the authors and speakers for their cooperation in making the conference possible and for their excellent contributions, which appear in this proceedings. I also express special thanks to my session cochairmen, Stanley J. Klima and Edward R. Generazio, both of NASA Lewis Research Center, for keeping the sessions moving at a lively pace and assuring ample audience interaction with the speakers.

It is well known that undertaking a conference of the scope of this one requires the participation of a number of individuals working behind the scenes. I was fortunate in having the following individuals on the Conference Committee who gave the support, talents, enthusiasm, and energy needed to assure its success: Harold E. Kautz, Gloria J. O'Donne11, Don J. Roth, and Shelly J. White. Finally, I express my thanks to Carol A. Vidoli whose expertise and sharp editorial eye piloted the compilation of this volume through the treacherous straits of publication.

\author{
Alex Vary \\ Conference Chairman \\ Head, Nondestructive Evaluation Section \\ NASA Lewis Research Center
}


ULTRASONIC NONDESTRUCTIVE MATERIALS CHARACTERIZATION

Robert E. Green, Jr., The Johns Hopkins University . . . . . . . . . 1

ULTRASONIC CHARACTERIZATION OF MICROSTRUCTURE IN POWOER METAL ALLOY

B.R. Tittmann, L.A. Ahlberg, and K. Fertig,

Rockwell International Science Center ............. 31

STUDIES OF ACOUSTIC EMISSION FROM POINT AND EXTENDED SOURCES

Wolfgang Sachse, Kwang Yul Kim, and C.P. Chen, Cornell University . . 49

RESIDUAL STRESS MEASUREMENTS IN CARBON STEEL

Joseph S. Heyman, NASA Langley Research Center, and Min Namkung,

College of William and Mary ..................

ULTRASONIC MEASUREMENT OF POROSITY IN CASTS AND WELDS

Laszlo Adler and Shaio-Wen Wang, The Ohio State University . . . . . 75

ANALYTICAL ULTRASONICS FOR CHARACTERIZATION OF METALLURGICAL

MICROSTRUCTURES AND TRANSFORMATIONS

Moshe Rosen, The Johns Hopkins University . . . . . . . . . . . 83

ANALYTICAL ULTRASONICS FOR STRUCTURAL MATERIALS

David S. Kupperman, Argonne National Laboratory . . . . . . . . . 103

ULTRASONIC CHARACTERIZATION OF STRUCTURAL CERAMICS

Stanley J. Klima and George Y. Baaklini, NASA Lewis Research Center . . 117

ON THE INTERACTION OF ULTRASOUND WITH CRACKS: APPLICATIONS TO

FATIGUE GROWTH

O. Buck, R.B. Thompson, and D.K. Rehbein, Iowa State University . . . 127

ANALYTICAL ULTRASONICS FOR EVALUATION OF COMPOSITE MATERIALS RESPONSE

PART I: PHYSICAL INTERPRETATION

Edmund G. Henneke II and John C. Duke, Jr., Virginia Polytechnic

Institute and State University. . . . . . . . . . . . . 141

ANALYTICAL ULTRASONICS FOR EVALUATION OF COMPOSITE MATERIALS RESPONSE

PART II: GENERATION AND DETECTION

John C. Duke, Jr., and Edmund G. Henneke II, Virginia Polytechnic

Institute and State University. . . . . . . . . . . . . 153

INFERENCE OF STRESS AND TEXTURE FROM ANGULAR DEPENDENCE OF

ULTRASONIC PLATE MODE VELOCITIES

R.B. Thompson, J.F. Smith, and S.S. Lee, Iowa State University . . . . . 169

EFFECT OF STRESS ON ULTRASONIC PULSES IN FIBER REINFORCED COMPOSITES

John H. Hemann, Cleveland State University, and George Y. Baaklini,

NASA Lewis Research Center............... 181 
PATTERN RECOGNITION CHARACTERIZATIONS OF MICROMECHANICAL AND

MORPHOLOGICAL MATERIALS STATES VIA ANALYTICAL QUANTITATIVE ULTRASONICS

James H. Williams, Jr., and Samson S. Lee, Massachusetts Institute

of Technology . . . . . . . . . . . . . . . . . . . . .

ULTRASONIC VERIFICATION OF MICROSTRUCTURAL CHANGES DUE TO HEAT TREATMENT

Edward R. Generazio, NASA Lewis Research Center . . . . . . . . . . . 207

ULTRASONIC MATERIAL PROPERTY DETERMINATIONS

Steven Serabian, University of Lowell . . . . . . . . . . . . . . . . . 219

DYNAMIC MODULI AND LOCALIZED DAMAGE IN COMPOSITES

L.S. Fu, The Ohio State University . . . . . . . . . . . . . . . . . . . 233

TRANSFER FUNCTION CONCEPT FOR ULTRASONIC CHARACTERIZATION OF

MATERIAL MICROSTRUCTURES

Alex Vary and Harold E. Kautz, NASA Lewis Research Center . . . . . . . 257

ADHESIVE JOINT EVALUATION BY ULTRASONIC INTERFACE AND LAMB WAVES

S.I. Rokhilin, The Ohio State University . . . . . . . . . . . . . . . . 299

INPUT-OUPUT CHARACTERIZATION OF AN ULTRASONIC TESTING SYSTEM

BY DIGITAL SIGNAL ANALYSIS

James H. Williams, Jr., Samson S. Lee, and Hira Karagulle,

Massachusetts Institute of Technology

QUANTITATIVE FLAW CHARACTERIZATION WITH SCANNING LASER ACOUSTIC

MICROSCOPY

Edward R. Generazio and Don J. Roth, NASA Lewis Research Center . . . 341 


\title{
ULTRASONIC NONDESTRUCTIVE MATERIALS CHARACTERIZATION
}

\author{
Robert E. Green, Jr. \\ The Johns Hopkins University \\ Baltimore, Maryland 21218
}

\begin{abstract}
A brief review of ultrasonic wave propagation in solid materials is presented with consideration of the altered behavior in anisotropic and noninear elastic materials in comparison with isotropic and Iinear elastic materials. Some experimental results will be described in which ultrasonic velocity and attenuation measurements give insight into materials microstructure and associated mechanical properties. Recent developments with laser beam non-contact generation and detection of ultrasound will be presented. The results of several years of experimental measurements using high-power ultrasound will be discussed, which provide substantial evidence of the inability of presently accepted theories to fully explain the interaction of ultrasound with solid materials. Finally, a special synchrotron x-ray topographic system will be described which affords the possibility of observing direct interaction of ultrasonic waves with the microstructural features of real crystalline solid materials for the first time.
\end{abstract}

\section{INTRODUCTION}

Although historically nondestructive techniques have been used almost exclusively for detection of macroscopic defects in structures after they have been in service for some time, it has become increasingly evident that it is both practical and cost effective to expand the role of nondestructive evaluation to include all aspects of materials production and application. Currently efforts are directed at developing and perfecting nondestructive evaluation techniques which are capable of monitoring and controliing the materials production process; the materials stability during transport, storage, and fabrication; and the amount and rate of degradation during the materials inservice life. Ultrasonic techniques afford very useful and versatile nondestructive methods for evaluating the microstructures, associated mechanical properties, and macroscopic flaws in solid materials.

\section{ULTRASONIC WAVES IN SOLID MATERIALS}

The use of ultrasonic waves as nondestructive probes has as a prerequisite the careful documentation of the propagational characteristics of the ultrasonic waves themselves ( $r$ ef. 1). Since in nondestructive evaluation applications it is not desirable for the ultrasonic waves to alter the material through which they pass, it is necessary to work with very low amplitude waves, which normally are regarded to obey linear elasticity theory. Although most practical uses of ultrasonics are applied to solid materials which are polycrystalline aggregates 
and therefore assumed to be isotropic, with real crystalline solids the condition of ideal isotropy is extremely difficult if not impossible to attain. The prime reason for this is that the solidification or forming and heating processes associated with material processing does not permit a random distribution of crystallographic orientations among the aggregation of grains and often does not even permit a uniformity of grain size. Therefore, most real polycrystalline aggregates possess a "texture" and this texture strongly influences the mechanical properties of the anisotropic material including ultrasonic wave propagation.

\section{LINEAR ELASTIC WAVE PROPAGATION}

In general three different linear elastic waves may propagate along any given direction in an anisotropic material. These three waves are usually not pure modes since each wave generally has particle displacement components both parallel and perpendicular to the wave normal. However, one of these components is usually much larger than the other; the wave with a large parallel component is called quasi-longitudinal while the waves with a large perpendicular component are called quasi-shear. In the event that sufficient symmetry prevails such that the direction of propagation is elastically isotropic or if the material is elastically isotropic in general, then all modes become pure modes, i.e. the particle displacements are either parallel or perpendicular to the wave normal, and the two quasi-shear modes degenerate into one pure shear mode. Also of great practical importance to elastic wave propagation in anisotropic materials is the fact that the direction of the flow of energy per unit time per unit area, the energy-flux vector, does not in general coincide with the wave normal as it does in the isotropic case, i.e. the ultrasonic beam exhibits refraction even for normal incidence.

Figure 1 serves to illustrate several aspects of ultrasonic wave propagation in anisotropic materials. This figure is one of a set of four photographs originally published by Merkulov and Yakovlev (ref. 2) who used the optical schlieren technique to visualize the sound field due to ultrasonic waves propagating in optically transparent quartz crystals. In Fig. 1 a single $25 \mathrm{MHz}$ $x$-cut quartz piezoelectric transducer attached to a $y$-face of a single crystal quartz block is shown to simultaneously generate a quasi-longitudinal and a quasi-shear wave, both of which exhibit energy-flux deviation from the wave normal, i.e. refraction. In general, a piezoelectric transducer generates both longitudinal and shear waves, but depending on the particular piezoelectric properties of the specific transducer one type of mode will be dominant. It should be noted, that since most real structural materials exhibit some degree of anisotropy, great care must be exercised in locating flaws in these materials using ultrasonic techniques. For example, Fig. 2 illustrates schematically how anisotropy caused by the strong materials texture of the test object results in a marked deviation of the energy-flux (the ultrasonic beam) such that it detects a crack which is not in the direct line of sight beneath the transducer. If the degree of energy-flux deviation was not determined prior to this test and the assumption was made that no deviation occurred, subsequent investigation would not reveal the crack in the calculated position beneath the transducer, while the real crack location would not be confirmed. 
Here is a good place to call attention to the fact that, although many duthors and investigators draw a close analogy between electromagnet1c wave propagation and elastic wave propagation in solid materials, great caution should be exercised in doing so. The behavior of anisotropic materlals with respect to propagation of elastic waves is much more complicated than the propagation of electromagnetic waves, since the materlal constitutive equations required to properly descrlbe elastio waves are of higher order tensor rank than those required to describe electromagnetic waves ( $r$ ef. 3 ).

\section{ATTENUATION OF NEARLY LINEAR ELASTIC WAVES}

For all read solids, the assumption of pure linear elastiolty is only an approximation since all real ultrasonic waves are attenuated as they propagate. If one considers this more realistic case, one finds, to the lowest order of approximation, that the general propagational characteristics of such waves in solid materials are ldentical with the linear elastic case considered previously, as regards wave speeds, particle displacements, energy flux vectors, and diffraction spread. However, as a result of various mechanisms, there will be energy loss from these propagating waves.

Although geometrical effects can cause energy to be lost from the ultrasonic beam, such losses are not indicative of intrinsic loss mechanisms associated with the microstructure. Once proper precautions are taken to either eliminate or control these geometrical effects, ultrasonic attenuation measurements serve as a very sensitive indicator of internal loss mechanisms caused by microstructures and microstructural alterations in the material (ref. 4). This sensitivity derives from the ability of ultrasonic waves to interact with volume defects such as cracks, mlorocracks, included particles, and precipitates; surface defects such as grain boundaries, interphase boundarles, and magnetic domain walls; and dislocation line defects. Moreover, ultrasonic waves are sensitive to the interaction of point defects such as impurity atoms and vacancies with dislocations.

Since a nearly linear elastic wave propagating in a crystal has a stress field represented by a second-order tensor, and since dislocation motion in crystals is primarily confined to certain directions in specific planes, the interaction of ultrasonic waves with dislocations in single crystals is anisotropic. Calculation of the stress on the dislocations is obtained by resolving the nine components of stress associated with the elastic wave into the appropriate slip direction and then summing.

Figure 3, taken from the work of Swanson and Green (ref. 5), illustrates the experimental agreement with the orientation dependence of ultrasonic attenuation caused by dislocation damping and also exhibits the extreme sensitivity of such ultrasonic waves as probes to measure plastic deformation. The data to plot the curves shown in this figure was obtained by measuring the change of attenuation of specifically polarized shear waves propagating along the axis of two similar cylindrical aluminum crystals while the orystals were subjected to uniaxial compressive deformation. Figure $3(a)$ shows the stress-strain curve and the change in attenuation-strain curve for a quasi-transverse wave polarized with a large component of particle displacement 
perpendicular to the projection of the primary-slip direction onto the end surface of the crystal specimen. With such a polarization, there is only a very small interaction between the stress field of the ultrasonic wave and dislocations on the primary-slip system. As can be seen, the attenuation rises steadily as the specimen is loaded to the yield point. During easy glide of the dislocations, the attenuation remains relatively constant, but starts to increase again as other slip systems become active.

Figure $3(\mathrm{~b})$ shows the stress-strain curve and the change in attenuation-strain curve for a quasi-transverse wave polarized with a large component of particle displacement parallel to the projection of the primary-slip direction onto the end surface of the crystal specimen. With such a polarization, there is a maximum interaction between the stress field of the ultrasonic wave and dislocations on the primary-slip system. In this case, the attenuation increased with increasing strain up to the yield point at which time there was a sharp decrease. After the sudden decrease associated with the yield point the attenuation continued to increase with increasing strain. These results show that ultrasonic attenuation measurements are extremely orientation dependent and that such measurements are much more sensitive to the early stages of plastic deformation than are conventional stress-strain measurements.

Sachse and Green, (ref. 6), conducted additional experiments which illustrate the extreme sensitivity of ultrasonic attenuation measurements to microstructural alterations. Figure 4 shows the results of an experiment in which an aluminum crystal, which had previously been loaded to a plastic load of $180 \mathrm{~kg}$, was reloaded to $60 \mathrm{~kg}$ and maintained at this load for one minute, unloaded to $45 \mathrm{~kg}$ and maintained for one minute, unloaded to $30 \mathrm{~kg}$ and maintained for one minute, unloaded to $15 \mathrm{~kg}$ and maintained for one minute, and then unloaded completely. Upon subsequent reloadirg to $70 \mathrm{~kg}$ and unloading, the ultrasonic attenuation displayed dips at those load values which had been maintained for a short time during the previous cycle. Note that even though the attenuation "remembered" the discontinuous nature of the first load-unload cycle, the load-unload curve (or equivalently the stress-strain curve) gave no indication of it. The observed results were attributed to the pinning of dislocation loops by point defects which were preferentially located at the position they occupled when the load was maintained constant for the one minute intervals. Upon reloading, these point defects pinned the dislocation loops again when the dislocations arrived at the location of the defects thus causing the dips in attenuation. These results show that ultrasonic attenuation measurements are sensitive to migration of point defects and their interaction with dislocations.

Figure 5 demonstrates the ability of ultrasonic attenuation measurements to detect extremely small microstructural alterations during fatigue testing and hence give early warning of fatigue-induced failure. This figure was taken from the work of Joshi and Green (ref. 7) who used ultrasonic attenuation measurements as a continuous monitor of fatigue damage in cold-rolled 1015 polycrystalline steel specimens which were cycled in reverse bending as cantilever beams to fracture at $30 \mathrm{~Hz}$. Note that the ultrasonic attenuation change indicated microstructural alterations, probably microcrack formation, prior to detection of an additional ultrasonic pulse on the A-scan display as is used in conventional nondestructive ultrasonic evaluation to detect crack formation. 


\section{NONLINEAR ELASTIC WAVE PROPAGATION}

Nonlinear effects associated with ultrasonic wave propagation may also be used to advantage for nondestructive materials characterization (ref. 1). These nonlinear effects may arise from several causes. First, the ultrasonic wave amplitude may be sufficiently large so that finite strains arise. Second, a material may behave in a nonlinear fashion, even when very low amplitude ultrasonic waves are propagated, provided that a sufficient amount of external stress is superimposed or provided that a residual stress (strain) is present in the material.

Although $x$-ray diffraction techniques have historically been the nondestructive method most often used in actual practice to measure residual stress (strain), they are not optimally suited for field applications partially because the necessary equipment is heavy and bulky and, perhaps more importantly, they suffer from the fact that they only serve to determine the state of stress (strain) in a surface layer of a material, while in many practical cases a knowledge of the bulk stresses (strains) is desired. Ultrasonic residual stress (strain) measurements have been made far less frequently, probably because of lack of familiarity with nonlinear elasticity theory and the experimental techniques necessary to obtain the required ultrasonic velocity measurement accuracy.

Most ultrasonic measurements of residual stress (strain) have been based on stress induced acoustical birefringence of a homogeneous isotropic solid. When linear elastic shear (transverse) waves are propagated through a homogeneous isotropic solid at right angles to the direction of applied or residual stress, the shear wave with particle displacement parallel to the direction of the stress propagates with a faster velocity than the shear wave with particle displacement perpendicular to the direction of the stress. Other ultrasonic techniques can also be used to make such measurements. For example, five of the equations derived originally by Hughes and Kelly (ref. 8) relate a linear elastic wave velocity to a stress component in the material. These equations can be used either singly or in appropriate combination to determine stress components from experimental measurements of various ultrasonic wave velocities. Moreover, additional features of nonlinear elastic wave propagation, such as harmonic generation, temperature dependence of ultrasonic velocity, change in velocity as a function of applied magnetic field, or ultrasonic wave dispersion, have been proposed for residual stress determinations. Details of most of these measurement techniques, as well as a survey of theoretical considerations, may be found in two recent review articles (refs. 9 and 10 ).

The major difficulty associated with reliable (strain) measurement is that the change in linear

ultrasonic residual stress elastic wave velocities in solid materials due to residual stress is small and other factors, which can cause greater velocity changes, may mask residual stress effects. The prime factor in this regard is the fact that all real structural materials are initially anisotropic and not isotropic. Therefore, the ultrasonic residual stress (strain) measurement problem becomes one of measuring changes in anisotropy before and after stressing rather than the commonly treated theoretical problem of stress-induced anisotropy of an originally isotropic solids. 
Additional problems arise with ultrasonic wave propagation in inhomogeneous materials. The presence of a single bounding surface complicates the propagational characteristics of ultrasonic waves in solid materials and can lead to erroneous interpretation of velocity and attenuation measurements. The presence of many bounding surfaces, such as may occur in composites, complicates the propagational characteristics even more and, except in a few special cases, the problems have not been solved analytically. However, solution of these problems will permit proper ultrasonic measurements to be an invaluable tool in characterizing composite materials.

\section{CONTACT OR WATER COUPLED TRANSDUCERS}

Historically, piezoelectric crystals such as quartz were predominantly used as transducer materials. Currently, poled ferroelectric ceramics are most of ten used. Depending on the particular cut (crystallographic orientation) of the piezoelectric crystal or the particular geometry and poling configuration of the ferroelectric ceramic, the active elements may vibrate so as to generate longitudinal, shear, surface, or a variety of different guided wave modes. A number of shaped piezoelectric elements and electrode configurations have been developed in order to cause the generated ultrasonic beam to have the profile desired such as straight or focused. Unless these special precautions are taken, the ultrasonic beam generated will experience diffraction spread which increases with increasing distance from the transducer and is a function of transducer diameter and ultrasound wavelength. Figure 6 serves to illustrate the fact that even though this diffraction spread may only be moderate in an isotropic material, it can be quite pronounced in anisotropic material. In this figure, taken from the work of Liu (ref. 11), the diffraction spread of $10 \mathrm{MHz}$ longitudinal waves in quartz were measured experimentally using a laser scan technique. The half-width of the ultrasonic beam is plotted as a function of the distance from the piezoelectric transducer, indicated by cross-hatching at the top of the figure. It should be noted that the diffraction spreads for the case of elastically isotropic fused quartz and the elastically isotropic z-axis direction in a quartz crystal are the same within the limits of the experimental parameters used, while the diffraction spread for the case of wave propagation along the anisotropic $x$-axis direction in the quartz crystal is much greater. This illustrates once more the importance of properly considering the effects due to anisotropy of real materials and makes questionable the commonly used method of determining ultrasonic transducer beam profiles using a tank of water as the propagation medium.

A major problem associated with conventional ultrasonic techniques is the requirement that either the generating and receiving transducers be acoustically bonded to the test structure with some sort of acoustical impedance matching coupling medium such as water, oll, or grease; or often more harmful, particularly to polymer based composite materials, is the necessity of immersing the entire structure to be tested in a tank of water or coupling the transducers to the workpiece using water squirter systems. Although the couplant allows acoustical energy to propagate into the structure, it causes several problems in addition to potential harm to the test object. For velocity measurements the 
coupling medium can cause transit time errors on the order of one percent of the measured values. Due to partial transmission and partial reflection of sound energy in the couplant layer, there may be a change of shape of the waveform which can further affect velocity measurement accuracy. This can also lead to serious errors in absolute attenuation measurements of up to twenty percent of the measured values. This latter fact is the reason that so few rellable absolute measurements of attenuation are reported in the scientific literature. The requirement of physical contact between transducer and test structure places limitations on ultrasonic testing in structural configurations which possess geometries with difficult to reach areas. Limitations are also placed on the testing of materials at elevated temperatures or in the environment of outer space.

\section{NON-CONTACT TRANSDUCERS}

A method of non-contact generation and detection of ultrasound is therefore of great practical importance. Several such techniques are presently available in various stages of development, namely capacitive pick-ups, electromagnetic acoustic transducers (EMAT'S), and laser beam optical generators and detectors. However, as the name implies, capacitive pick-ups cannot be used as ultrasonic generators and, even when used as detectors, the air gap required between the pick-up and test structure surface is extremely small, which in essence causes the device to be very nearly a contact one. EMAT'S, on the other hand, have already been successfully used for material defect characterization particularly in metal bars, tubes, pipes, and plates. They have also been used for material property measurement such as material anisotropy (texture) and internal stress (strain) state. As useful as these devices have proven, they still have not been optimized because they are very inefficient ultrasound generators and require very large power supplies in order to operate properly. Additional problems are that they can obviously only be used for examination of electrically conducting materials and the efficiency of ultrasound generation and detection rapidly decreases with lift-off distance between the EMAT face and the surface of the test object as well as with increasing temperature of the test object. Laser beam ultrasound generation and detection overcomes all of these problems and affords the opportunity to make truly non-contact ultrasonic measurements in both electrically conducting and non-conducting materials, in materials at elevated temperatures, in corrosive and other hostile environments, in geometrically difficult to reach locations, in outer space, and do all of this at relatively large distances, 1.e. meters, from the test object surface.

\section{LASER BEAM ULTRASOUND GENERATION}

As early as 1963, White (ref. 12) reported the generation of elastic waves in solid materials by transient surface heating and research has continued in this area up to the present time (refs. 13-22). Three different mechanisms have been proposed to account for the generation of ultrasonic waves by the impact of pulsed laser beams, namely radiation pressure, ablation, and thermoelasticity. Radiation pressure, which is caused by momentum transfer from the incident electromagnetic pulse, is the least efficient of the three proposed 
mechanisms and is therefore of little importance for practical applications. At the other extreme, when a laser pulse possessing sufficient power density strikes the surface of a material the electromagnetic radiation is absorbed in a very thin layer of the material and vaporizes it. The amplitude of the ultrasonic wave generated in the material by this ablation process can of ten be increased by placing a proper coating of different thermal properties on the surface of the test object. Although the surface of the test object is slightly damaged when ablation occurs, in certain cases the amount of damage is acceptable when such a generation process is the only way to obtain ultrasonic waves of sufficient amplitude in a non-contact manner. The thermoelastic process consists of absorption of a laser pulse possessing moderate energy in a finite depth of the material under investigation such that thermal expansion causes a volume change and consequently an elastic wave. Thus, the thermoelastic process is the only process which is truly nondestructive and still capable of generating an ultrasonic wave of sufficient amplitude for nondestructive evaluation purposes.

\section{LASER GENERATION PIEZOELECTRIC DETECTION}

Initial work with laser generation of ultrasound was performed using piezoelectric transducers as detectors since the various investigators had prior experience with this type of transducer and associated electronic equipment. Figure 7, (ref. 21), shows an example of an ultrasonic waveform generated by a Q-switched Nd:YAG pulsed laser in a graphite-epoxy composite laminate. As shown, the laser pulse was incident normal to the laminate plies and the resulting waveform was detected by a plezoelectric transducer mounted on the opposite side of the specimen. It should be noted that even though the laser energy delivered to the specimen generated an ultrasonic pulse of sufficient amplitude to undergo multiple reflections through the specimen, absolutely no damage was done to the specimen surface by the laser beam.

\section{LASER BEAM INTERFEROMETRIC ULTRASOUND DETECTORS}

A number of investigators have used laser beam interferometers for noncontact detection of acoustic emission and ultrasonic waves in solid materials (refs. 13, 15-17, 19-25). Figure 8 is a schematic diagram of one of the most sensitive broadband laser interferometric detectors of acoustic waveforms constructed to date (ref. 26). This device is a modified Michelson interferometer which includes active stabilization of the relative optical path lengths of the signal and reference arms of the interferometer. It possesses a flat frequency response from $D C$ to $60 \mathrm{MHz}$ and drops of to the $3 \mathrm{~dB}$ point at 160 $\mathrm{MHz}$. Since the detection signal-to-noise ratio is inversely proportional to the square root of the detection bandwidth, increasing the bandwidth can degrade detection of small amplitude signals. The use of balanced photodiodes as optical detectors and an improved laser power supply has permitted detection of signals with amplitudes as small as $0.1 \mathrm{~nm}$ ( 1 Angstrom) while retaining detection bandwidth. The use of beam steering mirrors, along with a telescoping lens assembly to focus the signal component of the laser beam on the specimen surface, makes adjustment of the interferometer very convenient. It can also 
accomodate a wide range of orientations of the specimen surface major readjustment.

\section{LASER GENERATION LASER DETECTION OF ULTRASOUND}

Although a number of investigators have reported both generation and detection of ultrasonic waves in solld materials using laser beams, only two investigations have been conducted using low-power laser beams. This is especially important for many non-contact nondestructive evaluation applications where portability and power limitations are a necessity. In 1983, Dewhurst (ref. 17) constructed a hand-held laser generator of ultrasonic pulses based on components taken from a laser rangef inding device. A photograph of this laser generator is reproduced in Fig. 9. Operating at a wavelength of 1.06 microns, the hand-held laser has provided optical pulses on a single shot basis with energies of 3-10 millijoules in 10 nanoseconds. This optical output was sufficient to generate both surface and bulk ultrasonic waves with an aluminum specimen.

Most recently, Bourkoff and Palmer (ref. 20) have succeeded in generating ultrasonic pulses in metals and composite materials using a low-energy tunable dye laser emitting 6 nanosecond pulses with $20-210$ microjoule energies. The ultrasonic waveforms were detected optically with a modified in-line Fizeau calibrated laser interferometer having improved signal-to-noise ratio. The interferometer possesses improved fringe stability due to an electronic control system, which enables it to be operated near common low frequency noise sources. High frequency noise due to the interferometer laser source itself is also reduced. The overall sensitivity is about $50 \mathrm{pm}$ over a $10 \mathrm{MHz}$ bandwidth. Figures $10(a)$ and $10(b)$ show the system and detected waveforms, respectively. This work has shown that it is entirely feasible to use a portable laser generation laser detection system for non-contact characterization of composite materials. To the best of the present authors knowledge this is the first report of laser beam generation and detection of ultrasound in composite materials.

\section{HIGH-POWER ULTRASONIC WAVES}

In the event that the wave amplitude is increased to very large values, ultrasonic waves propagating through a metal specimen may cause the metal to deform plastically. The three mechanisms that have been proposed to account for this plastic deformation are superposition of the dynamic high-power ultrasonic stress on the quasi-static tensile stress, heating of the specimen due to the applied high-power ultrasound, and interaction of the high-power ultrasonic waves with dislocations in localized regions of the test specimens (ref. 27).

Figure 11 shows the results of an experiment which illustrates the influence of high-power ultrasound on the load-time curve of an aluminum crystal. The crystal was subjected to a continuous uniaxial tensile test, while intermittently insonating it with a high-power ultrasonic horn operating at 20 $\mathrm{kHz}$. Each load drop was produced by an $0.03 \mathrm{sec}$ insonation period at constant horn input power. The dashed curve drawn through the minimum loads attained during insonation corresponds with the load-time curve, or equivantly the 
stress-strain curve, that would have been obtained if the high-power ultrasound had been applied continuously during the tensile test.

In 1979, Mignogna and Green (ref. 29) developed a multiparameter experimental system which, for the first time, permitted simultaneous measurement of sufficient quantities to test all of the proposed mechanisms for the influence of high-power ultrasound on the mechanical properties of metal specimens. The various parameters which could be either controlled or measured during both tensile elongation and insonation of a variety of single crystal and polycrystalline metal specimens are applied tensile load, specimen elongation, contact pressure between high-power ultrasonic horn ( $20 \mathrm{kHz}$ ) and test specimen, electrical power supplied to the horn, high-power insonation time, test specimen surface temperature distribution, low-power ultrasonic ( $8 \mathrm{MHz}$ ) wave velocity (directly related to elastic moduli) and attenuation (directly related to dislocation motion). A schematic diagram of the high-power ultrasonic horn assembly, specimen, gripping system, low-power nondestructive ultrasonic transducer, linear variable differential transformer, and support cage are shown in Fig. 12.

Figure 13 shows a set of typical data obtained from an aluminum single crystal specimen subjected to high-power insonation at $20 \mathrm{kHz}$, but not subjected to any tensile load. The insonation was applied at a constant power level for different time periods: $I=0.03 \mathrm{sec}$, II $=0.63 \mathrm{sec}$, III $=1.23 \mathrm{sec}$, IV $=1.83$ sec, $V=2.43 \mathrm{sec}$, and VI $=3.63 \mathrm{sec}$. Depicted are change in specimen length, relative change in low-power nondestructive ultrasonic velocity and change in low-power ultrasonic attenuation as a function of time. The changes in low-power ultrasonic velocity indicate small changes in the elastic moduli during insonation. The changes in low-power ultrasonic attenuation indicate that high-power ultrasound is capable of causing large scale dislocation motion and the resulting plastic deformation.

The temperature of the test specimens was monitored in all of the early experiments by epoxying copper-constantan thermocouples to the central region of the specimen gauge length. Infrared thermovision scans of the test specimens' surfaces (ref. 30) revealed extremely rapid temperature increases at the point of attachment of thermocouples to the specimens' surfaces, which casts doubt on all past temperature measurements and which has undoubtedly led to misunderstanding about the mechanism responsible for the observed load drops.

High-power ultrasonic insonation induced localized heating at displacement nodes in brass, copper, steel, and polymer specimens of resonant lengths. Hot zones detected at saw-cuts, drilled holes, fatigue cracks, and grain boundaries, coupled with the high-power ultrasound caused bending of a large grain polycrystalline specimen subjected to insonation alone, the ultrasonic attenuation results, and scanning electron microscope observations of the specimens' fracture surfaces clearly indicate that high-power ultrasound can interact with material inhomogeneities many orders of magnitude smaller than the ultrasonic wavelengths. Of particular importance for the present paper is the fact that the similarity of the ultrasonic attenuation results, as shown in the lower portion of Fig. 13 and the lower left portion of Fig. 4, provide strong evidence for the ability of sufficiently large amplitude long wavelength ultrasound to physically move dislocations causing plastic deformation of the test specimens. 
$X$-ray diffraction topography is the generic name given to a number of experimental techniques which permit images to be recorded of crystal lattice defects located in the volume of single crystals as well as those located in the surface layers. Defects ranging in size from dislocations upward may be Imaged over large areas of relatively thick specimens. Although the images recorded by $x$-ray diffraction topography do not possess the high resolution obtainable with transmission electron microscopy, they may be recorded under normal atmospheric conditions with no special specimen preparation which is particularly important for nondestructive evaluation of materials. Review articles describing x-ray diffraction topographic techniques may be found in references 31 and 32.

In 1976, Boettinger el al. (ref. 33) described a crystal topographic (ACT) system, which utilized the Bragg diffraction condition to obtain an expanded, highly collimated, monochromatic $x-r a y$ beam. Among the advantages of this expanded beam system are superior sensitivity to lattice imperfection induced strain fields. A portable $x$-ray image intensifier detector was routinely used to obtain rapid $x$-ray topographic images. A similar ACT system was constructed at Johns Hopkins and has been used to record $x$-ray back-reflection and transmission topographs of defect structure in a variety of metallic, semiconductor, and polymeric crystals.

In 1971, Green published a review paper (ref. 34) which described the design and performance characteristics of electro-optical systems for direct display of x-ray diffraction images. Subsequently, Green (ref. 35) in 1977 and Winter and Green (ref. 36) published other papers related to this subject. Figure 14 shows schematic drawings of one type of commonly used first generation portable $x$-ray image intensifier and a more recently developed second generation miniature $x$-ray image intensifier. These electro-optical systems have been used at Johns Hopkins to orient single crystals, to study crystal lattice rotation accompanying plastic deformation, to measure the rate of grain boundary migration during recrystallization annealing of cold-worked metals, to determine the physical state of exploding metals, to monitor the amorphous to crystalline phase transformation of rapidly solidified metals, to rapidly measure residual stress, to study the dynamics of structural phase transitions in ferroelectric crystals, and to record topographic images of lattice substructure and defects. An example, taken from the work of Chikawa (ref. 38), of the application of an electro-optical system to image dislocations in a silicon crystal is reproduced in Fig. 15.

Of primary importance to the present paper is the work of Itagaki (ref. 39) who used the Lang $x$-ray diffraction topographic method to study the vibratory motion of charged dislocations in single crystal ice caused by the application of an alternating electric field. The experimental system developed by Itagaki made it possible to distinguish the dislocations vibrating under the influence of the electric field as well as their amplitude and sign of their changes.

In 1979 a Synchrotron Topography Project (STP) was established with the goal of designing and constructing an x-ray topography station on the National Synchrotron Light Source (NSLS) at Brookhaven National Laboratory. This project 
has proceeded in cooperation with NSLS and the system has nearly been completed on beam line $\mathrm{X}-19$. A description of this fully dedicated synchrotron topography station is given in ref. 40. Combination of appropriate components of the multiparameter high-power ultrasonic system, described previously, with synchrotron $x$-ray diffraction topography, using $x$-ray image intensifier detectors coupled with solid state image digitizing television cameras and subsequent computerized signal processing ( $r$ ef. 36 ), affords the possibility of direct in situ observation of the interaction of ultrasonic waves with the microstructural and defect features of metallic, optically opaque ceramic and semiconductor materials for the first time.

\section{CONCLUSIONS}

Optimization of the use of ultrasonic techniques for nondestructive materials characterization has as a prerequisite a thorough understanding of the fundamental principles of the propagational characteristics of the ultrasonic waves in real materials. This understanding must take into account both linear and nonlinear elastic waves; quasi- and pure mode waves; energy flux deviations from the wave normals; attenuation due to absorption and scattering; mode conversion at bounding surfaces; and isotropic, anisotropic, homogeneous and inhomogeneous materials. Advances in this field will be made by the use of non-contact transducers particularly laser beam generation and detection systems. Combination of high-power ultrasound systems with synchrotron $x-r a y$ diffraction topography, using $x$-ray image intensifier detectors coupled with solid state image digitizing television cameras and subsequent computerized signal processing, affords the possibility of observing direct interaction of ultrasonic waves with the microstructure of real crystalline materials for the first time.

\section{REFERENCES}

1. Green, R.E., Jr., Ultrasonic Investigation of Mechanical Properties. Vol. III Treatise on Materials Science and Technology, Academic Press, New York (1973).

2. Merkulov, L.G. and Yakovlew, L.A., Propagation and Reflection of Ultrasonic Beams in Crystals, Akust. Zh. 8, 99-106 (1962) [Sov. Phys.-Acoust. 8, 72-77 (1962).

3. Henneke, E.G., II and Green, R.E., Jr., Light-Wave/Elastic-Wave Analogies in Crystals, J. Acoust. Soc. Amer. 45, 1367-1373 (1969).

4. Green, R.E. Jr., Effect of Metallic Microstructure on Ultrasonic Attenuation, in Nondestructive Evaluation:Microstructural Characterization and Reliability Strategies, 0. Buck and S.M. Wolf (eds.), The Metallurgical Society of AIME, Warrendale, PA, (1981) pp. 115-132. 
5. Swanson, R.A. and Green, R.E. Jr., Ultrasonic Attenuation Study of Dislocation Motion Part II. Experimental, Trans. Metallurgical Soc. AIME 230, 1390-1394 (1964).

6. Sachse, W. and Green, R.E. Jr., Experimental Study of the Orientation Dependence of Dislocation Damping in Aluminum Crystals, Trans. Metallurgical Soc. AIME 242, 2185-2190 (1968).

7. Joshi, N.R. and Green, R.E. Jr., Ultrasonic Detection of Fatigue Damage, Engr. Fract. Mech. 4, 577-583 (1972).

8. Hughes, D.S. and Kelly, J.L., Second-Order Elastic Deformation of Solids, Fhys. Rev. 92, 1145-1149 (1953).

9. Allen, D.R., Cooper, W.H.B., Sayers, C.M. and Silk, M.G. The Use of Ultrasonics to Measure Residual Stresses, in Vol. VI Research Techniques in Nondestructive Testing, R.S. Sharpe (ed.), Academic Press, Orlando, FLA, (1983) pp. 151-209.

10. Pao, Y-H, Sachse, W. and Fukuoka, H., Acoustoelasticity and Ultrasonic Measurements of Residual Stresses, in Vol XVII Physical Acoustics, W.P. Mason and R.N. Thurston (eds.), Academic Press, Orlando, FLA, (1984) pp. 61-143.

11. Liu, J., Mechanics Department, The Johns Hopkins Unfversity, Baltimore, MD (Unpublished Work).

12. White, R.M., Generation of Elastic Waves by Transient Surface Heating, J. Appl. Phys. 34, 3559-3567 (1963).

13. Bondarenko, A.N., Drobat, Yu. B., and Kruglov, S.V., Optical Excitation and Detection of Nanosecond Acoustic Pulses in Nondestructive Testing, Soviet J. NDT 12, 655-658 (1976).

14. Ledbetter, H.M. and Moulder, J.C., Laser-Induced Rayleigh Waves in Aluminum, J. Acoust. Soc. Amer. 65, 840-842 (1979).

15. Calder, C.A. and Wilcox, W.W., Non-Contact Materlal Testing Using Laser Energy Deposition and Interferometry, Mater. Eval. 38, 86-91 (1980).

16. Wellman, R.L., Laser System for the Detection of Flaws in Solids, Harry Diamond Laboratories, Report No. HDL-TR-1902 (1980).

17. Dewhurst, R.J., A Hand-Held Laser-Generator of Ultrasonic Pulses, NDT Communications 1, 93-103 (1983).

18. Rudd, M.J. and Doughty, J.A., Laser Generation of Ultrasound, Naval A1r Development Center, Report No. NADC-81067-60 (1983).

19. Alndow, A.M., Dewhurst, R.J., Palmer, S.B., and Scruby, C.B., Laser-Based Non-Destructive Testing Techniques for the Ultrasonic Characterization of Subsurface Flaws, NDT International 17, 329-335 (1984). 
20. Bourkoff, E. and Palmer, C.H., Low-Energy Optical Generation and Detection of Acoustic Pulses in Metals and Nonmetals, Appl. Phys. Lett. 46, 143-145 (1985).

21. Friant, C.L. and Green R.E., Jr., (Unpublished Work).

22. Rosen, M., Analytical Ultrasonics for Characterization of Metallurgical Microstructures and Transformations, (This Publication).

23. Palmer, C.H. and Green, R.E., Jr., Optical Detection of Acoustic Emission Waves, Appl. Opt. 16, 2333-2334 (1977).

24. Kline, R.A., Green, R.E., Jr., and Palmer, C.H., A Comparison of Optically and Piezoelectrically Sensed Acoustic Emission Signals, J. Acoust. Soc. Amer. 64, 1633-1639 (1978).

25. Palmer, C.H. and Green, R.E., Jr., Optical Probing of Acoustic Emission Waves, in Nondestructive Evaluation of Materials, J.J. Burke and V. Weiss (eds.), Plenum Publishing, New York (1979 pp.347-378.

26. Murphy, J.C. (Private Communication) Applied Physics Laboratory, The Johns Hopkins University, Laurel, MD 20707.

27. Green, R.E., Jr., Non-Linear Effects of High-Power Ultrasonics in Crystalline Solids, Ultrasonics, 13, 117-127 (1975).

28. Mignogna, R.B. and Green, R.E., Jr., Effects of High Frequency Loading on Materials, in Ultrasonic Eatigue, J.M. Wells, O. Buck, L.D. Roth, and J.K. Tien (eds.), Metallurgical Society of AIME, Warrendale, PA (1982) pp.63-85.

29. Mignogna, R.B. and Green, R.E., Jr., Multiparameter System for Investigation of the Effects of High-Power Ultrasound on Metals, Rev. Sci. Instrum. 5e, $1274-1277$ (1979).

30. Mignogna, R.B., Green, R.E., Jr., Duke, J.C., Jr., Henneke, E.G., II, and Reifsnider, K.L., Thermographic Investigation of High-Power Ultrasonic Heating in Materials, Ultrasonics 19, 159-163 (1981).

31. Armstrong, R.W, and Wu, C.C., X-ray Diffraction Microscopy, in Microstructural Analysis: Tools and Techniques, J.L. McCall and W.M. Mueller (eds.), Plenum Publishing, NY (1973) pp.169-219.

32. Tanner, B.K., X-ray Diffraction Topography, Pergamon Press, New York (1976).

33. Boettinger, W.J., Burdette, H.E., Kuriyama, M. and Green, R.E., Jr., Asymmetric Crystal Topographic Camera, Rev. Sci. Instrum. 47, 906-911 $(1976)$.

34. Green, R.E., Jr., Electro-Optical Systems for Dynamic Recording of X-ray Diffraction Images, in Advances in X-ray Analysis, C.S. Barrett, J.B. Newkirk, and C.0. Ruud (eds.), Vol.14, Plenum Press, NY (1971) pp.311-337.

35. Green, R.E., Jr., Direct Display of X-ray Topographic Images, in Advances in 
X-ray Analys1s, H.f. MoMurdie, C.S. Barrett, J.B. Newk1rk, and C.O. Ruud (eds.), Vol.20 Plenum Publishing, NY (1977) pp.221-235.

36. Winter, J.M. and Green, R.E., Rapid Imaging of X-ray Topographs, In Applications of $X$-ray Toposraphic Methods to Materials Sclence, $S$. Welssmann, F. Ballbar, and J.-F. Petroff (eds.), Plenum Publishing, NY (1984) pp. 45-58.

37. Rosemeler, R.G. and Green, R.E. Jr., A New Miniature Microchannel Plate X-ray Detector for Synchrotron Radiation, Nuclear Instrum. \& Methods 196, 299-301 (1982).

38. Chikawa, J., Fujimoto, I, and Abe, T., X-ray Topographic Observation of Moving Dislocations in Silicon Crystals, Appl. Phys. Lett. 21, 295-298 (1972).

39. Itagaki, K., X-ray Topographic Study of Vibrating Dislocations in Ice Under an AC Electric Field, in Advances in X-ray Analysis, Plenum Press, NY (1970) pp.526-538.

40. Bilello, J.C., Chen, H., Helo, A.B., Liu, J.M., Birnbaum, H.K., Herley, P.J. and Green, R.E., Jr., The Synchrotron Topography Project (STP) at the National Synchrotron Light Source, Nuclear Instrum. and Methods 215, 291-297 (1983). 


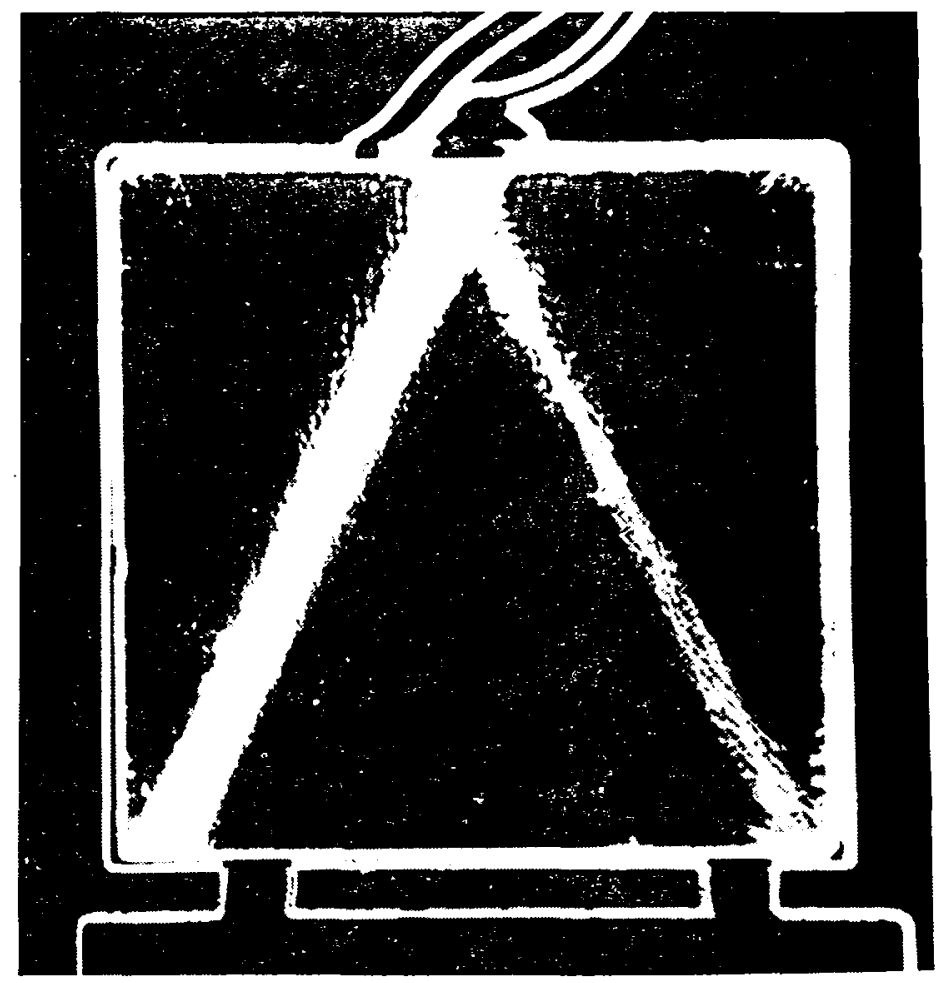

Figure 1. Schlieren photograph of ultrasound field associated with simultaneous generation of quasilongitudinal and quasi-shear waves in a quartz crystal from a single piezoelectric transducer [2]

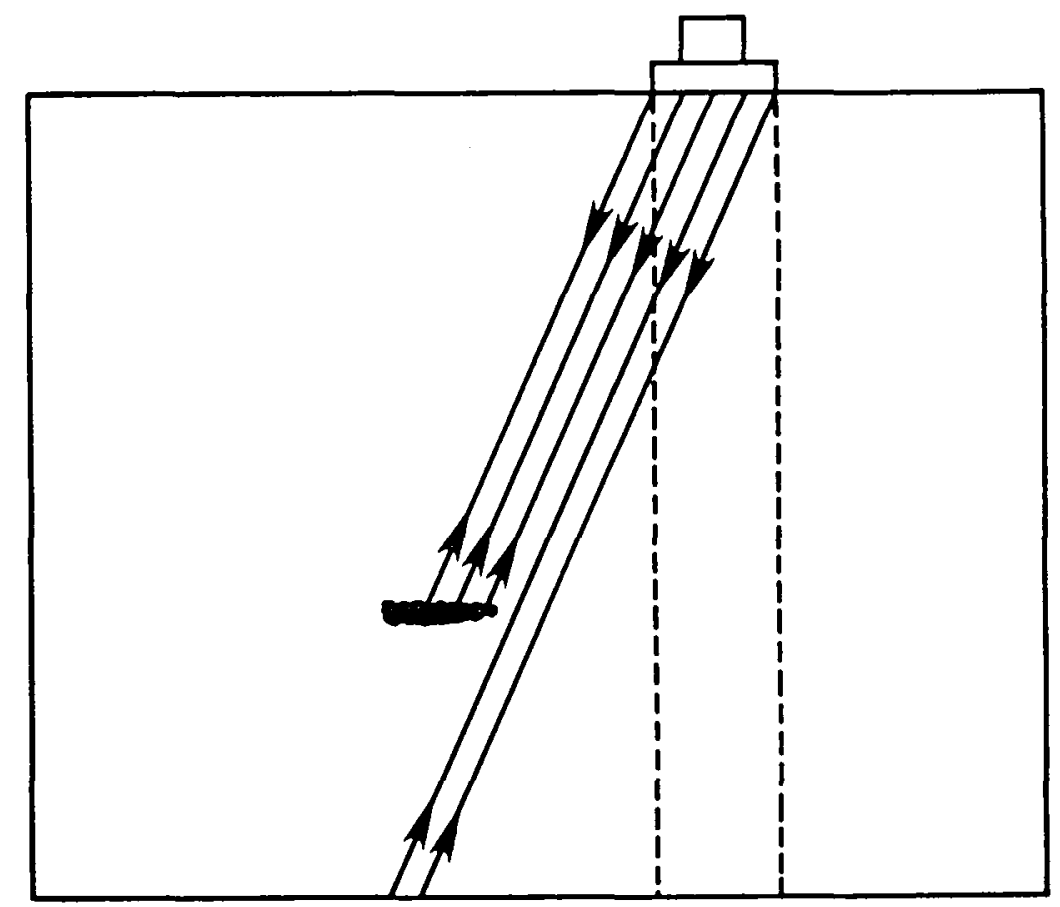

Figure 2. Schematic illustration of energy-flux deviation (refraction) causing misjudgement of crack location in anisotropic solid. 

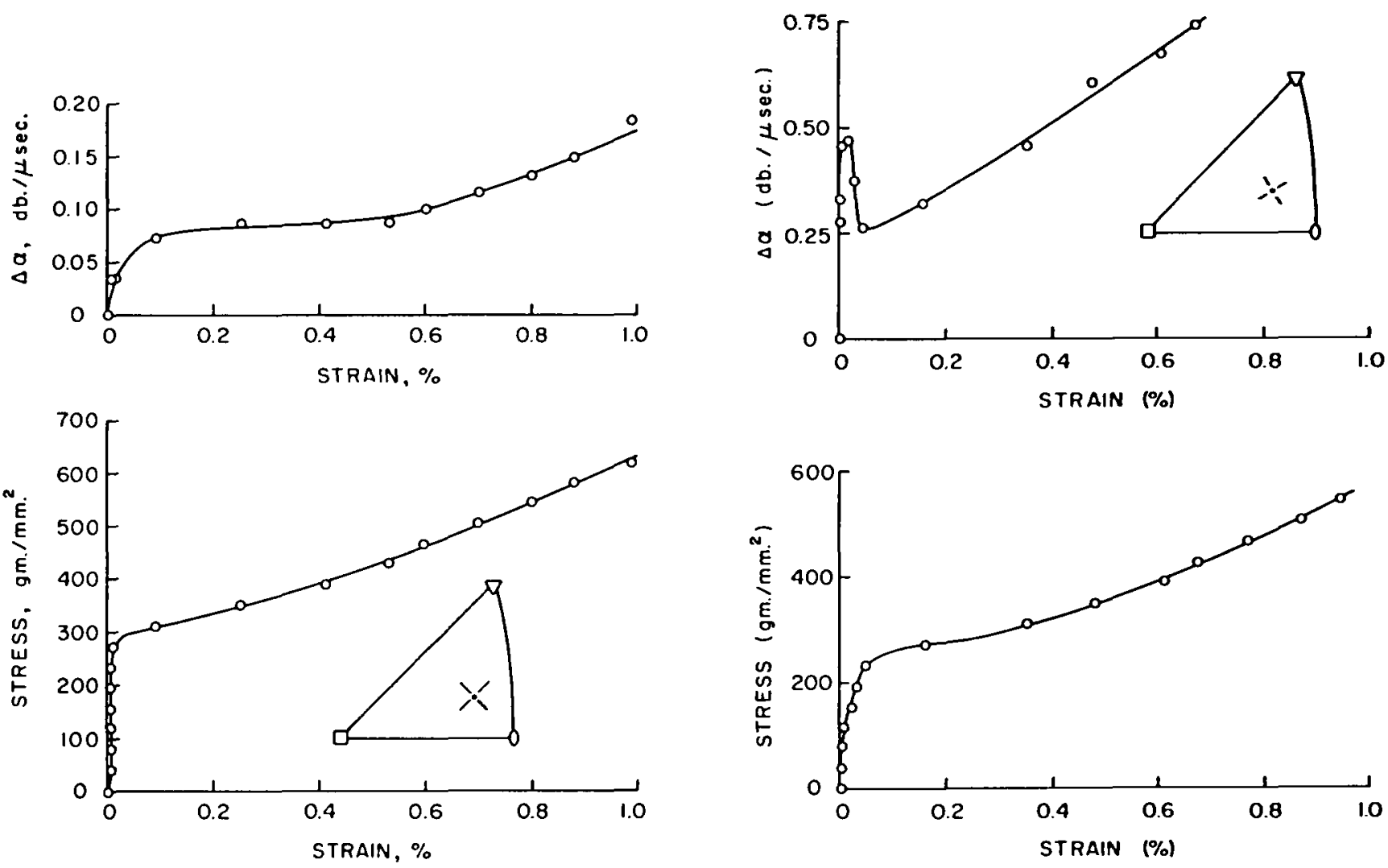

Figure 3. Stress and change in ultrasonic attenuation as a function of strain for quasi-shear waves polarized with large component of particle displacement (a) perpendicular and (b) parallel to projection of the primary-slip direction onto the end surface of the crystal specimen [5]. 


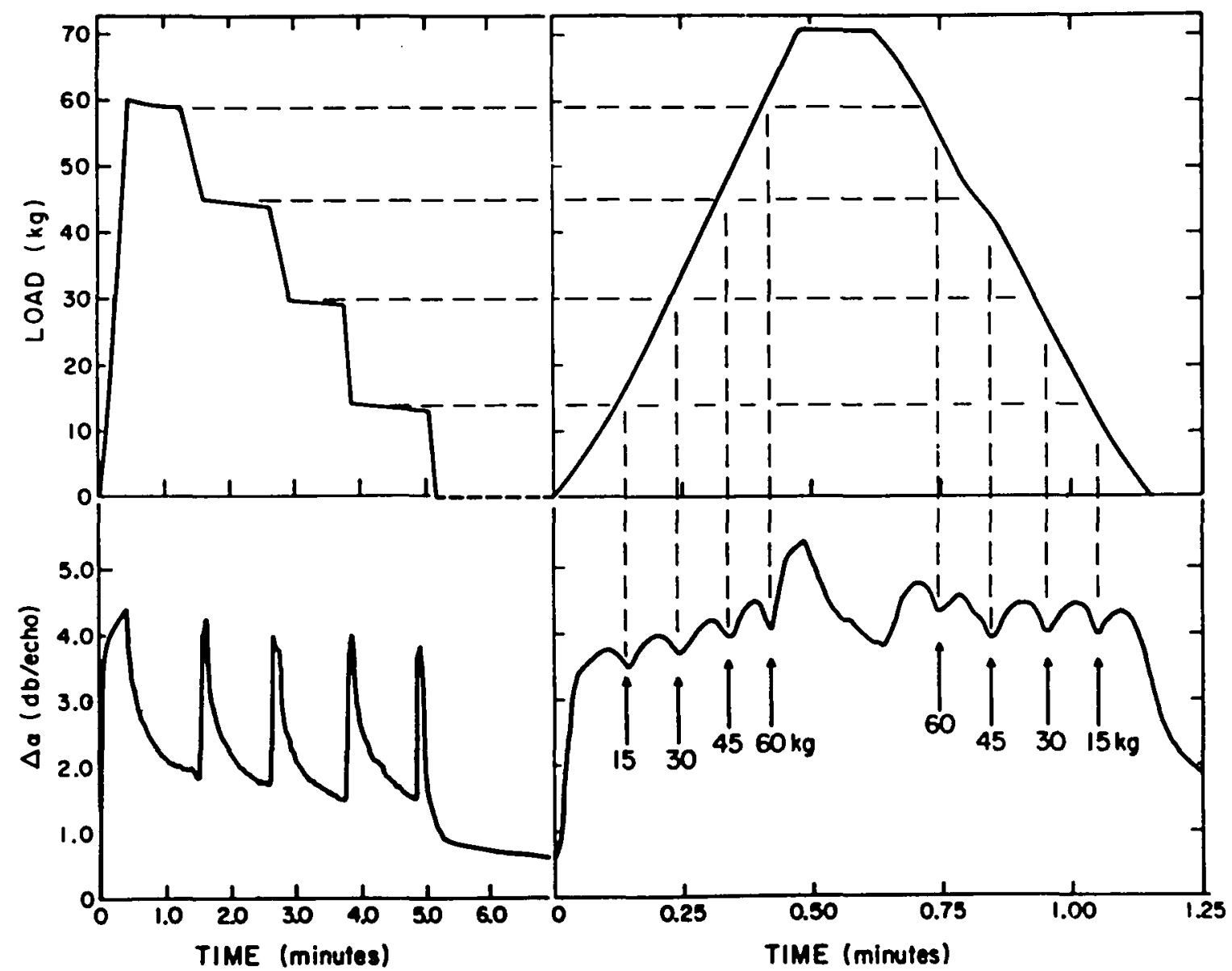

Figure 4. Load versus time and ultrasonic attenuation change versus time plots obtained during a loadhold-unload-hold sequence (left), followed by $a$ reload-unload sequence ( $r$ ight) conducted on an aluminum single crystal [6]. 


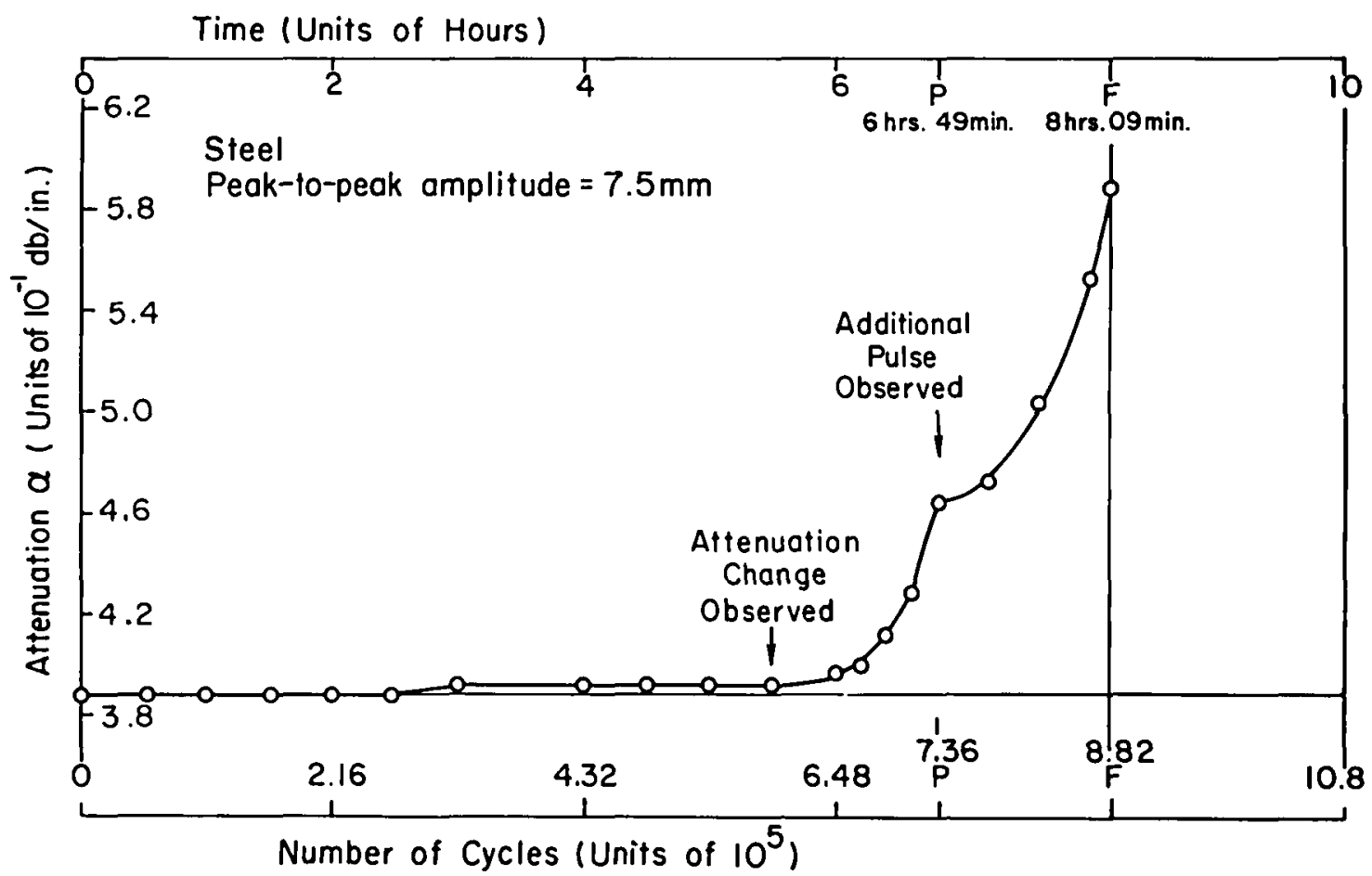

Figure 5. Longitudinal ultrasonic wave attenuation versus number of fatigue cycles for polycrystalline steel [7]. 


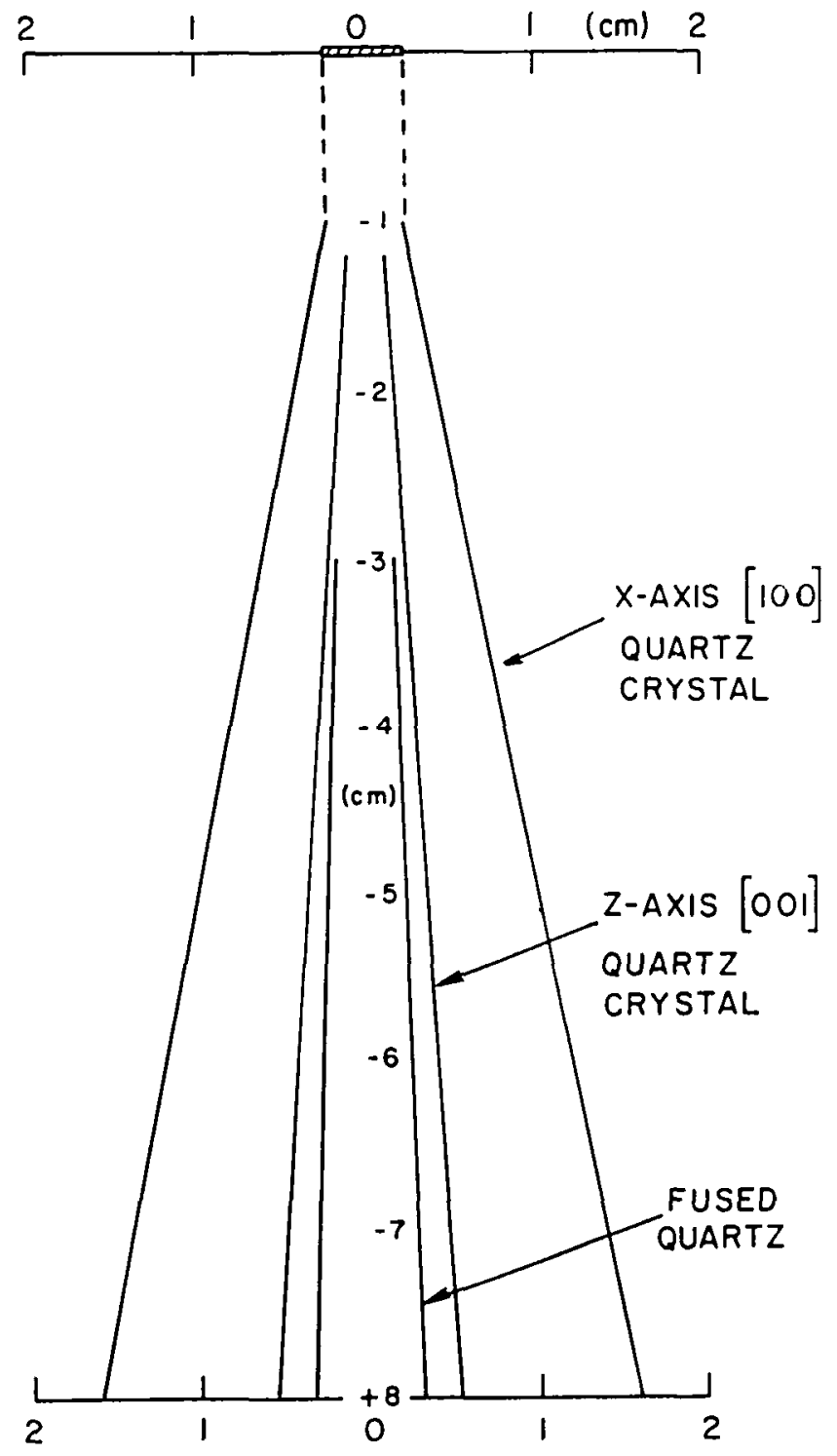

Figure 6. Dependence of diffraction spread of $10 \mathrm{MHz}$ longitudinal waves in quartz on anisotropy of propagation direction [11]. 

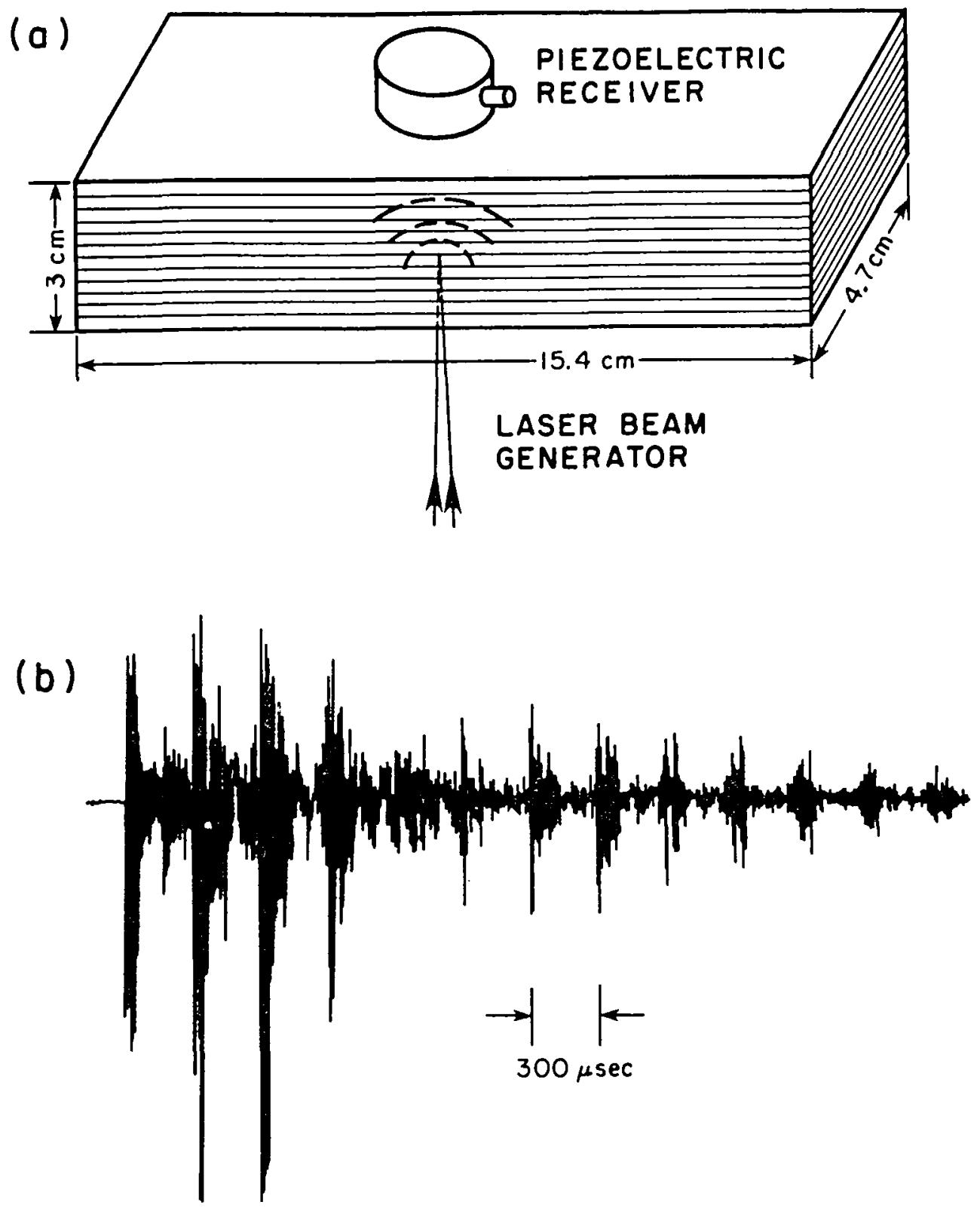

Figure 7. Laser generation of ultrasonic pulse in graphiteepoxy composite laminate [21].

(a) experimental configuration 


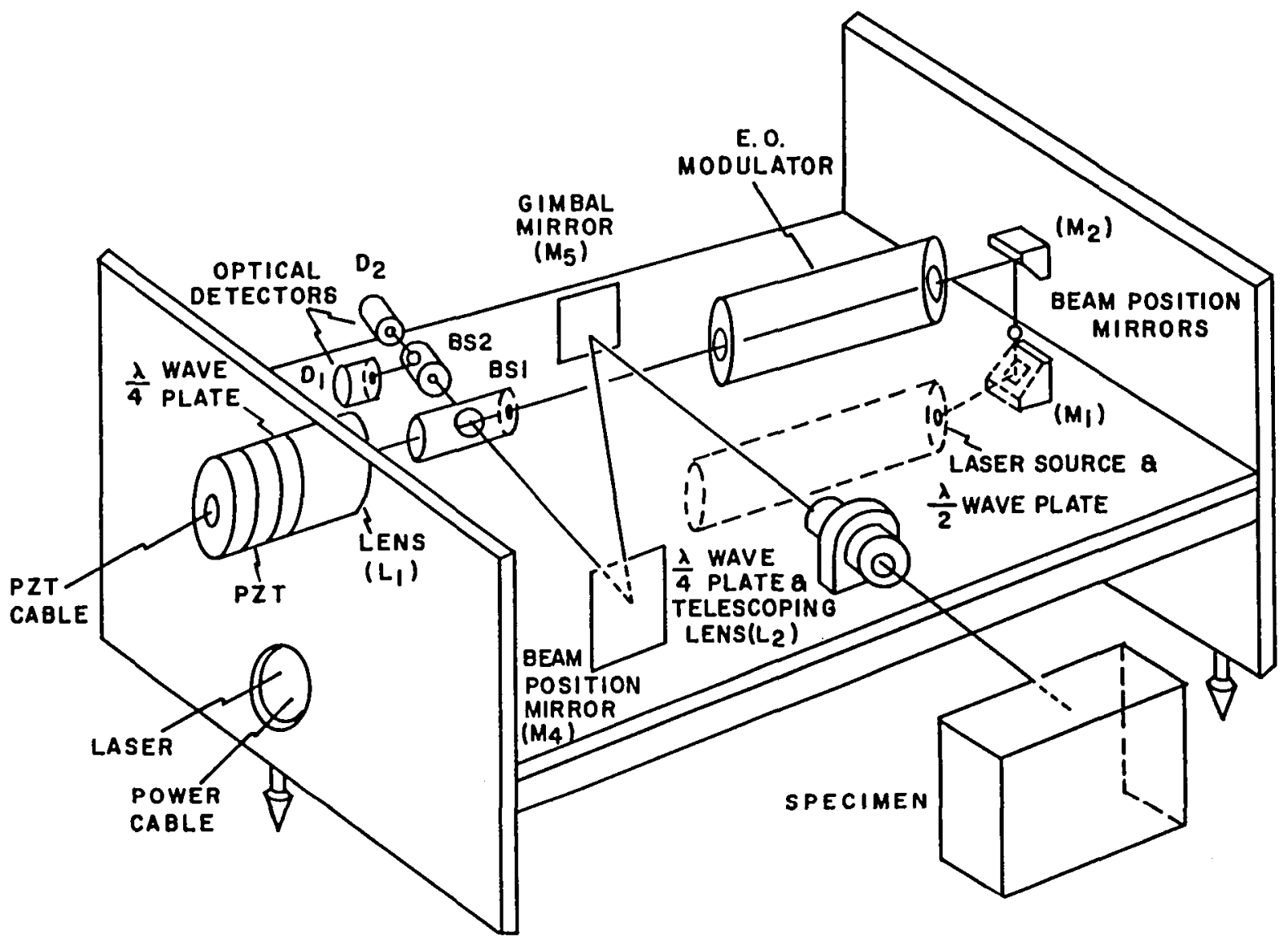

Figure 8. Schematic diagram of very sensitive broadband laser interferometer detection of acoustic waveforms [26]. 

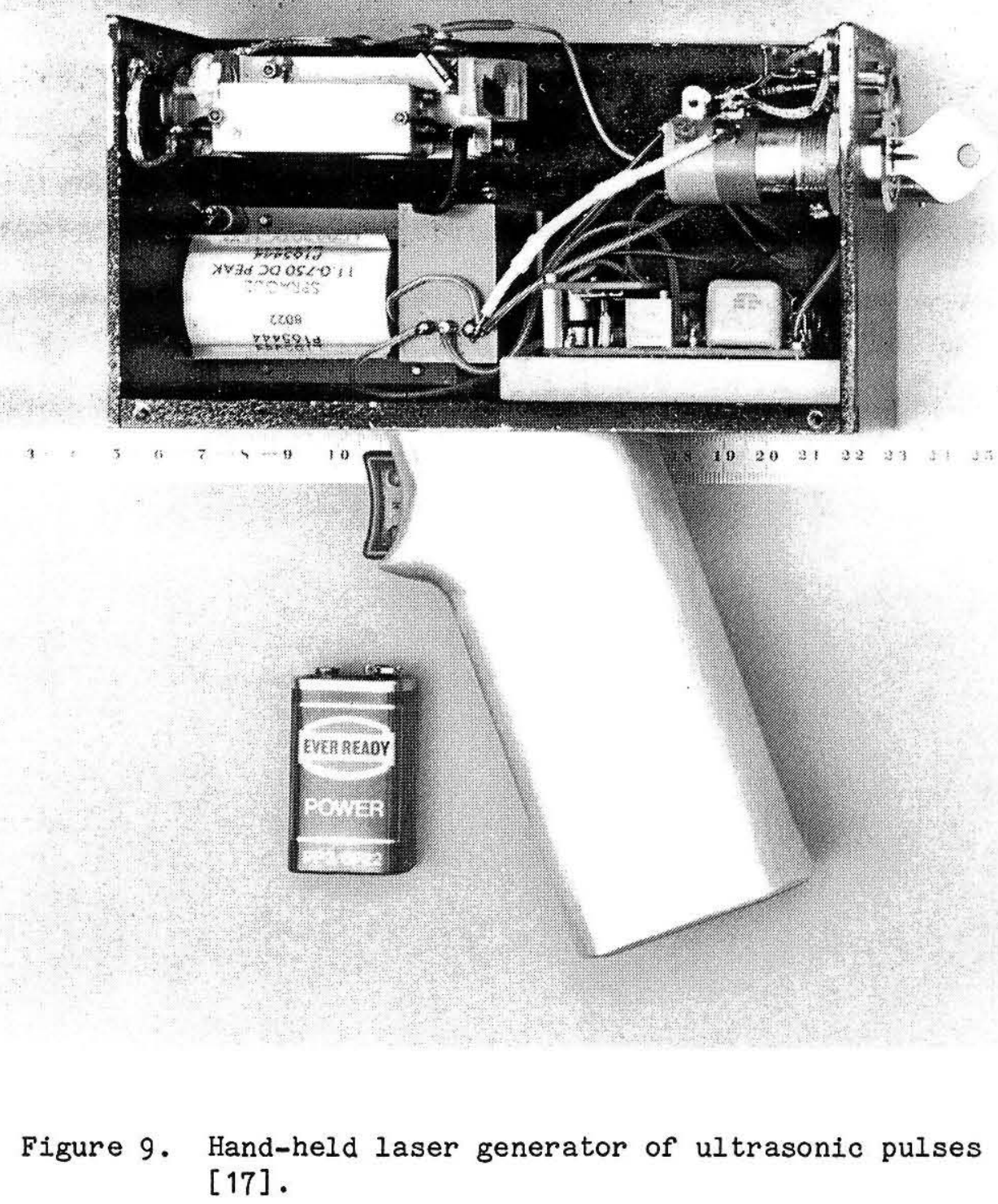

[17]. 

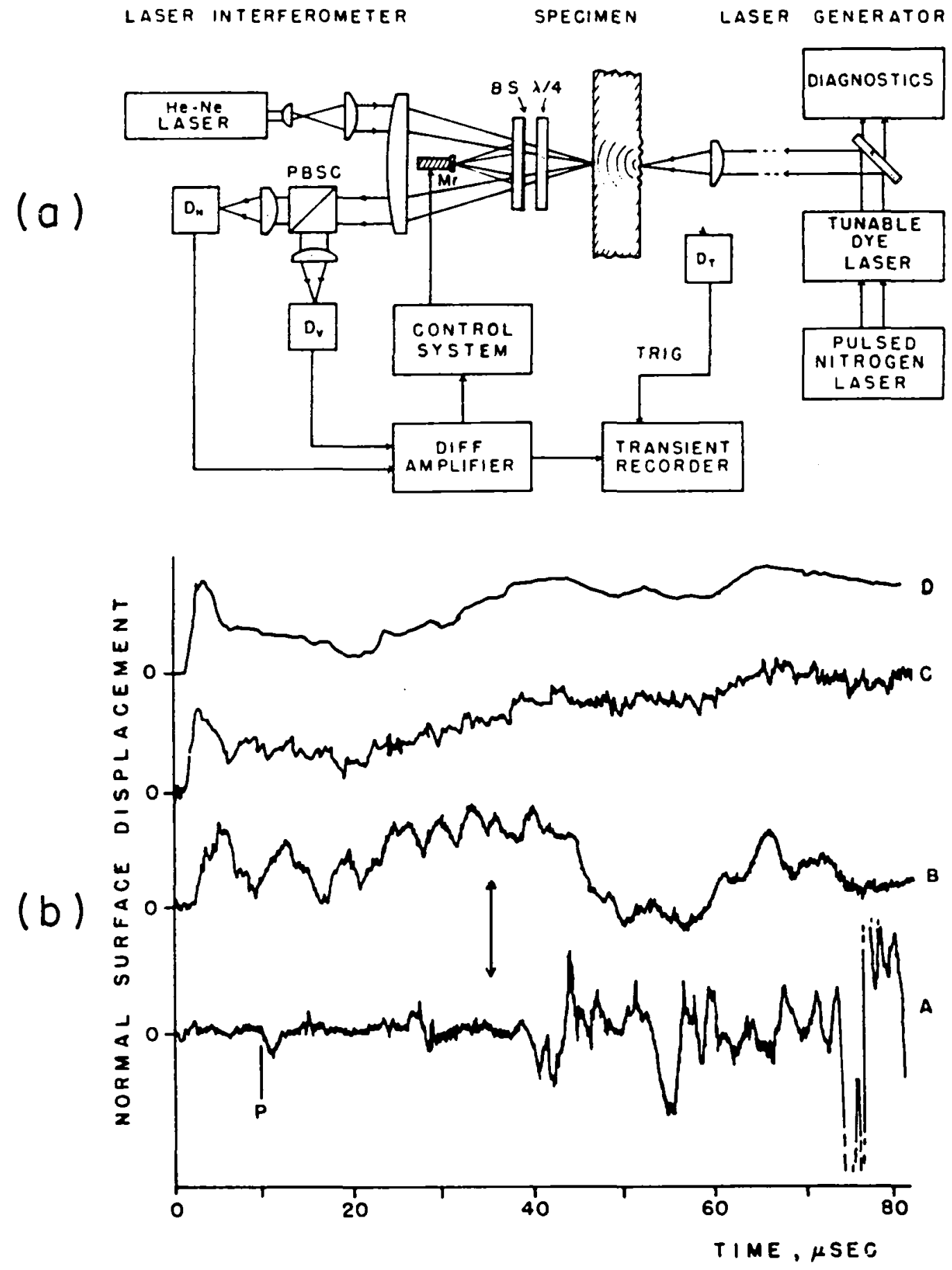

Figure 10. Low energy laser generation of ultrasonic pulses [20]

(a) interferometer system

(b) waveforms: A-6061-T6 uluminum, B-polyimideglass-fiber composite, $C$ and D-graphiteepoxy composite. 


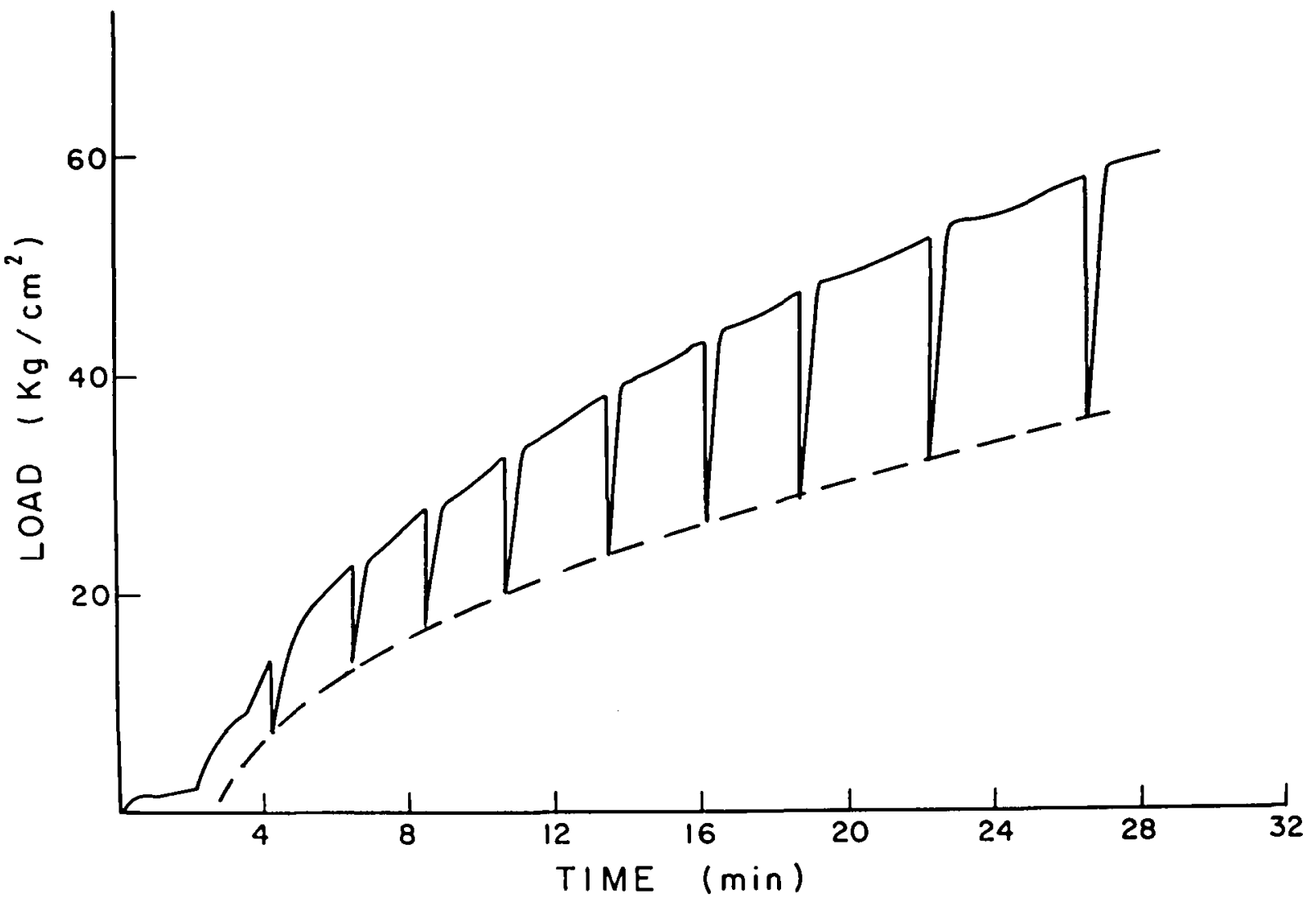

Figure 11. Load drops during tensile elongation of aluminum single crystal due to a sequence of $0.03 \mathrm{sec}$ insonation periods at the same high-power ultrasonic power level [28]. 


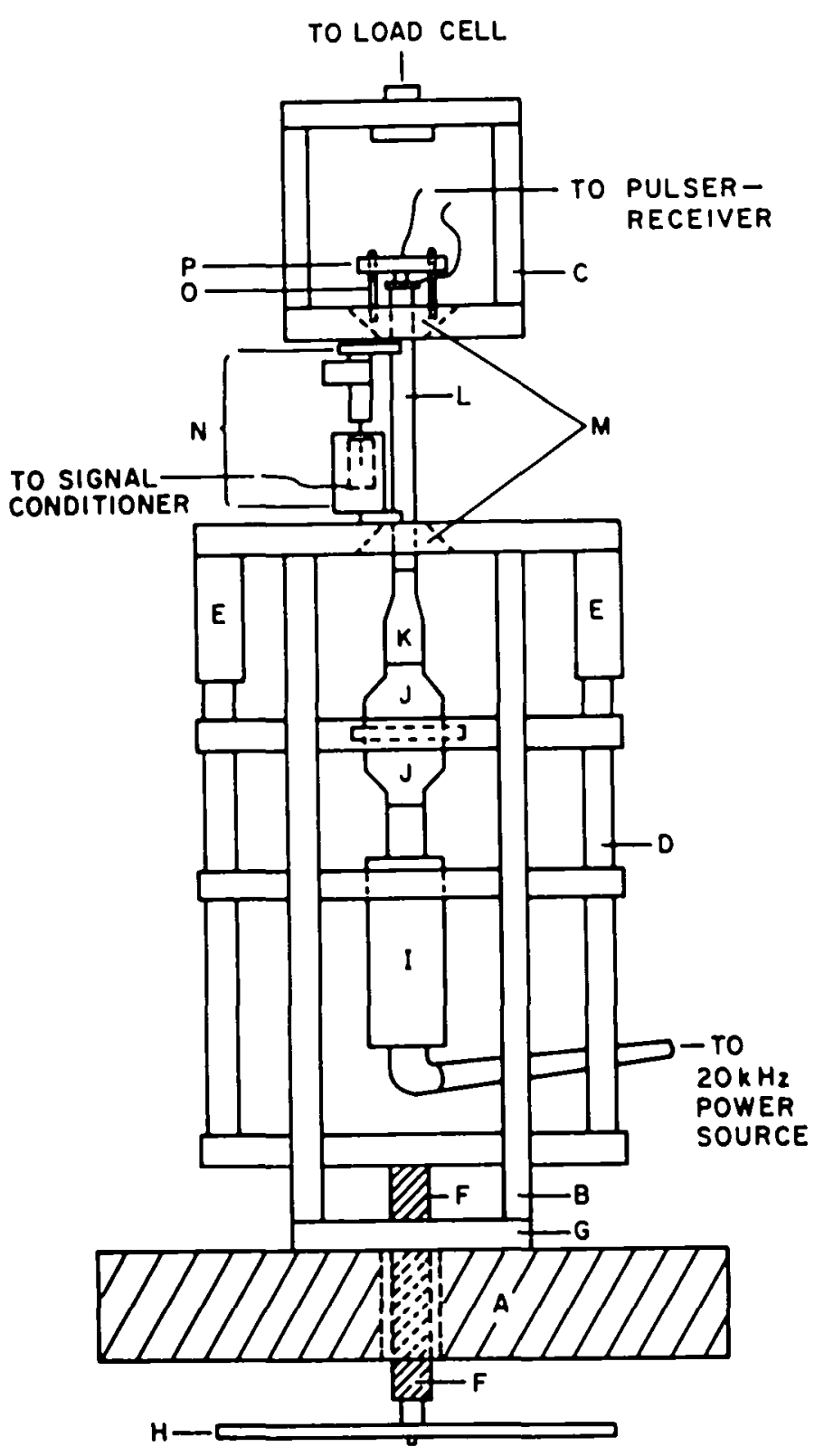

Figure 12. Schematic diagram of high-power ultrasonic horn assembly and test apparatus: A-moving crosshead; B-bottom grip cage; C-upper grip cage; D-mount for ultrasonic horn converter assembly; E-guide tubes; F-horn positioning screw; G-lower plate of bottom grip cage; H-pulley; I-converter; Jbooster; K-catenoidal horn; L-specimen; M-split contcal change; N-LVDT; $0-8 \mathrm{MHz}$ transducer, $\mathrm{P}-$ transducer holder [29]. 


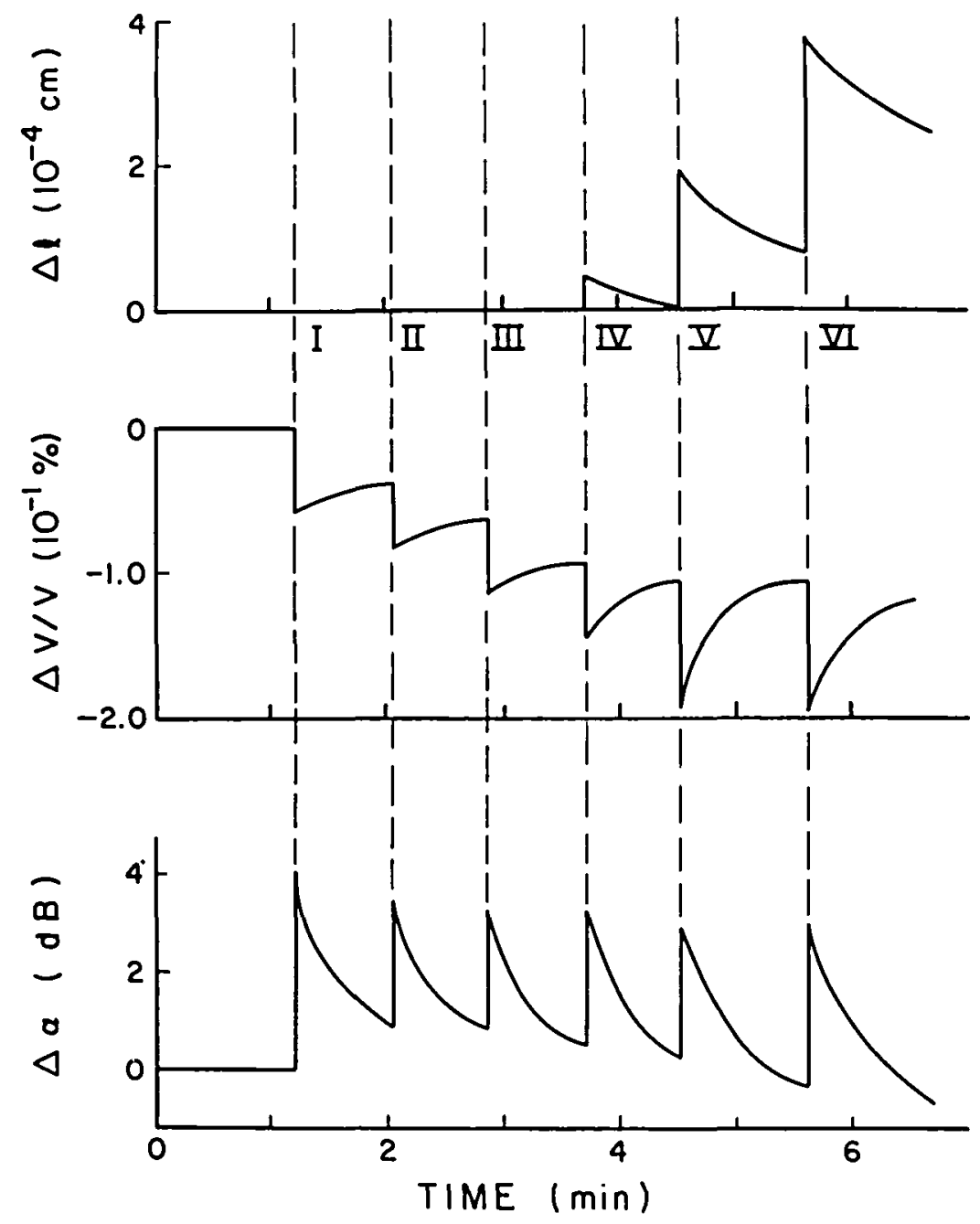

Figure 13. Data obtained from an aluminum single crystal subjected to high-power insonation only at a constant power level for different time periods [28]: $\quad$ I $=0.03 \mathrm{sec}$, II $=0.63 \mathrm{sec}$, III $=1.23$ $\mathrm{sec}, I V=1.83 \mathrm{sec}, V=2.43 \mathrm{sec}, V I=3.63 \mathrm{sec}$. $\Delta l=$ change in specimen length $\Delta v / v=$ relative change in ultrasonic velocity $\Delta \alpha=$ change in ultrasonic attenuation 


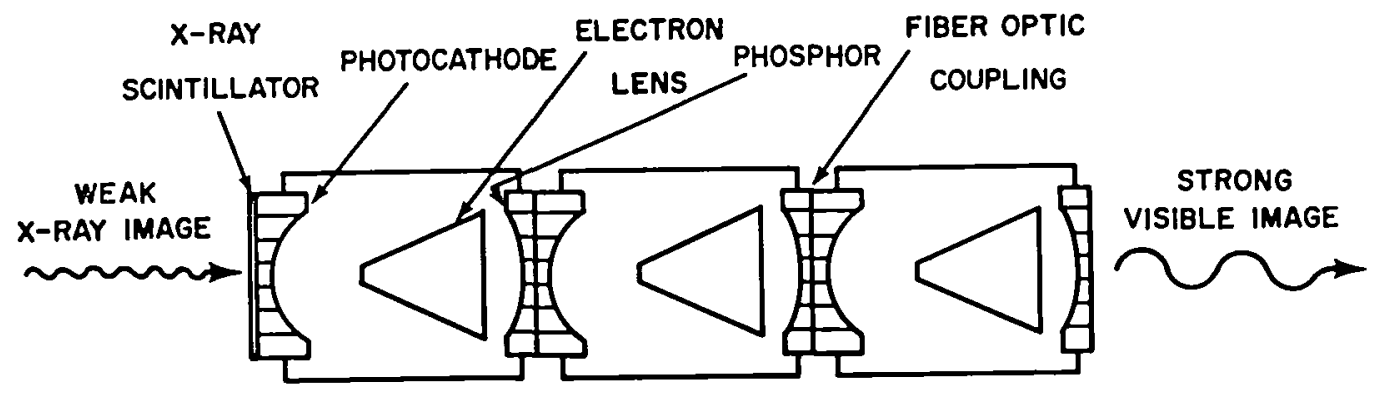

PORTABLE IMAGE X-RAY INTENSIFIER

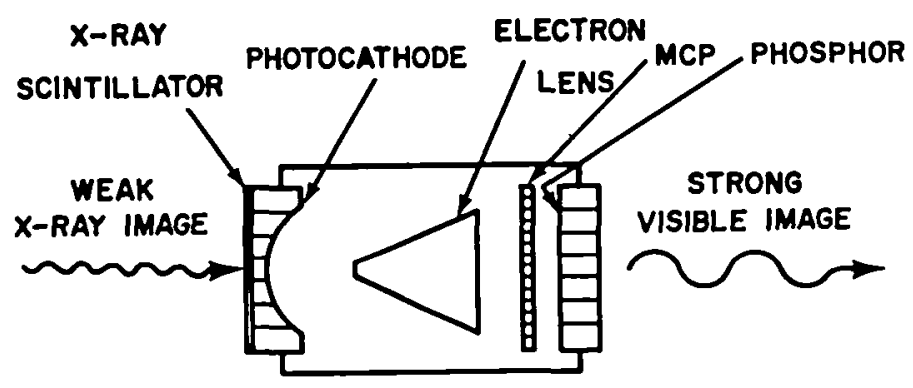

MINIATURE IMAGE X-RAY (MINIX) INTENSIFIER

Figure 14. Schematic drawings of first generation (upper) and second generation (lower) $x$-ray image intensifier tubes for rapid display of $x-r a y$ diffraction images [37]. 


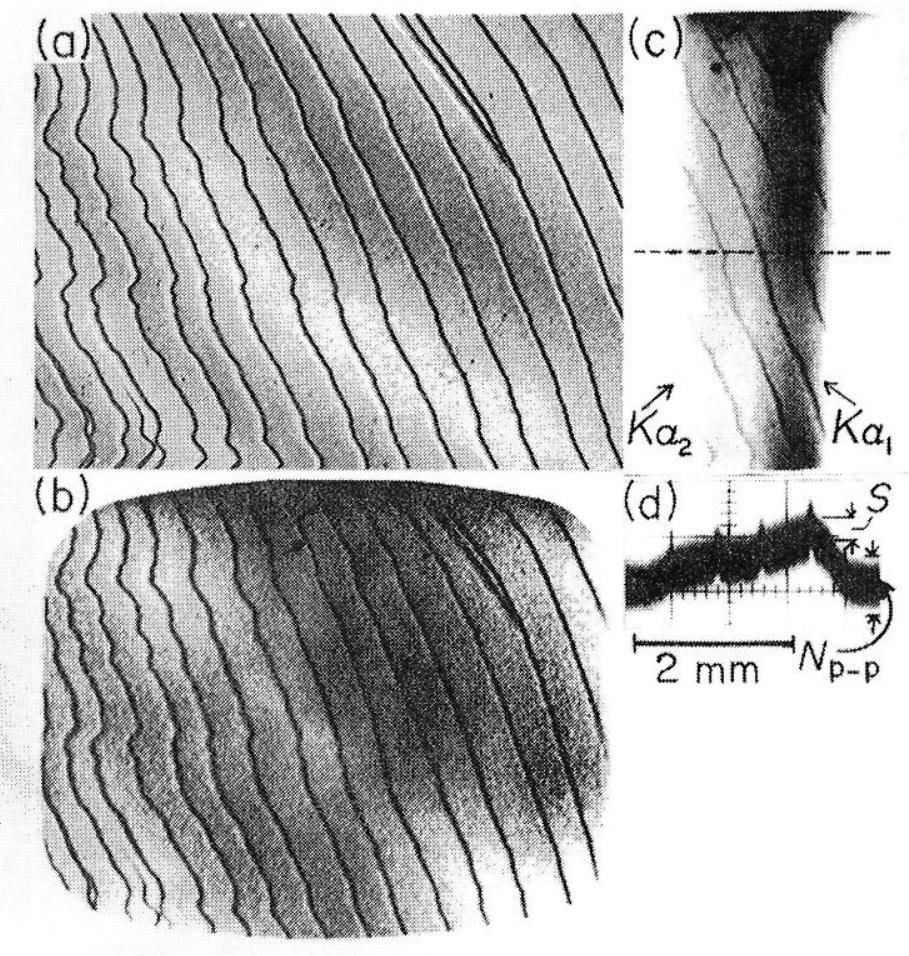

Figure 15. X-ray topographic images of static dislocations in a silicon crystal wafer [38]. (a) Scanning topograph recorded on nuclear emulsion plate (b) Video image of the same area. 



\title{
ULTRASONIC CHARACTERIZATION OF MICROSTRUCTURE IN POWDER METAL ALLOY*
}

\author{
B.R. Tittmann, L.A. Ahlberg, and K. Fertig \\ Rockwell International Science Center \\ Thousand Oaks, California 91360
}

The ultrasonic wave propagation characteristics were neasured for $1 N-100$, a powder metallurgy alloy used for aircraft engine components. This material was selected as a model system for testing the feasibility of characterizing the microstructure of a variety of inhomogeneous media including powder metals, ceramics, castings and composites. The data were obtained for a frequency range from about 2 to $20 \mathrm{MHz}$ and were statistically averaged over numerous volume elements of the samples. Micrographical examination provided size and number distributions for grain and pore structure. The results showed that the predominant source for the ultrasonic attenuation and backscatter was a dense $\left(\sim 100 / \mathrm{mm}^{3}\right)$ distribution of small micropores ( $10 \mu \mathrm{m}$ radius). Two samples with different micropore densities were studied in detail to test the feasibility of calculating from observed microstructural paraneters the frequency dependence of the microstructural backscatter in the regime for which the wavelength is much larger than the size of the individual scattering centers. Excellent agreement was found between predicted and observed values so as to demonstrate the feasibility of solving the forward problem. The results suggest a way towards the nondestructive detection and characterization of anomalous distributions of micropores when conventional ultrasonic imaging is difficult. The findings are potentially significant toward the application of the early detection of porosity during the materials fabrication process and after manufacturing of potential sites for stress induced void coalescence leading to crack initiation and subsequent failure.

\section{INTRODUCTION}

The acquisition and interpretation of ultrasonic data for the inference of microstructural information is not new and has been carried out by several researchers in the recent past [ref. 1-7]. The objective of this work is to apply these techniques to the types of powder metallurgy alloys used in the manufacture of aircraft engine disc components. The particular application motivating this work is the development of an approach to the nondestructive detection and characterization of anomalous microstructure when conventional ultrasonic imaging is difficult. Such an approach could be important tracking porosity during the materials process as

\footnotetext{
*This work was sponsored in part by Rockwell International Research and Development Funds and in part by the Center for Advanced Nondestructive Evaluation, operated by the Ames Laboratory, USDOE, for the Defense Advanced Research Projects Agency and the Air Force Wright Aeronautical Laboratories/Materials Laboratory under Contract No. W-7405-ENG-82.
} 
well as during service as a finished part in the early locating of potential sites for crack initiation.

The material chosen for these tests is $\mathrm{IN}-100$, a Ni-base alloy prepared by Pratt \& Whitney Aircraft Co. This material is used in the F-15 and F-16 fighter aircraft engine at a rate of $1000 \mathrm{lb}$ of billet material per engine to manufacture three compressor discs, four turbine discs and two spacers. The following sections discuss the experimental approach, the analytical models and the comparison between theory and experiment.

\section{SAMPLE CHARACTERIZATION}

This section treats the micrographical and ultrasonic characterization of the samples, the determination of bulk microsructure parameters from the 2D optical micrographs and he ultrasonic measurement technique [ref. 8].

\section{Micrographical Characterization}

Suitable samples were prepared from a number of different parent samples supplied by Pratt \& Whitney Co. Selected sample surfaces were polished and examined under the optical microscope. Figure 1 shows an optical micrograph of the grain structure. Figure 2 gives histogram data displaying the grain size distribution for two samples. These data show a uniform grain size of about $2-4 \mu \mathrm{m}$.

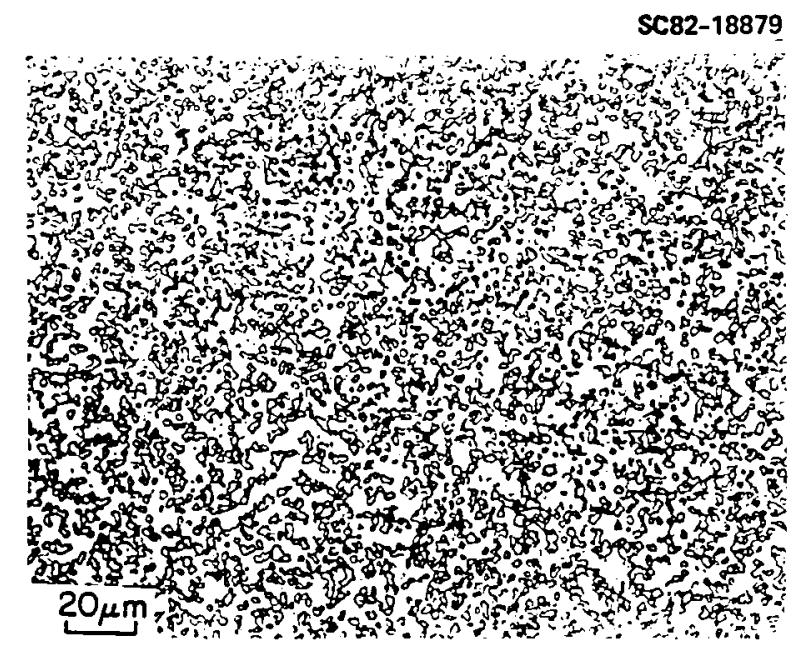

IN 100 GRAIN

Figure 1

Photomicrograph of $\mathrm{IN}-100$ grains.
SC82-18888

\section{GRAIN SIZE IN 100}
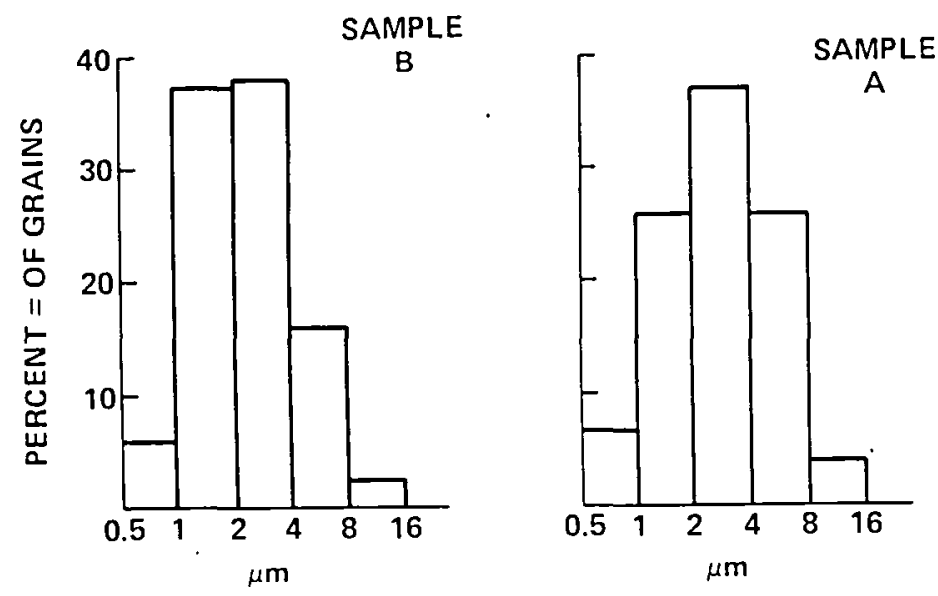

Figure 2

Grain size distribution in IN-100.

One of the samples tested (sample C) is a section of a used F100 engine 2nd disc which was pulled out for spin pit tests. The section is shown in cross-section 
In figure 3 together with lines drawn to indicate traverses with the microscope to study surface features. Figure 4 shows sample microphotos taken along some of these traverses indicating the presence of micropores. The microporosity is a by-product of the powder metallurgy process and has its origin in hollow powder particles with argon gas trapped during the atomization process. Although a sparse, uniform distribution of micropores is not thought to be the limiting factor in low cycle fatigue strength, the micropores do contribute significantly to the ultrasonic noise background and must be taken into account. Table 1 presents a summary of the micropore populations found along the various traverses. Statistical analysis of the micrograph data indicate that the pore distribution is not random but show a tendency towards clustering. Figure 5 presents the information of Table 1 in graphical manner. Figures $6 a$ and $6 \mathrm{~b}$ give pore size distribution histograms for two of the samples tested. Sample A was found to have an approximate micropore density of $330 / \mathrm{mm}^{3}$ whereas sample $C$ had $90 / \mathrm{mm}^{3}$. For sample $C$ the majorlty of micropores fall in the range of between 5 and $10 \mu \mathrm{m}$ in diameter whereas in sample $C$ the diameters range from $\sim 1 \mu \mathrm{m}$ to $50 \mu \mathrm{m}$.

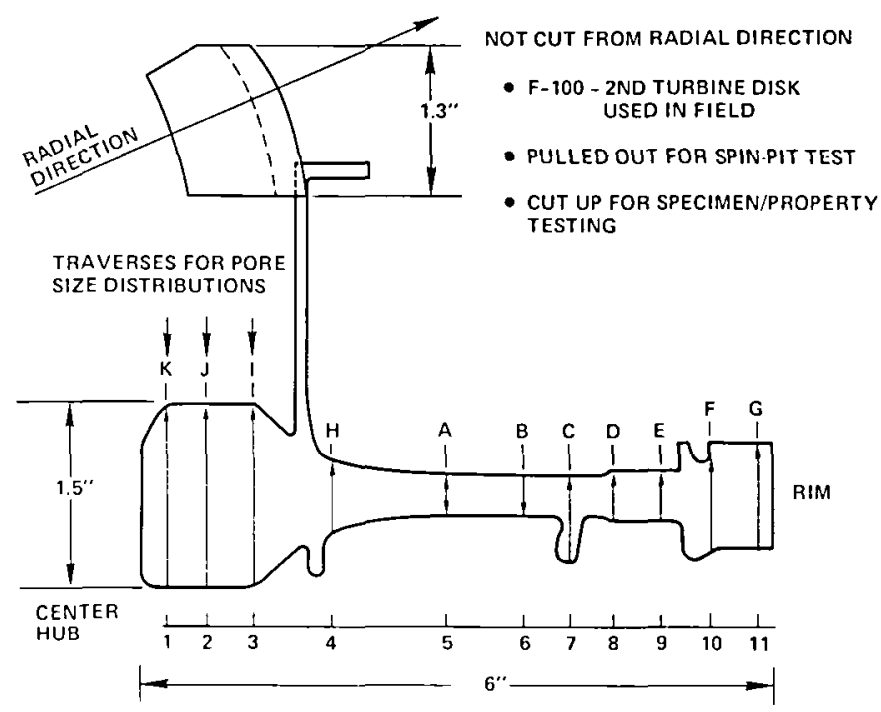

SC82.19519

Figure 3 Section of F100 engine disc.

In order to employ the histogram data obtained from the optical examinations to estimate volume microstructural information, the expressions for the mean volume porosity $\rho_{v}$ and the expectation value for the sixth power of the pore radius $E\left(r^{6}\right)$ were derived:

$$
\begin{aligned}
& \rho_{v}=\frac{1}{\pi} \int_{0}^{\infty} \frac{n_{2}\left(r^{\prime}\right)}{r^{\prime}} d r^{\prime}=\frac{1}{\pi} \sum_{j} \frac{n_{j}}{r_{j}} \Delta r_{j} \\
& E\left(r^{6}\right)=\frac{1}{\rho_{v}} \int_{0}^{\infty} r^{6} n_{3}(r) d r \doteq \frac{8}{\rho_{v}} \sum_{l}\left(\Delta r_{j}\right)\left(r_{j}\right)^{5}\left(n_{j}\right)
\end{aligned}
$$




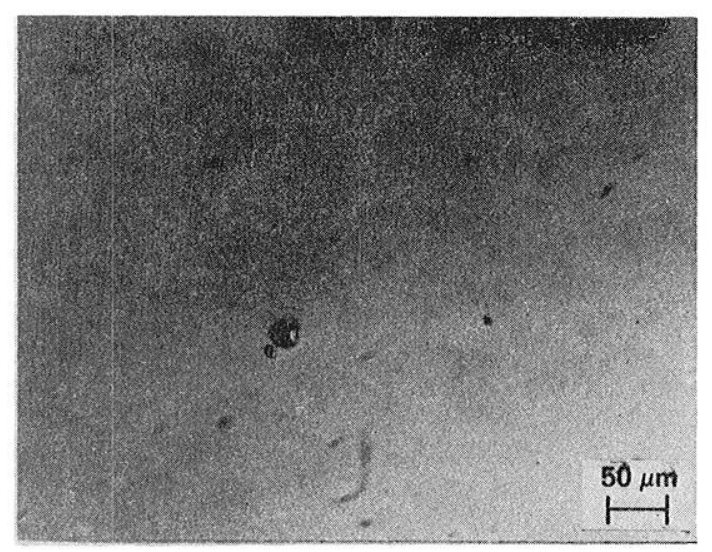

K71

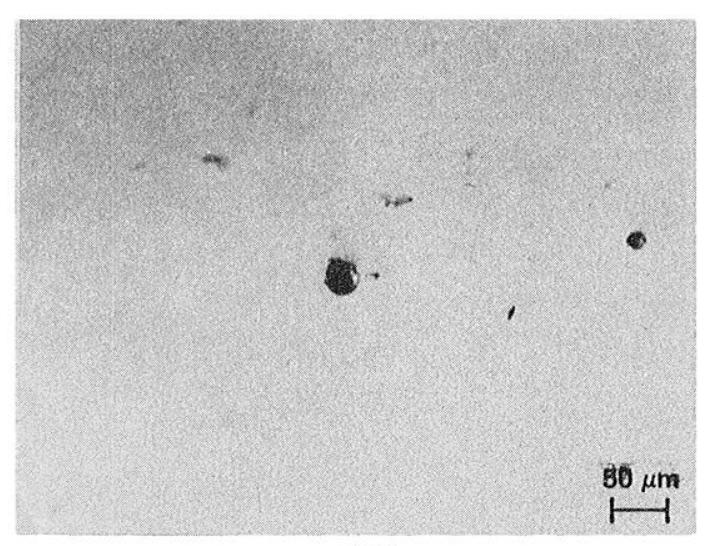

K57

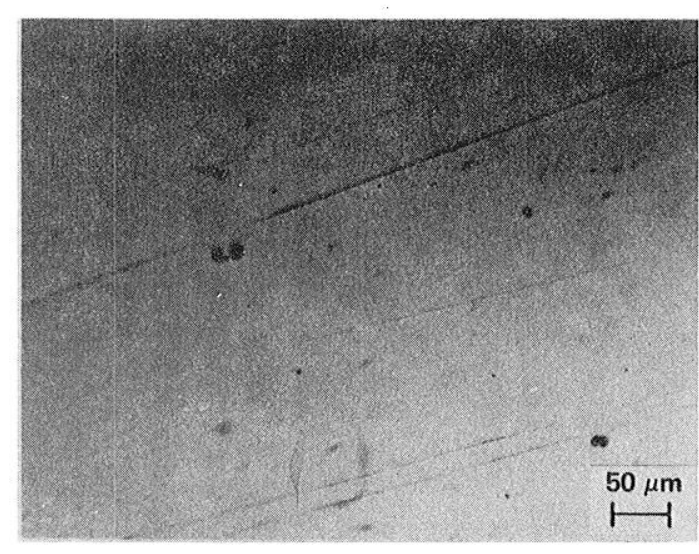

J46

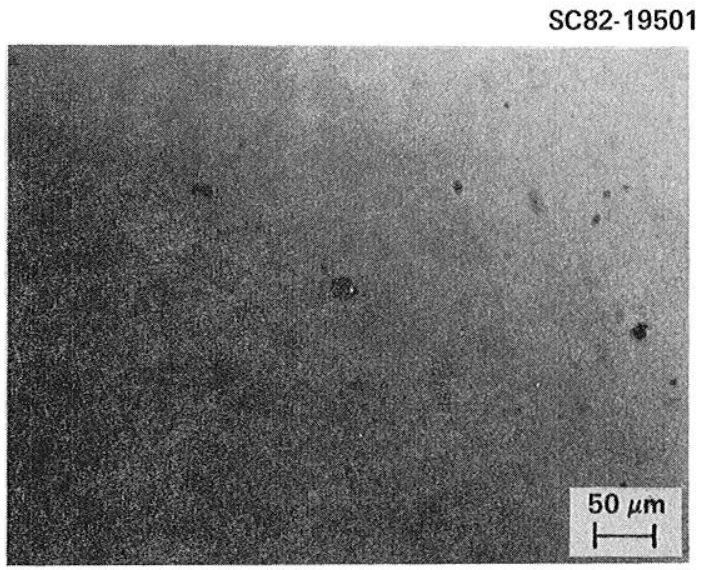

H29

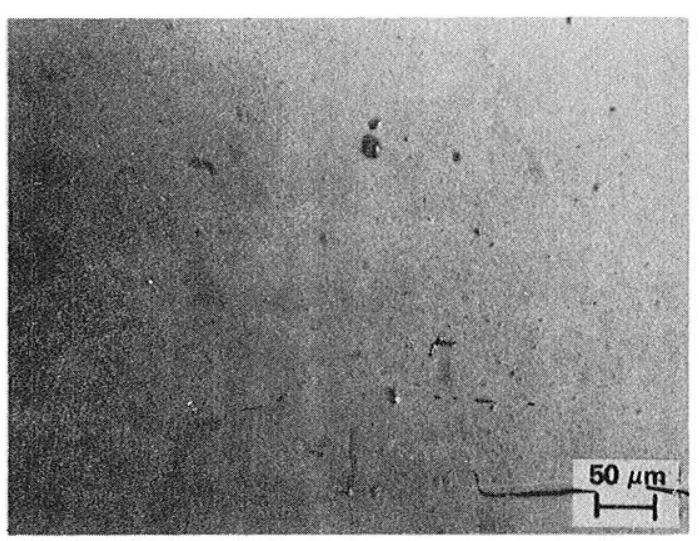

D6

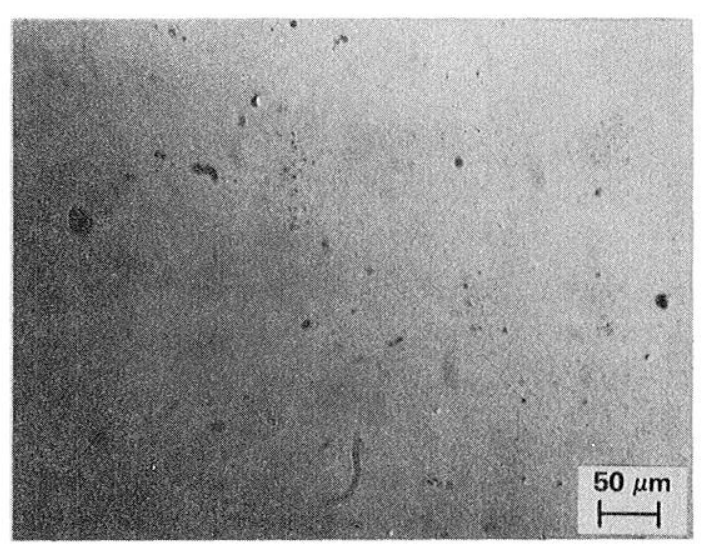

E4

Figure 4 Microphotos of area elements (along tranverses of figure 3) showing micro porosity. 


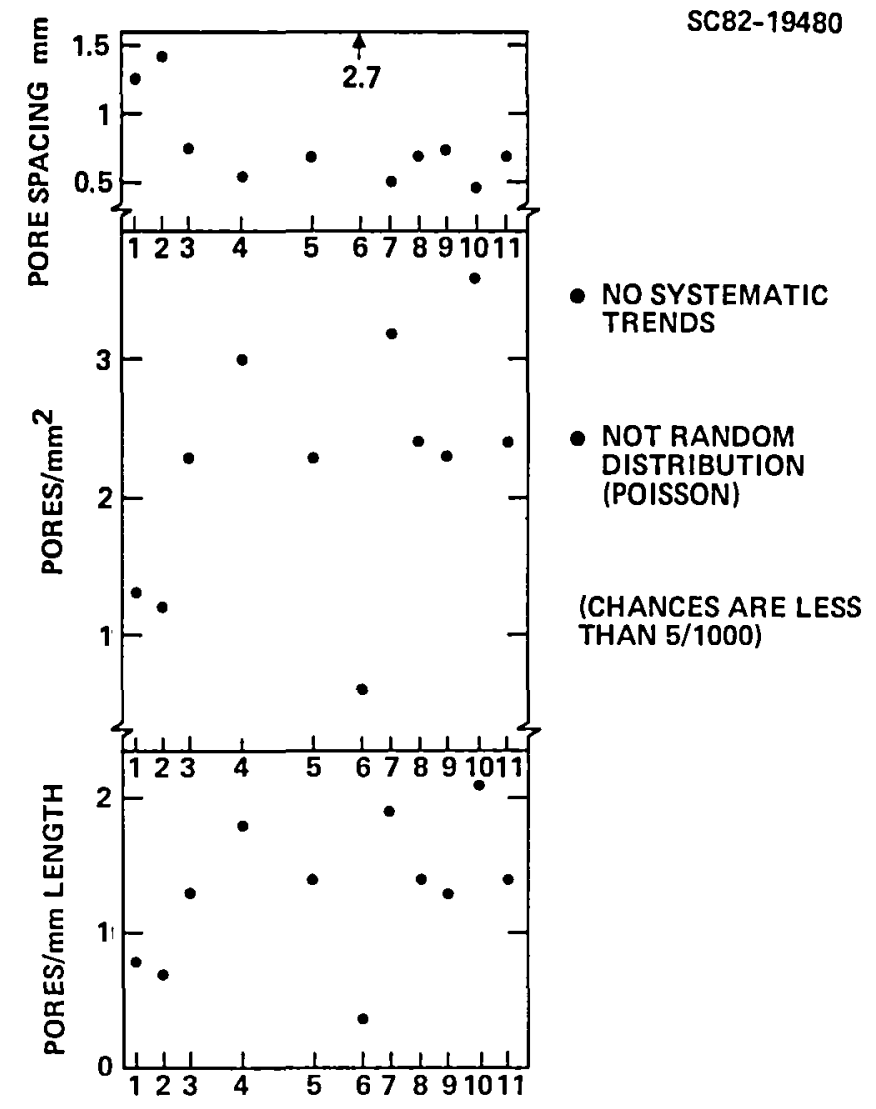

Figure 5 Graph of number of pores per unit length, number of pores per unit area and pore separation plotted vs distance from disc center to run (as indicated by traverse number, see Fig. 3).

SC83-23334
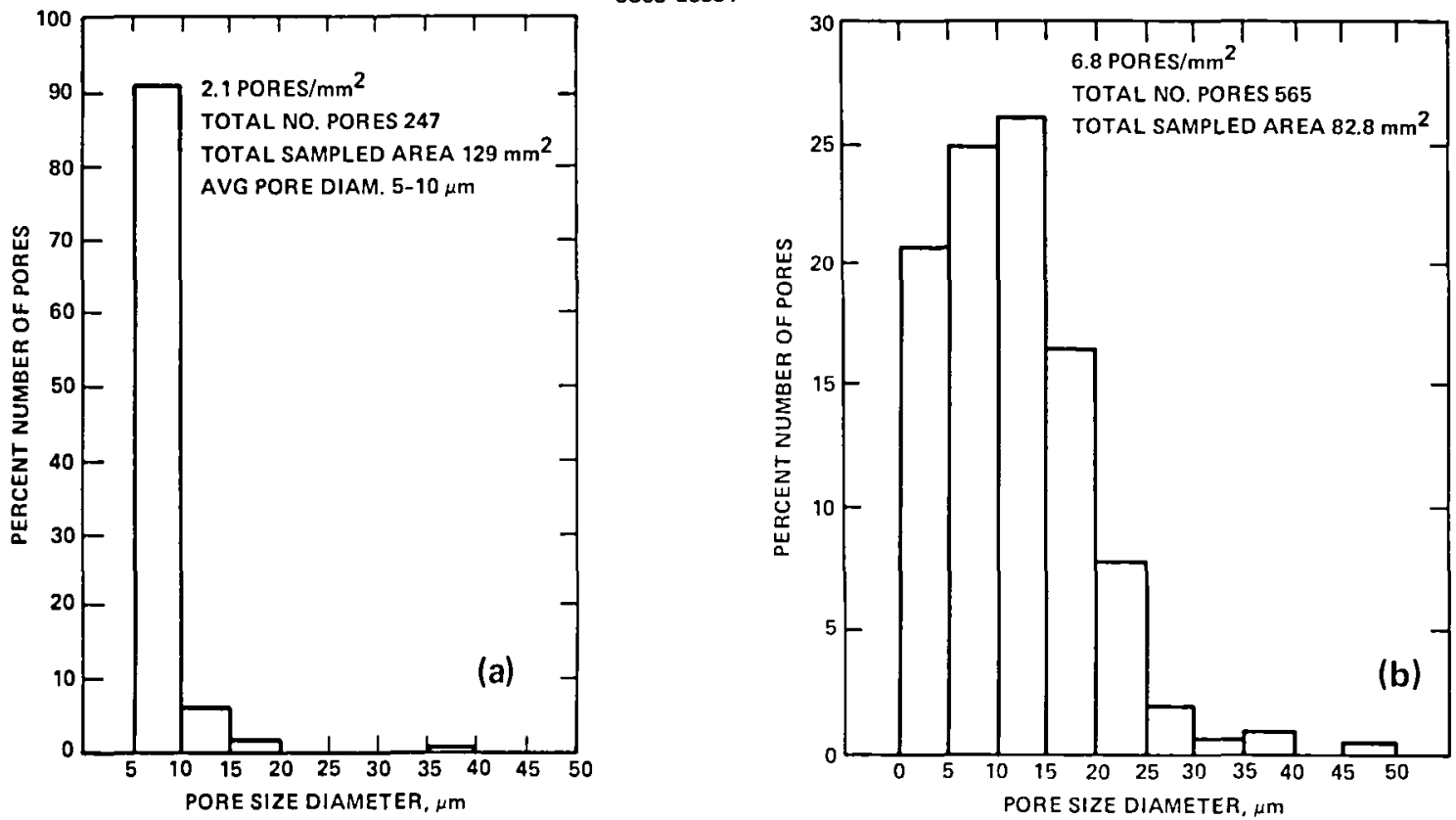

SC83-23338

Figure 6 Pore size distribution (a) sample C - IN-100, (b) sample A - IN-100. 
Table 1. Summary of Observations in Spin Test Sample

\begin{tabular}{|c|c|c|c|c|c|c|c|c|c|}
\hline $\begin{array}{l}\text { Traverse } \\
\text { Number }\end{array}$ & \multicolumn{2}{|c|}{\begin{tabular}{cc}
\multicolumn{2}{c}{ Traverse } \\
Traverse Length \\
Symbol & $\mathrm{mm}$
\end{tabular}} & $\begin{array}{l}\text { Sample } \\
\text { Length } \\
\mathrm{mm}\end{array}$ & $\begin{array}{l}\text { Number } \\
\text { of } \\
\text { Frames }\end{array}$ & $\begin{array}{l}\text { Sample } \\
\text { Area } \\
\left(\mathrm{mm}^{2}\right)\end{array}$ & $\begin{array}{l}\text { Number } \\
\text { of } \\
\text { Pores }\end{array}$ & $\begin{array}{c}\text { Number } \\
\text { of } \\
\text { Pores/ } \\
\text { Linear } \\
\text { mm }\end{array}$ & $\begin{array}{c}\text { Number } \\
\text { of } \\
\text { Pores } \\
\mathrm{mm}^{2}\end{array}$ & $\begin{array}{l}\text { Pore } \\
\text { Spacing } \\
\text { (Av) } \mathrm{mm}\end{array}$ \\
\hline 1 & K & 40 & 36.9 & 81 & 21.9 & 29 & 0.79 & 1.3 & 1.27 \\
\hline 2 & $\mathrm{~J}$ & 40 & 39.6 & 87 & 23.5 & 28 & 0.7 & 1.2 & 1.4 \\
\hline 3 & I & 40 & 40.6 & 89 & 24 & 54 & 1.3 & 2.3 & 0.75 \\
\hline 4 & $\mathrm{H}$ & 16 & 15 & 33 & 8.9 & 27 & 1.8 & 3.0 & 0.55 \\
\hline 5 & A & 8 & 7.3 & 16 & 4.3 & 10 & 1.4 & 2.3 & 0.7 \\
\hline 6 & B & 8 & 8.2 & 18 & 4.9 & 3 & 0.36 & 0.6 & 2.7 \\
\hline 7 & c & 19 & 18.2 & 40 & 10.8 & 35 & 1.9 & 3.2 & 0.5 \\
\hline 8 & D & 10 & 10.5 & 23 & 6.2 & 15 & 1.4 & 2.4 & 0.7 \\
\hline 9 & $E$ & 10 & 10.5 & 23 & 6.2 & 14 & 1.3 & 2.3 & 0.75 \\
\hline 10 & $\mathrm{~F}$ & 21 & 16 & 31 & 9.5 & 34 & 2.1 & 3.6 & 0.47 \\
\hline 11 & G & 21 & 15.5 & $\underline{34}$ & 9.2 & $\underline{22}$ & 1.4 & 2.4 & 0.7 \\
\hline \multicolumn{3}{|c|}{ Total Sample } & 218 & & 129 & 271 & 1.27 & 0.87 & \\
\hline \multicolumn{10}{|c|}{ Pore Size Distribution $(\mu \mathrm{m})$} \\
\hline \multicolumn{3}{|c|}{$5-10$} & $10-15$ & $15-20$ & $20-25$ & $25-30$ & \multicolumn{2}{|c|}{$30-35$} & $35-40$ \\
\hline \multirow{2}{*}{\multicolumn{2}{|c|}{$\begin{array}{l}\text { Number of Pores } \\
\text { Percentage }\end{array}$}} & & 18 & 5 & 0 & 0 & & 0 & 1 \\
\hline & & 1.1 & 6.6 & 1.8 & 0 & 0 & & 0 & 0.4 \\
\hline
\end{tabular}

where $n_{2}\left(r^{\prime}\right) d r^{\prime}$ is the number of circles per unit area (with radil between $r^{\prime}$ and $r^{\prime}$ $+\mathrm{dr}^{\prime}$ as measured from micrographs. Each circle represents a cut through a pore by the polished sample surface. $n_{3}\left(r^{\prime}\right)$ is the number of micropores per unit volume element, $\Delta r_{j}$ is the bin width of the pore radius histogram, $r_{j}$ is the average pore radius for each bin and $n_{j}$ is the number of pores in each bin.

These formulae were used for calculations in the detailed examination of two demonstrative samples discussed below.

\section{U1trasonic Characterization}

The samples were studied in two ways. The ultrasonic attenuation was measured In the frequency range from about 5 to $100 \mathrm{MHz}$ on samples $A$ and $B$ with nominally similar pore size distributions. The shear wave backscattered power was measured in the frequency range $2-20 \mathrm{MHz}$ on samples $\mathrm{C}$ and A described in detail in the text.

In the ultrasonic attenuation measurements the data were obtained in the $10 \mathrm{MHz}$ to $100 \mathrm{MHz}$ range of frequencies in several smaller sub-bands. A low attenuation buffer (Al or fused silica) was typically used to obtain the transducer response and the reflection coefficient of the sample interface. The technique and calculations are illustrated in figure 7 for the case of longitudinal waves. For the case of shear waves, an additional technique was employed for rapid data acquisition and is illustrated in figure 8. Here the buffer is water and the angle of incidence is such that the longitudinal waves in the water produce shear waves in the sample. 

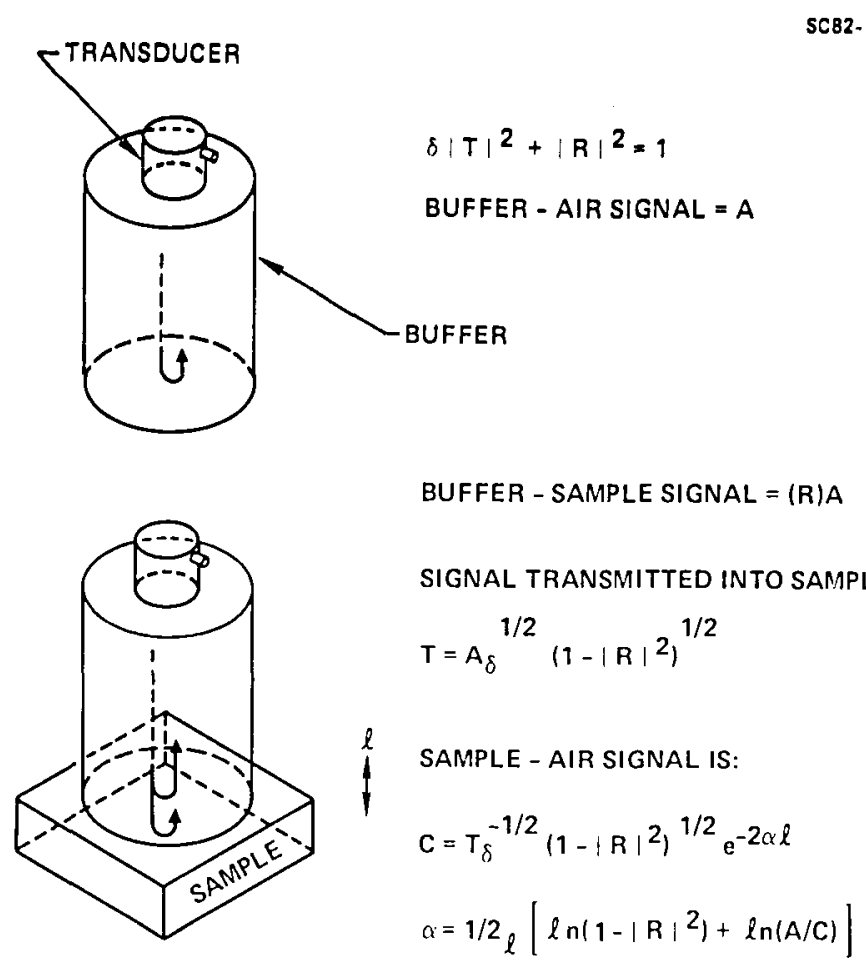

Figure 7 Sample configuration for measurement of longitudinal wave attenuation.

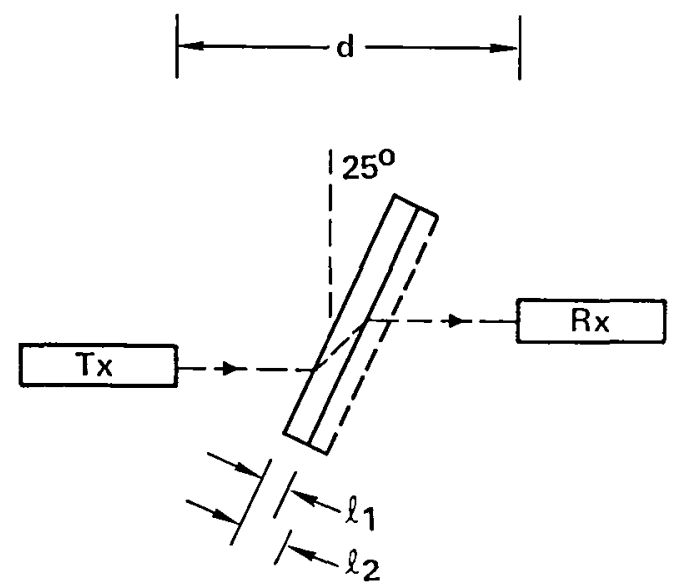

$\operatorname{SC} 82-18891$

$A_{1}=T_{01} T_{10} \exp -\left(\alpha_{s} I_{s 1}+\alpha_{w} I_{w 1}\right)$

$A_{2}=T_{01} T_{10} \exp -\left(\alpha_{s} I_{s 2}+\alpha_{w} I_{w 2}\right)$

$\alpha_{s}=\frac{1}{\Delta l_{s}}\left(\ln \frac{A 1}{A 2}\right)+\alpha_{w}$

Figure 8 Sample configuration for measurement of shear wave attenuation.

Because of the possible generation of leaky waves generated at the interfaces and the non-circular beam spreading associated with non-normal incidence, the measurements were augmented with calibration measurements, carried out with narrow band quartz shear wave transducers in direct tightly bonded contact with the sample. 
In reference to figure $8, \mathrm{~T}_{01}, \mathrm{~T}_{10}$ are the water-sample, sample-water transmission coefficients; $\alpha_{S}$ is the sample shear wave attenuation in nepers $/ \mathrm{cm}, \alpha_{W}$ is the water longitudinal attenuation in nepers/cm, $1_{s 1}, 1_{s 2}$ are the shear wave paths in the sample under test, $1_{w 1}, 1_{w 2}$ are the wave path length in water.

The measurements are summarized in figures 9 and 10 which display attenuation values as a function of frequency for two different samples and for three different locations for the same sample.

$\operatorname{SC} 82-18878$

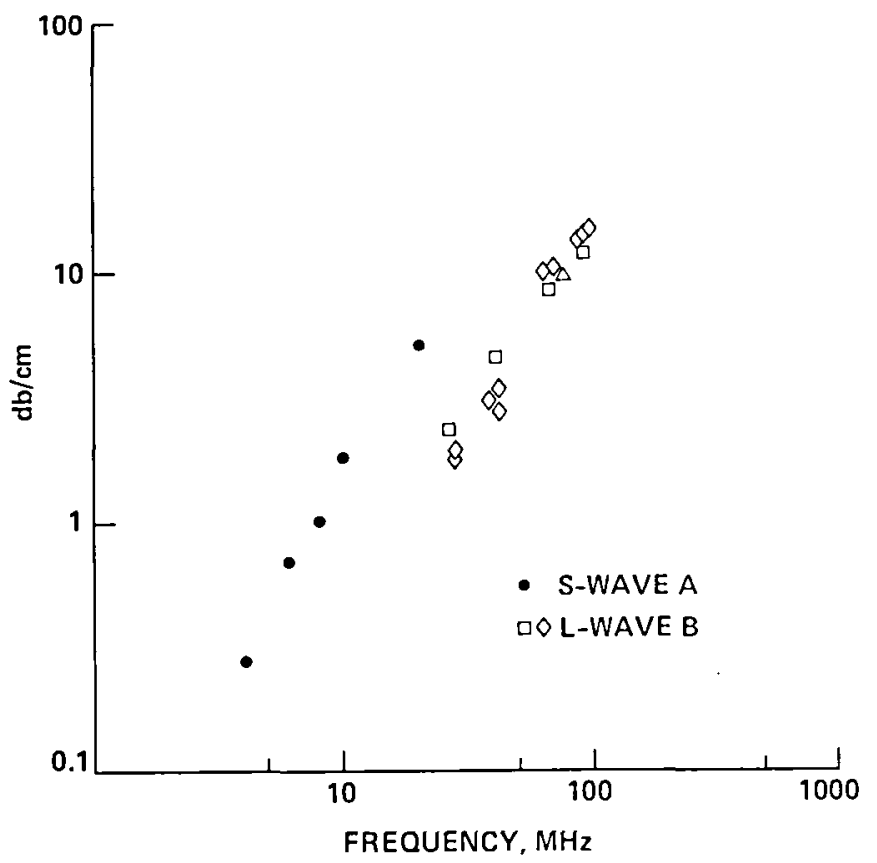

Figure 9

Longitudinal ( $L$ ) and shear (S) wave attenuation for 2 samples of $\mathrm{IN}-100$.

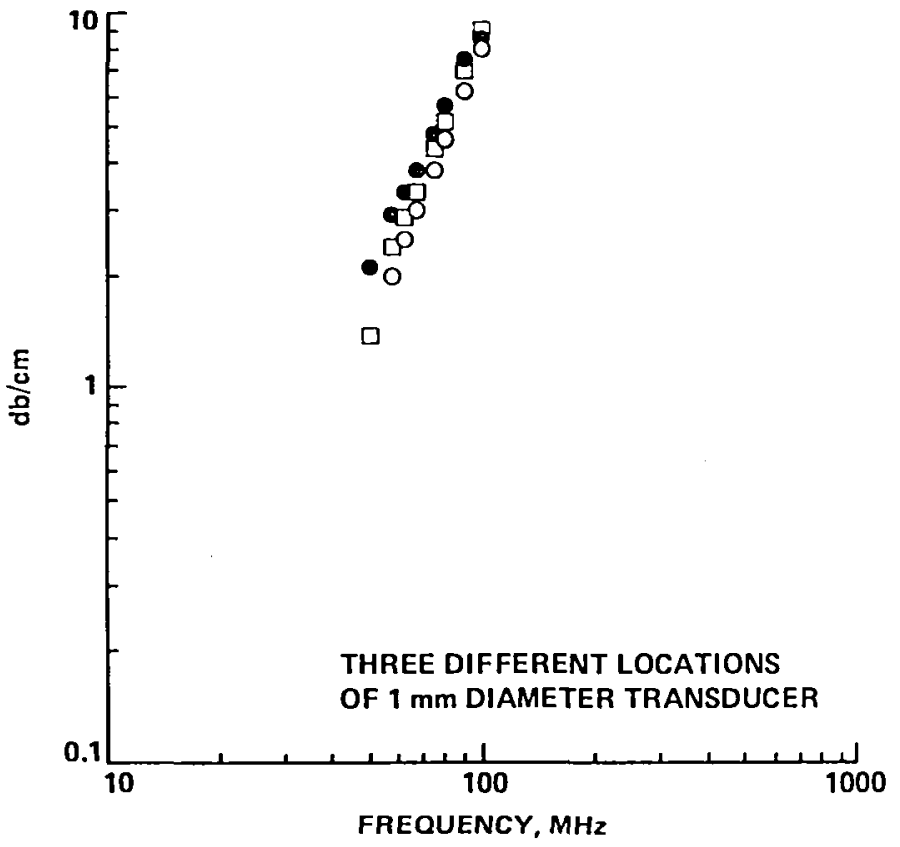

Figure 10

Longltudinal wave attenuation at three different locations on sample $C$.

Table 2 summarizes the information collected by given representative values for attenuation and velocity for two powder metal alloys $\mathrm{IN}-100$ and $\mathrm{T} 1-6246$ compared to a common engine disc material of nonpowder fabrication, 1.e., Waspalloy.

Table 2 .

\begin{tabular}{|c|c|c|c|c|c|c|c|c|}
\hline \multirow[t]{2}{*}{ Sample } & \multicolumn{2}{|c|}{$\begin{array}{l}\text { Velocity } \\
(\mathrm{mm} / \mu \mathrm{m})\end{array}$} & \multirow{2}{*}{$\begin{array}{l}\text { Grain } \\
\text { Size } \\
(\mu \mathrm{m})\end{array}$} & \multicolumn{2}{|c|}{$\begin{array}{l}\text { Atten. } \\
(\mathrm{dB} / \mathrm{cm})\end{array}$} & \multirow[t]{2}{*}{$\begin{array}{l}\text { Freq } \\
\text { (MHz) }\end{array}$} & \multirow{2}{*}{$\begin{array}{c}\text { Freq. } \\
{ }^{\alpha_{L}}\end{array}$} & \multirow{2}{*}{$\begin{array}{l}\text { Dep. } \\
\qquad \alpha_{S}\end{array}$} \\
\hline & $\mathrm{v}_{\mathrm{L}}$ & $\mathrm{v}_{\mathrm{S}}$ & & $\alpha_{L}$ & $\alpha_{S}$ & & & \\
\hline Waspaloy & 6.04 & 3.23 & 100 & 4 & 11 & 10 & $\mathrm{f}^{2.2}$ & $f^{2: 5}$ \\
\hline IN -100 & 6.52 & 3.29 & 2 & $4 \pm 1$ & 20 & 50 & $f^{2}$ & $\mathrm{f}^{2.2}$ \\
\hline $\mathrm{T} 1-6246$ & 6.11 & 3.16 & $2-4$ & 10 & - & 100 & & $\mathrm{f}^{3.5}$ \\
\hline
\end{tabular}




\section{ANALYSIS}

This section presents the analytical developments leading up to the calculations for a comparison between the experimental observations and the predicted frequency dependencles of back-scattering.

The model for the microstructural backscatter (nolse) spectrum we w111 use 18 taken from the work of Fertig and Richardson [ref. 9]. Th1s model accounts for the statistical nature of the scattering process as well as the diffraction assoclated with the measurement system. Consider flgure 11 below. We assume that the time window, $T$, assoclated with the experiment is small enough so that the variation in the sound beam along the z-axis is negliglble within the sampled part. If we consider a scatterer at position $\vec{r} j=\left(\underline{r}_{j}, z_{j}\right)$, with $\underline{r}_{j}=\left(x_{j}, y_{j}\right)$, then the contribution to the Fourier transform of $n(t)$, the measured time dependent wave form, from this scatterer will be

$$
p\left(\omega, \underline{r}_{j} ; z_{1}\right) \exp \left(i \omega \tau_{j}\right) A_{j}(\omega)
$$

where $\tau_{j}=\left(z_{j}-z_{l}\right) /(2 c)$ is the relative time delay of the $j$ th scatterer, $A_{j}(\omega)$ is its scattering amplitude and $p\left(\omega, \underline{r}_{j}\right)$ (suppressing the dependence of $z_{1}$ ) is the system response function.

- SYSTEM RESPONSE FUNCTION FOR DISTRIBUTED SCATTERERS

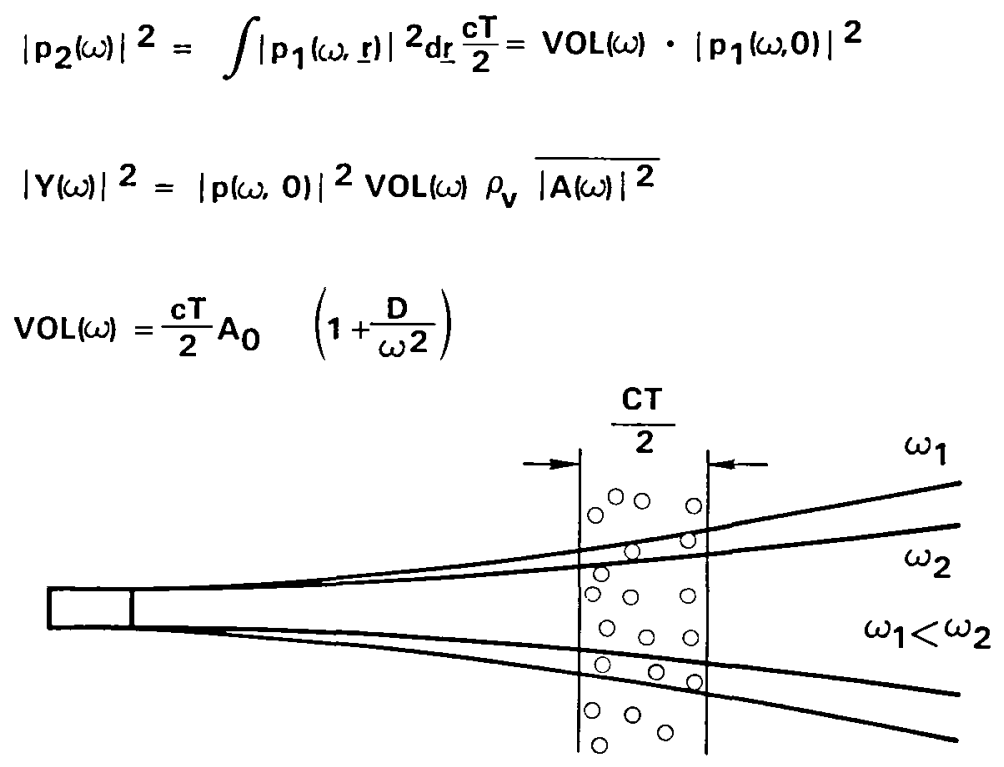

Figure 11 Diagram of pulse echo experiment measuring the backscatter from a region a distance $z_{1}$ from the transducer. 
The spectral density of $n(t)$ is found by taking the expectation of $n(\omega) n *(\omega)$ over the distribution of scatterer sizes and positions. If we assume that the scatterers are uniformly distributed in real space, that multiple scattering is negligible, and we restrict the frequency mesh to $\omega_{k}=2 \pi k / T$, we obtain

$$
E\left(n(\omega) n^{*}\left(\omega^{\prime}\right)\right)=\delta_{\omega \omega^{\prime}} \frac{c T}{2}\left(\iint_{-\infty}^{\infty}|p(\omega, \underline{r})|^{2} d^{2} \underline{r}\right) \rho_{v} E|A(\omega)|^{2},
$$

where $\rho_{\mathrm{v}}$ is the expected number of scatterers per unit volume, $\delta$, is the Kronecker delta, and $E|A(\omega)|^{2}$ is the expected squared scattering amplitude for a randomly chosen scatterer. For piston-source transducers, the integral of $|p(\omega, r)|^{2}$ is not analytically tractable. For Gaussian transducers, however, it is quite easy. In either case, we identify the "volume", vol $(\omega)$, of the beam, as a frequency dependent entity which may be defined by

$$
\operatorname{vol}(\omega)=(\mathrm{cT} / 2) \iint_{-\infty}^{\infty}|\mathrm{p}(\omega, \underline{\mathrm{r}})|^{2} \mathrm{~d}^{2} \underline{\mathrm{r}} /|\mathrm{p}(\omega, \mathrm{o})|^{2} .
$$

Fertig and Richardson [ref. 9] give approximate expressions for vol( $\omega)$ for which oblique incidence and curved surfaces are allowed. The term $p(\omega, 0)$ is the on-axis diffraction correction and may be determined from the work of Thompson and Grey [ref. 10, 11] for piston sources. These latter authors give explicit forms for $p(\omega, 0)$ which allow for the inclusion of a reference calibration wave, $R(\omega)$, which enables one to relate a pressure profile on the face of the transducer to a voltage reading. In the case in which the scatterers are spherical and for which the Rayleigh limit holds, one obtains

$$
\begin{aligned}
& E|n(\omega)|^{2} /|R(\omega)|^{2}= \\
& \left.\qquad \frac{2 C^{2}\left(z_{1}, z_{2}, \omega\right)}{k_{2} a^{2} D\left(z_{1}, z_{2}, \omega\right)}\right]^{2} \frac{1}{R_{1}^{2} R_{2}^{2}} \operatorname{vol}(\omega) \omega^{4} \eta \rho_{v} E\left(r^{6}\right)
\end{aligned}
$$

Here $C$ and $D$ are diffraction correction functions for the experiment and calibration, $R_{1}$ and $R_{2}$ are corner reflection coefficients associated with the calibration, $E\left(r^{6}\right)$ is the expected value of the sixth power of the pore radius, and $\eta$ is a frequency and size independent parameter related to the scattering of spheres in the host material. In the far fleld limit, the expression in brackets above is Independent of Erequency.

\section{COMPARISON BETWEEN THEORY AND EXPERIMENT}

The experimental approach centers around a broadband transducer which is used to launch a short pulse of shear wave energy into the material in many different directions as shown schematically for the spin-pit test sample in figure 12 . During its passage through the material, the microstructural inhomogeneities reflect energy back toward the transducer which is now used as a receiver to detect these small echoes which form a train of waveforms which have amplitudes and phase relationships depending on where and how strong the sources are. Several time traces recorded for a variety of regions for both samples $A$ and $C$ are shown in figure 13 . It is clear 


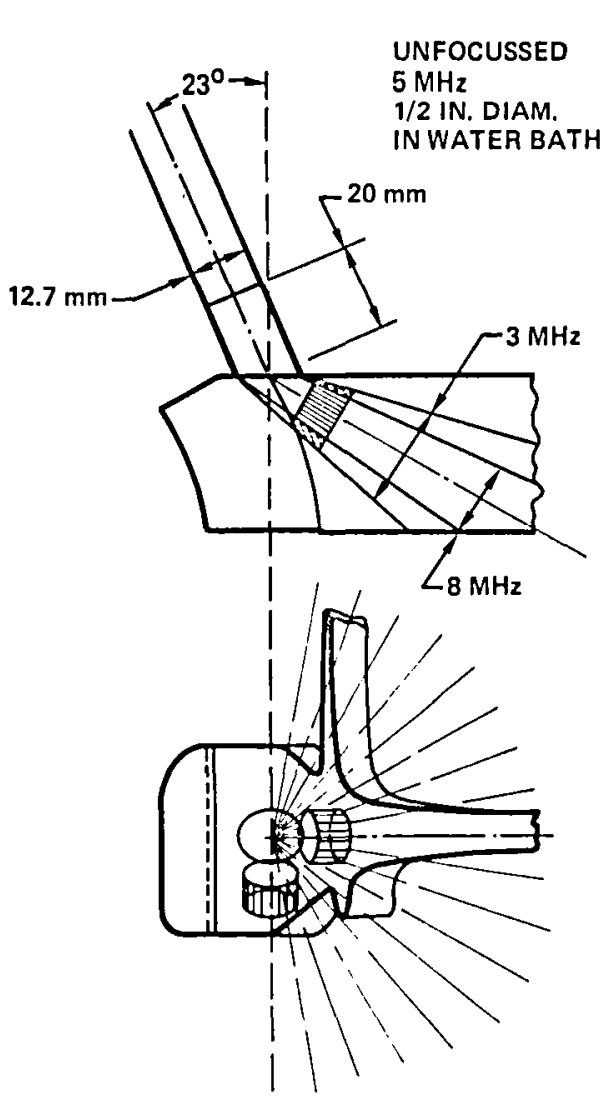

$\operatorname{SC83-23337}$

Figure 12 Schematic diagram of spin pit test specimen and ultrasonic shear wave beam.

that these signals are more or less random and form a background. In a typical run, the backscattered background signals are windowed at 10 different sections of the time trace corresponding to 10 different regions of the sample. Each waveform is time averaged over 512 transmission pulses to decrease electronic noise in the recelver equipment. The 10 waveforms are deconvolved with a reference signal to remove the transducer characteristics and averaged to produce one statistically representative waveform for the sample material from which now the power frequency spectrum may be calculated. Figure 14 shows the backscatter power spectra for samples $A$ and $C$ before deconvolution whereas figure 15 shows the same data after deconvolution and therefore presents the results in final form.

As Indicated by the dashed lines in figure 15 the predicted backscatter spectra have slopes which agree well with those obsrved, 1.e., a 3.25 power law for the frequency dependence of the backscattered intensity.

Another, more stringent, test of the data and model is to see whether a key microstructural feature may be predicted and compared with micrographical data. This is attempted in Table 3. Here the values of the estimated mean power densities for the two samples and the ultrasonic backscatter intensities at $10 \mathrm{MHz}$ are inserted into the model to calculate values of $\left\langle r^{6}\right\rangle^{1 / 6}$ which are then compared to corresponding values estimated from the micrographical examination. The comparisons are shown in the last two columns of the table. Considering that the micrographical examination was limited to the surfaces of the samples, the agreement is reasonable. The calculation demonstrated also that small errors in the count of the large pores introduces large errors in the final estimates. 


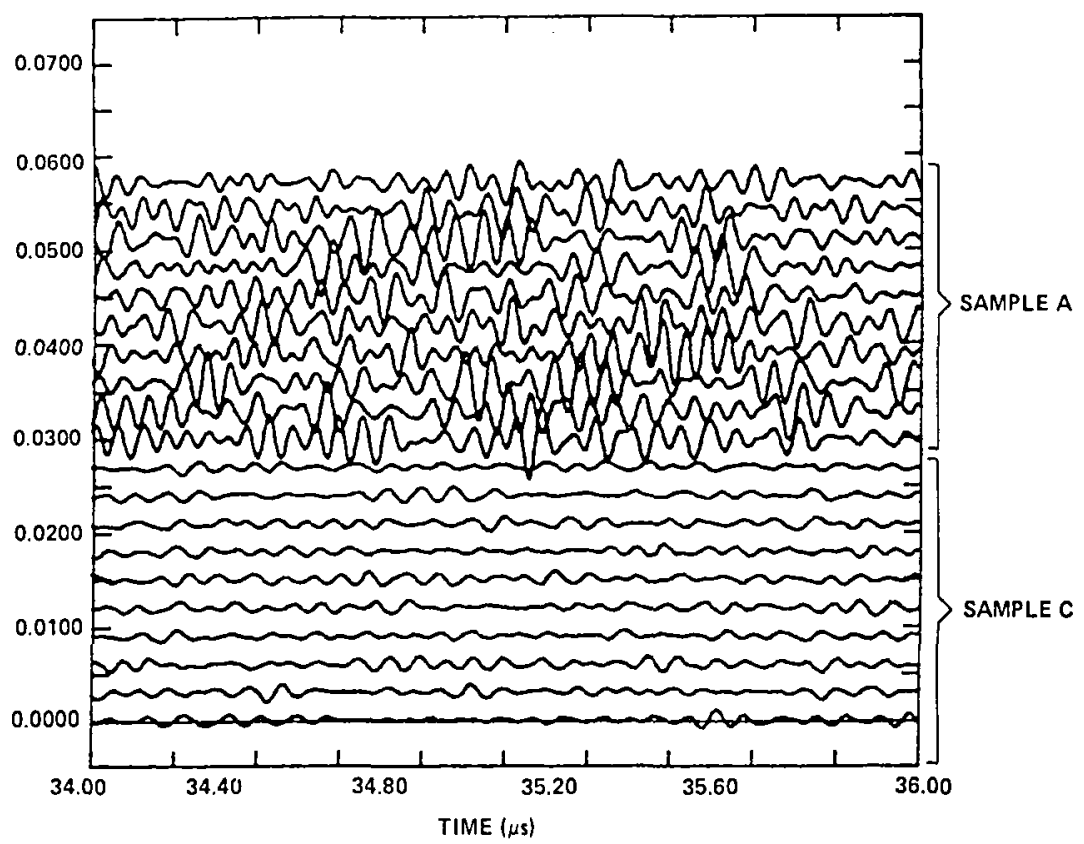

F1gure 13 U1trasonic backscattering noise in IN-100.

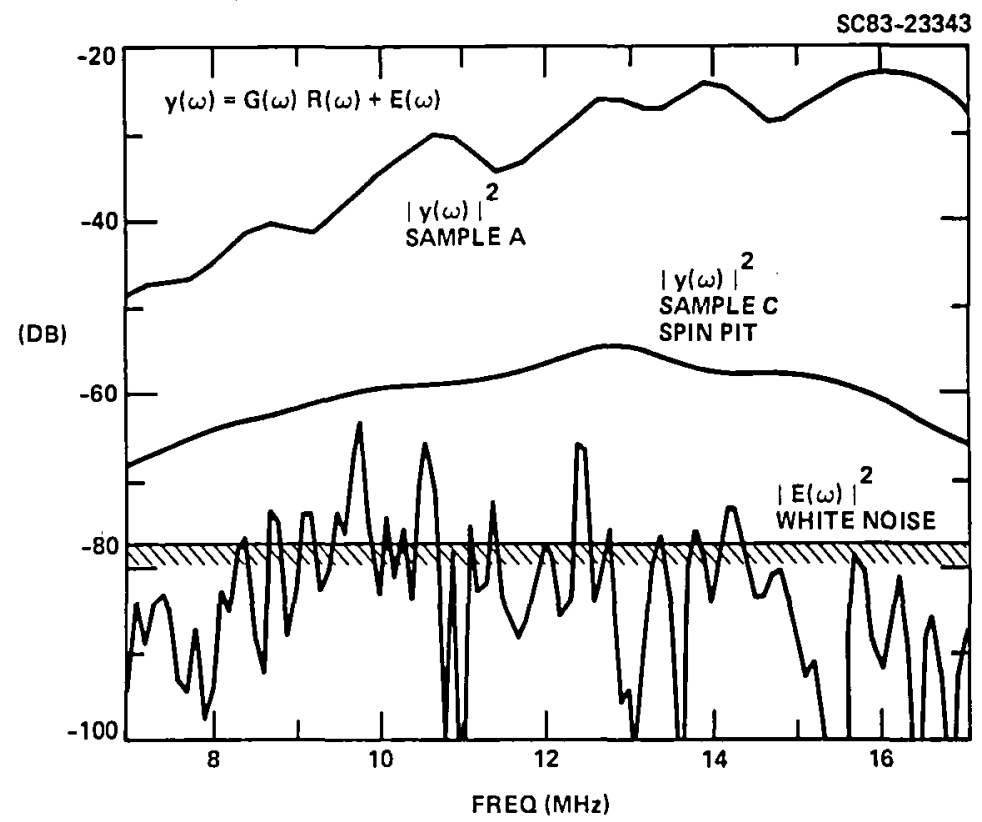

Figure 14 Ultrasonic backscatter power spectra for two samples of IN-100 before deconvolution. 


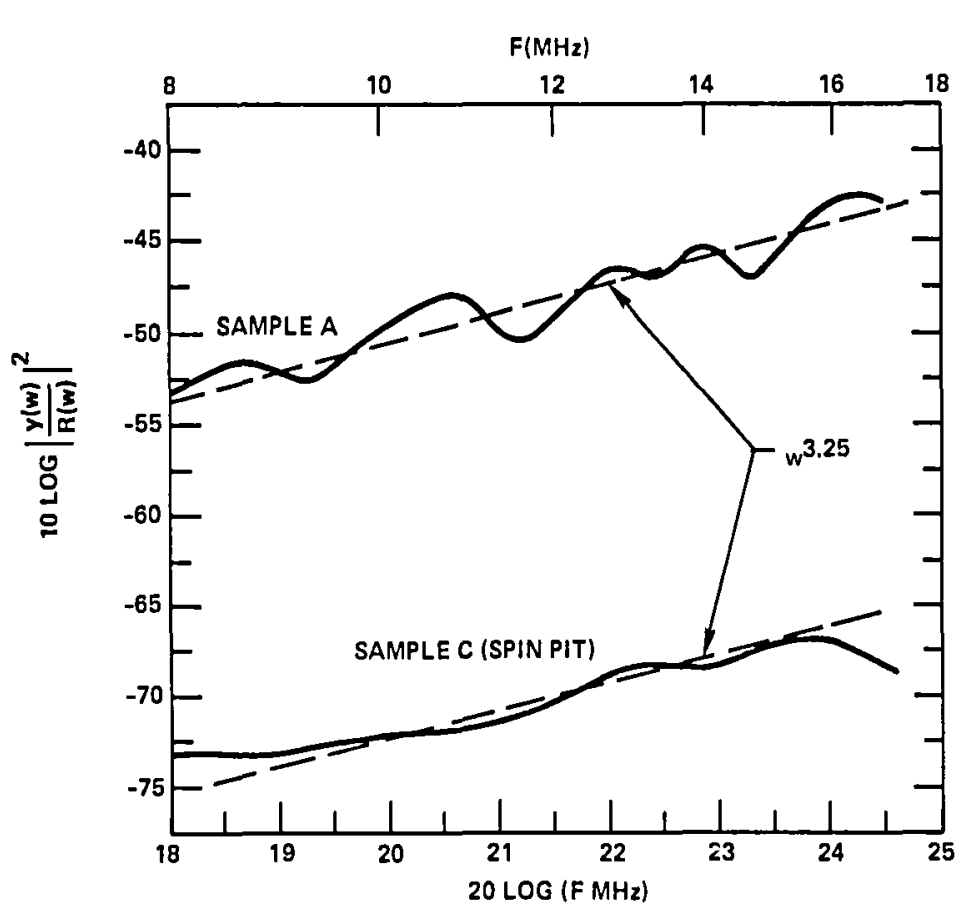

Figure 15 Ultrasonic backscattering spectra for two different samples of IN-100.

Table 3

\begin{tabular}{|c|c|c|c|c|c|}
\hline & $\begin{array}{l}\text { Pore Dens1ty } \\
\qquad\left(\mathrm{mm}^{-3}\right)\end{array}$ & $\begin{array}{c}\text { Pores in } \\
5 \mu \mathrm{s} \mathrm{Beam} \\
\text { Vol }-0.23 \mathrm{~cm}^{3}\end{array}$ & at $10 \mathrm{MHz}$ & $\begin{array}{l}\text { Scattering } \\
\langle r\rangle \\
(\mu \mathrm{m})\end{array}$ & $\begin{array}{c}\text { Micrograph } \\
\langle r\rangle \\
(\mu \mathrm{m})\end{array}$ \\
\hline $\begin{array}{l}\text { Research } \\
\text { Sample A }\end{array}$ & 329 & $\begin{array}{l}80,000 \\
(-50 \mathrm{~dB})\end{array}$ & $1 \times 10^{-5}$ & 5.34 & $4.1-19.5$ \\
\hline $\begin{array}{l}\text { Spln P1t } \\
\text { Sample C }\end{array}$ & 89 & $\begin{array}{l}20,000 \\
(-73 \mathrm{~dB})\end{array}$ & $5.01 \times 10^{-8}$ & 2.74 & $2.4-10.0$ \\
\hline
\end{tabular}

FORMAL APPROACH TO INVERSE PROBLEM

Whereas we have not attempted to solve the full inverse problem in this paper, it is appropriate to consider how one might attack this problem. For this purpose, it is convenient to formulate the problem in terms of a new set of random variables, called $v_{j i}$, which represent the number of spheres whose centers lie in a volume element $j k^{\prime}$ located at $\vec{r}_{j}$ in the material and who have radil in the range $\left(a_{k-1}, a_{k}\right)$ If we let $\hat{j}_{j}\left(a_{k}\right)$ represent the "counting" process equaling the actual number of spheres per unit volume with centers in $\delta V_{j}$ and radil less than or equal to $a_{k}$, we see

$$
v_{j k}=\delta V_{j}\left(N_{j}\left(a_{k}\right)-N_{j}\left(a_{k-1}\right)\right)
$$


Again, if we take the case of negligible multiple scattering, the Fourier transform of the time windowed measured waveform takes the form

$$
y(\omega)=\sum_{j k} \sum_{p}\left(\omega, \vec{x}_{j}\right) A\left(\omega, \tilde{a}_{k}\right) v_{j k}+\varepsilon
$$

where $\varepsilon$ is additive noise (e.g., electronic), and $A\left(\omega, \tilde{a}_{k}\right)$ is the scattering amplitude of a sphere with nominal radius $\tilde{a}_{k,} \varepsilon\left(a_{k-1}, a_{k}\right)$. The term $p\left(\omega, \vec{r}{ }_{j}\right)$ is the system response function for position $\vec{r}_{j}$, the "center of $\delta V_{j}$. The above formulation assumes that the $\delta V_{j}$ and $\delta a_{k}=a_{k}-a_{k-1}$ are all suitably small.

Contrary to the "usual" inversion problem wherein information on an isolated scatterer of interest is contained in the expected value of $y(\omega)$, for this case, the information on the statistics of the $v_{j k}$ is contained in the variance of $y(\omega)$.

We make the following assumptions for the observation time $T$,

$$
\begin{aligned}
& \text { 1. } p(\omega, \vec{r}) \doteq p(\omega, \underline{r}) e^{i \omega \tau}, \quad v=2 z / c . \\
& \text { 2. } v_{j k} \sim \text { stationary Polsson process } \\
& \text { 3. } \varepsilon(\omega) \sim \text { zero mean stationary process, independent of } v_{j k} \text {. }
\end{aligned}
$$

Then as $\delta \mathrm{V}_{j} \rightarrow 0, \Delta \mathrm{a}=\max \left|a_{k}-a_{k-1}\right| \rightarrow 0$, we have on the special frequency mesh $\omega_{\mathrm{k}}=2 \pi \mathrm{k} / \dot{\Phi}$,

$$
\begin{aligned}
& E\left(y\left(\omega_{k}\right)\right)=0 \\
& E\left(y\left(\omega_{k}\right) y *\left(\omega_{k},\right)\right)=\delta_{k k^{\prime}}\left[\frac{c T}{2} \int d^{2} \underline{r}\left|p\left(\omega_{k}, \underline{r}\right)\right|^{2}\right. \\
& \left.\cdot \int d a \rho(a)|A(\omega, a)|^{2}+C_{\varepsilon}(k) T\right],
\end{aligned}
$$

where $\delta_{\mathrm{kk}^{\prime}}$ is the Kronecker delta, $\rho(a)$ is the number density of spheres of radius $a$, and $C_{\varepsilon}(k) T$ is the variance of the $k^{\prime}$ th frequency component of the additive noise.

By inversion, we mean based on a set of samples of $y(\omega)$, estimate $p(a)$. Specifically, we are interested here in estimating the underlying statistics of the pore distribution, not the actual size and location of a specific set of pores in a particular region of the specimen. This latter question is, of course, of interest in many applications. Here, however, we are interested in the former. As is well known, this is typically an i11-posed problem. Large changes in $\rho(a)$ can result in small changes in $y(\omega)$. For that reason, it will probably be necessary to parameterize $\rho$. To that end, define $\rho_{3}$ as the number density of spheres and $\bar{F}_{3}(a ; \underline{\theta})$ as the fraction of spheres with radil greater than a. Thus 


$$
\rho_{3} \bar{F}_{3}(a ; \underline{\theta})=\int_{a}^{\infty} \rho(a) d a
$$

The subscript 3 is meant to reinforce the fact that we are estimating volume densitles of particles and not area densitles. The latter often forms the raw data from sectioning experiments. The inversion problem is to estimate $\rho_{3}$ and the parameter $\theta$ (possibly vector-valued). One approximation to use for estimation of $\rho 3$ and $\theta$ is to assume that $y(\omega)$ is a Gaussian process. This will be the case if the spheres are "dense" relative to the system response function so that no scatterer 1s "Isolated" in time. In the Gaussian case, the posterlor probability of p3 and $\theta$ given $y$ is

$$
\begin{aligned}
\ln P\left(\rho_{3}, \underline{\theta} \mid y\right)= & k-\frac{1}{2} \sum_{k} y^{*}\left(\omega_{k}\right) B_{k k} y\left(\omega_{k}\right) \\
& +\ln P\left(\rho_{3}, \underline{\theta}\right)+\frac{1}{2} \sum_{k} \ln B_{k k},
\end{aligned}
$$

where $K$ is a normalization constant, $P\left(\rho_{3}, \theta\right)$ is the prior distribution of $\rho_{3}$ and $\underline{\theta}$, and $B_{k k}$ is the inverse of the diagonal of the variance of $y\left(\omega_{k}\right)$. It is given by

$$
B_{k k}^{-1}=T C_{\varepsilon}\left(\omega_{k}\right)+M\left(\omega_{k}\right) \int \rho_{3} f_{3}(a ; \underline{\theta})\left|A\left(\omega_{k}, a\right)\right|^{2} d a,
$$

where

$$
M(\omega)=c T / 2 \int|p(\omega, \underline{r})|^{2} d^{2} \underline{r}
$$

and $f_{3}(a ; \underline{\theta})=-d \bar{F}_{3}(a ; \underline{\theta}) / d a$ is the probability density function of the sphere sizes.

Two classical sets of estimators present themselves. The first set is $\left\{\rho_{3}, \theta\right\}$ which maximizes the posterior density $P\left(\rho_{3}, \theta \mid y\right)$. These estimators define the mode of this distribution and represent the most probable values. The second set estimators is $\left\{\rho_{3}, \underline{\theta}\right\}$ defined by the means of marginals of $P\left(\rho_{3}, \underline{\theta} \mid y\right)$. Thus

$$
\begin{aligned}
& \hat{\rho}_{3}=\int \rho_{3} P\left(\rho_{3}, \underline{\theta} \mid y\right) d \rho_{3} d \underline{\theta} \\
& \hat{\hat{\theta}}=\int \underline{\theta} P\left(\rho_{3}, \underline{\theta} \mid y\right) d \rho_{3} d \underline{\theta}
\end{aligned}
$$

These estimators minimize the mean square error.

In either case, the posterior variance of the estimators will be reduced if multiple waveforms $y_{r}(\omega), r=1, \ldots, R$ are avallable. Having a small number of waveforms places a greater reliance on the prior density $P\left(\rho_{3}, \underline{\theta}\right)$ to keep the problem well-posed. 
The microstructure of a nickel-base powder metal alloy IN-100 has been studied with the aid of micrographical and ultrasonic techniques. The material showed grains of small diameter with a narrow distribution in the range of 2-4 $\mu \mathrm{m}$ and sparsely distributed micropores with diameter in the 5-20 $\mu \mathrm{m}$ range. The micropores dominated the ultrasonic attenuation and backscattering characteristics. The ultrasonic backscattering was selected to carry out quantitative measurements and calculations for comparison of theory and experiment. The frequency dependence from 8 to $18 \mathrm{MHz}$ of the backscattered signal was in good agreement with calculations which included the contributions from on-axis diffraction, back-surface reflection, the frequency dependent beam, the Rayleigh scattering and the distribution of scatterers. In addition to the forward problem, the inverse problem was addressed in two ways (1) by estimating the mean pore size $\left\langle\mathrm{r}^{6}\right\rangle^{1 / 6}$ from an approximate count of the pore density. This was found to give reasonably good agreement with the pore size estimated from micrographical information. (2) A formal approach for a stochastic Inversion was developed.

The results point the way toward the nondestructive detection and characterization of anomalous distributions of micropores where conventional ultrasonic imaging is difficult. Such information may be useful in the early detection of potential fallure sites where stress induced void coalescence leads to crack initiation.

\section{REFERENCES}

1. M. O'Donne11 and J.G. Miller, "Quantitative Broadband Ultrasonic Backscatter: An Approach to Nondestructive Evaluation in Acoustically Inhomogeneous Materials," J. Appl. Phys. 52, 1056 (1981).

2. K. Goebbels, "Method of Quantitatively Determining the Grain Size of Substances," United States Patent No. 4026,157, May 31, 1977.

3. E. M. Papadak1s, J. Acoust. Soc. A, 37, 711 (1965).

4. W. P. Mason and H. J. McSkimin, J. Appl. Phys. 19, 940 (1948).

5. I. M. Lifshitz and G. D. Parkhomovsk11, J. Expt. Theoret. Phys. (USSR) 20,175 (1950).

6. A. G. Evans, B. R. Tittmann, L. Ahlberg, B. T. Khun-Yakub, G. S. Kino, "Ultrasonic Attenuation in Ceramics," J. Appl. Phys. 49, 1669 (1978).

7. L. Adler, Proceedings of the Review of Progress in Quantitative NDE (edited by D.0. Thompson and D. Chiment1) Plenum Publishing Company, (1984).

8. B.R. Tittmann, L.A. Ahlberg and K. Fertig, Proceedings of the Review of Progress in Quantitative NDE (edited by D.0. Thompson and D. Chimenti) Plenum Publishing Company, 1983 and 1982.

9. K.W. Fertig and J.M. Richardson, "Computer Simulation of Probability of Detection," 1982 AF/DARPA Review of Quantitative NDE, Plenum Publishing Company. 
10. R.B. Thompson, "Diffraction Corrections for Scattering Measurement," Internal Report, Center of Advanced Nondestructive Evaluation, Ames Laboratory, Iowa State University (1982).

11. R. B. Thompson and T.A. Gray, "Analytical Diffraction Corrections in Ultrasonic Scattering Measurements," Proceedings of the Review of Progress In Quant1tative NDE, (edited by D.0. Thompson and D. Chiment1) Plenum Publish1ng Company, August 1-6 (1982). 



\title{
STUDIES OF ACOUSTIC EMISSION FROM POINT AND EXTENDED SOURCES
}

\author{
Wolfgang Sachse, Kwang Yul Kim, and C.P. Chen \\ Cornell University \\ Ithaca, New York 14853
}

The use of simulated and controlled acoustic emission signals forins the basis of a powerful tool for the detailed study of various deformation and wave interaction processes in materials. We report the results of experiments and signal analyses of $A E$ resulting from point sources such as various types of indentation-produced cracks in brittle materials and the growth of fatigue cracks in 7075-T6 aluminum panels. Fur thermore, we describe our recent work dealing with the modeling and subsequent signal processing of an extended source of emission in a material. Results of the forward problem and the inverse problem are presented with the example of a source distributed through the interior of a specimen shown.

\section{INTRODUCTION}

The use of acoustic elnission (AE) signals for real-tine structural integrity monitoring applications has long been sought. Developments over the last several years have gone far towards obtaining a better understanding of the $A E$ process in actual structural components (Refs. 1-5). These include the development of a model consisting of a repeatable source of known type, a structure for which the propagation of transient elastic waves can be computed, sensors whose transfer characteristics are either known or can be deterinined experimentally and the development of new signal processing algorithrns for analyzing the detected signals.

The associated problem, sometines called the "inverse source problem", has as its aim the characterization of a source in terms of its location in the structure, its type, specified in terms of force or moment tensor components, its spatial distribution, if the source is extended, and its temporal or dynamic characteristics. This paper gives an overview of the work recently completed in our laboratory on aspects of this topic.

\section{THEORY AND WAVEFORM PROCESSING}

The displacement signals $u_{k}$ detected at a receiver location, $x$, in a structure from an arbitrary source $f\left(x^{\prime}, t\right)$ located at ${ }^{k} x^{\prime}$ having source volume $v_{0}$ can be written as

$$
u_{k}=\int_{V} f_{j}\left(x^{\prime}, t\right) * G_{j k}\left(x / x^{\prime}, t\right) d V
$$

where the term $G_{j k}$ represents the Green's function of the structure. Insight into this formulation can be obtained if one makes a Taylor series expansion of the Green's function about the source point, $x^{\prime}$, located at, say 0 , to give

$$
u_{k}(x, t)=\int_{V_{0}} f_{j}(0, t) * G_{j k}(x / 0, t) d V+\int_{V} x_{0}^{x} f_{j}(0, t) * G_{j k, i}(x / 0, t) d V^{\prime}
$$


The first term in this equation, representing the monopole contribution, can be rewritten in the form

or

$$
u_{k}^{(m)}=\left\{\int_{V} f_{0}(0, t) d V\right\} * G_{j k}(x / 0, t)
$$

$$
u_{k}^{(m)}=s_{j}(t) * G_{j k}(x / 0, t)
$$

The second term in Eq. (2), representing the dipole contribution, can be rewritten in a similar fashion as

or

$$
u_{k}^{(d)}=\left\{\int_{V_{0}^{x_{i}^{\prime}}} f_{j}\left(x^{\prime}, t\right) d V^{\prime}\right\} * G_{j k, i}(x / 0, t)
$$

$$
u_{k}^{(d)}=M_{i j}(t) * G_{k, i}^{\prime}(x / 0, t)
$$

The term, $M_{i j}$, called the moment tensor of the source, can be used to represent any combination of double forces (Ref. 3) to model the source. It can be shown that the moment tensor can be written in terms of the crack's displacement jump and its area in a material (Ref. 6). If each of the components of the moment tensor of the source have the same time dependence, then the spatial and temporal characteristics of the source are separable. Thus, the moment tensor can be written as a product of the moment density tensor, $\mathrm{m}_{\mathrm{ij}}$, and the and the source-time function, $S(t)$,

$$
\mathrm{M}_{\mathrm{ij}}=S(t) \cdot \mathrm{m}_{\mathrm{ij}}
$$

In recovering the characteristics of a dipolar source from the measured signals, $u_{z}$, one uses knowledge of the Green's function $G$ and inverts Eq. (4) to recover $S(t)$ and the components $\mathrm{m}_{\mathrm{ij}}$. In the most complicated deformation processes and for an arbitrary crack orientation, all six components of the mornent density tensor may need to be evaluated. As of now, this has not yet been done. Here we show the results obtained for several simpler cases.

The simplest case is that of a point source of known type for which only the temporal aspects of the source are to be recovered from the detected signals. In that case, Eq. (3) can be rewritten as a simple convolution equation in time

$$
u(t)=S(t) * G(t)
$$

This equation has been modified to include the transfer characteristics of a non-displacement transducer, whose response is band-limited. Recovery of the source function, $S(t)$, then involves two measurements, one of which is a calibration experiment using a known step excitation source applied at the source position. Examples of processing near-field and farfield signals from an axisymınetric source have been published previously (Refs. 7-9).

For the case described by Eq. (4) in which both the time-function and moment-density tensor components of a source are to be recovered, several processing algorithms have been developed. One of these is based on an algebraic scheme in which the amplitudes of particular ray arrivals in the detected signals are directly related to the amplitudes of the Green's function (Ref. 10). Once these are determined, the source tirne-function can be recovered as before. The problems related to this scheme result principally from the amplitude 
measurernent precision required of specific features on a waveform. An alternative is a double iterative scheme demonstrated by Michaels and Pao (Ref. 9). The procedure is initiated with a guess of the moment tensor magnitudes from which a solution of the source time-function is obtained. This solution is used, in turn, to evaluate a new set of moment tensor arnplitudes, and so on. The iteration process is continued until the results obtained in the iterations converge to a final answer. The procedure appears to be initial guess dependent and it may also converge slowly for certain configurations of the source moment tensor. It has been found that an appropriate initial guess for the double-iterative scheme can be obtained by making use of the results obtained from the algebraic processing scheme.

\section{RESULTS}

Demonstration of any signal analysis procedure for AE source characterization must begin with a controlled deformation process in a material. Reproducible, deformation-related AE sources whose location is known precisely, include indentation-produced cracks in brittle materials and fatigue-generated cracks in thin specimens and these were investigated in this work.

\section{a. Fatigue Cracks}

In detailed fatigue experiments, conventional test parameters were recorded in combination with electrical crack length measurements and a total of five AE waveforms, detected at points around the crack region. Two sample signals recorded equidistant from the crack front are shown in Figure l. The four equivalent forces or moment tensor components by which an arbitrary dipolar source in a thin specimen can be modelled are shown schematically in Figure 2. It is found that the dynamic Green's functions corresponding to the $M_{12}$ and $M_{21}$

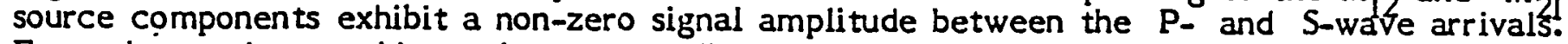
From observations as this, each corresponding to an event, it is concluded that only two signals are needed to determine without ambiguity the characteristics of the source. It was found that some of the events could be adequately described in terms of only two double forces, i.e.; $M_{11}$ and $M_{22}$. Other events required also a contribution from the $M_{12}$ and $M_{21}$ components. Table 1 shows the results obtained for two events one of each type. Hore than thirty-eight signals were processed in a typical test. In Figure 3 is the recovered source-time function obtained with the double-iterative processing scheme. It appears that the crack displacement profile had a risetime of approximately $400 \mathrm{nsec}$. The risetime may actually be shor ter than this, since the recovered value corresponds to the upper bandwidth limit of the transducer/amplifier system.

\section{b. Indentation Cracks}

The pressing of an indentor onto the surface of a flat plate results in a stress field configuration which depends on the shape of the indenter and the mechanical properties of the plate specimen. If the specimen is a brittle material, cracks of specific shape for $m$ when and in those regions where the stress field exceeds the fracture strength of the material. The dynamic force released in forming the crack is emitted as an AE signal. A typical epicentral AE signal obtained with a spherical-tipped indenter is shown in Figure 4(a). Typical Hertzian cone cracks are obtained when such indenters are pressed against a specimen. It has been shown that the associated dynamic source is equivalent to a vertical axisymmetric force acting on the structure (Ref. 11). On unloading, one or more lateral vent cracks may form whose associated $A E$ signals exhibit completely different characteristics as shown in Figure 4(b). In some cases, these may be adequately modelled by a single horizontal dipole acting near the surface of the plate. This appears to be the case, when only one lateral vent crack forms. The detected AE 
signals resemble those obtained when a conical indenter is pressed against the specimen. In the latter situation, a single penny-shaped crack is formed. Evaluation of the stress field under the conical indenter, obtained from the Boussinesq solution (Ref. 12), shows regions of tensile and compressive hoop stresses. The crack is generated in the region of tensile hoop stresses whose contours resemble a penny.

Experiments were carried out with an eight-channel $A E$ system consisting of point piezoelectric transducers with a $1.2 \mathrm{~mm}$ active area situated at various angles at a distance approximately $2 \mathrm{~h}$ ( $\mathrm{h}$ - plate thickness) from the indenter. Sensors whose response was nearly uniform in amplitude were selected for the array. The signals were amplified by $60 \mathrm{~dB}$ with preamplifiers whose bandwidth ranged from $10 \mathrm{kHz}$ to $2 \mathrm{MHz}$. They were subsequently digitized in transient waveform recorders operating at a sampling rate of $30 \mathrm{MHz}$ with 10-bit resolution.

Evaluation of the moment tensor components of the penny-shaped crack via one of the procedures. listed in the previous section often did not yield reliable results, as they were strongly initial guess dependent. It was clear, however, when comparing the detected waveforms to synthesized $A E$ signals, that the model of a tensile crack given in terms of three or thogonal dipoles of strength, $\lambda+2 \mu, \lambda$ and $\lambda$, where $\lambda$ and $\mu$ are the Lame constants, may not be appropriate to model an indentation-produced crack in a small specimen as described here.

An alternative approach for recovering the ratios of the moment tensor components involves measurement of the radiated field of the AE signal from the source. The radiated amplitude measured at a distance $R$ from a dipolar source, can be written in terms of the displacement amplitude, $u_{z}$, and this is expected to be of the form

$$
u_{z}(R, t)=a_{1}(t)+a_{2}(t) \cos ^{2} \theta
$$

where $\theta$ is the orientation of the normal to the crack. For a single horizontal dipole source, aligned parallel to the crack normal, the term $a_{1}(t)$ is expected to be near zero. For threedipole crack model, its value is expected to be approximately 0.28 . Eq. (7) can be rewritten to account for an arbitrary crack orientation relative to the sensor array

$$
u_{z}(R, t)=a_{1}(t)+a_{2}(t) \cos ^{2}\left(\theta-\theta_{c}\right)
$$

where $\theta_{c}$ is the orientation of the normal of the crack relative to the orientation of the sensor array.

By a proper choice of angular distribution of sensors around the indenter, an eight-channel $\mathrm{AE}$ system is adequate to determine the field characteristics of the indentation-generated cracks. Each channel of the entire system was calibrated simultaneously by breaking a glass capillary $0.05 \mathrm{~mm}$ in diameter at the crack position on the specimen. The result was an axisymmetric step excitation source. The amplitudes of both the first P- and S-wave arrivals were measured. The corresponding amplitudes in the crack-generated signals were normalized with the calibration amplitudes. The result obtained after one indentation is shown in Figure 5. The solid and dasined lines correspond to the results produced from a non-linear least-square fit of Eq. (8) to the measured data. From the curve fit, the parameters of the radiated field can be extracted. Once these are known, the measured amplitudes at each transducer can be reflected across the various symmetry planes to generate image data points with the results obtained shown in Figure 6. Inspection of the amplitude ratio $a_{1} / a_{2}$ shows that the tensile cracks produced by the conical indenter are best modelled as resulting principally from a single 
horizontal dipole. The orientation of the $\mathrm{crack}$, relative to the array configuration, is recovered immediately when Eq. (8) is fit to the amplitude data. The difference between the observed and recovered crack orientation angle from seven indentations was always less than $6^{\circ}$ and on average less than $3^{\circ}$.

The radiated field of the signals emitted from lateral vent unloading cracks was also measured. It was found that the formation of multiple cracks results in a radiated field which radiate more uniformly in all directions and clearly, the radiation does not resenble that of a single crack.

It is possible to process an epicentral displacement signal with the double-iterative processing scheme to recover the individual magnitudes of the moment tensor as well as their time function. Figure 7 shows the recovered source time function for an indentation crack.

\section{EXTENDED SOURCE}

Both the forward and inverse problems of the extended source have been solved using synthetic data. A series of examples dealing with two source types and several linear distributions on the surface or through the interior of a plate specimen have been computed (Ref. 13). In Figure $8(a)$ is shown the case of a distribution of horizontal dipole sources, extending to $0.75 \mathrm{~h}$ into the interior of the plate and whose time function was a Heaviside step function. It has been proposed that such a source may be used to model the interaction of intense synchrotron-generated pulses of $x$-rays striking a specimen and penetrating into its interior (Refs. 14-15). Figure 8(b) shows a comparison between the signal from a single dipole very close to the top surface of the specimen and the signal synthesized from forty source points whose strength distribution along the $z$-axis was given by $e^{-0.5 z}$. After only ten iterations in the double-iterative processing scheme, the input source function was recovered from the synthetic input data within a percent and the corresponding recovered source time function was indistinguishable from the input.

\section{CONCLUSIONS}

Acoustic emission signals detected from cracks formed during indentation and fatigue loading experiments have been processed to recover the moment tensor components of the crack source and their time function. Several signal processing schemes were demonstrated including one based on ray amplitude measurements, a double-iterative processing scherne and an AE radiation pattern analysis method. A signal processing algorithm was also demonstrated with synthetic AE waveforms corresponding to an extended source of emission by which the signals can be processed to recover both the spatial extent of the source and its time function.

\section{ACKNOWLEDGMENTS}

This work was supported by the National Science Foundation through grants to the Materials Science Center and to the College of Engineering at Cornell University. A significant portion of the equipment used in the experiments described was acquired through a DoD Instrumentation Grant awarded through the Office of Naval Research. 


\section{REFERENCES}

(1) R. F. Breckenridge, C. E. Tschiegg and M. Greenspan, "Acoustic Emission: Some Application of Lamb's Problem", J. Acoust. Soc. Am., 57, 626-631 (1975).

(2) N. N. Hsu, J. Simmons and S. C. Hardy, "An Approach to Acoustic Emission Signal Analysis - Theory and Experiment", Matls. Eval., 35(10), 100-106 (1977).

(3) Y. H. Pao, "Theory of Acoustic Emission", in Elastic Waves and Non-destructive Testing of Materials, Y. H. Pao, Ed., AMD-Vol. 29, Am. Soc. Mech. Engrs., New York (1978), pp. 107-128.

(4) N. N. Hsu and S. C. Hardy, "Experiments in Acoustic Emission Waveform Analysis for Characterization of AE Sources, Sensors and Structures", in Elastic Waves and Nondestructive Testing of Materials, Y. H. Pao, Ed., AMD-Vol. 29, Am. Soc. Mech. Engrs., New York (1978), Pp. 85-106.

(5) H. N. G. Wadley and C. B. Scruby, "Quantitative Acoustic Emission Studies during Deformation and Fracture: A Review", Report AERE - R 10353, AERE Harwell, Oxfordshire (1981).

(6) K. Aki and P. G. Richards, Quantitative Seismology, Vol. 1, W. H. Freeman and Co., San Francisco (1980), Chapt. 4.

(7) J. E. Michaels, T. E. Michaels and W. Sachse, "Applications of Deconvolution to Acoustic Emission Signal Analysis", Materials Evaluation, 39(11), 1032-1036 (1981).

(8) P. N. Hsieh and W. Sachse, "Far-field Signals from Axis-symmetric Sources in Elastic Plates", in Ultrasonics International '83 - Conference Proceedings, Butterworth and Co. Publ., Sevenoaks, Kent, UK (1983), pp. 388-394.

(9) J. E. Michaels and Y. H. Pao, "Deconvolution of Source Time Functions of the Moment Density Tensor", in Review of Progress in Quantitative NDE, D. O. Thompson and D. E. Chimenti, Eds., Plenum Press, New York (1984), pp. 707-715.

(10) C. P. Chen, "Acoustic Emission Source Characterization of Fatigue Cracks in 7075-T6 Aluminum Alloy Thin Plate", Ph. D. Dissertation, Cornell University, Ithaca, NY (1985).

(11) K. Y. Kim and W. Sachse, "Characteristics of Acoustic Emission Signals of Hertzian and Unloading Cracks in Glass", J. Appl. Phys., 55, 2847-2856 (1984).

(12) I. N. Sneddon, "Boussinesq's Problem for a Rigid Cone", Proc. Cambridge Phil. Soc., 44, 492- (1948).

(13) C. Chang and W. Sachse, "Analysis of Elastic Wave Signals from an Extended Source in a Plate", J. Acoust. Soc. Am., 77, 1335-1341 (1985).

(14) K. Y. Kim and W. Sachse, "X-ray Generated Ultrasound", Appl. Phys. Letts., 43, 10991101 (1983).

(15) W. Sachse and K. Y. Kim, "Observation of X-ray Generated Ultrasound", 1983 Ultrasonics Symposium, Inst. Elect. Electr. Engs., New York (1983), pp. 677-680. 
$U_{2}=C_{11} G_{21,1}+C_{22} G_{22,2}+C_{12} G_{21,2}+C_{21} G_{22,1}$

Event \#34; Model: Two Double Forces

Algebraic; $C_{11}: C_{22}=1: 0.258$

Double Iteration; $C_{11}: C_{22}=1: 0.266$

Event \#35; Model: Two Double Forces, Two Single Couples

Algebraic ; $C_{11}: C_{22}: C_{33}: C_{44}=1: 0.324:-0.271: 0.237$

Double Iteration; $C_{11}: C_{22}: C_{33}: C_{44}=0.996: 0.308:-0.271: 0.209$

Table I 
FATIGUE CRACK: SENSIR \# 1

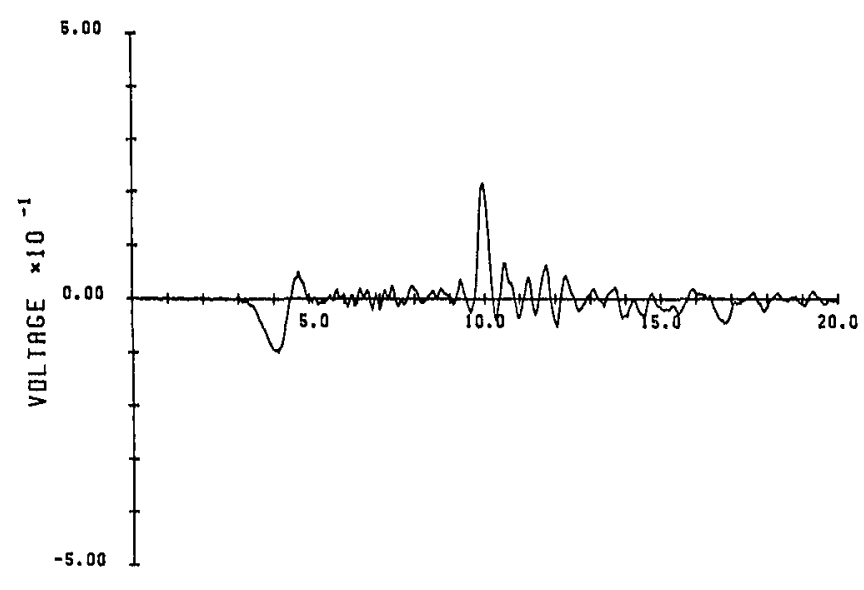

TIME (useo)

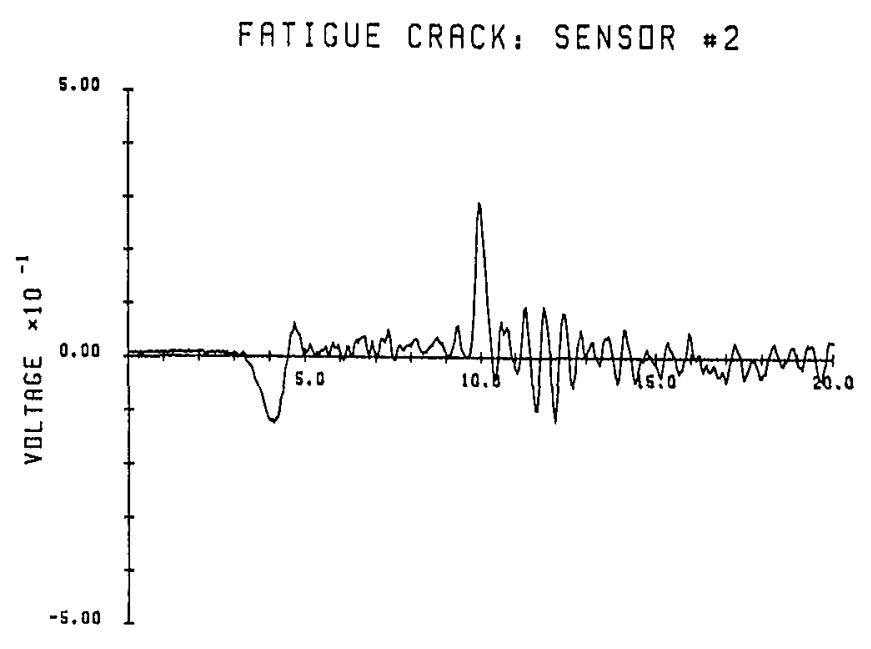

TIME (useo)

Figure 1

EQUIVALENT FORCES / MOMENT TENSOR COMPONENTS

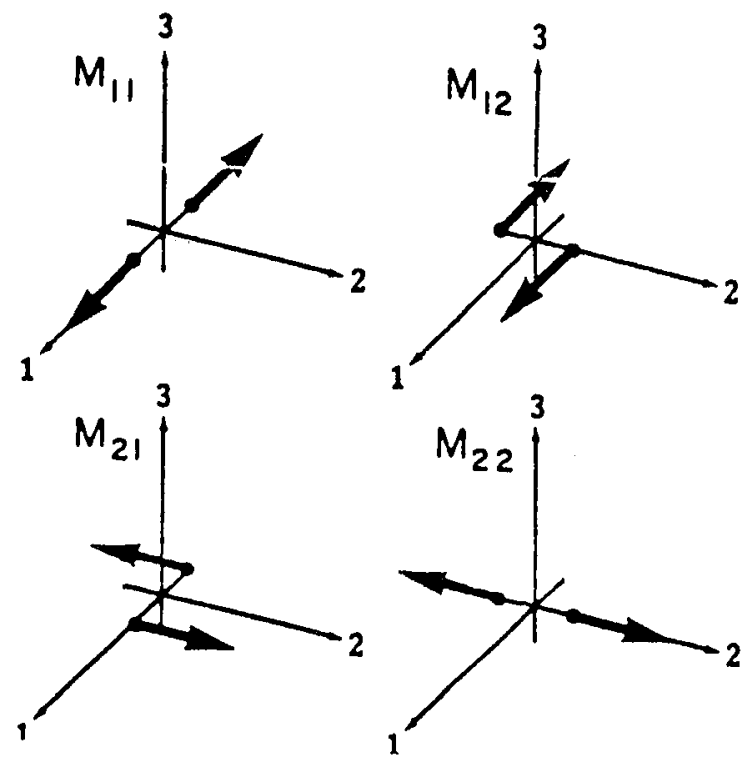

Figure 2 

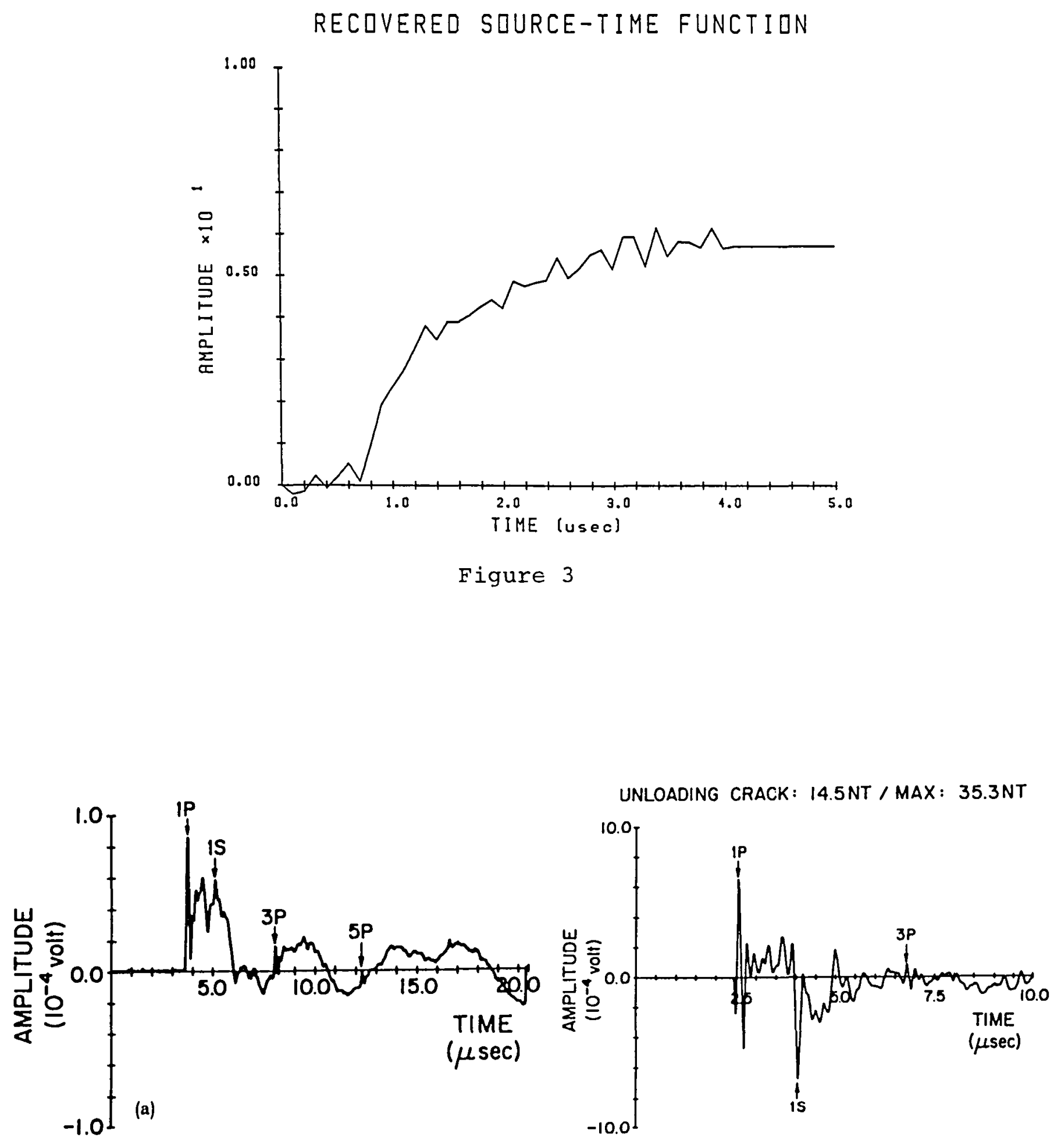

Figure $4(a)$

Figure 4(b) 
$120^{\circ}$ CONICAL INDENTER - DIPOLE RADIATION

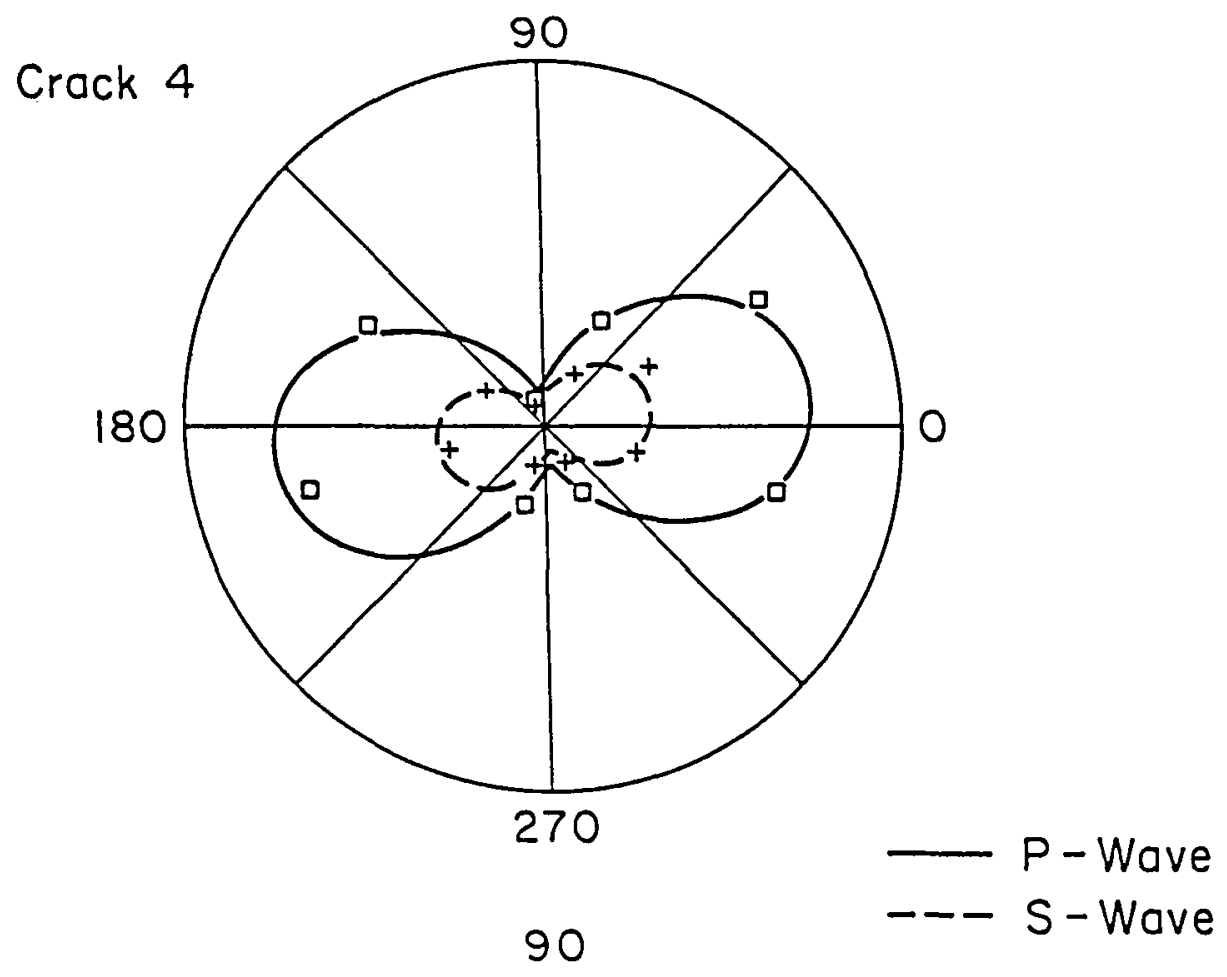

Figure 5

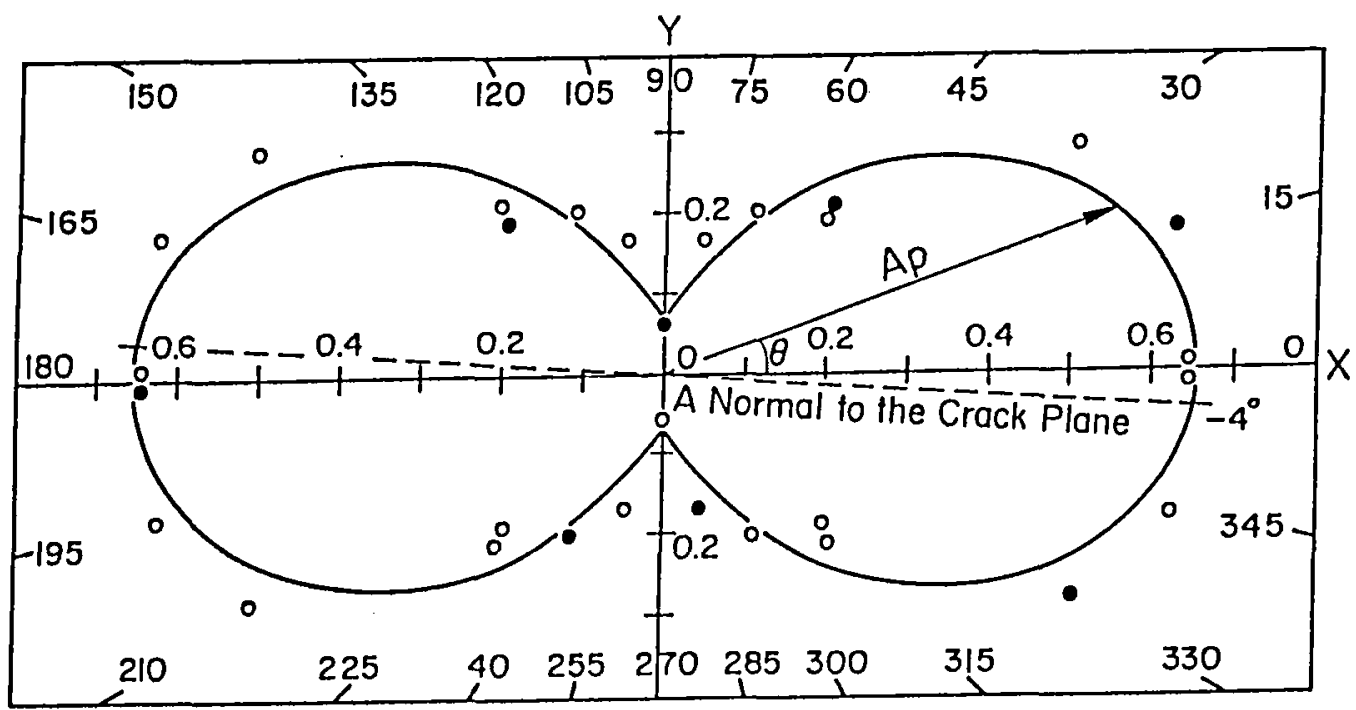

- -Real Data points, o-Images across $X, Y$ Axes; $A p=0.585\left(0.123+\cos ^{2} \theta\right)$

Figure 6 
RECOVERED SOURCE - TIME FUNCTION (CRACK 9)

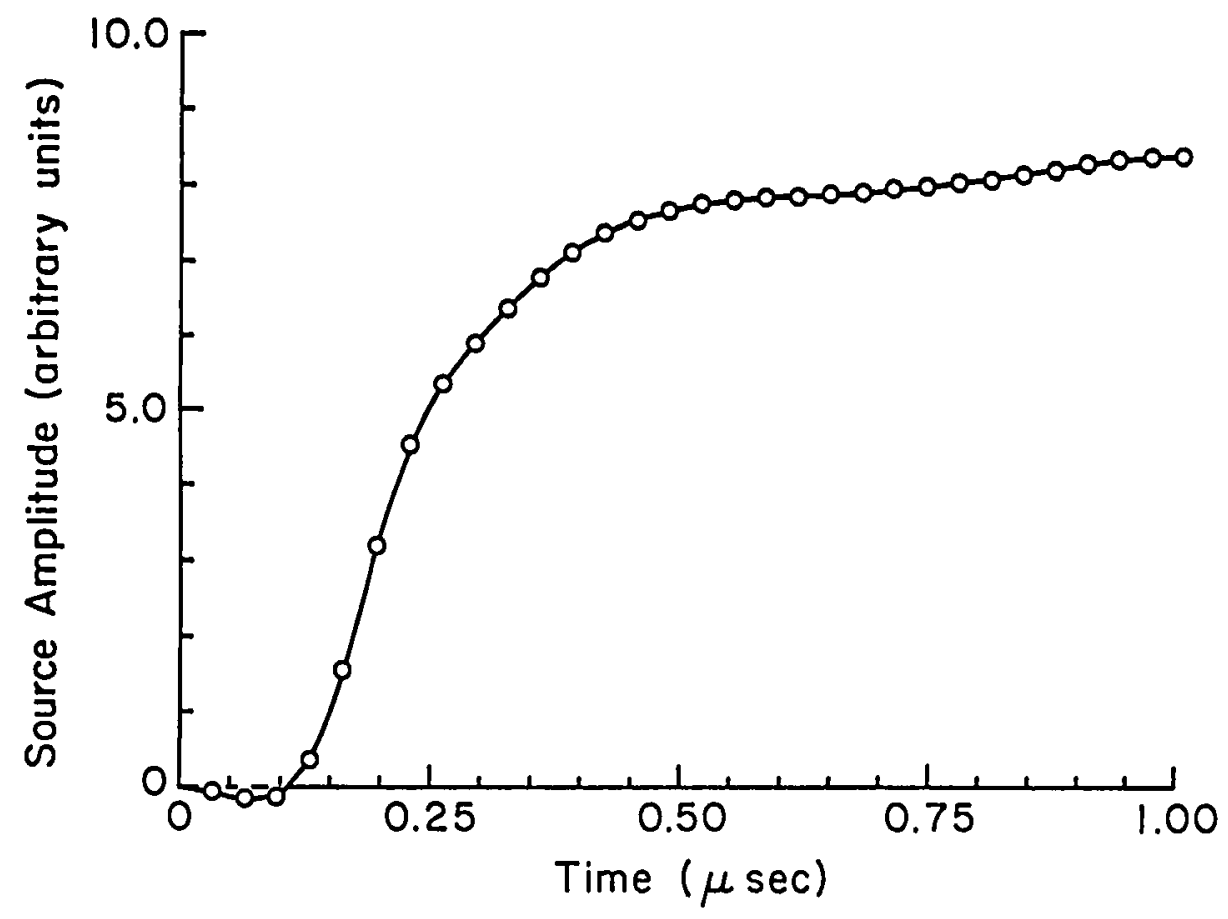

Figure 7

HORIZONTAL DIPOLES

$(0.0-0.75 h)$

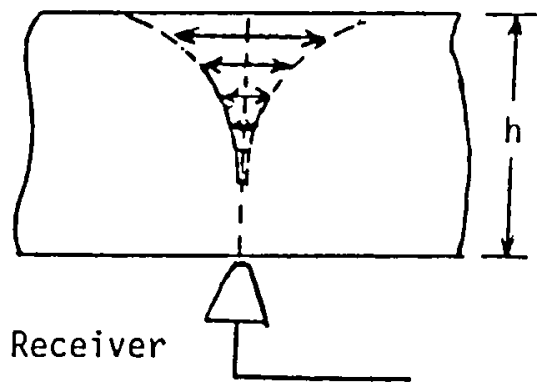

Extended dipole source model

Figure $8(a)$
DIPOLE SOURCES: EXPONENTIAL DISTRIB

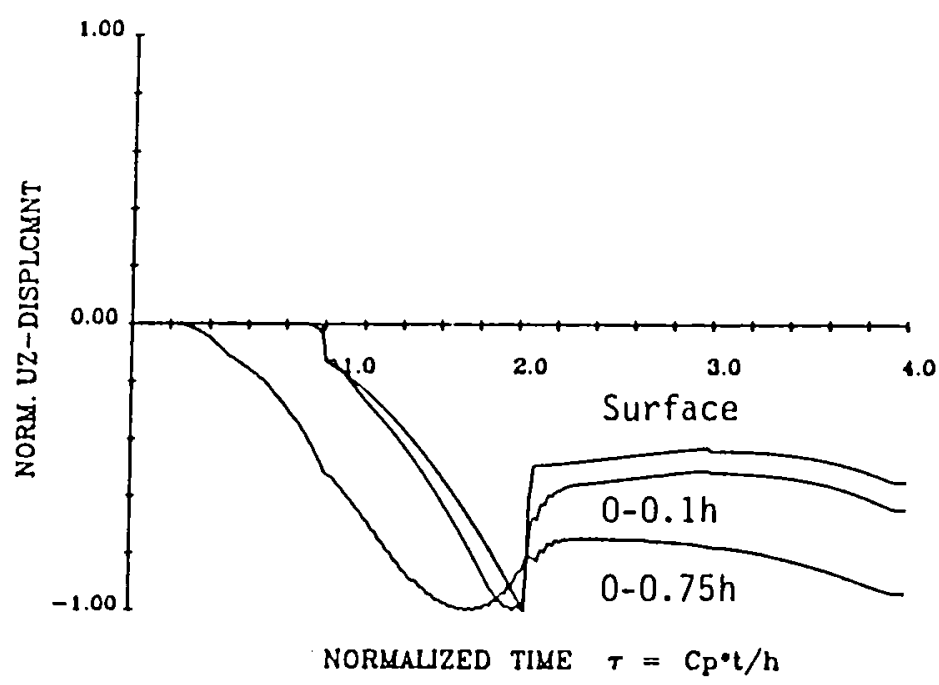

Synthetic normal displacement signals corresponding to several source distributions

Figure $8(\mathrm{~b})$ 



\title{
RESIDUAL STRESS MEASUREMENTS IN CARBON STEEL
}

\author{
Joseph S. Heyman \\ National Aeronautics and Space Administration \\ Langley Research Center \\ Hampton, Virginia 23665 \\ Min Namkung \\ College of William and Mary \\ Williamsburg, Virginia 23185
}

External de magnetic field-induced changes in natural velocity of Rayleigh surface waves have been measured in steel specimens under varlous stress conditions. The low field slopes of curves representing the fractional changes of natural velocity have been proved to provide correct stress information in steels with different metallurgical properties. The slopes of curves under uniaxial compression, exceeding about one third of the yield stress, fall below zero in all the specimens when magnetized along the stress axis. The slopes under tension vary among different steels but remain positive in any circumstances. The stress effect is observed for both applied and residual stress. A physical interpretation of these results is given based on the stress-induced domain structure changes and the $\Delta E$-effect. Most importantly, it is found that the influence of detalled metallurgical properties causes only secondary effects on the obtained stress information.

\section{INTRODUCTION}

The practical application of the currently available techniques for nondestructive bulk residual stress measurements in metals is severely limited mainly due to a lack of calibration standard. This is because too of ten the stress effect is inhibited by material properties, such as texture, grain size, defect structure and so on. As a result, even the determination of the sign of the bulk residual stress can be made only under very specific conditions. Thus a technique, insensitive to material characteristics other than stress state and capable of differentiating the effects of tension from those of compression, is in great demand. In our previous papers (refs. 1 and 2) we reported a recent method measuring the uniaxial stress dependence of ultrasonic natural velocity changes induced by external dc magnetic field showing that compression can be detected unambiguously without knowing the zero stress standard.

The use of an external magnetic field in the ultrasonic velocity measurement In steel is based on two phenomena; the stress-induced domain structure change and the domain-dependent elastic modulus of a ferromagnet.

The unit cells of cubic crystals are not exactly cubic but slightly deformed in the ferromagnetic state (ref. 3). Specifically, the body-centered cubic (BCC) unit cells of iron below the Curie temperature are tetragonal with the longer 
edges parallel to the domain magnetization vectors oriented in the one of six equivalent directions of $\langle 100\rangle$. The effect of further deformation of unit cells on domains by uniaxial stress can be described by the magnetoelastic interaction. This interaction energy is lowest (highest) in domains oriented paralle1 (perpendicular) to the stress axis when an iron crystal is under tension and is opposite under compression. As in the case of magnetization with external magnetic field, the energetically favored domains expand their volume and a complete domain alignment (with zero net magnetization) can be achieved with sufficient uniaxial stress (ref. 4). The experimental verifications of such a stress-induced domain alignment have been made not only in pure iron but also in steel (refs. 5 and 6).

The elastic modulus of a nonlinear solid is defined as the ratio between infinitesimal applied stress and induced strain. In a ferromagnetic material the stress-induced strain, in general, includes the magnetoelastic strain as well as the direct elastic strain. The former is the strain induced by domain structure change with stress which aligns the longer edges of iron unit cells in certain directions with respect to the stress axis. The magnetoelastic strain is always positive under tension and is negative under compression for iron base alloys with BCC structure (ref. 7). Hence, the elastic modulus of a ferromagnet depends explicitly on 1ts domain structure. The contribution of magnetoelastic strain to the resultant elastic modulus can be separated by altering the domain structure with an external magnetic fleld. Such a domain-dependent elastic modulus of a ferromagnet is called the $\Delta E$-effect and is most pronounced in the domain wall motion region of the magnetization process (refs. 5 and 8 ).

The inftial domain structure is determined uniquely at each stress level. The subsequent change in domains due to external magnetic field will proceed differently according to the material stress. Therefore, the simultaneous changes In domain-dependent elastic modulus provides the stress information. Uniaxial stress affects the domain structure of iron only. by domain wall motion. Thus the stress information is expected in only the early stage of the magnetization process where domain wall motion occurs. This must be true in steels unless the crystalline anisotropy energy is drastically reduced by various impurities.

\section{ACOUSTIC MEASUREMENTS}

The dc magnetic field-induced ultrasonic wave natural velocity changes were measured using a pulsed-phase-locked-loop (P2L2) system described in detail elsewhere (ref. 9). A block diagram of this system is shown in figure 1. The basis of the measurement is a phase feedback scheme using a voltage-controlled oscillator $(\mathrm{VCO})$. The VCO output is gated to produce a tone burst of several cycles to drive a broadband transducer. The received pulse is amplified and phase detected using the VCO as a reference. A logic system samples the phase signal at the preselected point and causes the frequency of the VCO to change until quadrature is achieved. Once locked, the P2L2 maintains the quadrature condition with the change in frequency related to the change in sample properties as follows :

$$
\Delta \mathrm{F} / \mathrm{F}=\Delta \mathrm{V} / \mathrm{V}-\Delta \mathrm{L} / \mathrm{L}
$$




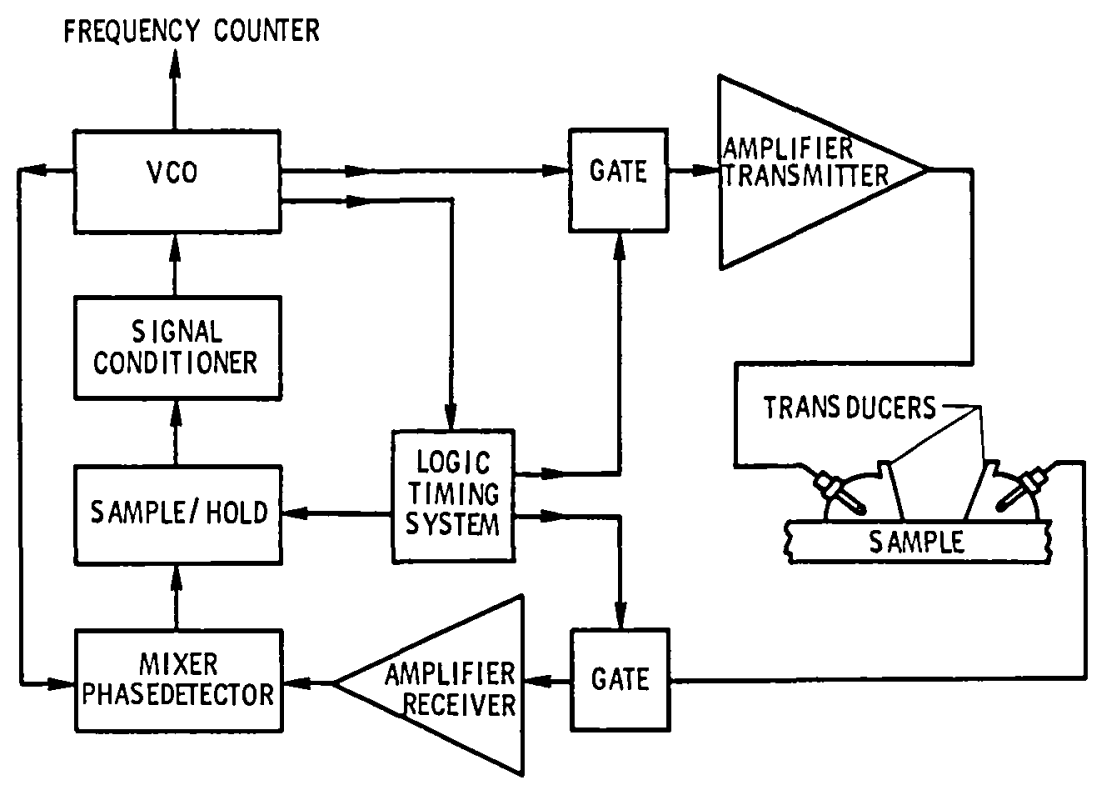

Figure 1. Block diagram of pulsed-phase-locked loop system.

where $V$ is the actual acoustic phase velocity and $L$ is the acoustic path length in the sample. $\Delta \mathrm{F} / \mathrm{F}$ is the fractional change in ultrasonic natural velocity. With the acoustic natural velocity, one does not have to measure the change in sample length during the measurement (refs. 10 and 11).

\section{PRELIMINARY EXPERIMENTS AND RESULTS}

Steel plates $(0.635 \mathrm{~cm} \times 5.08 \mathrm{~cm} \times 45.72 \mathrm{~cm})$ with unknown impurity composition were chosen to check the uniaxial stress effect on the domaindependent elastic modulus. Through this experiment surface waves propagated in the direction of the magnetic field which was parallel to the stress axis. A pair of longitudinal wave transducers, with a center frequency of $2.25 \mathrm{MHz}$, were mounted on variable angle blocks and used in the pitch-catch mode. Untaxial stress was applied to the specimen by four-point bending and the induced surface strain was measured with wire-type strain gauges. A major portion of the plate was magnetized by forming a closed magnetic circuit with a C-magnet. Each end surface of magnet was rounded to keep the contact area constant when the plate was bent. A Hall probe was inserted in a slit made on one of the C-magnet legs and the $\Delta F / F^{\prime} s$ were plotted against the measured field values.

To minimize any time-dependent effect, due to interstitial impurity migration or thermoelasticity, the following measurement procedure was employed. A sufficient relaxation time was allowed before the measurement at each stress level. The magnetic field was turned on and off while increasing the field strength to its maximum. The difference in the frequencies in the on and off states was divided by the off state value and taken as the $\Delta \mathrm{F}(\mathrm{H}) / \mathrm{F}$. After this, the specimen was demagnetized by a very low frequency ac field $(<\mathrm{Hz})$ with an exponentially decaying amplitude. 
The results are shown in figure 2 with the elastic surface micro-strain listed at the end of each curve. Here, the stress effect on $\Delta F(H) / F$ is clearly seen to lower its slope under compression and is opposite under tension. These measurements were repeated in many other specimens of the same material with the identical geometry. The position and the shape of curves varied somewhat from one specimen to another but the relative positions of curves and the stress effect were well reproducible.

The consistent effect of residual uniaxial stress on $\Delta F(H) / F$ was checked with permanently-bent plates (radius of curvature $=58 \mathrm{~cm}$ ) with the same initial geometry. It is well known from beam theory that the stretched side is under residual compression and the other side under residual tension when a plate is

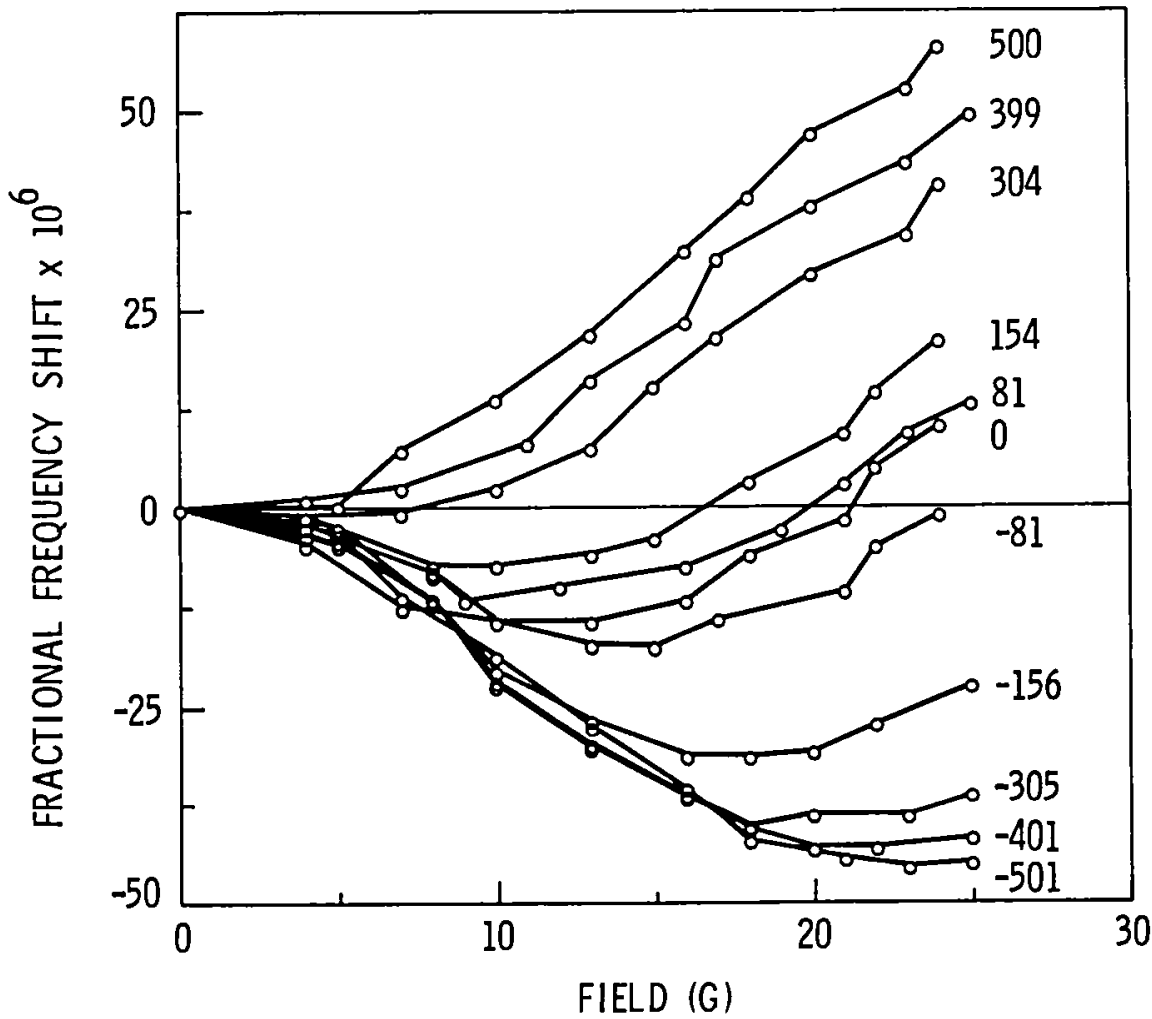

Figure 2. $\Lambda F(H) / F$ under applied tension and compression. Surface microstrain is labeled at each curve.

released after permanent bending with a finite radius of curvature. The results are shown in figure 3 for before and after bending. The curve labeled " $A$ " was taken in the surface stretched by bending and that labeled by " $B$ " was taken in the other surface. This result parallels the results for the applied stress state.

\section{MEASUREMENTS WITH CARBON STEELS AND RESULTS}

Steel samples with three carbon contents $(1020,1045$, and 1095) were used in this experiment. Two sets of samples were made for measurements with applied and residual uniaxial stress. 


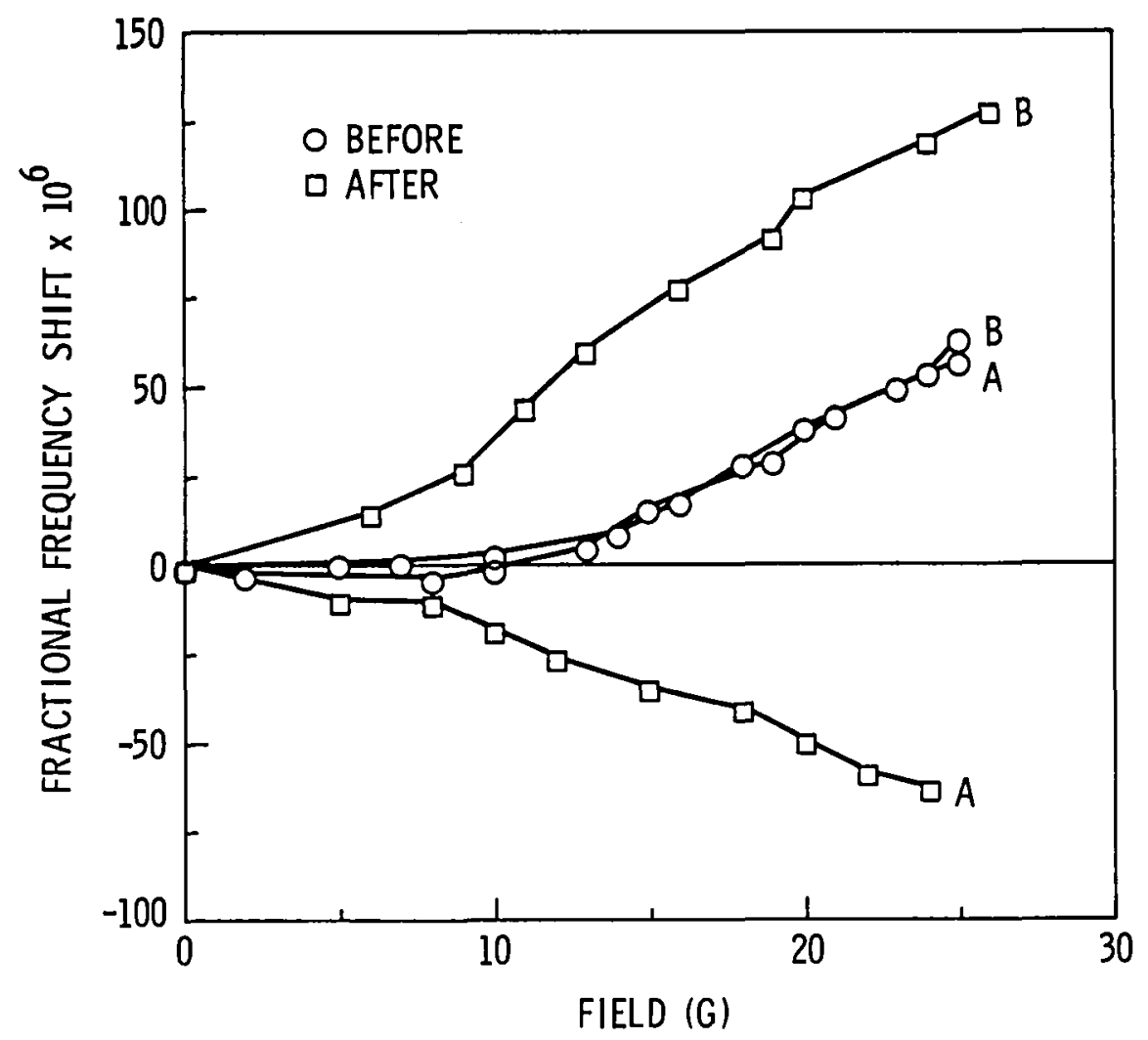

Figure 3. $\triangle \mathrm{F}(\mathrm{H}) / \mathrm{F}$ before and after permanent bending. For the square symbols, the label " $A$ " indicates the compressive surface and " $B$ " Indicates the tensile surface produced by permanent bending.

The samples were made from cylindrical rods of $2.54 \mathrm{~cm}$ in diameter and $30.3 \mathrm{~cm}$ in length. The center portion of these rods was machined to form a pair of parallel and flat surfaces $(1.8 \mathrm{~cm} \times 12 \mathrm{~cm})$. Both ends of each rod were threaded to fit into sample holders and placed in an INSTRON stress machine. Samples were magnetized lengthwise by a pair of water-cooled identical solenoids with C-shaped cores made of low carbon steel. Another set of samples were steel plates with the same carbon contents having dimensions of $3.8 \mathrm{~cm} \times 1.25 \mathrm{~cm} \times$ $30.3 \mathrm{~cm}$. Measurements were taken before and after permanently bending these plates with the radius of curvature $25.4 \mathrm{~cm}$. A specially designed C-magnet with rotatable arms was used to avold air gaps between the magnet ends and the curved surfaces. The total magnetic flux was measured by an integrating flux meter. The flux readings were callbrated with a big iron core magnet where the field Inhomogeneity was completely negligible for a region much larger than the dimension of the pickup colls.

The Raylelgh surface wave transducers with $5 \mathrm{MHz}$ and $2.25 \mathrm{MHz}$ center frequencies were used for rods and plates, respectively, in the pitch-catch mode. As before, ultrasonic waves propagated parallel to the field:applied in the stress axis. Measurements with external stress were taken in two different ways. First, the specimen was loaded to a desired stress level. Then, the magnetic field along the length of the specimen was monotonically increased to its maximum and decreased to zero while measuring $\Delta F / F$. At the end of this halfcycle measurement, the specimen was demagnetized. These measurements were 
repeated four or five times at each stress level. The previously mentioned discrete field on-off measurements were then made before stress was released. Such a sequence of measurement procedure was also used for the plates before and after permanent bending.

The continuous measurements exhibited a time dependence both when the external load was changed and in virgin specimens without load. The former was more pronounced and affected up to the first three measurements at each load level (each measurement took about 6 minutes) depending on the stress amplitude. This time dependence was caused by the combination of mechnical after effect (stressinduced directional ordering of elastic dipoles) (ref. 12), the magnetic after effect (mutual influence between ferromagnetic state and interstitial impurities) (ref. 13) and possibly the thermoelastic effect. The discrete measurements, on the other hand, gave almost time-independent results under any circumstances and the reproductbility was excellent. Hence, again the results with the discrete measurements will be presented here.

The results of $\triangle F / F$ in this section are plotted against the magnetic induction rather than internal magnetic field which is difficult to measure directly. Figures 4 and 5 show $\Delta F(B) / F$ in 1020 steel under external uniaxial

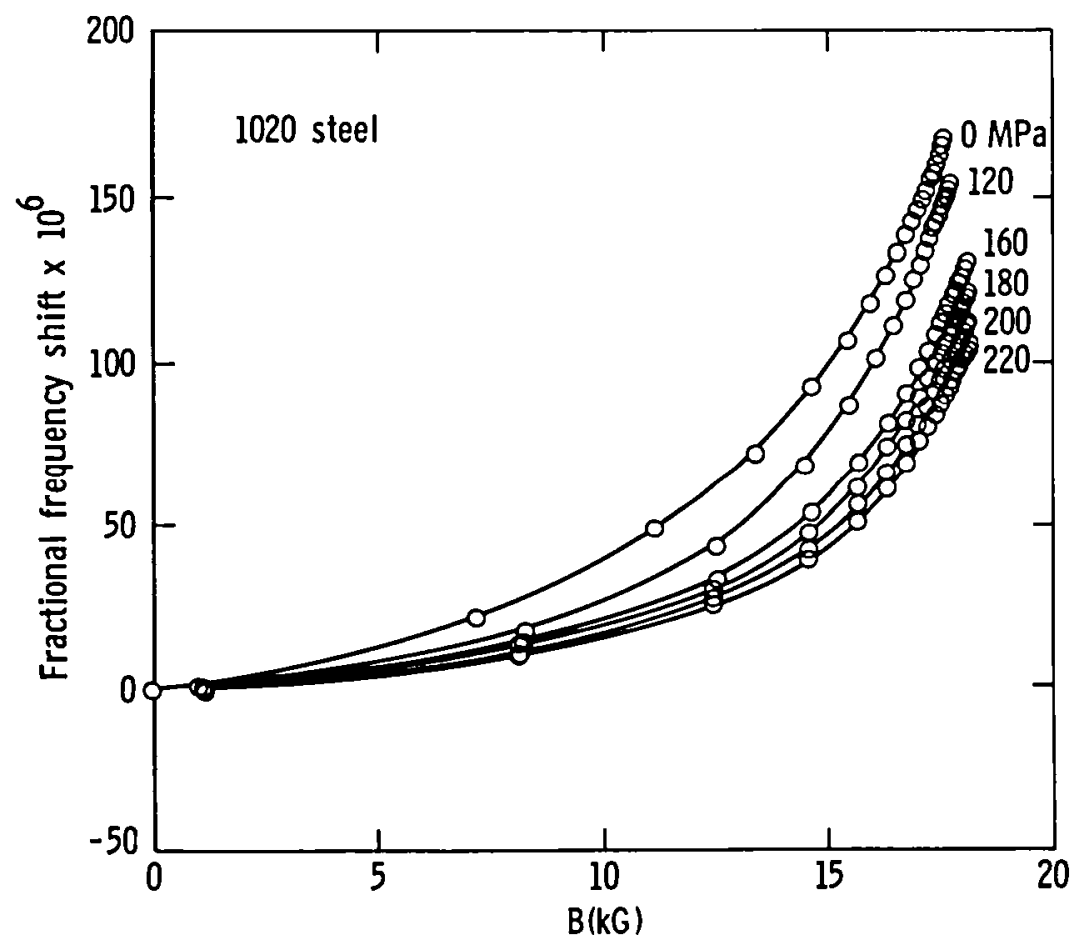

Figure 4. $\Delta F(B) / F$ in 1020 steel under applied tension.

tension and compression, respectively. Curves under high tension and those under low compression overlap. These curves move downward under tension but up to $220 \mathrm{MPa}$ the shape of curves remain very similar to that of curve without stress. Under compression, curves also move downward but, with increasing compression amplitude, their shape changes distinctively. At $-120 \mathrm{MPa}$ the negative initial slope begins to appear and 1 t becomes steeper under higher compression. 


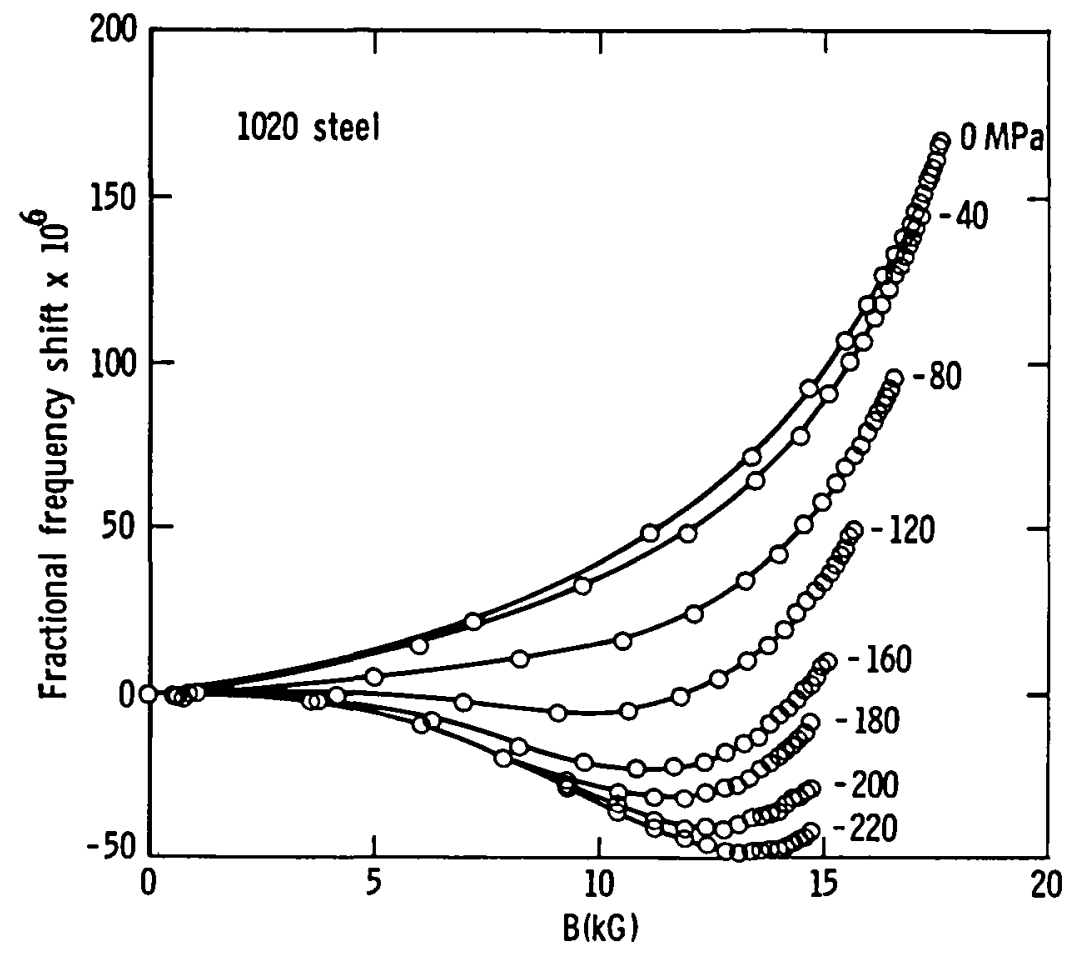

Figure 5. $\Delta \mathrm{F}(\mathrm{B}) / \mathrm{F}$ in 1020 steel under applied compression.

The results with 1045 rod are shown in figure 6. The tension curves in this specimen are distributed narrowly and all curves under $220 \mathrm{MPa}$ lie between the zero and maximum stress curves. Compression curves slightly overlap with tension curves and they spread downward with increasing compression magnitude. Here again, the negative initial slope begins to appear at $-120 \mathrm{MPa}$. Figure 7 shows the results with a 1095 rod. These results are very similar to those in the previous figures except that the negative initial slope of $\triangle F(B) / F$ begins to appear at a slightly higher value of compression.

The effect of tension on $\Delta F(B) / F$ has been seen to vary among different types of steels. This, as will be discussed later, depends on many metallurgical and structural properties of materials. The most important points are, however, the initial slope of $\Delta F(B) / F$ remains positive under tension and it only becomes negative under sufficient compression.

The consistent effect of residual stress was checked with permanently-bent plates with the same carbon contents. The measurements with the same plates before bending were made with an air core Helmholtz pair which was not able to magnetize properly the bent plates. Hence, the C-magnet with rotatable arms was used with the bent plates. Since total flux measured with the Helmholtz pair is different from the total flux in the plates only, measurements were repeated with other plates with the same carbon contents using the C-magnet. 


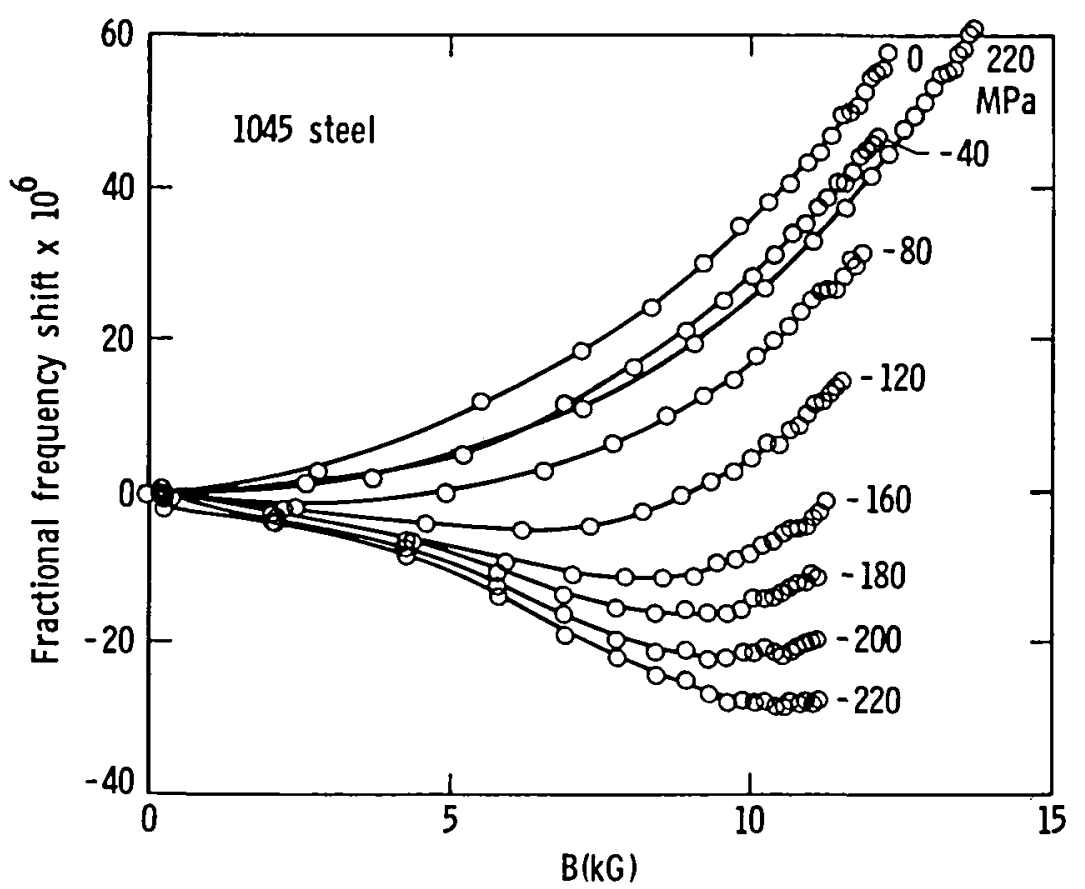

Figure 6. $\Delta F(B) / F$ in 1045 steel under applied tension and compression.

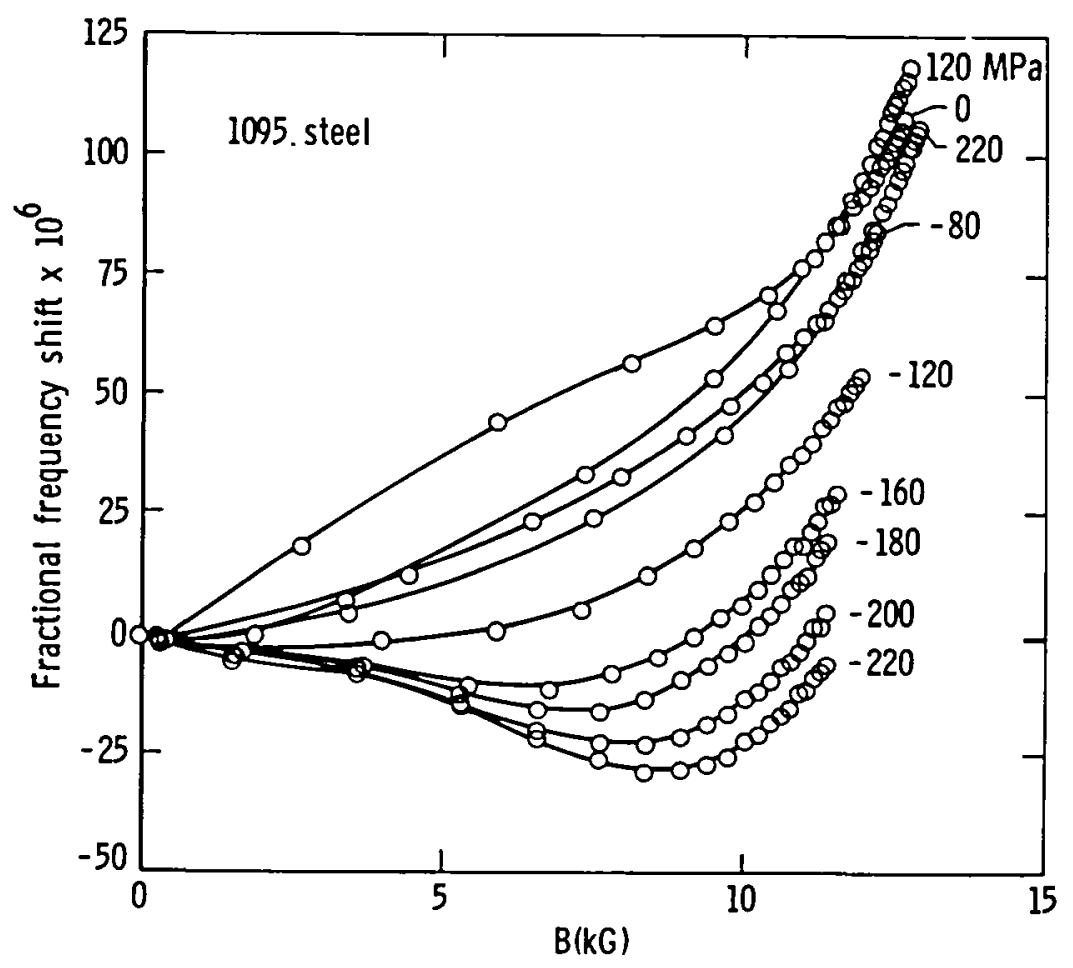

Figure 7. $\triangle F(B) / F$ in 1095 steel under applied tension and compression. 


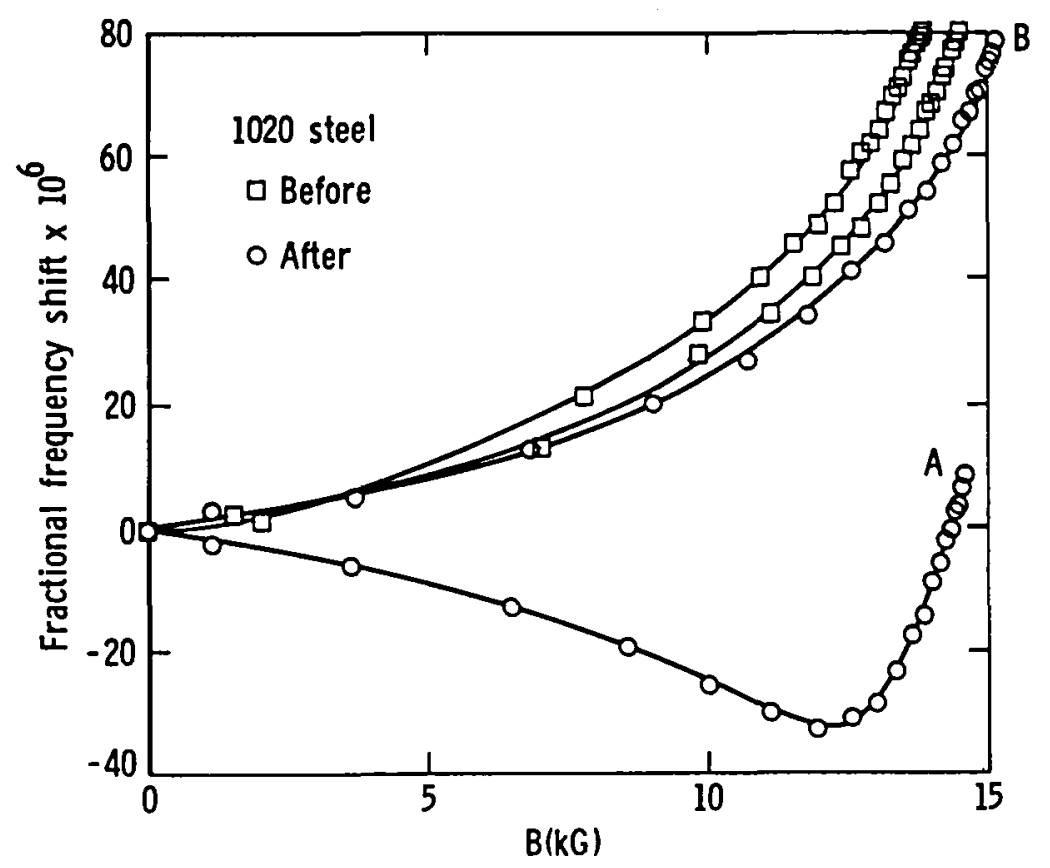

Figure 8. $\triangle F(B) / F$ in 1020 steel before and after permanent bending.

These results are shown in the next three figures for unbent and permanentlybent plates with the radius of curvature of $25.4 \mathrm{~cm}$. The stress effects on $\triangle F(B) / F$ generally follow what is expected from the results obtained with externally-applied uniaxial stress. In the 1045 plate the compression curve is almost a straight line passing through zero in the vertical axis indicating a very weak magnetization in that surface region. This is because magnetization depends on the stress state at each point in the material and it can be far lower in the surface under compression than that averaged over the sample cross section (ref. 7).

\section{DISCUSSION}

The presence of domain magnetization causes anisotropy of ultrasonic wave propagation in a crystalline solid (ref. 14). This is because the

ferromagnetic state is always accompanied by the spontaneous distortion of unit cells uniquely determined by the orientation of magnetization vector. This means that the wave velocity differs in different magnetic domains and in individual grains of a polycrystalline material. Hence, studies on the magnetoacoustic interaction have been limited to single crystalline materials beyond technical saturation. This certainly is not the present situation where we are interested in the magnetoacoustic phenomena during the domain wall motion only. For a qualitative discussion, however, the stress effect on $\Delta F(B) / F$ can be treated almost trivially by considering the following facts:

1. Uniaxial stress changes the domain structure in iron, and also in steel, only by domain wall motion. 


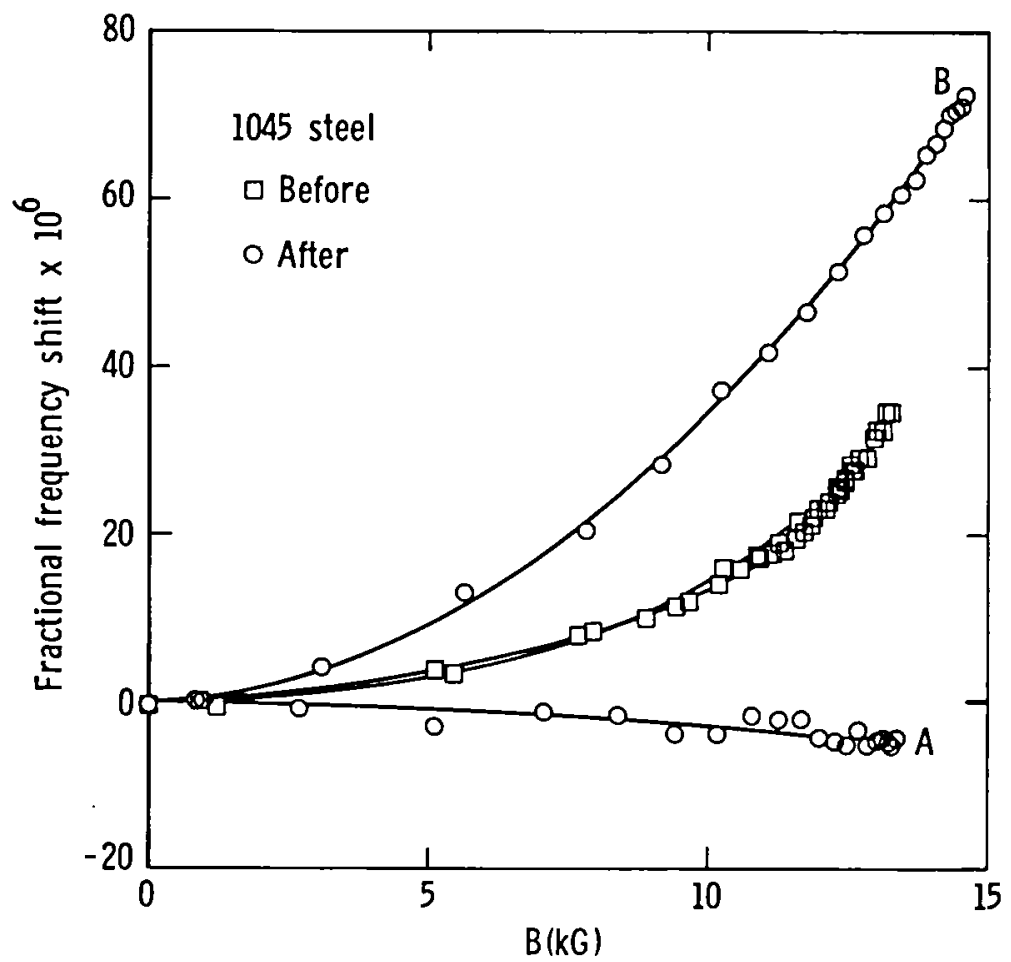

Figure 9. $\Delta F(B) / F$ in 1045 steel before and after permanent bending.

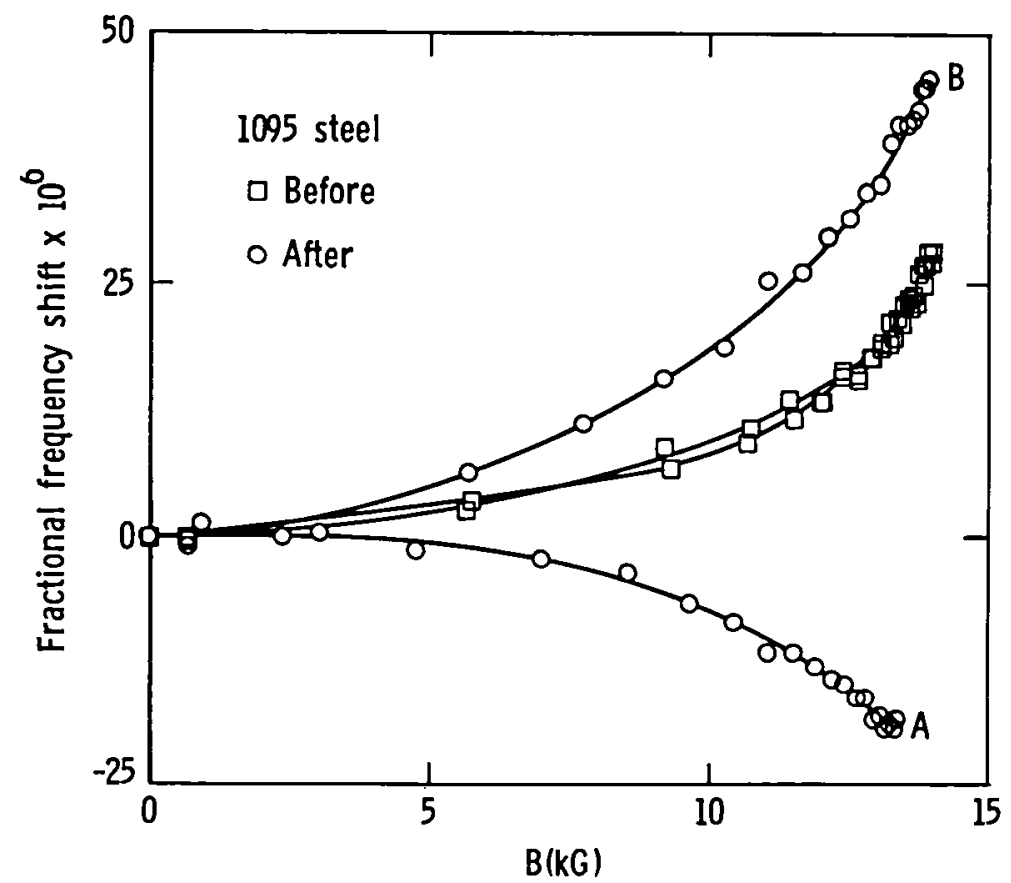

Figure 10. $\Delta F(B) / F$ in 1095 steel before and after permanent bending. 
2. The portion of $\Delta F(B) / F$ curves giving the stress information is solely contributed by field-induced domain wall motion.

Therefore, the discussion will focus on the slope of $\triangle F(B) / F$ with all the domains oriented in the easy axes of iron which are the six equivalent directions of $\langle 100\rangle$.

The mathematical description of magnetoelastic interaction gives the energy density in a domain subjected to uniaxial stress. This energy density is thermodynamically equivalent to a hydrostatic pressure acting on the domain boundary. The domain wall separating two domains feels a net pressure which is the difference in the magnetoelastic energy densities in the neighboring domains (ref. 15). As a result, the energetically-favored domains expand their volume in expense of less favored nelghboring ones by moving the domain wall. Among the two types of domaln walls in iron or steel, only 90-degree walls are moved by uniaxial stress while the positions of 180-degree walls are unaffected. Such a stressinduced 90-degree wall motion is resisted by various lattice imperfections (refs. 16 and 17 ).

Under sufficient tension, the majority of domain walls are those 180-degree walls close to the stress axis with a fraction of residual 90-degree walls remaining in the material. As the fleld is applied in the stress axis, the domains oriented close to the field axis expand their volume by moving both 180and 90-degree walls. The mobility of 180-degree walls is much higher than that of 90-degree walls not only because of the higher Zeeman energy difference across the walls but also the interaction with lattice imperfections is usually much less for 180-degree walls (ref. 18). Under sufficient compression, domains align perpendicular to the stress axis and the majority of walls are 180-degree walls. With the fleld applied in the stress axis, there is no Zeeman energy difference across 180-degree walls and only the residual 90-degree walls move. At the same time, the total area of 90-degree wall in the material grows. The area of 90-degree walls continues to grow until it reaches its maximum. After this point, as the magnetization proceeds, 90-degree walls begin to decrease and eventually vanish.

It has been already mentioned that the magnetoelastic strain is produced by aligning the longer side of iron unit cells into the applied tensile stress axis and is always positive. This, of course, is possible only by moving 90-degree domain walls. Hence, it is apparent that the presence of 90-degree walls effectively lowers the elastic modulus of the material.

The appearance of the negative slope of $\triangle F(B) / F$ in the early stage of magnetization along the uniaxial compressive stress axis is, therefore, well explained on the basis of initial domain alignment and the state of 90-degree walls. These, however, imply that curves under tension should be lower than that without stress because of less 90-degree walls to be removed by the field. This was seen at every stress level in 1020 and under high stress in other steels. The degree of stress-induced domain alignment, above al1, depends on many different material characteristics, such as saturation magnetization, spontaneous magnetostriction, local lattice strain and stress amplitude. There is a possibility that if the stress-induced domain alignment is difficult in a material then tensile stress merely helps the external field, in its own axis, remove 90-degree walls. Thus, the detailed shape of $\triangle F(B) / F$ curve depends on many different factors. The overall effect of sufficient compression causing the negative initial slope of $\Delta F(B) / F$, however, should be common in all materials of this type. 
In this paper, we presented clear experimental evidence of a technique capable of differentiating the effect of uniaxial compression from that of others both for applied and residual stresses. The Initial slope of natural velocity change in Rayleigh surface waves is negative under conpression and is positive otherwise when steel specimens are magnetized in the stress axis. The physical basis of this phenomena is provided by a simple model description. This is consistent with the results obtained in steels covering a fairly wide range of carbon contents. Hence, the effects of detailed material properties on the obtained stress information can be treated as secondary. 


\section{REFERENCES}

1. Namkung, M. and Heyman, J. S., Residual Stress Characterization with an Ultrasonic/Magnetic Technique, Nondest. Test. Comm., Vol. 1, 1984, pp. 175-186.

2. Namkung, M. and Heyman, J. S., Residual Stress Characterization with a Magnetic/Ultrasonic Technique, Proceedings of IEEE Ultrasonic Symposium, Nov. 1984, Dallas, Texas.

3. Kittel, C., Physical Theory of Ferromagnetic Domains, Rev. Mod. Phys., Vol. 21, 1949, pp. 541-584.

4. Namkung, M., A $\mu$ SR Study of Unlaxlal Stress Induced Symmetry Breaking in an Iron Single Crystal, Ph.D. Thesis, College of William and Mary, Oct. 1982.

5. Bozorth, R. M., Ferromagnetism, Van Nostrand, New York, 1951.

6. Dijkstra, L. J. and Martius, U. M., Domain Pattern in Silicon Iron Under Stress, U. M., Rev. Mod. Phys., Vol. 25, 1953, pp. 146-150.

7. Cullity, B. D., Introduction to Magnetic Materials, Addison-Wesley, Menlo Park, 1972.

8. Yamamoto, $M$. The $\Delta E$ Effect in Iron-Nickel Alloys, Sci. Rep., Tohoku Univ., Vo1. A11, 1955, pp. 102-119.

9. Heyman, J. S., Allison, S. G., and Salama, K., Influence of Carbon Content on Higher Order Ultrasonic Properties of Steel, Proceedings of IEEE Ultrasonic Symposium, Nov. 1983, Atlanta, Georgia.

10. Thurston, R. N. and Brugger, K., Third-Order Elastic Constants and the Velocity of Small Amplitude Elastic Waves in Homogeneously Stressed Media, Phys. Rev., Vol. 133, 1964, pp. Al604-1610.

11. Cantre11, J. H., Jr., Anharmonic Properties of Solids from Measurements of the Stress Acoustic Constant, J. Test. Eval., Vol. 10, No. 5, 1982, pp. 223-229.

12. Nowick, A. S. and Berry, B. S., Anelastic Relaxation in Solids, Academic Press, New York, 1972.

13. De Vries, G., Van Geest, D. V., Gersdorf, R., and Rathenau, G. W., Determination of the Magnetic Anisotropy Energy Caused by Interstitial Carbon or Nitrogen in Iron, Phisica, Vol. 25, 1959, pp. 1131-1138.

14. Alers, G. A., Neighbours, J. R., and Sato, H., Dependence of Sound Velocity and Attenuation on Magnetization Direction in Nickel at High Fields, J. Phys. Chem. Solids, Vol. 9, 1958, pp. 21-27.

15. Brown, W. F., Jr., Irreversible Magnetic Effects of Stress, Phys. Rev., Vo1. 75, 1949, pp. 147-154. 
16. Morrish, A. H., The Physical Principles of Magnetism, John Wiley and Sons, New York, 1965.

17. Craik, D. J., Structure and Properties of Magnetic Materials, Pion Limited, London, 1971.

18. Trauble, H., The Influence of Crystal Defects on Magnetization Processes in Ferromagnetic Single Crystals, in Magnetism and Metallury, Vol. 1, Edited by Berkowitz, A. E. and Kneller, E., Academic Press, New York, 1969. 


\title{
ULTRASONIC MEASUREMENT OF POROSITY IN CASTS AND WELDS
}

\author{
Laszlo Adler and Shaio-Wen Wang \\ The Ohio State University \\ Columbus, Ohio 43210
}

Porosity, i.e. voids and gas bubbles, of casting and welds significantly effect the mechanical properties and acceptability. Porosity may occur in castings via two major mechanisms (ref. 1), (a) gas evolution or capture during solidification and (b) shrinkage during solidification. While nondestructive techniques, such as ultrasonic methods are available and used for the detection and characterization of large (i.e. greater than $1 \mathrm{~mm}$ ) pores in many alloys, only destructive techniques are currently available to quantitatively characterize smaller pores in cast alloys. This paper describes the development of a quantitative nondestructive method which involves ultrasonic attenuation measurements in frequency domain to determine volume fraction of porosity in aluminum cast. The aluminum alloy A357 casting samples were produced at The Ohio State University Foundry with controlled porosity contents ranging from $0 \%$ to $6 \%$. A computer controlled system was used to direct ultrasonic beam to a test sample to different places to conduct ultrasonic attenuation measurements. The plot of attenuation coefficients as a function of frequency was then evaluated based on existing theories to determine volume fraction of porosity and pore size.

\section{SAMPLE PREPARATION}

The A357 aluminum alloy used in this study has a composition (weight \%) given below (ref. 2).

$\begin{array}{cccccccccc}\mathrm{Si} & \mathrm{Fe} & \mathrm{Cu} & \mathrm{Mn} & \mathrm{Mg} & \mathrm{Zn} & \mathrm{Ti} & \mathrm{Be} & \text { others } & \mathrm{A} 1 \\ 6.5-7.5 & .2 & .2 & .1 & .40-.70 & .1 & .10-.20 & .04-.09 & .15 & \text { ba1- } \\ \text { ance }\end{array}$

This alloy has a melting and freezing range of about $50 \mathrm{C}$ (Solidus $=557 \mathrm{C}$ and Liquidus $=612 \mathrm{C})$. Various techniques were used to produce A357 aluminum alloy casting to have porosities ranging from $0 \%$ to $6 \%$ by volume.

In order to produce zero gas content samples which could be used as zero gas content standards, the melting, pouring and solidification were performed in an evacuated chamber at a pressure of about $1 \mathrm{~mm}$ mercury. After successfully producing low gas content (i.e. less than $0.2 \%$ gas voids by volume) samples via the vacuum casting experiments, castings were made in which varying amounts of gas porosity were introduced throughout variations in the melting environment and mold conditions. The A357 alloy was melted in air in both induction and gas-fired crucible furnaces. For some samples, moisture was deliberately introduced to the melt and/or casting by placing a moist towel on top of the melt, placing moisture on the mold surface, or using a moist brick as the support for the permanent mold and allowing contact be- 
tween the liquid alloy and the noist brick base.

Samples were then sectioned fron each casting at various locations and inilled to forn a regular parallel pipe. The side dinensions and weight were neasured to obtain density for each sample. The volume average porosity based on the density measureinents was then calculated using the equation:

$$
\text { Porosity } \%=\left(\rho_{\text {the }}-\rho_{\text {measured }}\right) / 100
$$

where the theoretical density of the A357 aluninum alloy was taken as $2.667 \mathrm{~g} / \mathrm{cc}$. Two faces of the sample were then polished and photonicrographed. The Inicrographs provide representative statinents of the porosity in the sample (Fig. 1) and also could determine the porosity quantitatively. The areas of pures in the picture was cut and both the removed sections and total section were weighted. Porosity was then deternined by

Porosity $\%=$ (weight of pures/weight of total section) $\times 100$

Only samples which have agreeable porosities outcome from both density and photomicrograph were used in ultrasonic study.
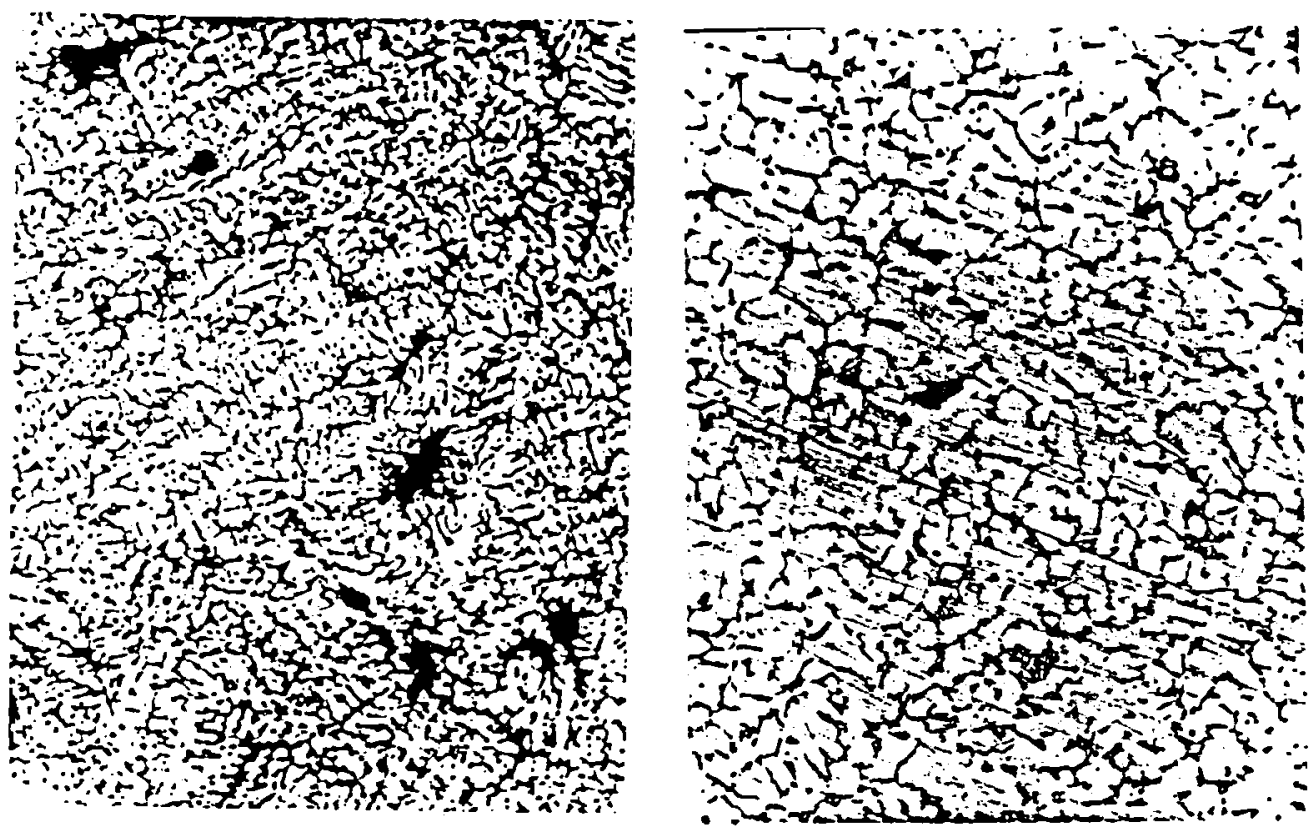

FIG. 1. Metallography: Cast AI

\section{THEORETICAL CONSIDERATIONS}

A theoretical approach to use ultrasonic scattering in porous naterials has recently been formulized by Gubernatis and Domany (ref. 3) and by Rose (ref. 4). They calculate the attenuation coefficient of elastic waves due to scattering from pores. The assumption is that the pores are far apart that no inultiple scattering will take place. The attenuation coefficlent is calculated as a function of frequency for various concentrations of pores. 
In the Rayleigh region where the wavelength $(\lambda)$ is inuch larger than the pore size (a), the attenuation $(\alpha)$ is proportional to the third power of the frequency while in the diffusive region $(\lambda<<a)$, i.e. for high frequencies the attenuation coefficlent is independent of the frequency. There is, however, a connecting region at $\mathrm{k}_{\mathrm{O}} \mathrm{a} \simeq 1$, where $\mathrm{k}$ is the wave number, is a turning point. At that point the attenuation coefficient $\alpha \simeq k_{0} C K$. $C$ is the volume fraction of porosity and $k$ is dependent on the elastic constants of the host inaterials.

The behavior of the predicted frequency dependent attenuation curve is shown on Fig. 2. In the next section, the experinental systern and procedures will be described to obtain such frequency dependent attenuation curves in aluminum cast naterials.

\section{EXPERIMENTAL SYSTEM AND PROCEDURE}

The aluminum cast naterials were machined to rectangular blocks on inch thick and a surface area nuch larger than the diameter of the transducer. The aluminum cast block is subinerged in a water bath. A broadband transducer is placed in its desired position in the water bath by rotation around two perpendicular axes situated in a horizontal plane.

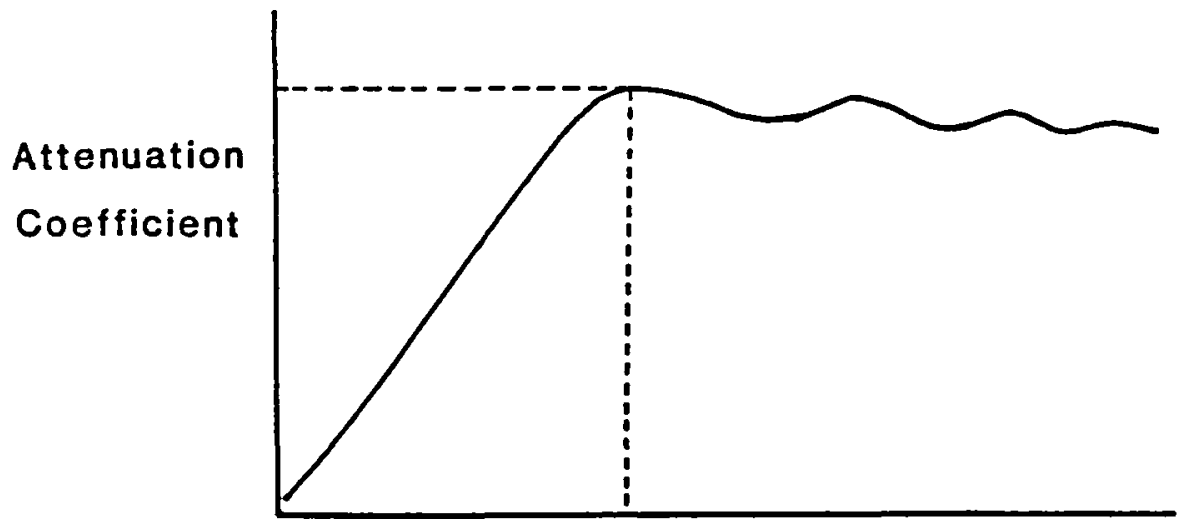

Frequency

Fig. 2. Theoretical plot of attenuation as a function of frequency for inhomogeneous material.

\section{U1trasonic Spectroscopy Systen}

The ultrasonic spectroscopy system, which is schenatically displayed in Fig. 3, is assembled around a PDP II/34A minicomputer. A broadbandwidth ultrasonic pulse is produced by exciting an untuned ceranic transducer with a fast rise-tine, high-voltage pulse. Reflected signals are received by the sane transducer (pulse-echo configuration). The electrical pulse generated by the received waveform is filtered and amplified.

The time domain signal can either be fed to a conventional spectruin analyzer or be sampled and converted into digital data to be processed by a conputer. For processing by the spectruin analyzer, a stepless gate is used to select a portion of the received signal. The receiver output as well as the gated waveform are displayed. The anplitude spectrum of the gated waveform is displayed on a spectrum analyzer. 


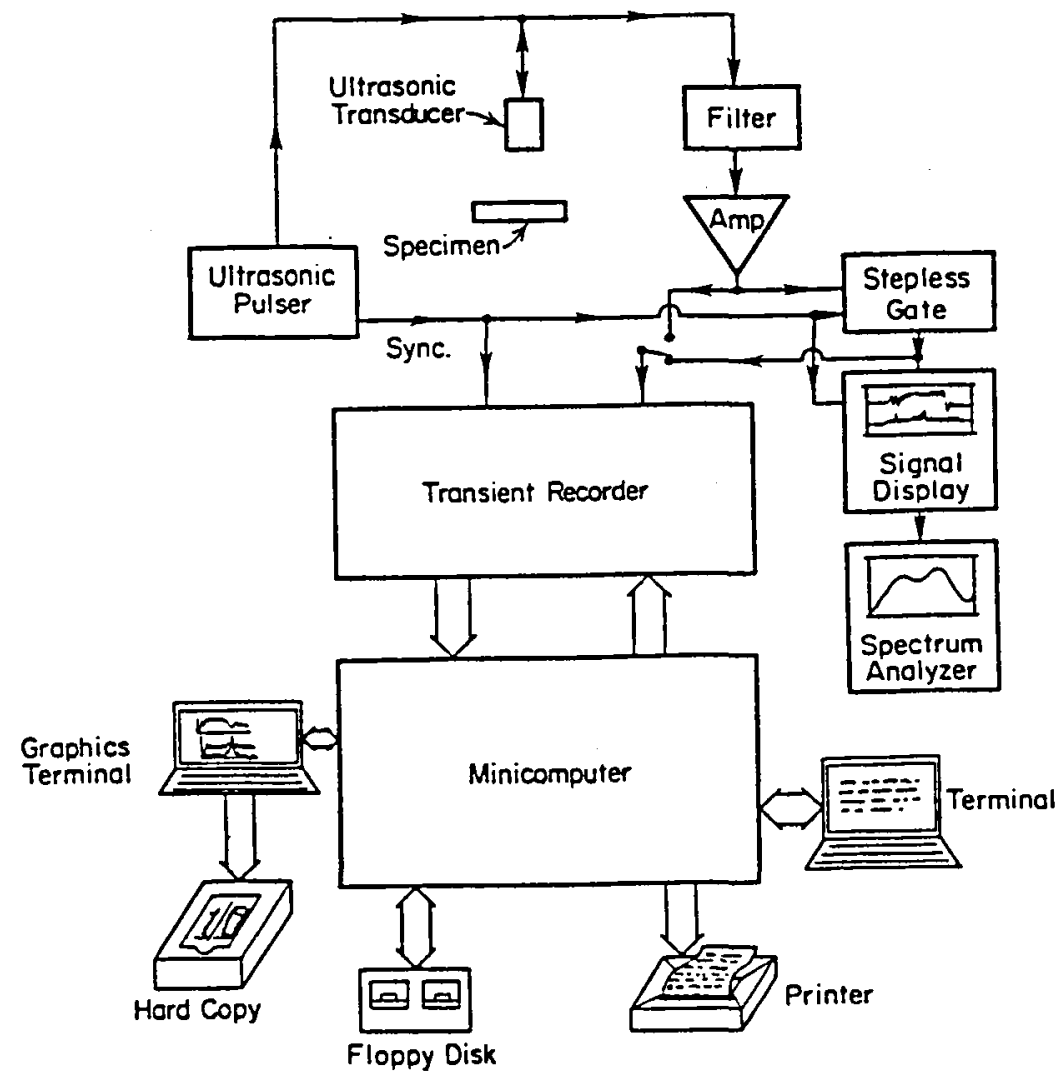

FIG. 3 Scheinatic diagram of the ultrasonic spectroscopy systen.

For conversion to digital data, a high-speed transient recorder is used to store the signal amplitude at discrete times in its digital memory. The computer controls the acquisition of the ultrasonic pulse data and then transfers the digitally represented signal from the recorder to the minicomputer menory. The RF signal data nay be permanently recorded on floppy disks. Processing of the ultrasonic signal is performed on the ininicomputer and includes the following operations: gating, autocorrelation, averaging, Fourier transform (Fast Fourier Transforn procedure), deconvolution and plotting. Plots in the time domaln as well as in the frequency domain are displayed on a graphics terininal. Displays may be recorded permanently by utilizing a hard copy unit.

\section{Attenuation Measurements and Results}

To obtain attenuation coefficients as functions of frequency of the given sample, a deconvolution process was used. The spectrum of a normally reflected signal from the back surface of the sample was deconvolved with the spectrum obtained from the front surface. This procedure is shown schernatically on Fig. 4 . On Fig. $4 \mathrm{a}, \mathrm{b}, \mathrm{c}$ the front, the back and the deconvolved spectruin is shown respectively. Correction for other losses such as bean spread and interface losses are also incorporated into the program to obtain the attenuation/unit length as a function of frequency.

The experinental results of the averaged ultrasonic attenuation coefficient as a function of frequency were displayed in Fig. 5 for low, mediun and high porosity samples. The location of the turning point was then used to determine the mean pore 
and the porosity contents from the following equations:

$$
\begin{array}{ll}
\text { Pore size: } & a=(V / 2 \pi) \cdot\left(1 / f_{p}\right) \\
\text { Porosity: } & c=(V / 2 \pi \cdot K) \cdot\left(\alpha_{p} / f_{p}\right)
\end{array}
$$

where $V$ is the longitudinal velocity of each sample, $K$ is a constant which depends on the inaterial used, $f_{p}$ and $\alpha$ are the frequency and the attenuation coefficient corresponding to the location of the turning point. Table 1 shows the calculated results. The pore radii are close to those obtalned from photonicrographs. As to the porosity contents, the results from ultrasonic experinents agree well with those from density measurements for low and inediun porosity samples. For samples with high porosity such as cast AI 1920, the agreenent is off a little bit. This may be caused by a inultiple scattering due to the existence of nore pores, which contradict the theoretical assuintion of individual scattering.

\section{Broad Bano}

\section{Transducer}
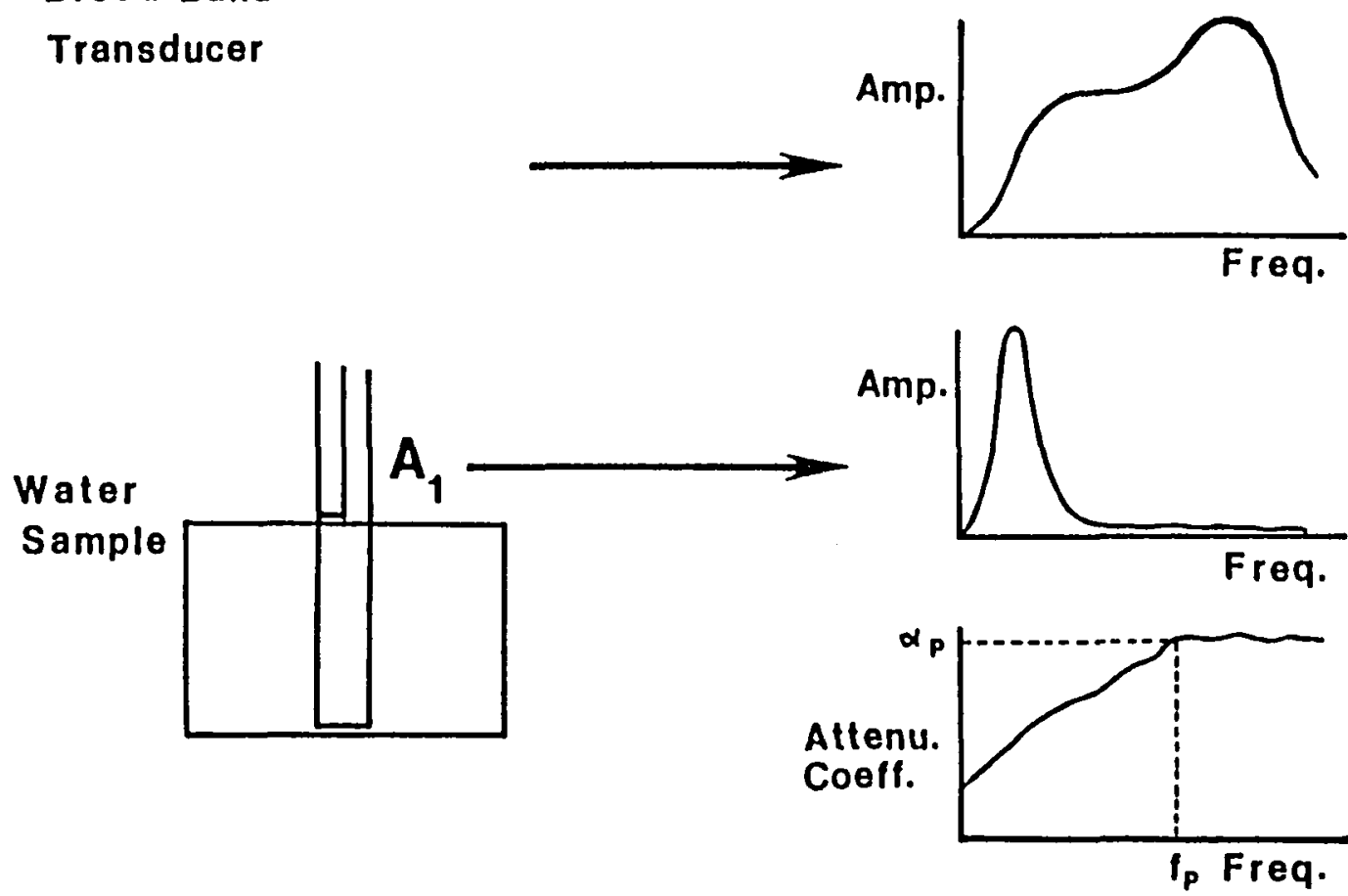

FIG. 4. Ultrasonic Determination of Porosity. 
Table 1. Experimental Results of Porosity and Pore Size from Ultrasonic Measurements.

Sample

Cast Al 013

Cast Al 1010

Cast A. 1210

Cast Al $1410 \mathrm{H}$

Cast AI 141UL

Cast A1 1510

Cast $A 11810$

Cast A1 1820

Cast Al 1830

Cast Al 1850

Cast AI 1920

\section{Attenuation}

Coefficient

(NP/cm)
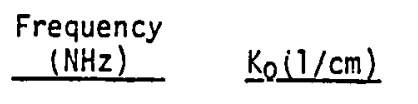

Pore
Radius (um)

Exp.

Porosity

Density

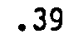

15

.22

.20

12

8

13

10

.20

1.12

9

1.00

10

1.25

12

1.20

11.5

1.00

11

2.85
142

114

76

123

95

85

95

114

109

104

76
70

87

131

80

105

116

105

87

91

$9 b$

131

$\begin{array}{cl}.24 & -.- \\ .17 & 0 \\ .23 & .22 \\ .74 & 1.3 \\ .19 & 1.3 \\ 1.18 & 2.18 \\ .95 & .9 \\ .99 & 1.05 \\ .99 & 1.20 \\ .86 & 1.08 \\ 3.4 & 4.6\end{array}$




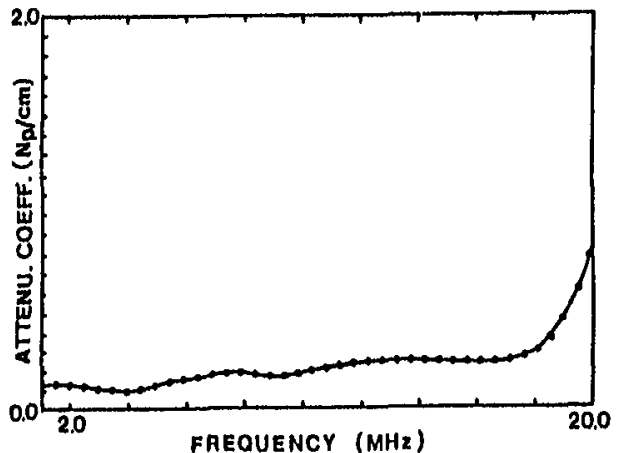

(a)

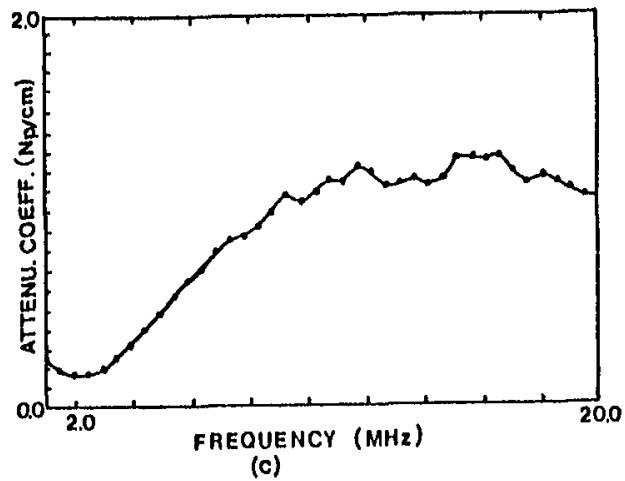

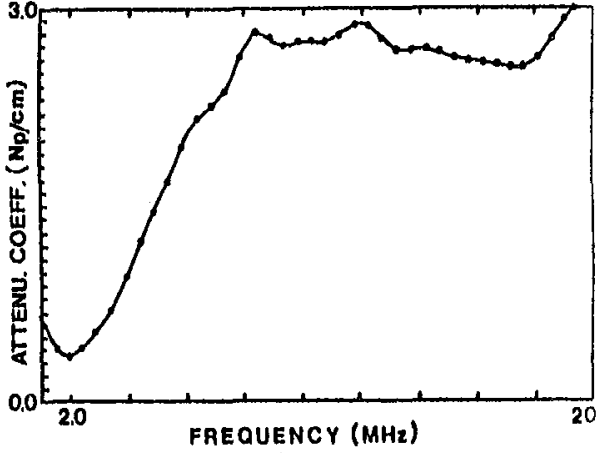

(b)

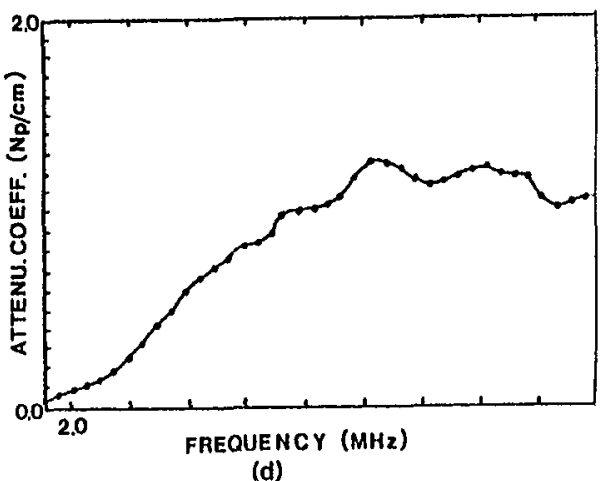

(d)

FIG. 5 Attenuation Coefficient as a function of frequency for cast AI Samples: (a) 1010 , .2\% porosity; (b) $1920,5 \%$ porosity; (c) $1 \%$ porosity; (d) 1830. $1 \%$ porosity.

\section{REFERENCES}

1. Pisonka, T.S. and Flemings, M.C., "Pore Formation in Solidification:, Trans. Met. Soc., AIME, Vol. 236, August 1966, p. 1157.

2. Standards for Aluninum Sand and Permanent Mold Castings, 8th ed., Aluminum Association, 1980.

3. Gubernatis, J.E. and Donany, E., "Effects of Microstructure on the Speed and Attenuation of Elastic Waves: Some Results for Porous Materials, "in Review of Progress in Quantitative Nondestructive Evaluation (1983), ed. by D.O. Thompson and D.E. Chinenti.

4. Rose, Janes H., "Ultrasonic Characterization of Porosity: Theory", to be published in Review of Progress in Quantitative Nondestructive Evaluation 1984.

* This work was sponsored by the Center for Advanced Nondestructive Evaluation, operated by the Anes Laboratory, USDOE, for the Aire Force Wright Aeronautical Laboratories/Materials Laboratory under Contract No. W-7405-ENG-82 with Iowa State University. 



\title{
ANALYTICAL ULTRASONICS FOR CHARACTERIZATION OF METALLURGICAL
}

\section{MICROSTRUCTURES AND TRANSFORMATIONS}

\author{
Moshe Rosen \\ The Johns Hopkins University \\ Baltimore, Maryland 21218
}

\begin{abstract}
Application of contact (piezoelectric) and noncontact (laser generation and detection) ultrasonic techniques for dynamic investigation of precipitation hardening processes in aluminum alloys, as well as crystallization and phase transformation in rapidly solidified amorphous and microcrystalline alloys will be discussed. From the variations of the sound velocity and attenuation the precipitation mechanism and kinetics were determined. In addition, a correlation was established between the observed changes in the velocity and attenuation and the mechanical properties of age-hardenable aluminum alloys. The behavior of the elastic moduli, determined ultrasonically, were found to be sensitive to relaxation, crystallization and phase decomposition phenomena in rapidly solidified metallic glasses. Analytical ultrasonics enables determination of the activation energies and growth parameters of the reactions. Therefrom theoretical models can be constructed to explain the changes in mechanical and physical properties upon heat treatment of glassy alloys. The composition dependence of the elastic moduli in amorphous $\mathrm{Cu}-\mathrm{Zr}$ alloys was found to be related to the glass transition temperature, and consequently to the glass forming ability of these alloys. Dynamic ultrasonic analysis was found to be feasible for on-line, real-time, monitoring of metallurgical processes.
\end{abstract}

\section{INTRODUCTION}

Ultrasonic nondestructive evaluation has traditionally been concerned with the search and location of flaws in materials structures and the determination of their distribution and orientation. A considerable body of knowledge has also been accumulated from ultrasonic scattering studies in assessing grain size and orientation effects in materials. Recently, it has become widely recognized that ultrasonic measurements can be used to characterize materials structures and hence properties such as strength, toughness, effect of residual stresses so as to supplement, or even replace, the conventional destructive techniques employed in metallurgy. Ultrasonic nondestructive characterization offers distinct advantages in that materials properties can be verified on actual components of engineering structures. The scientific literature is extremely scarce in dynamic nondestructive characterization (NDC) whereby ultrasonic techniques are applied to on-line, real-time, monitoring of microstructures for the control of metallurgical processes. Inherent difficulties related to these types of measurement methodologies include operation at elevated temperatures and in hostile environments. Furthermore, there 
are little basic data available on the relationship between the metallurgical microstructures and measured ultrasonic responses. Therefore, the full potential of ultrasonic techniques in this field is yet to be realized.

Recent studies have addressed the specific area of dynamic, real-time nondestructive characterization of metallurgical microstructures, e.g., precipitation hardening of aluminum alloys and crystallization of amorphous alloys (ref. 1-5). Conventional bulk ultrasonic techniques were adapted for application to continuously monitor changes of the microstructure and the effect on physical properties. However, in attempting to dynamically characterize metallurgical processes in amorphous ribbons or structurally modified thin surface layers, the conventional ultrasonic techniques were found to be inadequate. Thus, a new approach for contactless generation and detection of acoustic waves, using a laser generation and laser interferometric detection system, was developed (ref. 6).

\section{ULTRASONIC WAVES: GENERATION AND DETECTION TECHNIQUES}

The relationship between experimentally determined sound wave velocities (longitudinal and transverse) and the elastic moduli, is expressed in terms of the equations of motion of elastic waves in a solid, and Hooke's law for an isotropic medium.

Thermodynamic formalism shows that a solid should exhibit an anomalous elastic behavior ("softening effect") in the vicinity of a phase transformation point. Anomalous changes in the elastic moduli are expected to occur, to a different degree and magnitude, in the proximity of metallurgical reactions involving precipitation processes, segregation of solute, magnetoelastic effects accompanying magnetic ordering and other phenomena.

The propagating sound waves in the solid undergo a series of loss processes due to scattering by reflection and refraction from grain and phase boundaries, thermoelastic losses including elastic anisotropy effects in polycrystals (Zener effect), as well as attenuation of the sound wave energy due to interaction of the proagating wave with electrons and phonons. Interaction with dislocations in the crystal lattice is also responsible for the observed sound-wave damping effects. In instances where dislocation movement plays a prominent role, e.g., in martensitic phase transformations, the behavior of the ultrasonic attenuation may contain important information with respect to the transformation mechanism. During precipitation hardening of 2024 aluminum alloy the main contributions to observed changes in attenuation ( $r$ ef. 1) were found to be due to geometric scattering effects by newly formed precipitates of a certain critical size distribution, as well as to resonant interaction between the propagating sound waves and the dislocation loops generated around precipitates.

For bulk ultrasonics, where the wavelength of the propagating waves is much smaller than the specimen dimensions, the velocity and attenuation of ultrasound in specimens can be determined through the use of a pulse-echo overlap technique (ref. 7). The ultrasonic transit time, between the opposite faces of a flat and parallel sample, is determined by overlapping two successive members of an echo train on an oscilloscope display. This method, by stroboscopic identification, allows the time interval between pulses to be determined to within 1 part in $10^{4}$. The logarithmic decay of the amplitude of successive pulses determines the ultrasonic attenuation. The apparatus for measuring both velocity and attenuation is shown in figure 1 . 
VELOCITY

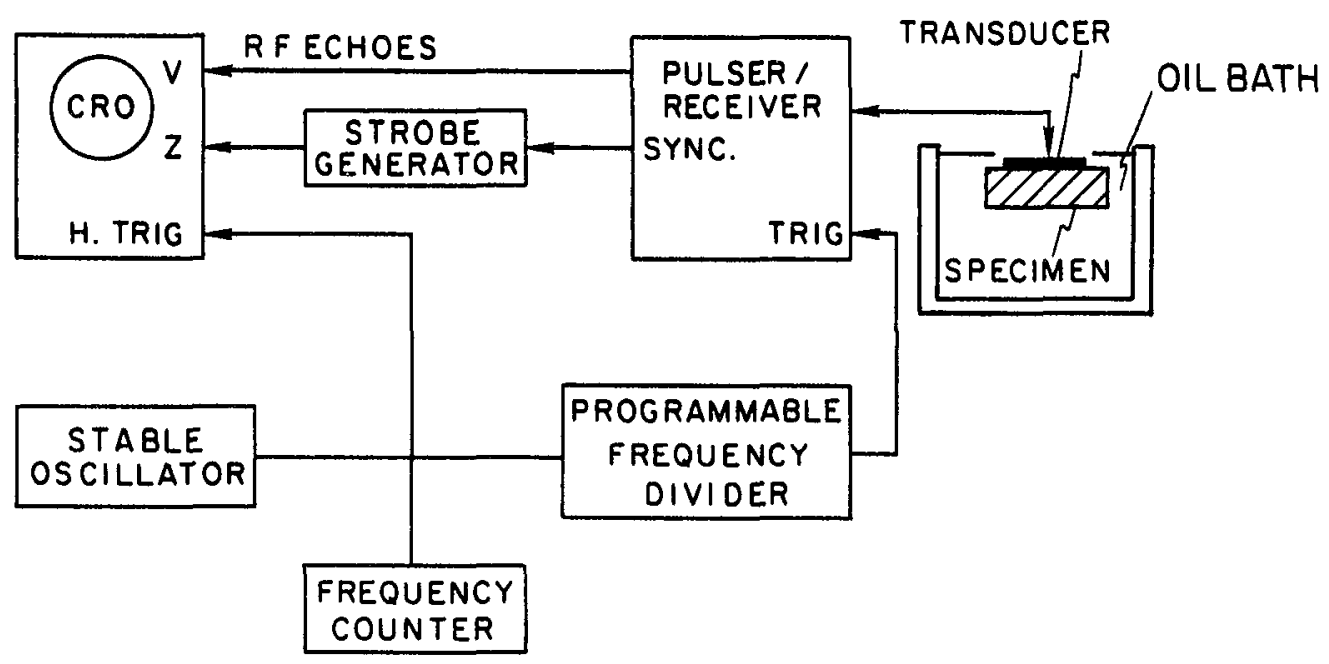

ATTENUATION

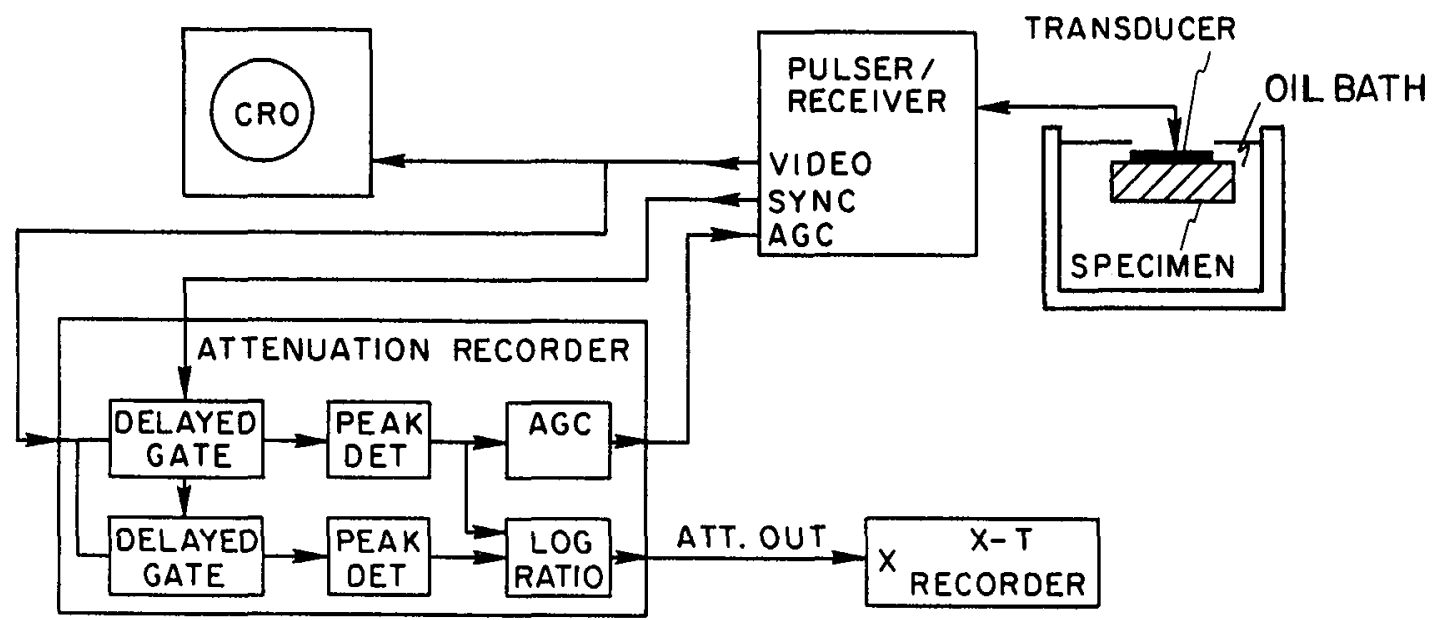

ATTENUATION AND VELOCITY MEASURING SYSTEMS

Figure 1. 
Dynamic measurements of sound velocity and ultrasonic attenuation, during metallurgical changes in the temperature interval between the ambient and $250^{\circ} \mathrm{C}$, were performed while the specimen and the ultrasonic sensors were in direct contact in an oil bath. For static ultrasonic measurements, carried out at room temperature, with the purpose of correlating ultrasonic data with mechanical properties, both the samples and the sensors were immersed in a distilled-water tank at a constant separation. Careful adjustments were made to ascertain an exponential decay of the amplitude of successive echoes, combined with a maximal number of echoes in the pulse train.

The growing interest in metallic glasses has created the need for the determination of their physical and mechanical properties. Some of these properties, especially the mechanical ones, cannot be measured conveniently by traditional ultrasonic methods as the high cooling rates required to form metallic glasses restrict their physical shapes to shallow layers or thin ribbons. Alternate measurement methodology needed to be developed in order to accurately assess these properties. One of the techniques employs a coll to magnetostrictively launch and detect extensional waves in a ribbon or magnetic material, called the "driver." The specimen can be coupled to the end of the driver, producing an echo pattern from which the extensional velocity in the material can be derived by a pulse-superposition technique (ref. 7). A pulse-receiver was constructed to feed high current pulses to the coil and to amplify the electrical signals produced in the coil by the acoustic waves.

The noncontact feature of both generation and detection of ultrasonic waves can be very advantageous in situations requiring physical separation between the measuring system and the material under investigation, e.g., when high temperatures or hostile atmospheres are involved. Furthermore, the contactless generation and detection precludes interaction with, and modification of, the wave propagation pattern under study. In addition, laser generation of acoustic waves yields a wide variety of propagation modes (longitudinal, transverse and plate modes, Rayleigh waves) over a wide frequency range, thus enhancing the amount of information obtained from a single measurement.

Compressive stress waves that propagate in a material can be generated by transient loads applied by rapid energy transfer from a single-pulse Q-switched high-energy Nd:Yag laser, (fig. 2). Propagation of ultrasonic waves in a medium causes surface displacements on the material that can be measured optically by exploiting the phase shift of an optical beam reflected from the surface of the material. When the reflected beam is combined with a reference optical beam, from a helium-neon laser, optical phase changes are converted into amplitude phase changes that are detectable by a sensitive photodiode. These variations in amplitude are proportional to the surface displacements on the specimen. Potential problems arlsing from the fact that phase changes also result from relative motion among optical components of the system and from temperature and pressure fluctuations of the ambient air, are prevented by appropriate design of the interferometer. An optical scheme due to Fizeau is particularly suitable for our specific purpose. Two optical probes are separated to allow accurate measurements of travel time of an ultrasonic wave in the material over a well-defined distance. Furthermore, the variation in magnitude of the surface displacements detected by the two interferometers determines the ultrasonic attenuation in the material. Thus, both velocity and attenuation can be measured simultaneously, fig. 3. The interferometer was built at Johns Hopkins University by Dr. Harvey Palmer.

Compared with other sensors, optical interferometers offer several advantages. 


\section{Overall Optical System}

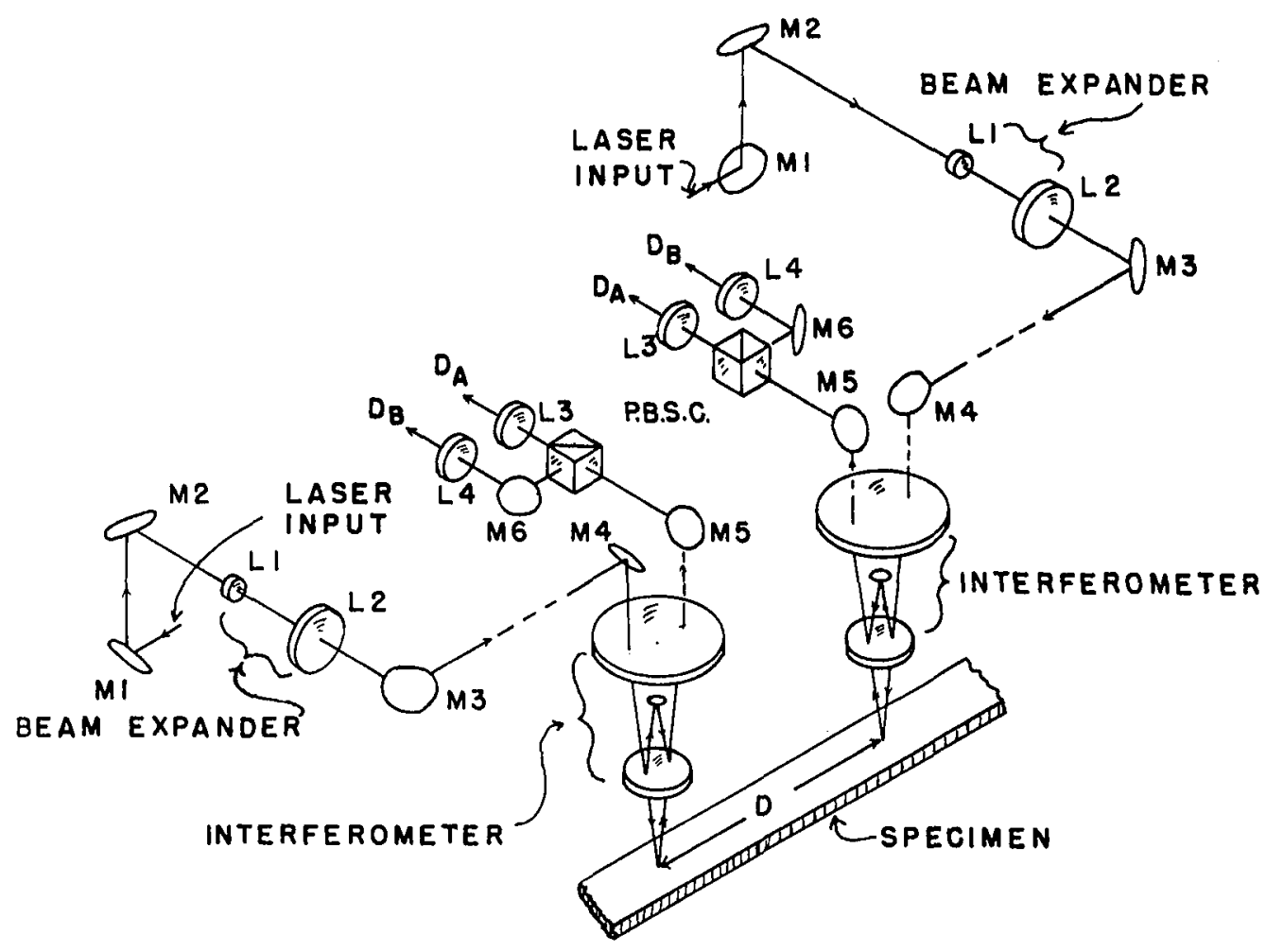

DUAL LASER INTERFEROMETER.

Figure 2.

\section{SOUND VELOCITY MEASUREMENT SYSTEM}

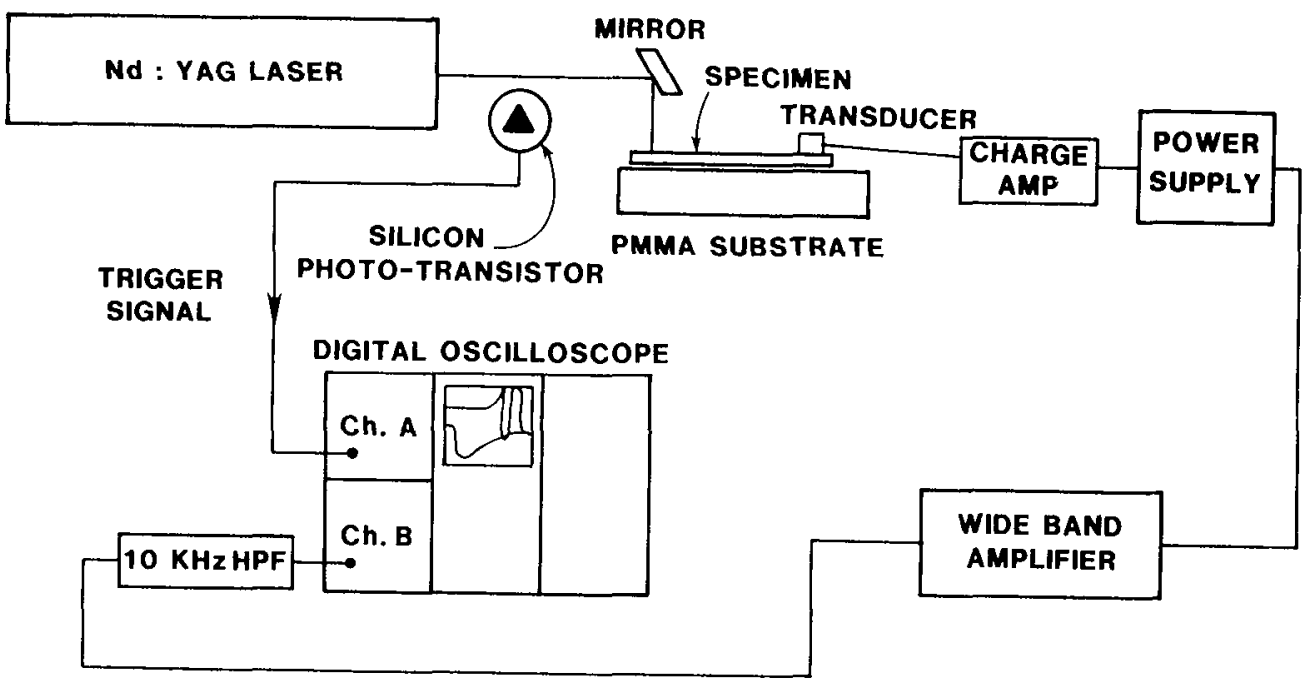

Figure 3. 
The sensitive area can be made very small, a few micrometers in diameter, for highly localized measurements. Consequently, the surface of the specimen does not necessarily have to be flat optically. The highly focused optical beams permit utilization of specimens with conventionally machined surfaces. The measured quantity is linear displacement. Independent methods of absolute calibration are applicable. Bandwidth, determining the fidelity of reproduction of signal waveforms, is not limited by the character of the transduction process but by the electronics of the interferometer detectors. Therefore, performance can largely exceed that of conventional plezoelectric transducers. Small signal resolution and bandwidth are related, thus linear displacements of a few angstroms are detectable at $7 \mathrm{MHz}$ bandwidth.

Laser pulse irradiation produces a stress pulse of short duration (15 ns) and relatively high amplitude (up to $200 \mathrm{~mJ}$ power) making the investigation of very thin (about $20 \mathrm{\mu m}$ ) and highly attenuating specimens possible. Since a multitude of acoustic wave propagation modes is generated the dual interferometer provides important information concerning the elastic and inelastic properties of the material under static or dynamic conditions.

The contactless generation and detection of acoustic waves is extremely advantageous because specimens can be studied while they are subjected to thermomechanical processing under adverse environmental conditions. No transducer protection (e.g., from elevated temperatures) is necessary, and the detected ultrasonic waveform is unperturbed by extraneous effects due to physical contact between specimen and transducer. Data acquisition is straightforward and real-time or post-test analysis is feasible. The capability to obtain the frequency dependence of both sound velocity (in dispersive regimes) and ultrasonic attenuation of fers new opportunities in solid state and physical metallurgy research where the elastic properties and acoustic energy absorption play a prominent role in the characterization of the processes.

Laser and electron beam irradiation techniques are being extensively applied for the modification of surface properties of metallic structures ( $r e f .9,10$ ). The nature of the modified surface zones is not amenable to conventional nondestructive characterization. However, the analysis of Rayleigh wave velocities, and the determination of the elastic moduli, may lead to a better understanding of the properties of the modified surface layers. Laser or plezoelectrically generated Rayleigh surface waves probe preferentially the near-surface region of the sample, and are extremely sensitive to variations in the elastic properties and ultrasonic attenuation of the material medium, figure 4. The extent of penetration of the Rayleigh surface wave normal to the surface of the sample depends on the frequency of the propagating Rayleigh waves and the exponential decay of their intensity. For a typical metallic phase, the Rayleigh wave velocity, V, is about $3000 \mathrm{~ms}^{-1}$. For a frequency of $10 \mathrm{MHz}$, the Rayleigh wavelength is, therefore, $300 \mu \mathrm{m}$. Theory shows that 90 percent of the energy of the Rayleigh wave is contained within one wavelength from the surface. Thus, the sub-surface region can be monitored with a high degree of accuracy. Moreover, Fourier analysis of the frequency content of the Rayleigh waves, when the dual-laser interferometer is used to contactlessly detect the propagating surface waves, allows the precise determination of the thickness of the surface layer. This gauging procedure is possible because of the unique property of the Rayleigh wave velocity that is independent of frequency.

Two properties of the Rayleigh surface wave make its detection possible by optical means. One, is the surface microcorrugation (microdistortions) as the Rayleigh wave propagates through the material medium. Second, is the periodic 


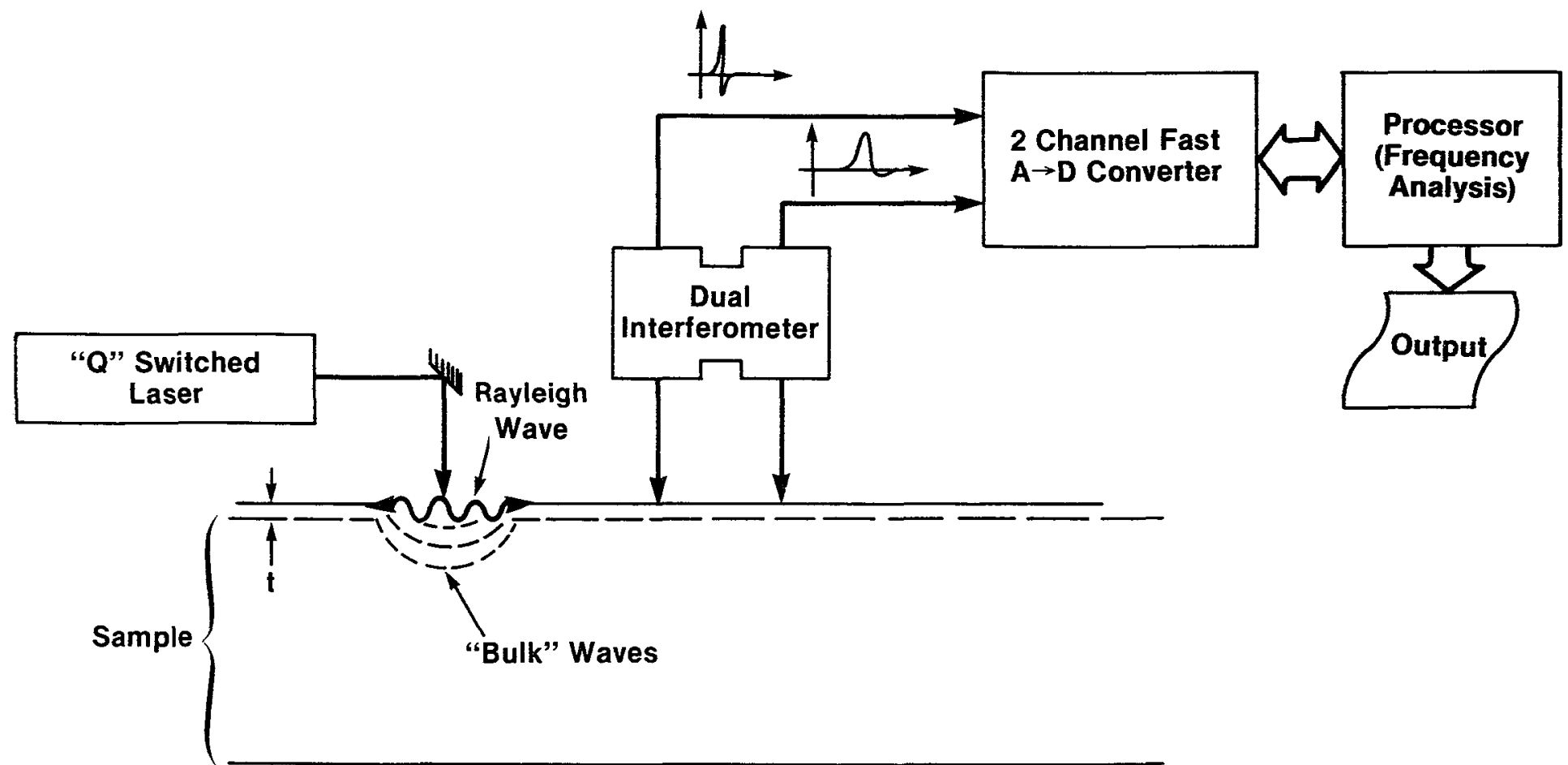

- Wideband Ultrasonic Pulse Generated by Laser Pulse.

- Vertical Displacement v.s. Time is Measured at Two Different Points by Two Wide Band Optical Detectors (Interferometers).

- Frequency Analysis of the Two Pulses Gives Attenuation v.s. Frequency and Velocity v.s. Frequency Curves.

- Since the Penetration of Rayleigh Waves is Frequency Dependent, the Thickness $t$ of the Amorphous Layer can be Evaluated From These Curves.

RAYLEIGH WAVE VELOCITY MEASUREMENTS IN MICROSTRUCTURALLY

MODIFIED LAYERS EMPLOYING LASER GENERATION AND LASER-

INTERFEROMETRIC DETECTION OF ULTRASONIC WAVES

Figure 4. 
variation of the index of refraction while the wave propagates on the surface. By means of optical interferometry, e.g., dual Fizeau interferometer, these waves can easily be detected in a contactless fashion. Thus, the contactless generation and detection of Rayleigh waves enables one to dynamically study the near-surface modifications of the material while the samples are contained in a hostile environment, and are subjected to programmed thermomechanical treatments subsequent to laser or electron beam alloying and microstructural modification.

\section{NONDESTRUCTIVE CHARACTERIZATION OF PRECIPITATION HARDENING PHENOMENA IN ALUMINUM} ALLOYS

Precipitation hardening, or aging, is an important metallurgical process whereby the material's strength and hardness can be augmented, in several instances, by an order of magnitude. Precipitation hardening is a thermally-activated, diffusion-controlled, process of particular importance in copper, iron, and aluminum-base alloys. The improved mechanical and physical properties of these alloys depend, largely, on the microstructure, spacing, size, shape, and distribution of the precipitated particles, as well as on the degree of structural and crystallographic coherency of the particles with the matrix. For example, the precipitation hardening process in aluminum alloys involves formation of Guinier-Preston (G.P.) zones, $\theta n$ and $\theta$ ' particles that are generally coherent or semicoherent with the matrix and contribute to the strenghthening of the alloy. At higher temperatures incoherent particles form, that dramatically reduce the strength and hardening of the alloy. Of particular importance in aluminum-base alloys are the $\theta$ ' particles, initially semicoherent with the matrix, that grow in size as the precipitation process progresses. At a certain critical size, when the stress fields surrounding these precipitates are maximal, dislocation rings (Orowan loops) form around them causing the coherency to be weakened. At this point overaging, or softening, begins.

of great technological importance is the development of a nondestructive probe that will permit: (a) determination of the extent of matrix supersaturation in alloys prepared by new processes such as rapid solidification, and (b) real-time monitoring of the precipitation hardening process to determine particular events such as the instant when particles lose coherency with the matrix and overaging begins. of course, solution of the latter problem implicitly addresses the former.

Figure 5 shows the time dependence of the changes in the sound velocity of 2219 aluminum alloy during isothermal aging at three temperatures. The nondestructive characterization of the aging process, as manifested in figure 5 and corroborated with optical and electron microscopy, hardness, strength, and eddy currents conductivity data, shows a series of dips that correspond to the maximal rate of formation of $\theta \boldsymbol{n}$ and $\theta$, particles.

The prominent peaks in ultrasonic attenuation, figure 6 , correspond to the peaks in hardness and represent the loss of coherency of $\theta$ ' particles with the matrix and beginning of the softening process. The peaks in ultrasonic attenuation arise from an interaction between the acoustic vibrations of the ultrasonic waves propagating through the material and the interface dislocations surrounding the $\theta$, particles. The strength of the interaction will depend on the total volume of the $\theta$, particles, their size, shape, and distribution. The peak value of attenuation can be qualitatively related with the total volume of coherent precipitates that lost coherency with the matrix. The temperature dependence indicated by the shift in the 


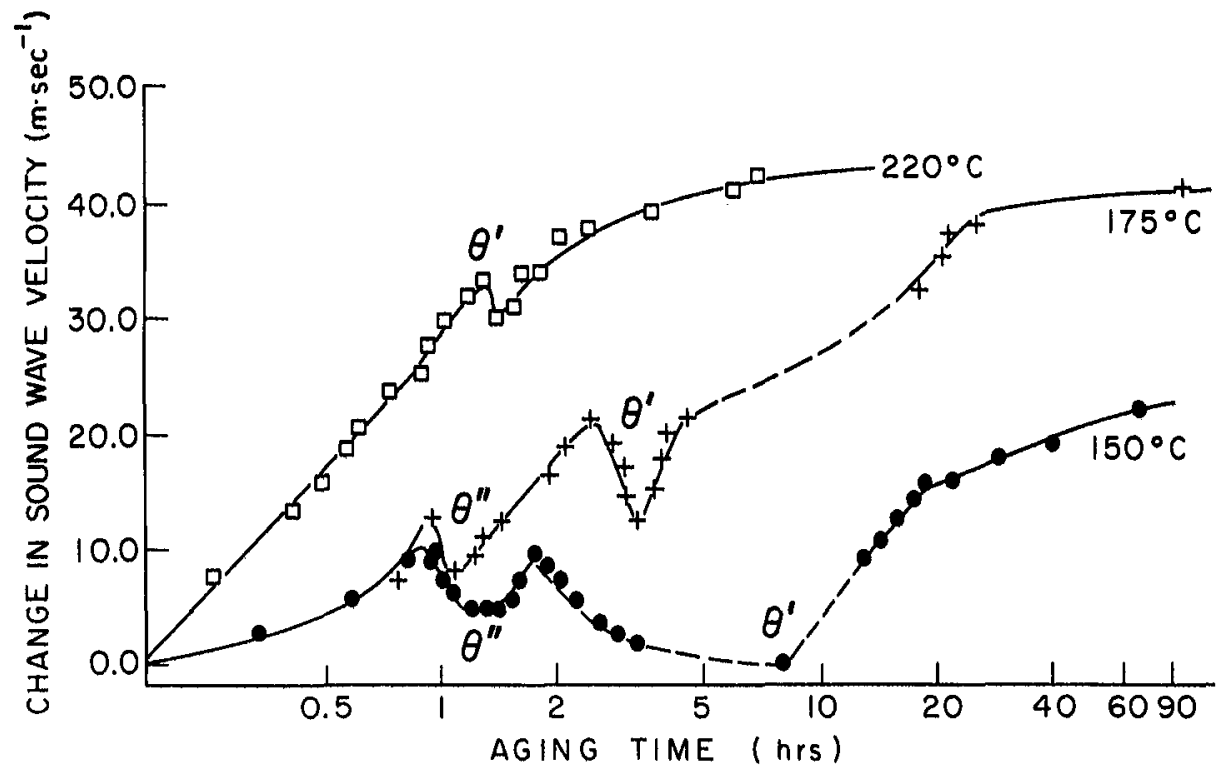

ULTRASONIC VELOCITY DURING THREE ISOTHERMAL HEAT

TREATMENTS OF 2219 ALUMINUM ALLOY

Figure 5.

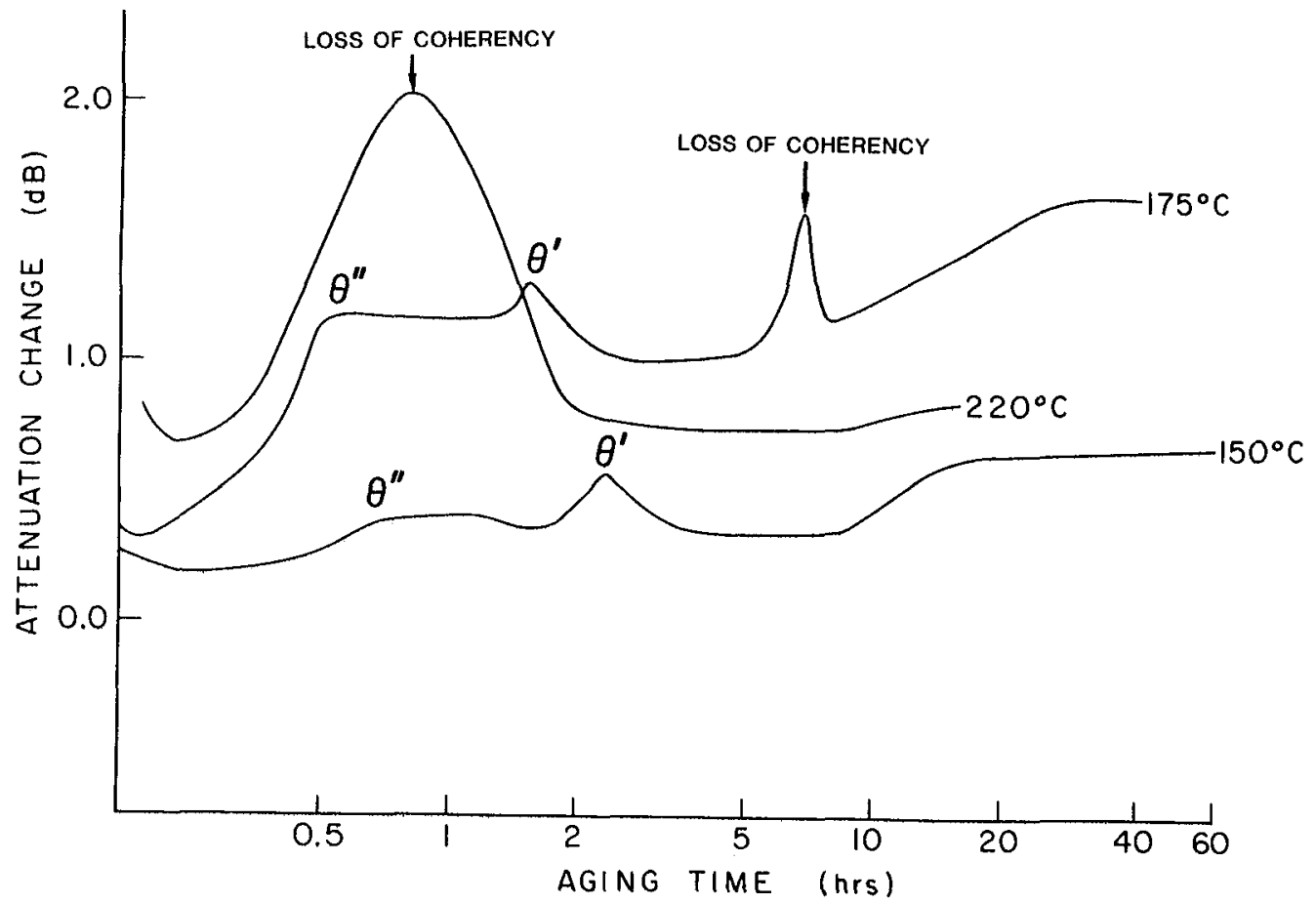

ULTRASONIC ATTENUATION DURING THREE ISOTHERMAL HEAT

TREATMENTS OF 2219 ALUMINUM ALLOY

Figure 6. 
attenuation peaks, figure 6, permits the determination of an activation energy that is found to be very close to that obtained from the shift of the hardness peaks (ref. 1). Corroborative evidence was obtained by means of electron microscopy and substantiates the proposed mechanism of coherency loss in this alloy.

This study demonstrates the feasibility of the ultrasonic NDC technique to determine the time of formation of the various precipitates, the kinetic parameters of the process, as well as to establish, by means of ultrasonic attenuation, the time when the alloy begins to suffer a loss of its mechanical properties due to overaging.

Nondestructive characterization measurements have been carried out on $160 \mathrm{Al}-\mathrm{Cu}$ alloy samples subjected to a series of different preaging heat treatments prior to thermal processing to different tempers. For each temper the maximum hardness of the alloy was found to correlate to a particular value of the sound wave velocity. In addition, ultrasonic attenuation was found to consistently decrease in a linear fashion as hardness is increased, figures 7 and 8 . This investigation demonstrates the potency of ultrasonic NDC for in-process monitoring and control of the mechanical properties (hardness, strength) of age-hardenable aluminum alloys. The correlation suggests that within a range of thermomechanical treatments the hardness of the alloy can be uniquely determined by means of sound velocity and ultrasonic attenuation.

\section{NDC OF CRYSTALLIZATION PROCESSES IN AMORPHOUS MATERIALS}

Amorphous alloys, or glassy metals, are solids with frozen-in liquid structures. The absence of translational periodicity in the amorphous state along with the macroscopic compositional homogeneity are the main reasons for their improved proerties, e.g., high mechanical strength, good corrosion resistance, and excellent magnetic behavior. Their unusual mechanical, chemical, and physical properties have stimulated extensive scientific and technological interest. One serious problem in the processing and utilization of amorphous alloys that may limit their future technological applications is their low thermal stability. When thermomechanical conditions are appropriate, metallic glasses relax structurally and ultimately crystallize into more stable structures resulting in drastic variations in properties. The factors governing the thermal stability of these alloys and their effect on properties are not well understood. For example, upon crystallization, amorphous alloys undergo very large changes in the elastic ( 40 percent) and anelastic properties with accompanying reduction in plastic properties (embrittlement). For this reason, availability of a nondestructive ultrasonic characterization technique for both property determination and metallurgical process control can be extremely useful.

There is also ample evidence that microstructural modifications of near-surface layers, with concurrent improvements in properties, can be achieved by laser and electron beam irradiation of bulk metallic materials. Rapid melting and resolidification of a surface layer can produce refined structures and more homogeneous materials, or, in some instances, new microstructures and phases. Examples of the latter include extended solid solubility and formation of metallic glasses.

Ultrasonic NDC, in particular the contactless generation and detection of Rayleigh surface waves by laser interferometry, can be very useful for determination of the properties and geometrical dimensions of structurally modified layers while in the formative or post solidification stage. 


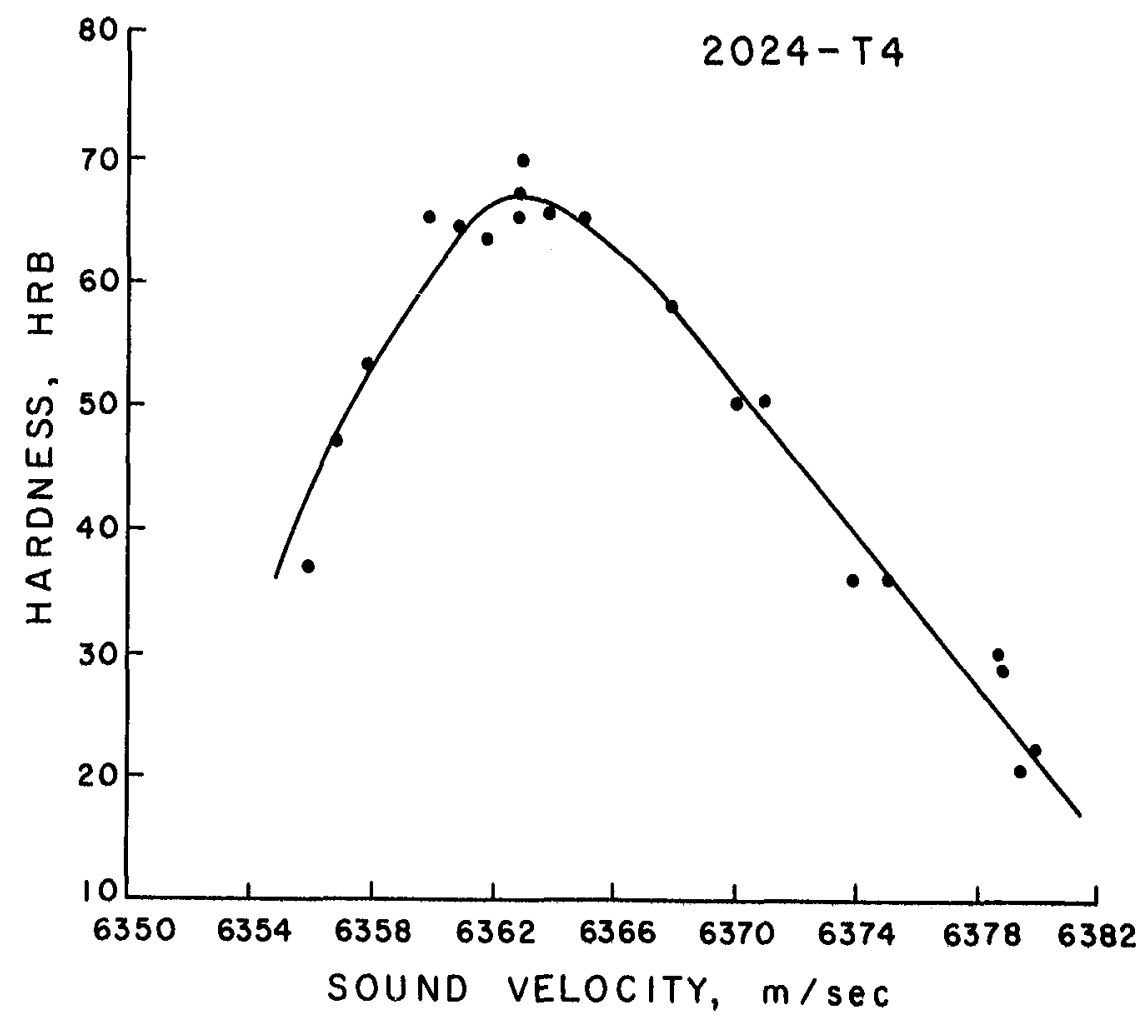

VARIATION OF THE SOUND WAVE VELOCITY

WITH HARDENESS IN AGE HARDENED SAMPLES

Figure 7.

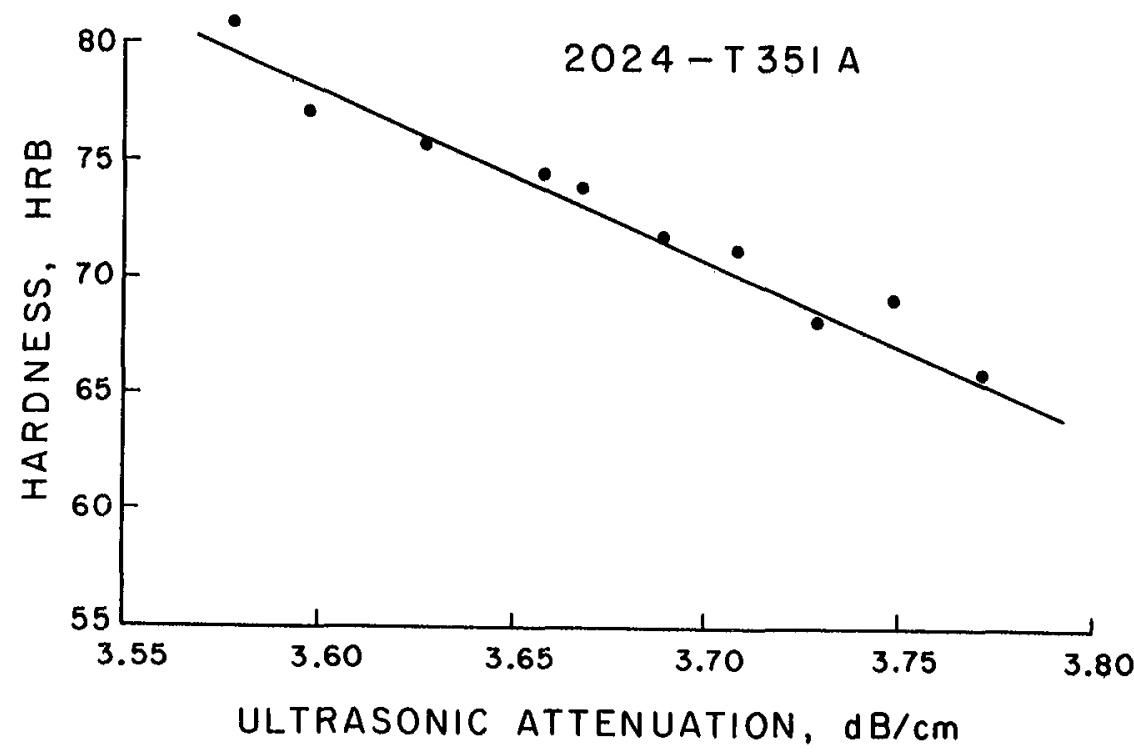

VARIATION OF THE ULTRASONIC ATTENTUATION WITH HARDNESS IN AGE HARDENED SAMPLES

Figure 8. 
Metallic glass ribbons of PdCuSi and CuZr alloys were prepared by the melt spinning technique. The samples were approximately $40 \mu \mathrm{m}$ thick and $1.25 \mathrm{~mm}$ wide. Ultrasonic, x-ray, and metallographic examination prior to the crystallization treatment revealed no crystallinity. Crystallization was performed in evacuated quartz capsules at predetermined isothermal holding temperatures. Optical metallography indicated that the crystallization process in PuCuSi ribbons is not homogeneous in the bulk. Two coexlsting growth processes were observed. Frontal growth from the free surfaces of the ribbon towards the interior, and homogeneous nuoleation and growth in the bulk.

The velocity of the ultrasonic extentional waves was determined by measuring the transit time of a single pulse generated by a laser and detected by a piezoelectric quartz-crystal tranducer located at a distance of about $200 \mathrm{~mm}$ from the spot on the ribbon irradiated by the laser. The transient load was applied by rapid deposition of energy from a single-pulse of a Q-switched neodymium YAG laser wtih a wavelength of $1.06 \mu \mathrm{m}$. The laser-pulse duration was $15 \mathrm{~ns}$, and the pulse energy, for this specific series of measurements, was about $20 \mathrm{~mJ}$. The elastic stress wave propagates along the specimen and may be detected by a piezoelectric transducer. The laser pulse was line-shaped to produce a nearly plane wave. A photodetector was used to trigger a transient pulse recorder as the laser pule was generated. Thus, the transit time of the extensional sound wave propagating along the ribbon could be determined to within better than 1 part in $10^{4}$.

Figure 9 shows the variation of the extensional wave velocity, $V$ as a function of crystallization time at three isothermal holding temperatures, 380,390, and 400 ${ }^{\circ} C$. The initial sound-wave velocity, in the amorphous state, was found to be 2970 $\mathrm{ms}^{-1}$, whereas in the fully crystallized state it reached an asymptotic value of 3490 $\mathrm{ms}^{-1}$. Taking 10.52 and $10.69 \mathrm{~g} \mathrm{~cm}^{-3}$ as the density of the amorphous and crystalline

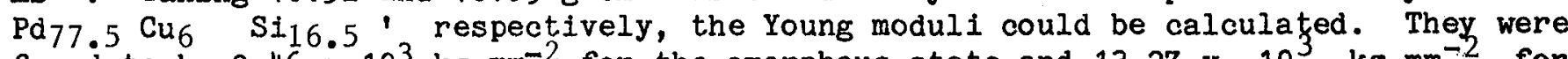
found to be $9.46 \times 10^{3} \mathrm{~kg}-\mathrm{mm}^{-2}$ for the amorphous state and $13.27 \times 10^{3} \mathrm{~kg}-\mathrm{mm}^{-2}$ for the crystalline state, $1 . e ., E$ of about 40 percent. Figure 9 exhibits a relatively sharp increase in the sound velocity during the first five minutes of the isothermal holding. The variation of the extensional wave velocity with crytalization time manifests the dramatic changes in the elastic properties with an increase of about 40 percent in Young's modulus. The sigmoid curves represent the crystallization kinetics which are a thermally activated process. Thus, the kinetic parameters, i.e., activation energy and the growth regime parameters can be determined form the NDC data (ref. 3, 4). The obtained values were found to be in satisfactory agreement with calorimetric data (ref. 11) of activation energies for crystallization of amorphous alloys (ref. 12). Furthermore, the time dependence of the crystallized volume fraction was found to be compatible with a diffusion controlled growth mechanism (ref. 13, 14) for a plane boundary growth that is preceded by a rapid nucleation process (ref. 15).

The effect of copper content on the Young's modulus of $\mathrm{ZrCu}$ alloys in the crystalline and amorphous state indicates that the lowest value of the Young's modulus is for pure crystalline zirconium, whereas the highest value is for pure crystalline copper. A maximum and a minimum are found for alloys with compositions near the intermetallic compounds $\mathrm{Zr}_{2} \mathrm{Cu}$ and $\mathrm{ZrCu}$, respectively. The variation of the Young's modulus with composition of the amorphous alloys, between 30 and 70 percent copper, is a smooth S-curve with an inflection point at 50 a/o copper. It is conjectured that the amorphous state can be interpreted in terms of short range order structure where the mixing pairs $\mathrm{Zr}-\mathrm{Zr}, \mathrm{Cu}-\mathrm{Cu}$, and $\mathrm{Cu}-\mathrm{Zr}$ are of equal probability at the composition of $50 \mathrm{a} / \mathrm{o} \mathrm{Cu}$. The inflection point on the Young's modulus versus composition curve will then represent the point for maximal configurational entropy 
of the amorphous alloy. Analysis of the temperature dependence of the sound wave velocity of $\mathrm{Zr}-\mathrm{Cu}$ alloys in the composition range between 30 and 70 a/o Cu allowed determination of the activation energies of the diffusional processes governing the crytallization kinetics. The activation energy increases from 90 to $110 \mathrm{kcal} / \mathrm{mole}$ as copper content exceeeds 50 a/o. The change in crytallization mechanism as copper content exceeds 50 a/o may be related to the configurational entropy change as the population of $\mathrm{Cu}-\mathrm{Cu}$ pairs increases. The relationship between the behavior of the Young's modulus as a function of composition, and the variation of the activation energy of the crystalization process with copper content is yet to be elucidated. It is belleved that this relationship is of basic importance in the understanding of the transformation of the amorphous state into coexisting compounds upon isothermal crytallization (ref. 5).

The initial increase in the extensional sound velocity, of about 3.7 percent, 1.e., 7.4 percent in the Young's modulus of PdCuS1 and a similar increase in the $\mathrm{Zr}-\mathrm{Cu}$ amorphous alloys, appears to be related to a structural relaxation that precedes the crytalization process in the amorphous state (ref. 14, 16). Structural relaxation was found to be particularly enhanced in glasses obtained at high quenching rates, where greater structural disorder is to be expected (ref. 17). The lower elastic stiffness in the glassy state has been attributed to the interatomic displacements inherent in a disordered structure (ref. 18). The elastic stiffness should, therefore, increase with increased local ordering as a consequence of the structural relaxation. Microhardness measurements on the ribbon edge, perpendicular to the length vector of the ribbon, revealed a dramatic increase in hardness while the material is still in the amorphous state, before crystallization ensues, figure 10. The embrittlement mechanism in the amorphous alloy can be related to the removal of the quenched-in free volumes (vacancy clusters). Initially, the amorphous alloy has a disordered packing of the constituent atoms. The reduced coordination of the atom complexes, due to the presence of excess free volumes, allows shear transformations to be sustained. The free volumes are analogous to dislocation core segments. Consequently, the material is very ductile in the disordered amorphous state. Heat treatment in the amorphous region at temperatures below the glass transition temperature, $\mathrm{Tg}$, or for relatively short periods of time at temperatures above $\mathrm{Tg}$ causes structural and compositional relaxation. The free volumes are redistributed by short range shuffling of the atoms, thus a more rigid close-packed atomic distribution of high coordination is achieved. The dense random packing of hard spheres (Bernal structure) leads to significant embrittlement of the amorphous phase.

The topological and compositional relaxation processes occurring in the amorphous state can be investigated by analyzing the behavior of the shear and compressional components of the ultrasonic attenuation, and their frequency dependence. From the frequency dependence of the ultrasonic attenuation, obtained by means of the dual-laser interferometer, the behavior of the bulk and shear viscosities can be characterized. Consequently, it is possible to establish the functional relationship between the appropriate relaxation times and the diffusional reactions leading to topological and compositional changes in the amorphous state. This specific phase of the investigation is currently in progress. It can be realized through the capability of the laser generation-laser interferometric detection of propagating sound waves, and the subsequent analysis of their frequency dependence within the framework of the viscoelastic theory. 


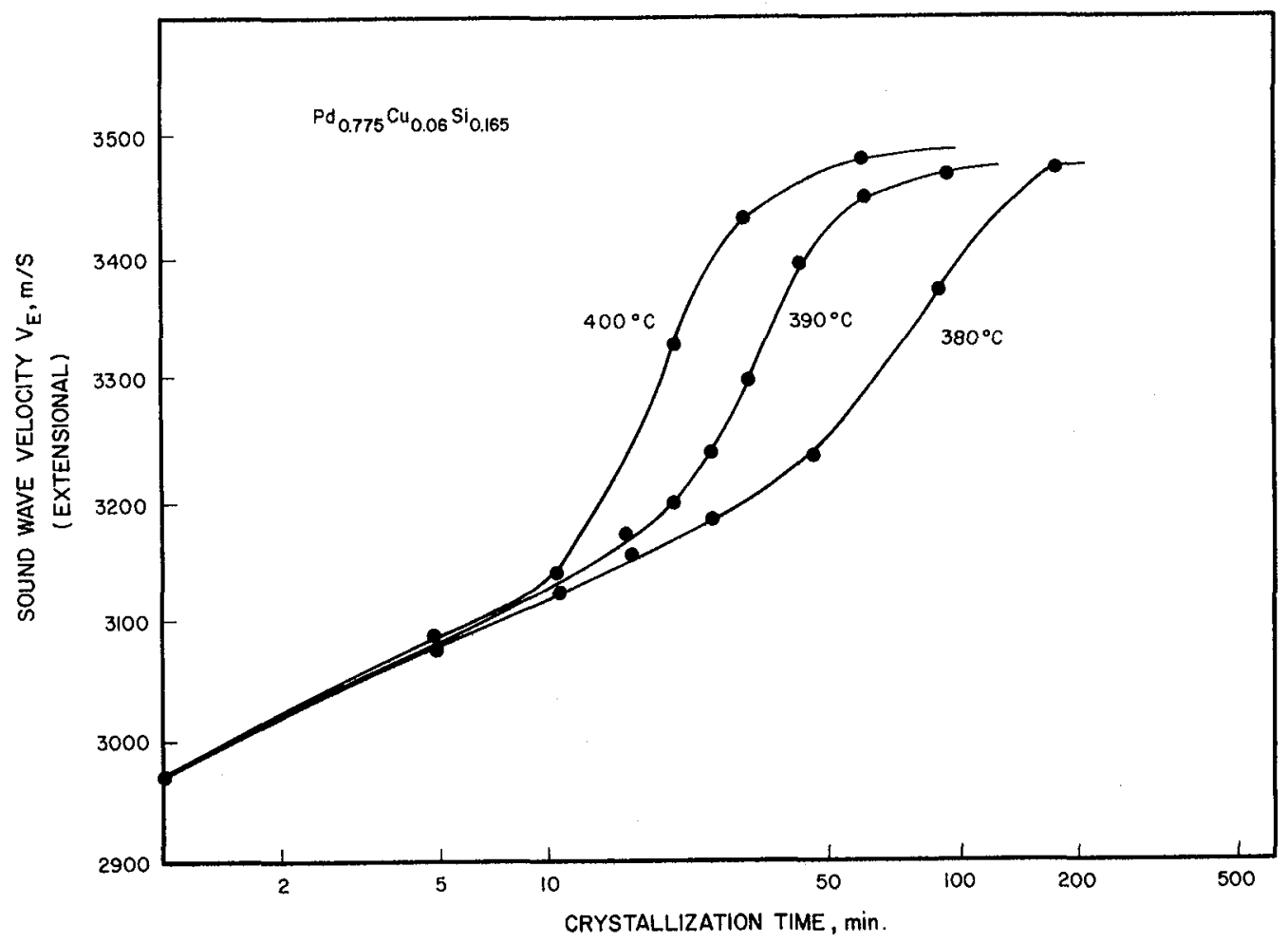

VARIATION OF EXTENSIONAL WAVE VELOCITY IN PdCU'Si DURING TRANSITION FROM AMORPHOUS TO CRYSTALLINE STATE

Figure 9.

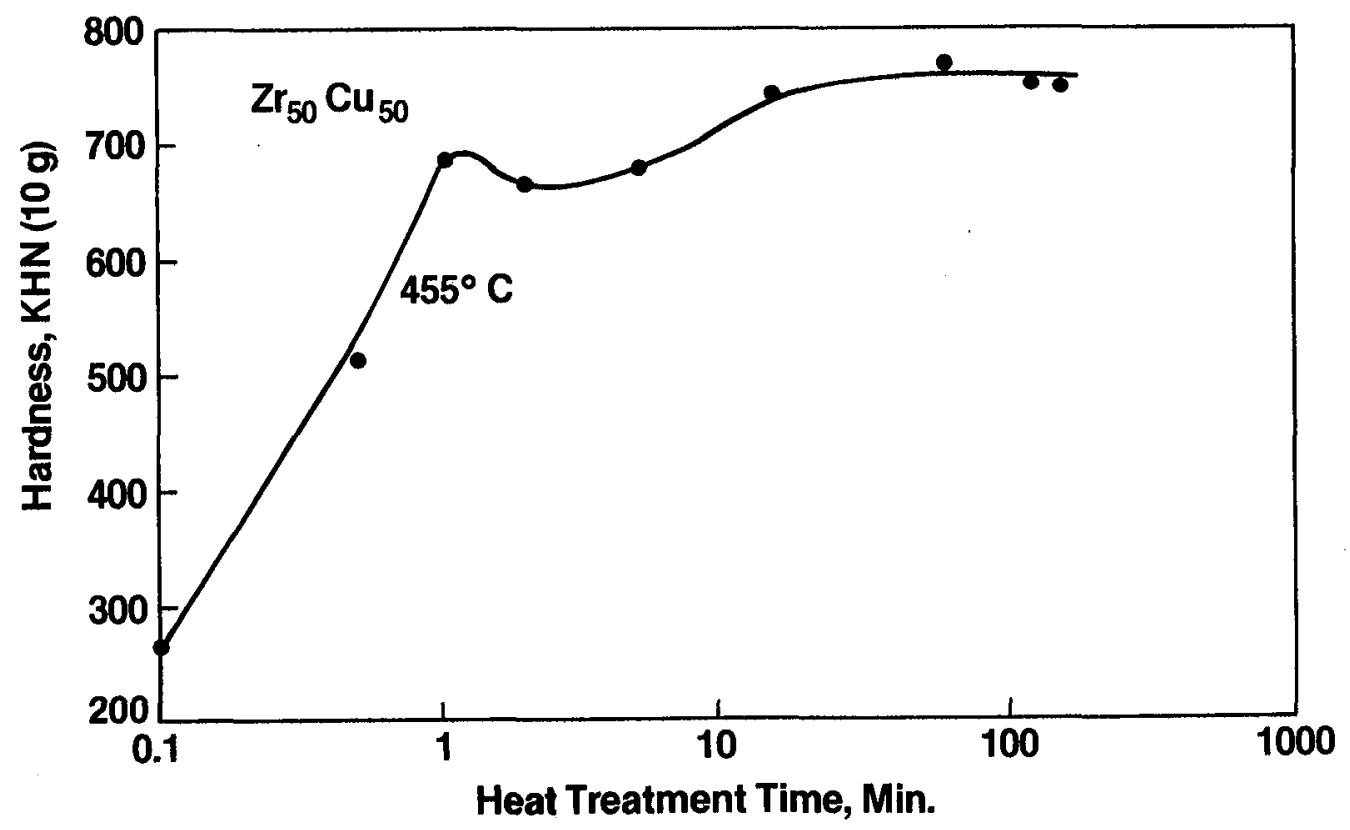

VARIATION OF MICROHARDNESS DURING CRYSTALLIZATION OF AN AMORPHOUS ZrCU ALLOY

Figure 10. 
High power lasers and electron beams have recently been applied for modifying metal surfaces and improving their physical, chemical, and mechanical properties (ref. 9, 10). Modification of surface properties may involve incorporation of alloying elements into the surface layer or localized thermomechanical treatment leading to formation of microstructures of desired characteristics. The focused laser or electron beam energy is deposited in such a fashion as to melt a thin layer while the bulk of the material provides the rapid quenching effect. Typical quenching rates may be of the order of $10^{6} \mathrm{ks}^{-1}$ or higher (fig. 11). Control of the developing microstructures and their characterization is crucial to the understanding of the operating mechanisms responsible for the near-surface reactions and for the exploitation of the great potential of this emerging technology for specific applications. Nondestructive characterization may be of prime importance for the on-line, real-time mechanical and physical evaluation of the surface properties, or as basic parameters for engineering design. Demands for safety and quality control have emphasized the lack of an acceptable NDE technique for quality assurance and process control. In a recent review (ref. 19) various NDE methods were discussed for application on surface coatings: electrical, magnetic, optical, and acoustic. Only those techniques based on acoustic properties showed sufficient promise for engineering applications.

Electron beam glazing has been applied in the course of the present research to develop modified metallurgical microstructures such as amorphous layers on crytalline substrates of PdCuSi ( $\mathrm{fig}$. 12), deposition of copper on 1100 aluminum samples followed by electron beam melting in an attempt to form an aluminum-copper surface layer on the aluminum bulk, and formation of metastable martensitic microstructures on a pearlitic bulk of AISI 1045 steel ( $f$ ig. 13).

Figure 12 shows an amorphous layer of $1 \mathrm{~mm}$ average depth produced by electron beam melting and rapid solidification of a dendritic crystalline substrate. The heat affected zone is apparent as well as the grain structure of the original polycrystalline material.

Ultrasonic NDC of the thermally modified peripheral layer was determined by means of Rayleigh surface waves, using the device depicted in figure 14 . As mentioned previously, the Rayleigh wave velocity is frequency independent. However, the extent of penetration of Rayleigh waves normal to the material surface is frequency dependent. Frequency analysis of the apparent Rayleigh velocities enables determination of the elastic properties of the layer, and the gauging of its average thickness. The Rayleigh surface wave velocity was measured by means of a wedge device (fig. 14) which converts longitudinal waves into Rayleigh waves, and vice versa. In the case of an amorphous PdCuSi layer, in which the Rayleigh surface wave velocity is about 30 percent smaller than the Rayleigh wave vélocity in polycrystalline material, this technique was found to be particularly potent. Samples of 1053 carbon steel in the pearlitic state were electron beam heat treated to obtain a microstructurally modified surface layer of martensite. The Rayleigh velocity of the uniform pearlite was found to be higher by more than $2 \%$ of that of martensite. By measuring the Rayleigh wave velocity over a frequency range, thus varying the penetration depth of the Rayleigh surface waves, the modified layer depth could be nondestructively determined (fig. 15). The Rayleigh velocity remains constant as long as the Rayleigh waves probe a uniform material. When the Rayleigh waves, due to decreasing frequency or increasing wavelength, begin to sample simultaneously both the modified martensite layer and the pearlite substrate, the 


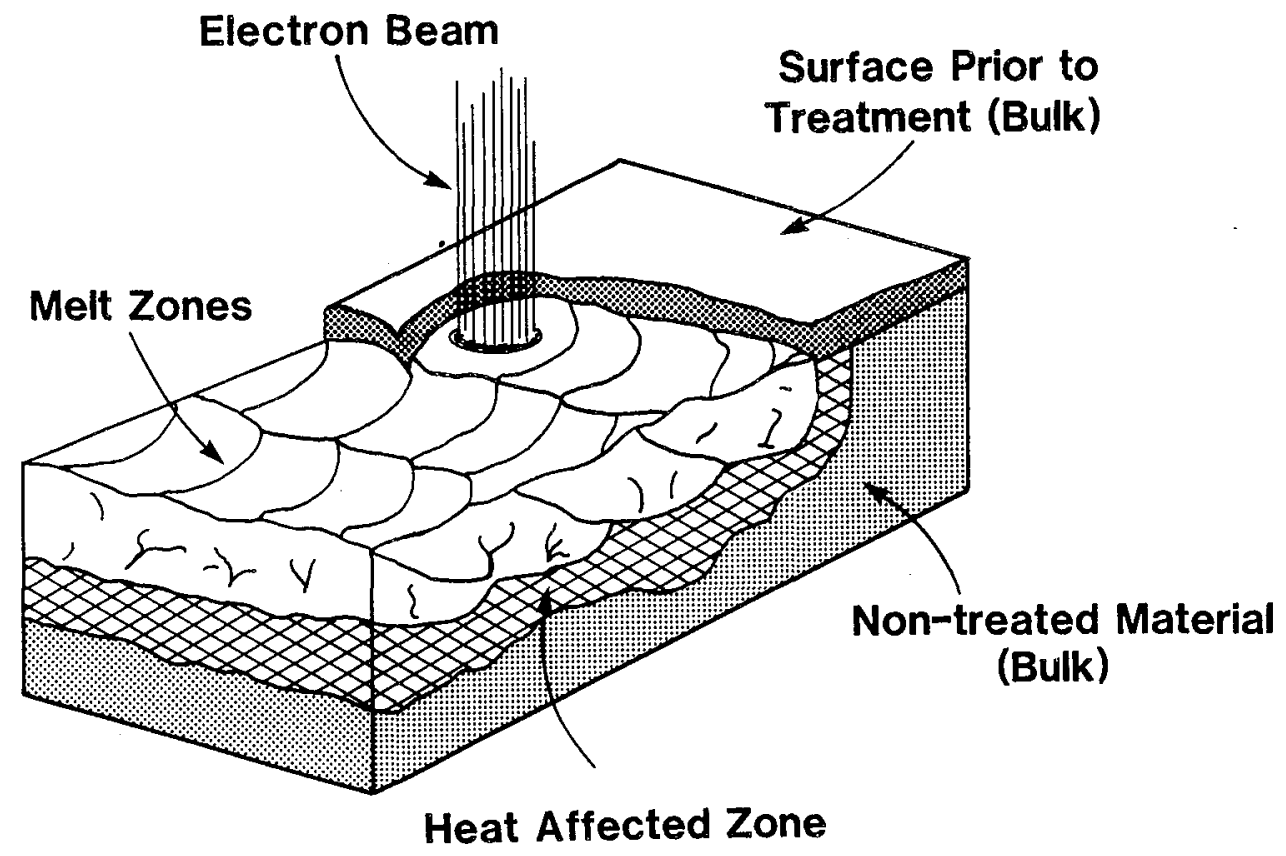

ELECTRON BEAM SURFACE MODIFICATION PROCESS

Figure 11.

\section{$500 \mu \mathrm{m}$

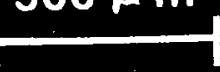

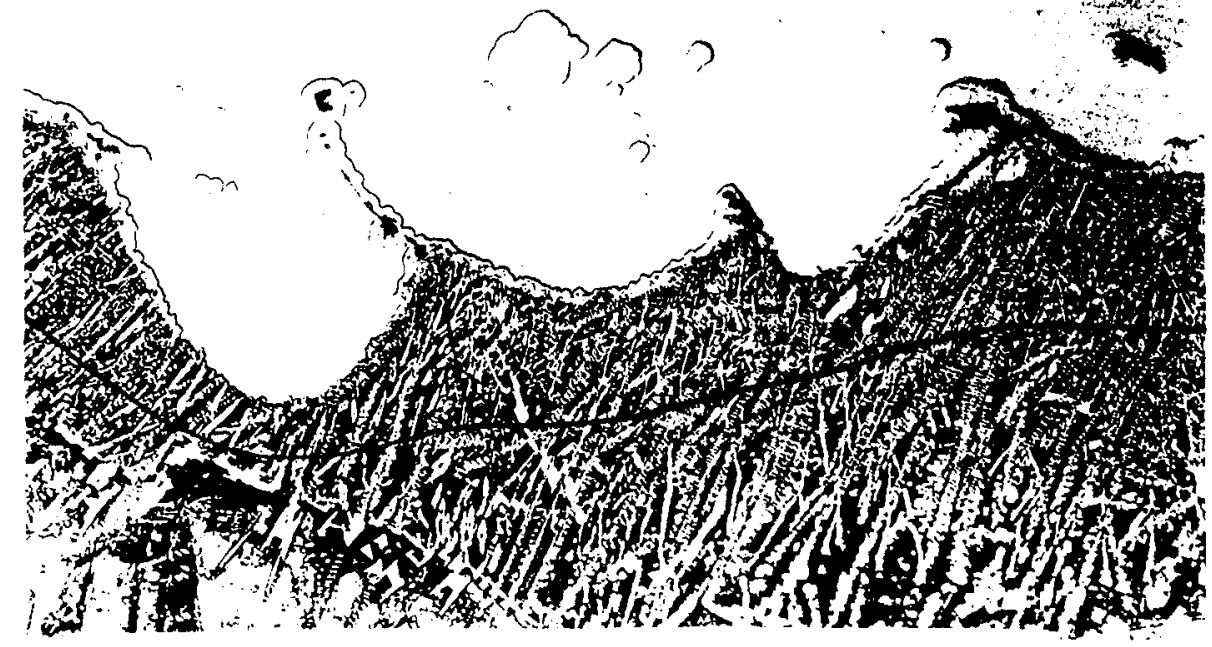

AMORPHOUS PACUST LAYER FORMED ON A CRYSTALLINE SUBSTRATE BY ELECTRON BEAM SURFACE TREATMENT

Figure 12. 


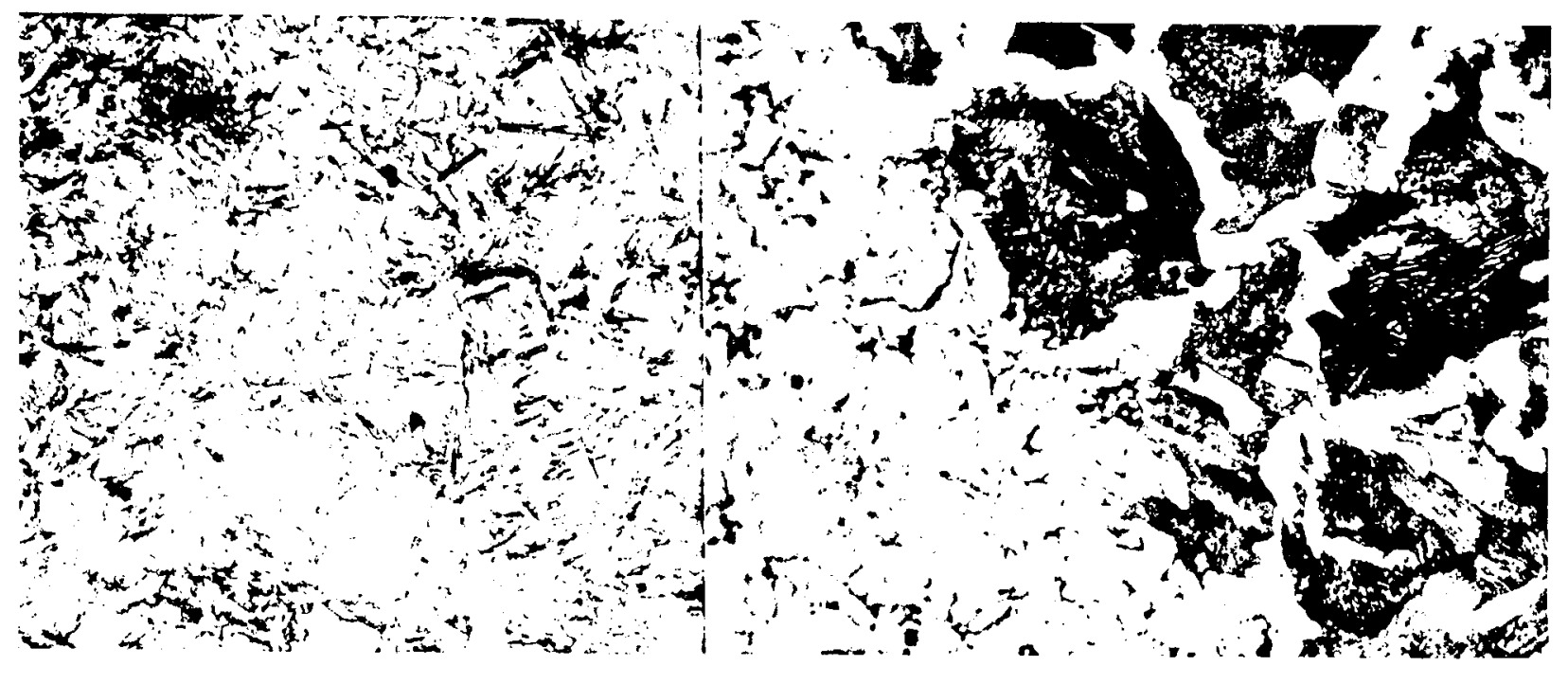

SURFACE LAYER OF MARTENSITE (LEFT) OF AISI 1045 STEEL OBTAINED BY ELECTRON BEAM IRRADIATION OF THE PEARLITIC MICROSTRUCTURE OF THE SUBSTRATE (RIGHT)

Figure 13.

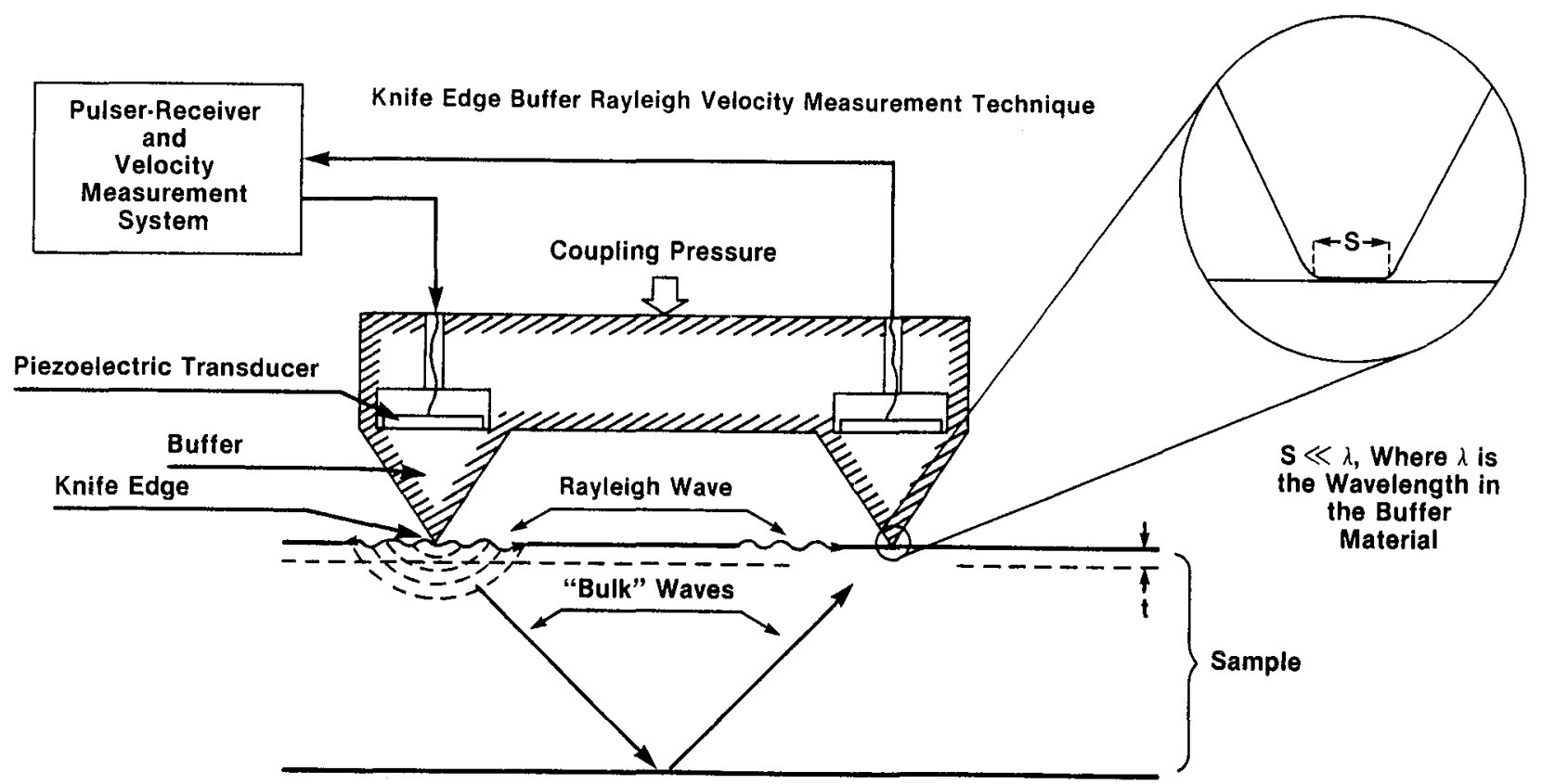

- The Amplitude of the Rayleigh Wave Decreases Only Due to Attenuation Whereas the Amplitude of the Cylindrical "Bulk" Wave Decreases as $1 / \mathrm{Nr}$, Where $r$ is the Propagation Distance.

RAYLEIGH WAVE VELOCITY MEASUREMENTS IN MICROSTRUCTURALLY MODIFIED SURFACE LAYERS USING THE PIEZOELECTRIC GENERATION AND DETECTION BY MEANS OF KNIFE EDGE BUFFERS

Figure 14 . 


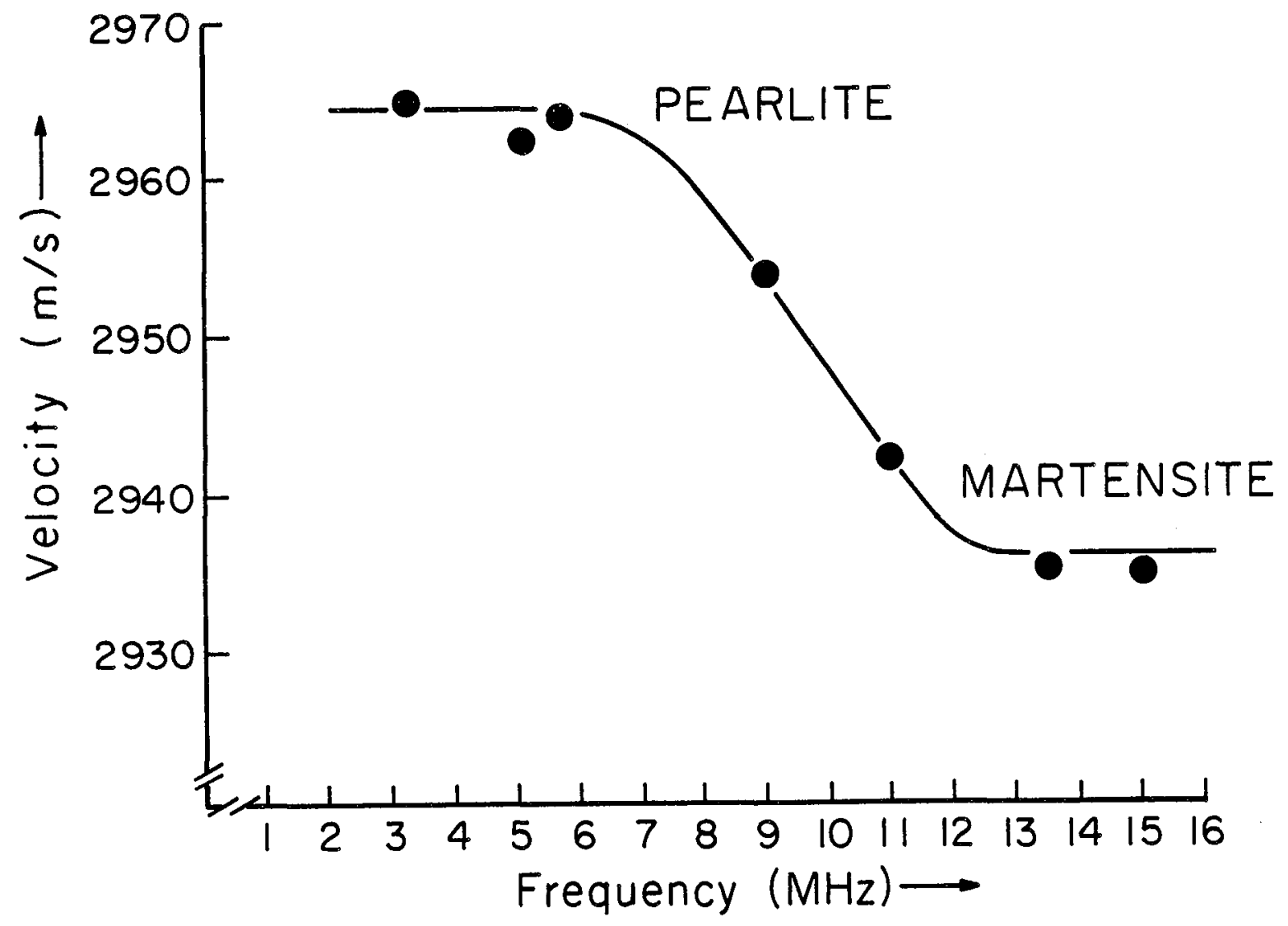

VARIATION OF RAYLEIGH WAVE VELOCITY WITH ULTRASONIC FREQUENCY, (RECIPROCAL OF DEPTH OF PENETRATION) IN AISI 1045 STEEL. THREE REGIONS ARE OBSERVED; a) AT HIGH FREQUENCIES (SMALL PENETRATIONS) WHERE THE VELOCITY IS CONSTANT AS LONG AS THE WAVES PROBE THE UNIFORM MARTENSITIC LAYER (ABOUT $700 \mu \mathrm{m}) ; \mathrm{b}$ ) AT LOW FREQUENCIES, IN ADDITION TO THE THIN MARTENSITIC LAYER, THE PEARLITIC BULK IS PROBED; C) THE INTERMEDIATE REGION, WHERE BOTH MARTENSITE AND PEARLITE IS PROBED RESULTING IN A VARIATION OF VELOCITY, SINCE BOTH MICROSTRUCTURES ARE SAMPLED

Figure 15 . 
measured Rayleigh velocity is expected to increase. This behavior is apparent in figure 15. The lower curve should increase and merge asymptotically into the upper curve (straight line) that exhibits the constant Rayleigh velocity in the pearlitic substrate. These measurements demonstrate the feasibility of the technique to nondestructively characterize the properties of a modified surface layer, and to evaluate its thickness. Preliminary studies have shown that the noncontact, laser generation and laser-interferometric detection of Rayleigh surface waves can be applied for nondestructive, noncontact, evaluation of surface layers. Development of an in-process, real-time method would be of great technical importance.

\section{REFERENCES}

1. Rosen, M.; Fick, S.; Reno, R.; Horowitz, E.; and Mehrabian, R.: Kinetics of Precipitation Hardening Process in 2219 Aluminum Alloy Studied by Sound Velocity and Ultrasonic Attenuation. Mater. Sci. Eng., vol. 53, 1982 , pp. 163.

2. Rosen, M.; Swartzendruber, L.; Fick, S.; Horowitz, E.; and Mehrabian, R.: Aging Process in 2024 Aluminum Alloy Studied by means of Eddy Currents. Mater. Sci. Eng., vol. 53, 1982, pp. 191.

3. Rosen, M.; Wadley, H.N.G.; and Mehrabian, R.: Crystallization Kinetics Study of Amorphous PdCuSi by Ultrasonic Measurements. Scripta Metall., vol. 15, 1981, pp. 1231.

4. J.C.; Nadeau, F.; Rosen, M.; and Mehrabian, R.: Crystallization Kinetics Study of Amorphous $\mathrm{Zr}_{50} \mathrm{Cu}_{50}$ by Means of Ultrasonic Measurements and Microhardness. Scripta Metall., vol. 16, 1982, pp. 1073.

5. Nadeau, F.; Rosen, M.; and R. Mehrabian: Effects of Composition on the Elastic Properties of Amorphous and Crystalline Cu-Zr Alloys. Johns Hopkins University Report CMR-NDE-7, 1982.

6. Palmer, C.H.; Fick, S.; and Rosen, M.: A Dual-probe Laser Interferometer for Contactless Determination of Ultrasonic Wave Velocities and Attenuation. Johns Hopkins University Report CMR-NDE-11, 1983.

7. MeSkimin, M.J.: J. Acoust. Soc. Am., vol. 33, 1961, pp. 12.

8. Morse, R.W.: J. Acoust. Soc. Am., vol 22, 1950, pp. 219.

9. Breinan, E.M.; and Kear, B.K.: Rapid Solidification Laser Processing of Materials for Control of Microstructures and Properties. In Proceedings of the 1st International Conference on Rapid Solidification Processing, Reston, VA, 1977, R. Mehrabian; B.H. Kear; and M. Cohen, Eds., Claitors Publ., 1978, vol. I., pp. 87-103.

10. Tucker, T.R.; and Ayers, J.D.: Surface Microstructures Produced by Electron Beams. In Proceedings of the 2nd International Conference Scanning Solidification Processing, Reston, VA, 1980, R. Mehrabian; B.K. Kear; and M. Cohen, Eds., Claitors Publ. 1981, vol II, pp. 206-211. 
11. Bagley, B.A.; and Vogel, E.M.: J. Non-Cryst. Solids, vol 18, 1975, pp. 29.

12. Lubrosky, F.E.:Mater. Sci. Eng., vol. 28, 1977, pp. 139.

13. Johnson, W.A.; and Mehl, R.F.: Trans. AIME vol. 135, 1939, pp. 416.

14. Christian, J.W.: Physical Metallurgy. R.W. Cahn, Ed., North-Holland Press, Amsterdam, London, 1970, pp. 471.

15. Cahn, J.W.: Acta Metall., vol. 4, 1956, pp. 449.

16. Kursumovic, A.;Scott, M.A.; Girt, E.; and Cahn, R.W.: Scripta Metall., vol. $14,1980, \mathrm{pp} .1303$.

17. Chen, H.S.: Rep. Prog. Phys., vol. 43, 1980, pp. 355.

18. Weatre, D.; Ashby, M.F.; Logan, J.; and Weins, M.S.: Acta Metallogr., vol. 19,1979 , pp. 799 .

19. Bucklow, I.A.: Proceedings of the International Conference on the Advances in Surface Coating Technology, London, 1978, p. 61. 


\title{
ANALYTICAL ULTRASONICS FOR STRUCTURAL MATERIALS
}

\author{
David S. Kupperman \\ Argonne National Laboratory \\ Argonne, Illinois 60439
}

The application of ultrasonic velocity and attenuation measurements to characterize the microstructure of structural materials is discussed. Velocity measurements in cast stainless steel are correlated with microstructural variations ranging from equiaxed (elastically isotropic) to columnar (elastically anisotropic) grain structure. The effect of the anisotropic grain structure on the deviation of ultrasonic waves in cast stainless steel is also reported. Field-implementable techniques for distinguishing equiaxed from columnar grain structures in cast stainless steel structural members are presented. The application of ultrasonic velocity measurements to characterize structural ceramics in the green state is also discussed.

\section{INTRODUCTION}

The reliability of ultrasonic techniques used in the inspection of structural materials is often limited by the peculiar microstructure of the material. This is the case for cast stainless steel (CSS) and for structural ceramics in the "green" (unfired) state. The research described in this paper is directed toward understanding the wave propagation characteristics of these materials so that ultrasonic NDE techniques can be reliably applied to them.

\section{ULTRASONIC CHARACTERIZATION OF CAST STAINLESS STEEL}

\section{Background}

It is well known that the coarse grain size and elastic anisotropy of CSS make ultrasonic inspection difficult. Although the ASME code requires inspection of CSS piping, it has not been possible to demonstrate that current inspection techniques are adequate. Measures that may increase the reliability of ultrasonic inspection in the near term include (a) the development of methods to establish the microstructure of the material (to help optimize the inspection technique), (b) calibration standards that are more representative of the material to be inspected, and (c) the use of cracked CSS samples for training purposes. For the long term, it will be necessary to establish (a) the variability of the microstructure of CSS, (b) the effect of microstructure on inspection reliability, (c) the degree of improvement possible with techniques and equipment designed specifically for CSS, e.g., focused

*Work funded by the U.S. Nuclear Regulatory Commission, Office of Nuclear Regulatory Research and the U.S. Department of Energy, Advanced Research and Technology Development Fossil Energy Materials Program. 
transducers and lower frequencies than those used conventionally, and (d) qualification of requirements for CSS inspections. Recent work carried out at ANL to address some of these points is presented below.

Technical Progress

\section{Variation of Sound Velocity with Microstructure}

When CSS material is isotropic (composed of equiaxed grains), the variation in velocity with wave propagation direction can be small $(<2 \%)$, whereas for anisotropic material (composed of columnar grains) the variation in velocity may be large (as much as $100 \%$ for shear waves). The magnitude of the sound velocity may also be used as a measure of the degree of anisotropy and thus as an indicator of microstructure. Relatively low longitudinal-wave velocities indicate a columnar grain structure, whereas high velocities may indicate an equiaxed (isotropic) structure. Intermediate values may indicate the presence of both microstructures.

The validity of this concept has been demonstrated on CSS samples with different microstructures. Figure 1 shows the longitudinal-wave velocittes for seven $400 \mathrm{x}$ $180 \times 60-\mathrm{mm}$ samples of a 28-in. pipe provided by Battelle PNL and 18 samples of a comparable large-diameter pipe provided by Westinghouse. The Battelle samples were fabricated from a weldment in which material with a well-defined equiaxed grain structure was joined to material with a well-defined columnar grain structure. The Westinghouse samples were also made from a weldment. These specimens, however, were machined flat and have a coarse and poorly defined grain structure. Sound velocity was measured by standard pulse-echo methods with a 37-mm-dia, 1-MHz transducer. Echo transit times were measured with a Tektronix oscilloscope. In all cases the longitudinal waves propagate in a radial direction through the pipe wall. As shown in figure 1, the equiaxed and columnar sides of the Battelle samples can be easily distinguished by measurements of the longitudinal-wave velocity. Also, the sampleto-sample variation is relatively smal1. The Westinghouse samples, however, show large variations in sound velocity from sample to sample as well as within a sample (two measurements were made on each sample). The wide range of the values is indicative of large variations in the microstructure of these samples. This complex microstructure could cause significant, unpredictable distortion of the ultrasonic waves used to interrogate the material and could lead to an unreliable result.

A second set of experiments was performed during a visit to Commonwealth Edison's Byron Station. Sound velocity was measured in the pulse-echo mode in a CSS/carbon steel reference block and two CSS elbows (one each on the steam-generator and pump side of the loop 4 crossover).

On the cast portion of the $7.6-\mathrm{mm}$-thick reference block, the longitudinal sound velocity was measured at 11 points covering about $0.01 \mathrm{~m}^{2}$. No significant difficulties were encountered in making these measurements. The average velocity was $6090 \mathrm{~m} / \mathrm{s}$, with a variation of about $+1.5 \%$ due to the microstructure. The relatively high sound velocity strongly suggests that the reference block has an equiaxed grain structure, which may be significantly different from the grain structure of the plant piping and elbows. As a check on the accuracy of the measurement, the carbon steel portion of the reference block was also examined. A sound velocity of $5890 \mathrm{~m} / \mathrm{s}$, which is consistent with previous data for this material, was obtained. 


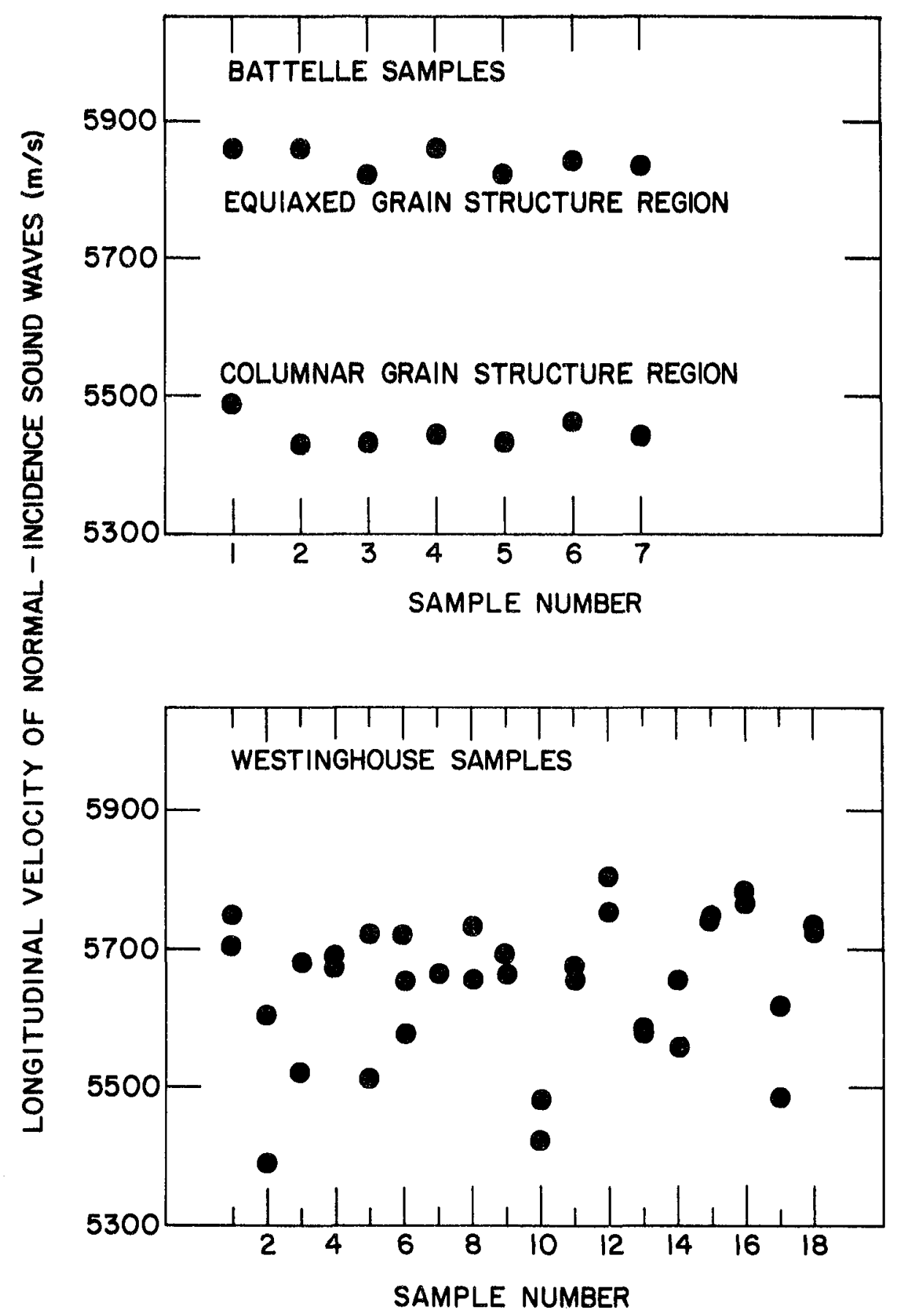

Fig. 1. Variation of Longitudinal-Wave Velocity in Large-Diameter CSS Pipe Sections. (Upper panel) samples with both columnar and equiaxed regions; (lower panel) samples with a poorly defined, coarse grain structure. Two measurements were made on each sample. Sample wall thicknesses are nominally $60 \mathrm{~mm}$; 1-MHz longitudinal waves were propagated normal to the pipe outer surface in a pulse-echo mode. 
The surfaces of the CSS elbows were, in most areas, too rough for efficient propagation of ultrasonic waves. Only the smoothly ground areas adjacent to the elbow-to-pipe welds were amenable to pulse-echo examination; ultrasonic backwall echoes could be detected in most, but not a11, of these areas. On the pump-side elbow, echoes were detectable at several locations away from the top of the elbow. Since the elbow and pipe appeared to have the same wall thickness, the wall thickness stamped on the pipe section was used as a "best guess" value for the elbow wall thickness. On the basis of this assumption, the sound velocity in the elbow varied from 5200 to $5860 \mathrm{~m} / \mathrm{s}$. This suggests that the microstructure of the elbow differed considerably from that of the reference block. The wall thickness of the generatorside elbow could not be estimated because of the elbow geometry, so absolute velocities were not determined; however, the velocity appeared to vary by about $5 \%$. The sound velocity in the straight (wrought) section between the two elbows was found to be $5760 \mathrm{~m} / \mathrm{s}$, a value consistent with previous measurements on stainless stee1.

Attempts were also made to propagate $1-\mathrm{MHz}, 45^{\circ}$ longitudinal waves in the cast material with two transducers in a pitch-catch mode. No backwall echo was detected in the reference block; this finding is consistent with the conclusion that the material is equiaxed. No echo signal could be detected on the pump-side elbow, but echoes were present in the generator-side elbow. Thus, the two elbows have distinctly different wave propagation characteristics. However, in both cases there was considerable ultrasonic noise.

These results are consistent with the suppositions that ultrasonic-wave propagation characteristics vary considerably from component to component and within an individual component, and that reference-block material may not be representative of the material to be inspected.

\section{Microstructure and Deviation of Ultrasonic Beams}

In an elastically isotropic material (composed of equiaxed grains), the energy in an ultrasonic beam propagates in the direction of the wavefront normal, as expected. However, in elastically anisotropic material (with columnar grain structure), the direction of propagation of ultrasonic energy in a beam can be different from the direction of the wavefront normal. Because of this phenomenon, it may be possible to distinguish columnar from equiaxed grain structures nondestructively by examining the propagation behavior of $45^{\circ}$ shear or longitudinal waves in the material. Figure 2 shows the predicted shear-wave propagation behavior in 60-mm-thick specimens. For an equiaxed spectmen with the transmitter and receiver placed on opposite sides (fig. 2a), the maximum acoustic signal will be detected $\sim 60 \mathrm{~mm}$ from the point directly opposite the beam entry point. For a specimen with columnar grains (fig. 2b), the energy in the beam deviates markedly from the expected $45^{\circ}$ path, such that the maximum signal will be detected at only $\sim 16 \mathrm{~mm}$ (see analysis below) from the point opposite the beam entry point. As shown in figure $2 \mathrm{c}$, the beam in the equiaxed material will be reflected to a point $~ 120 \mathrm{~mm}$ from the transmitting transducer (following the expected "full-V" path), while the maximum reflected-beam intensity in the columnar material will be found much closer to the transmitting transducer ( $\sim 32 \mathrm{~mm}$ away). 


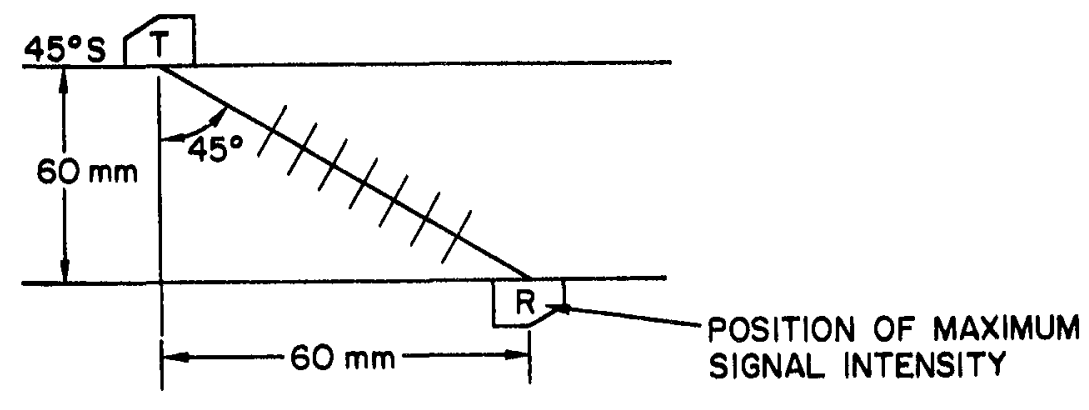

(b) THROUGH - TRANSMISSION (COLUMNAR GRAINS)

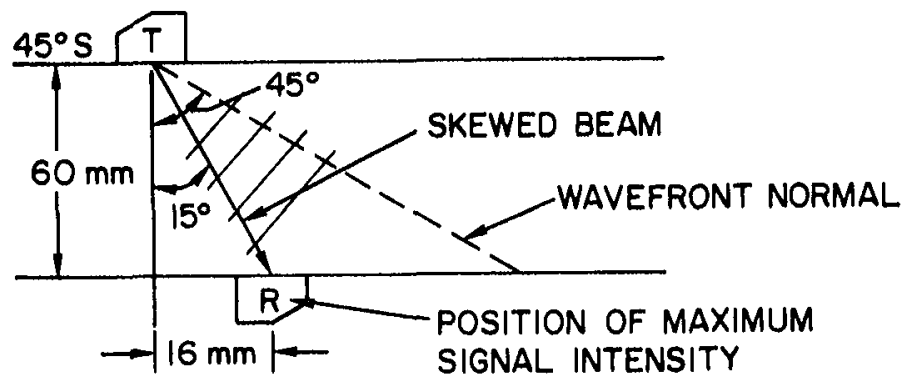

(c) PITCH-CATCH MODE ("FULL V")

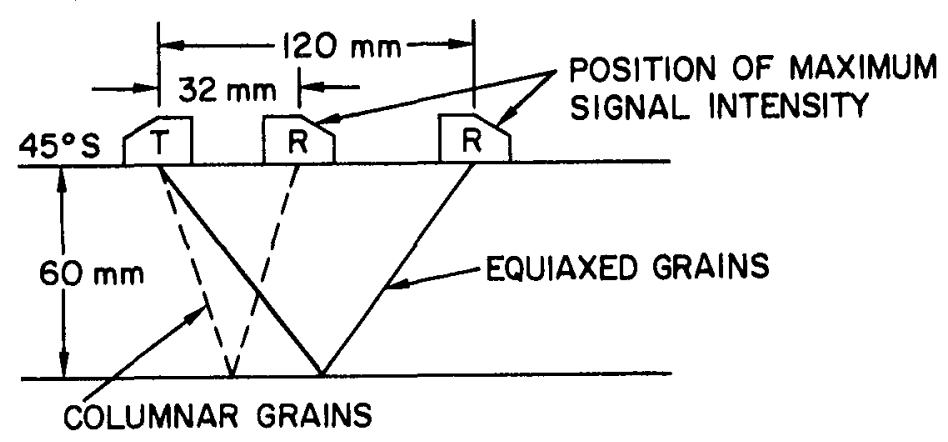

Fig. 2. Schematic Representation of the Path of Ultrasonic Energy in Cast Stainless Steel with Equiaxed vs Columnar Grain Structure. (a) Transmit from outer surface and receive at inner surface (equiaxed grains), (b) transmit from outer surface and receive at inner surface (columnar grains), and (c) transmit and receive at outer surface. 
The analysis leading to this prediction is given below. If the angular deviation of longitudinal waves as a function of propagation angle (angle of refraction, In this case) is known, the point of maximum signal intensity for the pitch-catch configuration of figure 2 can be calculated from Snell's law:

$$
\frac{\sin \theta_{w}}{V_{w}}=\frac{\sin \theta_{C S S}}{V_{C S S}(\theta)} \text {, }
$$

where $\theta_{\mathrm{W}}$ and $\mathrm{V}_{\mathrm{W}}$ are the angle of incidence and the velocity, respectively, of the sound wave in the wedge, and $\theta_{\text {CSS }}$ and $V_{\text {CSS }}$ are the angle of refraction and longitudinal velocity of sound in the cast sEalnless steel. In the case shown, $\sin \theta_{\mathrm{w}} / \mathrm{V}_{\mathrm{w}}=0.22$.

The angular dependence of the velocity of sound must be known in order to calculate the angle of refraction. The orthotropic elastic constants for the columnar-grain CSS were calculated from velocity measurements in two different directions (ref. 1) by use of the Christoffel equations. These constants were then used to calculate the velocity as a function of the angle of incidence, and hence, to calculate the angle of refraction from equation (1). For the wedge used in the velocity measurements, the calculated angles of refraction are $36^{\circ}$ for the columnargrain CSS and nearly $45^{\circ}$ for the equiaxed material. The angle between the velocity vector and the direction of maximum energy propagation is $21^{\circ}$ for CSS. Hence, the energy of the beam propagates in a direction inclined $15^{\circ}$ (i.e., $36^{\circ}-21^{\circ}$ ) to the surface normal, and in a 60-mm-thick sample, the reflected wave should have a maximum signal intensity at the transmitting surface $\sim 32 \mathrm{~mm}$ from the transmitting probe. In the case of the equiaxed specimen, the calculated separation distance for maximum signal intensity is $2127 \mathrm{~mm}$ (the angle of refraction is actual1y $47^{\circ}$; hence, the calculated separation distance is slightly larger than the value of $120 \mathrm{~mm}$ expected for a $45^{\circ}$ angle).

To verify these predictions, experiments were carried out on one columnar and one equiaxed pipe region from a sample simflar to the Battelle pipe sections mentioned earlier. Two $0.5-\mathrm{MHz}$, nominally $45^{\circ}$ shear-wave transducers $(25 \times 25 \mathrm{~mm}$ in size) were placed on the outer surface of each pipe section, and the separation between the two transducers was varied to maximize the received signal. Table I compares the optimum separations determined in these experiments with the corresponding predicted values. The agreement is reasonable.

Table I. Transducer Pair Separation ${ }^{2}$ for Maximum Received Signa1 on Outer Surface of $60-\mathrm{mm}$-Thick CSS Pipe Sections

\begin{tabular}{ccc} 
Grain Structure & $\begin{array}{c}\text { Predicted Value, } \\
\mathrm{mm}\end{array}$ & $\begin{array}{c}\text { Measured Value, } \\
\mathrm{mm}\end{array}$ \\
\hline Columnar & 32 & 42 \\
Equiaxed & 127 & 105 \\
\hline
\end{tabular}

$\mathrm{a}_{45^{\circ}}$ shear waves in pitch-catch "full-V" configuration at $0.5 \mathrm{MHz}$. 
The distortion of an ultrasonic beam as it propagates through weld metal can be visualized by use of a beam profile analyzer (courtesy of Sonoscan, Inc., Bensenville, II). Figure 3 shows the schematic arrangement of this commercially available device. A transducer and sample are placed in a water bath. The ultrasonic beam (pulsed) travels through the specimen and strikes a gold-plated plastic plate. A laser beam scans the gold film and, by use of an optical interferometer, monitors the intensity of the beam in the plane of the film. The light-beam spot is $250 \mu \mathrm{m}$ across and has a field coverage of $10 \times 10 \mathrm{~cm}$. The oscilloscope images discussed below were produced with a 1-cm-dia (2.25 MHz) transducer; the gain of the system was adjusted to make the maximum detected intensity of the ultrasonic beam the same in all cases.

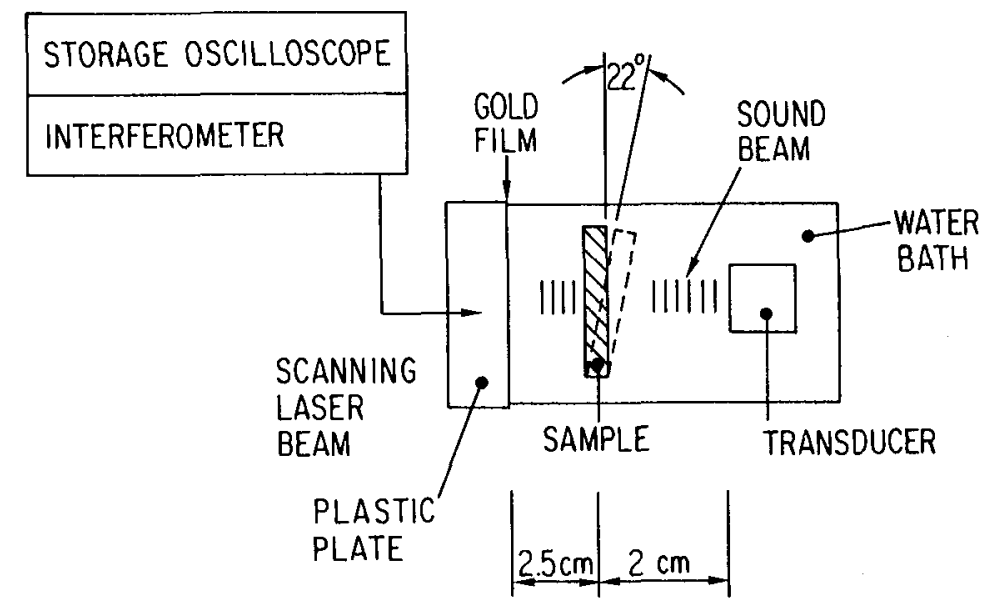

Fig. 3. Schematic Arrangement of Beam Profile Analyzer with U1trasonic Beam Passing through Weld-Metal Sample.

Longitudinal waves are expected to converge when propagating along a direction $45^{\circ}$ to the grain axis in columnar CSS or SS weld metal. Figures $4 a$ and $b$ show the images produced by identical $2.25-\mathrm{MHz}$ longitudinal waves traveling through water alone and through $12 \mathrm{~mm}$ of weld metal at $45^{\circ}$ to the grain axis, respectively. In the latter case, the incident ultrasonic beam was normal to the specimen surface. Despite the short path, the beam was distorted as it passed through the weld metal, with focusing of the beam in the horizontal (110) plane, but not in the orthogonal plane. The difference in the way the velocities vary with propagation direction accounts qualitatively for the elliptical shape of the beam emerging from the specimen.

Figures $4 \mathrm{c}$ and $\mathrm{d}$ show the ultrasonic beam distortion produced for longitudinal waves propagating parallel and perpendicular, respectively, to the columnar grains. Again, the incident beam was normal to the specimen surface. In both cases, the emerging beam was weak relative to the one propagated at $45^{\circ}$ to the grains (fig. $4 \mathrm{~b}$ ), and was distorted more in the horizontal plane than the vertical. Figure 4e shows the cross section of a beam propagated through a 4-mm-thick slice from a weld-metal specimen, at an angle of incidence of about $22^{\circ}$. With this sample orientation, 


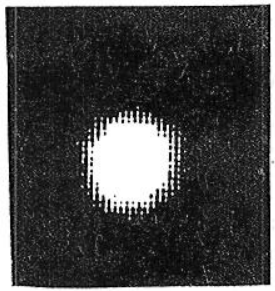

(a)

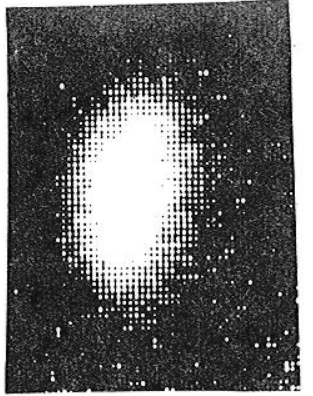

(b)

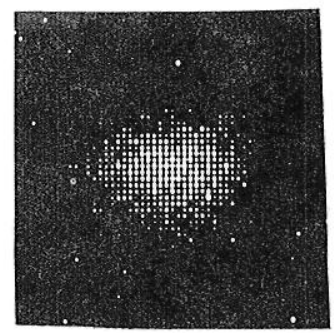

(c)

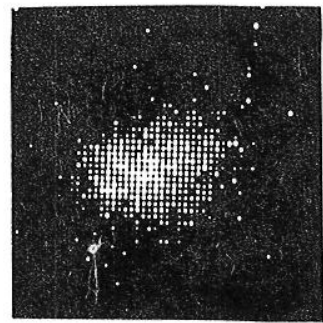

(d)

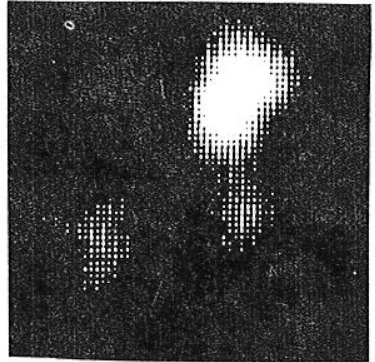

(e)

Fig. 4. Cross Section of Longitudinal Beam Passing through (a) Water; (b-e) Weld Metal Oriented with the Grain Axis (b) at $45^{\circ}$ to the Beam Axis; (c) Paralle1 to the Beam Axis; (d) Perpendicular to the Beam Axis; and (e) at an Angle That Allows Only Shear Waves to Travel through the Weld Metal.

longitudinal waves were mode converted to shear waves which then propagated approximately perpendicular to the columnar grain axes. (The shear waves mode-converted back to longitudinal waves in the water.) In this case, the propagation in weld metal produced a large, nonuniform distortion. In contrast, a beam propagated through a similar sample of isotropic metal emerged from the sample with a highly uniform cross section (data not shown). This effort demonstrates that visualization of ultrasonic beams can lead to better insight into the distortion of ultrasonic waves as they pass through transverse isotropic material.

\section{ULTRASONIC CHARACTERIZATION OF GREEN CERAMICS}

\section{Background}

Development of NDE methods for green (unfired) ceramics could result in considerable cost savings in fabricating structural components, as the final firing stage would be eliminated for defective components. This section describes a preliminary investigation to study the propagation of ultrasonic waves in green ceramics 
and to assess the potential of ultrasonic NDE methods as a source of useful quantitative information on defects, cracking, delaminations, agglomerates, inclusions, regions of high porosity, and anisotropy.

The difficulties associated with ultrasonic examination of green ceramics are formidable. For example, low-frequency sound waves are not scattered sufficiently to allow the detection of flaws much smaller than $1 \mathrm{~mm}$ in size; on the other hand, sound waves with frequencies of $\lambda^{3} \mathrm{MHz}$ generally undergo excessive attenuation in samples $>3 \mathrm{~mm}$ thick. Also, ordinary couplants (e.g., water or glycerol) are often absorbed by green ceramics; this not only mitigates their coupling function, but may also affect the subsequent fabrication process. This problem can be avoided by using pressure alone to couple the transducer to the specimen, but great care is required because the applied pressure can affect the measured velocity and attenuation, particularly for high-frequency longitudinal waves, or even result in breakage of the fragile specimen.

In spite of these difficulties, studies of ultrasonic attenuation and acoustic velocity (including dispersion and frequency spectra) in green ceramics may provide useful information related to density variations, porosity content, presence of agglomerates and delaminations, elastic anisotropy, and material quality in general; some examples of promising approaches are presented below. Further development of ultrasonic testing techniques, coupled with signal enhancement techniques, may lead to improved flaw detection sensitivity.

Technical Progress

Green ceramic specimens were provided by the Ceramics Group of the Materials Science and Technology Division at Argonne National Laboratory (ANL) and the Materials Chemistry Division of the National Bureau of Standards (NBS). The specimens were generally 50-60\% of theoretical density and very fragile. The main experimental effort was carried out with silicon nitride disks $3.3 \mathrm{~cm}$ in diameter and 0.6 $\mathrm{cm}$ thick and magnesium aluminate spinel disks $3.7 \mathrm{~cm}$ in diameter and $0.6 \mathrm{~cm} \mathrm{thick}$. Some other materials were also included. The specimens were cold pressed with various loading pressures and additions of polyviny1 alcohol (PVA) or Carbowax (CW) binder.

\section{Elastic Anisotropy}

The elastic anisotropy of a green ceramic specimen can be determined from the change in sound velocity that occurs when a shear-wave transducer is rotated with respect to the specimen, thus varying the polarization of the shear waves propagating in a particular direction. Sound veloctty data were acquired for $\mathrm{Si}_{3} \mathrm{~N}_{4}$, $\mathrm{MgAl}_{2} \mathrm{O}_{4}$, $\mathrm{MgO}$, and $\mathrm{YCrO}_{3}$ specimens with a Panametric 5052UAX ultrasonic transducer analyzer and a Tektronix 7904 oscilloscope with 7 B85 and 7B80 time bases. Panametric 2. 25-MHz normal-incidence shear-wave transducers (13 $\mathrm{mm}$ in diameter) and Aerotech 2. 25-MHz alpha transducers (6 mm in diameter) were employed for the measurements. The velocity was measured by overlapping successive echoes in the pulse-echo mode and determining the time delay from the oscilloscope. The time base was calibrated by checking the oscilloscope readings against a sequence of precisely timed pulses from a Tektronix type 184 time mark generator. With the Mgo specimens, which have a 
high content of $\mathrm{CW}$ binder, Panametric shear-wave couplant was successfully used for both longitudinal and shear waves. With the chalk-1ike $\mathrm{YCrO}_{3}$ specimens (<1\% PVA binder), no couplant was needed for either type of wave.

Figure 5 shows the echo pattern for 2.25- $\mathrm{MHz}$ longitudinal and shear waves in a 3.8-mm-thick $\mathrm{YCrO}_{3}$ specimen. (About 6 Hs separate the first and second longitudinal echoes; about $10 \mu \mathrm{s}$ separate the shear echoes.) Apparent attenuation is clearly rather high, on the order of $10-20 \mathrm{~dB} / \mathrm{cm}$. Furthermore, the effect of polarization on shear-wave velocity shows that the material is elastically anisotropic: A maximum velocity variation of about $3 \%$ was reproducibly observed as the shear-wave transducer was rotated about its axis while remaining over the same point on the sample. (The pressure of the transducer on the sample was controlled so that the velocity variations could not be attributed to variations in transducer loading.) Table II summarizes the sound velocity data for the $\mathrm{YCrO}_{3}$ sample. The low value of Poisson's ratio implies that under stress, the volume of the specimen is significantly reduced; this is consistent with the porous, low-density nature of the sample. Since the modulus of elasticity is related to the velocity of sound, variations in shear velocity with polarization can indicate variations of modulus with direction. This variation in modulus could affect the performance of a component made from such a sample.

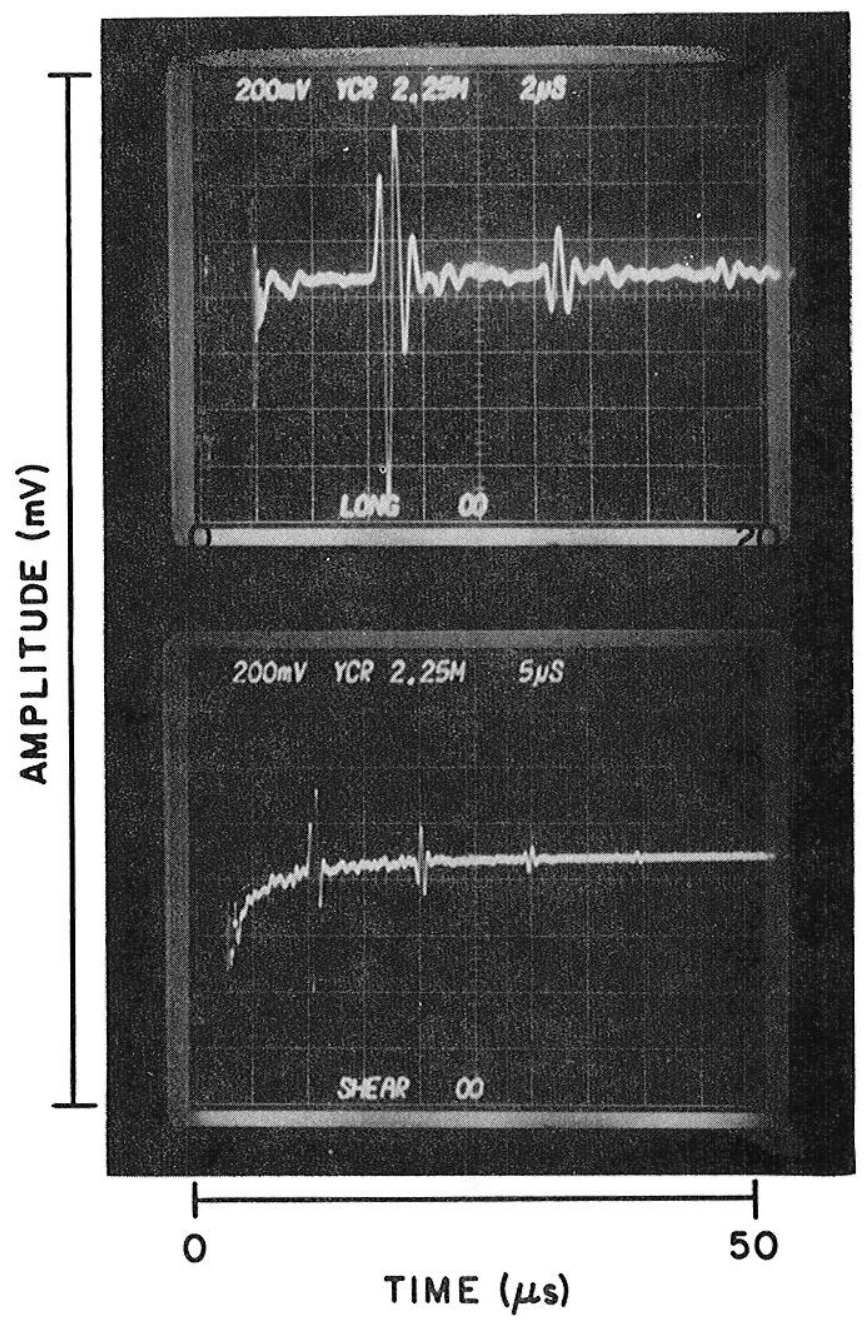

Fig. 5. Echoes from a 3.8-mm-Thick YCrO Sample Insonified with (Top) Longitudinal Waves and (Bottom) Shear Waves at $2.25 \mathrm{MHz}$. Axis dimensions are as follows: Ordinate - $200 \mathrm{mV} / \mathrm{div}$; abscissa - $2 \mu \mathrm{s} / \mathrm{div}$. in upper pane1, $5 \mathrm{\mu s} / \mathrm{div}$. in lower pane1. 
Table II. Sound Velocity in $\mathrm{YCrO}_{3}$ Sample with PVA Binder

\begin{tabular}{ccc}
$\begin{array}{c}\text { Longitudinal Velocity } \\
\left(10^{5} \mathrm{~cm} / \mathrm{s}\right)\end{array}$ & $\begin{array}{c}\text { Shear Velocity } \\
\left(10^{5} \mathrm{~cm} / \mathrm{s}\right)\end{array}$ & $\begin{array}{c}\text { Poisson's Ratio, } \\
\sigma\end{array}$ \\
\hline 1.43 & $0.947 \mathrm{max}$ & 0.11 \\
1.43 & $0.919 \mathrm{~min}$ & 0.15 \\
\hline
\end{tabular}

Another example of variation with polarlzation was observed in a 3-mm-thick section of a splnel disk, insonifled with shear waves propagating in the plane of the disk. With polarlzation parallel to the disk axis, the velocity (error $<0.5 \%$ ) was about $3.5 \%$ higher than with polarization perpendicular to the disk axis. No variation of velocity with polarization was found for wave propagation parallel to the disk axis. This implies that the shear modulus is greatest in the pressing direction.

\section{Vartation of Velocity with Density}

The velocity of longitudinal waves has been measured as a function of density for MgO specimens with $20 \mathrm{wt} . \% \mathrm{CW}$ binder. Density variations among these spectmens result from differences in loading pressure, i.e., in porosity. Figure 6 shows a plot of density vs sound velocity for four samples. One of the samples is anomalous (it has a visible delamination). From the results for the other three samples, a linear relationship between velocity and density is apparent; the variation in density is $1 / 3$ the varlation in velocity. Also shown in figure 6 is the nominal variation in density for three of the samples (rectangles around the data points), determined from variations in velocity measurements taken at five different points on each sample. Variations in density of up to $2 \%$ were observed. These results suggest that not only can the density (and thus porosity) of a sample be determined from sound velocity measurements, but if sufficiently small transducers were aval1able, anomalous microstructures could be detected by mapping local variations in density.

The maximum density ( $0 \%$ porosity) of $\mathrm{MgO}-20 \mathrm{wt} . \% \mathrm{CW}$, calculated from the densities of the two constituents, is $2.7 \mathrm{~g} / \mathrm{cm}^{3}$. The velocity obtained at this density by extrapolating the curve of figure 6 is $8.1 \times 10^{5} \mathrm{~cm} / \mathrm{s}$. This velocity can be compared to theoretical models that predict upper and lower bounds for sound velocity in a homogeneous composite material with a soft matrix and hard filler, as a function of the volume fraction of one of the constituents. Theoretical values for the longitudinal velocity were calculated as a function of volume fraction by using both the Voigt model (which assumes constant strain and gives an upper bound for velocity) and the Reuss model (which assumes constant stress and gives a lower bound). The velocities were calculated according to the following relationships (ref. 2):

$$
\begin{aligned}
& \rho v_{l_{v}}^{2}=f_{1} \rho_{1} v_{l_{1}}^{2}+f_{2} \rho_{2} v_{l_{2}}^{2} \text { (Voigt velocity), } \\
& \frac{1}{\rho v_{l R}^{2}}=\frac{f_{1}}{\rho_{1} v_{l_{1}}^{2}}+\frac{f_{2}}{\rho_{2} v_{l_{2}}^{2}} \text { (Reuss velocity), }
\end{aligned}
$$




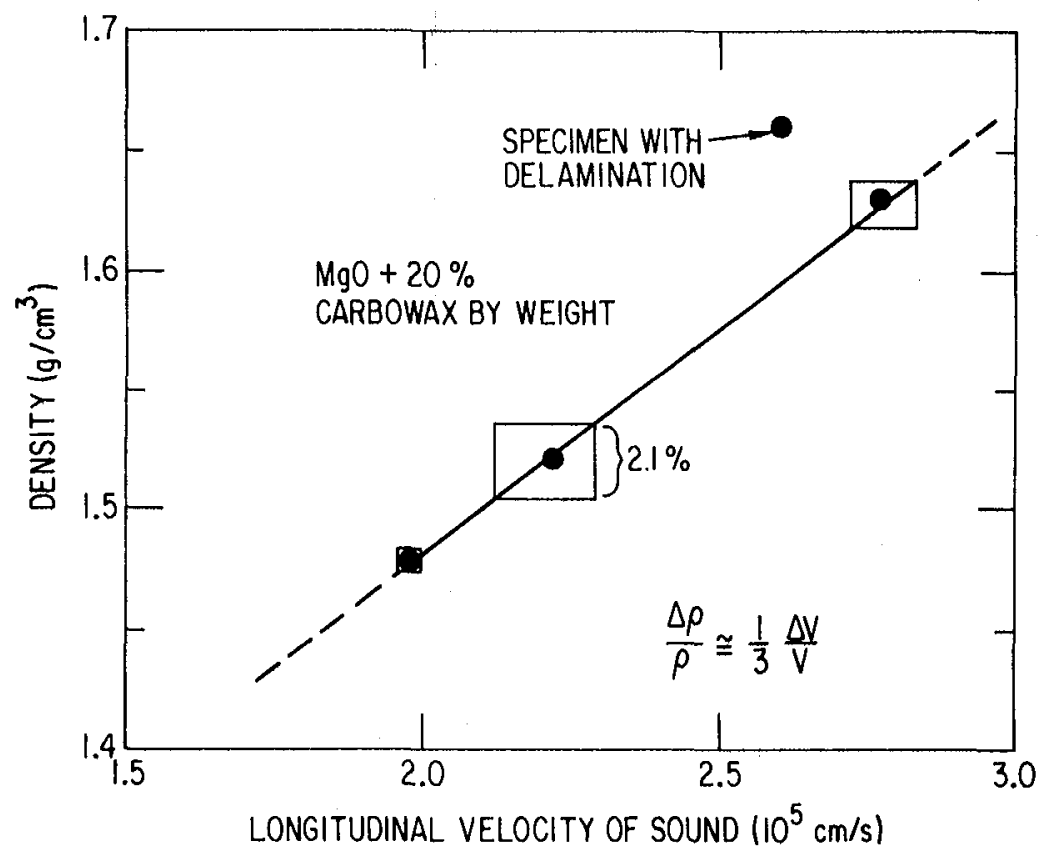

Fig. 6. Sample Density vs Longitudinal Velocity of Sound for $\mathrm{MgO}+20 \%$ Carbowax. Rectangles show variation within each sample.

where $f_{1}, f_{2}$ are fractional volumes, $\rho_{1}, \rho_{2}$ are densities, and $v_{\ell_{1}}, v_{\ell}$ are longitudinal velocities for constituents 1 and 2 , respectively, and $\rho=f_{1} \rho_{1} 1_{1} f_{2} \rho_{2}$. Figure 7 shows the resultant upper (Voigt) and lower (Reuss) velocity 1 lmits for $\mathrm{MgO} / \mathrm{CW}$ composites vs the volume fraction of $\mathrm{CW}$. The extrapolated velocity for a volume fraction of $37.5 \% \mathrm{CW}$ (the amount used in the ANL pellets) is indicated. This value is within the theoretical bounds and is close to the Voigt limit.

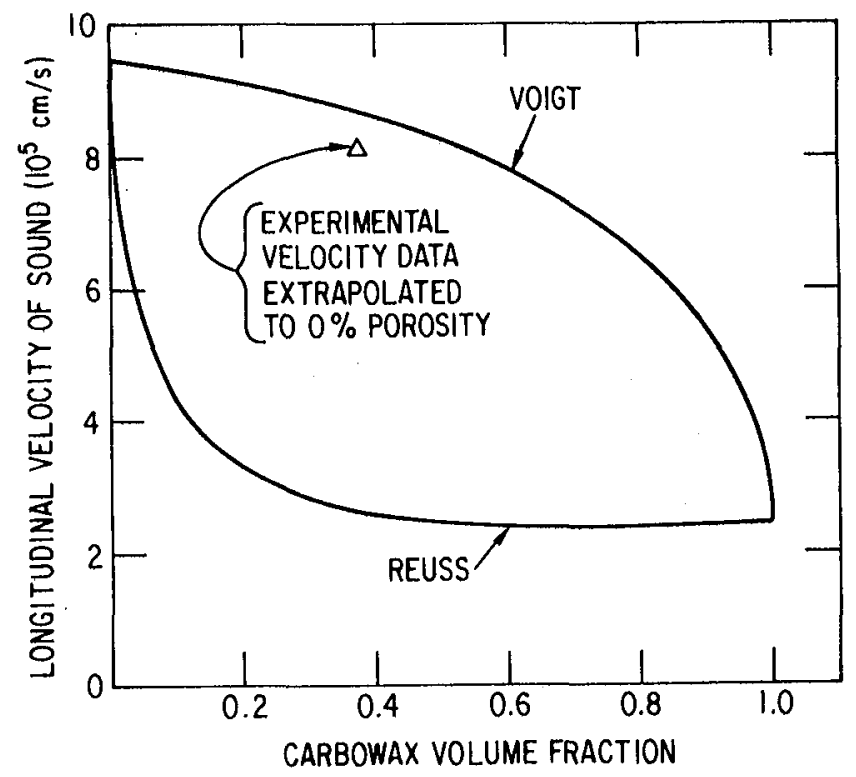

Fig. 7. Theoretical Upper and Lower Bounds for Longitudinal Velocity vs Carbowax Volume Fraction. Experimental value determined from extrapolation of data in Fig. 6 is also shown. 
These results Indicate that sound velocity measurements can provide quantitative Information on porosity and local fluctuation in density, and can perhaps indicate anomalous microstructures. The analysis also indlcates that data acquired in this manner may be predicted by models for composites, further suggesting that sound velocity data and relationships to anomalous microstructures may be understood from a fundamental point of view.

\section{REFERENCES}

1. Kupperman, D. S.; ReImann, K. J.; and KIm, D. I.: U1trasonic Characterization and Microstructure of Stalnless Steel Weld Metal. Nondestructive Evaluation: Microstructural Characterization and Reliability Strategles, 0 . Buck and S. M. Wolf, Eds., The Metallurglcal Soclety of AIME, 1981, pp. 191-216.

2. Lees, S. and Davidson, C. L.: Ultrasonic Measurement of Some Mineral Filled Plastics, IEEE Trans. Sonics Ultrason. SU-24(3), 222 (May 1977). 



\title{
ULTRASONIC CHARACTERIZATION OF STRUCTURAL CERAMICS
}

\author{
Stanley J. Klima and George Y. Baaklini \\ National Aeronautics and Space Admintstration \\ Lewis Research Center \\ Cleveland, Ohto 44135
}

Uitrasonic velocity and attenuation measurements were used to characterize density and microstructure in monolithic silicon nitride and silicon carbide. Research samples of these structural ceramics exhibited a wide range of density and microstructural variations. It was shown that bulk density variations correlate with and can be estimated by velocity measurements. Variations in microstructural features such as grain size or shape and pore morphology had a minor effect on velocity. However, these features had a pronounced effect on ultrasonic attenuation. The ultrasonic results are supplemented by low-energy radiography and scanning laser acoustic microscopy.

\section{INTRODUCTION}

The energy-saving potential of ceramics in heat engines is based on higher permissible engine operating temperatures as well as reduced weight, inertia, and friction. Estimates of potential efficiency improvements for automotive engines with major ceramic components range from 30 to 50 percent over current engine technology (ref. 1).

Structural ceramics such as silicon carbide and silicon nitride are leading candidates for engine hot-section components because of their relatively light weight, excellent oxidation and thermal shock resistance, and good hightemperature strength. They are also composed of nonstrategic materials that are inexpensive when mass produced. However, their wide variation in strength properties and relatively low fracture toughness have inhibited a large-scale move toward replacing many metal engine parts with ceramics. The variable strength and low toughness are generally attributed to discrete defects such as voids, inclusions, and cracks introduced during processing (refs. 2 and 3 ). Current cost-effective fabrication procedures also frequently produce ceramics containing bulk density variations and microstructural anomalies that can adversely affect performance (ref. 4). Therefore it is essential to have nondestructive evaluation (NDE) methods available that can not only detect discrete flaws but also provide information regarding the material's physical and mechanical properties and uniformity. By incorporating NDE technology into a ceramic materials technology development program, information may be obtained regarding the source of defects so that steps can be taken to minimize their occurrence through improved fabrication procedures. Thus NDE may help to reduce the effort expended in a materials research program and shorten the time needed to develop strong, reliable structural ceramics. With these considerations in mind, a program for NDE of ceramics was established to provide direct support to the ceramic materials development program at the NASA Lewis Research Center. 
The objectives of the Lewis NDE program are to identify and develop techniques for application to ceramic research materials and heat engine components and to establish NDE methodology for ceramic process control, properties verification, and degradation assessment. This paper describes the preliminary use of uitrasonic techniques for characterizing density and microstructure in monolithic silicon carbide and silicon nitride research samples. The ultrasonic work is supplemented by other nondestructive techniques such as radiography and scanning laser acoustic microscopy.

\section{MATERIALS AND TEST PROCEDURE}

\section{Materials}

The materials investigated were structural-grade monolithic ceramics of alpha silicon carbide and silicon nitride. The silicon carbide was procured from a commercial source, and the silicon nitride was fabricated in-house from commercially avallable powders.

Specimens of alpha silicon carbide with densities ranging from 2.83 to $3.18 \mathrm{~g} / \mathrm{cm}^{3}$ were used for this study. Blocks measuring 5.0 by 5.0 by $0.64 \mathrm{~cm}$ were procured in the as-sintered condition from three separate lots. Selected blocks from lot $A$ were sliced into two equal parts. One half of each block was hot isostatically pressed (HIP) at either 1950 or $2050{ }^{\circ} \mathrm{C}$. Whole blocks from lot B were HIP at either 1950 or $2050{ }^{\circ} \mathrm{C}$. All lot A and B blocks were subsequently machined into rectangular bend-test specimens measuring 0.31 by 0.62 by $2.5 \mathrm{~cm}$. The blocks from lot C were HIP at $2040{ }^{\circ} \mathrm{C}$ but were otherwise unaltered. Densities were calculated from bulk weight and volume measurements of the final test specimens.

The silicon nitride specimens were made by mixing $\mathrm{Si}_{3} \mathrm{~N}_{4}$ powder with $\mathrm{Y}_{2} \mathrm{O}_{3}$ powder ( 6 percent) and ball milling to obtain a uniform mix and to reduce the particle size. The $\mathrm{Y}_{2} \mathrm{O}_{3}$ is a sintering aid used to promote densification in the absence of external pressure. The milled powder was then dry pressed to near net shape, sintered at $2140{ }^{\circ} \mathrm{C}$, and diamond ground to final dimensions of 0.31 by 0.62 by $2.75 \mathrm{~cm}$. Bulk densities were calculated from total weight and volume measurements of the final test specimens. A detailed description of the fabrication procedure is given in reference 5 .

\section{Velocity Measurements}

The ultrasonic velocity is given by $v=4 d / 2 t$, where $d$ is the thickness of the test bar and $t$ represents the time for a sound wave to make a roundtrip through the thickness. The specimen thickness, nominally $0.31 \mathrm{~cm}$, was measured to the nearest $2.5 \mathrm{um}$. Velocity measurements were made at three locations along the length of the test bar, averaged, and plotted against the average buik density of the bar.

A broadband longitudinal wave transducer with a $0.63-\mathrm{cm}$-diameter active element and a 20-MHz center frequency was used for the velocity measurements. The active element was bonded to a fused silica delay buffer, which was coupled to the specimen with glycerine. The materials studied exhibited sufficientiy 
low ultrasonic attenuation that recovery of multiple echoes from the highfrequency, short-wavelength signals was not a problem even in the lowest density samples. The pulse-echo overlap method for measuring time of travel was used for all velocity determinations. A detailed description of the technique and an estimate of its accuracy are given in references 6 and 7 . The heart of the system is a pulser-receiver that drives the ultrasonic transducer and provides an intensified sweep capability that highlights the signals of interest while ignoring all others. An oscillator driving a horizontal amplifier in a dual-trace oscilloscope provides for overlap of the intensified signals (the first and third back-surface reflections) on the CRT. Overlap occurs when the oscillator frequency equals the reciprocal of the time interval between the intensified signals. A digital counter was used to display either the oscillator frequency or the measured time of travel of the sound wave.

\section{Attenuation Measurements}

Attenuation measurements were obtained by the ultrasonic pulse-echo technique using a broadband longitudinal wave transducer with a center frequency of $100 \mathrm{MHz}$. The $0.63-\mathrm{cm}$-diameter active element was bonded to a fused silica buffer coupled to the specimen surface with glycerine. The first and second back-surface reflections within the sample were digitized, transformed into the frequency domain by the Fourier method, and compared to obtain ultrasonic attenuation as a function of frequency. The method of calculating the attenuation coefficient takes into account the frequency dependence of the reflection coefficient at the specimen-buffer interface. The method used to measure ultrasonic attenuation is described in detail in reference 8.

\section{RESULTS AND DISCUSSION}

\section{Microstructures}

Microstructural examination of some alpha silicon carbide specimens revealed three distinct microstructures, typified in the photomicrographs presented in figure 1. These microstructures were not intentionally induced and were observed while performing destructive and nondestructive characterization of the materials under investigation. All of the material from lot A exhibited a relatively fine microstructure consisting of small, equiaxed grains and fine, uniformly distributed porosity. Although the photomicrograph in figure 1 (a) is of a specimen that was hot isostatically pressed at $2040{ }^{\circ} \mathrm{C}$, the microstructure is also typical of both the as-sintered materials and the material HIP at $1950{ }^{\circ} \mathrm{C}$. The only significant difference was a decrease in average pore size and population in the HIP specimens. The lot B silicon carbide was procured at a later date. Two-thirds of the specimens from this group had a duplex microstructure consisting of small, equiaxed grains mixed with a substantial proportion of larger, elongated grains (fig. $1(b)$ ). The average grain size was slightly greater than that observed for lot $A$ material, but the porosity was again fine and uniformly distributed. The remaining one-third of lot B specimens exhibited a duplex structure with much larger grains, most of which appeared to be elongated (fig. $1(\mathrm{c})$ ). In addition, the voids were larger and fewer and many contained free carbon. The lot B material was available only in the HIP condition; therefore microstructural comparisons could not be made with as-sintered material. 
Significant variations in bulk density and micropore size and distribution were also found in the silicon nitride specimens. X-ray films of modulus-ofrupture (MOR) specimens selected from a single batch of material are reproduced in figure 2. The radiographs exhibit density variations indicative of bulk material density gradients in the samples after sintering and grinding to final dimensions. The darkest regions in the figure represent zones of highest density; the lighter shades of gray delineate zones having proportionally lower density. Except for a few bars such as the one on the left, all of the specimens are characterized by a low-density core surrounded by a high-density case. The case-core configurations are generally unique to each specimen and appear to be related to temperature gradients experienced in the sintering furnace. Figure 3 shows three views of a polished cross section and fractographs of a typical MOR bar after mechanical testing. The macrophotograph of the polished cross section reveals different shades of gray coincident with the shading observed in the transmission radiographs in the previous figure, physical evidence of the variation in density in these specimens. The population of voids in the core far exceeds the number in the case. The fractographs exhibit surface morphologies that reflect differences in the way the crack propagated through the specimen. The fractograph of the case shows a rough surface texture indicative of a tortuous fracture path; the core appears smooth and faceted, suggestive of a structure with low crack propagation resistance.

\section{Velocity-Density Relations}

The velocity of ultrasonic waves in a solid is related to the elastic properties of the material as shown in the well-known expression:

$$
v_{\ell}^{2}=\frac{E}{\rho} \frac{(1-\mu)}{(1+\mu)(1-2 \mu)}
$$

where $v_{\ell}$ is the velocity of a longitudinal wave, $E$ is Young's modulus, $\rho$ is material density, and $\mu$ is Poisson's ratio. The elastic properties in turn depend on the level of porosity as well as certain pore characteristics. For example, reference 9, which summarizes the results of several investigations, shows that Young's modulus increases exponentially with increasing density (i.e., as porosity decreases):

$$
E=E_{0} \exp (-b P)
$$

where $E_{0}$ is Young's modulus for the nonporous material, $P$ is volume fraction porosity, and $b$ is a porosity correction factor determined by pore size, shape, location, etc. It was also reported that Poisson's ratio generally increases with increasing density, but the effect is small. Thus it was expected that velocity will be an increasing function of density in spite of the inverse relation in equation (1). This expectation was borne out by the results of this investigation.

The relation between ultrasonic velocity and the bulk density of alpha silicon carbide is shown in figure 4. These data were obtained for two blocks from lot A material, which exhibited a fine, equiaxed grain structure. Six specimens from each block were used to generate base-line ultrasonic and density information for the as-sintered condition. A variation in density of approximately 1 percent is revealed in both blocks. The horizontal scale on 
the plot was expanded to better illustrate how this relatively small but significant variation in density is reflected in the ultrasonic velocity measurements. The remaining six specimens from each block were HIP at either 1950 or $2050{ }^{\circ} \mathrm{C}$. These data show that both the bulk density and velocity increased proportionally with HIP temperature. The small spread in the $2050{ }^{\circ} \mathrm{C}$ data indicates that densification was nearly complete and uniform at the higher HIP temperature and that spot ultrasonic velocity measurements can accurately estimate the density. The plotted velocity readings are an average of only three measurements at equaliy spaced intervals along the specimen length. Thus the good correlation with density could be expected only with samples whose properties are uniform throughout their bulk. The samples HIP at $1950{ }^{\circ} \mathrm{C}$ exhibited a lower degree of densification. The spread in average bulk density between specimens, although quite small at 0.5 percent, implies that the samples do not have uniform properties. A better correlation with ultrasonic velocity could only be achieved by making a greater number of measurements over the breadth of individual specimens. A large number of measurements, each on a small-volume sample at regularly spaced intervals, would also provide information regarding density gradients within a given specimen.

The alpha silicon carbide from lot B was not avallable for ultrasonic measurements in the as-sintered condition. Data obtained for the HIP material are presented in figure 5. After hot isostatic pressing at $1950{ }^{\circ} \mathrm{C}$ densification was moderate and substantial variability existed between specimens. Com-. parison with lot A material HIP at the same temperature (fig. 4) shows that the lot $B$ material with nearly the same grain size was more variable and less dense on average. After hot isostatic pressing at $2050{ }^{\circ} \mathrm{C}$ the smal1-grained material from lot $B$ was essentially equivalent to the lot A material in terms of density (99 percent of theoretical) except for a single specimen. Again, ultrasonic velocity provided a good measure of density for a 11 specimens. However, the large-grained material performed somewhat differently, as can be seen in figure 5. The density was not as high nor as uniform as that for the sma11grained material HIP at $2050{ }^{\circ} \mathrm{C}$. Also, the ultrasonic velocity was somewhat higher than might have been predicted from data obtained with the small-grained specimens. This observation implies that the large-grained specimens had a higher modulus of elasticity. However, according to reference 9 , elastic properties have no basic dependence on grain size. If this is indeed the case, it must concluded that the higher measured values are related to the pore size and distribution evident in the specimens with the larger grain size. For specimens with equivalent bulk density this would have to be accounted for by the value assigned to the porosity correction factor $b$ in equation (2).

All of the silicon carbide data obtained in this investigation are summarized in figure 6 . The upper half of the plot contains only the extreme values of velocity and density from each block or material condition in lots $A$ and $B$. This was done to 1 llustrate the degree of scatter while minimizing the number of plotted points. The four data points in the lower half of the figure were obtained from the two blocks in lot $c$ that were intentionally sintered to provide unusually low initial density levels for this composition. The pair at the lower left are measurements taken from one of the blocks before and after not isostatic pressing at $2050^{\circ} \mathrm{C}$. Little or no densification was accomplished, indicating that porosity was continuous and open to the specimen surface. The two data points at densities between 2.9 and $3.0 \mathrm{~g} / \mathrm{cm}^{3}$ were obtained from the other block and reveal a small beneficial effect of hot isostatic pressing. It is evident from this figure that a strong functional relation exists between ultrasonic velocity and the bulk density of alpha 
silicon carbide over a wide density range. Although the individual effects of different microstructural features on elastic modulus were not uniquely determined, it appears that velocity measurements can serve to screen out specimens and components with abnormally low bulk density and modulus. With appropriate calibration, bulk densities can be estimated to within approximately 1 percent and better for specimens with essentially similar microstructures.

Ultrasonic velocity measurements were made on sintered silicon nitride to determine the utility of this technique for measuring bulk density and modulus on another candidate heat engine material. Figure 7 shows radiographs of three MOR bars with different mean bulk densities. The top specimen was relatively uniform and fully dense. The others contained a low-density core that varied inversely in size with the average bulk density. The figure shows a 6 percent difference in both density and velocity between the top and bottom specimens. However, although the middle specimen was 3 percent less dense than the top specimen, the velocity was only 1 percent less. This demonstrates that in specimens containing steep density gradients such as these, a large number of velocity measurements, each on a relatively small-volume sample, should be made for precise quantitative material characterization. A detailed velocity map could thus be produced to characterize quantitatively the material in terms of density.

To determine the magnitude of density variation within a specimen, the relatively high-density case was machined off the bottom specimen in figure 7 , using the radiograph to determine the amount to be removed. The core (which was not necessarily uniform of itself) was approximately 3 percent less dense than the average for the total specimen. The core of the bottom specimen, how-. ever, was approximately 8 percent less dense than the fully dense top specimen. These differences are reflected in the ultrasonic velocity measurements. The complementary nature of the $x$-ray and ultrasonic results illustrates the usefulness of applying more than one technique for nondestructive characterization of materials.

Scanning laser acoustic microscopy in the interference mode (ref. 10) can also be used to obtain information regarding bulk density variations. In a specimen with constant thickness the time of travel of the ultrasonic wave provides an indication of relative velocity. SLAM interferograms can be used to image velocity variations that exist within the viewing field, as shown in figure 8. Any deviation in time of travel will cause the interference lines to bend to the right (higher velocity) or to the left (lower velocity). The relatively straight lines in figure $8(\mathrm{a})$ indicate that, within the field of view, the density of the ceramic specimen is relatively uniform. In figure 8 (b) however, the density at the bottom (near the edge of the specimen) is significantly greater than that at the top of the image, which is located just over $2 \mathrm{~mm}$ away from the edge. These observations agree with observations made on radiographs shown previously.

\section{Ultrasonic Attenuation}

Although significant variations in the microstructure of alpha silicon carbide had a relatively small effect on ultrasonic velocity, the same variations had a profound effect on attenuation of high-frequency ultrasonic waves. Uitrasonic attenuation was plotted as a function of frequency (fig. 9) for eight SiC specimens selected because their bulk densities were nearly equal. 
All specimens were from lot $B$ and were divided equally into sets with two widely differing microstructures (fig. 9). Attenuation in the large-grained material was at least four times that in the small-grained material. Although grain size was the most obvious microstructural difference between the two sample groups, there was also a sharp contrast in the void morphology. On the basis of density considerations the two groups had the same net average porosity. The photomicrographs show that the voids in the small-grained specimens were smaller but more numerous than those in the large-grained material.

Modulus-of-rupture test results show that the material with the fine microstructure was significantly stronger at room temperature than the coarser material. These preliminary results suggest that ultrasonic attenuation measurements may be useful as a quality control tool to verify material strength to the extent that it is controlled by microstructure.

\section{CONCLUDING REMARKS}

Ultrasonic velocity and attenuation measurements were determined to be useful for characterizing ceramics in terms of density and microstructure. Bulk densities of silicon nitride and silicon carbide correlated with and could be estimated by velocity measurements. Although variations in certain microstructural features had a minor effect on velocity, they had a strong effect on ultrasonic attenuation. It appears that attenuation measurements may be useful in estimating material strength, to the extent that it is determined by microstructure.

\section{REFERENCES}

1. Johnson, O.R.; et al.: Ceramic Technology for Advanced Heat Engines Project. Am. Ceram. Soc. Bul1., vol. 64, no. 2, Feb. 1985, pp. 276-281.

2. Rice, R.W.; et al.: Failure Causing Defects in Ceramics; What NDE Should Find. NRL-MR-4075, Naval Research Laboratory, Oct. 1979. (AD--A078234.)

3. Baumgartner, H.R.; Brockelman, R.H.; and Hanson, P.M.: Development of Nondestructive Testing Techniques for High Performance Ceramics. AMMRC-TR-78-71, Army Materials and Mechanics Research Center, Jan. 1978. (AD-A059063.)

4. Lange, F.F.: Fracture Mechanics and Microstructural Design. Fracture Mechanics of Ceramics, Vol. 4, R.C. Bradt, D.P.H. Hasselman, and F.F. Lange, eds., Plenum Press, 1978, pp. 799-819.

5. Sanders, W.A.; and Mieskowski, D.M.: Strength and Microstructure of Sintered $\mathrm{Si}_{3} \mathrm{~N}_{4}$ with Rare-Earth-0xide Additions. Am. Ceram. Soc.

Bull., vol. 64, no. 2, Feb. 1985, pp. 304-309.

6. Klima, S.J.; et al.: Ultrasonic Velocity for Estimating Density of Structural Ceramics. NASA TM-82765, 1981.

7. Papadakis, E.P.: Absolute Accuracy of the Pulse-Echo Overlap Method and the Pulse-Superposition Method for Ultrasonic Velocity. J. Acoust. Soc. Am., vol. 52, no. 3, pt. 2, Sept. 1972, pp. 843-846. 
8. Generazio, E.R.: The Role of the Reflection Coefficient in Precision Measurement of Ultrasonic Attenuation. Mater. Eval., vol. 43, no. 8, July 1985, pp. 995-1004.

9. Rice, R.W.: Microstructure Dependence of Mechanical Behavior of Ceramics. Properties and Microstructure, R.K. MacCrone, ed., Academic Press, 1977, pp. 199-381.

10. Kessler, L.W.; Yuhas, D.E.; and Vorres, C.L.: Acoustic Microscopy of Ceramics. Proceedings of the DARPA/AFWAL Review of Progress in Quantitative Nondestructive Evaluation, AFWAL-TR-81-4080, pp. 128-135, (AD-A108741.)

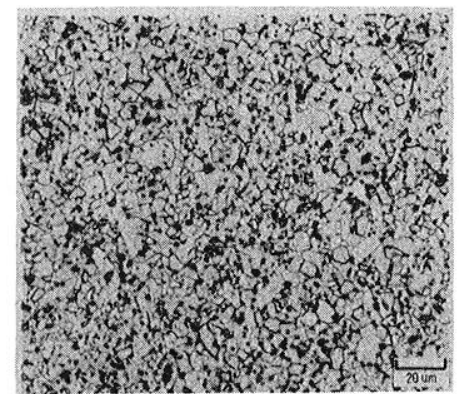

(a) Lot A material: small, equiaxed grains.
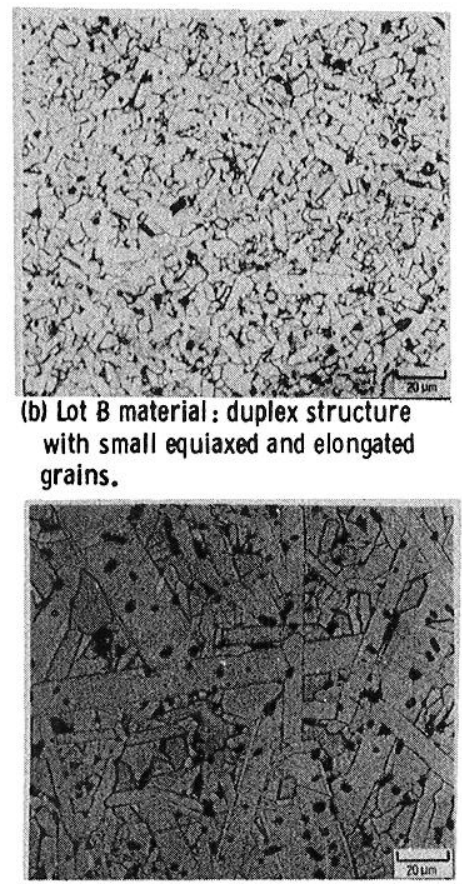

(c) Lot B material: duplex ștructure with large, elongated grains.

Figure 1. - Microstructures in alpha silicon carbide, showing variations in grain size and pore morphology. All specimens shown were hot isostatically pressed at $2050^{\circ} \mathrm{C}$.

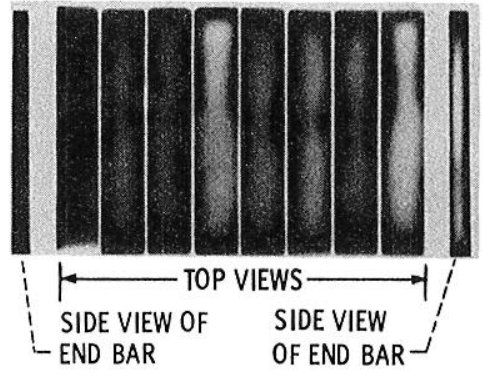

Figure 2. - Radiographs of modulus-ofrupture bars showing density variations within single lot of sintered silicon nitride. Specimens were ground to uniform thickness. 


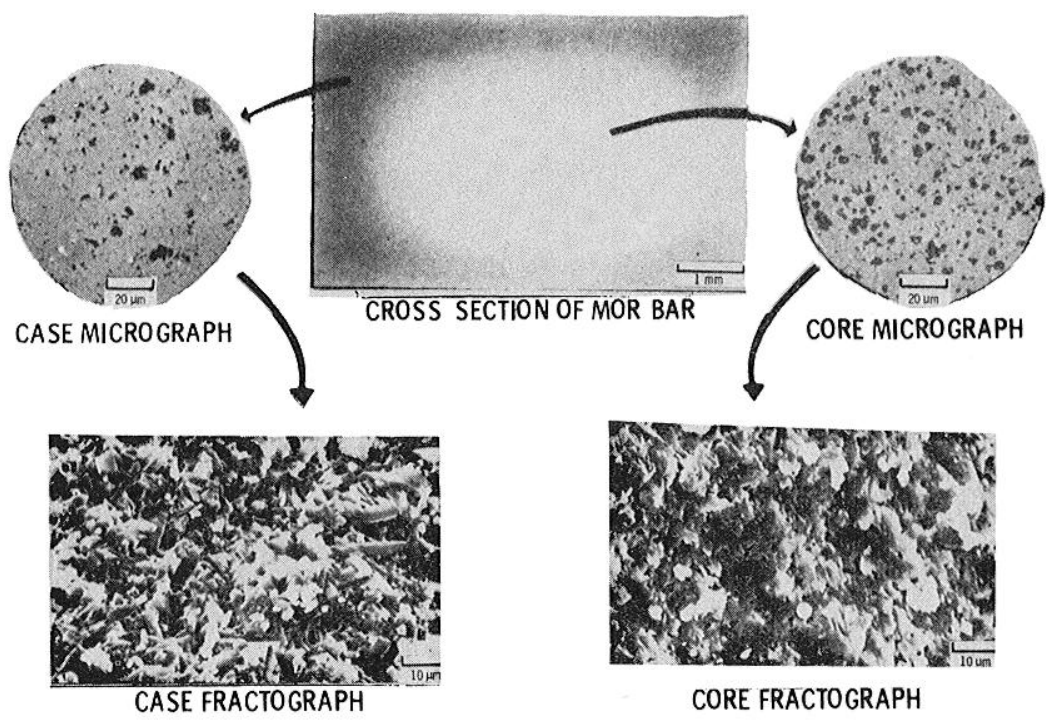

Figure 3. - Unetched cross section of modulus-of-rupture bar showing high-density case and low-density core. SEM fractographs show differences in fracture surface morphology in the two zones.

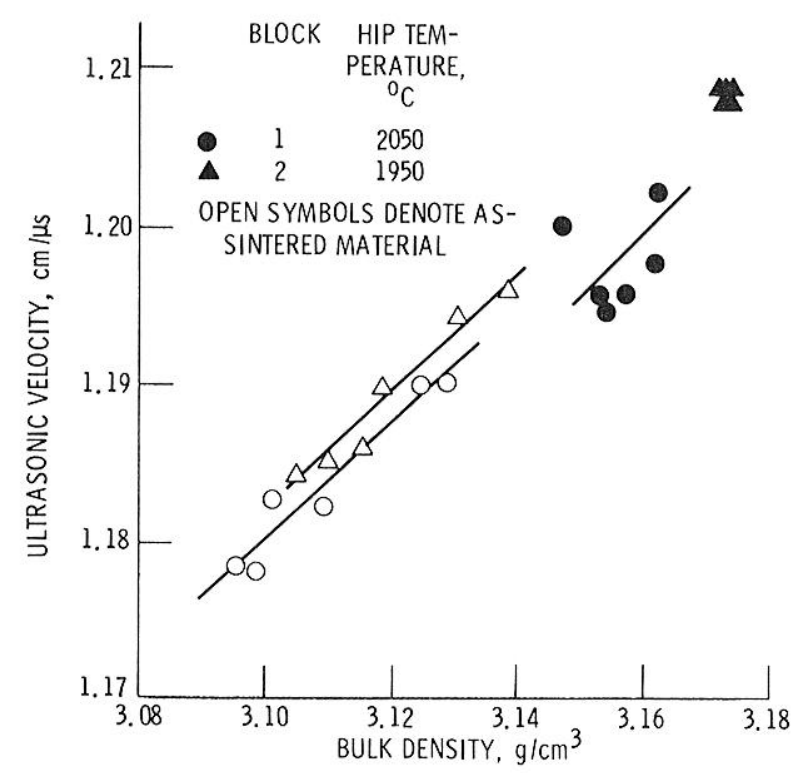

Figure 4. - Relation between ultrasonic velocity and bulk density of alpha silicon carbide from lot A before and after hot isostatic pressing.

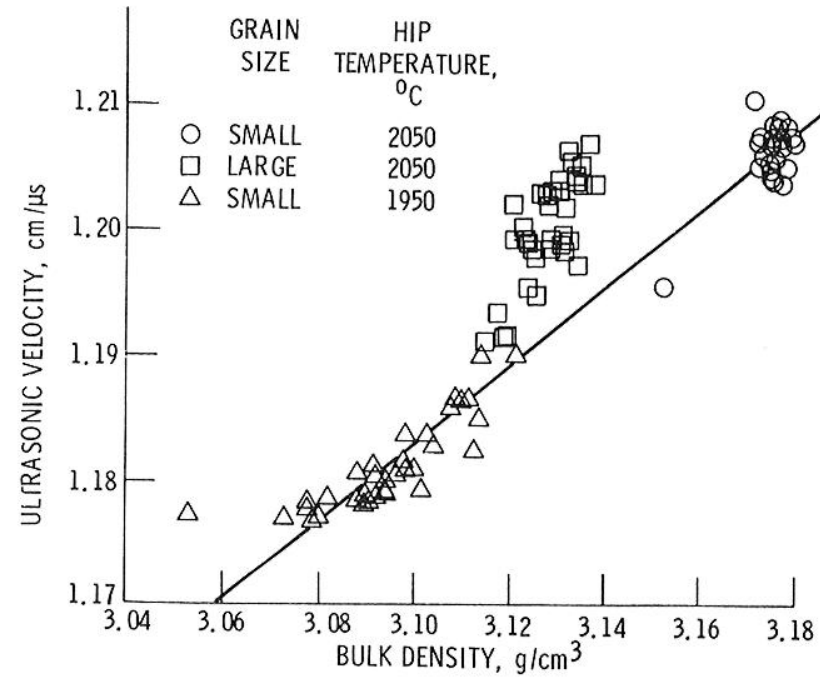

Figure 5. - Effect of microstructure on ultrasonic velocity and bulk density of hot isostatically pressed alpha silicon carbide from lot $B$. 


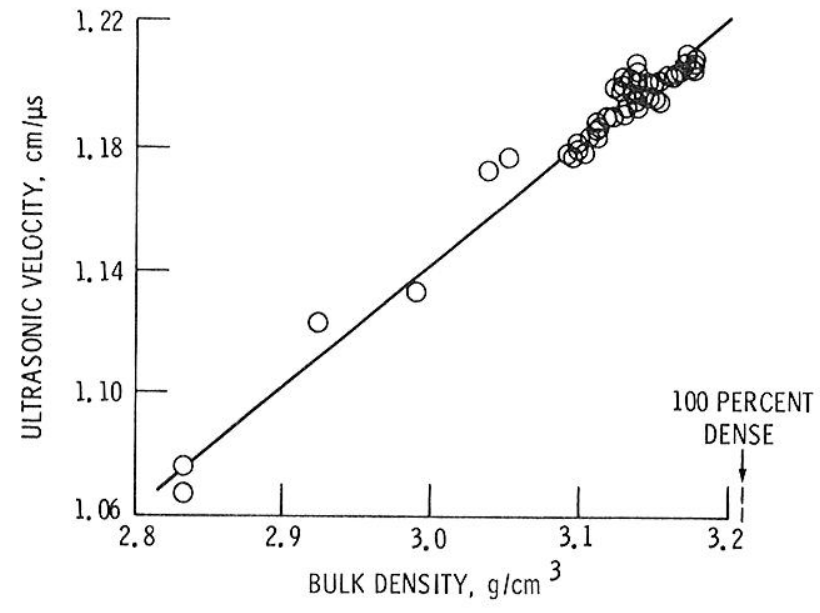

Figure 6. - Relation between ultrasonic velocity and bulk density of alpha silicon carbide. Data for material in as-sintered and hot isostatically pressed conditions and three grain sizes.

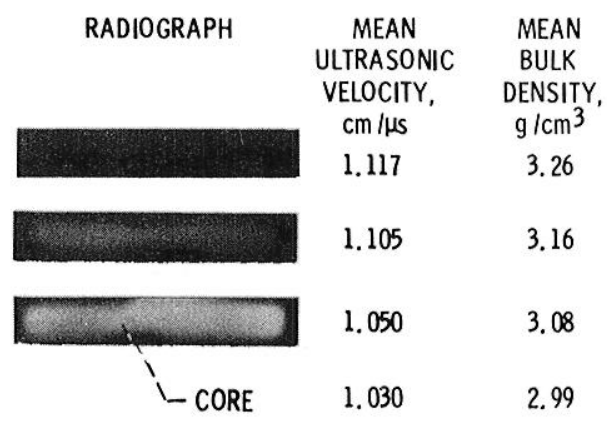

Figure 7. - Ultrasonic velocity measurements for three silicon nitride specimens with variable densities.

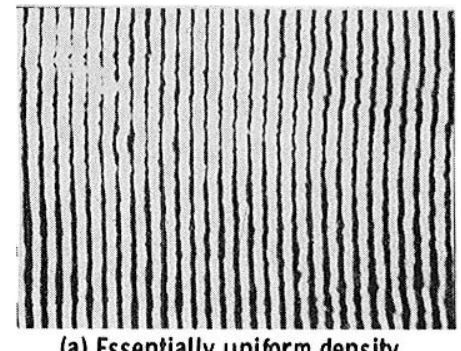

(a) Essentially uniform density.

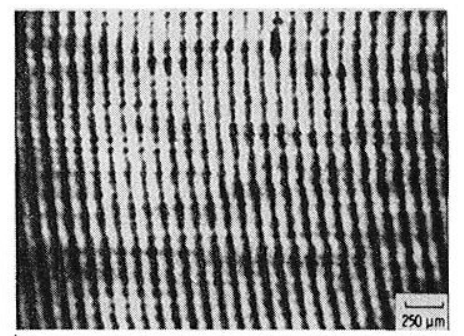

(b) Density increasing from top to bottom.

Figure 8. - Interferograms produced by scanning laser acoustic microscope, illustrating capability for imaging density gradients in silicon nitride modulusof-rupture bars.

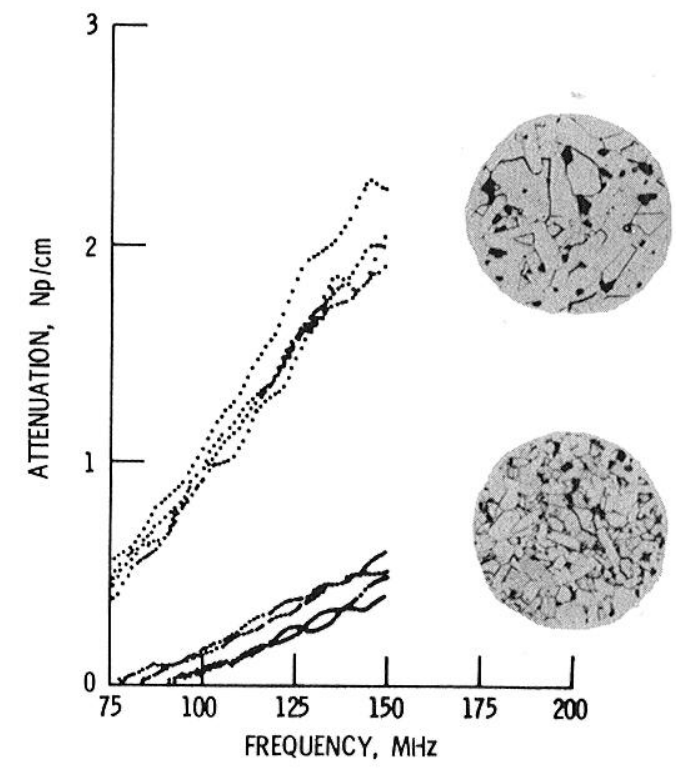

Figure 9. - Effect of microstructure on ultrasonic attenuation in sintered alpha silicon carbide. Bulk density, 3.11 to $3.12 \mathrm{~g} / \mathrm{cm}^{3}$. 
ON THE INTERACTION OF ULTRASOUND WITH CRACKS:

\title{
APPLICATIONS TO FATIGUE CRACK GROWTH
}

\author{
O. Buck, R.B. Thompson, and D.K. Rehbein \\ Iowa State University \\ Ames, Iowa 50011
}

Partial contact of two rough fatigue crack surfaces leads to transmission and diffraction of an acoustic signal at those contacts. This paper deals with recent experimental and theoretical efforts to understand and quantify such contact in greater detail. The final objective is two-fold: 1. To develop an understanding of the closure phenomenon and its application to the interpretation of fatigue data, in particular the R-ratio, spike overload/underload and threshold effects on crack propagation. 2. To obtain an understanding of the effects of closure on the detection probability of fatigue cracks, which reflects strongly on the capability for accurate life prediction. In the present paper only the first objective will be discussed.

\section{INTRODUCTION}

It has been pointed out (ref. 1) that the ultrasonic interrogation of components for the detection and sizing of defects has advantages over other techniques in that both surface as well as subsurface defects can be probed. However, it has become clear that this is not a simple problem since the phenomenon of crack closure or crack surface contact can contribute to the transmission of ultrasound across the crack surfaces which reduces the detectability and leads to erroneous crack sizing (ref. 2). Originally, the term "crack closure" was used to describe the observation of a nonlinearity in the crack opening displacement as a specimen, containing a crack, is cyclically loaded (ref. 3). It has been demonstrated that this is mainly a result of individual contact points (asperities) caused by a mismatch of the fracture surfaces (ref. 4). As this contact occurs, the stresses ahead of the crack will be redistributed such that the driving force for crack propagation becomes significantly smaller than would be expected from a simple calculation of the stress intensity range $\Delta \mathrm{K}=\mathrm{K}_{\max }-\mathrm{K}_{\min }($ refs. 2,3$)$. Therefore, crack closure will affect crack propagation rates (refs. $3,5,6$ ) in addition to the acoustic effects mentioned above.

*This work was supported by USDOE, Office of Basic Energy Sciences, Division of Materials Sciences under contract No. W-7405-Eng-82. 
Using ultrasonic techniques, the characterization of the size and spatial variation of the local contacts, as we11 as the local contact stresses, is now in progress (refs. 7-15). As soon as these quantities have been fully determined it will be possible to calculate the residual stresses along the fracture surface (refs. 16, 17) which have to be overcome by the external forces in order to open the crack fully. This residual stress field is compressive and will have to be continuous with that of the plastic zone due to reverse yielding (ref. 18). Eventually it will yleld full information on the "effective" stress intensity range (ref. 19), as indicated in this paper.

The purpose of the present paper is to review briefly the status of this work and to speculate on further developments which may provide valuable information on the "state" of a fatigue crack, including the "effective" stress intensity range. Only large cracks formed in non-corrosive environments will be considered here. Modifications of the closure models due to formation of corrosion debris (ref. 20) and effects of these modifications on the interaction of ultrasound with the crack have not been considered as yet.

\section{EXPERIMENTAL TECHNIQUES}

Basically two types of ultrasonic experiments have been conducted on specimens, which can be loaded in tension and compression, to study asperity contact. The first method uses the diffraction of bulk (refs. 7, 11) or surface (Rayleigh) waves (ref. 21) from locations where the contact occurs. Individual contact points can be observed in a time domain display of the signal arriving at the recelving transducer. A schemat1c descrịption of a bulk wave diffraction experiment (ref. 7 ) is shown in Figure 1. The second method uses mainly the transmission and reflection coefficient of bulk waves (refs. 8, 10, 12-15). In this case a broadband transmitter is used. The received signal is Fourier analyzed in order to obtain information on the asperity contact. In addition, mode conversion at the asperities leads to a diffraction effect which can also be studied (ref. 15). Figure 2 shows this arrangement schematically. The longitudinal transmitted signal is obtained at $\theta=$ $0^{\circ}$, the diffracted signals at $\theta=45^{\circ}$.

The above experiments are special cases of a more general one in which the angle of incidence and the angle of observation may be arbitrarily chosen for all types of mode conversions. It should be pointed out here that a theory for this general case has not been developed yet. However, to provide guidance for such a theory, an experiment has been devised (see fig. 3) in which such measurements can be performed on "mode1" cracks with known asperity size and separation (refs. 15, 22). A (cylindrical) specimen was produced by pressing together two blocks. One of the blocks contains a photolithographically produced roughness, simulating asperity contact at the interface. Such a specimen seems to be well suited to study transmission and reflection, as well as diffraction for a wide range of angles for different roughnesses (periodic or random).

\section{OBSERVATIONS AND INTERPRETATIONS}

Making use of signals diffracted at asperities (fig. 1) seems to be the most direct way to determine the location of these asperities, at least if they are relatively large. Golan and Arone (ref.11) recently published first results on 
such an Investigation using a compact tension specimen. These authors measured the signal amplitude of shear waves diffracted at each contact point as a function of applied load. Thus, knowing at whlch external load each individual contact opens up (determined by a loss of the diffracted slgnal) and assuming that the contact is elastic allowed these authors to evaluate the contact forces using a set of torque balance equations. The results indicate that the contacts do not open in a "perfect" sequence (the opening of the contacts is unrelated to the distance from the crack tip). Consequently the contact forces do not vary in a systematic way. The sizes of the contact areas have not been determined so that the individual contact stresses can not be estimated at the present time.

The usefulness of the transmission and reflection coefficlent to determine asperity contact has been demonstrated by Haines (ref. 8) (based on data by Wooldridge (ref. 23)), Thompson et al. (refs. 10,12,13), and Rehbein et al. (ref. 15). In either case, a quasi-static model for the interaction of an ultrasontc wave with partially contacting surfaces is used. It is assumed that, when the ultrasonic wavelength is large with respect to the dimensions and separations of the contacts, their influence on an ultrasonic wave can be modeled by a pair of effective boundary conditions

$$
\begin{aligned}
& \sigma_{1}+\sigma_{r}-\sigma_{t}=0 \quad \text { (for the stresses) } \\
& u_{1}+u_{r}-u_{t}=U \text { (for the displacements) }
\end{aligned}
$$

where the Indices $1, r$, and $t$ refer to Incldent, reflected, and transmitted waves, respectively and $U$ is the elastic displacement the asperities experience due to $\sigma_{t}$, as will be discussed below. By applying standard analysis the (frequency dependent) transmission and reflection coefficients can be calculated. Figure 4 shows, as an example, a comparison of the calculated (ref. 8) and measured (ref. 23) reflection coefficients as a function of applied stress, $\sigma_{0}$, obtained on steel plates with given roughness. Qualitatively good agreement has been obtalned. The major difference between the Haines and Thompson approaches is in the interpretation of the quantity $U$. Haines (ref. 8) makes use of a detalled model of the contact between real surfaces as required to understand the physics of tribology. In this case

$$
U=\frac{\sigma_{t} \pi d p_{m}}{2 \sigma_{0} k^{*} E}
$$

where $d$ is the mean diameter of the contact area, (which can be related to surface roughness), $p_{m}$ is the "flow pressure" (usually three times the ultimate tensile strength), $E$ is the Young's modulus, $\sigma_{0}$ is the average static stress across the interface, and $k^{*}$ is a constant $(* 2)$. Thompson et al. (ref. 10), on the other hand, uses an analytical solution for the additional displacement which an interface experiences if this interface consists of individual contacts. For a simple strip mode1* one obtains

$$
\mathrm{U}=\frac{\sigma_{\mathrm{t}}}{\kappa}
$$

with

\footnotetext{
*A modification of this strip model to describe the more realistic situation of individual contact points will be discussed later.
} 


$$
K=\frac{E}{2 \alpha s}\left\{1.071\left(\frac{1}{\alpha} \ln \frac{1}{1-\alpha}-1\right)+0.25 \alpha-0.357 \alpha^{2}+0.121 \alpha^{3}+\ldots\right\}
$$

where $\alpha=1-w / s$, and $w$ and $s$ are the width and separation of the asperity contacts. Using focused, broadband acoustic transducers (see fig. 2), Thompson and Fiedler (ref. 13) determined the transmission coefficient for a fatigue crack (fig. 5a). The results can be compared with calculated values ( $f 1 g .5 b$ ) under the following assumptions. The beam profile is Gaussian and frequency dependent and the Kirchhoff approximation can be applied. $K$ (and therefore $w$ and $s$ ) changes gradually from $\infty$ to 0 over a certain distance away from (but containing) the crack tip. A good fit to the experimental results has been obtained choosing an exponentially decaying $k$. The result is that the crack length appears to be larger for the higher frequency components than for the lower ones. The independent variables $w$ and $s(E q$. 4) have not been determined separately as yet. A second type of experiment will have to be performed.

On the other hand, it should be mentioned that the frequency dependent transmission and reflection coefficients, as calculated by this model (ref. 10), have been compared to exact solutions for periodic arrays of strip contacts, as calculated by Angel and Achenbach (ref. 24). It was found that the agreement between these exact solutions and those obtained by Thompson et al. (ref. 10) is excellent when the wavelength is large with respect to the contact spacing, a condition under which most of the experiments have been performed to date. It is noteworthy, however, that the exact solutions (ref. 24) show large changes in the transmission and reflection coefficients when the wavelength is equal to the contact spacing, thus providing direct information on contact spacing.

The quasi-static model was expanded recent1y (refs. 15, 25) for the case of a longitudinal wave at normal incidence to the fracture surface. The model now predicts diffracted longitudinal and mode-converted transverse waves, given by the normalized signal

$$
\Gamma^{N}=C \int_{-\infty}^{\infty} d x\left[\frac{1}{1+j \alpha}\right] e^{-\left(x-x_{1}\right)^{2} / p^{2}} e^{-j k\left(x-x_{l}\right) \sin \theta}
$$

where $C$ is a normalizing constant involving the wave velocities, beam amplitude, beam widths and an angular term, $p$ is a beam width parameter, $k$ is the wave vector, $x_{1}$ is the position of the beam center and $\theta$ is the angle of the receiver with respect to the transmitter axis. For all forward transmission experiments, $\theta$ is equal to $0^{\circ}$; all tip diffracted experiments were performed at $\theta=45^{\circ}$ so far. The normalization is executed with respect to the through transmission signal received from the uncracked region. The bracketed factor in the equation represents the interface transmissivity and the other factors describe the beam magnitude and phase overlaps. The factor $\alpha$ in the bracketed term is

$$
\alpha=\pi \rho v f / K(x)
$$

where $\rho$ is the material density, $v$ the transmitted wave velocity, $f$ is the frequency and $k(x)$ is the distributed spring constant. Again, choosing $k(x)$ to be a continuous exponential, comparison of theory and experiment at $\theta=0^{\circ}$ was favorable, as reported elsewhere (refs. 12,13). Alternatively, the experimentally observed longitudinal and shear wave signals diffracted in the closure region of a fatigue crack, could not be predicted by the model. The more severe deviation of experiment from the model is for the shear wave case, as shown in figure 6 . The frequency dependence of the signals from the real crack is much greater than those from the 
Ideal crack for both the model and experiment. In addition, the peak amplitude of the experimental data has decreased significantly from the corresponding peak for the 1deal crack. The frequency dependence of the theoretical predictions is quite pronounced, and at the higher frequencles, the theory is several orders of magnitude too small. It would appear, therefore, that the closure region plays an even more slgniflcant role for tip diffracted shear waves than for longitudinal waves, either tip diffracted or through transmission. The model was therefore extended to (approximately) include the effects due to discrete contact points of varying diameter. In equation (5), the interface transmissivity is given by the term $1 /(1+j \alpha)$. In a first attempt to assess the significance of discrete contacts, the following assumptions were made regarding the form of the dynamic crack opening displacement (COD). (1) The spatial average of the COD is the same as that predicted by the quasi-static spring model. (2) The local COD assumes the value of zero over an effective contact of diameter $d$ and is a constant elsewhere. (3) In evaluating the ensuing scattering expressions, integrals over the small circular contact areas (with dimensions much less than a wavelength) may be approximated by the value of the integrand times the area of a circle of twice the contact diameter. When the approximate COD is substituted into the representation integral for ultrasonic scattering, the result is equivalent to that which would be obtained when the interface transmissivity is replaced by the factor

$$
\frac{1}{1+j \alpha}+\left(\frac{1}{1+j \alpha}\right)\left\{1-\frac{j 8 \pi f \rho d v}{E^{\prime}\left(1-N \pi d^{2}\right)}+\frac{1}{N^{1 / 2}} \sum \frac{j 8 \pi f p v d \delta\left(x-x_{1}\right)}{E^{\prime}\left(1-N \pi d^{2}\right)}\right\}
$$

where $\mathrm{N}$ is the contact density (assumed to be a constant over the closed region), $\mathrm{d}$ is the diameter of an individual contact, $E^{\prime}=E /\left(1-v^{2}\right)$ and the sum is to be evaluated over the $x$-coordinate of the crack.

If one views $k(x)$ as being known independently, e.g. froin measurements of forward transmission of longitudinal waves, then $d$ and $N$ are related according to

$$
\mathrm{d}(\mathrm{x})=8 \kappa(\mathrm{x}) / \mathrm{N} \pi \mathrm{E}^{\prime}
$$

Thus, In this mode1, two parameters are needed to fully define the scattering, $\kappa(x)$ and $N$. The previously reported longltudinal forward transmission measurements agreed well with the continuous spring model (refs. 12, 13). Hence those measurements can be considered as an experimental determination of $\kappa(x)$. If the tip diffraction measurements are sensitive to $N$, they would provide an experimental means of determining this independent parameter of the partially contacting closure zone. Knowledge of both $K(x)$ and $N$ would allow the contact stresses to be calculated (refs. 12, 14).

To test this possibility, the modified model was used to calculate the $45^{\circ}$ tip diffracted shear wave signal, as shown in figure 7 . As can be seen, a change in the contact density has a marked effect on the amplitude of the peak for the $4 \mathrm{MHz} t \mathrm{ip}$ diffracted wave. At the lowest density considered, $\mathrm{N}^{1 / 2}=250$ contacts $/ \mathrm{cm}$, the theoretical predictions have increased by three orders of magnitude from the continuum limit and are approaching the level observed experimentally. In contrast, the corresponding results for longltudinal through transmission showed an indistinguishable change. It is concluded that it is essential to include discreteness of contacts in a description of tip diffracted waves and that the above scenario for directly measuring contact stress appears to hold considerable promise.

Unexpectedly large scattering effects at "model cracks", as described earlier, have been observed (refs. 15, 22). Figure 7 shows a polar plot of the signa1 
received for an L-wave striking an interface, with random roughness, at $45^{\circ}$. The comparison with the signals received from a reference block shows a surprisingly strong scattered signal in the $90^{\circ}$ observation direction, as well as the specularly reflected signal in the $270^{\circ}$ observation direction, obtained on the block with interface. The quasi-static model will thus have to be extended again to include the transmissivity and diffraction of crack surfaces, if the incident wave is off normal.

An interesting effect of a crack's stress history on the transmissivity was discovered using a 7075-T651 aluminum compact tension specimen. The specimen was precracked to a length of approximately $0.65 \mathrm{~cm}$. At that point, the load cycling was stopped and the specimen was allowed to age for an undetermined time. The crack was then extended an additional $1 \mathrm{~cm}$. The through transmission data from this specimen is shown in figure 9. In addition to the normal closure region at the tip of the crack, a second peak in the transmission coefficient data at the position of the earlier fatigue interruption can be seen. The peak becomes narrower with increasing frequency because of the smaller beam width, as at the $t$ ip of the crack. In addition, its peak value decreases at high frequencies due to the lower value of the interface transmissivity. Our current speculation is that the secondary closure peak in this data occurs in the region of the tip of the original precrack and is due to a stress overload condition that was placed on the crack when it was extended.

\section{CONCLUSIONS}

The preceeding discussions indicate that, through the use of ultrasound, it will become possible to determine the nature of asperity contact along fracture surfaces, including contact response to an external stress. Diffraction experiments (ref. 11) show that contact forces vary greatly from asperity to asperity. Transmission experiments (refs. 12-15, 25) indicate that the $k$ decreases with distance away from the crack tip. From Haines' model (ref. 8) and the newly developed distributed spring model (refs. 15, 25) which also uses discrete contact points, an evaluation of the contact stress present across the crack faces may now be possible through comparison of experimental data and model predictions to determine the density of asperity contacts. Once this objective is achieved, the data can be used to evaluate several characteristics of the fatigue crack, such as $\sigma_{0}$, the average static stress across the fracture surface (eq. 2) and, more importantly, the "effective" stress intensity range, driving the crack during propagation, as indicated below.

Combining eqs. (2) and (3) yields the average static stress to be

$$
\sigma_{0}=\frac{\pi d p_{m^{k}}}{2 k^{*} E}
$$

and, since (ref. 8)

$$
(d / 2)^{2}=\frac{\sigma_{0}}{p_{m}{ }^{N \pi}}
$$

thus 


$$
\sigma_{0}=\left(\frac{k}{k^{*} E}\right)^{2}\left(\frac{\pi}{N}\right) p_{m}
$$

where $\mathrm{N} 1 \mathrm{~s}$ the (local) number of asperities per untt area and therefore related to $\mathrm{w}$ and $s$ in equation (4). The decrease in $k$ with distance from the crack tip suggests a slmilar behavior for $\sigma_{0}$, as one would expect for an unloaded specimen, knowing the residual stress fleld in front of the crack tip and the remanent crack mouth opening.

Using a simple model, Beevers et al. (ref. 18) showed that the contact of an asperity (viewed as an infinite strip) leads to a stress intensity factor $K_{I}$ (local) at the crack $t 1 p$ for an unloaded specimen. The model $1 s$ shown in figure $10 \mathrm{a}$ and yields

$$
\mathrm{K}_{I}(\text { local })=\left(\frac{2}{\pi}\right)^{1 / 2} \frac{\mathrm{P}}{\mathrm{BC}^{1 / 2}}
$$

where $C$ is the distance from the crack tip to the asperity, $B$ the specimen thickness and $\mathrm{P}$ the contact load. Using the definitions introduced earlier, the above quantities may be replaced by $C \approx s$ and $P \approx \bar{\sigma}$ Bw since $b * w_{0} \bar{\sigma}$ represents the mean stress at the contact, which is related to the average static stress $\sigma_{0}$ by, roughly, $\bar{\sigma} \approx \frac{s}{w} \sigma_{0}$ so that

$$
K_{I}(\text { local }) \approx\left(\frac{2}{\pi}\right)^{1 / 2}\left(\frac{K_{k}^{*}}{k^{*}}\right)^{2}\left(\frac{\pi}{N^{3 / 2}}\right) p_{m}
$$

As an external load $Q$ is applied, $K_{I}$ (local) decreases and the compact tension specimen stress intensity factor

$$
K_{I}(g l o b a 1)=\frac{f(a) Q}{B \sqrt{a}}
$$

increases, as shown in $f$ Igure $10 \mathrm{~b}$, where $f(a)$ is the standard geometry factor, a the crack depth, and $Q$ the external load. If crack closure effects are not taken into account, the driving force, $\Delta \mathrm{K}$, on the crack in fatigue would be

$$
\Delta \mathrm{K}=\mathrm{K}_{\operatorname{Imax}}-\mathrm{K}_{\mathrm{Imin}}
$$

Crack closure (ref. 3) reduces this stress intensity range $\Delta \mathrm{K}$ to $\Delta \mathrm{K}_{\text {eff, the "effec- }}$ tive" stress intensity range, which can now be determined by

$$
\Delta \mathrm{K}_{\text {eff }}=\mathrm{K}_{\mathrm{Imax}}-\mathrm{K}_{\mathrm{I}} \text { (local) }
$$

where $\mathrm{K}_{\mathrm{I}}$ (local) is to be evaluated (acoustically) at the minimum load applied to the specimen and $\mathrm{K}_{\mathrm{Imax}}$ is given by the geometry of the specimen, the maximum external load $Q_{\max }$ and the crack depth a by eq. (14).

The usefulness of the acoustic measurements to determine the actual crack depth $a$, thus providing information on $\mathrm{K}_{\mathrm{Imax}}$, and the term $\mathrm{K}_{\mathrm{I}}$ (1ocal) thus seems to be obvious. Its application to determine the fatigue crack propagation rate

$$
\mathrm{da} / \mathrm{dN}=\mathrm{A}\left(\Delta \mathrm{K}_{\mathrm{eff}}\right)^{\mathrm{m}}
$$

as suggested orlginally by Elber (ref. 3), is a goal of the future. Particularly interesting will be to study cases in which the materials parameters $A$ and $\mathrm{m}$ should be independent of the applied fatigue conditions, such as for stress ratio and spike overload effects. 
1. Silk, M. G., "Research Techniques in Non-Destructive Testing, Vol. I", ed. R. S. Sharpe, Academic Press, 1977, p. 51.

2. Buck, 0., and Tittman, B. R., "Advances in Crack Length Measurement", ed. C. J. Beevers, Engineering Materials Advisory Services (EMAS), Cradley Heath, Warley, West Midlands, U. K., 1982, p. 413.

3. Elber, W., "Damage Tolerance in Aircraft Structures", ASTM STP 486, 1971, p. 230.

4. Bowles, C. Q., and Schijve J., "Fatigue Mechanisms: Advances in Quantitative Measurement of Physical Damage", ASTM STP 811,1983, p. 400.

5. Buck, 0., Frandsen, J. D., and Marcus, H. L., "Fatigue Crack Growth Under Spectrum Loads", ASTM STP 595, 1976, p. 101.

6. Suresh, S., and Ritchie, R. 0., Scripta Met 17, 1983, p. 595.

7. Golan, S., Adler, L., Cook, K. V., Nanstad, R. K., and Bolland, T. K., J. Nondestructive Evaluation 1, 1980, p. 11 .

8. Haines, N. F., "The Theory of Sound Transmission and Reflection at Contacting Surfaces", Report RD/B/N4711, Central Electricity Generating Board, Research Division, Berkeley Nuclear Laboratories, Berkeley, England, 1980 .

9. Wooldridge, A. B., Revue du CETHEDEC 17-4, NS 80-2, 1980, p. 233.

10. Thompson, R. B., Skillings, B. J., Zachary, L. W., Schmerr, L. W., and Buck, 0., "Review of Progress in Quantitative Nondestructive Evaluation, Vol 2A", eds. D. 0. Thompson and D. E. Chimenti, Plenum Press, 1983, p. 325.

11. Golan, S., and Arone, R., "New Procedures in Nondestructive Testing", ed. P. Höller, Springer Verlag, 1983, p. 587.

12. Thompson, R. B., Fiedler, C. J., and Buck, 0., "Nondestructive Methods for Materials Property Determination", eds. C. O. Ruud and R. E. Green, Plenum Press, 1984, p. 161.

13. Thompson, R. B., and Fiedler, C. J., "Review of Progress in Quantitative Nondestructive Evaluation, Vol. $3 A^{\prime \prime}$, eds. D. 0. Thompson and D. E. Chimenti, Plenum Press, 1984, p. 207.

14. Buck, 0., and Thompson, R. B., "Fatigue 84", ed. C. J. Beevers, Engineering Materials Advisory Services, Cradley Heath, Warley, West Midlands, U. K., 1984 , p. 667 .

15. Rehbein, D. K., Thompson, R. B., and Buck, 0., "Interaction of Ultrasonic Waves with Simulated and Real Fatigue Cracks", in "Review of Progress in Quantitat1ve Nondestructive Evaluation, Vol. 4", eds. D. 0. Thompson and D. E. Chimenti, Plenum Press (in press).

16. Westergaard, H. M., 1939, Trans. ASME (J. App1. Mech.) 61, 1939, p. A-49. 
17. Buck, 0., Skil11ngs, B. J., and Reed, L. K., "Review of Progress 1n Quant1tative Nondestructive Evaluation, Vol. $2 \mathrm{~A}^{\prime \prime}$, eds. D. 0. Thompson and D. E. Chimenti, Plenum Press, 1983, p. 345.

18. Rice, J. R., "Fatigue Crack Propagation", ASTM STP 415, 1967, p. 247.

19. Beevers, C. J., Be11, K., Carlson, R. L., and Starke, E. A., Eng. Fract. Mechanics 19, 1984, p. 93.

20. Suresh, S., Parks, D. M., and Ritchie, R. 0., "Fatigue Thresholds: Fundamentals and Englneering Applications", eds. J. Bäcklund, A. F. Blom and C. J. Beevers, Englneerlng Materials Advisory Services (EMAS), Cradley Heath, Warley, West Midlands, U. K., 1981, p. 391.

21. Tittmann, B. R., Ahlberg, L. A., and Buck, 0., "Revlew of Progress in Quantitative Nondestructive Evaluation, Vol. 1", eds. D. 0. Thompson and D. E. Chiment1, Plenum Press, 1982, p. 551.

22. Buck, 0., Fledler, C. J., Reed, L. K., Lakin, K. M., and Thompson, R. B., "Review of Progress in Quantitative Nondestructive Evaluation, Vo1. 3A", eds. D. 0. Thompson and D. E. Chimenti, Plenum Press, 1984, p. 199.

23. Wooldridge, A. B., "The Effects of Compressive Stress on the U1trasonic Response of Steel-Steel Interfaces and of Fatigue Cracks", Report NW/SSD/RR/42/79, Central Electricity Generating Board, Northwestern Region, Manchester, England, 1979 .

24. Ange1, Y. C., and Achenbach, J. D., "Reflection and Transmission of Elastic Waves by an Array of Microcracks" in "Review of Progress in Quantitative Nondestructive Evaluation, Vo1. 4", eds. D. O. Thompson and D. E. Chimenti, Plenum Press (in press).

25. Thompson, R. B., Fiedler, C. J., and Rehbein, D. K., Ames Laboratory, private communication. 

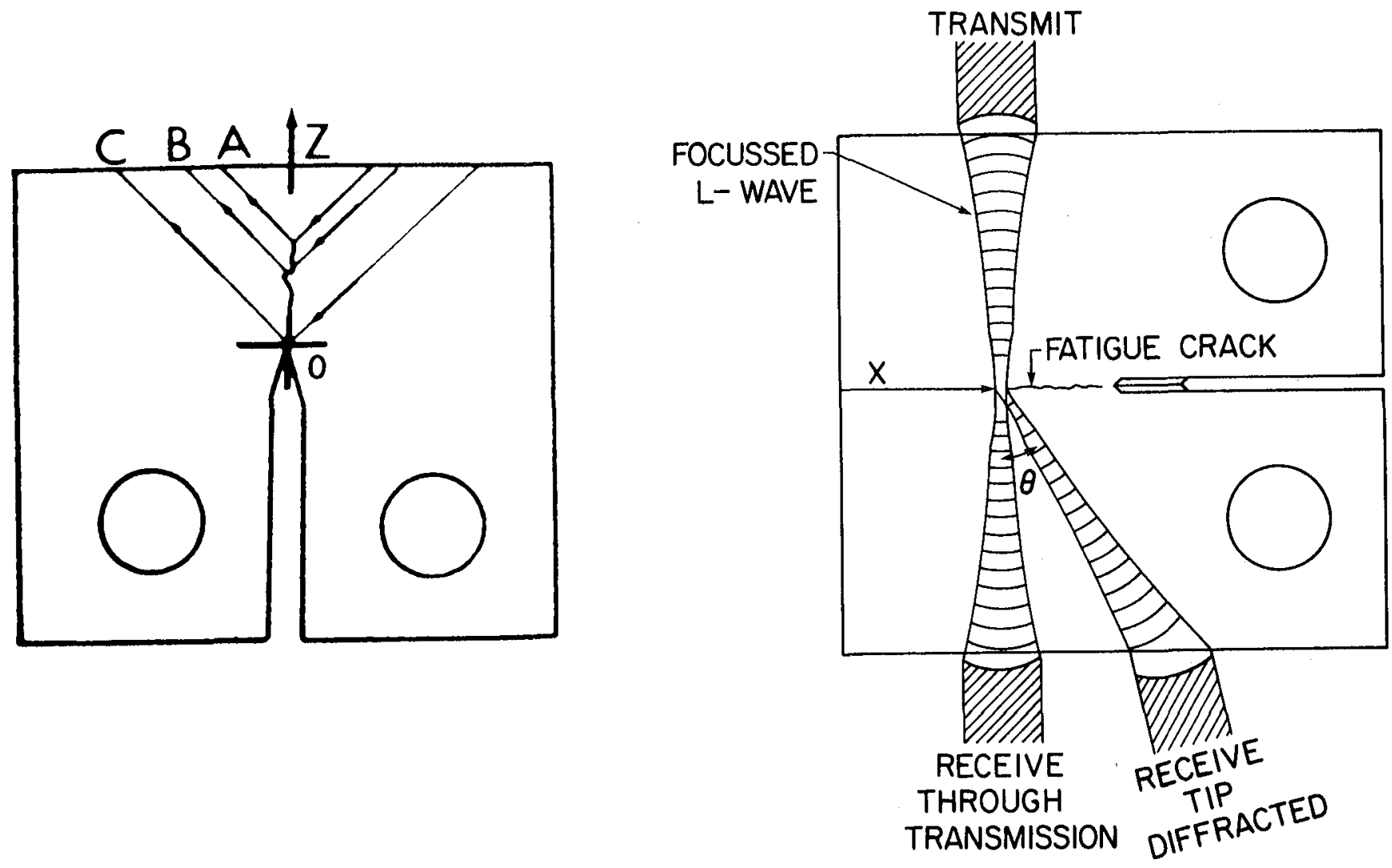

Fig. 1 Signals diffracted at

Fig. 2 Setup for obtaining crack contacts along the crack (ref. 7). and reference signal.

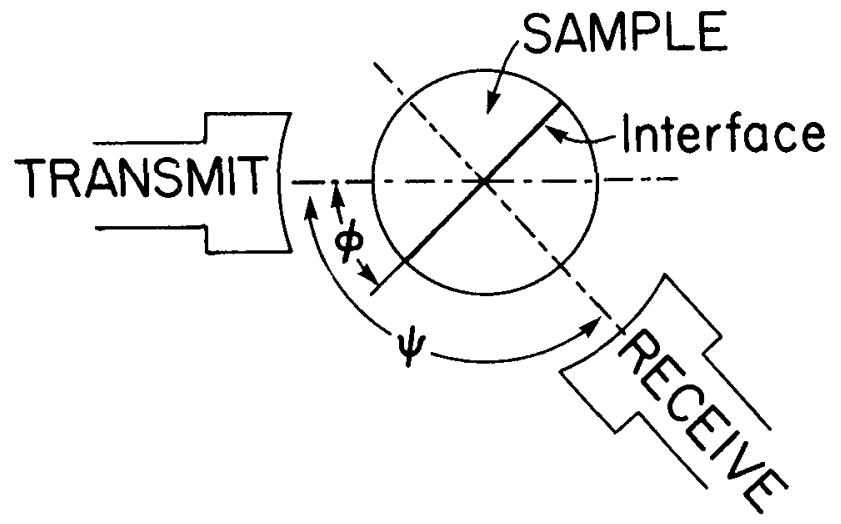

Fig. 3 Experimental setup using focussed transducers to determine scattering from interfaces in a cyclindrical sample (ref. 22). 


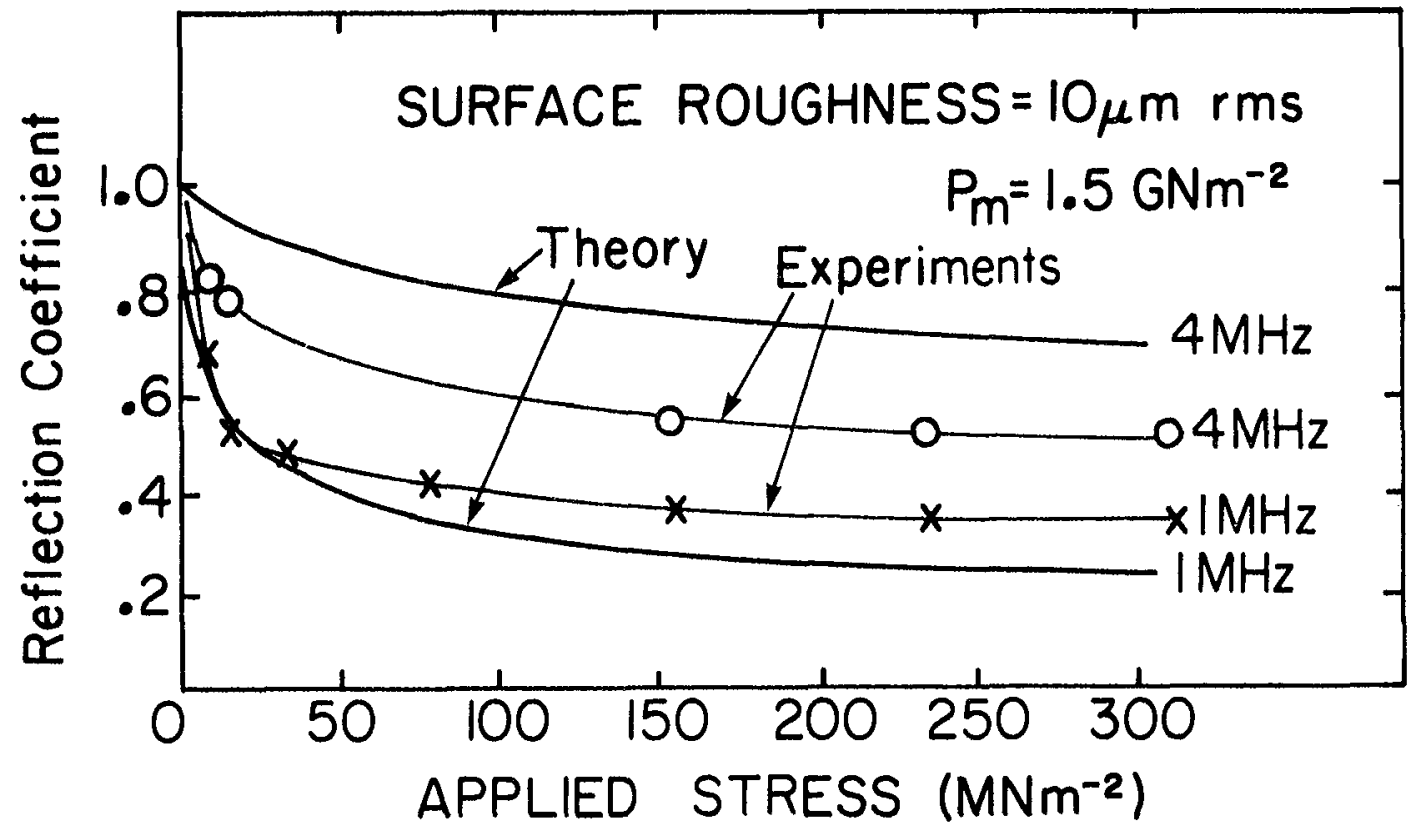

Fig. 4 Comparison of calculated and measured reflection coefficients as a function of $\sigma_{0}$, using shear waves (refs. 8 and 23 ).
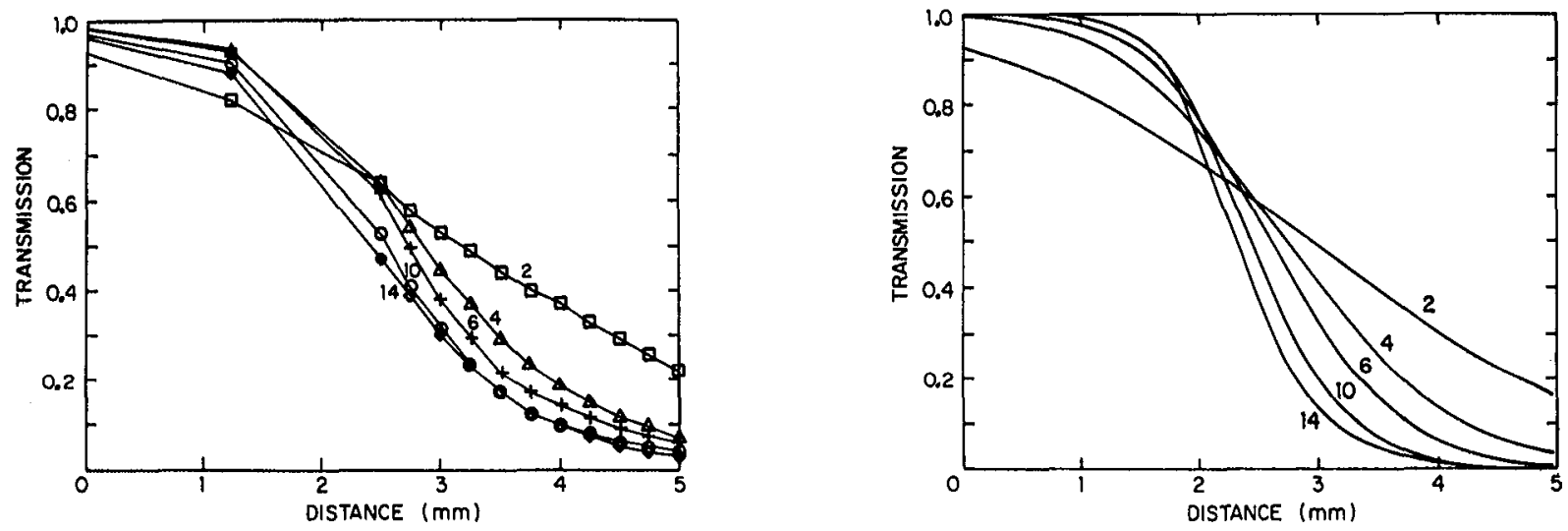

Fig. 5 U1trasonic transmission past a fatigue crack at 2, 4, 6, 10 and $14 \mathrm{MHz}$ (ref. 13). (a) experiment; (b) theory. 


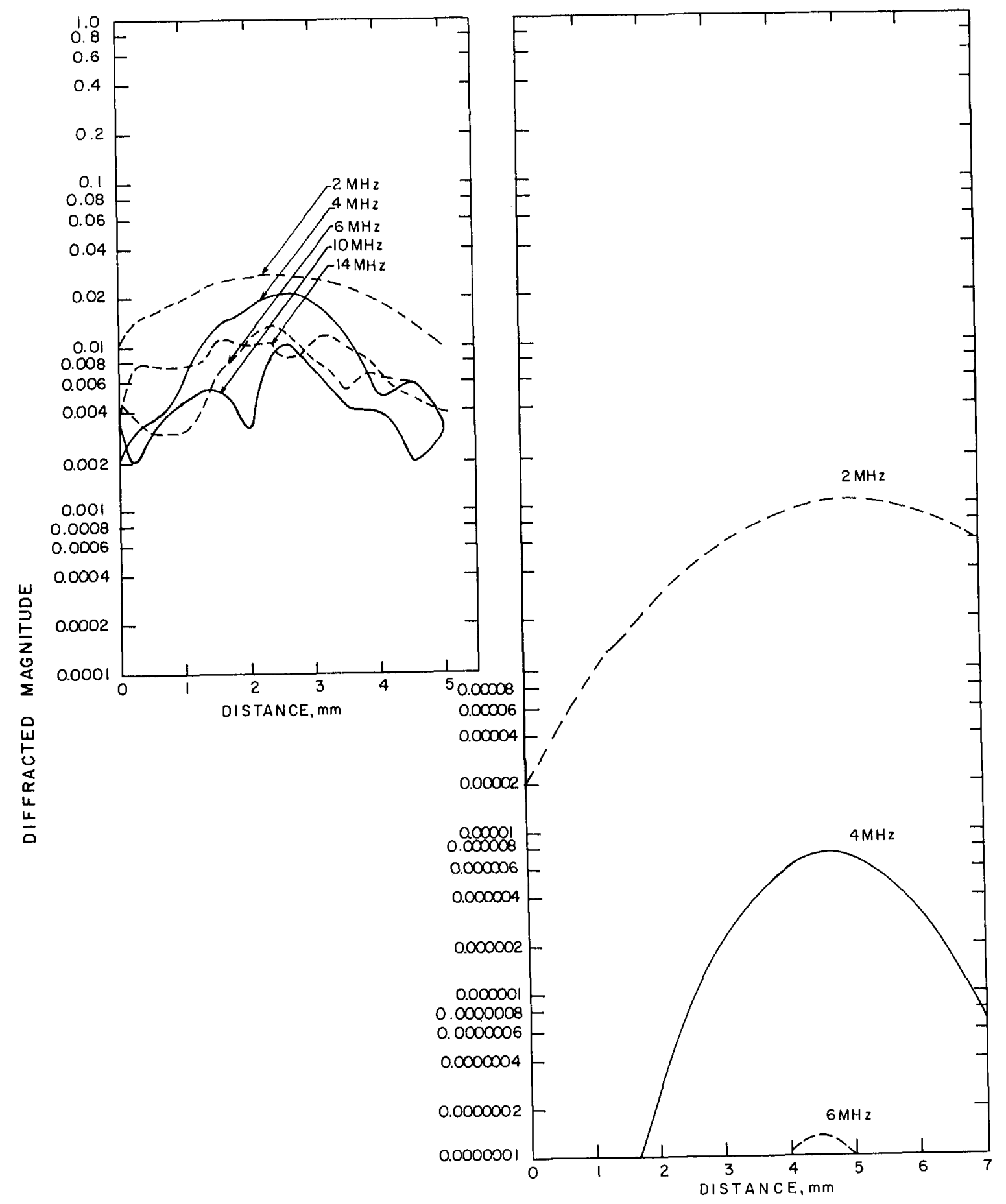

F1g. 6 Shear wave signal, mode converted in the closure region of a real crack (ref. 15). (a) experiment; (b) theory. 


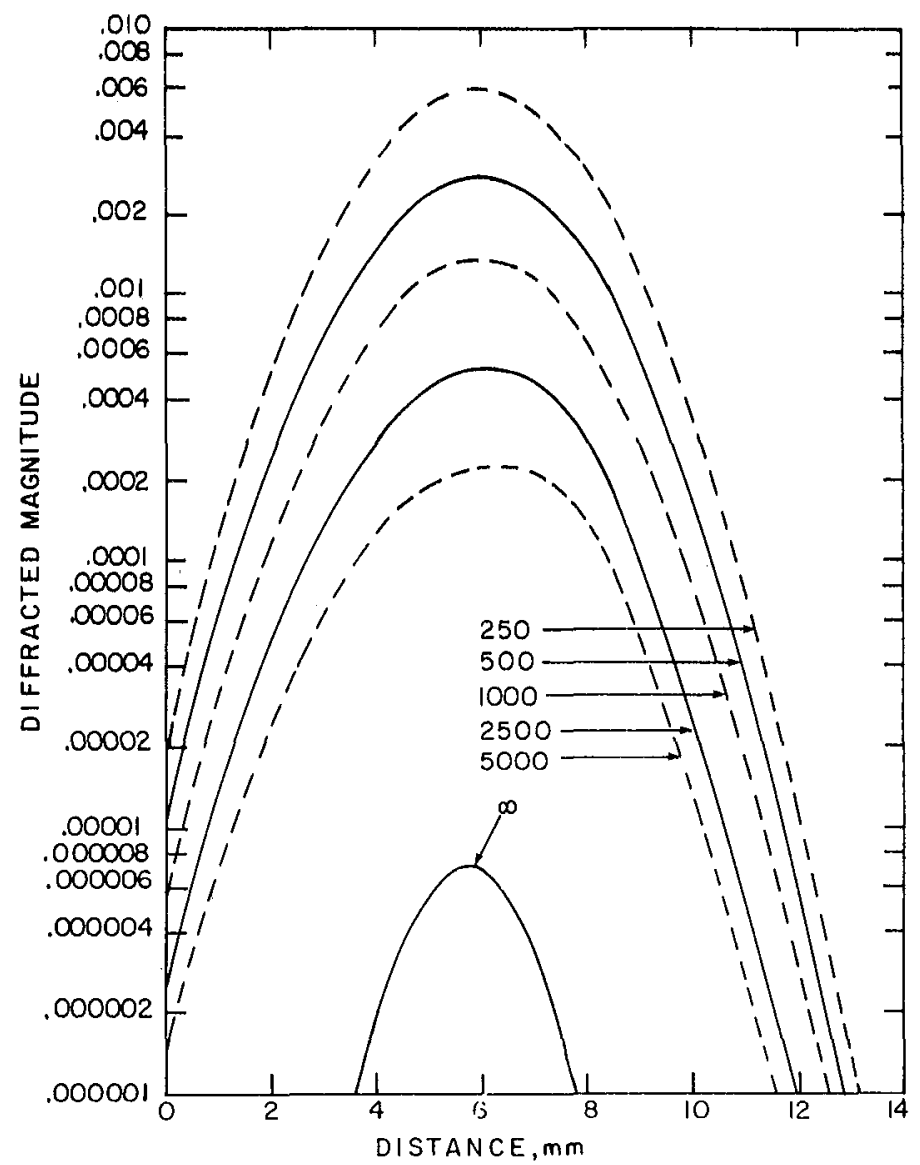

Fig. 7 Effect of contact density on the shear wave signal, mode converted in the closure region of a real crack theory (ref, 15).

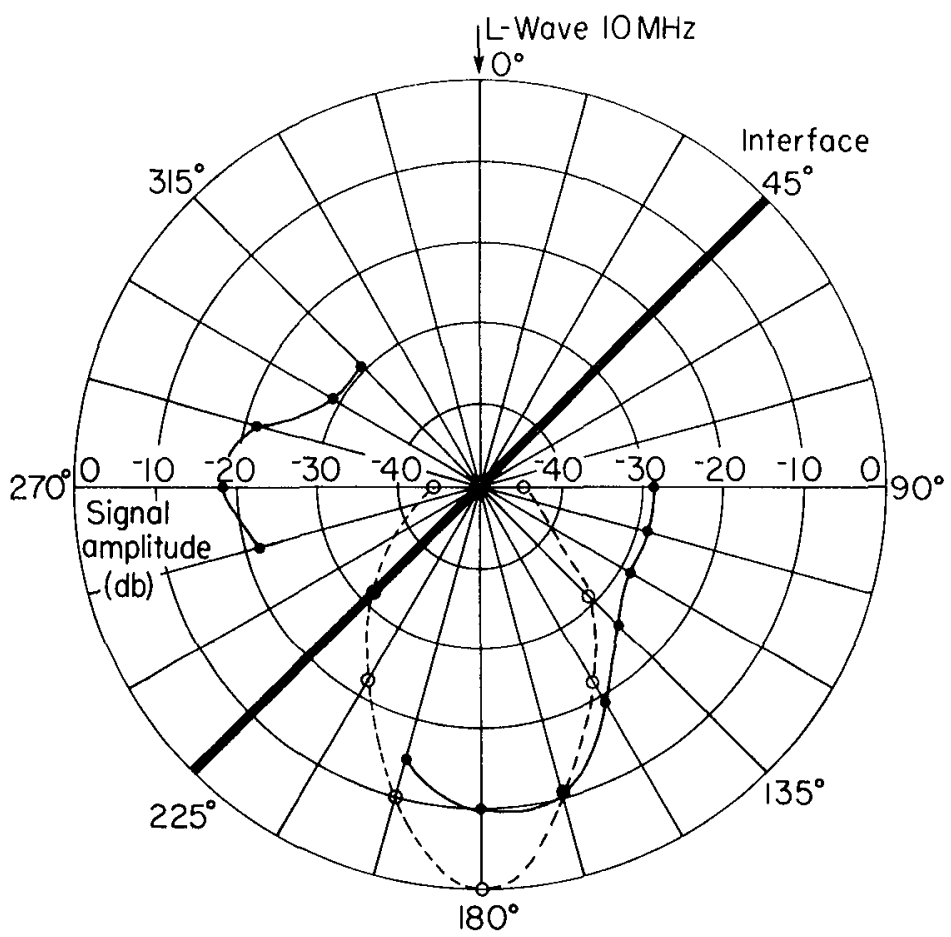

Fig. 8. Amplitudes of scattered signal (ref. 22). _ block with interface of random roughness. - - reference block (no interface). 


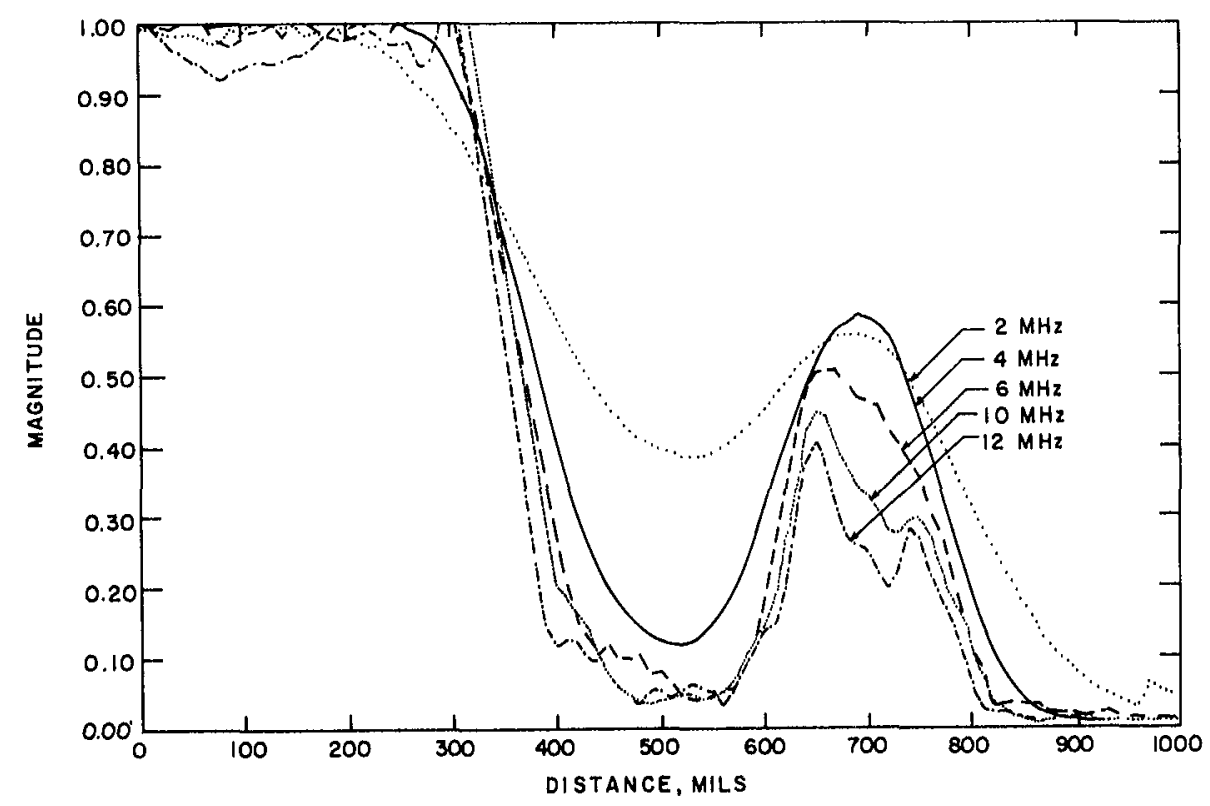

Fig. 9 Through transmission response of a fatigue crack showing partial closure far away from the crack tip (ref. 15).
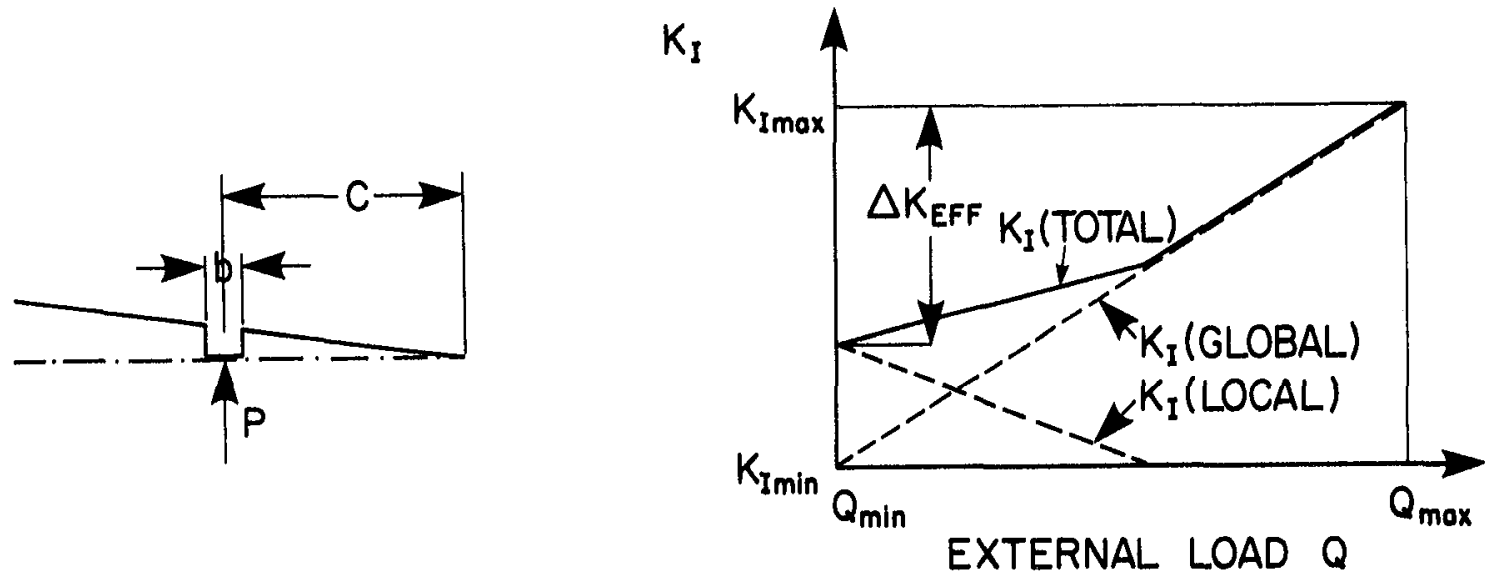

F1g. 10 (a) Model for $K_{I}$ (global) and (b) Stress Intensity factor as a function of external load $Q$. 


\title{
ANALYTICAL ULTRASONICS FOR EVALUATION OF COMPOSITE MATERIALS RESPONSE
}

\author{
PART I: PHYSICAL INTERPRETATION \\ Edmund G. Henneke II and John C. Duke, Jr. \\ Virginia Polytechnic Institute and State University \\ Blacksburg, Virginia 24061
}

The prediction of the mechanical performance of engineering structures made from composite materials is an issue receiving great attention from designers, builders, and users of these items. The need for nondestructive evaluation techniques and procedures to fulfill the requirements for such predictive capabilities is obvious. Ultrasonics, and in particular, analytical ultrasonics, is one NDT method which has the potential to meet all of the demands necessary to develop predictive physical and mathematical models. However, to maximize utilization of ultrasonics, it is necessary that we have complete understanding of the capabilities and limitations of the method. This paper will review some of the basic aspects of wave propagation in anisotropic materials from the viewpoint as to how these basic physical properties place fundamental constraints upon what we may ultimately expect to learn from ultrasonic interrogation of a composite. Then, a brief discussion is given concerning the characterization of various physical properties of the composite by appropriate quantifiable ultrasonic signal parameters. The physical properties of major interest here are those which influence the mechanical response of the material.

\section{INTRODUCTION}

Composite materials are the materials of the future. Just as solid state semiconductor materials have made a revolution in the electronics industry, advanced composite materials will likely one day play a major role in the way we design and use materials for a wide range of engineering structural components. Composites have the ability to be designed from the outset to take advantage of stiffness and strength in the load carrying directions. In addition, they have the advantage of very 1 arge strength-to-weight and stiffness-to-weight ratios. Before these materials can be employed with full utilization of their capabilities and advantages, and lead to the revolution predicted, much knowledge must be obtained concerning their mechanical behavior. While a great amount of work has been performed on the study of the mechanical behavior of these materials, there is still missing from the literature one most important piece of information -- a knowledge of the final failure processes which lead to catastrophic rupture of the material. This is of especial importance, of course, for the formulation of failure theories which can be used for design purposes. A failure theory something like fracture mechanics for homogeneous materials has been the subject of many investigations reported in the literature, yet it is obvious that no equivalently useful models have at present been formulated.

To answer the needs of the modelers in formulating a failure theory for composite materials, much experimental information has been gathered and reported in the literature. Most of this information has been gathered by experimental 
techniques which, if used by themselves, would be classified as nondestructive. The gathered information has revealed that the failure modes leading up to final rupture of composite laminates is quite complex (ref. 1). Figure 1 is an X-ray radiograph of a graphite epoxy laminate after many cycles of tension-tension fatigue. It is obvious from this radiograph that many different damage modes operate before final rupture, including transverse cracking, longitudinal splitting, and local delamination. Not obvious from the radiograph but known to occur also before final rupture are fiber breakage and fiber-matrix debonding. One thing that is apparent from many such studies is that final failure is not the result of the propagation of a single crack in a self-similar fashion as it is for a homogeneous material. To obtain useful data on such a complex damage state using nondestructive testing methods, and to develop nondestructive evaluation procedures based upon such information, it is necessary that an approach be used different from that which has been followed for homogeneous materials. Rather than developing NDT methods which look for single cracks and attempt to size and orient these, it is necessary to develop NDT methods which can interact with the entire, integrated state of damage in the composite. Several different NDT techniques are capable of doing this; most notably, perhaps, is that of ultrasonics.

Analytic, or quantitative, ultrasonics is also a subject which has received extensive attention in the recent literature. In general, this terminology has been applied to the search for ultrasonic methods which locate and size cracks as mentioned previously. Here we intend it to mean something even more general. We refer to analytic ultrasonics as those ultrasonics techniques which are capable of producing quantitative experimental parameters that are somehow descriptive of the material state, and, in particular, the material damage state. To apply analytic ultrasonics to composite materials, a great deal of care must be expended to understand the capabilities and limitations of the method. This paper reviews some of the basic aspects of wave propagation in anisotropic materials from the viewpoint. as to how these basic physical properties place fundamental constraints upon what we may ultimately expect to learn from ultrasonic interrogation of a composite.

\section{WAVE PROPAGATION IN ANISOTROPIC MATERIALS}

The propagation of waves in anisotropic, homogeneous materials has been covered well in several texts (see, for example, refs. 2-5). The propagation of ultrasonic waves can be approached by the same techniques covered in those texts as long as the frequency of the waves is below, say, the gigahertz region. (For frequencies in and above this range, the wavelengths begin to approach the size of interatomic spacing and the material can no longer be treated strictly as a continuum.) Composites are, of course, not homogeneous materials, and to treat the propagation of waves in composites by theories established for such materials is not rigorously correct. However, many approaches to the mechanics of composite materials begin with the simplifying assumption that a composite material may be treated as a homogeneous, anisotropic continuum with the underlying symmetry of the composite, usually taken to be orthotropic. Here, we will begin with this assumption also and review the solution to wave propagation in anjsotropic, homogeneous materials as if it could be applied to composites. As we shall see, the difficulties encountered in interpreting wave propagation in anisotropic materials are complex enough from this simplified viewpoint. Complete understanding will be required of this solution before we attempt to extend it to heterogeneous materials.

The discussion of wave propagation in homogeneous anisotropic materials begins with the wave equation for a continuum: 


$$
\sigma_{i j, j}=\ddot{u}_{i}
$$

where $\sigma_{i j}$ are the stress tensor components, $\rho$ is the material mass density, and $u_{i}$ are the components of the particle displacement vector. The next step is to consider a constitutive law such as the generalized Hooke's Law for an anisotropic elastic material:

$$
\sigma_{i j}=C_{i j k 1} \cdot \varepsilon_{k l}=C_{i j k 1} u_{k, 1}
$$

where $\varepsilon_{k 1}$ are the components of the linear strain tensor. Then, for mathematical convenience, a plane wave solution can be assumed of the form:

$$
u_{i}=A_{i} \exp i\left(k_{i} x_{i}-\omega t\right)
$$

where $A_{j}$ are the amplitudes of the particle displacements in the directions of the coordinate axes parallel to the symmetry axes of the anisotropic material, $k_{j}$ are the components of the wave vector, and $\omega$ is the angular frequency of the wave ( $x_{j}$ and $t$ are the independent position and time variables, respectively). For an anisotropic material having orthotropic symmetry, nine independent elastic constants are required to specify the constitutive relation between stress and strain, (eqn. (2)). In so-called reduced notation, these elastic constants are: $C_{11}, C_{22}$, $\mathrm{C}_{33}, \mathrm{C}_{12}, \mathrm{C}_{13}, \mathrm{C}_{23}, \mathrm{C}_{44}, \mathrm{C}_{55}$, and $\mathrm{C}_{66}$. Complete wave solutions to the anisotropic wave equation for orthotropic materials have been developed and have appeared in the literature (ref. 6). For our present purposes it will suffice to discuss the general aspects of the solution to the wave equation in anisotropic materials. Particular points applicable to orthotropic materials will be referred to as necessary.

First, as is well know, the plane wave solution to the wave equation is found to describe the possibility of three wave modes, in general. These wave modes travel, generally, with three distinct phase speeds; see, for example, figure 2 for an orthotropic material. These modes do not generally have particle displacements which are purely parallel or perpendicular to the direction of the wave vector (i.e., the direction of propagation of the wave). Thus the modes of propagation in anisotropic materials are generally neither longitudinal nor transverse as the modes in isotropic materials are typically described (fig. 3). Certain particular directions in the material, often coincident with directions of high symmetry but not always restricted to such directions, do allow for the propagation of pure modes, but these are the exception. In other instances, one mode may be purely transverse for all directions (fig. 3). This occurrence is not of no consequence for the experimental application of ultrasonics. All mechanical ultrasonic transducers generate waves by the development of vibrations produced by the piezoelectric effect. These transducers are manufactured to generate either inplane displacement vibrations (for the production of "transverse waves") or out-ofplane perpendicular displacement vibrations (for the production of "longitudinal waves"). The previous words have been placed in quotes because transducers are generally manufactured for the generation of waves in isotropic materials, in which case the transducers behave as advertised. Figure 4 schematically represents the introduction of longitudinal and transverse waves into an isotropic material. However, for anisotropic materials the directions of particle displacements preferred by the solution to the wave equation (and as ordained by the normal to the 
surface of the specimen on which the ultrasonically generated wave is incident, which must, by necessity, be the direction of wave propagation) are not in general either parallel or perpendicular to the surface normal. Thus a mechanical transducer, in general, will generate multiple modes simultaneously in an anisotropic material as the incident particle displacement vibrations will have components in all three directions of particle displacements mandated by the solution to the wave equation.

This phenomenon is of special consequence to the ultrasonic investigation of thin composite laminates since typically the wave propagation time in the material is insufficient to separate the various modes in time. Hence the observed wave may be a combination of multiple modes and not a single mode. This may lead to misinterpretation of travel times or attenuation measurements. It should be noted here, however, that if the incident wave is in the direction normally called the $z$-direction for the composite (i.e., perpenducular to the lamina plane), then only one wave is established in the laminate, as this is a special direction for the laminate symmetry along which pure modes may propagate. Any deviation from exact normality, however, as may occur in a c-scan tank due to non-normal wave incidence or focused beams, will cause multiple wave generation in the composite.

A second aspect of wave propagation is of importance in anisotropic materials and not at all in isotropic materials. The energy flux vector associated with each wave mode is, by definition, the rate at which energy is propagated across a surface normal to the direction of wave propagation per unit area. Mathematically, this quantity is defined by the contracted product of the stress tensor and the particle velocity:

$$
E_{i}=-\sigma_{i j} \dot{u}_{j}
$$

In general, the energy flux does not coincide with the direction of wave propagation for an anisotropic material (refs. 1-5). This leads to a different set of wave surfaces (fig. 5) often called the group velocity surfaces since these can be shown to be related to the group velocity of the wave. This phenomenon has many consequences for wave propagation in anisotropic materials that are never seen in isotropic materials. For example, if a wave mode is established in a single crystal of finite dimensions, it is possible that the wave flux will cause incidence of the wave upon the lateral surface of the specimen (see fig. 6, for example), thereby leading to mode conversion or longer times of travel in the specimen than anticipated (see also refs. 5 or 8). A second consequence of the phenomenon of energy flux deviation is that, for certain directions in an anisotropic material, one may observe the apparent propagation of more than three wave modes. As many as five distinct modes may be observed in certain directions in the material. The wave normals are not all coincident, the apparent propagation of five modes is due to the deviation of the energy flux vectors associated with mode propagation in other material directions (ref. 4). Again this occurrence in the ultrasonic study of anisotropic materials such as composite laminates may lead to misinterpretation of results unless one is aware of it and is ready to take it into account.

The deviation of the energy flux from the direction of the plane wave normal can be seen easily in a simple experiment that can be performed with a unidirectional composite laminate. If two transducers are mounted on wedge blocks to cause non-normal incidence on the surface of a laminated plate, and the plane containing the normal to the plate surface and the direction of the incident wave is chosen to be at an angle to the fiber direction, then the receiving transducer 
cannot be placed in this same plane if a received signal is to be observed. Rather, the receiving transducer must be placed outside of this plane. The angle between this plane and the plane containing the surface normal and the direction of the received wave corresponds to the direction of the energy flux vector for that wave node. If the two transducers are not set up at precisely the correct angle, the receiver will not detect the maximum signal from the transmitter, or unforeseen constructive or destructive interference patterns may result.

The phenomenon of energy flux deviation also plays an important role when discussing the reflection-refraction problem in an anisotropic material. The reflection-refraction problem for plane waves incident upon a plane boundary has received some attention in the literature for anisotropic materials (ref. 8). Generally, a plane wave incident upon a plane boundary in anisotropic media will reflect as three distinct wave modes. (Figure 7 is an example of the use of slowness surfaces to study the reflection problem in anisotropic media.) Similarly, a wave incident externally upon a plane surface boundary of an anisotropic material will refract in the material as three distinct wave modes. The wave vectors for the incident and reflected (or refracted) modes and the surface normal must all lie in the same plane. The angles of the reflected, refracted, and incident modes relative to the surface normal obey a general statement of Snell's Law. However, the velocities of propagation for each of the reflected waves are dependent on the direction of propagation and hence Snell's Law cannot simply be applied, since there are two unknowns in Snell's relation -- the direction of reflection or refraction, and the velocity of propagation. Hence more involved solution procedures are required (see, for example, refs. 2, 3, and 8). The important point to be made here is that once again the anisotropy of the material leads to complications which are not present in the more familiar problem of reflection in isotropic materials. In the latter case, mode conversion also occurs, but only two waves are reflected or refracted, and the directions can be found immediately from Snell's Law. Furthermore, to belabor the point, in anisotropic materials, the reflected or refracted waves are neither longitudinal nor transverse, in general. Also, the critical angle phenomenon in anisotropic materials is dependent upon the energy flux direction and not the direction of the reflected wave vector as is normally assumed (ref. 9). This fact is of importance to those experimental techniques which utilize measurement of critical angles of reflection of external waves to measure wave velocities in solid materials.

The previous discussion has dealt with plane wave solutions to the wave equation in anisotropic materials. Other types of wave fronts, such as spherical, may always be treated locally as a plane wave. Hence a spherical wave which arises from a point source will display the same propagation characteristics as plane waves propagating in all possible directions simultaneously. Thus for the case of transducers which are focused and thus will have considerable divergence on the far side of the focalpoint, or for the study of uitrasonic waves emanating from acoustic emission sources, very careful consideration must be given to anisotropic wave propagation phenomena, as all of the previously described phenomena, as well as others not yet mentioned, will likely be present.

A number of other interesting phenomena, such as internal and external conical refraction, pure mode axes not coinciding with high symmetry directions, etc., might be discussed. However, for the purposes of this paper, we might close this discussion with a few general observations concerning the experimental application of ultrasonic techniques to composite materials. As long as one is sending an ultrasonic wave in the direction perpendicular to the plane of the laminate, which 
indeed is the most frequently used experimental configuration, few of the complexities discussed above need to be considered. However, there will always be some degree of beam divergence and hence even an incident "normal" wave will have some portion of the sound beam which is not strictly perpendicular to the surface and hence will generate some off-axis waves which are of different modes than the purely longitudinal or transverse waves usually expected. Also, other ultrasonic techniques such as the stress wave factor method developed by Vary, et al... (refs. 10-11) establish waves which propagate in the direction of the laminate plane. The laminate plane is, of course, a plane of high anisotropy, and all of the phenomena described previously will occur in this plane. It is unclear at this point (at least to the present authors) how this statement might be modified by a quasi-isotropic laminate such as a $[0,90,+45,-45]_{s}$ stacking sequence. It seems likely, based upon experimental observation, that some influence of the anisotropy of the individual lamina will influence the propagation of stress waves in the plane of the laminate, and even in this case, some of the phenomena caused by the anisotropy of the material will be evidenced.

Finally, it must be clearly understood that the discussion to this point has dealt with homogeneous, anisotropic materials. Composite materials may be considered as such a material only as a very rough first approximation, although it is true that this is a good approximation in certain wavelength and frequency regimes. When the wavelengths become of the same order of magnitude as laminae thicknesses, fiber diameters, fiber spacings, or other physical size characteristics, then the material must be treated as inhomogeneous. A significant literature has also been developed for such discussions, but will not be referred to further here.

\section{CHARACTERIZATION OF PHYSICAL PROPERTIES BY ULTRASONICS}

The characterization of the physical properties of composite materials by various ultrasonic techniques has received a great deal of attention in the literature. For the purposes of this paper, and to illustrate potential utilization of some of the information presented in the previous section, we will discuss some of those works which have directly utilized the phenomena of wave propagation in anisotropic materials for the study of composites. A more complete study would require an extensive review paper and hence is beyond the scope of what we would like to accomplish here.

Perhaps one of the most basic mechanical properties of composite laminates which has been studied by ultrasonics is the elastic constant tensor. Several authors have measured elastic moduli by measuring wave speeds, but perhaps the most complete work in a composite laminate is that done by Kriz and Stinchcomb (ref. 12). Kriz and Stinchcomb determined the complete set of nine elastic constants for a graphite epoxy composite by constructing a $25.4 \times 25.4 \times 150 \mathrm{~mm}$ block of unidirectional material. They then sliced from this block several specimens with orientations appropriate for making nine independent measurements so that all nine constants corresponding to an orthographic material were determined. While one or two of the elastic constants were somewhat in doubt (because the corresponding calculation required taking the difference of two numbers which were nearly the same, and hence small measurment errors were multiplied), the results of this experimental effort were in line with predictions made by the rule of mixtures for composites. 
Kriz has suggested that the deviation of the energy flux vector from the direction of wave propagation can be used to determine the moisture content in polymer matrix composites (ref. 13). It is well known that the ingression of water into the matrix of a composite, even in relatively small amounts, can alter the values of the material elastic constants by a few percent. Kriz has performed calculations which show that the deviation of the energy flux vector from the direction of wave propagation changes by measurable amounts when the elastic constants change by the small amounts caused by the presence of moisture (fig. 8). He has verified this by making experimental measurements on graphite epoxy material before and after exposure to moisture in the environment. His results indicate that moisture content can indeed by determined in a laminate if before and after angular deviation measurements are made on the same specimen.

\section{CONCLUSIONS}

The phenomena associated with the propagation of elastic waves in anisotropic materials are many and varied, as we have tried to illustrate here. For example, wave modes propagating in general directions relative to the material coordinate system are not purely longitudinal nor transverse. Hence the generation of ultrasonic waves by common piezoelectric transducers will generate multiple modes to some extent. The received signals will likely be a combination of different modes. When using two transducers to send and receive ultrasonic waves, deviation of the energy flux vector may reduce the apparent value of the received signal unless the proper orientation of the two transducers with respect to one another is taken into account. And application of reflection from plane boundaries for the purposes of making certain measurements may lead to misinterpretation of results unless one is aware of the differences in multiple mode generation and critical angle phenomena between isotropic and anisotropic materials. When studies or characterizations of composite materials by ultrasonics are to be performed, these phenomena must be taken into consideration so that proper and correct application and interpretation of the measurements can be made. Finally, attention must be drawn again to the fact that composite materials are heterogeneous by definition. The results discussed here have been determined for homogeneous materials only. While the assumption of homogeneity appears to be valid for certain wavelength ranges in composites, future work must continue to study the phenomena of wave propagation in anisotropic, nonhomogeneous materials. 


\section{REFERENCES}

1. Jamison, R. D.: Advanced Fatigue Damage Development in Graphite Epoxy Laminates, PhD Dissertation, College of Engineering, Virginia Polytechnic Institute and State University, Blacksburg, VA, Aug. 1982.

2. Fedorov, F. I.: Theory of Elastic Waves in Crystals (Plenum Press, New York, 1968).

3. Musgrave, M. J. P.: Crystal Acoustics (Holden-Day Inc., San Francisco, 1970).

4. Auld, B. A.: Acoustic Fields and Waves in Solids, Vol. I \& II (John Wiley \& Sons, New York, 1973).

5. Green, R. E. Jr.: Treatise on Materials Science and Technology, Vol. 3, Ultrasonic Investigation of Mechanical Properties (Academic Press, New York, 1973).

6. Kriz, R. D.: Mechanical Properties for Thick Fiber Reinforced Composite Materials Having Transversely Isotropic Fibers, Masters Thesis, College of Engineering, Virginia Polytechnic Institute and State University, Blacksburg, Virginia, 1976.

7. Kriz, R. D. and Ledbetter, H. M.: Elastic Representation Surfaces of Unidirectional Graphite Epoxy Composites, 2nd US-Japan Conf. on Composite Materials, 1983, ASTM STP (American Society for Testing and Materials, 1985).

8. Henneke, E. G.: Reflection-Refraction of a Stress Wave at a Plane Boundary Between Anisotropic Media, J. of Acous. Soc. Amer. 51, 1972, pp. 210-217.

9. Henneke, E. G.: Critical Angles for Reflection at a Liquid-Solid Interface in Single Crystals, J. Acous. Soc. Amer. 59, 1976, pp. 204-205.

10. Vary, A. and Lark, R. F.: Correlation of Fiber Composite Tensile Strength with the Ultrasonic Stress Wave Factor, J. Testing and Eval. 7, July 1979, pp. 185-191.

11. Vary, A.: Acousto-Ultrasonic Characterization of Fiber Reinforced Composites, Mat1s. Eval. 40, 1982, pp. 650-654.

12. Kriz, R. D. and Stinchcomb, W. W.: Elastic Moduli of Transversely Isotropic Fibers and Their Composites, Exp. Mechs. 19, 1979, pp. 41-49.

13. Kriz, R. D.: Absorved Moisture and Stress-Wave Propagation in Graphite/Epoxy, Composites Technology Review 3 Winter 1981, pp. 154-155. 


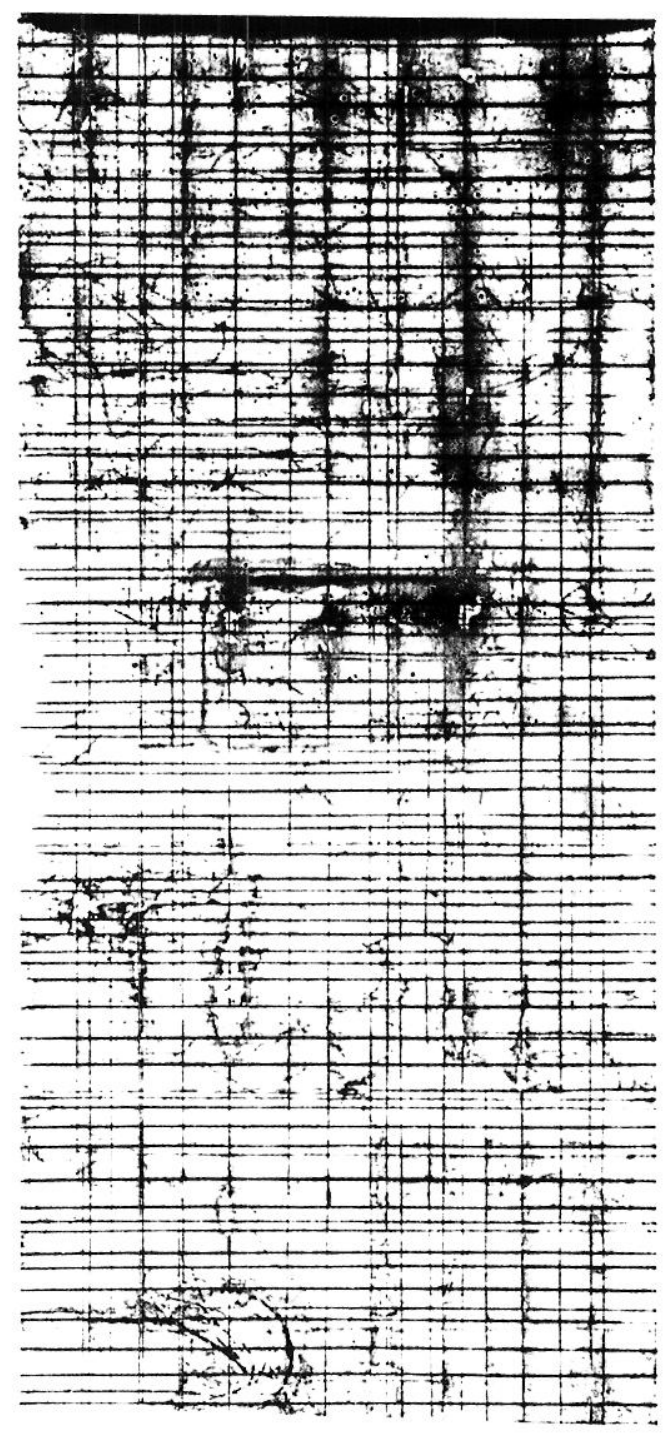

Fig. 1. X-ray radiograph showing advanced state of damage in a fatigued graphite epoxy composite 1 aminate.

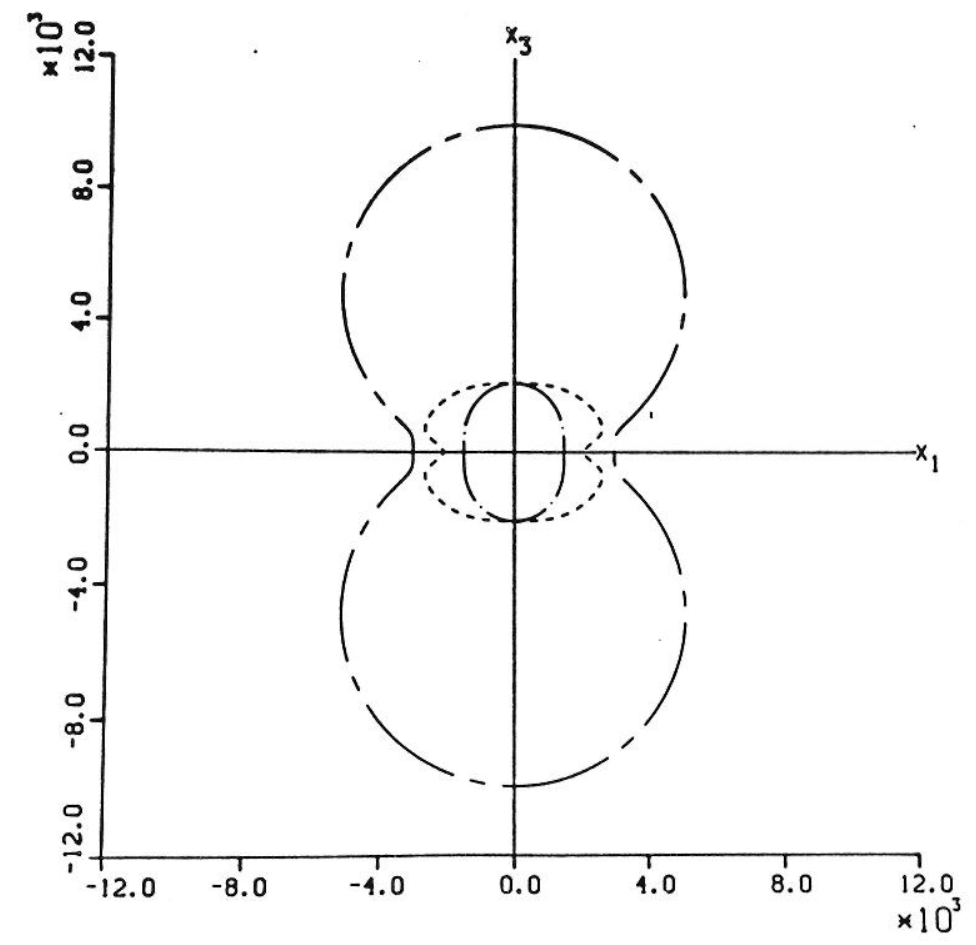

Fig. 2. Phase velocity surface for graphite epoxy composite (after Kriz (ref. 7)). 


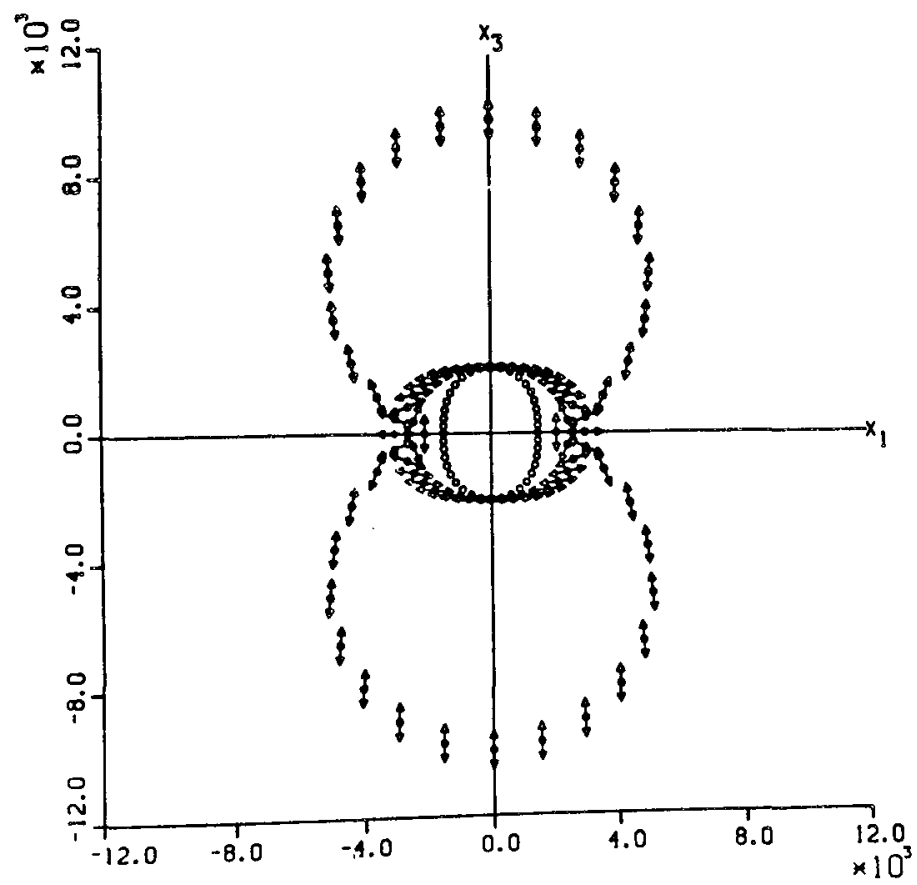

Fig. 3. Arrows indicate relative directions of particle displacements with respect to direction of wave propagation (which is position vector from origin to center of double arrows) (after Kriz (ref.7)).

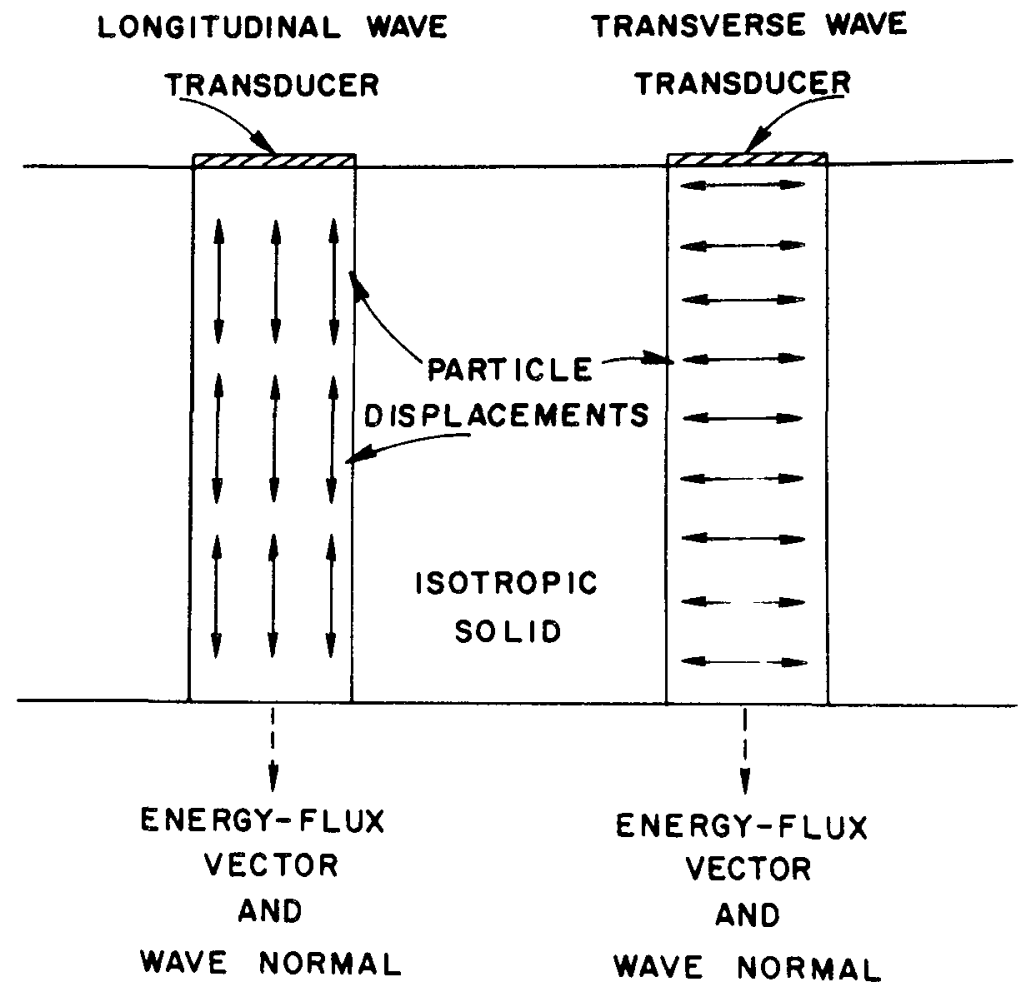

Fig. 4. Schematic representation of longitudinal and transverse waves being generated in an isotropic material (after Green (ref. 5)). 


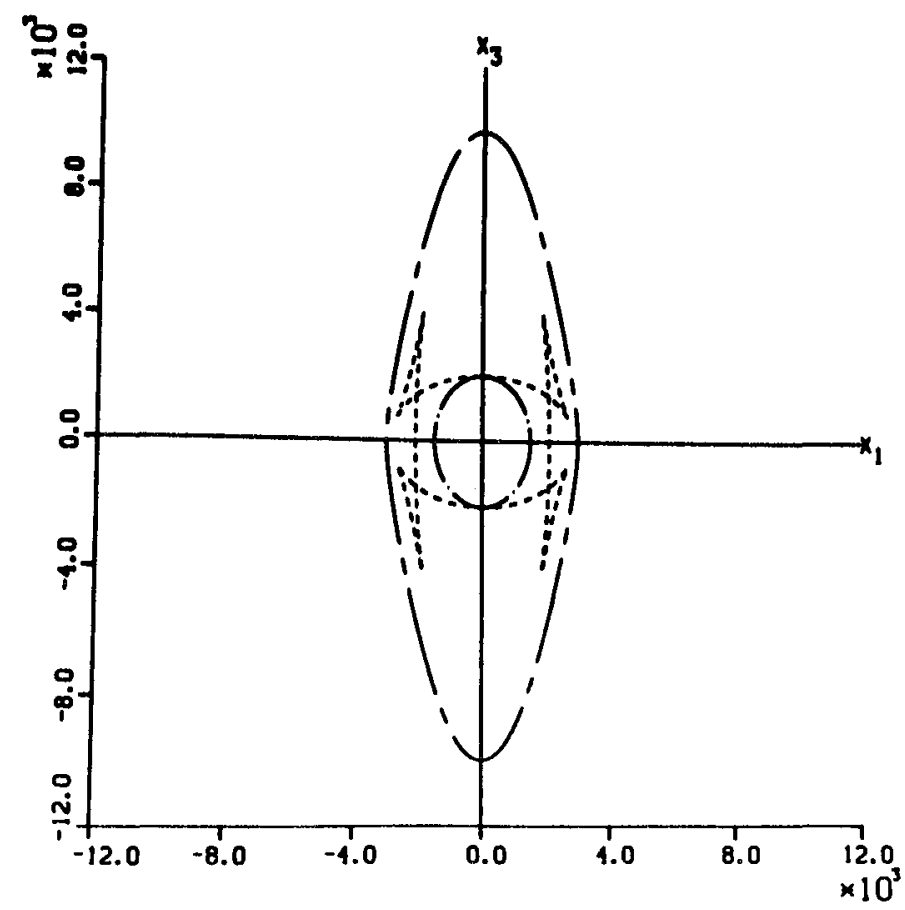

Fig. 5. Group velocity surface for graphite epoxy composite (after Kriz (ref. 7)). NONSYMMETRY DIRECTION

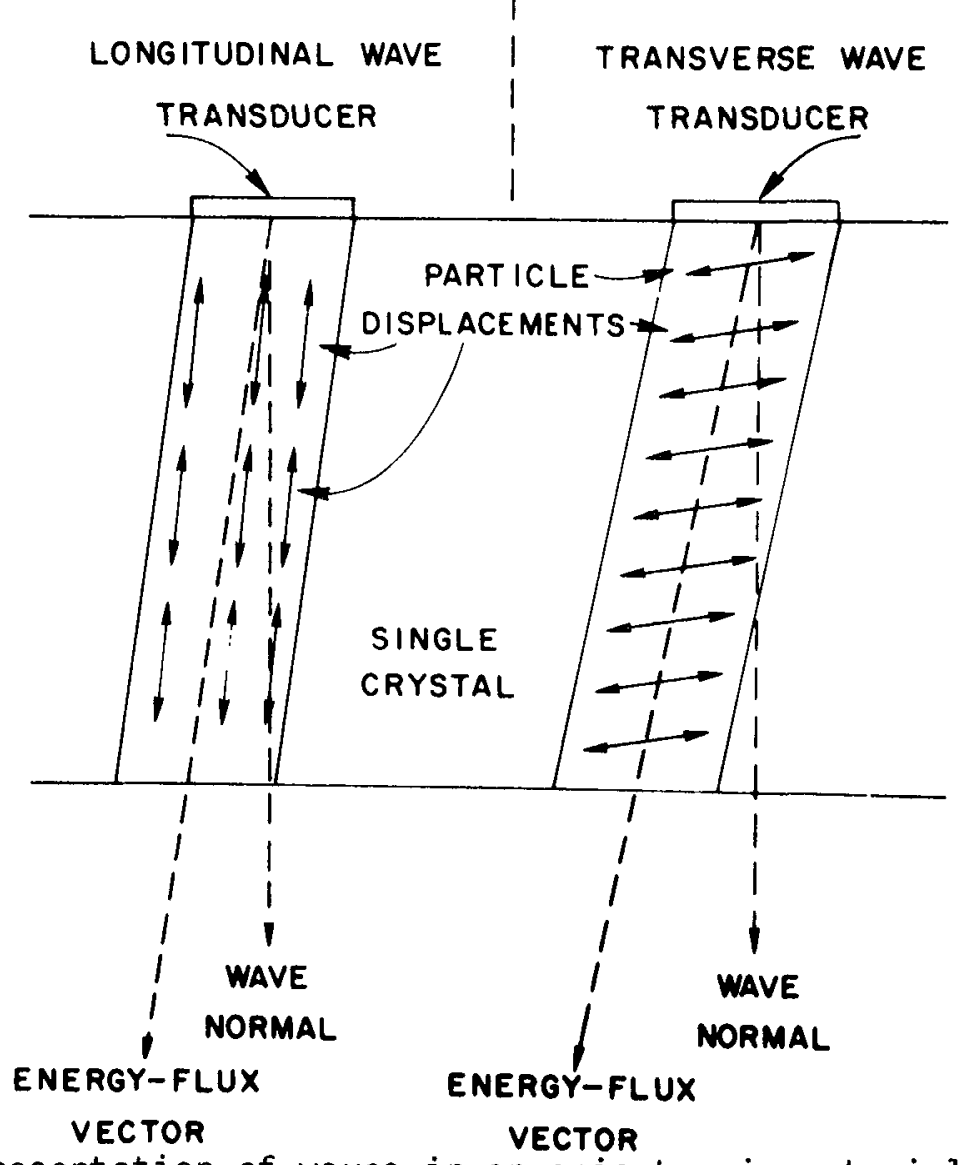

Fig. 6. Schematic representation of waves in an anisotropic material with energy flux deviation occurring for the transverse mode (after Green (ref. 5)). 


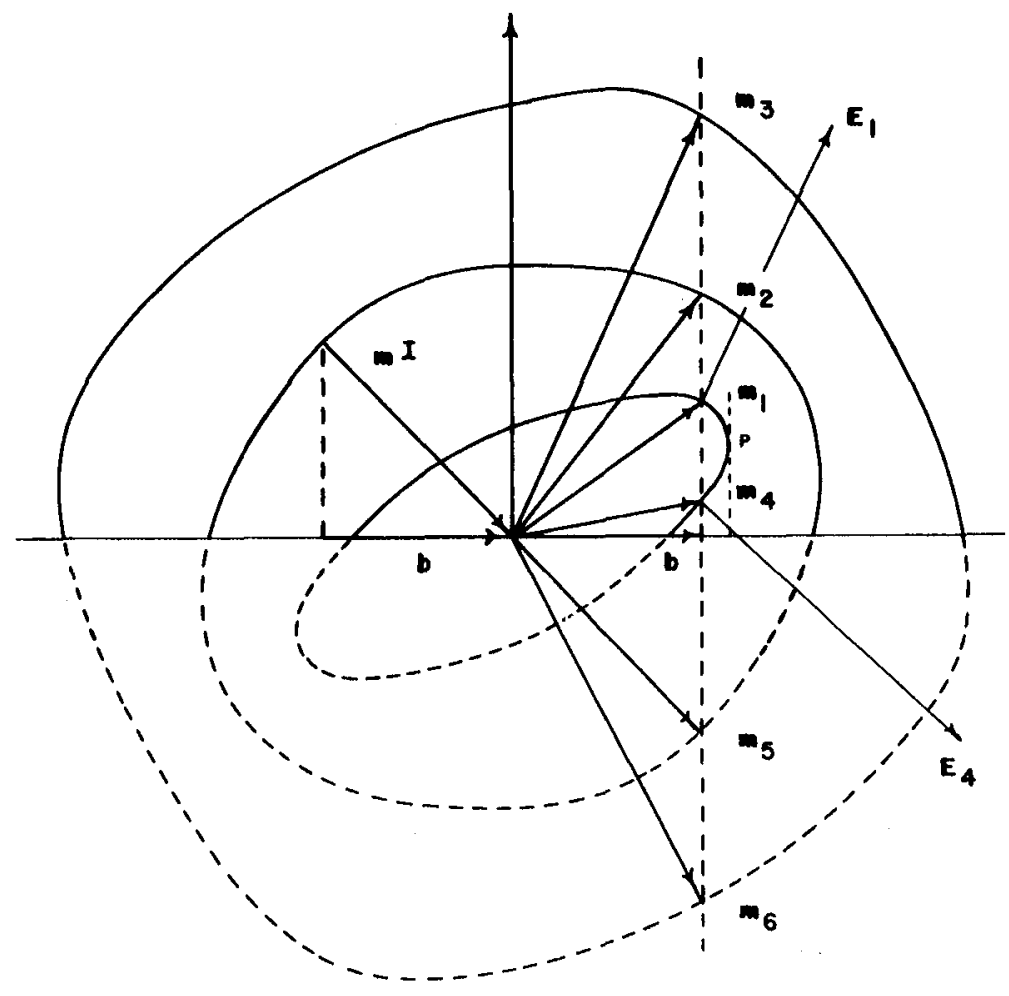

Fig. 7. Reflection of a plane wave at a stress free boundary in an anisotropic material using ray analys is (after Henneke (ref. 8)).

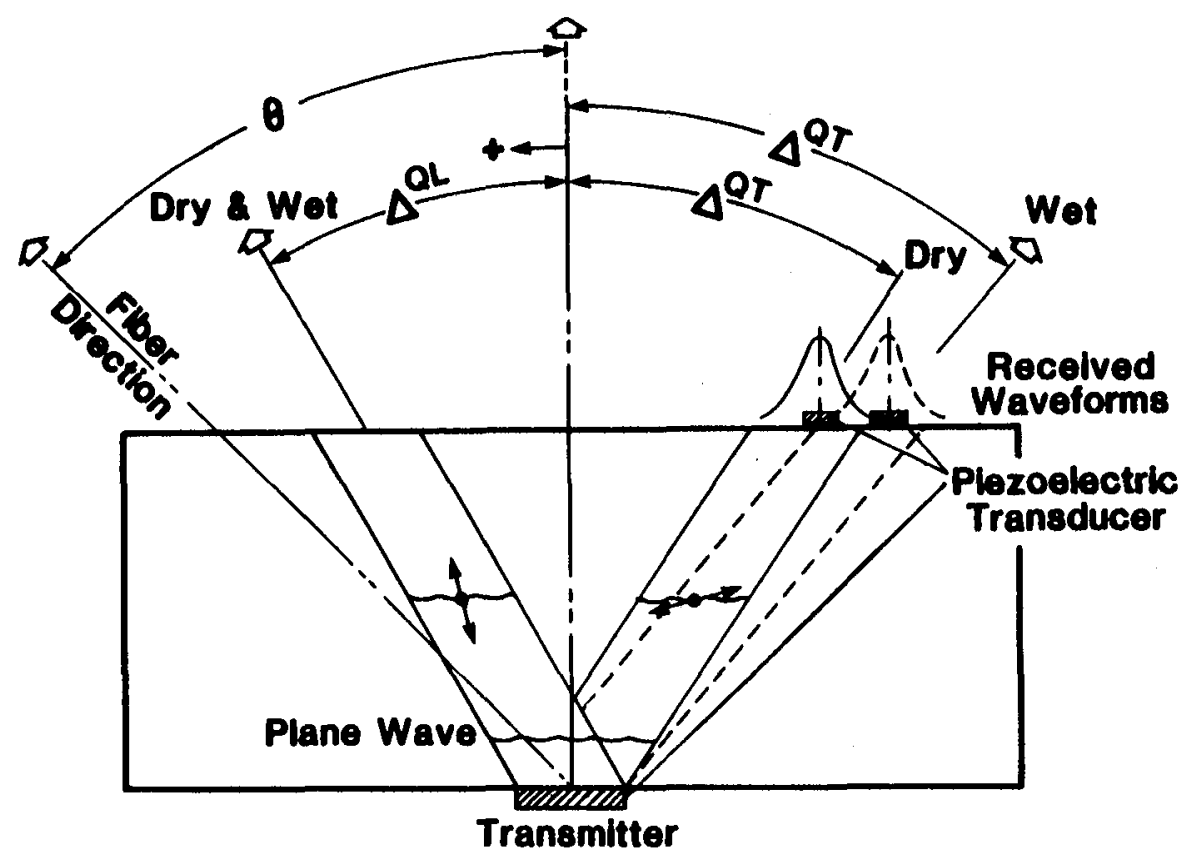

Fig. 8. Schematic representation of changes in energy flux deviation due to moisture in a composite (after Kriz (ref. 14)). 


\title{
ANALYTICAL ULTRASONICS FOR EVALUATION OF COMPOSITE MATERIALS RESPONSE
}

\author{
PART II: GENERATION AND DETECTION \\ John C. Duke, Jr., and Edmund G. Henneke II \\ Virginia Polytechnic Institute and State University \\ Blacksburg, Virginia 24061
}

To evaluate the response of composite materials, it is imperative that the input excitiation as well as the observed output be well characterized. This characterization ideally should be in terms of displacements as a function of time with high spatial resolution. Additionally, the ability to prescribe these features for the excitation is highly desirable. This paper examines various methods for generating and detecting ultrasound in advanced composite materials. Characterization and tailoring of input excitation is considered for contact and noncontact, mechanical, and electromechanical devices. Type of response as well as temporal and spatial resolution of detection methods are discussed as we11. Results of investigations at Virginia Tech in application of these techniques to characterizing the response of advanced composites are presented.

\section{INTRODUCTION}

Accurate characterization of mechanical performance of materials is crucial for engineering application. Destructive mechanical testing is capable of establishing a range of lower limit values for a limited number of states of stress. Generally this type of testing seeks to establish a uniform state of stress and to monitor the magnitude of load causing deformation or failure. Such testing is of little use for characterizing materials which are inhomogeneous by design or for materials which are created in a structural form. Modern tailored fiber reinforced composite materials fall into both of these categories. In that composites are being used frequently in high performance applications, appropriate means of mechanical characterization are a vital need.

Traditionally, in the engineering arena, ultrasonic techniques have been relegated to detecting flaws in components. In recent years, proponents of their use for characterizing material properties have been receiving greater attention. Rightly so, in that ultrasonic techniques possess the potential for local examination as well as incorporating a variety of parameters that are sensitive to subtle changes in material condition. These features are especially attractive for characterization of composite materials which may be composed of random or oriented reinforcement with varying conditions of reinforcement, matrix, reinforcement to matrix bonding, and layer to layer bonding.

Work supported in part by NASA Grants NSG-3-172 and NAG-3-323. 
This paper is Part II of a pair of papers examining the application of ultrasound for characterizing composite materials. Part I has examined from a theoretical perspective the restrictions on the applications as well as provided insight into some yet unexplored areas. This paper examines the experimental methods of generating ultrasound in materials as well as detecting the associated resulting material response. A brief survey of existing methodologies is presented to serve as a basis for discussion with no attempt being made to fully review the extensive work in any of these areas. The primary objective here is to look to the future as regards new or improved methods of analytical ultrasonic evaluation of composite materials. In particular, the issue of characterizing materials of this, class, which are constructed deliberately to be inhomogeneous, and those containing damage, is examined.

\section{EXPERIMENTAL METHODS OF ULTRASOUND GENERATION}

The prevailing philosophy in the generation of ultrasound has been to develop a generating device having an output ultrasound field with prescribed characteristics, these characteristics being a smooth -- either plane or curved -- wavefront; a specified amplitude, frequency spectrum, and duration. The subsequent interaction of the ultrasound field with the material was inferred from theory with recognition that problems with insertion, such as scattering and reflection, existed. Recently these difficulties although minimized, have still been seen as unacceptable. As a result, methods of creating ultrasonic disturbances within the material of interest, have begun to be explored. Some features of both types of approaches are presented, with attention to control and specification of the disturbance in the material being characterized.

\section{METHODS OF EXTERNAL ULTRASOUND GENERATION}

\section{Piezoelectric Devices}

Piezoelectric devices are used more frequently than any other for generation of ultrasound. They are simple in construction, rugged, and easy to use. 0f these, piezoelectric ceramic materials are most prevalent; however, quartz, a naturally occurring piezoelectric material, does offer some advantages. These include good linearity of performance, little degradation with age, and pure mode generation, when appropraitely cut. Quartz is less active than its ceramic counterparts. Consequently, in most commercial devices, ceramics are used. Recently, the development of polymeric piezoelectric materials has provided some additional flexibility in structural and electronic configuration of devices. That is to say, that the response of the element at any point is constrained by its surroundings and the electrode configuration. Consequently, the element responds as a whole to imposed voltages but the response from point to point may not be the same. The sound field generated mimics this variation. A reduction in this variation can be achieved by shaping and mechanically coupling the element and by careful manufacturing control; neither problem can be completely eliminated. Frequently, the variation in the sound field is diminished by focusing the field into a smaller beam for application where the fine structure of the sound field is of little importance.

Through the use of electrode configuration or element arrays, it is possible to tailor the sound field (ref. 1). Claus and Zerwekh, in reference 2, for example, have used a single piezoelectric element and a concentric array of annular electrodes to develop a two-dimensional Gaussian field profile (as measured in water). Such development is nearly always demonstrated in water which is isotropic and homogeneous. 
Some work has been done with the use of shock waves created by the ionization of gases when a spark arcs a gap between two electrodes. The shock wave is directed at the surface of the material of interest. Little attempt is made as regards modifying the shock, as the primary objective of such methods is to create short duration disturbances which may be reproduced.

For all of these methods, the control and specification of the disturbance introduced in the material being examined is indirect. It is directly influenced by the surface condition, the differences in mechanical impedance between external media and that of the material, the isotropy, and homogeneity of the material. 0f special significance, however, is the aspect of direction of propagation. The direction of incident wave propagation determines in large measure the subsequent direction of propagation in the material; additional wave modes that may not be desired will accompany non-normal incidence.

\section{METHODS OF INTERNAL ULTRASONIC MECHANICAL WAVE GENERATION}

It is possible to avoid the problems associated with creating an external ultrasound field and coupling it into a material. This may be accomplished by creating a mechanical disturbance within the material directly. Such an approach incorporates the advantages of avoiding problems related to coupling media and the constraints of the generating device mentioned above. Often these advantages are offset by the fact that there is no way to characterize the resulting ultrasonic disturbance parat from the material being examined. Under ideal conditions it may be possible to experimentally charactrerize the disturbance caused by such a method. It should be noted, however, that the use of a characterized source does not necessarily mean that the ultrasonic disturbance it creates is characterized.

\section{Electromagnetic Acoustic Transducers (EMAT)}

The recognition that an eddy current induced in a conducting material would interact with an external magnetic induction to cause a Lorentz force on the particles of the conductor and thus an elastic disturbance lead to the development of electromagnetic acoustic transducers. Consequently, it is possible by appropriate choice of orientation of the electric and magnetic fields to induce a variety of different wave modes in conducting materials or materials on which a conducting layer has been deposited. EMAT induced ultrasonic disturbances are of course still influenced by the isotropy and homogeneity of the material, but now this includes both mechanical and electromagnetic considerations. A good review of work in this area has been made by Frost (ref. 3 ).

For these devices, the size does not control the frequency response; however, the electrical coil geometry does influence the overall response and it may not be varied arbitrarily. This limits somewhat the spatial variations that may be achieved in the induced ultrasound field.

\section{Laser Generation}

Rapid localized heating of the surface of a material may be accomplished using a pulsed laser. The associated rapid thermal expansion causes a mechanical disturbance which, upon cooling, is reversed, giving rise to ultrasonic waves within the material. It is suggested in reference 4 that since the surface is unconstrained in 
the normal direction, that the main resultant thermoelastic stresses are in-plane. The area of the beam can be in practice quite small, on the order of millimeters, and shaped as desired. Factors influencing the induced disturbance include the energy distribution of the laser beam, the isotropy of thermal conductivity and expansion, and homogeneity of the material.

In composite materials the thermal properties of the constituents are often different and the material is inhomogeneous on the scale of the beam diameter. This would certainly influence the ultrasonic waves created.

\section{Impulsive Methods}

A number of other methods which have as their basis mechanical impulsive loading of the material surface have been proposed and demonstrated. These include the use of a helium gas jet (ref. 5), tapping (or other forms of impact), pencil lead breaking (ref. 6), and glass capillary breaking (ref. 7). The advantage of such techniques is that the disturbances they cause are highly localized and often broadband in frequency content. The direction of propagation as well as the mode and frequency content are not controlled, but rather are determined by the response of the material to the impulsive load. It is imperative in the use of such methods that the impulsive source be mechanically characterized. This must be accomplished in a material in which the ultrasonic wave propagation is well characterized, which in general implies a homogeneous and isotropic material. The application of such sources to anisotropic, inhomogeneous materials is particularly useful for providing insight to efforts for modeling the wave propagation in such materials.

\section{EXPERIMENTAL METHODS OF DETECTING ULTRASOUND}

A number of methods have been developed for detecting ultrasonic mechanical waves in materials; however, not all of these methods provide information in a form that may be interpreted in terms of mechanical response. This limitation does not preclude effective application of these methods for imaging imperfections and other situations for which a precise description of particle displacement and velocity are not needed.

\section{Piezoelectric Devices}

Until very recently, the output electrical signal of piezoelectric transducers, although extremely sensitive to ultrasonic motion in materials, could not be closely related to the motion of the material. Proctor, building on the work of colleagues at the National Bureau of Standards, developed a piezoelectric device which, because of its configuration, provides an electrical output that may be shown to be related closely to the out-of-plane displacement of the material caused by the ultrasonic distrubance. Its sensing area is on the order of $2 \mathrm{~mm}^{2}$ and is coupled to the surface of the material; more recently, development of a similar device for detecting in-plane displacements is underway. These devices are somewhat massive, making them difficult to utilize in many configurations, causing loading of the surface and thereby influencing the response of the material. They also suffer from all of the normal problems associated with coupling. Nevertheless, in application to the study of wave propagation in composites, they are capable of providing output information which, in conjunction with a well characterized input disturbance, provides the experimental data necessary to verify theoretical models of such behavior. 
A special application of piezoelectric devices worth mentioning is that of the acoustic microscope (ref. 8). The construction of such a system is an enormous challenge, but the capabilities are powerful. It is possible with such systems to work at gigahertz frequencies which correspond to wavelengths of sound comparable to that of light. With appropriate design of the transmitter and receiver, extremely fine spatial resolution is possible. Application to composite materials has shown that images of mechanical reflectivity or transmissivity are possible (fig. 1); comparative quantitative evaluation is also possible.

\section{Capacitance Displacement Measurement}

A relatively simple application of the concept of a parallel plate capacitor may be used to detect out-of-plane displacements of a material caused by an ultrasonic disturbance. Such a device has been used to study wave propagation in composite plates; the details of this work are described in a later section. Such devices, although electrically capable of wide band response, are practically limited by their size at lower frequency if their gap is maintained by support on the material being examined. If support is independent of the material, the dynamic range of detectable displacements is limited since ideally for optimal sensitivity the separation of the "plates" should be similar to the size of the displacements. The size of such devices may be reduced, but the biasing potential must be increased to maintain sensitivity; the breakdown potential for arcing across the gap thus provides a limit. Such devices are insensitive to in-plane displacements.

\section{Optical Methods}

A number of interferometric methods have been developed with good success, although primarily development has been limited to the situation of out-of-plane displacement. A typical configuration finds the material acting as the mirror of one or, at times, both legs of a Michelsen Interferometer. Displacement of the surface causes interference to occur which may be determined precisely in terms of the wavelength of the laser light used. Such methods are normally sensitive to vibration of the material that may not be associated with the ultrasonic disturbance of interest. The area of examination is generally 1 to $2 \mathrm{~mm}^{2}$.

A method of imaging the distortion of a reflecting surface caused by a continuous ultrasonic beam having passed through the material of interest has been developed and marketed by SonoScan, Inc. (ref. 9). The primary application is for detection of poor transmission of ultrasound within the material. Quantitative comparative interpretation of the wave propagation of the material from this method is possible. It is similar to the method of acoustical holography which in principal is similar to optical holography; this method also finds its principal applications to imaging of regions of imperfect ultrasound transmission.

Recently, Wagner (ref. 10) has utilized a pulsed laser holographic method for providing full field out-of-plane displacement images of a propagating ultrasonic disturbance caused by an explosive impulse, and by laser generation as well. Such a procedure is extremely beneficial for providing insight into the modeling of the material behavior in inhomogeneous anisotropic materials.

In general, full field visualization methods sacrifice temporal resolution because of the response of the recording media; however, technological advances are remedying this situation. 
Some work has been done with measuriny ultrasonic amplitudes in small volumes of material by using $X$-ray measurements (ref. 11). In principle, this method relies on the fact that the ultrasonic wave decreases the diffracted $x$-ray beam intensity. For composites composed of crystalline constituents, this method would appear to have potential.

\section{DATA ANALYSIS}

Ideally, the decision regarding the method of generation and detection of the ultrasound disturbance should be made with a mind toward the intended physical interpretation of the results. Certainly there is no possibility of explaining directly a quantitative observation, in terms of material performance, if the cause is unknown. An indirect explanation may, however, be possible in specific situations. Although it is desirable to be able to prescribe the full displacement and velocity, at every point as a function of time arbitrarily, it is not presently possible. If one desires to evaluate the performance of the material without regard to a certain scale of inhomogeneity, it is presently possible. Velocity measurement for determining material constants is such a situation. The fact that the property determined may not be constant along the path, or across the beam of the ultrasonic wave, is generally not a concern unless the difference is severe. In such instances, a reflection due to an impedance discontinuity is often observed; gross changes could occur however without discontinuity. In such a fashion, considerable information may be determined experimentally through the use of a constant but uncharacterized source, if the homogeneity and isotropy of the material are known.

This approach may be extended and applied to advantage in characterizing the condition of composite materials in conjunction with other nondestructive and destructive methods. It, however, requires considerable effort as regards determining how the observed behavior is modified by various independently determined material conditions. Extension to other similar materials is of higher probability, the more similar the material. This type of approach, sometimes called adaptive learning, is not ultimately a substitute for an understanding of the actual materials response, and should only be used as a stepping stone to a more complete understanding. (From an engineering point of view, the advent of high speed computers makes this approach attractive for routine problems.)

If the assumption of a constant source is reasonable, considerable artificial data interpretation is possible in certain special cases. Such is the case with ultrasonic tomography and other methods of spatial data averaging.

The method of data analysis proposed by the National Bureau of Standards, which serves in part as the basis for their method of transducer calibration, is that of deconvolution (ref. 12). The approach supposes that the material, and its structural configuration, act as a linear invariant system. Then, using a wellcharacterized point source to create a white noise ultrasonic disturbance, which ultimately is detected at a point by a quantifiable detector, the influence of the material/structure can be quantified. This quantification is in the form of a Green's function which mathematically describes the material condition in some average way; it is not unique as regards the material condition. The technique is useful in certain 1 imited situations for characterizing the source. This characterization is limited by the information obtained by the detector, i.e., if the detector measures only out-of-plane displacement, then the characterization of the 
source as regards in-plane displacements is not possible. Additionally, the process of calculating the Green's function is not trivial; however, recently Eitzen, et al., in reference 13, have suggested that by modifying the input source from the Dirac-delta function associated with a step force unloading at a point to an inverse Gaussian waveform, the calculation could be simplified.

Work employing finite difference methods or finite elements is underway; however, it is questionable as to whether, at this time, it is possible to properly pose this problem for analysis by these methods. It is certain that careful experimental investigation of this problem is needed to aid in these modeling processes.

\section{EXPERIMENTAL INVESTIGATION OF WAVE PROPAGATION IN COMPOSITE MATERIALS}

Composite materials are anisotropic and inhomogeneous. The inhomogeneity occurs on at least three scales:

$i$. on the scale of the fiber,

ii. on the scale of the laminae,

iii. on the scale of the laminate fiber orientation established by design. Although during the fabrication of composite material structures manufacturing flaws occur, routine application of inspection methods is often sufficient for locating and sizing large flaws. On the other hand, during the fabrication, the material itself is created and its condition is often of concern. In general, the concern is with regard to the quality of the mechanical properties and their uniformity, where often the causes of poor properties are related to subtle variations in the material that are not detected by conventional flaw detection methods. Even if they were detected, the need is to characterize the performance, not to place the blame. Uitrasonic methods of determining materials constants are available. Unless the condition of concern is uniformly distributed, the "properties" measured reflect an integrated response that may, in fact, not represent any place in the material precisely. Furthermore, since the damage in fiber reinforced composite materials begins to occur at relatively low loads and is globally distributed, the interpretation of velocity measurements must be carefully undertaken. Quite often the properties of the composite formed may suffer substantial change. It might be contended that such changes are not changes in material but rather changes in structure. It is, however, this structure which synergizes the properties of the constituents into a unique material. The task of characterization therefore incorporates both material and structural features.

In 1979, Vary and Bowles (ref. 14) suggested a method which interrogated both the material and structural properties of the composite materials examined. This acousto-ultrasonic method employed an ultrasonic transmitting transducer and an acoustic emission type receiving transducer, both coupled to the same side of the material. This pitch-catch arrangement in the limit of zero transducer separation is the conventional pulse echo technique. Vary and co-workers subsequently have shown correlation of sound transmission measured in this way with tensile strength. In addition, regions of poor ultrasonic stress wave transmission, as measured in this way, were found to be at the location of final failure in uniaxial tension test coupons. Henneke et al. (ref. 15) have continued to explore the use, and develop the understanding of the method for characterizing composite materials.

As has been suggested above, in order to quantitatively describe the nature of wave propagation in composite materials resulting from a mechanical disturbance, it is imperative that the source of the disturbance be known. Further, in order to validate the prediction of any theory regarding the wave propagation resulting from 
a known disturbance, it is necessary to employ a detector which can be quantitatively interpreted in terms of mechanical response. As part of an overall effort to model the mechanical behavior of composite materials with and without mechanically induced damage, Rebello and Duke have utilized a point load with step function time dependence as a source (pencil lead break) and an out-of-plane displacement sensor (capacitive transducer) to study the behavior of laminated composite plates (ref. 16).

Plates of aluminum and graphite epoxy composite were examined with various boundary conditions. Figure 2 displays the experimental arrangement employed to study the response. Figure 3 is the spectrum observed for an aluminum plate simply supported on all four edges when subjected to a pencil lead break in the center of the plate. Table 1 shows the comparison between the frequencies predicted by an exact solution and a finite element solution. Figure 4 is the spectrum observed for a graphite epoxy plate simply supported on all four edges when subjected to a pencil lead break in the center of the plate. Table 2 is the comparison between the exact and finite element solution for this case.

In both cases, despite the broadband content of the source, resonances of the plate were observed. The conclusion drawn was that if the response is observed for a period of time much longer than the source event duration, or the distance from the source is such that interaction of the disturbance with the structure will occur, the spectral content of the observed response will be dominated by induced structural resonances; changes in structural resonances caused by damage might be observed. Further characterization of the source is possible only if the disturbance propagates directly to the detector and the period of observation is short enough to ignore portions of the disturbance propagating from other directions. Generally this is extremely difficult to achieve, however, material, rather than source, characterization is the objective.

Since the capacitive transducer is capable of detecting only out-of-plane displacement, development of optical methods for detecting dynamic in-plane -and out-ofplane displacements are being developed. The primary effort is being directed at the in-plane measurements, with the method for out-of-plane measurement being adapted from other workers. The long range objective is to be able to introduce a known disturbance, predict the response at various positions in the structure, observe the actual response, and explain any difference from prediction in terms of the damaged material condition. Ideally this description would facilitate directly or indirectly the assessment of stiffness and strength or life.

In parallel with efforts to quantitatively measure displacements are efforts to study the influence of damage on the wave propagation in composites. This activity is utilizing the approach discussed above involving a constant but uncharacterized source. In particular, a conventional ultrasonic transducer is used to generate a disturbance in the material. At a fixed distance away on the same side of the material, another similar transducer is used to observe the disturbance (ref. 17). The material is at the same time subjected to mechanical loading which induces damage; independent means are employed to characterize the damage, including stiffness monitoring and penetrant-enhanced radiography. Figure 5 shows the spectrum of the observed disturbance at various stages of deformation. Of particular significance are the changes in the spectrum which appear to be related to the occurrence of particular damage mechanisms. This apparent frequency sensitivity raises the possibility of differentiating modes of damage by the observed spectral response. At the same time it appears to be advantageous to be able to influence the frequency 
content of the source in order to increase the range of damage development which may be monitored and to make possible the detection of damage that might interact with frequencies not already present.

By quantifying the observed response in a way related to the root mean squared energy, a correlation with stiffness degradation resulting during cyclic loading has been observed. This measure appears more sensitive than that of stiffness (f'ig. 6) but the reason is as yet unexplained.

Recently, experiments have been performed, replacing the receiving transducer with a Proctor out-of-plane displacement sensor (ref. 18). Figure 7 shows the spectrum of the observed disturbance caused by the same transmitting transducer used for the observations of Fig. 5. An extremely high resolution FFT has been performed; an explanation in terms of channeled plate waves (Lamb waves) is being sought.

\section{FUTURE DIRECTIONS}

Ultrasonic techniques, in that they are physically based on mechanical disturbances, possess the greatest potential for determining mechanical behavior of advanced materials. Consequently, in the future, efforts to understand the wave propagation in composites must be continued, as ultrasonic methods possess the potential for characterizing the mechanical behavior of these increasingly important engineering materials. Special emphasis must be given to characterizing the undamaged behavior of inhomogeneous materials, since conventional testing methods are inadequate. The ability to characterize, not simply identify, damage in composites must be a top priority, especially since there materials are very damage tolerant and may still perform satisfactorily even though severely damaged. At present the use of broadband ultrasonic disturbances appear to be most promising, but the need to measure quantitatively the displacement fields caused by such disturbances must be given priority. In addition, attention must be given to the means of creating such disturbance since the composites are inhomogeneous on a scale comparable to the area of the disturbance. A possible alternative to the broadband type of pulse disturbance which might lend itself to material characterization is to utilize a wide beam single frequency sound field, as is the procedure with the "acoustic microscope" of Sonoscan, but analyze the disturbance subsequent to additional interaction with the structure.

It should be anticipated that improvement of ultrasonic equipment and computer algorighms for data analysis will, in the future, lead to a greatly increased capability to image damage or regions of material property imperfection. However, for materials with complex or extensive damage, an image may afford little assistance in ultimately describing the anticipated performance of the material. 


\section{REFERENCES}

1. Rose, J. L., Ultrasonic Field Analysis and Approximation Parameters, Br. J. Nondestructive Testing 17, p. 109, 1975.

2. Claus, R. 0. and Zerwekh, P. S., Ultrasonic Transducer with a Two-Dimensional Gaussian Field Profile, IEEE Trans. Sonics and Ultrasonics 30, no. 1, p. 36 , 1983.

3. Frost, H. M., Electromagnetic Ultrasonic Transducers: Principles, Practice, and Applications, Physical Acoustics 14, p. 179, 1979.

4. Scruby, C. B., Wadley, H. N. G., Dewhurst, R. J., Hutchins, D. A. and Palmer, S. B., A Laser-Generated Standard Acoustic Emission Source, Mat1s. Eval 39, no. 13, p. 1250,1981 .

5. McBride, S. L. and Hutchison, T. S., Can. J. Physics 54 p. 1824, 1976.

6. Hsu, N. N., U.S. Patent $3,924,444,1977$.

7. Breckenridge, F. R., Tschiegg, C. E. and Greenspan, M.. J.Acoustical Soc. 57, p. $626,1977$.

8. Ilett, C., Somekh, M. G. and Briggs, G. A. D., Acoustic Microscopy of Elastic Discontinuities, Proc. R. Soc. Lond. A 393, pp. 171-183, 1984.

9: Kessler, L. W. and Yuhas, E. E., Acoustic Microscopy 1979, Proc. IEEE 67, no. 4, p. 526, 1979 .

10. Wagner, J. W., Full Field Visualization of Surface Acoustic Waves, to be presented at the 11th World Conf. on NDT, Las Vegas, 1985 (address: The Johns Hopkins University).

11. Saccocio, E. J., Lopez, M. A. and Lazara, K. J., J. Appl. Physics 38, p. 309, 1967.

12. Hsu, N. N. and Breckenridge, F. R., Characterization and Calibration of Acoustic Emission Sensors, Matls. Eval. 39, 1981.

13. Eitzen, D., Hsu, N., Carasso, A. and Proctor, T., Deconvolution by Design -- An Approach to the Inverse Problem of U1trasonic Testing, Proc. of the Review of Progress in Quantitative NDE, LaJolla, 1984. (The suggestion, that the Diracdelta function may be replaced by the inverse Gaussian function to simplify the deconvolution process, seems mathematically sound. However, the experimental realization would replace a point force step unloading by a transducer generating the Fourier equivalent of the inverse Gaussian. It is questionable whether the disturbance created will in fact be that desired.)

14. Vary, A. and Bowles, K. J., An Ultrasonic-Acoustic Technique for Nondestructive Evaluation of Fiber Composite Quality, Polymer Eng. and Sc. 19, no. 5, p. 373, 1979. 
15. Henneke, E. G. II, Duke, J. C. Jr., Stinchcomb, W. W., Govada, A. and Lemascon, A., A Study of the Stress Wave Factor Technique for the Characterization of Composite Materials, NASA Contractor Report 3670, 1983.

16. Rebello, C. J. and Duke, J.C. Jr., Factors Influencing the Ultrasonic Stress Wave Factor Evaluation of Materials, to be published. Presented at the 1984 ASME Winter Annual Meeting, New Orleans.

17. Govada, A., Duke, J. C. Jr., Henneke, E. G. II and Stinchcomb, W. W., A Study of the Stress Wave Factor Technique for the Characterization of Composite Materials, Interim Report of work on NASA Grant NSG-3-172, CCMS-84-13, Virginia Tech, 1984.

18. Lubnow, T., Senior Project, Engineering Science and Mechanics Dept., Virginia Tech, 1984 . 
TABLE 1

Aluminum Frequencies, SSSS

\begin{tabular}{|l|c|c|c|l|}
\hline $\begin{array}{c}\text { Frequency } \\
f_{1 j}\end{array}$ & $\begin{array}{c}\text { Finite } \\
\text { Element } \\
\mathrm{Hz}\end{array}$ & $\begin{array}{c}\text { Exact } \\
\mathrm{Hz}\end{array}$ & $\begin{array}{c}\text { Percentage } \\
\text { Change in } \\
\text { Frequencies }\end{array}$ & \multicolumn{1}{|c|}{$\begin{array}{c}\text { Experimental } \\
\text { Observation } \\
\text { (Source-Sensor) }\end{array}$} \\
\hline$f_{11}$ & 0.2206 & 0.2170 & 1.6 & $(\mathrm{C}, \mathrm{C}),(\mathrm{A}, \mathrm{C}),(\mathrm{B}, \mathrm{C}),(\mathrm{A}, \mathrm{A}),(\mathrm{B}, \mathrm{B})$ \\
$\mathrm{f}_{12}, \mathrm{f}_{21}$ & 0.5773 & 0.5425 & 6.0 & $(\mathrm{~A}, \mathrm{C}),(\mathrm{B}, \mathrm{C}),(\mathrm{A}, \mathrm{A}),(\mathrm{B}, \mathrm{B})$ \\
$\mathrm{f}_{22}$ & 0.9284 & 0.8679 & 6.5 & $(\mathrm{~A}, \mathrm{~A}),(\mathrm{B}, \mathrm{B}),(\mathrm{C})$ \\
$\mathrm{f}_{13}, \mathrm{f}_{31}$ & 1.2736 & 1.0849 & 14.8 & $(\mathrm{C}, \mathrm{C}),(\mathrm{A}, \mathrm{C}),(\mathrm{B}, \mathrm{C})$ \\
$\mathrm{f}_{23}, \mathrm{f}_{32}$ & 1.6140 & 1.4104 & 12.6 & $(\mathrm{~A}, \mathrm{~A}),(\mathrm{B}, \mathrm{B})$ \\
\hline
\end{tabular}

TABLE 2

Graphite-Epoxy Frequencies, SSSS

\begin{tabular}{|c|c|c|c|c|}
\hline $\begin{array}{c}\text { Frequency } \\
\mathbf{f}_{1 \mathrm{j}}\end{array}$ & $\begin{array}{l}\text { Finite } \\
\text { Element } \\
\mathrm{Hz} \\
\end{array}$ & $\begin{array}{c}\text { Exact } \\
\mathrm{Hz}\end{array}$ & $\begin{array}{l}\text { Percentage } \\
\text { Change in } \\
\text { Frequencies } \\
\end{array}$ & $\begin{array}{c}\text { Experimental } \\
\text { Observation } \\
\text { (Source-Sensor) } \\
\end{array}$ \\
\hline $\begin{array}{l}\mathbf{f}_{11} \\
\mathbf{f}_{12} \\
\mathbf{f}_{13} \\
\mathbf{f}_{21} \\
\mathbf{f}_{22} \\
\mathbf{f}_{41} \\
\mathbf{f}_{23} \\
\mathbf{f}_{24} \\
\mathbf{f}_{31} \\
\mathbf{f}_{33}\end{array}$ & $\begin{array}{r}64.3 \\
99.1 \\
197.4 \\
256.8 \\
271.3 \\
326.1 \\
389.2 \\
473.9 \\
629.7 \\
637.9\end{array}$ & $\begin{array}{r}61.7 \\
93.6 \\
167.8 \\
239.2 \\
252.0 \\
281.7 \\
292.2 \\
374.3 \\
535.4 \\
567.0\end{array}$ & $\begin{array}{r}4.0 \\
5.6 \\
15.0 \\
6.9 \\
7.1 \\
13.6 \\
24.9 \\
21.0 \\
15.0 \\
11.1\end{array}$ & $\begin{array}{l}(C, C),(A, C),(B, C) \\
(C, C),(A, C),(B, C),(A, A),(B, B) \\
(A, A),(B, B),(B, C),(A, A),(B, B) \\
(C, C),(A, C),(A, A),(B, B) \\
(C, C),(A, C),(A, C) \\
(C, C),(A, C),(B, C)\end{array}$ \\
\hline
\end{tabular}

\begin{tabular}{|cc|}
\hline &. $\mathrm{A}$ \\
. $\mathrm{C}$ & \\
\hline
\end{tabular}




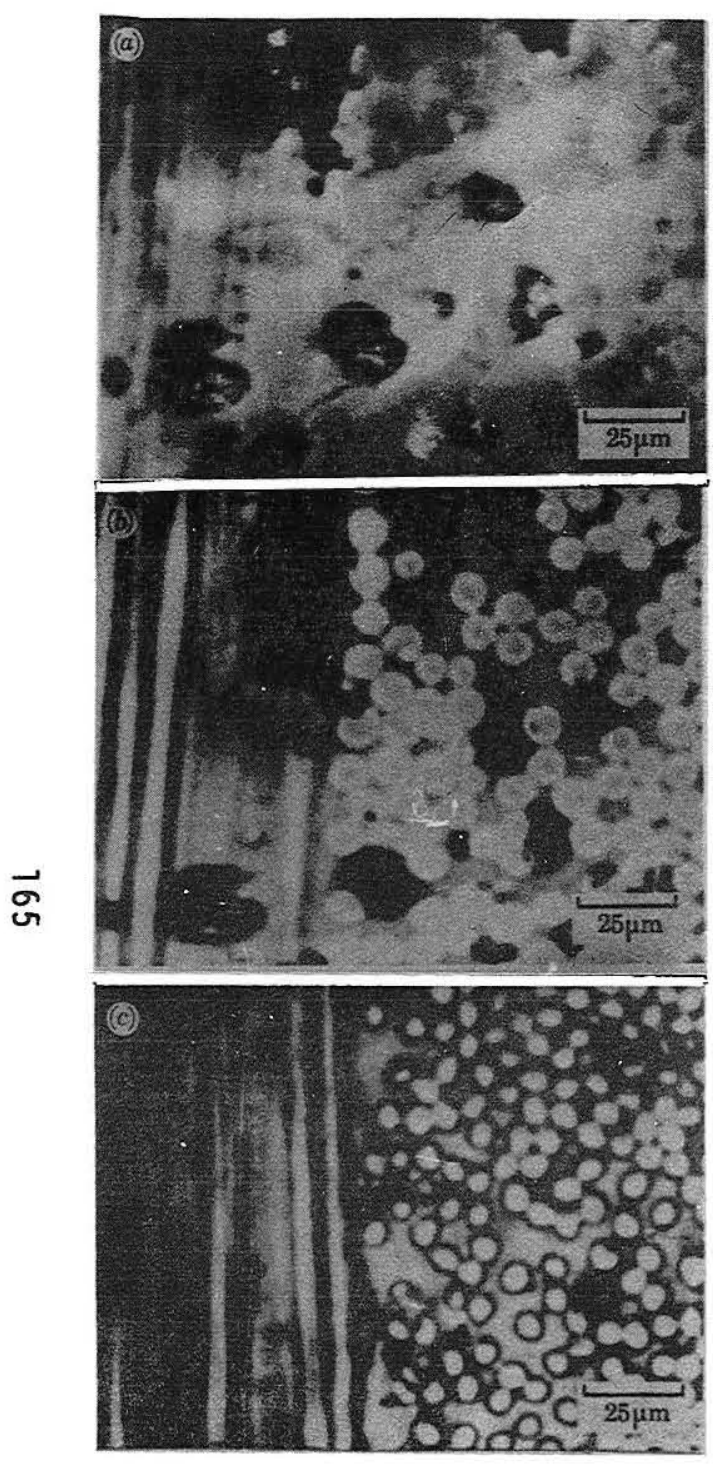

Figure 1 Comparison of Scanning Electron Micrograph (a) and Acoustic Micrograph (b focused), (c modest defocus) (from reference 8)

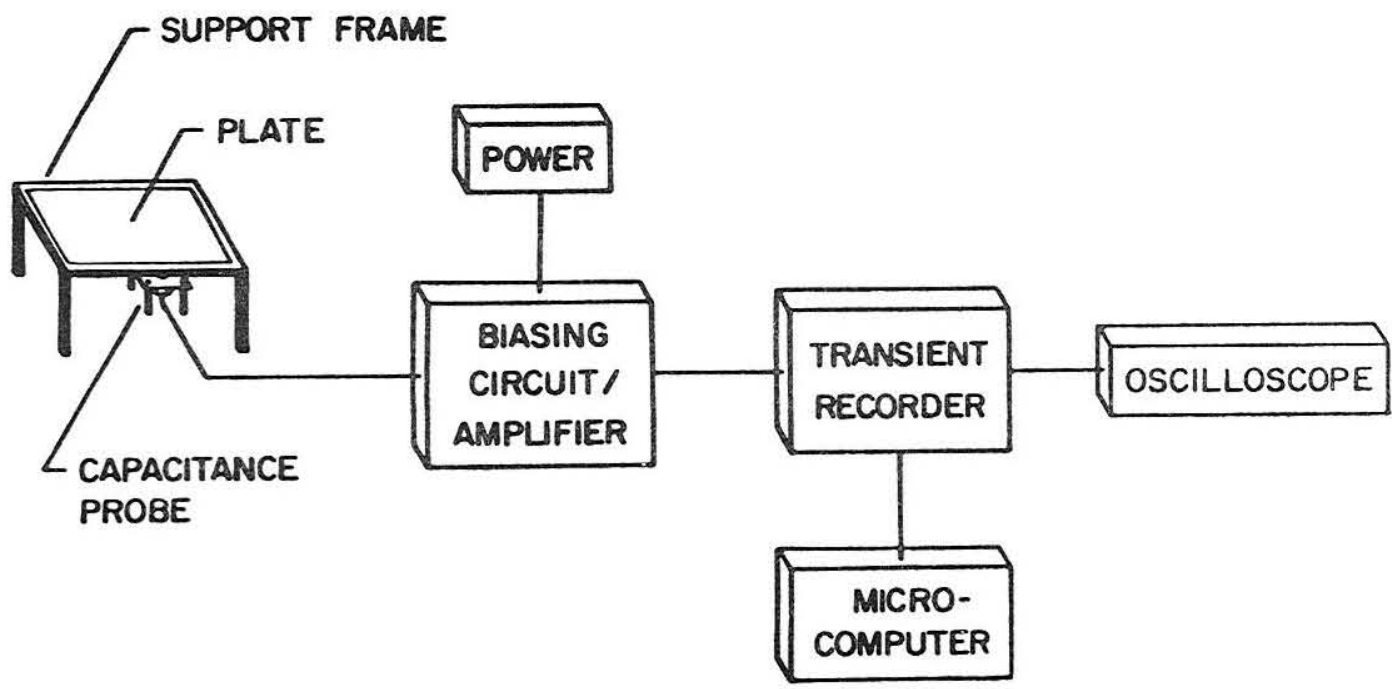

Figure 2 Experimental arrangement used to study the response of plates to point load with step function time dependence. 


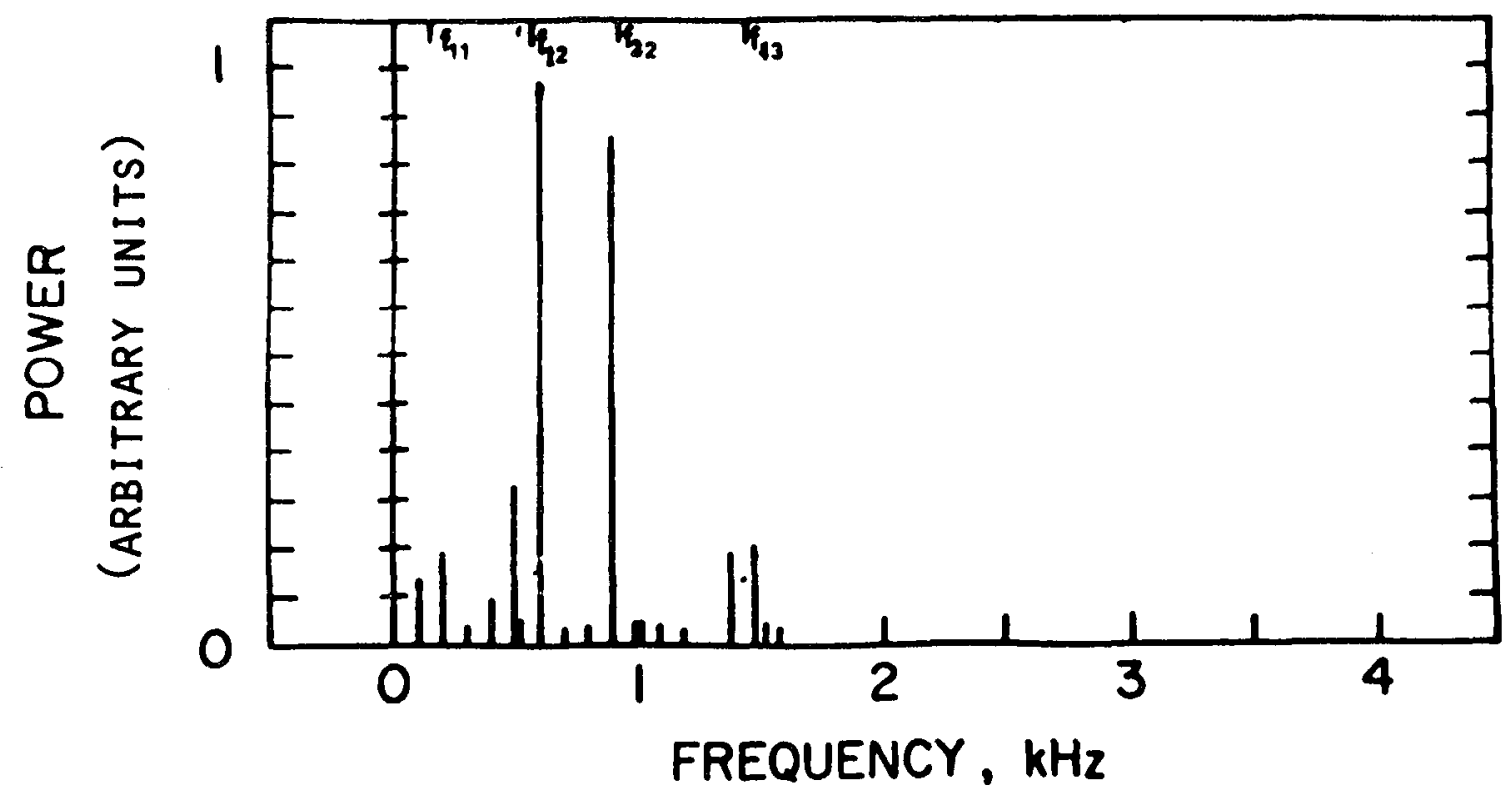

Figure 3 Frequency spectrum for an aluminum plate simply supported on a 11 four edges when subjected to pencil lead break in the center of the plate.

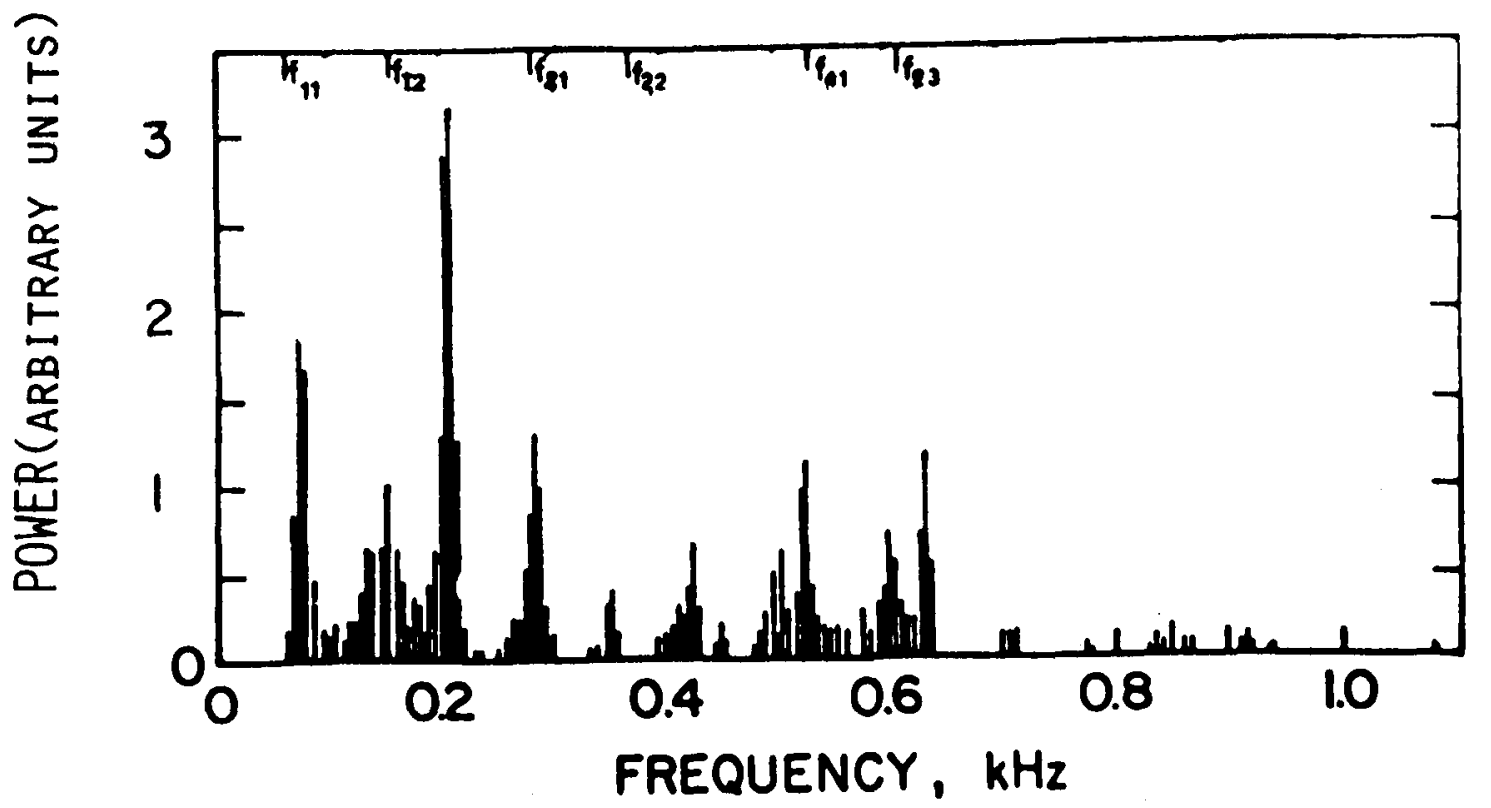

Figure 4 Frequency spectrum for a graphite epoxy plate simply supported on a 11 four edges when subjected to a pencil lead break in the center of the plate. 

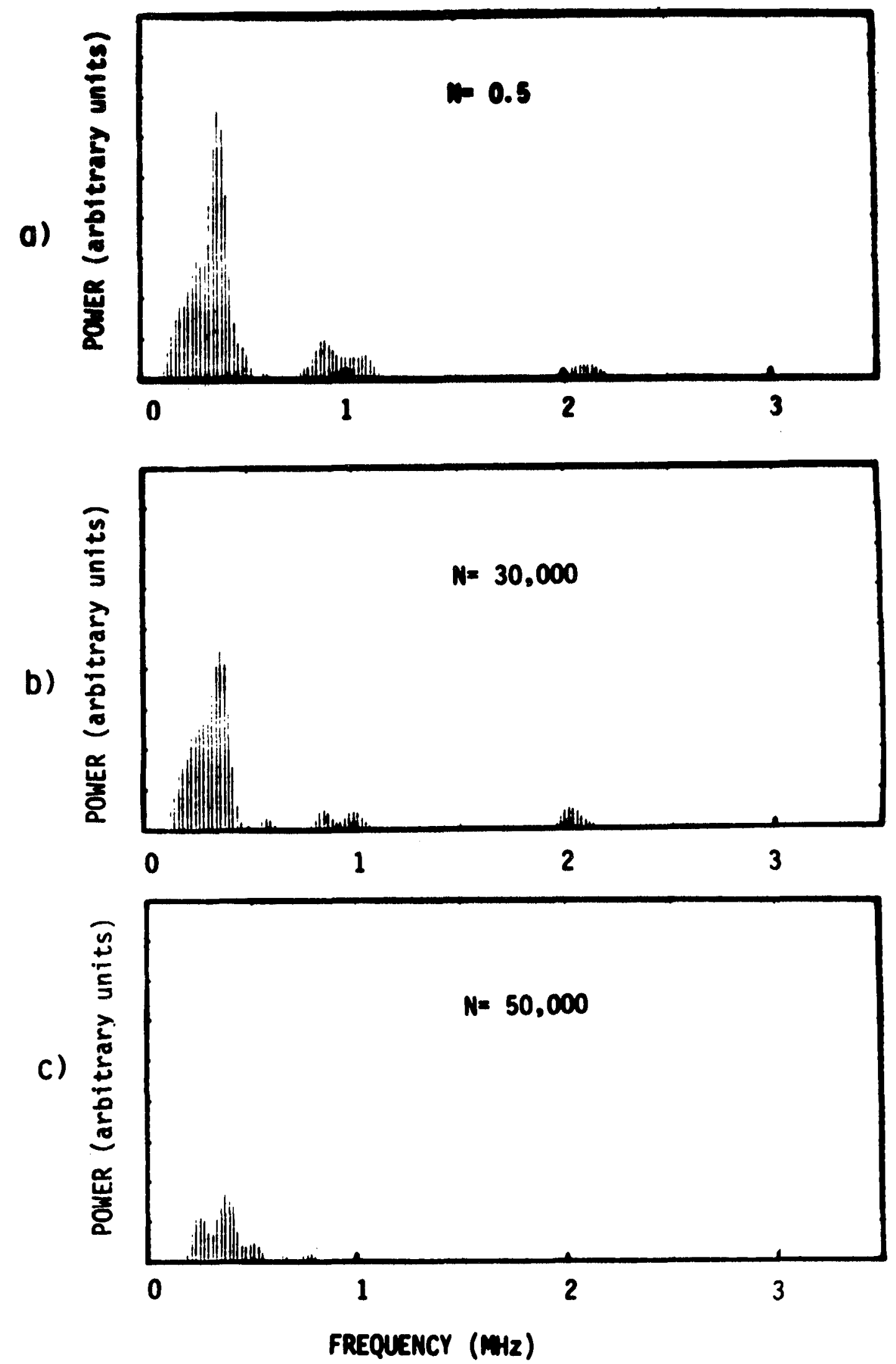

Figure 5 Frequency spectra at various stages of damage induced by cyclic loading. 


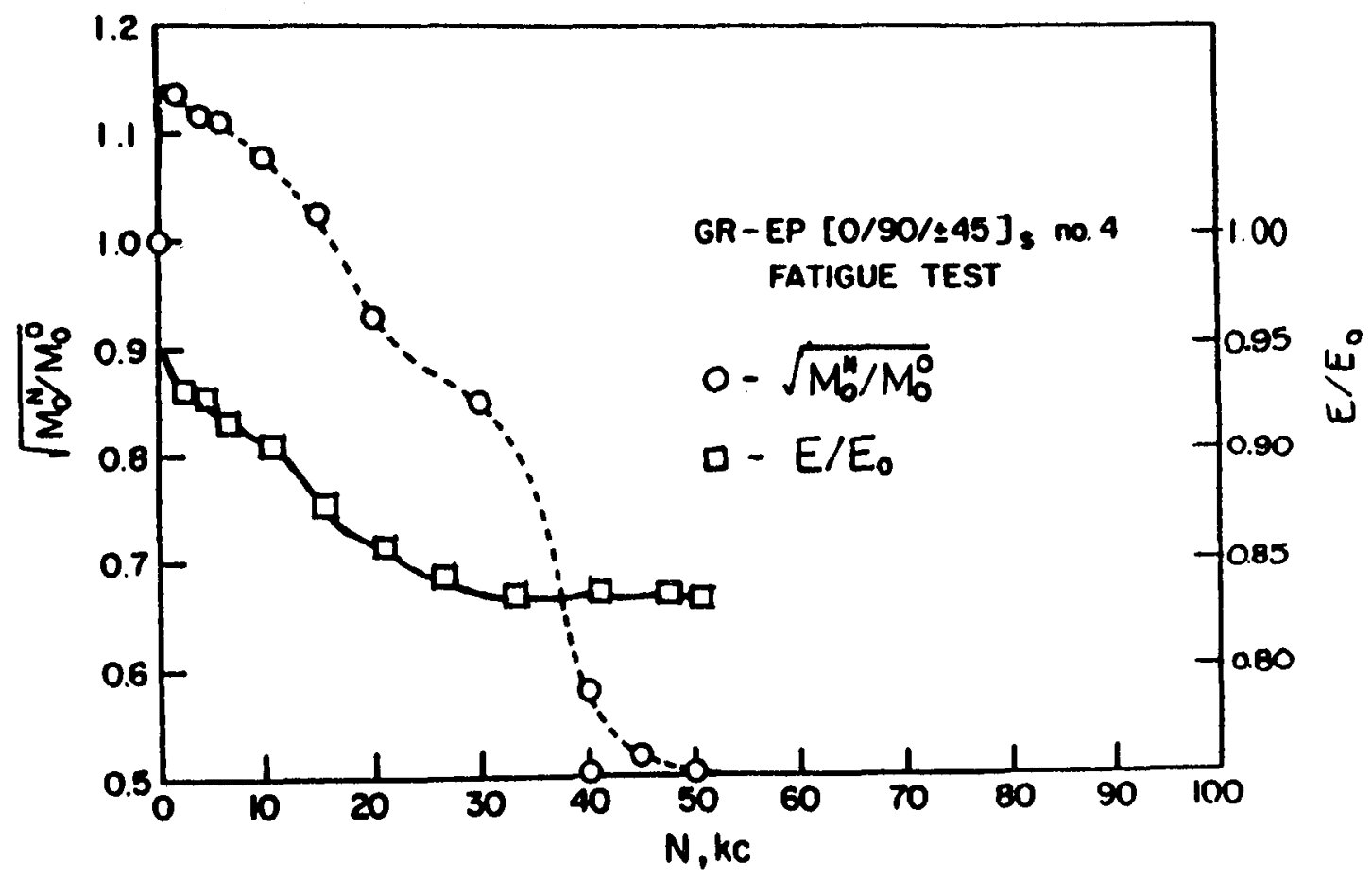

Figure 6 Change of normalized stiffness $E / E_{0}$ and the root of the normalized first spectral moment $M / M_{0}$ versus cycles of loading.

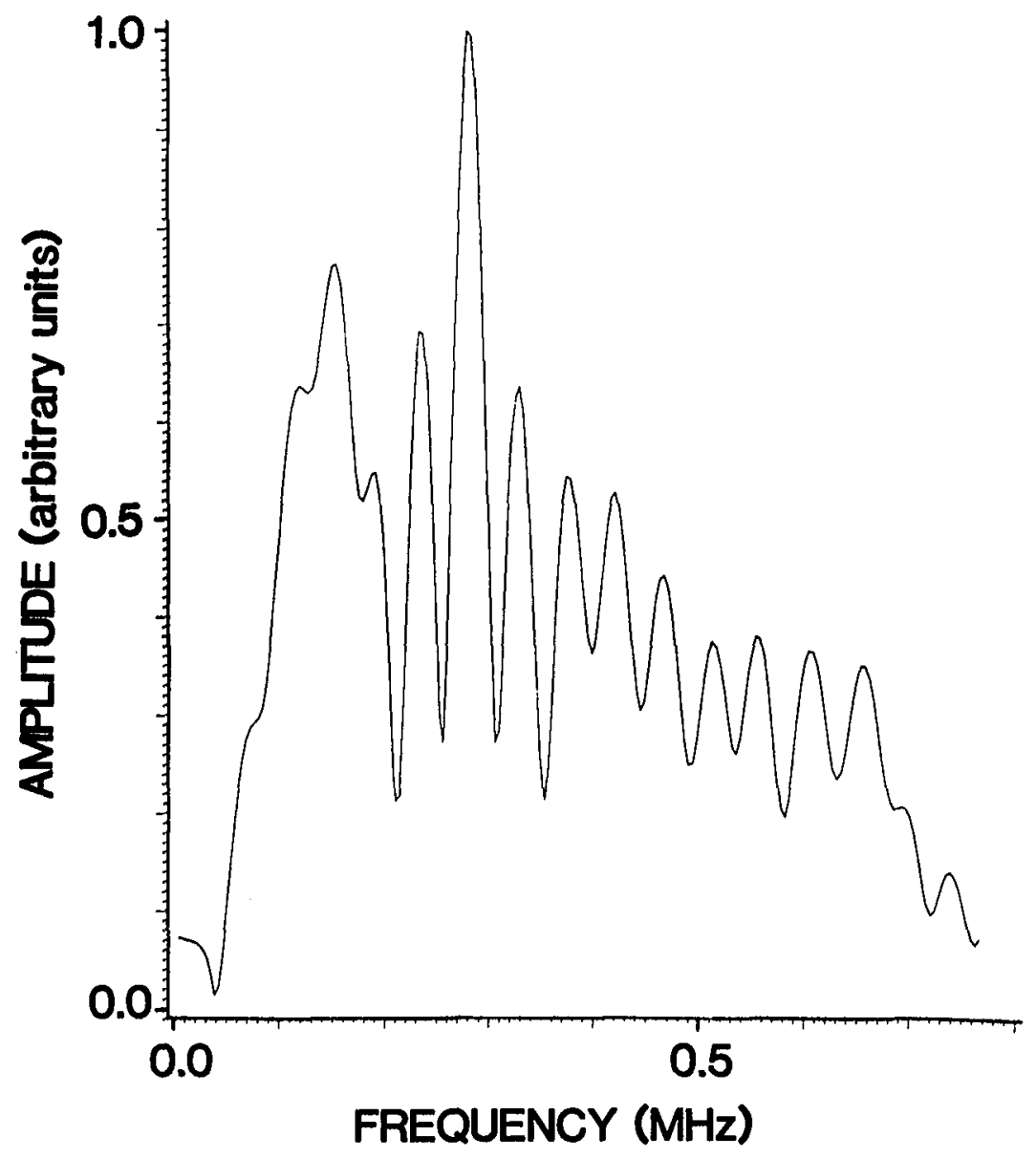

Figure 7 Output signal from a "Proctor" transducer used as a receiver in the acoustoultrasonic measurement procedure 


\title{
INFERENCE OF STRESS AND TEXTURE FROM ANGULAR DEPENDENCE OF
}

\author{
ULTRASONIC PLATE MODE VELOCITIES \\ R.B. Thompson, J.F. Smith, and S.S. Lee \\ Iowa State University \\ Ames, Iowa 50011
}

The theory for the angular dependence of the ultrasonic wave velocity in a symmetry plane of an orthorhombic, stressed materlal is presented. The two waves having polarizations in this plane are shown to have velocitles which can be est1mated from measurements of the $\mathrm{SH}_{\mathrm{O}}$ and $\mathrm{S}_{\mathrm{O}}$ guided modes of a thin plate: the relationshlp beling exact for the $\mathrm{SH}_{0}$ mode and requiring a $10 \%$ correction for the $\mathrm{S}_{\mathrm{o}}$ mode at long wavelength. It is then shown how stress and texture can be independent1y inferred from various features of the angular dependence of these two velocities. From the $\mathrm{SH}_{\mathrm{O}}$ data, the ablifty to determine the directions and differences in magnitudes of principal stresses is described and supported by experimental data on several materials. From a combination of the $\mathrm{SH}_{\mathrm{O}}$ and $\mathrm{S}_{\mathrm{O}}$ data, a procedure is proposed for determining the coefficients $W_{400}, W_{420}$, and $W_{440}$ of an expansion of the crystallite orientation distribution function in terms of generalized Legendre functions. Possible applications in process control are indicated.

\section{INTRODUCTION}

Successful process control requires the nondestructive characterization of a number of structural properties. Included are hardness, grain size, ductility, strength, preferred orientation, and stress. This paper discusses techniques whereby significant information related to the latter two quantities in rolled plate can be inferred from the angular dependence of the velocity of ultrasonic waves propagating In the plane of the plate.

\section{THEORY OF THE ANGULAR DEPENDENCE OF ULTRASONIC VELOCITY}

Consider a rolled plate and a coordinate system in which the 1, 2, and 3 axes colncide, respectively, with the rolling direction, the transverse direction, and the thickness direction, as shown in figure 1. Assume that the material can be represented as an elastic continuum with orthorhombic symmetry, and imagine that a biaxial stress is present in the plane of the plate with principal values $\sigma_{a}$ and $\sigma_{b}$ and orientation angle $\Omega$ as shown in figure 2 . The velocity of ultrasonic waves propa-

\footnotetext{
*This work was performed for the U.S. Department of Energy, Office of Basic
} Energy Sciences, Division of Materials Sclences, under contract No. W-7405-Eng-82. 


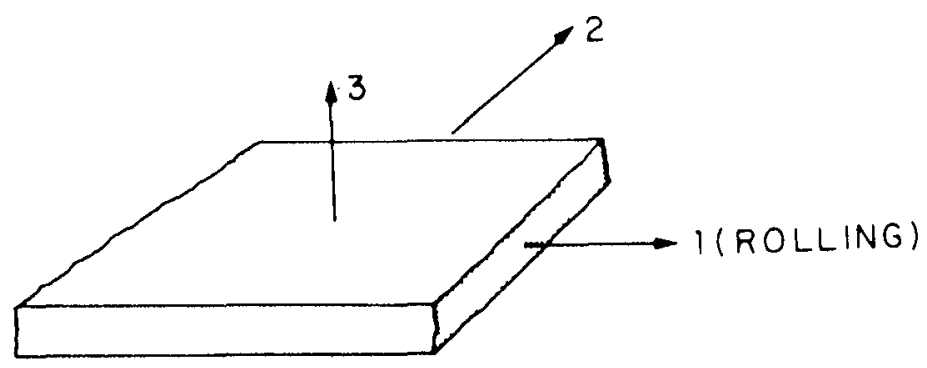

F1g. 1. Coordinate system.

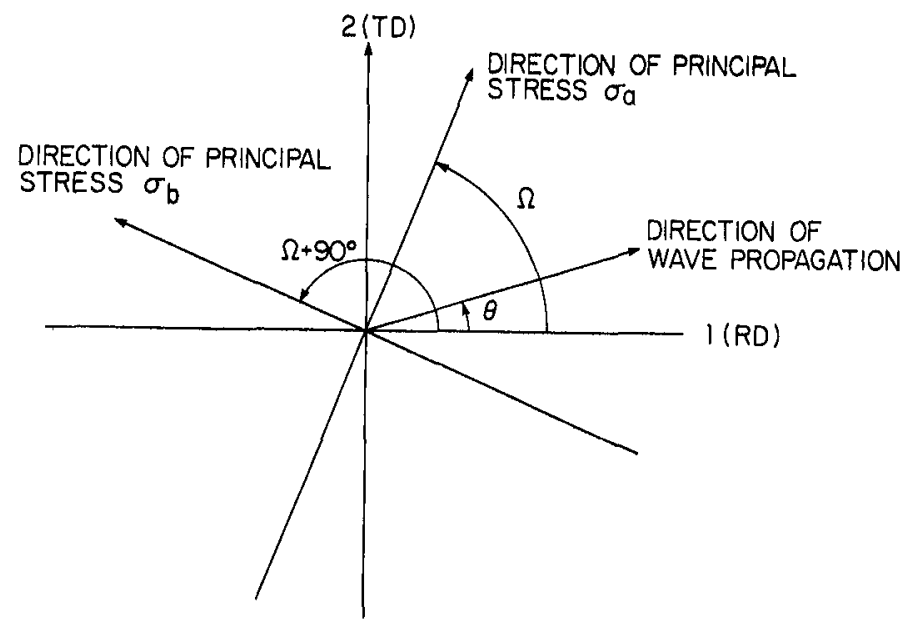

Fig. 2. Orientation of stress and wave propagation direction.

gating in the plane of the plate, 1.e. guided modes of the plate, must be obtained by solving the nonlinear, anisotropic equations of dynamic elasticity subject to the stress free boundary conditions on the two surfaces of the plate. The formidable job can be simplified by first neglecting the plate surfaces and treating plane wave propagation in an unbounded medium. Using an analysis, the solutions for the 1ongitudinal and transverse modes, to second order in the elastic anisotropy, are found to be of the form

$$
\begin{aligned}
\rho V_{L}^{2} & =\bar{C}_{L}+T+\frac{\bar{\alpha}}{2} \bar{C}_{L} \cos 2 \theta-\frac{\bar{\beta}}{2} \bar{C}_{T}(1-\cos 4 \theta)-\frac{\bar{\beta}^{2} \bar{C}_{T}^{2}}{\left.4 \bar{C}_{L}-\bar{C}_{T}\right)}\left[(1-\cos 4 \theta)^{2}\right. \\
& -2(1-\cos 4 \theta)]+\frac{\bar{\alpha} 2}{32} \frac{\bar{C}_{L}^{2}}{\left(\bar{C}_{L}-\bar{C}_{T}\right)}[1-\cos 4 \theta] \\
& +\frac{\overline{\alpha \beta}}{4} \frac{\bar{C}_{L} \bar{C}_{T}}{\left(\bar{C}_{L}-\bar{C}_{T}\right)}[(1-\cos 4 \theta)(\cos 2 \theta)]+\left(\bar{C}_{16}+\bar{C}_{26}\right) \sin 2 \theta \\
& +\frac{1}{2}\left(\bar{C}_{16}-\bar{C}_{26}\right) \sin 4 \theta
\end{aligned}
$$




$$
\begin{aligned}
\rho V_{T}{ }^{2} & =\bar{C}_{T}+T+\frac{\bar{\beta}}{2} \bar{C}_{T}(1-\cos 4 \theta)+\frac{\bar{\beta}^{2} \bar{C}_{T}^{2}}{4\left(\bar{C}_{L_{L}}-\bar{C}_{T}\right)}\left[(1-\cos 4 \theta)^{2}-2(1-\cos 4 \theta)\right] \\
& -\frac{\bar{\alpha}^{2}}{32} \frac{\bar{C}_{L}{ }^{2}}{\left(\bar{C}_{L}-\bar{C}_{T}\right)}[1-\cos 4 \theta]-\frac{\overline{\alpha \beta}}{4} \frac{\bar{C}_{L} \bar{C}_{T}}{\left(\bar{C}_{L}-\bar{C}_{T}\right)}(1-\cos 4 \theta)(\cos 2 \theta) \\
& -\frac{1}{2}\left(\bar{C}_{16}-\bar{C}_{26}\right) \sin 4 \theta
\end{aligned}
$$

Here $\rho$ is density, $V_{L}, T$ is the wave velocity of longltudinal and transverse waves, $\theta$ is the angle of propagation with respect to the 1-direction, $\bar{C}_{\mathrm{T}} \overline{\mathrm{C}}_{66}, \overline{\mathrm{C}}_{\mathrm{L}}=\left(\overline{\mathrm{C}}_{11}+\overline{\mathrm{C}}_{22}\right) / 2, \alpha=\left(\overline{\mathrm{C}}_{11}-\overline{\mathrm{C}}_{22}\right) / \mathrm{C}_{\mathrm{L}}, \bar{\beta}=\left[\left(\mathrm{C}_{\mathrm{L}}-\overline{\mathrm{C}}_{12}\right) / 2-\overline{\mathrm{C}}_{\mathrm{T}}\right] / \bar{C}_{\mathrm{T}}$. The latter two parameters are measures of longltudfinal and shear wave velocity anisotroples, respectively. In addition,

$$
\mathrm{T}=\left(\sigma_{\mathrm{a}}+\sigma_{\mathrm{b}}\right) / 2+\left[\left(\sigma_{\mathrm{a}}-\sigma_{\mathrm{b}}\right) / 2\right] \cos 2(\Omega-\theta)
$$

where $\sigma_{a}$ and $\sigma_{b}$ are the princlpal stresses with the former acting along an axis inclined at an angle $\Omega$ with respect to the 1-direction. In the absence of stress, the $\bar{C}_{1 f}$ are equal to the anisotropic elastic stiffnesses of the orthorhombic continuum, for which $\bar{C}_{16}=\bar{C}_{26}=0$. Hence there are nine independent values (ref. 1 ). When stress is present, there will be small shifts in the values of these nine constants (ref. 2). If $\Omega \neq 0$ the constant $\bar{C}_{16}$ and $\bar{C}_{26}$ will no longer vanish due to the lowering of the effective symmetry of the problem from orthorhombic to tricilnic.

The solution $\mathrm{V}_{\mathrm{L}}$ corresponds to a quasi-longltudinal wave which is polarized in the 1-2 plane, while the solution $V_{T}$ corresponds to a quasi-transverse wave polarized in the same plane. A third solution, polarized along the 3-direction, also exists which will not be discussed here.

The relationship of these plane wave solutions for an unbounded medium will next be related to wave propagation in a plate. Consider first the shear wave solution given by equation (2). Examination of the full solution shows that all stress components vanish on planes $\mathrm{Z}=$ constant. Hence the plane wave results are also exact solutions in the plate geometry. Since they are uninfluenced by the position of the plate faces, they are non-dispersive. This solution is known as the $\mathrm{SH}_{0}$ mode of the plate (ref. 3).

The plane waves whose velocities are given by equation (1) are not exact solutions in the plate geometry since the dynamic stress component $\sigma_{33}$ does not vantsh for these solutions. However, a simple approximation exists when the frequency is sufficlently low that the wavelength is large with respect to the plate thickness. In isotropic, linear elasticity, the fundamental, symmetric Lamb mode ref. 3), $S_{O}$, is known to propagate with a velocity of $v_{S_{O}}=v_{L_{0}}\left[1-\left(\frac{v}{I-\nu}\right)^{2}\right]^{1 / 2}$ in the low frequency 11mit. Here $V_{L_{o}}$ is the plane wave longltudinal veloclty and $\nu$ is Polsson's ratio. For a Poisson's ratio of $0.3, \mathrm{~V}_{\mathrm{S}_{\mathrm{o}}} / \mathrm{V}_{\mathrm{L}_{\mathrm{o}}}=0.90$.

In an orthorhombic material, this relationship generalizes to

$$
\mathrm{V}_{\mathrm{S}_{0}}=\mathrm{V}_{\mathrm{L}_{0}}\left(1-\mathrm{C}_{13}{ }^{\left.2 / \mathrm{C}_{11} \mathrm{C}_{33}\right)^{1 / 2}}\right.
$$


for propagation along the " $i$ " symmetry axis. Thus the correction relating the plate to plane wave velocities will depend on direction. The correction is the order of $10 \%$ as noted above, and it depends on elastlc constants which only vary by a few percent in modestly textured materials. Hence, in many cases, the angular dependence of the correction may be negligible and its isotropic value can be used. This point will be discussed further in the second section following.

As an experimental test of equation (2), consider the plot of $\Delta V / V$ versus for $\theta$ for $6061 \mathrm{Al}$ in figure 3 (ref. 4). In the upper plot, $\sigma_{a}=\sigma_{b}=0$ and the data are fitted well by the first and third term on the right hand side of equation (2). When $\sigma_{a} \neq 0$ and $\Omega=0$, the results shown in the lower plot were obtained. Here the theory includes the first three terms. $\bar{C}_{\mathrm{T}}$ was shifted slightly to fit the zero degree data, $\sigma_{a}$ was determined from load cell readings, and $\bar{\beta}$ was held equal to the unstressed value. Although some minor differences between theory and experiment are observed, the agreement is believed to be quite good. This is consistent with the theoretical expectation that the remaining omitted terms vary as a higher order in the anisotropy.

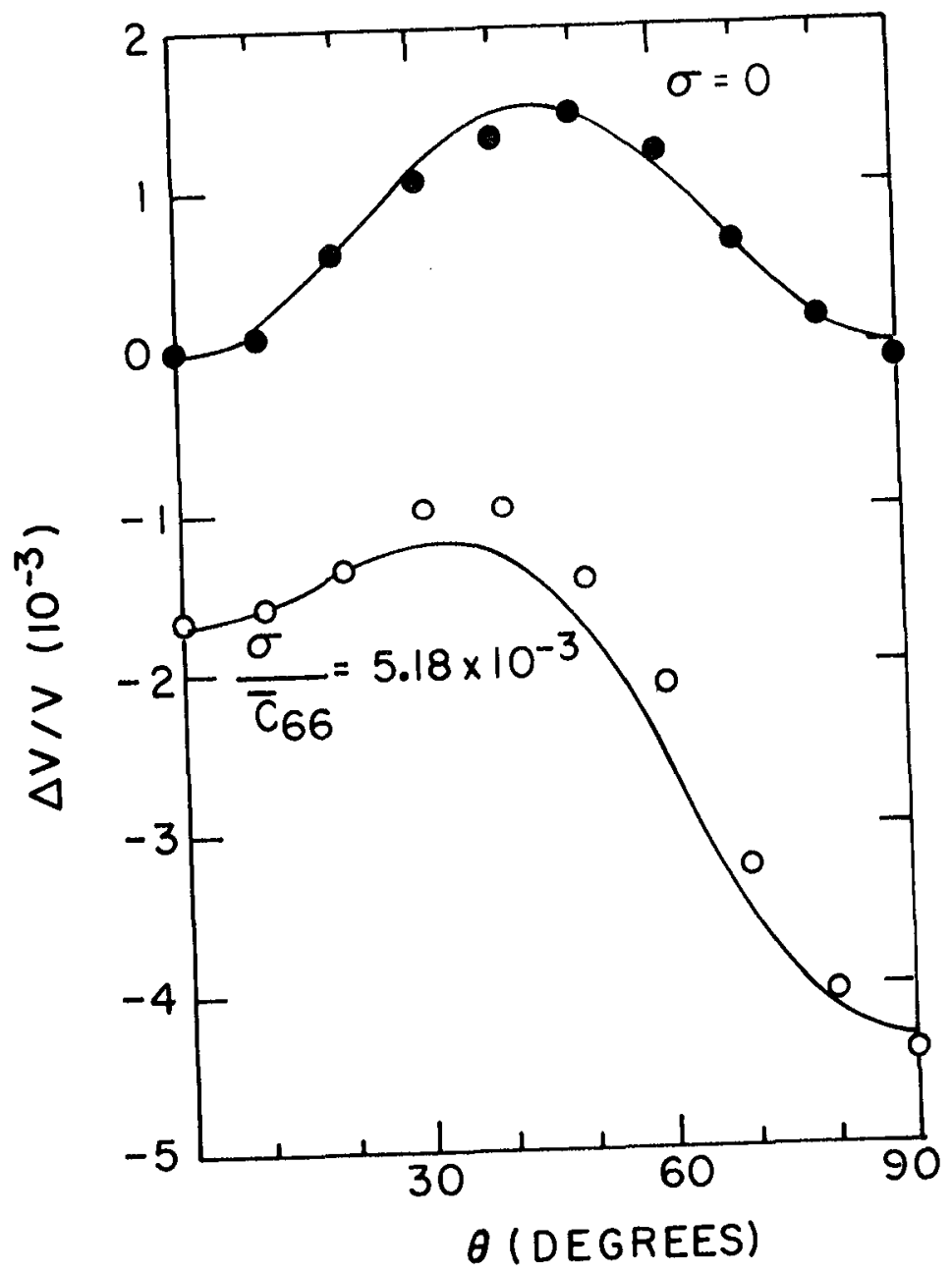

Fig. 3. Comparison of predicted and applied stress in 6061-T6 aluminum when stress is not parallel to material symmetry axes. Experimental configuration is shown in the inset and the method of comparison is described in the text. 
Stress may be determined from measurements of the shear wave velocity as predicted by equation (2). As the results are discussed in detall elsewhere (refs. 4-8), only a brief summary will be given. Consider first the expression for $T$ in equation (3). Note that no material constants influence the dependence of $T$ on $\sigma_{a}$ and $\sigma_{b}$. Thus, if the contributions of the terms following $T$ in equation (2) could be suppressed, the difference in principle stresses, $\sigma_{a}-\sigma_{b}$, could be determined from measurements of the angular dependence of $V_{T}$. This can be accomplished by noting that all but one of those terms has four-fold symmetry. Thus, if one measures $V_{T}(\theta)$ and $V_{T}\left(\theta+90^{\circ}\right)$ and takes the difference in ov 2 , it follows that

$$
\begin{aligned}
{\left[\mathrm{V}_{\mathrm{T}}(\theta)-\mathrm{V}_{\mathrm{T}}\left(\theta+90^{\circ}\right)\right] / \overline{\mathrm{V}}_{\mathrm{T}} } & =\frac{\left(\frac{1}{2 \rho \bar{V}_{\mathrm{T}} 2}\right)\left[\left(\sigma_{\mathrm{a}}-\sigma_{\mathrm{b}}\right) \cos 2(\Omega-\theta)\right.}{} \\
& \left.-\frac{\bar{\alpha} \bar{\beta} \overline{\mathrm{C}}_{\mathrm{L}} \overline{\mathrm{C}}_{\mathrm{T}}}{4\left(\overline{\mathrm{C}}_{\mathrm{L}}-\overline{\mathrm{C}}_{\mathrm{T}}\right)}(\cos 2 \theta-\cos 6 \theta)\right] .
\end{aligned}
$$

where $\bar{V}_{T} \Delta\left[V(\theta)+V_{T}\left(\theta+90^{\circ}\right)\right] / 2$. For small anisotropies, one can approximate $\rho \bar{V}_{T}{ }^{2}$ by $\overline{\mathrm{C}}_{\mathrm{T}}$ for further simplification.

The final term in equation (5) is second order in the elastic anisotropy. In cases in which it is negligible, the principal stress difference may be deduced by varying $\theta$ until the magnitude of the left hand side of equation (5) is maximized and setting that value equal to $\left(\sigma_{\mathrm{a}}-\sigma_{\mathrm{b}}\right) / 2 \mathrm{C}_{\mathrm{T}}$. As an example, figure 4 presents a comparison of measured and predicted stress in a second 6061-T6 aluminum plate having an unstressed $\mathrm{SH}_{\mathrm{O}}$ wave velocity anisotropy of $0.5 \%$, as distinct from the $0.15 \%$ anisotropy of the first sample shown in figure 3 . The stress was applied at $45^{\circ}$ with respect to the rolling direction, $\Omega=45^{\circ}$. The maximum value of velocity shift between the $\theta$ and $\theta+90^{\circ}$ directions was observed to occur when $\theta=45^{\circ}$, and a plot of predicted stress versus applied stress (not shown) closely followed the 1deal, unity slope line (ref, 8). However, that was not a critical test of the smallness of the final term in equation (5) since $\cos 2 \theta=\cos 6 \theta=0$ for $\theta=45^{\circ}$. Hence the measurement direction was then changed to $\theta=15^{\circ}$ so that the final term would no longer vanish 1dentically. In the plot shown in figure 4, the predicted stress was deduced on the basis of inserting the known values of $\Omega=45^{\circ}, \theta=15^{\circ}$ into equation (5) and neglecting the final term. The excellent agreement is consistent with the postulated small value of thls second order anlsotropy term, at least in the particular aluminum sample studied.

If the anisotropy term is not small, a procedure is st111 avallable for removing Its influence on the data. Note that equation (5) contains only one term with a $6 \theta-$ angular variation, which has as its coefficient the second order material anisotropy parameters. Determination of that coefficient from the value of the $6 \theta$ term in a Fourier series representation of $\left[V_{T}(\theta)-V_{T}\left(\theta+90^{\circ}\right)\right]$ should allow the entire anisotropy term to be subtracted from the data. The previously described procedure could then be applied to the corrected data.

Other than the example 1llustrated in figure 4, a detalled experimental study of this proposed procedure for non-parallel principal stress and material symmetry axes has not yet been completed. However, when $\Omega=0$, a number of samples have been studied (refs. 4, 6-8). Figure 5 presents, as an example, the results in a 304 


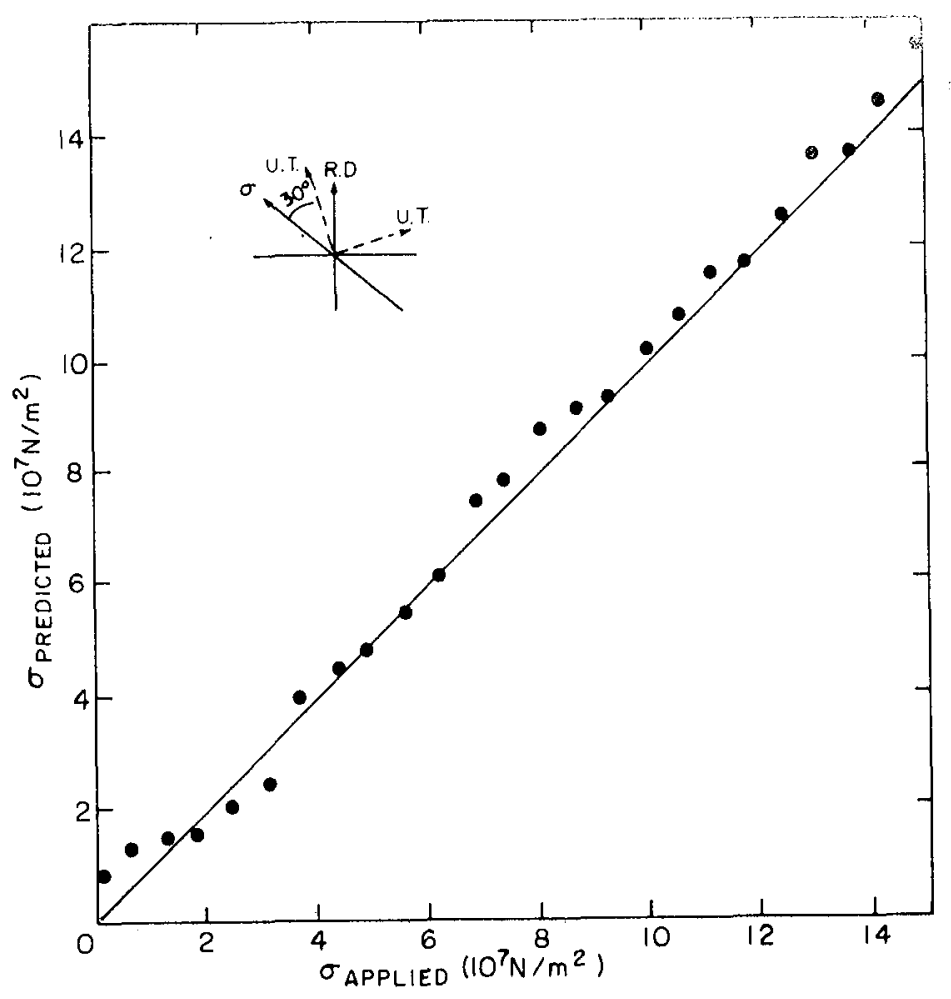

Fig. 4. Comparison of predicted and applied stress in 6061-T6 aluminum when stress is not parallel to material symmetry axes. Experimental configuration is shown in the inset and the method of comparison is described in the text

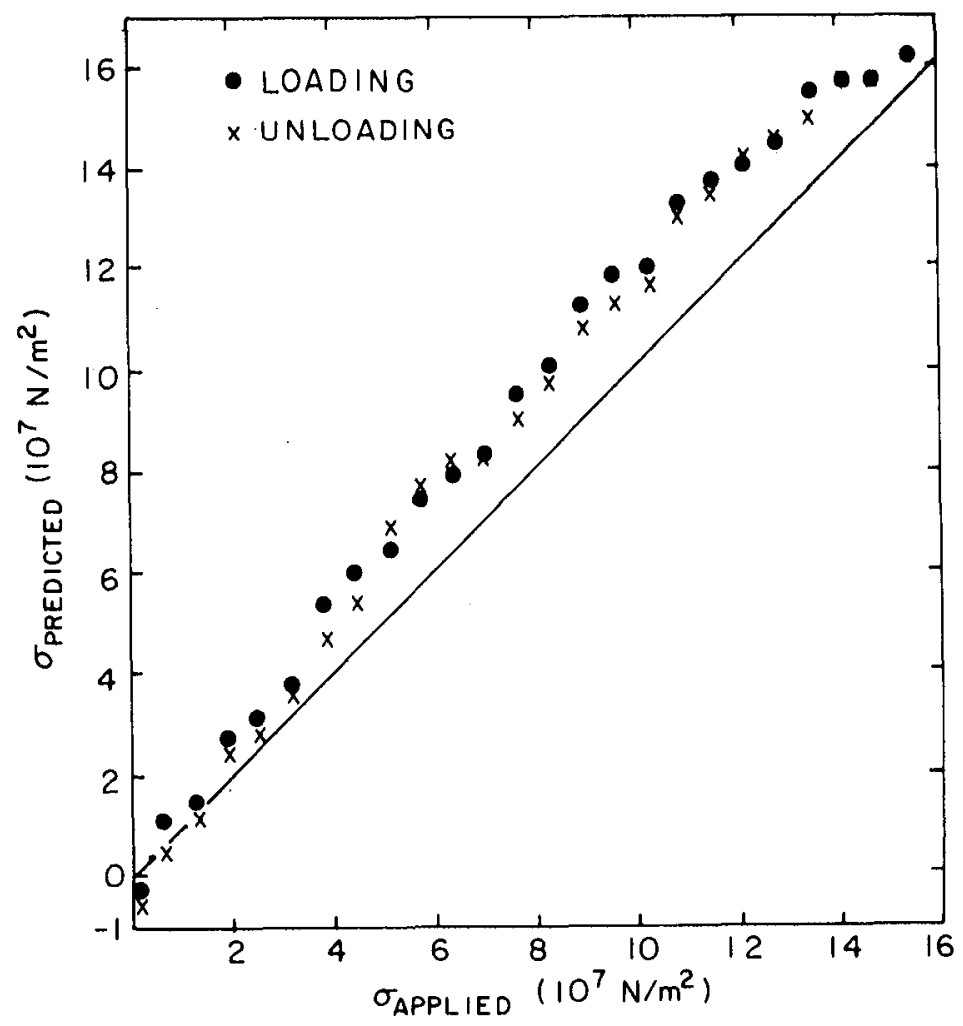

Fig. 5. Comparison of predicted and applied stress in 6061-T6 aluminum plate. 
stainless steel sample having an unstressed $\mathrm{SH}_{\mathrm{O}}$ velocity anisotropy of $1.5 \%$. Agreement of predicted and applied stress is excellent. Similar results have been obtained on 6061 aluminum, commerclally pure copper, and 1100 aluminum plate. In the latter case, tensile plastic deformation of $0.6 \%$ did not destroy the agreement. In an additional sample of titanium (ref. 8), errors occurred due to the absence of the assumed orthorhombic symmetry in the unstressed state.

\section{DETERMINATION OF TEXTURE}

In the measurement of stress, the effects of texture were suppressed by comparing the $\mathrm{SH}_{\mathrm{O}}$ velocities measured before and after rotating the propagation direction by $90^{\circ}$. However, those suppressed terms may be used to directly determine important parameters of the preferred orientation. The possibility of inferring preferred orientation from measurements of the anisotropies of the elastic constants or sound velocities has been demonstrated by several authors (refs. 9-11). The present discussion differs from the prior work in two regards. First, the nondestructive measurement of the velocity antsotropy can be made with couplant free EMAT transducers (ref. 12). Hence operation in an on-1ine, process control mode at room or elevated temperatures is possible (ref. 13). Second, using relationships recently reported by Sayers and Allen (ref. 2) the velocity anisotroples can be directly related to coefficients of the expansion of the crystallite orientation distribution function (CODF) in terms of generalized Legendre functions (refs. 13, 14).

For a material with unknown stress orfentations, the procedure would be as follows. First the techniques described in the above section should be employed to determine the principal stress directions and differences. This allows the angular variation of the term $T$ to be removed from equations (1) and (2). If one restricts attention to the velocities measured at $0^{\circ}, 45^{\circ}$, and $90^{\circ}$, one finds

$$
\begin{aligned}
& \overline{\mathrm{C}}_{\mathrm{T}}+\left(\sigma_{\mathrm{a}}+\sigma_{\mathrm{b}}\right) / 2=\rho \mathrm{V}_{\mathrm{T}}{ }^{2}\left(0^{\circ}\right)-\left[\left(\sigma_{\mathrm{a}}-\sigma_{\mathrm{b}}\right) / 2\right] \cos 2 \Omega \\
& \overline{\mathrm{C}}_{\mathrm{T}}+\left(\sigma_{\mathrm{a}}+\sigma_{\mathrm{b}}\right) / 2+\bar{\beta} \overline{\mathrm{C}}_{\mathrm{T}}-\frac{\bar{\alpha}^{2}}{16} \frac{\overline{\mathrm{C}}_{\mathrm{L}}{ }^{2}}{\left(\bar{C}_{\mathrm{L}}-\overline{\mathrm{C}}_{\mathrm{T}}\right)}=\rho \mathrm{V}_{\mathrm{T}}{ }^{2}\left(45^{\circ}\right)-\left[\left(\sigma_{\mathrm{a}}-\sigma_{\mathrm{b}}\right) / 2\right] \sin 2 \Omega \\
& \overline{\mathrm{C}}_{\mathrm{T}}+\left(\sigma_{\mathrm{a}}+\sigma_{\mathrm{b}}\right) / 2=\rho \mathrm{V}_{\mathrm{T}}{ }^{2}\left(90^{\circ}\right)+\left[\left(\sigma_{\mathrm{a}}-\sigma_{\mathrm{b}}\right) / 2\right] \cos 2 \Omega \\
& \overline{\mathrm{C}}_{\mathrm{L}}+\left(\sigma_{\mathrm{a}}+\sigma_{\mathrm{b}}\right) / 2+\bar{\alpha} \overline{\mathrm{C}}_{\mathrm{L}}=\rho \mathrm{V}_{\mathrm{L}}{ }^{2}\left(0^{\circ}\right)-\left[\left(\sigma_{\mathrm{a}}-\sigma_{\mathrm{b}}\right) / 2\right] \cos 2 \Omega \\
& \overline{\mathrm{C}}_{\mathrm{a}}+\underset{\mathrm{b}}{(\sigma+\sigma) / 2}-\overline{\mathrm{B}} \overline{\mathrm{C}}+\frac{\bar{\alpha}^{2}}{16} \frac{\overline{\mathrm{C}}_{\mathrm{L}}}{\left(\overline{\mathrm{C}}_{\mathrm{L}}-\overline{\mathrm{C}}_{\mathrm{T}}\right)}+\overline{\mathrm{C}}_{16}+\overline{\mathrm{C}}_{26}=\rho \mathrm{V}_{\mathrm{L}}{ }^{2}\left(45^{\circ}\right) \\
& -\left[\left(\sigma_{a}-\sigma_{b}\right) / 2\right] \sin 2 \Omega \\
& \bar{c}_{L}+\left(\sigma_{a}+\sigma_{b}\right) / 2-\frac{\bar{\alpha}}{2} \bar{c}_{L}=\rho V_{L}{ }^{2}\left(90^{\circ}\right)+\left[\left(\sigma_{a}-\sigma_{b}\right) / 2\right] \cos 2 \Omega
\end{aligned}
$$


In each of these equations, the experimentally observable velocities, and the inferred principal stress orientations and differences are placed on the right-hand side and can be considered as known quantities.

After noting that equations $(6 a)$ and $(6 \mathrm{c})$ are not independent because of the relationship between $\left[\mathrm{V}_{\mathrm{T}}{ }^{2}\left(0^{\circ}\right)-\mathrm{V}_{\mathrm{T}}{ }^{2}\left(90^{\circ}\right)\right]$ and $\left[\sigma_{\mathrm{a}}-\sigma_{\mathrm{b}}\right]$, one concludes that there are five equations in the six unknowns, $\bar{C}_{T}, \bar{C}_{L},\left(\sigma_{a}+\sigma_{b}\right)$, and $\left(\bar{C}_{16}+\bar{C}_{26}\right)$. Hence, a rigorous solution is not possible, and some further approximations will be required. Since $\bar{C}_{16}$ and $\bar{C}_{26}$ appear only in equation (6e) and are not of primary interest, this equation will be dropped leaving four equations in five unknowns. By elementary manipulations it can be concluded that

$$
\begin{aligned}
& \overline{\mathrm{C}}_{\mathrm{L}}+\left(\sigma_{\mathrm{a}}+\sigma_{\mathrm{b}}\right) / 2=\rho\left[\mathrm{V}_{\mathrm{L}}{ }^{2}\left(0^{\circ}\right)+\mathrm{V}_{\mathrm{L}}{ }^{2}\left(90^{\circ}\right)\right] / 2 \\
& \bar{\alpha} \overline{\mathrm{C}}_{\mathrm{L}}=\rho\left[\mathrm{V}_{\mathrm{L}}{ }^{2}\left(0^{\circ}\right)-\mathrm{V}_{\mathrm{L}}{ }^{2}\left(90^{\circ}\right)\right]-\left(\sigma_{\mathrm{a}}-\sigma_{\mathrm{b}}\right) \cos 2 \Omega \\
& \overline{\mathrm{C}}_{\mathrm{T}}+\left(\sigma_{\mathrm{a}}+\sigma_{\mathrm{b}}\right) / 2=\rho\left[\mathrm{V}_{\mathrm{T}}{ }^{2}\left(0^{\circ}\right)+\mathrm{V}_{\mathrm{T}}{ }^{2}\left(90^{\circ}\right)\right] / 2 \\
& \left.\bar{\beta} \overline{\mathrm{C}}-\frac{\bar{\alpha}^{2}}{\mathrm{~T}} \frac{\overline{\mathrm{C}}_{\mathrm{L}}{ }^{2}}{\left.\overline{(\overline{\mathrm{C}}}-\overline{\mathrm{C}}_{\mathrm{T}}\right)}=\rho\left[\mathrm{V}_{\mathrm{T}}{ }^{2}\left(45^{\circ}\right)-\mathrm{V}_{\mathrm{T}}{ }^{2}\left(0^{\circ}\right)\right]+\left[\sigma_{\mathrm{a}}-\sigma_{\mathrm{b}}\right) / 2\right][\cos 2 \Omega-\sin 2 \Omega]
\end{aligned}
$$

The stress terms on the left-hand side of equations (7a) and (7c) will generally be small with respect to $\overline{\mathrm{C}}_{\mathrm{L}}$ and $\overline{\mathrm{C}}_{\mathrm{T}}$ and can be neglected. However, those on the right-hand side must be retained as they appear in conjunction with the difference of two numbers, which could be of comparable magnitude. It should also be noted that, in general, $\bar{\alpha}, \bar{B}, \bar{C}_{T}$, and $\bar{C}_{L}$ are themselves weakly modified by stress through the stress dependence of the $\bar{C}_{i j}$. In general, these are small effects and will be neglected hereafter. In many applications, determination of texture would be done in the unstressed state and the above considerations are unnecessary. Finally, the term proportional to $\alpha^{2}$ in equation (7d) can be dropped for modest textures. The result is the simplified system

$$
\begin{aligned}
& \mathrm{C}_{\mathrm{L}} \cong \rho\left[\mathrm{V}_{\mathrm{L}}{ }^{2}\left(0^{\circ}\right)+\mathrm{V}_{\mathrm{L}}{ }^{2}\left(90^{\circ}\right)\right] / 2 \\
& \mathrm{C}_{\mathrm{T}} \cong \rho\left[\mathrm{V}_{\mathrm{T}}{ }^{2}\left(0^{\circ}\right)+\mathrm{V}_{\mathrm{T}}{ }^{2}\left(90^{\circ}\right)\right] / 2 \\
& \alpha_{\mathrm{L}} \cong \rho\left[\mathrm{V}_{\mathrm{L}}{ }^{2}\left(0^{\circ}\right)-\mathrm{V}_{\mathrm{L}}{ }^{2}\left(90^{\circ}\right)\right]-\left(\sigma_{\mathrm{a}}-\sigma_{\mathrm{b}}\right) \cos 2 \Omega \\
& \mathrm{BC}_{\mathrm{T}} \cong \rho\left[\mathrm{V}_{\mathrm{T}}{ }^{2}\left(45^{\circ}\right)-\mathrm{V}_{\mathrm{T}}{ }^{2}\left(0^{\circ}\right)\right]+\left[\left(\sigma_{\mathrm{a}}-\sigma_{\mathrm{b}}\right) / 2\right][\cos 2 \Omega-\sin 2 \Omega],
\end{aligned}
$$

which can readily be solved for $C_{L}, C_{T}, \alpha$, and $\beta$.

The coefficients of the CODF expansion are finally obtained from the relation ship between $\alpha, \beta, C_{\mathrm{L}}$, and $C_{T}$ and the coefficients $W_{\ell m n}$ as defined by the CODF expansion (refs. 2, 14)

$$
W(\xi, \psi, \Phi)=\sum_{\ell=0}^{\infty} \underset{m=-\ell}{\ell} \underset{n=-\ell}{\ell} W_{\ell m n} Z(\xi) e^{-i m \psi} e^{-i n \Phi}
$$


where $W$ is the CODF expressed in terms of the Euler angles $\xi, \psi, \Phi$ and $z_{\text {emn }}$ are generalized Legendre functions. Sayers and Allen have derived the relationship between the ultrasonic velocities and the $W_{\text {mn }}$ using the method of Voigt to compute polycrystalline averages (ref. 2). Following Bunge (ref. 15), they conclude that only three independent coefficients enter in the calculation of elastic constants for cubic crystallites, $W_{400}, W_{420}$, and $W_{440^{\circ}}$ One additional coefficient, $W_{000}$, is determined by normalization. In texture analysis, the preferred direction in equation (9) is generally taken to be the rolling direction, which leads to a different axis identification than that shown in figure 1 and used throughout the rest of this paper. After rotation to such a system, as employed by Sayers and Allen (ref. 2), in which the 1, 2, and 3 axes correspond respectively to the normal, transverse, and rolling directlons, one concludes

$$
\begin{aligned}
\rho\left[\mathrm{V}_{\mathrm{L}}{ }^{2}\left(0^{\circ}\right)\right. & \left.+\mathrm{V}_{\mathrm{L}}{ }^{2}\left(90^{\circ}\right)\right] / 2=\mathrm{C}_{11^{\circ}}-2 \mathrm{C}^{\circ}\left[\frac{1}{5}-\frac{11}{35} \sqrt{2} \pi^{2} \mathrm{~W}_{400}\right. \\
& \left.-\frac{4}{35} \sqrt{5} \pi^{2} \mathrm{~W}_{420}-\frac{2}{35} \sqrt{35} \pi^{2} \mathrm{~W}_{440}\right] \\
\rho\left[\mathrm{V}_{\mathrm{T}}{ }^{2}\left(0^{\circ}\right)\right. & \left.+\mathrm{V}_{\mathrm{T}}{ }^{2}\left(90^{\circ}\right)\right] / 2=\mathrm{C}_{44^{\circ}}+\mathrm{C}^{\circ}\left[\frac{1}{5}-\frac{16}{35} \sqrt{2} \pi^{2} \mathrm{~W}_{400}-\frac{16}{35} \sqrt{5} \pi^{2} \mathrm{~W}_{420}\right] \\
\rho\left[\mathrm{V}_{\mathrm{L}}{ }^{2}\left(0^{\circ}\right)\right. & \left.-\mathrm{V}_{\mathrm{L}}{ }^{2}\left(90^{\circ}\right)\right]-\left(\sigma_{\mathrm{a}}-\sigma_{\mathrm{b}}\right) \cos 2 \Omega \\
& =4 \mathrm{C}^{\circ}\left[\frac{5}{35} \sqrt{2} \pi^{2} \mathrm{~W}_{400}-\frac{4}{35} \sqrt{5} \pi^{2} \mathrm{~W}_{420}-\frac{2}{35} \sqrt{35} \pi^{2} \mathrm{~W}_{440}\right] \\
\rho\left[\mathrm{V}_{\mathrm{T}}{ }^{2}\left(45^{\circ}\right)\right. & \left.-\mathrm{V}_{\mathrm{T}}{ }^{2}\left(0^{\circ}\right)\right]+\left[\left(\sigma_{\mathrm{a}}-\sigma_{\mathrm{b}}\right) / 2\right][\cos 2 \Omega-\sin 2 \Omega]=\mathrm{C}^{\circ}\left[\sqrt{2} \pi^{2} \mathrm{~W}_{400}\right. \\
& \left.+\frac{28}{35} \sqrt{5} \pi^{2} \mathrm{~W}_{420}+\frac{2}{35} \sqrt{35} \pi^{2} \mathrm{~W}_{440}\right]
\end{aligned}
$$

where $\mathrm{C}_{j 1^{\circ}}, \mathrm{C}_{44^{\circ}}$, and $\mathrm{C}_{12^{\circ}}$ are single crystal elastic constants and $\mathrm{C}^{\circ}=\mathrm{C}_{11^{\circ}}-\mathrm{C}_{12^{\circ}}$ - $2 \mathrm{C}_{44}$. The $\mathrm{W}_{4 m o}$ are defined in a coordinate system with the 3-axis along the rolling direction, in contrast to the elastic constants appearing in previous equations in this paper. However, the experimental observables on the left hand side of equation (10) are unambiguous.

This constitutes an overdetermined system, since there are four equations in the three unknowns, $W_{4 m o}$ Either experimental error or inaccuracies in the Voigt averaging scheme would render an exact solution of all equations impossible. A detailed analysis of the most stable data reduction scheme has not yet been conducted. However, it can be speculated that equations (10c) and (10d) should be weighed heavily since the experimental quantities are relative velocities, rather than the absolute velocities appearing in equations (10a) and (10b). It is also likely that equation ( $10 \mathrm{~b}$ ) will prove more accurate than equation (10a) because no correction of plate to plane wave velocities is required. Whereas such a correction may be quite satisfactory for relative measurements, it may be inadequate for absolute measurements. Thus a first attempt at reduction to practice might involve disregarding equation ( $10 \mathrm{a}$ ) and solving the three remaining systems simultaneously.

As a check, one should use the computed $W_{\ln }$ values to test the accuracy of the isotroplc approximation to the correction relating the velocities $V_{L_{L}}$ and $V_{S_{o}}$ as
given by equation (4). For larger textures, an iterative solution might be required In which first estimates of the $W^{\prime} s$ are used to recalculate the velocities $V_{L_{0}}\left(0^{\circ}\right)$ and $V_{L_{0}}\left(90^{\circ}\right)$ using equation (4) and the polycrystalline averaged elastic constant expressions (ref. 2) 


$$
\begin{aligned}
& C_{13}=C_{12}^{\circ}+C^{\circ}\left[\frac{1}{5}-\frac{16}{35} \sqrt{2} \pi^{2}\left(w_{400}-\sqrt{5 / 2} w_{420}\right)\right] \\
& C_{23}=C_{12}^{\circ}+C^{\circ}\left[\frac{1}{5}+\frac{4}{35} \sqrt{2} \pi^{2}\left(W_{400}-\sqrt{70} w_{440}\right)\right]
\end{aligned}
$$

Again, a mixed notation has been used in this equation. The elastic constants appearing on the left hand side of equation (11) are defined in the coordinated system of figure 1. The $W_{4 m o}$ are defined after Sayers and Allen (ref. 2) as discussed above. As expected, the deviations from the isotropic assumptions will become greater when either the single crystal anisotropy, $C^{\circ}$, or the degree of preferred orientation, as indicated by the $\mathrm{W}_{4 m o}$, are large.

The above describes one approach to deduce the coefficients of the CODF from the angular dependence of the $\mathrm{SH}_{\mathrm{o}}$ and $\mathrm{S}_{\mathrm{o}}$ plate mode velocities. Smith et al. (ref. 16) have discussed a closely related approach in which the velocities of higher order, horizontally polarized plate modes, $\mathrm{SH}_{n}$, are measured and used to predict the anisotropic elastic constants. The experimental parameters of that approach could be related to the $W_{l m n}$ using arguments similar to the one presented above.

\section{CONCLUDING REMARKS}

It has been shown that measurements of the angular dependence of the $\mathrm{SH}_{0}$ and $\mathrm{S}_{\mathrm{O}}$ ultrasonic modes of a plate are influenced by both stress and texture. Data reduction schemes are presented for making independent estimates of a) the orientations and difference of in-plane principal stresses and b) the coefficients $W_{400}$, $W_{420}$, and $W_{440}$ of the CODF expansion. Since the data can be obtained with couplant free, EMAT probes, there appears to be a high potential for in-line process control applications (ref. 17). Figure 6 sketches the required measurements. Since the use

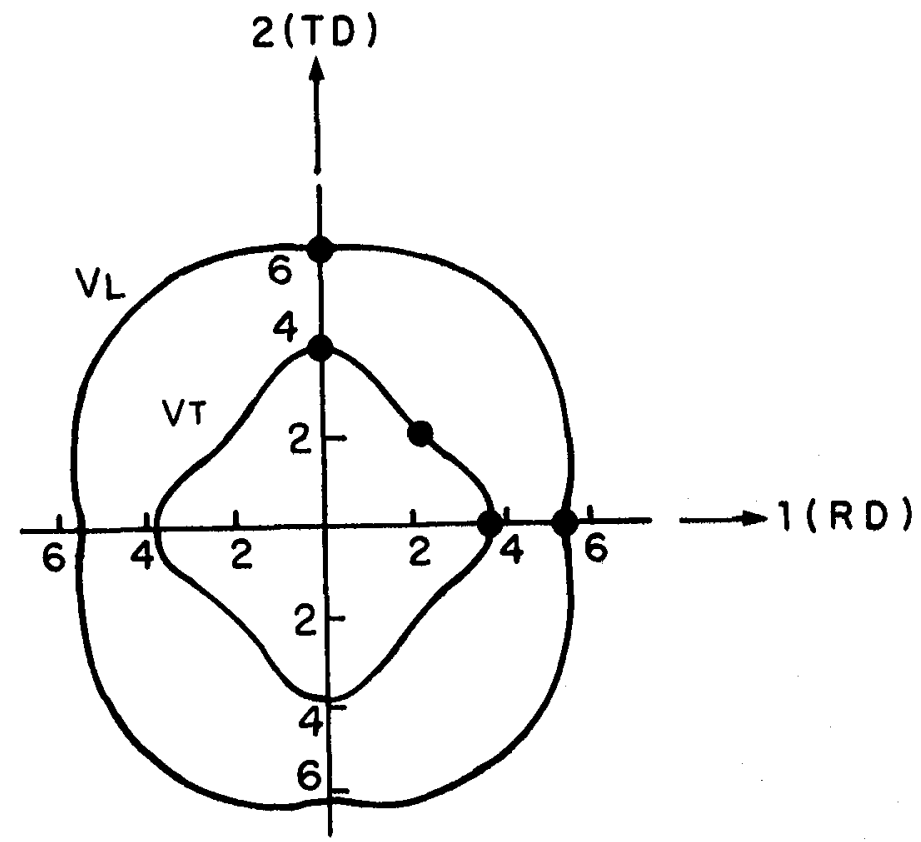

Fig. 6. Measurements required for nondestructive determination of stress and texture parameters. The solid lines schematically indicate the angular dependence of the velocities and the dots indicate the points at which data is required. 
of the CODF as a means of correlating texture with mechanical properties has shown considerable recent promise (ref. 18), such a technique might be of considerable value in process control.

\section{REFERENCES}

1. J. F. Nye: Physical Properties of Crystals, Oxford, 1957.

2. Sayers, C. M. and Allen, D. R. J.: Phys. D., vo1. 17, p. 1399.

3. B. A. Auld: Elastic Waves and Fields in Solids. .W1ley, NY, 1973.

4. Thompson, R. B., Lee, S. S., and Smith, J. F.: Review of Progress in Quant1tative Nondestructive Evaluation 3. D. 0. Thompson and D. E. Chimenti, Eds., Plenum Press, NY, 1984, p. 1311.

5. Thompson, R. B., Smith, J. F., Lee, S. S.: Review of Progress in Quantitative Nondestructive Evaluation 2. D. 0. Thompson and D. E. Chiment1, Eds., P1enum Press, NY, 1983, p. 1337.

6. Thompson, R. B., Smith, J. F., and Lee, S. S.: Appl. Phys. Lett., vol. 44, 1984, p. 296.

7. Thompson, R. B., Smith, J. F., and Lee, S. S.: Nondestructive Evaluation: Application to Materials Processing. O. Buck and S. M. Wolf, Eds., Amer. Soc. Metals, Metals Park, Ohio, 1984, p. 137.

8. Lee, S. S., Smith, J. F., and Thompson, R. B.: Evaluation of the Absolute Acoustoelastic Stress Measurement Technique. Review of Progress in Quantitative Nondestructive Evaluation 4, D. 0. Thompson and D. E. Chimenti, Eds., Plenum Press, NY, (in press).

9. Liu, Y. C., and Alers, G. A.: Trans. Met. Soc. AIME, vol. 236, 1966, p. 489.

10. Papadakis, E. P.: Trans. Met. Soc. AIME, vo1. 236, 1966, p. 1609.

11. Tittmann, B. R. and Alers, G. A.: Met. Trans., vo1. 3, 1972, p. 1307.

12. Thompson, R. B. and Alers, G. A.: Proc. 9th Symposium on Nondestructive Evaluation. South Texas Section of ASNT and Southwest Research Institute, San Antonio, 1973, p. 6 .

13. Thompson, R. B.: 1977 Ultrasontcs Sympostum Proceedings. IEEE, NY, 1977, p. 74 .

14. Roe, R. J.: J. Appl. Phys., vol. 36, 1965, p. 2024.

15. Bunge, H. J.: Krist. Tech., vol. 3, 1968, p. 431. 
16. Smith, J. F., Alers, G. A., Armstrong, P. E., and Eash, D. T.: Separation and Characterization of Stress Levels and Texture in Metal Sheet and Plate: I. Principles and Initlal Test. To be published in J. Nondestructive Eval.

17. Droney, B. E.: Nondestructive Methods for Material Property Determination. C. 0. Ruud and R. E. Green, Eds., Plenum Press, NY, 1984, p. 237.

18. Sowerby, R.: Deformation Textures in Some Non-Ferrous Meta1s. To be published in the proceedings of the symposium on texture of non-ferrous metals held as part of the 1984 Fa11 TMS-AIME Meeting, Sept. 16-20, 1984, Detroit, Michigan. 


\title{
EFFECT OF STRESS ON ULTRASONIC PULSES IN FIBER REINFORCED COMPOSITES
}

\author{
John H. Hemann \\ Cleveland State University \\ cleveland, ohto 44775 \\ George Y. Baaklint \\ National Aeronautics and Space Administration \\ Lewis Research Center \\ Cleveland, Oh10 44135
}

An Acoustical-Ultrasonic Technique was used to demonstrate that relationships exist between changes in attenuation of stress waves and tensile stress for an 8-p1y "0" degree graphite-epoxy fiber reinforced composite. A11 tests were conducted in the linear range of the material for which no mechanical or macroscopic damage was evident. Changes in attenuation were measured as a function of tensile stress in the frequency domain arid in the time domain. Stress wave propagation in these specimens was dispersive, i.e., the wave speed depends on frequency. Wave speeds varied from $2700 \mathrm{~m} / \mathrm{sec}$ to $6800 \mathrm{~m} / \mathrm{sec}$ as the frequency of the signal was varied from $150 \mathrm{KHZ}$ to $1.9 \mathrm{MHZ}$ which strongly suggests that flexural/Lamb wave modes of propagation exist. The magnitude of the attenuation changes depended strongly on tensile stress. It was further observed that the wave speeds increased slightly for all tested frequencies as the stress was increased.

\section{INTRODUCTION}

Non-Destructive Evaluation (NDE) techniques are being widely used today to characterize the physical state of solid materials. Vary, et.al., (refs. 1-6) have demonstrated that correlations do exist between ultrasonic measures and mechanical properties of metals, ceramics, and fiber composites. Williams, et.al., (refs. 7,8 ) have shown that ultrasonic attenuation is an indicator of fatigue iife for graphite-epoxy composites. UTtrasonic measurements in the area of composite materials have provided a nondestructive means for measuring variations in strength related properties, predicting interlaminar shear strength of fiber composite laminates, and ranking composite structures according to strength.

This research presents evidence on how well (NDE) techniques describe stress related effects. An ultrasonic attenuation study of a stress wave propagating through a "0" degree graphite-epoxy fiber reinforced composite specimen subjected to tensile loads was performed. This paper will provide not only qualitative data, but also quantitative data, showing the relationships that exist between changes in attenuation of stress waves and tensile stress for the 8-ply "0" degree specimen under consideration. Tensile testing was done in the linear range of the material, where linearity is defined by constant slope of the stress-strain curve in the range of the test conducted. Wave speeds were also measured showing that stress wave propagation is dispersive.

*This research was supported by a grant from the NASA-Lewis Research Center, NAG3-106. 
The demonstrated conclusions are:

1. Dispersion does occur, i.e., wave speeds are frequency dependent.

2. The attenuation is strongly dependent on tensile stress.

3. The wave speed is weakly dependent on tensile stress.

\section{EXPERIMENTAL PROCEDURE}

\section{Material}

The specimen used was an eight-ply "0" degree angle-ply graphite-epoxy fiber reinforced composite. An AS graphite fiber and a PR-288 exposy resin have been used to construct an AS/PR-288 preimpregnated fiber-resin ply. The fibers were coated with a polyvinyl alcohol to enhance fiber-matrix interface strength properties. A schematic of the unidirectional composite is shown in Figure 1. The physical properties of the specimen have been determined by Vary, et.al., (ref. 3).

\section{Apparatus}

A schematic of the specimen and transducers in uTtrasonic through transmission testing was shown in Figure 2. Broadband transducers of various center frequencies were used to gather all of the data over the various frequencies.

Aluminum semicylindrical buffers of $3.157 \mathrm{~mm}(.125 \mathrm{in})$ diameter were attached to the transducers in order to:

a. Provide a 7 ine contact of length $12.7 \mathrm{~mm}$ (.5 in) which is the width of the specimen.

b. Prevent bending from occurring as pressure was applied to the transducer holders.

c. Place the sending and the receiving transducer at specified location.

Strain gages were mounted on the specimen on both faces to measure the axial and transverse strains. Strain gages were 00 and 900 orthogonal back-to-back. Also strain gages were installed onto the transducer holders to measure the pressure applied to the transducers. Williams, et.al., (ref. 8) defined and measured a "saturation pressure" which is required for reproducible coupling of transducer to specimen; this pressure was monitored by the strain-gaged transducer holders.

An UTtragel II ultrasonic couplant was used between the aluminum buffer and the specimen. A very thin coating of couplant was used where effects of thickness of the couplant were neglected. A felt material was used on the backside of the transducer holder to insure that energy did not enter the transducer holder from the specimen.

The ultrasonic equipment and the tensile testing system are schematically shown in Figure 3. 


\section{Experimental Loading}

An ultrasonic wave was transmitted into the specimen by a sending transducer. Two inches away in the longitudinal direction, and on the same face, a receiving transducer was placed to sense the stress wave energy traversing the specimen. The receiving transducer output was displayed on the oscilloscope for real time analysis and on the spectrum analyser for frequency analysis.

The specimen, mounted in a tensile machine where care was taken to minimize off-axis loading or twisting, was pulled at an initial loading rate of approximately $17 \mathrm{\mu m} / \mathrm{sec}(.04 \mathrm{in} / \mathrm{min})$ to set the grips. After this initial loading to about $445 \mathrm{~N}$ (100 1bf) which was the reference load for attenuation measurements and change in attenuation measurements, further loading was done at mean rate of $4.2 \mu \mathrm{m} / \mathrm{sec}$ $(.01 \mathrm{in} / \mathrm{min})$.

The load was increased to $3560 \mathrm{~N}$ ( $800 \mathrm{lbf}$ ), 6675N (1500 $\mathrm{lbf}$ ), and in some experiments to $13350 \mathrm{~N}$ ( $3000 \mathrm{lbf}$ ) successively. Then the load was decreased to $6675 \mathrm{~N}, 3560 \mathrm{~N}$, and $445 \mathrm{~N}$. At the same time strain-load data was generated, photographs of the stress wave output and the frequency output were taken.

The loading-unloading cycle was repeated five to seven times for each setting of independent variables; the test usually was completed within a two-hour period. The number of cycles that were required was dictated by the reproducability and stability of the attenuation changes.

Typical output signals are shown in Figure 4 and Figure 5 for an entire loading-unloading cycle.

\section{RESULTS}

In this experimental research many observations were made concerning how various parameters effected the Uitrasonic output; only a few were examined and are presented in this paper because of time, equipment, and experimental system limitations. This chapter will present the dispersion phenomenon and the wave type, the attenuation as related to tensile load, and the wave speed as related to tensile load; the specimen was "0" degree graphtte-epoxy fiber reinforced composite.

\section{Dispersion}

Dispersion is understood to mean that wave speeds are frequency dependent. From the data collected and the measurements taken, a graph shown in Figure 6 was plotted showing speed versus frequency. The wave speed was determined by measuring the delay time between the output wave front and input wave front over a fixed distance. The graph shows that the wave velocity along the longitudinal direction is frequency dependent. Wave speeds varied from $2.7 \times 10^{5} \mathrm{~cm} / \mathrm{sec}$ to $6.8 \times 10^{5} \mathrm{~cm} / \mathrm{sec}$ as the frequency of the signal was varied from $150 \mathrm{KHZ}$ to $1.9 \mathrm{MHZ}$.

There are two types of dispersion. First is viscoelastic dispersion due to viscoelastic properties of the material. Second is a geometric dispersion due to the dimensions of the material. Williams (ref. 8) demonstrated that there is no viscoelastic dispersion in the graphite-epoxy composite for longitudinal and shear 
wave velocities in the range tested. Shear wave velocities were tested in the range of 0.48 to $3.0 \mathrm{MHZ}$ and longitudinal wave velocities were tested in the range of $0.225 \mathrm{MHZ}$ to $5 \mathrm{MHZ}$. The frequency range for the results presented in this paper is 0.15 to $1.9 \mathrm{MHZ}$, which is very close to the range of Williams' result.

For the wave propagating in the $x_{1}$ direction and having a particle motion in the $x_{7}$ direction, Williams (ref. 8) measured the longitudinal wave speed and found it to be 8.5 to $10^{5} \mathrm{~cm} / \mathrm{sec}$. This wave speed $C_{L}$ is plotted in Figure 6 and is used as a comparison since the material used in williams' research is very similar to the material used in the research reported here.

By comparing $C_{L}$ to the velocities obtained in this experiment as shown in Figure 6 , one can see that the wave velocities measured were not longitudinal wave velocities. The sending transducer inputs a longitudinal wave perpendicular to specimen which, because of many reflections, becomes a wave propagating along the length of the specimen. During this propagation shear waves are formed due to the reflections and interact with longitudinal waves. This interaction of waves propagating along a thin specimen produces Lamb waves (ref. 9). Dispersion does occur in the propagation of flexural/Lamb waves (ref. 10).

Lamb wave propagation may exist in a multimode state at a given frequency producing a finite number of wave speeds to exist simultaneously. Because of experimental equipment limitations, this research could not define which mode (fig. 6) and how many modes were superimposed upon each other, but this paper recognized the possible existence of different modes simultaneously in the stress wave output.

\section{Attenuation vs. Tensile Stress}

The relationship between attenuation and tensile stress was investigated by measuring the pulse amplitude change in the time domain and in the frequency domain. The tensile load applied to the specimen was in the linear range of the material where linearity is demonstrated by the constant slope of the stress-strain curve shown in Figure 7. Percent change in amplitude vs. tensile load in the frequency domain is shown in Fiugre 8 for each of the six frequencies used in the experiment. At $150,180,250$, and $1900 \mathrm{KHZ}$, a negative attenuation with increasing load is observed whereas at 850 and $1700 \mathrm{KHZ}$ the attenuation is positive. The slope of these lines remains constant with cycling, but a translation of the lines occurs in the first few cycles, after which the lines stabilize for subsequent cycles. The data needed to plot these lines come from direct measurements of amplitude changes from photographs of which Figure 4 is typical.

Figure 9 shows percent change in amplitude vs. tensile load in the time domain. At $150,180,250$, and $1900 \mathrm{KHZ}$ a negative attenuation with increasing load is observed whereas at $850 \mathrm{KHZ}$ a positive attenuation occurs. The slopes and translation of these lines with cycling behaves similarly to the frequency lines. Theoretically Figures 8 and 9 should be identical, but as one can see from Figure 5 the energy response in the time domain is stretched out into many small immeasurable spikes. The data plotted in Figure 9 reflects only the main part of the pulse. The $1700 \mathrm{KHZ}$ response could not be clearly separated from the $1900 \mathrm{KHZ}$ response in the time domain and hence was not plotted.

The data shown in Figures 8 and 9 behave in a generally similar manner. The 
data clearly indicates that the attenuation, both positive and negative, of the ultrasonic pulse depends on tensile stress.

\author{
Wave Speed vs. Tensile Load
}

The data at all frequencies clearly shows that the wave speed increases with increasing stress. This can be clearly seen in Figure 5 where all pulses are translated to the left (decreasing arrival time) with increasing load. The data showed wave speed changes as large as $8 \%$ at stress levels $30 \%$ of the ultimate stress.

\title{
CONCLUSION
}

It has been shown that dispersion did occur, i.e., wave speeds were frequency dependent. Moreover, it was found that flexural/Lamb waves existed, and a superposition of different modes existed in the stress wave output of the signal.

It has been proven that attenuation depended strongly on tensile stress. Also it was observed that attenuation was frequency dependent. load.

Finally, it was demonstrated that wave speeds were weakly dependent on tensile

Further research is needed using a pulsing system producing narrow band frequencies since all effects measured were frequency dependent.

\section{REFERENCES}

1. Vary, A., and Bowles, K. J.: Ultrasonic Evaluation of Strength of Unidirectional Graphite-Polyimide Composites. NASA Technical Memorandum TM-73646, April 1977.

2. Vary, A., and Bowles, K. J.: Use of an Ultrasonic-Acoustic Technique for Nondestructive Evaluation of Fiber Composite Strength. NASA Technical Memorandum TM-73813, February 1978.

3. Vary, A., and Lark, R. F.: Correlation of Fiber Composite Tensile Strength with the UTtrasonic Stress Wave Factor. NASA Technical Memorandum TM-78846, April 1978.

4. Vary, A.: Correlations Among UItrasonic Propagation Factors and Fracture Toughness Properties of Metallic Materials. Materials Evaluation, Vol. 36, No. 7 , June 1978, pp. 55-64.

5. Vary, A.: Quantitative U1trasonic Evaluation of Mechanical Properties of Engineering Materials. NASA Technical Memorandum TM-78905, June 1978.

6. Vary, A.: Correlations Between UTtrasonic and Fracture Toughness Factors in Metallic Materials. NASA Technical Memorandum TM-73805, June 1978. 
7. Williams, Jr. J. H., and Do11, B.: U1trasonic Attenuation as an Indicator of Fatigue Life of Graphite/Epoxy Composites. NASA CR-3179, 1979.

8. Williams, Jr. J. H., Nayeb-Hashemi, H., and Lee, S. S.: UTtrasonic Attenuation and Velocity in AS/3501-G Graphite/Epoxy Fiber Composite, NASA Contractor Report 3180, December 1979.

9. Lamb, H.: On Waves in an Elastic Plate. Proc. Rog. Soc. A.93, 114-28 (1917).

10. Meeker, T. R., and Meitzler, A. H.: Guided Wave Propagation in Elongated Cylinders and Plates. Physical Acoustics, edited by Mason, Warren P., Academic Press, New York and London, 1964. Part A, pp. 112-166. 


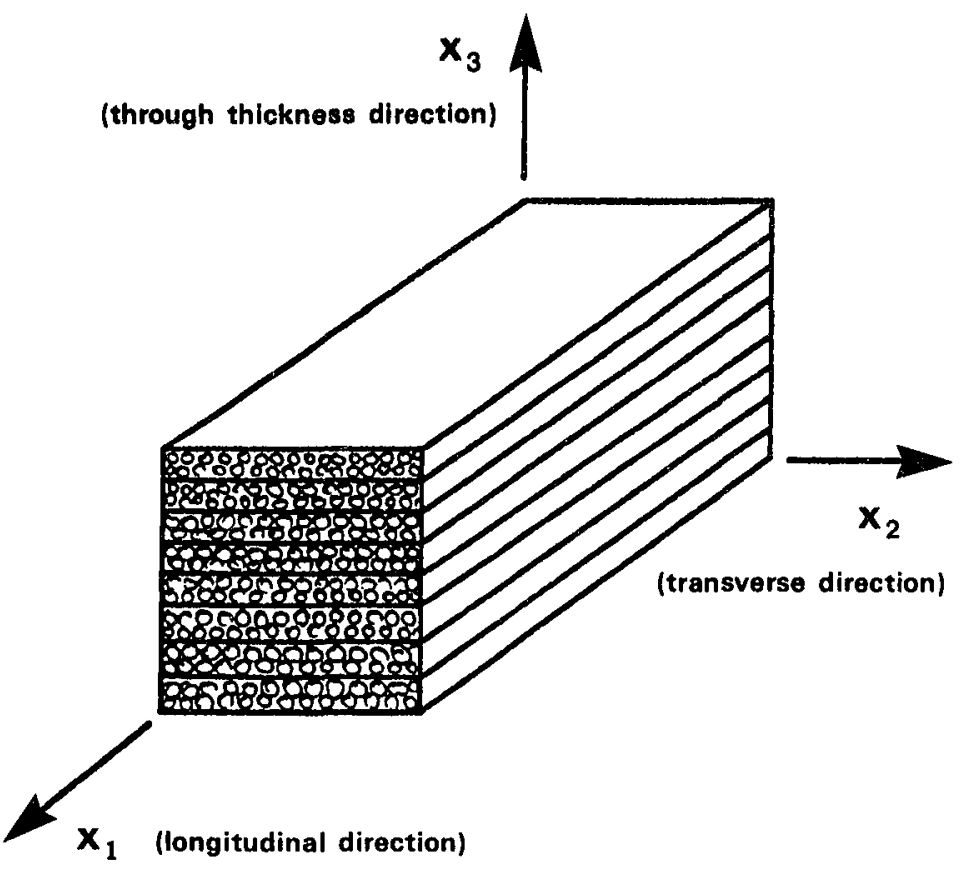

Figure 1. Principal directions of the " 0 " degree angle-ply specimen

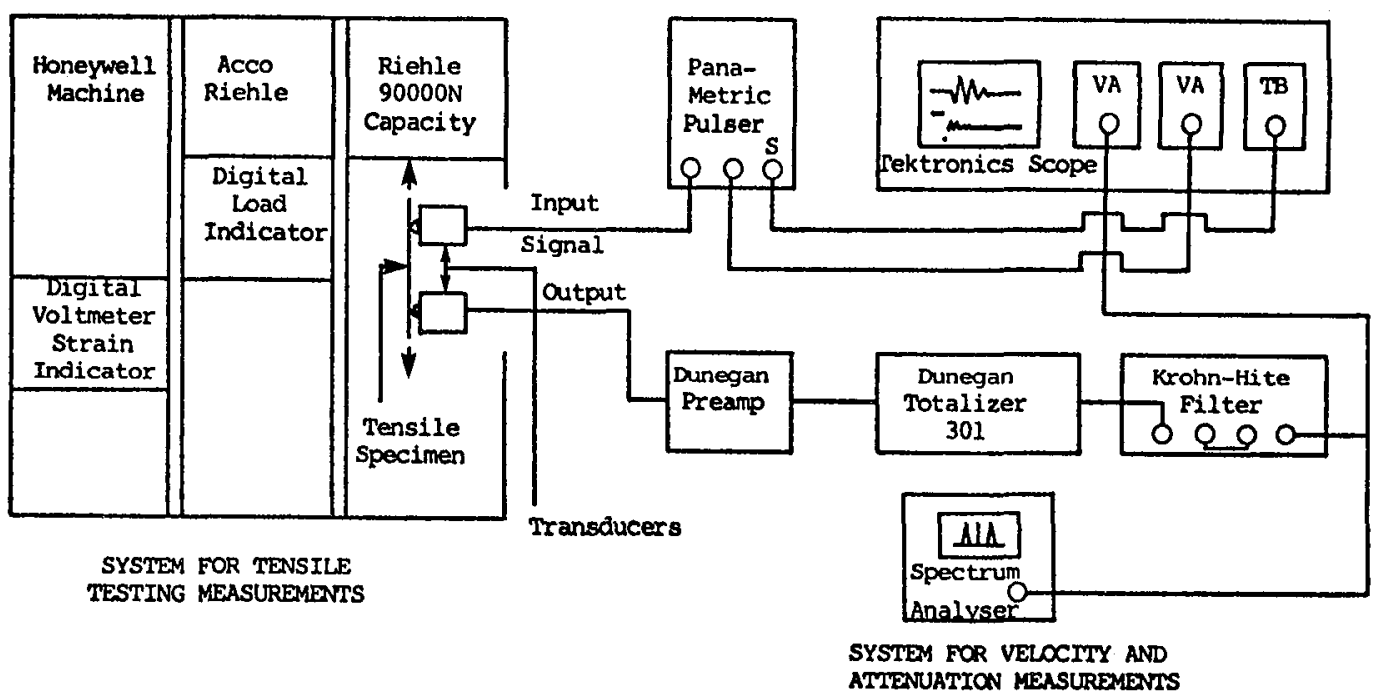

Figure 3. Experimental system

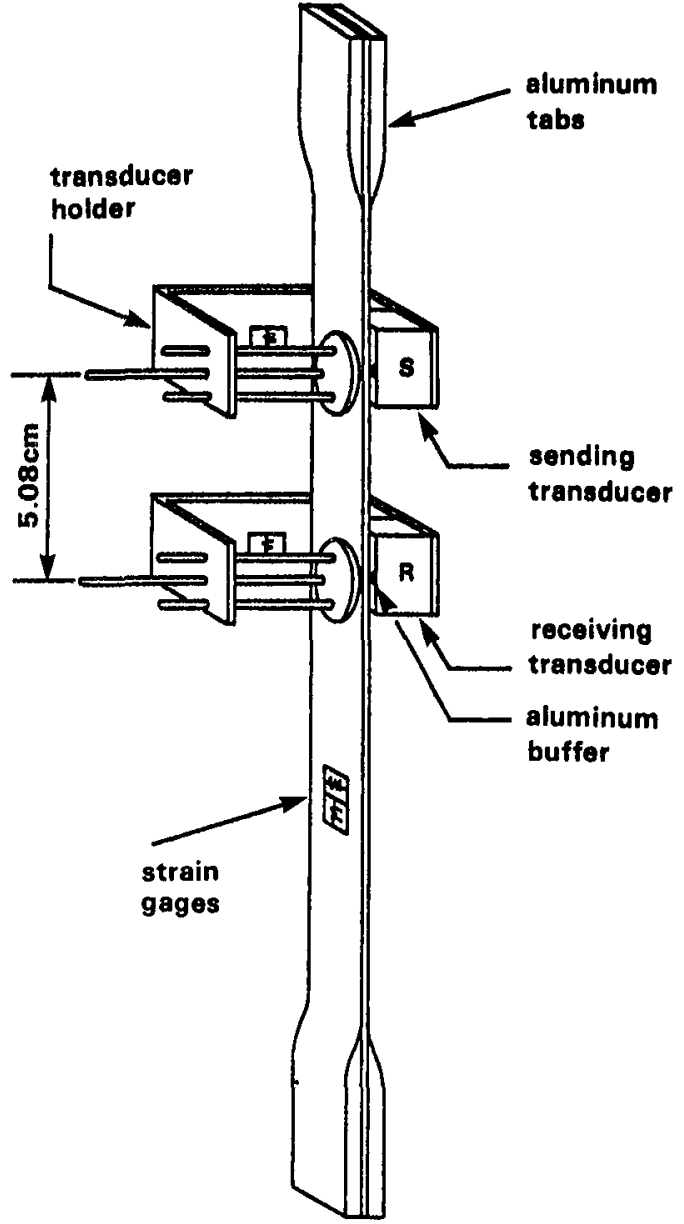

Figure 2. Schematic diagram specimen and transducers 


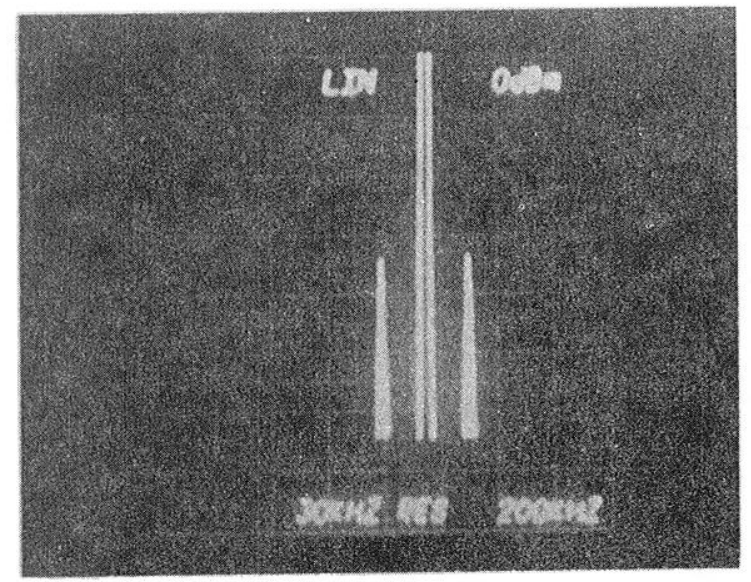

a. $507 \mathrm{~N}$

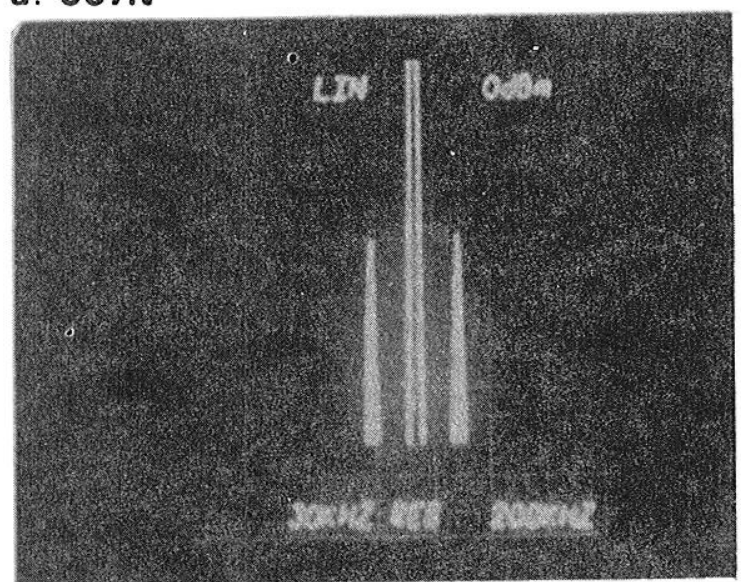

c. $6826 \mathrm{~N}$

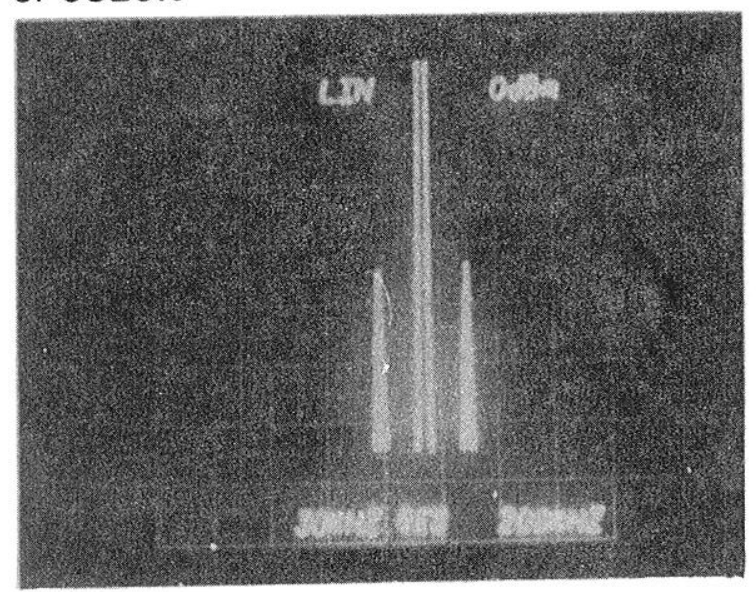

e. $525 \mathrm{~N}$

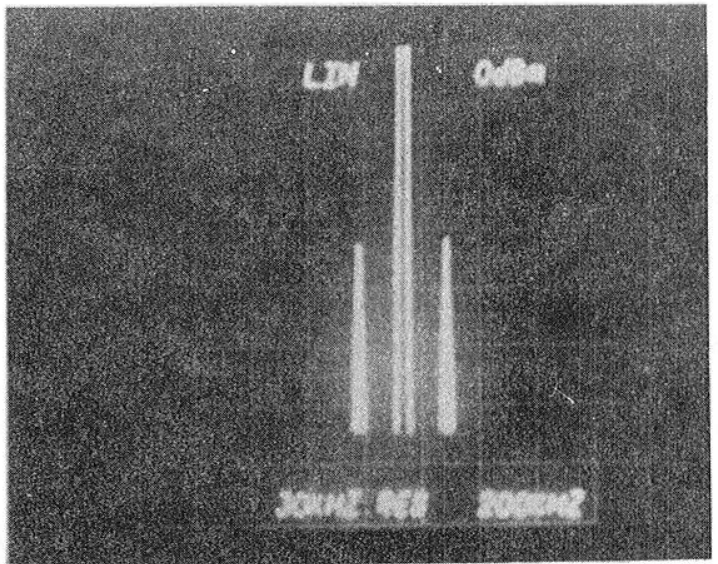

\section{b. $3658 \mathrm{~N}$}

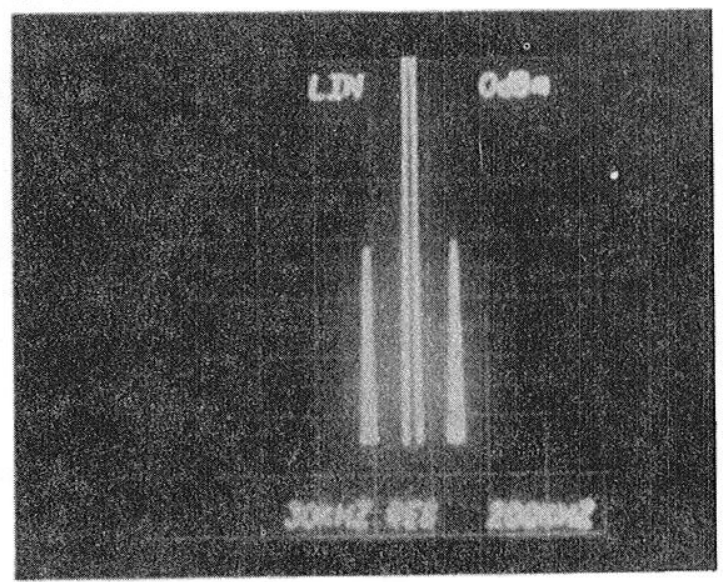

d. $3617 \mathrm{~N}$

Figure 4. - U1trasonic puTse attenuation versus load of $150 \mathrm{kHz}$ in frequency domai 


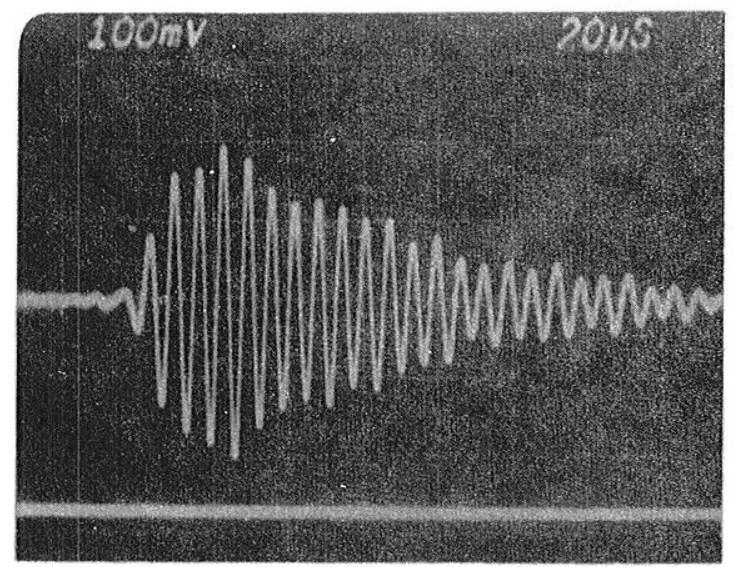

a. $507 \mathrm{~N}$

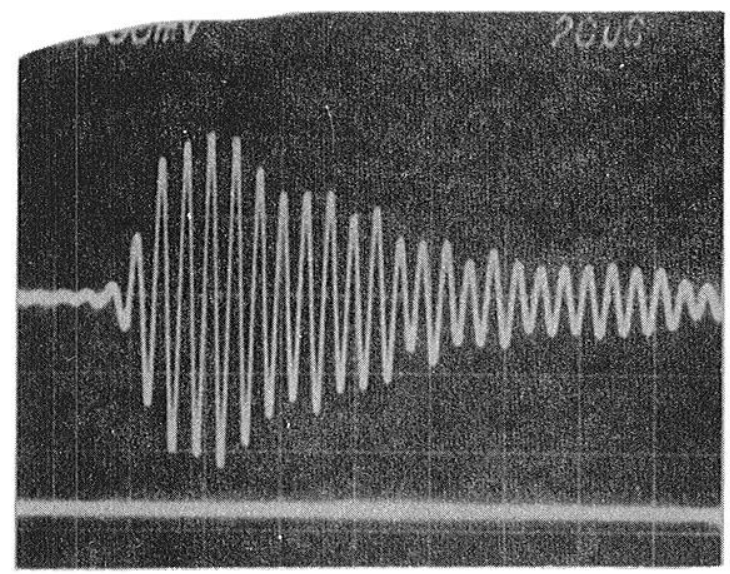

c. $6826 \mathrm{~N}$

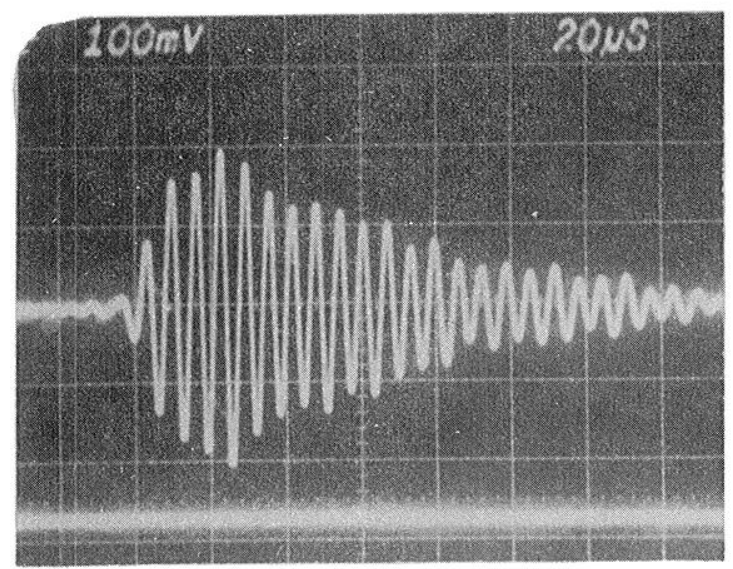

e. $525 N$

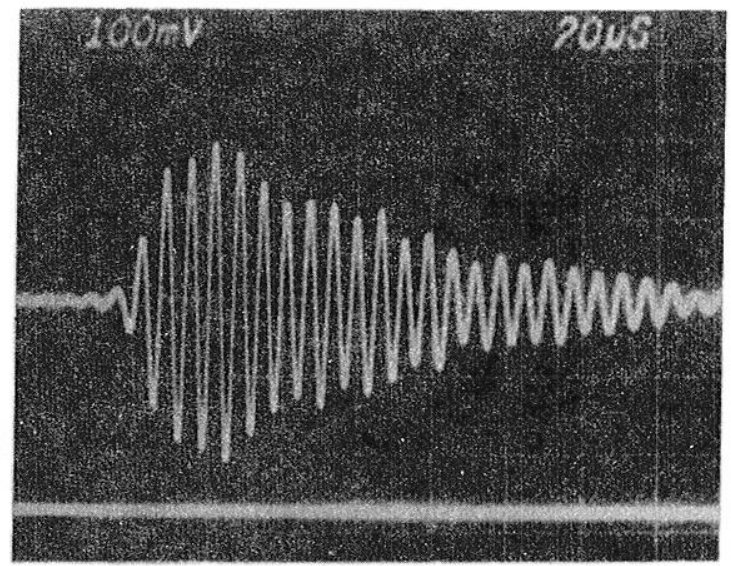

b. $3658 \mathrm{~N}$

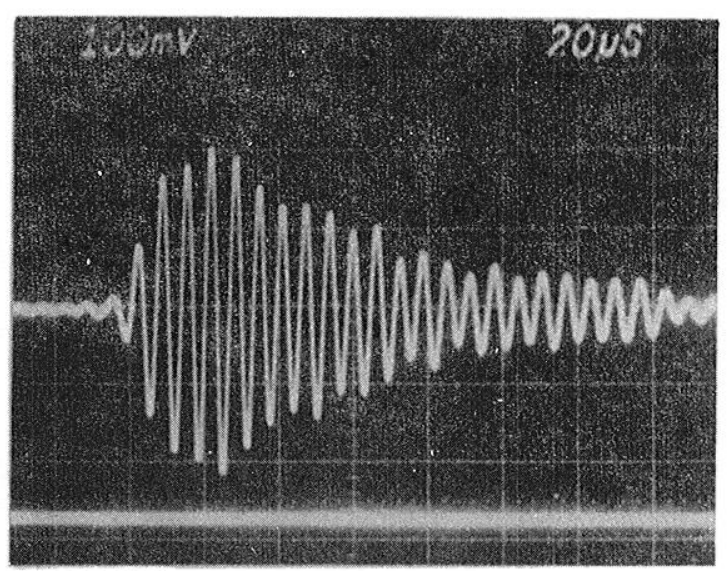

d. $3617 \mathrm{~N}$

Figure 5. - U1trasonic pulse attenuation versus load of $150 \mathrm{kHz}$ in time domain. 


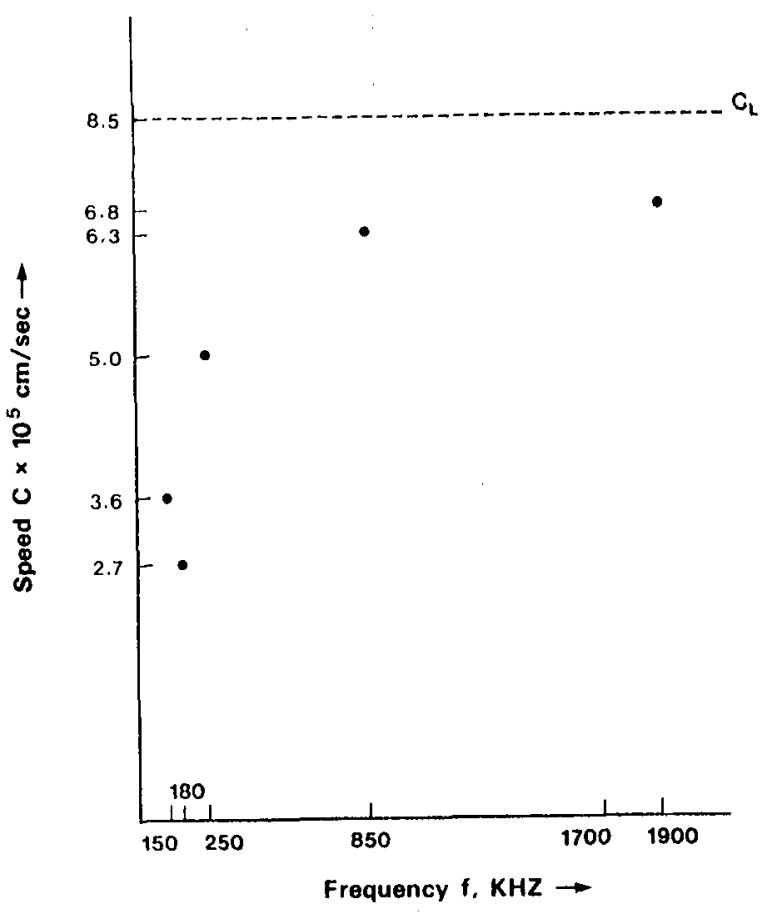

Figure 6. Wave speed vs. frequency

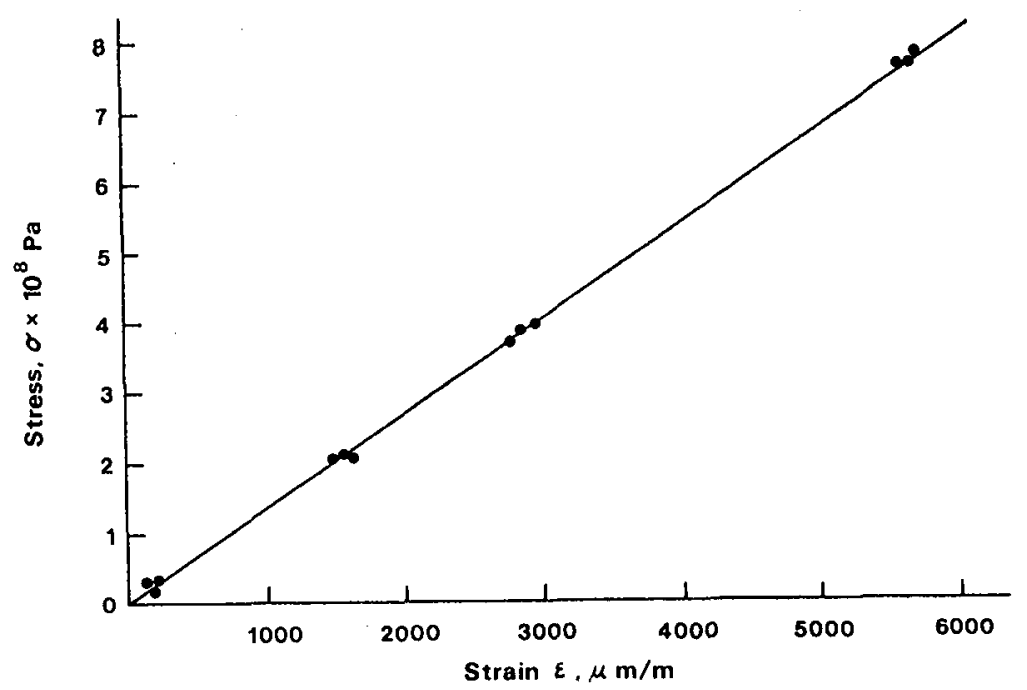

Figure 7. Axial stress vs. axial strain 


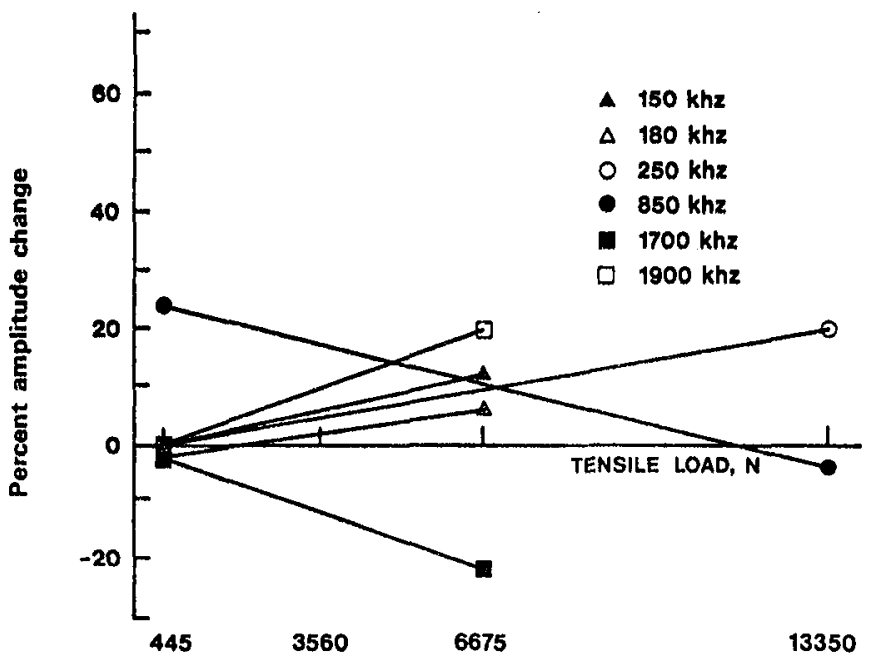

Figure 8. Percent change in amplitude vs.

tensile load in the frequency domain

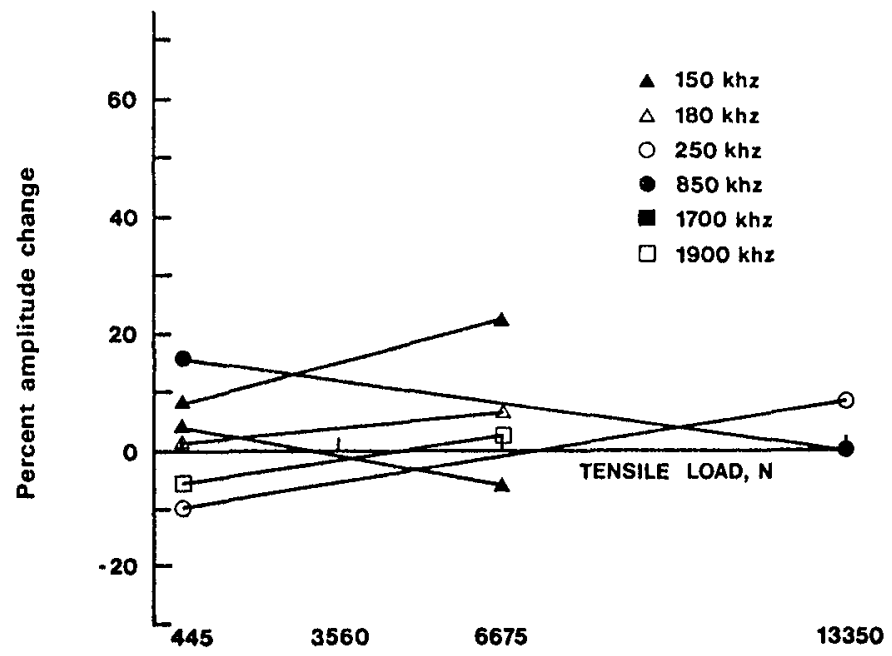

Figure 9. Percent change in amplitude vs. tensile load in the time domain 

PATTERN RECOGNITION CHARACTERIZATIONS OF MICROMECHANICAL AND MORPHOLOGICAL MATERIALS STATES VIA ANALYTICAL QUANTITATIVE ULTRASONICS

\author{
James H. Williams, Jr., and Samson S. Lee \\ Massachusetts Institute of Technology \\ Cambridge, Massachusetts 02139
}

\begin{abstract}
If an ultrasonic signal is introduced into or generated within a structure, the state of the structure governs its propagation and detection. The assessment of the state of the structure can be affected by material properties, geometrical properties, environmental conditions, and measurement conditions. Because of the large number of possible properties and conditions, the quantitative ultrasonic determination of a specific microstructural or morphological state, independent of all other states, is difficult.
\end{abstract}

To complicate matters further, many nondestructive evaluation (NDE) parameters can be measured via ultrasonic interrogation as specified frequencies by using the Fourier transform (e.g., signal amplitude, signal duration, stress wave factor, and signal strength). Thus a large amount of data can be generated from a single ultrasonic measurement. Large multivariate data sets are difficult to decipher; thus, methods of summarizing and extracting relevant information are necessary.

One potential approach to the quantitative acquisition of discriminatory information that can isolate a single structural state is pattern recognition. The pattern recognition characterizations of micromechanical and morphological materials states via analytical quantitative ultrasonics are outlined in this paper. The concepts, terminology, and techniques of statistical pattern recognition are reviewed. Feature extraction and classification and states of the structure can be determined via a program of ultrasonic data generation.

\title{
INTRODUCTION
}

In acoustic-ultrasonic nondestructive evaluation (NDE), an ultrasonic stress wave is introduced into, or generated within, the interrogated structure and detected after it has propagated through the structure. Stress wave propagation is affected by the micromechanical and morphological materials states of the medium of propagation. Thus, acoustic-ultrasonic NDE involves the characterization of the tested structure on the basis of information contained in the detected stress wave signal.

The state of the structure, which governs stress wave propagation and detection, can be described by a broad range of properties and conditions, some of which are

Material properties:

Geometrical properties: elastic modulus, density, attenuation, velocity,...

structural dimensions, discontinuities, microstructural and microstructural defect states, microstructural characteristic dimensions,... 
Environmental conditions: mechanical loading, structural boundary conditions, residual stresses, temperature, absorbed

moisture,...

Measurement conditions: location and size of transducers, sensitivity and frequency response of transducers, couplant, dynamic characteristics of electronic equipment,...

Even from this incomplete list, it is clear that the quantitative ultrasonic determination of a specific microstructural or morphological state, independent of all other states, is difficult.

To complicate matters further, many NDE parameters can be measured from an ultrasonic interrogation at specified frequencies by using the Fourier transform. A few of these are the maximum signal amplitude, signal duration, stress wave factor, and signal strength. Thus, a large amount of data can be generated from a single ultrasonic measurement. Large multivariate data sets are difficult to decipher; thus, methods of summarizing and extracting relevant information are necessary. Most often the summarizing and extraction are accomplished in an ad hoc qualitative manner.

One approach for the quantitative acquisition of discriminatory information that can often isolate a single structural state is pattern recognition. The objective of this study was to outline an approach for pattern recognition characterizations of micromechanical and morphological materials states via analytical quantitative ultrasonics. The concepts, terminology, and techniques of statistical pattern recognition are reviewed.

\section{CLASSIFICATION BY PATTERN RECOGNITION}

Determining the state of a sample via NDE by using pattern recognition techniques consists of three basic steps:

(1) Generating and processing NDE data

(2) Selecting significant features of the data

(3) Determining the sample state from the selected features

These three steps are illustrated in figure 1 as data generation, feature extraction, and classification, respectively.

\section{DATA GENERATION}

Data generation consists of ultrasonic NDE measurements that are expected to contain information for identifying the micromechanical and morphological states of a material or structure. Data generation may also involve data processing. Data processing involves signal conditioning or transformation of the collected data into various representations; an example of the latter is the acquisition of the frequency representation of a signal via its fourier transform.

The processed data are arranged in an ordered set called a pattern vector $\underline{z}$ as (ref. 1) 


$$
\underline{z}=\left\{\begin{array}{l}
z_{1} \\
z_{2} \\
\cdot \\
z_{m}
\end{array}\right\}
$$

whose components $z_{1}, z_{2}, \ldots, z_{m}$ may contain, for example, the maximum signal amplitude, signal duration, and signal strength at specified frequencies evaluated via the Fourier transform. The number of components in the pattern vector is at the discretion of the researcher. Usually only a few known or anticipated discriminatory components are retained for pattern recognition analysis. These are preferentially selected (for subsequent correlation with structural states) by using the feature selection schemes described in the next section.

\section{FEATURE EXTRACTION}

A subset of the pattern vector in equation (1) is selected and is called the feature vector $\underline{x}$ (refs. 1 and 2 )

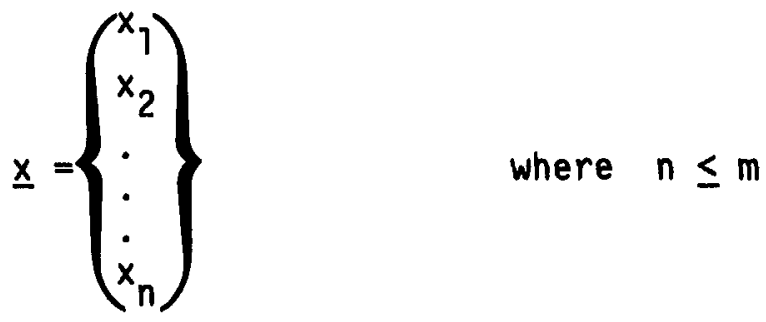

The attractiveness of dimensionality reduction from $m$ to $n$ is in simplifying the computational efforts necessary for classification.

The feature vector lies in a vector space called the feature space. Each component of the feature vector forms a dimension of the feature space. Thus, if the feature vector has $n$ components, the feature space is $n$-dimensional.

The components of the subset in equation (2) are selected in a very particular way so as to contain the most significant discriminatory components of the pattern vector. Interpreted graphically, the feature vectors are selected from the pattern vector such that feature vectors from distinct sample states (material properties, geometrical properties, environmental conditions, and measurement conditions) form distinct clusters in the feature space as illustrated in figure 2. Figure 2 shows a two-dimensional feature space formed by using the $j$ th and $j$ th components of a feature vector ( $i . e ., x_{i}$ and $x_{j}$, respectively). Ideally, the feature vectors from three distinct sample states form three distinct nonoverlapping clusters in this feature space. Those feature vectors of known sample states that are used to define the clusters are called training samples. All feature vectors corresponding to unknown sample states will be classified by comparing them with the training samples by using the techniques described in this study. 
A suitable feature vector produces the maximum separation between clusters (intercluster separation) as measured relative to the cluster size (intercluster dimension). The cluster size is defined by the spread of the feature vectors within the same sample state. The spread may be defined, for example, by the covariance of the feature vectors in a cluster.

Various distances can be used to measure the intercluster separation. If the covariances of samples in all clusters are similar and can be represented by a pooled sample covariance, the Mahalanobis distance $\mathrm{D}^{2}$ can be used (refs. 2 and 3 ). The Mahalanobis distance $D^{2}$ is defined as the square of the Euclidean distance between the sample cluster centroids (where the centroid is located at the mean vector of all feature vectors belonging to the cluster) normalized by an averaged cluster size (e.g., the covariance) (ref. 4). For example, the Mahalanobis $D^{2}$ between clusters $A$ and $B$ is (refs. 2 to 4 )

$$
\text { Mahalanobis } D^{2}=(\underline{\bar{A}}-\underline{\bar{B}})^{\top} \underline{S}_{\text {pooled }}^{-1}(\underline{\bar{A}}-\underline{\bar{B}})
$$

where $\bar{A}$ is the mean sample feature vector of cluster $A, \bar{B}$ is the mean sample feature vector of cluster $B$, and $\underline{S}$ pooled is the pooled sample covariance matrix of $A$ and $B$. The superscript $T$ denotes matrix transposition. Specifically, $\bar{A}, \underline{B}$, and $\underline{S}_{\text {pooled }}$ are defined as (ref. 3)

$$
\begin{gathered}
\underline{\bar{A}}=\frac{1}{N_{A}} \sum_{i=1}^{N_{A}} \underline{a}_{i} \\
\underline{\bar{B}}=\frac{1}{N_{B}} \sum_{i=1}^{N_{B}} \underline{b}_{i} \\
\underline{S}_{\text {pooled }}=\frac{1}{N_{A}+N_{B}-2}\left[\sum_{i=1}^{N_{A}}\left(\underline{a}_{i}-\underline{\bar{A}}\right)\left(\underline{a}_{i}-\underline{\bar{A}}\right)^{\top}+\sum_{i=1}^{N_{B}}\left(\underline{b}_{i}-\underline{B}\right)\left(\underline{b}_{i}-\underline{B}^{\top}\right]\right.
\end{gathered}
$$

where $\underline{a}_{j}$ and $\underline{b}_{j}$ are the feature vectors corresponding to clusters $A$ and $B$, respectively, and $N_{A}$ and $N_{B}$ are the numbers of feature vectors in clusters $A$ and $B$, respectively.

If the covariances of the samples in all of the clusters vary significantly, the chernoff distance can be used (ref. 2). The chernoff distance between clusters $A$ and $B$ is (ref. 2)

$$
\begin{aligned}
& \text { Chernoff distance }=\frac{1}{2} s(1-s)(\underline{\bar{A}}-\underline{\bar{B}})^{\top}\left[(1-s) \underline{S}_{A}-s \underline{S}_{B}\right]^{-1}(\underline{\bar{A}}-\underline{\bar{B}}) \\
&+\frac{1}{2} \ln \frac{\left|(1-s) \underline{s}_{A}+s \underline{S}_{B}\right|}{\left|\underline{S}_{A}\right|^{1-s}\left|\underline{S}_{B}\right|^{s}}
\end{aligned}
$$


where $s$ is a real number between zero and unity, $\underline{S}_{A}$ is the sample covariance of cluster $A$, and $\underline{S}_{B}$ is the sample covariance of cluster $B$. Specifically, $\underline{S}_{A}$ and $\underline{S}_{B}$ are defined as (ref. 3)

$$
\begin{aligned}
& \underline{S}_{A}=\frac{1}{N_{A}-1} \sum_{i=1}^{N_{A}}\left(\underline{a}_{i}-\underline{\bar{A}}\right)\left(\underline{a}_{i}-\underline{\bar{A}}\right)^{\top} \\
& \underline{S}_{B}=\frac{1}{N_{B}-1} \sum_{i=1}^{N_{B}}\left(\underline{b}_{i}-\underline{B}\right)\left(\underline{b}_{i}-\underline{\bar{B}}\right)^{\top}
\end{aligned}
$$

When $s=1 / 2$, the Chernoff distance is known as the Bhattacharyya distance (ref. 2).

The feature vector is selected from the pattern vector by maximizing the resulting intercluster distance. This can be accomplished analytically (ref. 1) or by trial and error with a computer search scheme (ref. 2).

\section{CLASSIFICATION}

A sample of unknown state is classified by determining the most likely sample state based on its feature vector. This is mathematically represented by the use of discriminant functions (refs. 1 to 3 ).

The discriminant function $g_{k}$ is determined from the training samples such that if $\underline{x}$ is a feature vector corresponding to sample state $k$,

$$
g_{k}(\underline{x})>g_{j}(\underline{x}) \quad \text { for all } j \neq k
$$

Equation (10) can be interpreted as follows: values of discriminant functions corresponding to all sample states can be evaluated by using a feature vector from sample state $k$. Then equation (10) states that the value of the discriminant function corresponding to sample state $k$ is the largest among values of discriminant functions corresponding to all other sample states.

The discriminant functions illustrated in figure 3 are based on the feature space represented in figure 2. Figure $3(a)$ shows discriminant functions $g_{1}, g_{2}$, and $g_{3}$ along a line $C-C$ in the feature space shown in figure $3(b)$. Within the region dominated by the cluster corresponding to sample state $1, g_{1}$ is greater than both $g_{2}$ and $g_{3}$, as required by equation (10). The points where the discriminant functions corresponding to sample states \#1 and \#2 have the same value; that is,

$$
g_{1}(\underline{x})=g_{2}(\underline{x})
$$

is a point on the so-called decision surface (ref. 1) separating the clusters corresponding to sample states \#1 and \#2. 
Figure 4 illustrates the decision surfaces separating the feature space into as many regions as there are distinct sample states. The sample whose feature vector lies within region $k$ should be classified as belonging to region $k$. Thus, the feature vector of unknown sample state in figure 4 classifies as belonging to sample state \#1. The main task in classification becomes the determination of discriminant functions or decision surfaces.

There are two approaches: (1) parametric and (2) nonparametric (refs. 1 and 2). Before discussing the methods of evaluating discriminant functions or decision surfaces, criteria for accessing the performance of the selected discriminant functions or decision surfaces will be described.

Assessing classification functions. - The ideal discriminant functions or decision surfaces minimize the sample misclassification rate. A misclassification occurs when a sample with sample state $k$ is classified as having a sample state other than $k$. (Sometimes it is not simply the misclassification rate that is important; certain misclassifications are more costly than others. Thus, it may be the cost of misclassification that should be minimized (ref. 3).)

One procedure to estimate the misclassification rate is to split the total training samples into two portions. One portion is used to establish the discriminant functions, and the other portion is used as validation samples to evaluate the misclassification of the resulting discriminant functions (ref. 3).

Another procedure to estimate the misclassification rate is called Lachenbruch's leaving-one-out method (refs. 2 and 3 ). Specifically, one training sample is left out in forming the discriminant functions; the left-out feature vector is then classified with the resulting discriminant functions and any misclassification is noted. Each training sample is omitted in turn, and the misclassification rate (or cost of misclassification) is evaluated.

Parametric methods. - If the probability distributions of the samples are known or can be assumed, parametric methods can be used to evaluate the discriminant functions (ref. 2). Denoting $P(k \mid \underline{x})$ as the conditional probability that a feature vector $\underline{x}$ belongs to sample state $k$, the discriminant function can be expressed as

$$
g_{k}(\underline{x})=P(k \mid \underline{x})
$$

Thus, for the feature vector $\underline{x}$ of unknown sample state, if

$$
P(k \mid \underline{x})>P(j \mid \underline{x}) \quad \text { for } a 11 j \neq k
$$

then $x$ is classified as belonging to sample state $k$. Equation (13) states that if the probability of a feature vector belonging to sample state $k$ is greater than the probabilities of the feature vector belonging to all other sample states, the feature vector is classified as belonging to sample state $k$. 'Bayes' theorem is often used to evaluate the probabilities expressed in equation (13). 
In any case, conditional probabilities of the samples must be known or must be assumed. Any remaining unknown parameters in the probabilities can be estimated by minimizing the misclassification rate.

Nonparametric methods. - If the probability distribution of the samples is not known or cannot be assumed, nonparametric methods must be used to evaluate discriminant functions or decision surfaces. There are many nonparametric classification schemes (refs. 1 and 2). Among these are the nearestneighbor, nearest-centroid, Fisher, and kerne] methods.

The nearest-neighbor method (ref. 2) consists of finding that training sample which lies closest to the unclassified feature vector and then classifying the unclassified feature vector to the same sample state as this nearest neighbor. The decision surface in this method lies equidistant between the boundaries of the clusters.

The nearest-centroid method (ref. 5) consists of finding the cluster whose mean feature vector (i.e., centroid) lies closest to the unclassified feature vector and then classifying the unclassified feature vector to the same sample state as this cluster. The decision surface in this method lies equidistant between the centroids of the clusters.

Fisher's method consists of assuming a linear discriminant function such that (refs. 1 and 3)

$$
g_{k}(\underline{x})=w_{1}^{(k)} x_{1}+w_{2}^{(k)} x_{2}+\ldots+w_{n}^{(k)} x_{n}+w_{n+1}^{(k)}
$$

where $x_{1}, x_{2}, \ldots, x_{n}$ are the components of the feature vector $\underline{x}$ and the scalar coefficients $w_{1}^{(k)}, w_{2}^{(k)}, \ldots, w_{n+1}^{(k)}$ correspond to sample state $k$. The linear combination of components of the feature vector given in equation (14) is simple to calculate. For the special case where the covariances of the samples in all clusters can be assumed to be equal, analytical values for the scalar coefficients exist (ref. 3 ). The scalar coefficients $w_{j}^{(k)}$ are evaluon the basis of the maximum separation between clusters and not the minimization of the misclassification rate.

The kernel method (ref. 6) consists of assuming kernel "potential" functions. Individual kernel functions are assumed to be centered at each training sample. The kernel function can be denoted by $k\left(\underline{x}, \underline{x}_{j}^{(k)}\right)$, where $\underline{x}_{j}^{(k)}$ is the feature vector of the $i$ th training sample defining sample state $k$. Then the discriminant function is defined as the superposition over all training samples of the same sample state $k$ as (ref. 1)

$$
g_{k}(\underline{x})=\frac{1}{N_{k}} \sum_{i=1}^{N_{k}} k\left[\underline{x}, \underline{x}_{i}^{(k)}\right]
$$

where $N_{k}$ is the number of training samples of the same sample state $k$. 
In one-dimensional feature space, equation (15) can be written as (refs. 1 and 2)

$$
g_{k}(x)=\frac{1}{N_{k} h_{k}} \sum_{i=1}^{N_{k}} k_{0}\left[\frac{x-x_{i}^{(k)}}{h_{k}}\right]
$$

where $K_{0}$ is the kernel shape, $h_{k}$ is the kernel size, $x$ is a position in the one-dimensional feature space, and $x_{i}^{(k)}$ is the position of the $i^{\text {th }}$ training sample defining sample state $k$.

There are many forms for $K_{0}$ in the literature. For example, the one-dimensional form of the exponential decay kernel can be written as

$$
k_{0}\left[\frac{x-x_{j}^{(k)}}{h_{k}}\right]=\exp \left[-\left|\frac{x-x_{i}^{(k)}}{h_{k}}\right|\right]
$$

The selection of the kernel shape and kernel size can be established by trial and error through a computer search scheme seeking to minimize the resulting misclassification rate.

\section{CONCLUSIONS}

Ultrasonic nondestructive evaluation (NDE) of structural states has been considered in this study. Because of the large number of possible properties and conditions describing the state of the structure and the large number of ultrasonic NDE parameters that can be considered, pattern recognition techniques have been suggested for identifying structural states from discriminatory information.

An outline has been provided for the pattern recognition characterizations of micromechanical and morphological materials states via analytical quantitative ultrasonics. The concepts, terminology, and techniques of statistical pattern recognition have been reviewed.

Determining the state of a sample by NOE with pattern recognition techniques consists of ultrasonic NDE data generation, feature extraction, and classification. Ultrasonic data generation consists of ultrasonic NDE measurements that are expected to contain information capable of identifying the micromechanical and morphological states of the material or structure. The collected data are organized in a pattern vector.

By using samples of known states, called training samples, the significant discriminatory components of the pattern vector are retained as a feature vector. The feature vectors are extracted such that training samples of distinct sample states form distinct clusters in the feature space. Then classification is achieved by defining discriminant functions or decision surfaces based on the training samples by using parametric or nonparametric methods. The ideal classification scheme will minimize the resulting misclassification rate (or the cost of misclassification). 
Thus, through a program of ultrasonic NDE data generation, feature extraction, and classification, the most likely materials states corresponding to an unknown sample can be determined. The pattern recognition techniques discussed in this study have broad applicability to various NDE procedures if samples of known states are available.

\section{REFERENCES}

1. H.C. Andrews, Introduction to Mathematical Techniques in Pattern Recognition, Wiley-Interscience, John Wiley \& Sons, Inc., NY, 1972.

2. D.J. Hand, Discrimination and Classification, John Wiley \& Sons, NY, 1981 .

3. R.A. Johnson and D.W. Wichern, Applied Multivariate Statistical Analysis, Prentice-Ha11, Inc., Englewood Cliffs, NJ, 1982.

4. D.F. Morrison, Multivariate Statistical Methods, McGraw Hill, NY, 1976.

5. M.R. Anderberg, Cluster Analysis for Applications, Academic Press, NY, 1973.

6. D.J. Hand, Kernel Discriminant Analysis, Research Studies Press, NY, 1982 . 


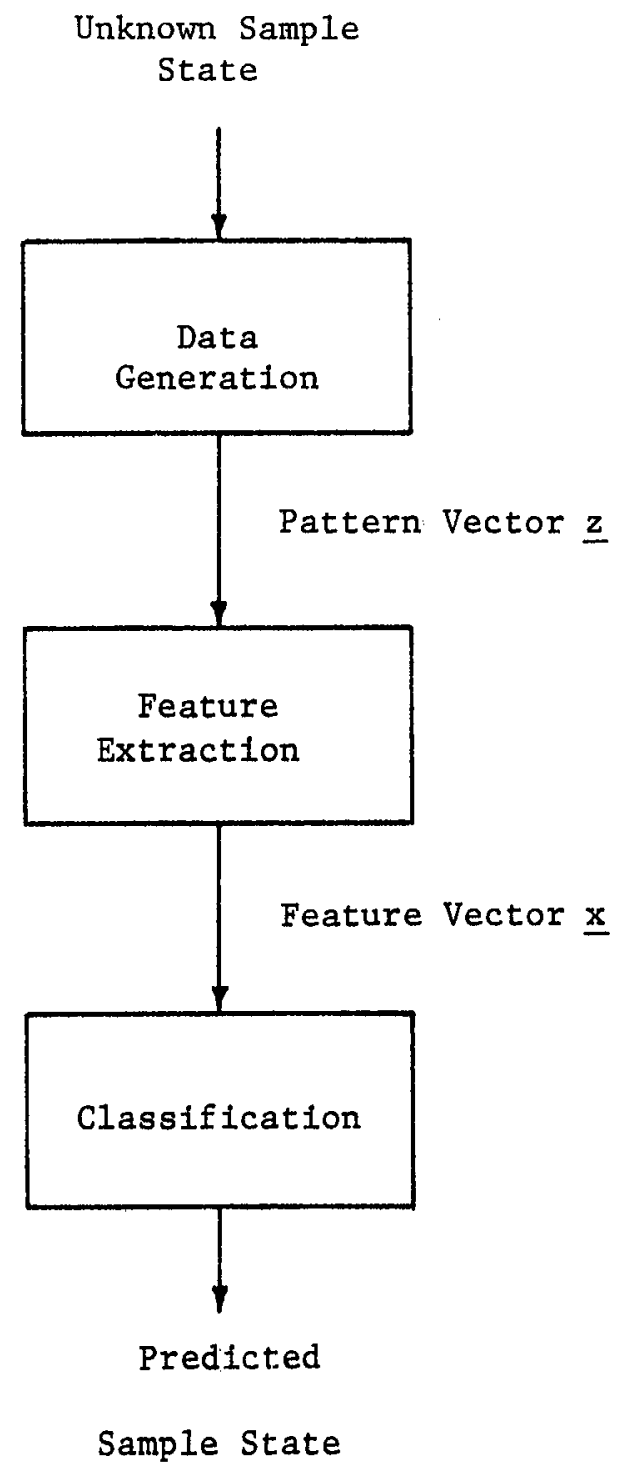

Fig. 1 Determination of state of unknown sample using pattern recognition techniques based on nondestructive evaluation data generation. 


\section{Feature Space}

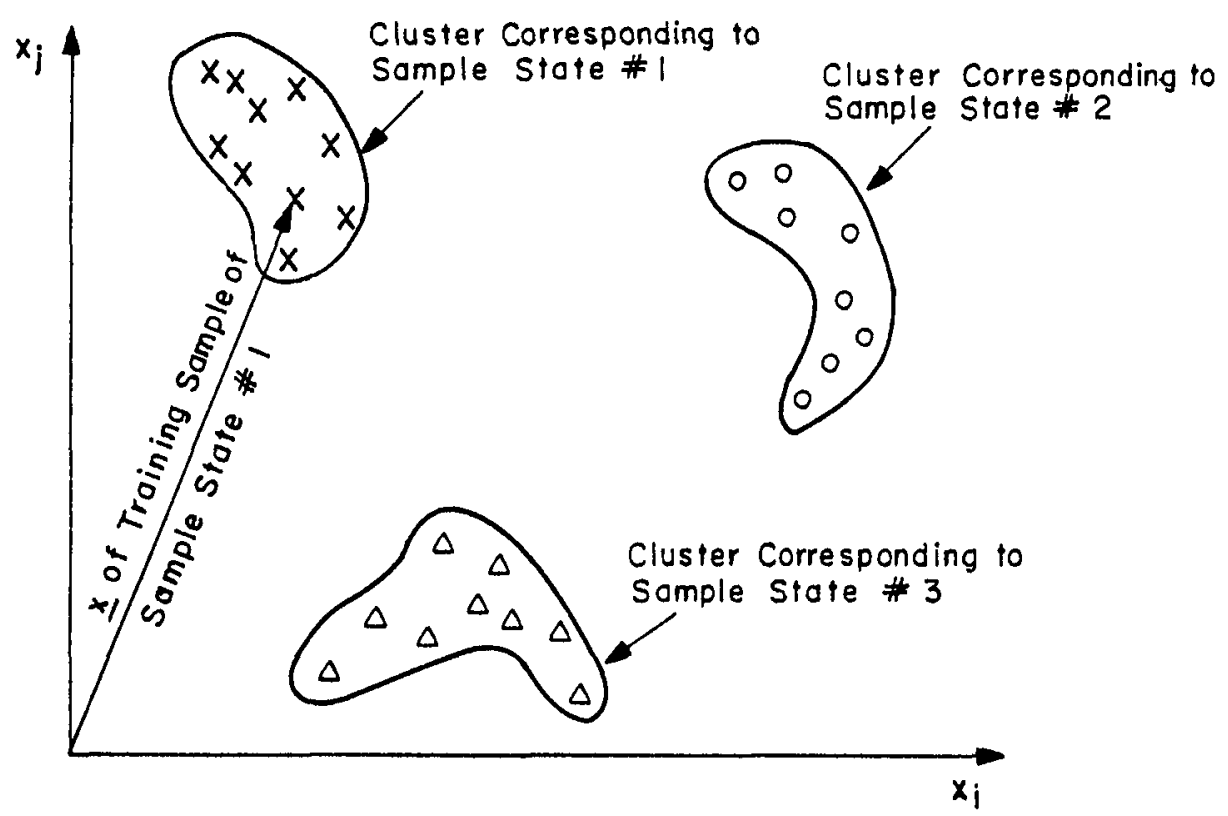

Fig. 2 Two-dimensional representation of feature space formed using $1-t h$ and $j-t h$ components of feature vectors, illustrating separate clusters of feature vectors $x$ of training samples having distinct sample states. 

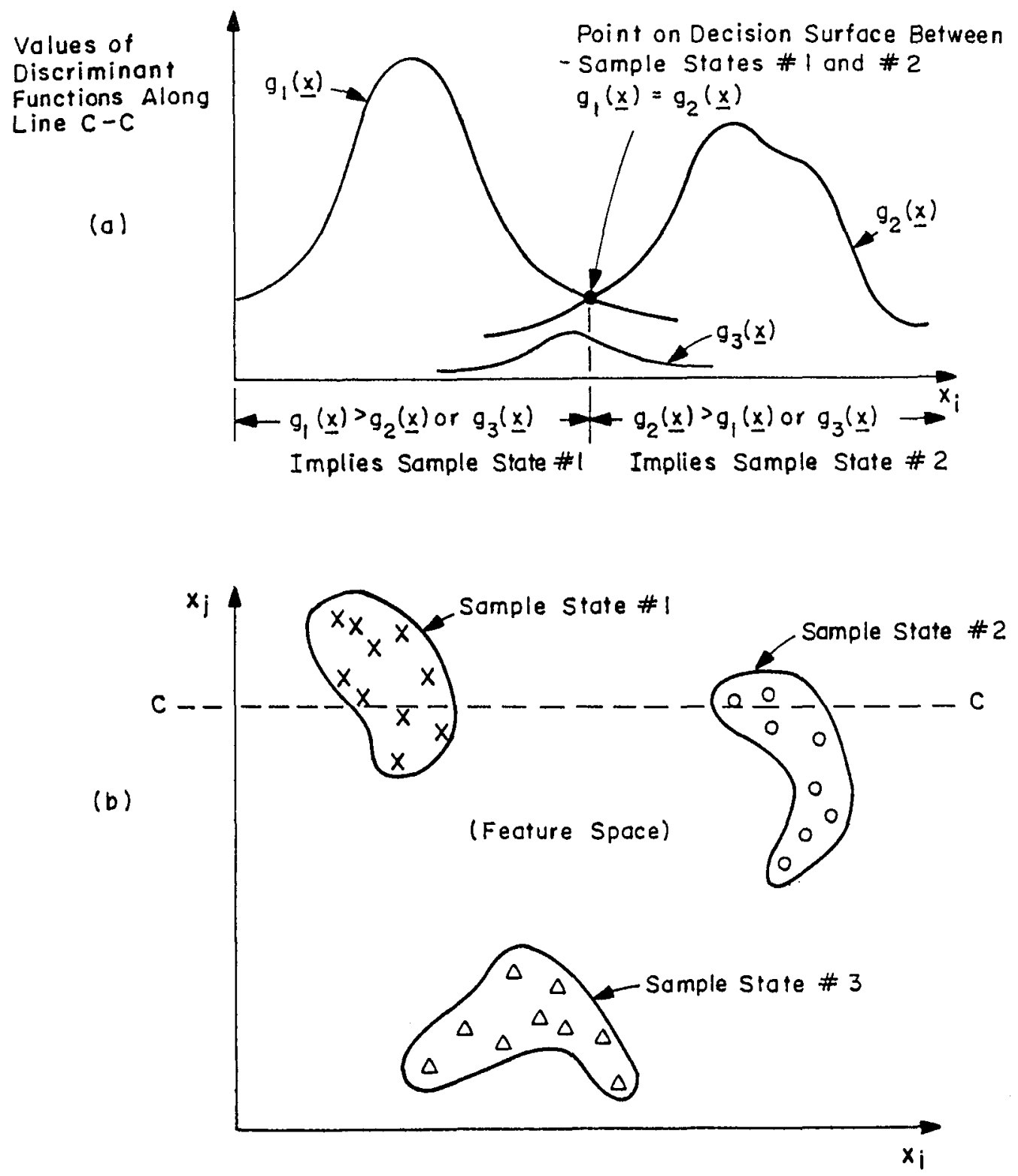

Fig. 3 Schematic illustrating (a) discriminant functions $g_{k}(x)$ along a line $C-C$ in feature space shown in (b). 


\section{Feapure Space}

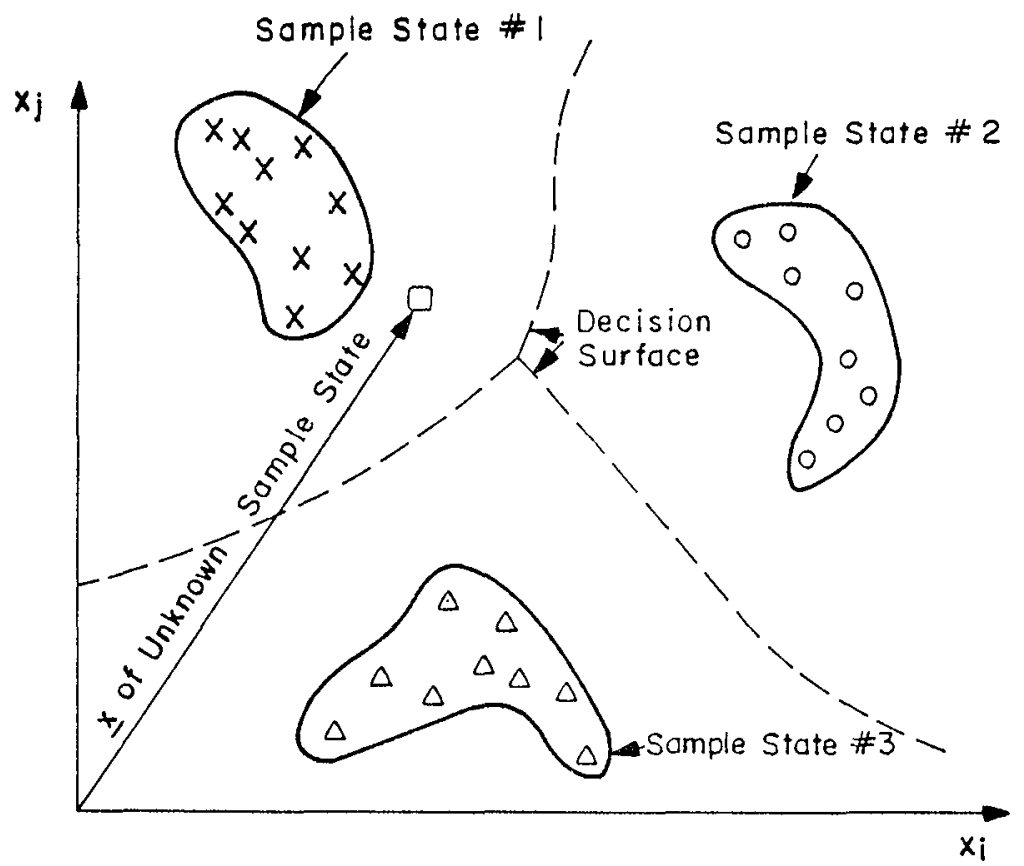

Fig. 4 Schematic illusţrating decision surfaces separating feature space into regions corresponding to distinct sample states, and also illustrating classification of unknown sample shown as belonging to sample state \#1. 



\title{
ULTRASONIC VERIFICATION OF MICROSTRUCTURAL CHANGES DUE TO HEAT TREATMENT
}

\author{
Edward R. Generazio \\ National Aeronautics and Space Administration \\ Lewis Research Center \\ Cleveland, Ohto 44135
}

\begin{abstract}
Ultrasonic attenuation was measured for polycrystalline samples of nickel and copper with varlous grain-size distributions produced by heat treatment. Attenuation as a function of frequency was determined for a sample having a known mean grain diameter. Once this function was determined, it could be scaled to determine the mean grain size of other samples of the same material with different mean grain diameters. These results were obtained by using broadband pulse-echo ultrasound in the 25- to $100-\mathrm{MHz}$ frequency range. The results suggest an ultrasonic, nondestructive approach for verifying heat treatment of metals.
\end{abstract}

\section{INTRODUCTION}

An extensive effort has been made to relate the observed uitrasonic attenuation to the mean grain diameter in polycrystalline solids (refs. 1 to 7 ). Although the experimental and theoretical results agree for some polycrystalline materials, many materials are so complex that they are not adequately described by existing theory (refs. 8 to 13). Because structural materials generally exhibit complex microstructures, verification of their microstructures is of high interest. As a practical matter, determining the mean grain size of both the simple and complex systems by nondestructive ultrasonic attenuation is preferable to the usual time-consuming, expensive, and inherently destructive metallographic methods.

Most polycrystalline solids are a collection of densely packed, inhomogeneous, and morphologically complicated scatters. Previous investigators have usualiy assumed that a particular mode of scatter attenuation (Rayleigh, stochastic, etc.) is dominant when comparing experimental and theoretical results. However, in many complex systems both Rayleigh and stochastic scattering may occur for a given range of frequencies (or wavelengths).

In general Rayleigh, stochastic, and diffusive scattering may occur simultaneously. The relative contribution of each scattering mechanism to the total observed attenuation is unknown for a complex microstructure. In this study no attempt was made to attribute attenuation to a particular scattering mode. We merely observed that a significant factor in comparing changes in microstructure is a ratio containing wavelength and mean grain diameter. It will be shown that any arbitrary wavelength can be used to establish a mean-grain-sizerelated scale factor between two polycrystalline microstructures. 
Expressions for uitrasonic attenuation coefficient $\alpha$ as a function of frequency $f$ have been developed for three regimes: the Rayleigh scattering regime, where wavelength $\lambda$ greatly exceeds mean grain size $\bar{D}(\lambda \gg \bar{D})$

$$
\alpha_{R}=C_{R} \bar{D}^{3} f^{4}, \mathrm{~Np} / \mathrm{Cm}
$$

the stochastic scattering regime, where $\lambda \simeq \overline{\mathrm{D}}$,

$$
\alpha_{s}=C_{s} \bar{D} f^{2}, \mathrm{~Np} / \mathrm{cm}
$$

and the diffusion scattering regime, where $\lambda \ll \bar{D}$,

$$
\alpha_{d}=\frac{C_{d}}{D}, \mathrm{~Np} / \mathrm{Cm}
$$

Here, $C_{R}, C_{S}$, and $C_{d}$ are constants that depend on material parameters such as elastic constants and longitudinal and shear velocities. Typical dimensions for a are nepers per centimeter. Equations (1) to (3) are generally valid only for homogeneous, regular, and equiaxed systems (ref. 10).

Note that each of the expressions (eqs. (1) to (3)) contains a coefficient that depends on $\bar{D}$, the mean grain size. Assume that the constants $C_{R}, C_{S}$, and $C_{d}$ are unaffected by changes in $\overline{\bar{D}}$. Also assume that the shape of the grain size distribution function and grain morphology are unaltered by changes in $\bar{D}$. Given these assumptions, the microstructure would simply change by a scale factor corresponding to the increase (or decrease) in $\bar{D}$. Substituting, $V / \lambda$, where $V$ denotes velocity, for $f$ and multiplying equations (1) to (3) by wavelength $\lambda$ yields a new set of equations in which $\bar{D} / \lambda$ is the key variable. Thus, given that velocity is not a function of frequency, we have for Rayleigh scattering

$$
\alpha_{\lambda}=C_{R}^{\prime}\left(\frac{\overline{0}}{\lambda}\right)^{3}, \text { Np/wave length }
$$

for stochastic scattering

$$
a_{\lambda}=C_{s}^{\prime}\left(\frac{\bar{D}}{\lambda}\right), N p / \text { wavelength }
$$

for diffusion scattering,

$$
a_{\lambda}=C_{d}^{\prime}\left(\frac{\lambda}{\delta}\right) \text {, Np/wave length }
$$

where $C_{R}^{\prime}, C_{s}^{\prime}$, and $C_{d}^{\prime}$ are constants. The attenuation coefficient is now expressed in nepers per wavelength for each regime. 
Evaluating equation (5) at some arbitrary wavelength $\lambda_{0}$ for a system $S_{0}$ having a mean grain diameter $\bar{D}_{0}$ (fig. $\left.1(a)\right)$, we obtain the attenuation coeffictent

$$
a_{\lambda}=\left.c_{s}^{\prime}\left(\frac{D}{\lambda}\right)\right|_{\delta_{0}, \lambda_{0}, c_{s}^{\prime}}=\alpha_{0} \text {, Np/wavelength }
$$

If the mean grain size and the wavelength are doubled (fig. $1(b)$ ), we obtain from equation (5),

$$
\alpha_{\lambda}=\left.c_{s}^{\prime}\left(\frac{\bar{D}}{\lambda}\right)\right|_{2 \bar{D}_{0}, 2 \lambda_{0}, c_{s}^{\prime}}=\alpha_{0} \text {, Np/wavelength }
$$

or the same numerical value for the attenuation coefficient as obtained in the previous case (simllar results can be obtained by using eq. (4) or (6)). This is shown graphically in figure 2(a). By varying the arbitrary wavelength $\lambda_{0}$, we can construct the attenuation-versus-wavelength functions $S_{0}$ and $S$ (fig. $2(b)$ ). Because the mean grain size of system $S$ is also arbitrary, we can construct the attenuation-versus-wavelength function for the $\mathrm{N}^{\text {th }}$ system $S_{n}$ having the mean grain size $\bar{D}_{n}$.

A scale factor can be defined as

$$
\sigma_{n} \equiv \frac{\lambda_{n}}{\lambda_{0}}=\frac{\delta_{n}}{\delta_{0}}=N^{\text {th }} \text { scale factor }
$$

where $\bar{D}_{n} / \bar{D}_{0}$ is the ratio of the mean grain size of the $N^{\text {th }}$ system to the mean grain size of the initial system. The scale factor is also represented as the ratio of wavelengths for which constant attenuation an is observed between two systems (1.e.. the ratio of wavelength $\lambda_{n}$ of the $N$ th system to the wavelength $\lambda_{0}$ of the initial system at constant attenuation).

The curve $s_{0}$ in figure $2(b)$ is the generating function from which the other curves can be obtained. To construct the generating function, a fourthdegree polynomial in frequency was fitted to the attenuation data by using the method developed by Marquardt (refs. 14 and 15). Here the attenuation is expressed as

$$
\alpha_{0}(f)=\sum_{m=1}^{4} A_{m} f^{m}, N p / c m
$$

where $A_{m}$ are constants. The terms in the expansion shown in equation (10) are not directly related to equations (1) to (3). Any other analytical expression that fits the attenuation data would be equally valid. The preceding curve-fitting function is made a function of wavelength by combining equation 10 with 


$$
V=f \lambda
$$

where $V$ is the velocity of sound, to obtain

$$
\alpha_{0}(\lambda)=\sum_{m=1}^{4} A_{m}^{\prime}\left(\frac{1}{\lambda}\right)^{m}, \mathrm{~Np} / \mathrm{cm}
$$

where $A_{m}^{\prime}$ are constants. By multiplying equation (12) by the variable wavelength, we obtain the generating function

$$
\alpha_{\lambda}=\sum_{m=1}^{4} A_{m}^{\prime}\left(\frac{1}{\lambda}\right)^{m-1} \text {, Np/wavelength }
$$

The theoretical attenuation for other systems (of larger grain size) is obtained from their respective generating functions by scaling the wavelength as indicated in figure 2. The scale factor $\sigma$ is varied until the generating function for the initial system with the scaled wavelengths reconstructs the attenuation for the $N$ th system. If the mean grain size of the initial system is known, the mean grain size $\bar{D}_{n}$ for the $N^{t h}$ system is given by equation (9).

\section{EXPERIMENT}

\section{Material Samples}

Two materials, nickel 200 and copper, were used in this investigation. The nickel 200 was 99.5 percent pure and the copper was 99.99 percent pure. Both were obtained as commerclally avallable rods. The nickel 200 was then cold rolled 50 percent and annealed in air at $690 \pm 9{ }^{\circ} \mathrm{C}$ and cold rolled again 50 percent. The copper sample was cold rolled 60 percent and annealed at $329^{\circ} \mathrm{C}$ in argon for $5 \mathrm{~min}$. Three nickel samples, cut from the rolled stock, had typical dimensions of 1 by 1 by $0.2540 \mathrm{~cm}$. Five copper samples, cut from the rolled stock, had typical dimensions of 1.2 by 0.8 by $0.18 \mathrm{~cm}$. Each sample was polished to a mirror finish with a final polish of 1.0-um-diameter diamond slurry.

\section{Microstructure Modification}

The mean grain size was increased in both the nickel and copper samples by annealing (refs. 16 and 17). Three nickel samples were annealed in atr at $800{ }^{\circ} \mathrm{C}$ for 1,2 , and $5 \mathrm{~min}$, respectively. Four of the five copper samples were annealed in argon for $5 \mathrm{~min}$ at $466,510,700$, and $800^{\circ} \mathrm{C}$, respectively. 


\section{Attenuation Measurement}

Before modifying the initial grain size, we made attenuation measurements on each sample. The methodology for making precision attenuation measurements is described in reference 18. These initial attenuation measurements were made to verify that all of the nickel and copper samples were nearly acoustically identical to the other samples of the same material. At $50 \mathrm{MHz}$ there was 4 and 6 percent variation in the attenuation measurement among nickel and copper samples, respectively. These variations included the effect of the imprecision in attenuation measurements that include the frequency-dependent reflection coefficient in the calculation (ref. 18).

After the mean grain size had been modified, attenuation measurements were again made on each sample. Since the attenuation coefficient (at fixed frequency) generally increases with mean grain size, samples exhibiting extremely high attenuation were ground thinner so that the uncertainty in the attenuation measurement was minimized (ref. 18). The results were reproducible with typical variations in measurement simflar to those for the initial attenuation measurement.

\section{RESULTS \\ Experimental Generating Functions}

In general, for a fixed frequency, the attenuation coefficient increased with annealing time (fig. 3 ). The solid curve is the best-fit curve (i.e., generating function) obtained by using equation (10), for the data for the nickel sample annealed for $1 \mathrm{~min}$. The data shown in figure 3 reappear in figure 4 as a function of wavelength. The solid curves for the samples annealed for 2 and $5 \mathrm{~min}$ were determined from the generating function by scaling the wavelength, as discussed earlier. The observed and theoretical results are shown in figure 5 as a function of frequency.

For fixed frequency the attenuation coefficient increased with annealing temperature (fig. 6) for the copper samples. The solid curve for the copper sample annealed at $329^{\circ} \mathrm{C}$ is the generating function for these samples. The data shown in figure 6 reappear in figure 7 as a function of wavelength. The solid curves were determined from the generating function by scaling the wavelength. The observed and theoretical results are shown in figure 8 as a function of frequency.

The scale factors for the data shown in figures 4 to 8 were determined by scaling the generating function to fit the frequency regime for which the most accurate data were obtained. This regime, although well defined (ref. 18), was different for each sample and depended on the sample thickness, the attenuation, and the frequency-dependent reflection coefficient.

Deviations existed between the experimental and theoretical results (figs. 4 to 8 ) at both the low and high frequencies ( $1 . e .$, long and short wavelengths). These deviations were due to the greater uncertainty in the measurement of attenuation at these extreme frequencies (ref. 18). 
Several methods are avallable for determining the mean grain size (refs. 12 and 19). For regular systems the standard line-intercept method appears to be adequate. However, this method is not applicable to systems with structuraliy complicated morphologies. More advanced theories that are based on spherical grains also are inherently not applicable to real, complicated systems. An alternative technique, the comparison method (ref. 19), is free of the theoretical bias imposed by the above-mentioned theories.

The simple comparison method allows for the grain structure to be visually compared with an ASTM standard chart. This technique is not directly applicable to complicated systems (i.e., the standard ASTM charts are for regular structures). We can modify the comparison method so that we may compare systems with dimensionally arbitrary scales but of similar complexity. This is done by taking photomicrographs of similar systems of different scales and subsequentiy magnifying the photomicrograph for the smaller system by the appropriate scale factor between the two systems. The magnified photomicrograph and the photomicrograph for the system of larger scale should appear similar. This is shown graphically in figures 9 and 10 for the nickel and copper samples, respectively. (Uitrasonic attenuation was measured along the vertical axis.)

From figure 4, the scale factor between the photomicrographs for the nickel systems annealed for 2 and $5 \mathrm{~min}$ (figs. 9(a) and (b)) is 1.85 . Figure $9(a)$ is shown magnified 1.85 times in figure $9(\mathrm{c})$, which is dimensionally and morphologically similar to figure $9(b)$. Similar results are shown in figure 10 for the copper samples annealed at 510 and $800^{\circ} \mathrm{C}$. Comparisons with the other systems show strikingly similar results.

\section{DISCUSSION}

The success of scaling a generating function over a wide frequency range to other systems of similar complexity but of different scale was quite remarkable in that only one free parameter was needed. This parameter is directly related to the relative scale between systems. Ideally, one would like to use (scale) a theoretical expression for uitrasonic attenuation that includes multiple grain-boundary scattering for morphologically complicated systems. However, such an expression is currentiy unavallable since the theory of grainboundary scattering for complicated systems remains underdeveloped (ref. 10).

The lack of a comprehensive theory can be bypassed by measuring the attenuation for an initial system as a function of frequency. The effects of grain distribution, morphology, orientation, and grain interaction are implicit. This experimentally measured attenuation also includes attenuation due to dislocation damping (refs. 6 and 20), thermoelastic effects (ref. 20), etc. These nonbarrier attenuation mechanisms will not scale as the grain-boundary scattering terms (eqs. (4) to (6)) do. We have assumed in the preceding analysis that these unscalable attenuation mechanisms are negligible relative to the observed attenuation. This, however, will not be true for materials that are in a mechanically worked state, where the dislocation densities are expected to be large (ref. 16). 
A major requirement for the scaling method to be successful is that the systems of interest must have similar morphology. This is a somewhat subjective judgment not quantitatively described. The lack of a comprehensive theory for determining the mean grain size for a real, complicated system has left us with no alternative, other than the comparison method, for making quantitative comparisons between theory and experiment. Although magnifying the photomicrographs (figs. 9 and 10 ) served to make these comparisons, it is not the best method for comparing grain structures. A more precise method would be to use a continuous ly variable magnification metallograph.

The theorles for both ultrasonic boundary scattering and grain size distribution are insufficientiy developed to provide quantitative results for comparing experiment and theory. This problem is temporarily resolved by using the observed attenuation and the observed microstructure to show this agreement. The full comprehensive theory for the ultrasonic attenuation that includes grain-boundary scattering for morphologically complicated systems and allows for interacting grains is expected to scale as the experimental results indicate here.

The simple concept of mean grain size is likely to be inappropriate for both acoustic and microstructure theory. A more complete description of the microstructure morphology would include the distribution of grain size (ref. 12) and grain boundary topology. This more complete description should be included in determining of the microstructure morphology and ultrasonic attenuation theory. Nevertheless, even in the form described in this paper the approach for determining microstructure scale is likely to be useful in verifying the heat treatment of most polycrystalline materials.

\section{CONCLUSIONS}

It has been shown that the ratio between wavelength and mean grain diameter is a significant factor in comparing changes in microstructure. This ratio can be used to determine a scale factor between two polycrystalline materials with similar morphologies but different mean grain diameters. These results were achieved by determining the attenuation as a function of frequency without reference to any particular conventionally accepted scattering mode. By this approach it was unnecessary to associate a particular wavelength with mean grain diameter. This allowed a generalized formulation for scaling meangrain-diameter variations among samples of a polycrystalline material that had undergone a range of heat treatments.

\section{REFERENCES}

1. Evans, A.G., et al.: Fallure Prediction in Structural Ceramics, Mater. Eval., vol. 35, no. 4, Apr. 1977, pp. 85-96.

2. Kesler, H.A.; and Shraifeld L.I.: Dispersion of Ultrasonic Waves in Polycrystalline Metals with Statistically Distributed Grain Size, Sov. J. Nondestr. Test (Engl. Trans1.), vol. 11, no. 1, Nov. 1975, pp. 76-80. 
3. Papadakis, Emmanuel P.: Ultrasonic Attenuation Caused by Scattering Polycrystalline Metals, J. Acoust. Soc. Am., vol. 37, no. 4, Apr. 1965, pp. $711-717$.

4. Serabian, S.; and Williams R.S: Experimental Determination of Ultrasonic Attenuation Characteristics Using the Roney Generalized Theory, Mater. Eval., vol. 36, no. 8; July 1978, pp. 55-62.

5. Papadakis, E.P.: Some Practical Aspects of Research with U1trasonic Waves, Int. J. Nondestr. Test., vol. 1, 1970, pp. 383-399.

6. Smith, R.L., et a1.: Ultrasonic Attenuation, Microstructure, and Ductile to Brittle Transition Temperature in Fe-C Alloys, Mater. Eva1., vol. 41, no. 2, Feb. 1983, pp. 219-222.

7. Klinman, R., et al.: Ultrasonic Prediction of Grain Size, Strength, and Toughness in Plain Carbon Stee 1, Mater. Eval., vol. 38, no. 10, Oct. 1980, pp. 26-32.

8. Bhatia, A.B.: Scattering of High-Frequency Sound Waves in Polycrystalline Materials, J. Acoust. Soc. Am., vol. 31, no. 1, Jan. 1959, pp. 16-23.

9. Bhatia, A.B.; and Moore, R.A.: Scattering of High Frequency Sound Waves in Polycrystalline Materials II, J. Acoust. Soc. Am., vol. 31, no. 8, Aug. 1959, pp. 1140-1141.

10. Papadakis, Emmanuel P.: Ultrasonic Attenuation Caused by Rayleigh Scattering by Graphite Modules in Modular Cast Iron, J. Acoust. Soc. Am., vo1. 70, no. 3, Sept. 1981, pp. 782-787.

11. Roth, W.: Scattering of Ultrasonic Radiation in Polycrystalline Metals, J. App 1. Phys., vol. 19, no. 10, Oct. 1948, pp. 901-910.

12. Papadakis, E.P.: From Micrograph to Grain-Size Distribution with U1trasonic Applications, J. Appl. Phys., vol. 35, no. 5, May 1964, pp. 1586-1594.

13. Smith, R.L.; Reynolds, W.N.; and Wadley, H.N.G.: Ultrasonic Attenuation and Microstructure in Low Carbon Stee1s, Met. Sc1., vol. 15, no. 11-12, Nov.-Dec. 1981, pp. 554-558.

14. Marquardt, D.W.: An Algorithm for Least-Squares Estimation of Nonlinear Parameters, J. Soc. Ind. App 1. Math., vol. 11, no. 2, 1963, pp. 431-441.

15. Bevington, R.P.: Data Reduction and Error Analysis for the Physical Sclences, Chapter Four, McGraw-Hi11, New York, 1969.

16. Dieter, G.E.: Mechanical Metallurgy, Chapter 5, McGraw-H 111 , New York, 1961 .

17. Metals Handbook, eighth Ed., vol. 7, American Society for Metals, Metals Park, Ohio, 1972. 


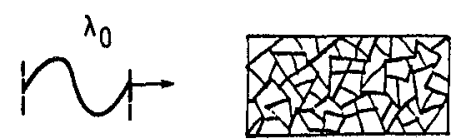

(a) Initial system $S_{0}$ with mean grain diameter $\bar{D}_{0}$.<smiles>[Te][Te]</smiles>
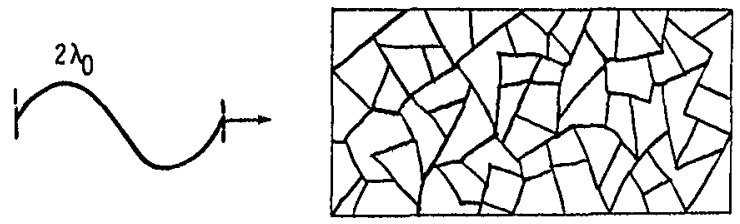

(b) Scaled system $\mathrm{S}$ with mean grain diameter $2 \bar{D}_{0}$.

Figure 1. - Initial and scaled systems.

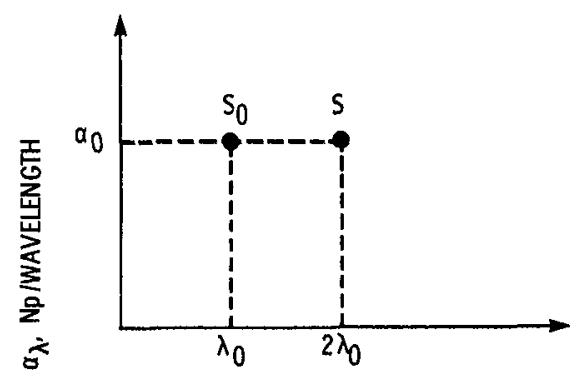

(a) Systems $S_{0}$ and $S$ at fixed wavelengths.

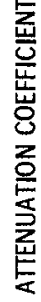

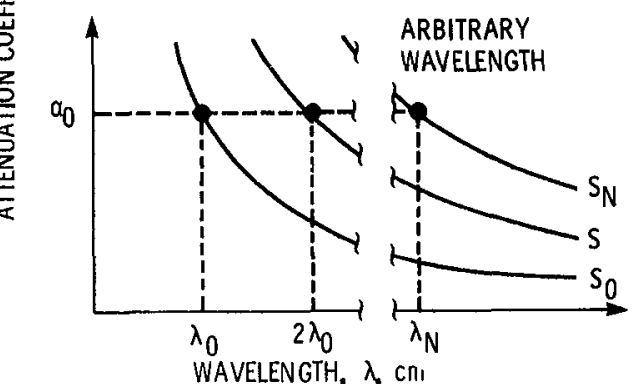

(b) Systems $S_{0}, S$, and $S_{N}$. Curve labeled $S_{0}$ is generating function.

Figure 2. - Attenuation coefficient as function of wavelength.

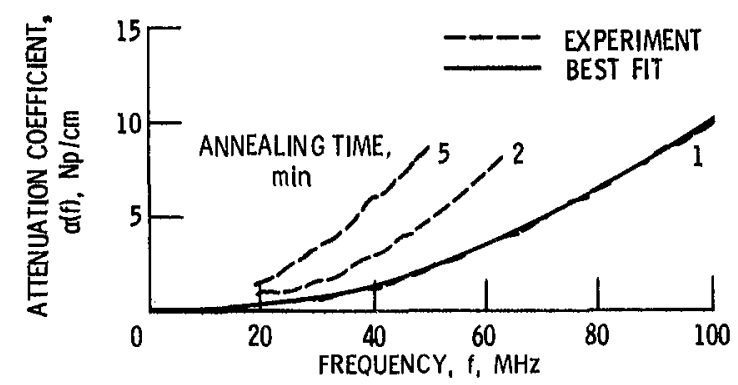

Figure 3. - Atten uation coefficient as function of frequency for nickel samples. Annealing temperature, $800^{\circ} \mathrm{C}$. Solid curve is generating function.

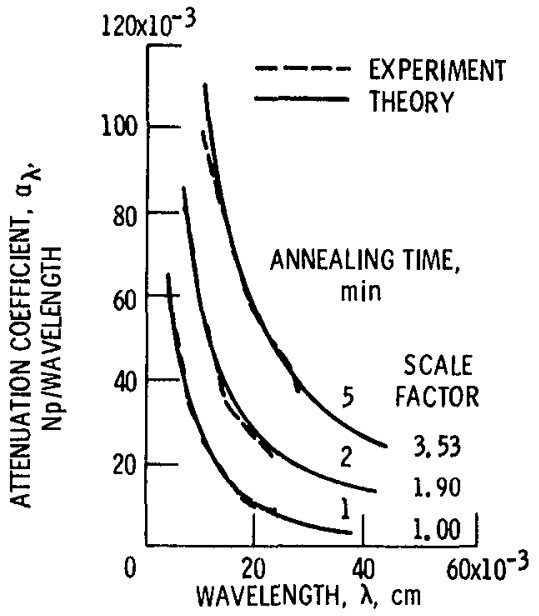

Figure 4. - Attenuation coefficient as function of wavelength for nickel samples. Annealing temperature, $800^{\circ} \mathrm{C}$. 


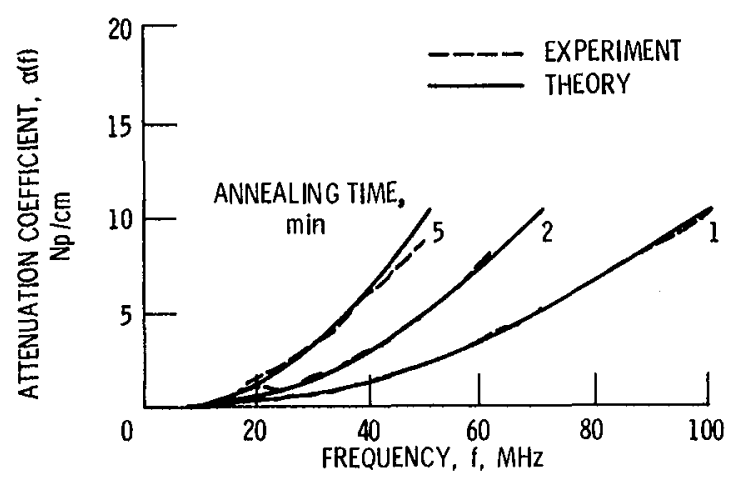

Figure 5. - Comparison between observed and theoretical attenuation coefficients as function of frequency for nickel samples. Annealing temperature, $800^{\circ} \mathrm{C}$.

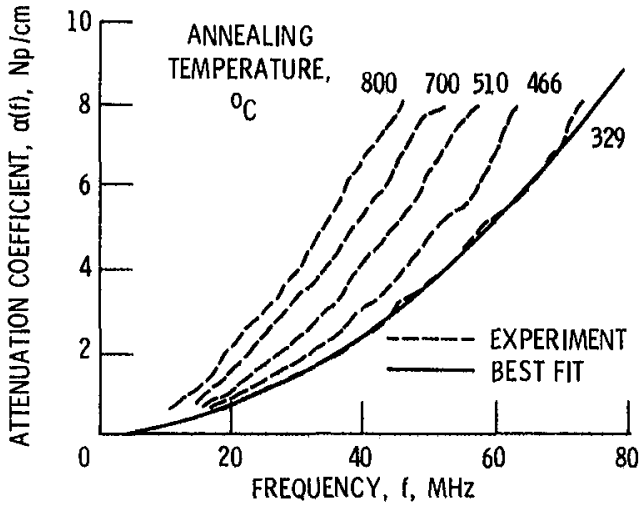

Figure 6. - Atten uation coefficients as function of frequency for copper samples. Annealing time, $5 \mathrm{~min}$. Solid curve is generating function.

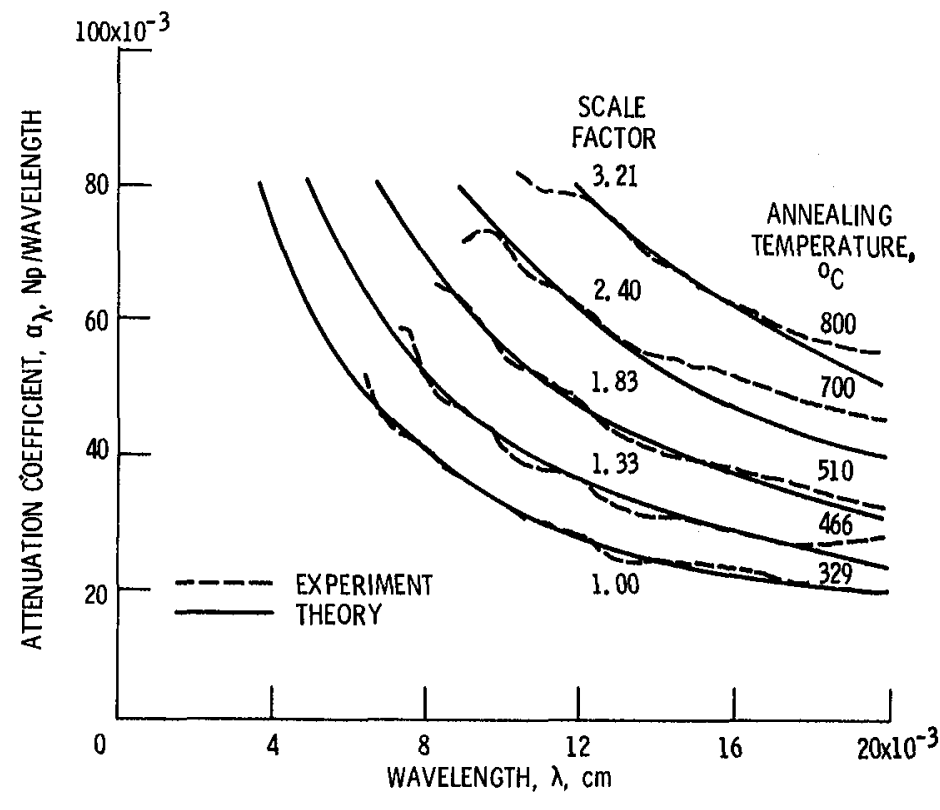

Figure 7. - Attenuation coefficient as function of wavelength for copper samples. Annealing time, $5 \mathrm{~min}$.

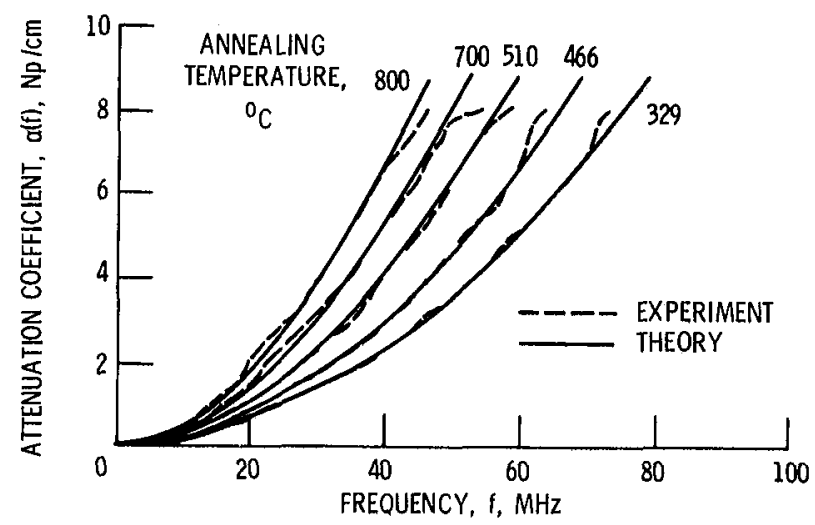

Figure 8. - Comparison between observed and theoretical attenuation coefficients as function of frequency for copper samples. Annealing time, $5 \mathrm{~min}$. 


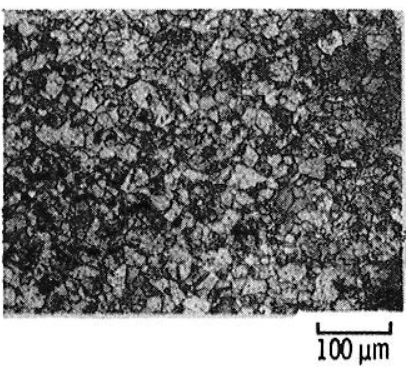

(a) Annealed for $2 \mathrm{~min}$ at $800^{\circ} \mathrm{C}$.

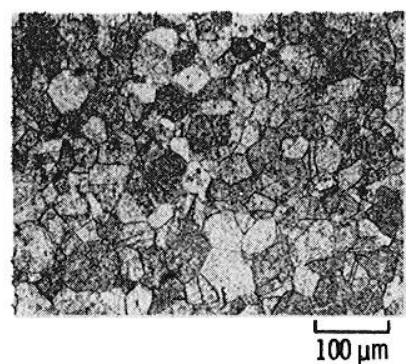

(b) Annealed for $5 \mathrm{~min}$ at $800^{\circ} \mathrm{C}$.
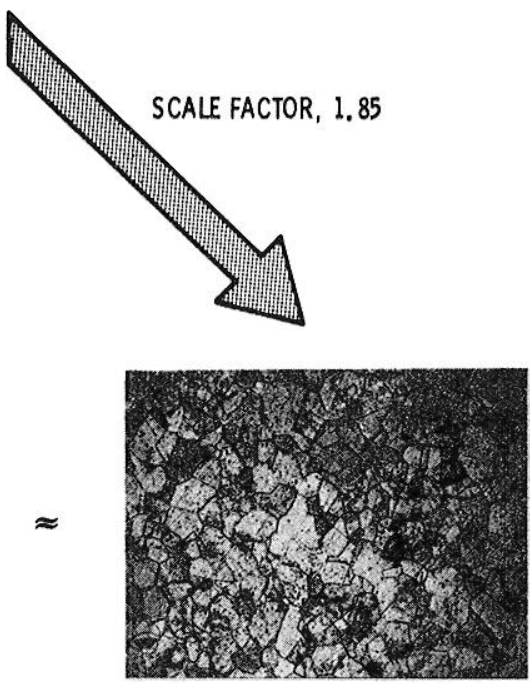

(c) 1.85 Magnification of figure $9(a)$.

Figure 9. - Photomicrographs of nickel sample.

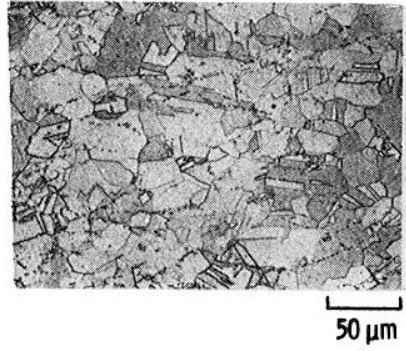

(a) Annealed for $5 \mathrm{~min}$ at $510^{\circ} \mathrm{C}$

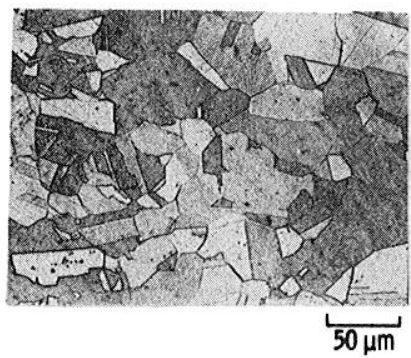

(b) Annealed for $5 \mathrm{~min}$ at $800^{\circ} \mathrm{C}$.
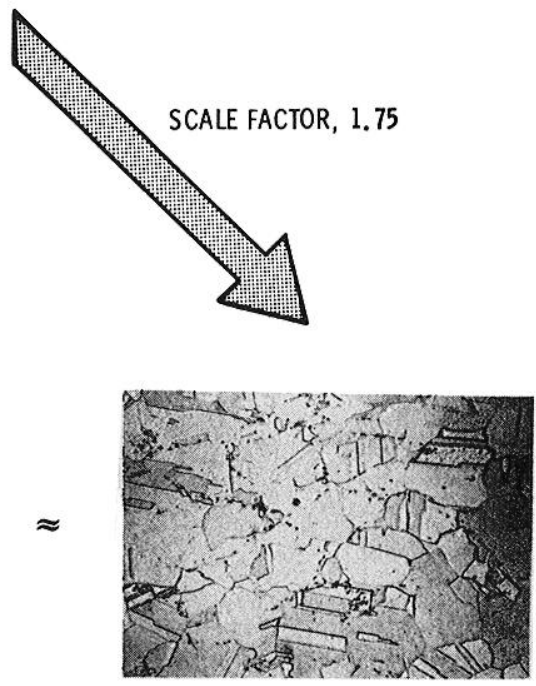

(c) 1.75 Magnification of figure 10(a).

Figure 10. - Photomicrographs of copper samples. 



\title{
ULTRASONIC MATERIAL PROPERTY DETERMINATIONS
}

\author{
Steven Serabian \\ University of Lowe 11 \\ Lowe 11, Massachusetts 01854
}

The use and potential offered by ultrasonic velocity and attenuation measurements to determine and/or monitor material properties is explored. The basis for such unique measurements along with examples of materials from a variety of industries will be presented.

\section{INTRODUCTION}

The ability of ultrasound to measure and/or monitor microstructure related properties has been established in the laboratory. A number of applications have been implemented for field use, however the full potential of such technologies has not been realized. Invariably one makes use of two of the propagational parameters of ultrasound - i.e., attenuation and velocity. The latter are intrinsic to the particular combination of material and processing under consideration. Absolute quantitative relations are usually unavailable, therefore empirical material property ultrasonic paràmeter correlations based upon qualitative reasoning are sought. In effect, a series of specimens containing the range of the material property of interest is necessary along with conventional destructive material property measurement schemes. obviously, such correlations are not universal and are only valid for the particular material and attendant processing being investigated. Correlation sensitivity is the prime factor that defines the accuracy and effectiveness of the generated correlation. Once the correlation has been established costly and time consuming conventional destructive evaluation procedures can be eliminated. In this paper a number of examples of correlations of significant material properties with ultrasonic parameters will be cited.

\section{VELOCITY BASED CORRELATIONS}

The original low pressure piping systems used by the utilities to transport gas were constructed of cast iron. Mechanical properties and susceptibility to corrosion renders cast iron undesirable for such an application. Economics precludes the total replacement 
by steel wraped pipes, thus one must resort to a gradual, scheduled replacement based upon a measure of quality. The determination of the necessary mechanical properties required the need for an extensive length of piping. This presented a costly as well as a limited number of pipe sections that can be evaluated during normal installation and maintenance programs. However, it is possible to trepan a $1-1 \frac{1}{2}$ " coupon without shutting down the piping system. The readily available large number of coupons would then present a greater sampling rate of the piping system. A program was initiated which would facilitate the use of the coupops for the determination of significant mechanical properties. (1) The latter along with other parameters such as soil condition, corrosion and road traffic activities are used to formulate pipe quality.

Industry wide practice consists of using the ASTM Talbot strip beam test as shown in Figure 1 to measure mechanical properties. The maximum deflection of the beam at fracture allows the measurement of the moduli of elasticity and rupture. Radiography was used to aid in the selection of the specimen location within any given pipe section to eliminate occasional structural anomalies such as voids and inclusions. Each beam specimen was characterized by velocity and attenuation profiles prior to the destructive phase, see Figure 2. The attenuation proved to be too sensitive to microstructure even at low frequencies. However, the velocity provided a consistent value for each specimen yet sufficient differences between specimens. It is for these reasons that velocity was selected as the ultrasonic parameter to be used in the correlations with material properties. The velocity measurement scheme involves locating the transmitted $R-F$ pulse on a calibrated time scale. The calibration is provided by using specimens with a range of thickness and a known velocity.

There is quantitative substantiation of a velocity-mechanical properties correlation provided by,

$$
\begin{aligned}
v_{L}^{2} & =\frac{E_{d}}{\rho} \frac{(1+\delta)}{(1+2 \delta)(1-\delta)} \\
& =\frac{E_{d}}{\rho} f(\delta)
\end{aligned}
$$

Where Ed is the dynamic modulus, $\hat{f}$ is the density and $\delta$ is Poisson's ratio. By noting that $f(\delta)$ is essentially unity for a brittle material such as cast iron and that there is a direct relationship between Ed and the secant modulus $\left(E_{s}\right)$, therefore equation 1 becomes,

$$
\mathrm{V}_{\mathrm{L}}^{2} \cong \frac{\mathrm{E}_{\mathrm{s}}}{\rho}
$$


For the correlation $V_{L}$ and density are taken adjacent to the fracture point $; V_{I}$ from ${ }^{L}$ a display typified by Figure 2 and density by the water $L$ displacement technique from a small section of the strip.

Figure 3 indicates the secant modulus. The data spread is $\pm 1 \times 10^{6}$ psi. At the midpoint of the modulus range considered this amounts to \pm 128 and is within the error associated with conventional destructive measurement procedures.

The other property of interest was the modulus of rupture (R). In a brittle material,

$$
E_{S}=\frac{R}{\varepsilon_{T}}
$$

Where $\varepsilon_{T}$ is the strain to failure. Since $\varepsilon_{\mathrm{T}}$ can only be measured destructively it was necessary to seek an indirect relationship involving a property that is more readily available. One such property is hardness $(\mu)$ or,

$$
\varepsilon_{\mathrm{T}}=\mathrm{c} \mu
$$

where $C$ is a constant. Incorporating Equations 3 or 4 into Equation 2 yields,

$$
R \approx \rho v_{L}^{2} \mu
$$

The hardness was measured adjacent to the fracture point. The results of this correlation is shown in Figure 4. The error for a modulus of rupture determination is $\pm 5,500$ psi. Viewing this error at the midpoint of the $R$ range translates into $\pm 10 \%$ which is a creditable determination.

Once the beam specimen correlations were established all the necessary measurements of density, hardness and ultrasonic velocity can be obtained from the parallel faced coupons. The need for the costly removable of large pipe sections and the associated destructive evaluations are eliminated. Moreover, the coupons, because of greater availability provides a more realistic overall assessment of the quality of the piping system and can be used as a guide for the pipe replacement program.

Concrete is another material that falls into the brittle category and is therefore susceptible to the determination of strength related properties by velocity based measurements. (2) 
A variety of water to cement ratios as well as curing times were considered. The cylindrical specimens were used for measurement of compressive strength and modulus while step wedges were used for the assessment of attenuation. Figures 5 and 6 indicate the results of the destructive phase for the compressive strength and secant modulus, respectively. The influence of the curing time and the water to cement ratio is as to be expected; both increase the compressive strength and the secant modulus. Figure 7 displays the axial longitudinal velocity as a function of the curing time and the water to cement ratio. The similarities between the data of Figures 5 and 6 with Figure 7 suggests that a correlation exists between these properties and ultrasonic velocity. Figure 8 shows the compressive strength correlation where it is significant to note that notations involving composition and curing time are lost. The accuracy ( \pm 500 psi) is within that expected by conventional destructive means. Much the same remarks are in order for the secant modulus correlation display as Figure 9 .

Some interesting observations regarding the state of cure can be noted by the use of attenuation. The attenuation in concrete is too excessive to measure by noting the decay of a multiple order back reflection. In this case the slope of an amplitude thickness display obtained by the step wedge specimen proved to be a valid technique. A torque wrench was used in a drill press arrangement to maintain a constant transducer pressure at each step. (1I) Figure 10 shows a typical determination for a water to cement ratio of 0.5 . It can be seen that the attenuation is high during the initial phase of cure. An attenuation decrease occurs as the structure becomes more rigid thus less energy is expended to propagate the ultrasound.

Another example of characterizing a material by velocity measurements is graphite of aerospace applications. (3) Figure 11 indicates a data display and serves to illustrate the sensitivity for density; the dynamic modulus was measured by resonant techniques. It should be noted that a variety of grades and velocity measuring directions are involved. Such a density sensitivity suggests that transport phenomena are susceptable to velocity correlations. Figure 12 supports this notion for the case of electrical resistivity.

\section{ATTENUATION BASED CORRELATIONS}

Let us now turn our attention to the use of attenuation for the characterization of materials; in particular to the polycrystalIine state. The functional dependency of the average grain size $(\bar{D})$ and frequency (f) upon attenuation is shown in Figure 13 where $\overline{\Delta K}$ is the direction related average change in elastic pro-

$\overline{\mathrm{K}}$ perties of the grains encountered by the ultrasound. (4) In Rayleigh scattering the whole grain acts as a scattering unit. 
For phase scattering one must be cognizant that each portion of the ultrasonic beam contends with a discrete set of grains, thus producing a variety of phases at the detector. The summation of these individual phases constitutes the phase scattering loss. In diffusion scattering one is essentially concerned with mean free path considerations - i.e., how many grain boundries are encountered. The dependency of these loss mechanics upon grain size and frequency can be shown experimentally. Figure 14 displays typical data for a steel that has been heat treated to obtain a range of grain size. The deviation from linearity at the lower frequencies for the less attenuating specimens is due to transducer beam spreading or diffraction losses. Appropriate corrections would, in effect, reduce each data point in this region to the linear extrapolated high frequency data. The slope of each attenuation - frequency display is the exponential frequency dependency $(n)$. Figure 15 shows the frequency exponent for a variety of materials with different grain sizes. (5) The spread in the data is primarily due to the fact that each specimen can have a range of grain size, thus more than one type of scattering mechanism is in effect.

By cross plotting the data of Figure 14 one can note the grain size dependancy (m) of attenuation. See Figure 16. Figure 17 shows such data for a number of materials and indicates that the grain size dependancy can range from zero to three. The data set is not capable of examining the diffusion type of loss $(m=-1)$.

Of importance for this discussion is the grain size dependency (m). Since for most engineering materials/structures the upper frequency limitation for propagation restricts scattering to the Rayleigh $(m=3)$ and phase $(m=2)$ loss mechanisms, thus attenuation is quite sensitive to grain size. One obvious use of this fact is the measurement of grain size. Figure 18 indicates the observed attenuation in a Timkin Alloy $(16-26-6)$ for a range of grain size. If the data is plotted in terms of the ratio of the average grain size to the dominate wavelength of a narrowband pulse of ultrasound, a single curve is obtained (Figure 19). This is in accordance with Roney's generalized scattering theory that allows one to consider attenuation in terms of a dimensionless parameter that obviates the need to consider both the grain size and wavelength individually. By making attenuation measurements at a variety of frequencies one can obtain an overall grain size determination.

The sensitivity of attenuation to grain size suggests the feasibility of using attenuation to measure any material property that is grain size dependent. Figures 20 and 21 indicate the

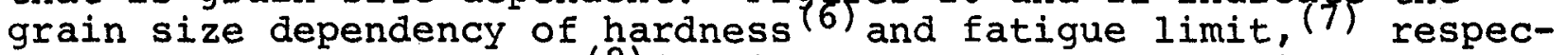
tively. The data of Low $(8)$ in Figure 22 shows the grain size dependency for strain in failure as well as fracture and yield stresses. As shown in Figure 23 Klinman(9) has utilized the grain size dependency of attenuation to determine transition temperature for a spectrum of carbon steels.

In addition to grain structure dependency attenuation is also 
influenced by other scatters such as porosity and inclusions of microscopic proportions. Such scattering losses contribute to the observed attenuation. Figure 24 attests to the sensitivity of attenuation to porosity in grain refined aluminum. (10) A uniform grain size throughout the specimen set assures that porosity is the dominate cause of the attenuation variations. The porosity, as verified in Figure 25, should effect the ultimate tensile strength and be measureable by attenuation.

Silicate inclusions are prevalent in 303 stainless steel and provides an example of microscopic inclusions acting as scatter sites. The specimens considered contained approximately the same grain size thus did not contribute to the observed attenuation variations. Inclusion counts were performed by a quantitative television microscope and were rated in accordance with length in six levels from 2 to 12 microns in 2 micron intervals. Using a weighting function of $\mathrm{n}^{2}$ to bring out the influence of the larger inclusions a measure of the inclusion severity was taken as $\sum^{2} n^{2} \mathrm{Sn}$ where $\mathrm{Sn}$ is the number of inclusions in the $\mathrm{n}$ th counting level. The results are shown in Figure 26.(12) As to be expected the higher frequencies present a greater sensitivity to the inclusion count. Such data can be used to quality grade the material.

Another example of inclusion produced attenuation is displayed in Figure 27. The structure is a large rotor forging used in large steam turbines. The center is the last to solidify, thus is the major recipient of the impurities. It is for this reason that a small borehole is cored out. The data shows the attenuation as a function of the axial length. The attenuation is measured by the decay in a multiple order bore refraction display and subsequently corrected for geometric effects originating from the curved surfaces encountered. It can be seen by the solid line that the initial measurement indicated an axial region that showed abnormally high attenuation. This was also verified by an attenuation measurement of the core material from this region as well as magnetic particle inspection of the bore surface. It was decided to subject the rotor to a minimal overboring operation. As evident by the dotted line display the attenuation decreased appreciably in the region of high inclusion count while the attenuation in other regions remained essentially unchanged. The latter lends credance to the notion that the high inclusion count was localized. While this example is highly qualitative it does serve to indicate that attenuation is susceptable to scattering losses due to microscopic inclusions.

\section{SUMMARY AND CONCLUSIONS}

Examples of the utilization of ultrasonic velocity and attenuartion for the measurement and monitoring of material properties have been presented. It is evident that a wide spectrum of activities in a number of industries are involved. It is significant to note that the measurement schemes and attendant instrumentation 
have, in general, minimal requirements of sophistication and complexity.

\section{REFERENCES}

1. S. Serabian and G. E. Lockyer, "Nondestructive Evaluation of Cast Iron Pipe" Univ. of Lowell Research Foundation, April 1971.

2. S. Serabian and J. Tsererides, "Property Measurements of Concrete", presented at annual ASNT meeting, 1972.

3. G. E. Lockyer, "Investigation of Nondestructive Methods for the Evaluation of Graphite Materials", Air Force Materials Laboratory, TR-65-113! 1965.

4. E. P. Papadakis, "Ultrasonic Attenuation Casued by Scattering in Polycrystalline Media", in Physical Acoustics (Volume IVB); W. P. Mason, Editor, $196 \overline{8}$.

5. S. Serabian, "Frequency and Grain Size Dependency of Ultrasonic Attenuation in Polycrystalline Materials", Brit. $\mathrm{J}$. of NDT, 22, 2, 69, 1980 .

6. R. W. Armstrong, "The Influence of Polycrystalline Grain Size on Several Mechanical Properties", Met. Trans. ASM, 44, 929, 1952 .

7. G. M. Sinclair and W. J. Craig, Trans. ASM, 44, 929, 1952 .

8. J. R. Low, "The Relation of Microstructure to Brittle Fracture", ASM, 1954.

9. R. Klinman, R. et al, "Ultrasonic Prediction of Grain Size, Strength and Toughness in Plain Carbon Steel", Material Evaluation, 38, 26, 1980 .

10. K. Chinnathambi and O. Prabhaker, "Quality Control of Aluminum Alloy Castings", Trans. Indian Institute Metals, 33, 4, 296, 1980 .

11. S. Serabian, "Influence of Attenuation Upon the Frequency Content of A Stress Wave Packet in Graphite", J. Acoust. Soc. Am., 42, 5, 1052, 1967.

12. S. Serabian, "Inclusion Count Determinations by ultrasonic Attenuation Measurements", Armco Steel Corp., Middletown, Ohio, Dec. 1976. 

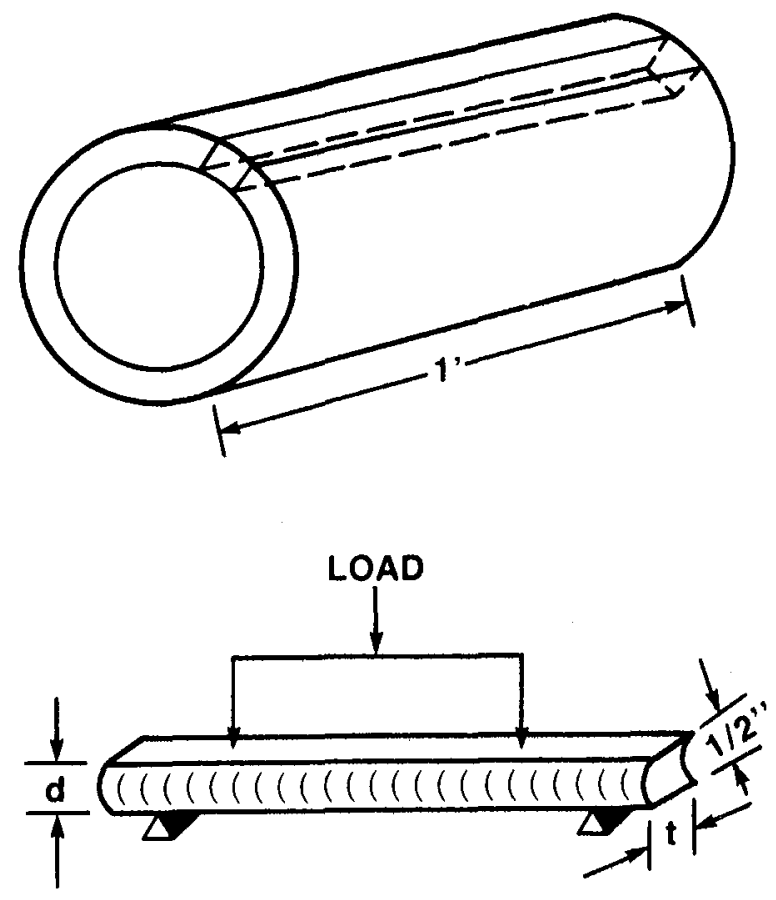

Fig 1 Talbot strip test.

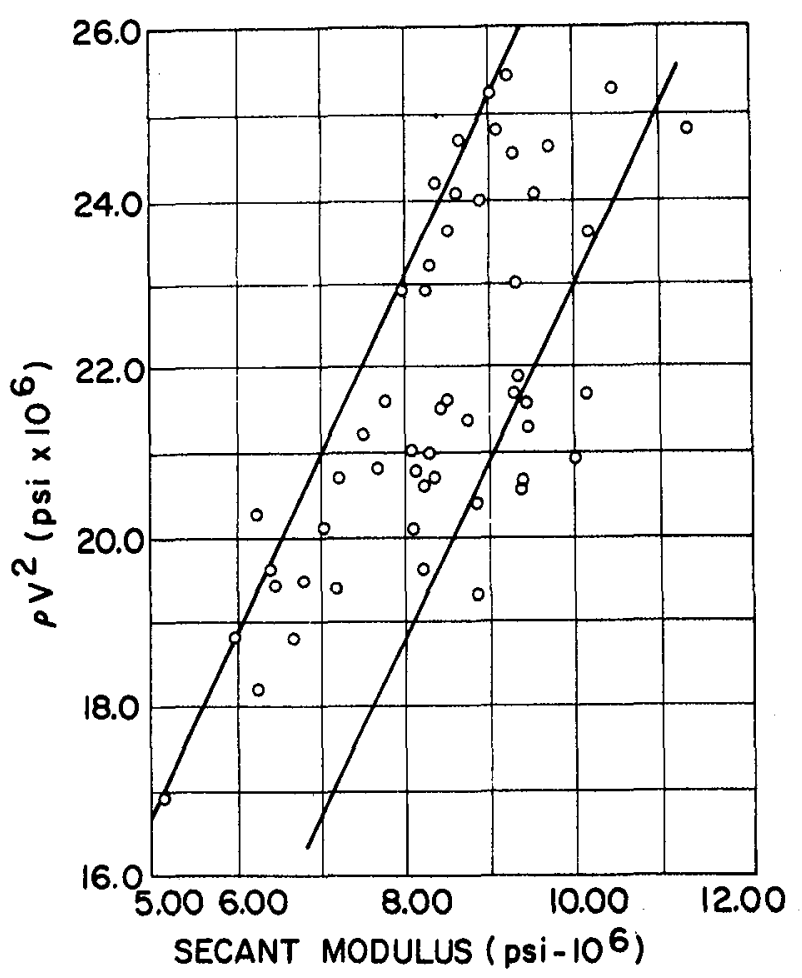

Fig 3 Secant modulus correlation in cast iron.
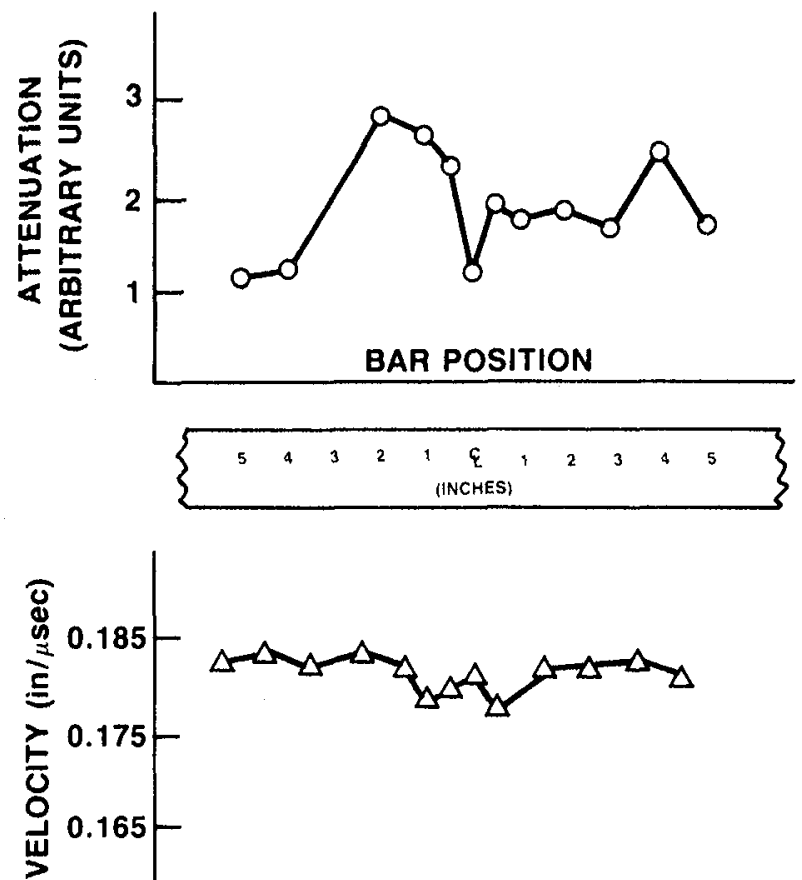

BAR POSITION

Fig 2 Velocity/attenuation characterization of each specimen.

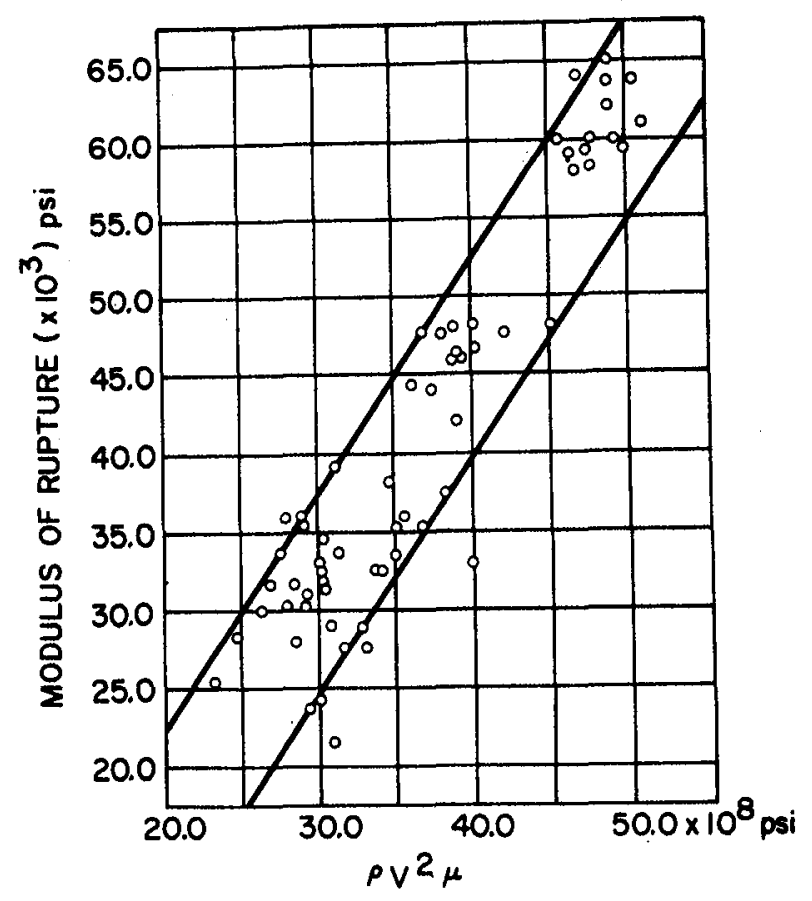

Fig 4 Modulus of rupture correlation in cast iron. 


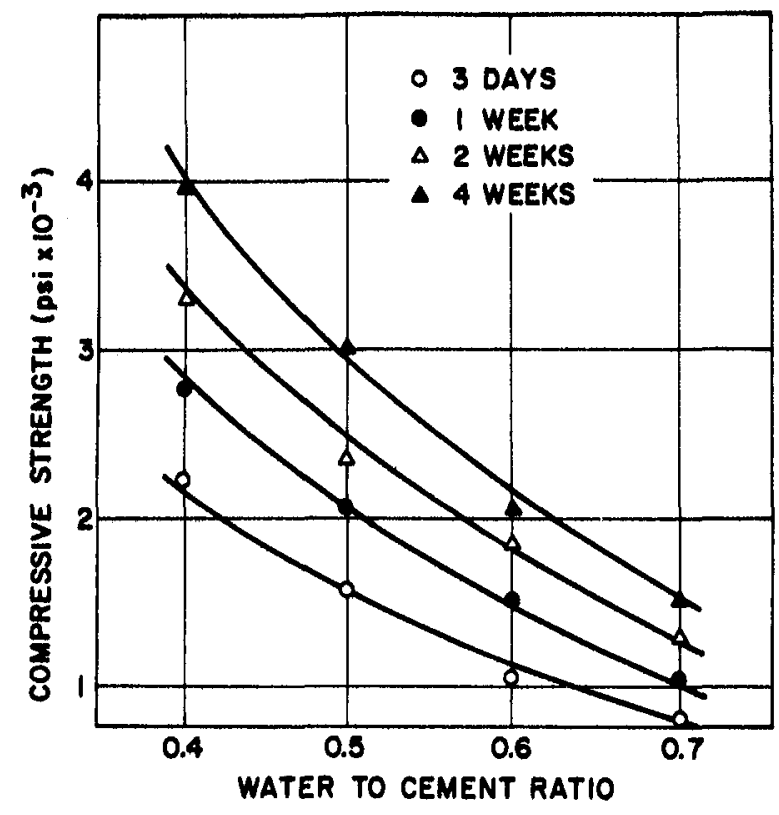

Fig 5 Concrete compressive strength vs water to cement ratio

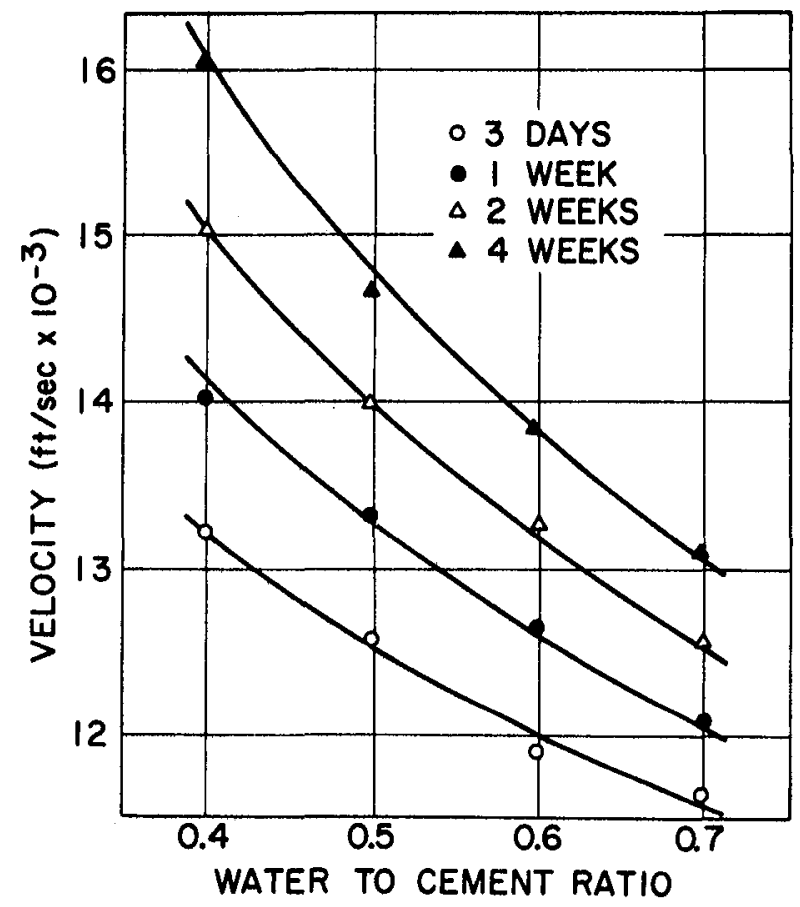

Fig 7 Compressive strength/velocity correlation in concrete.

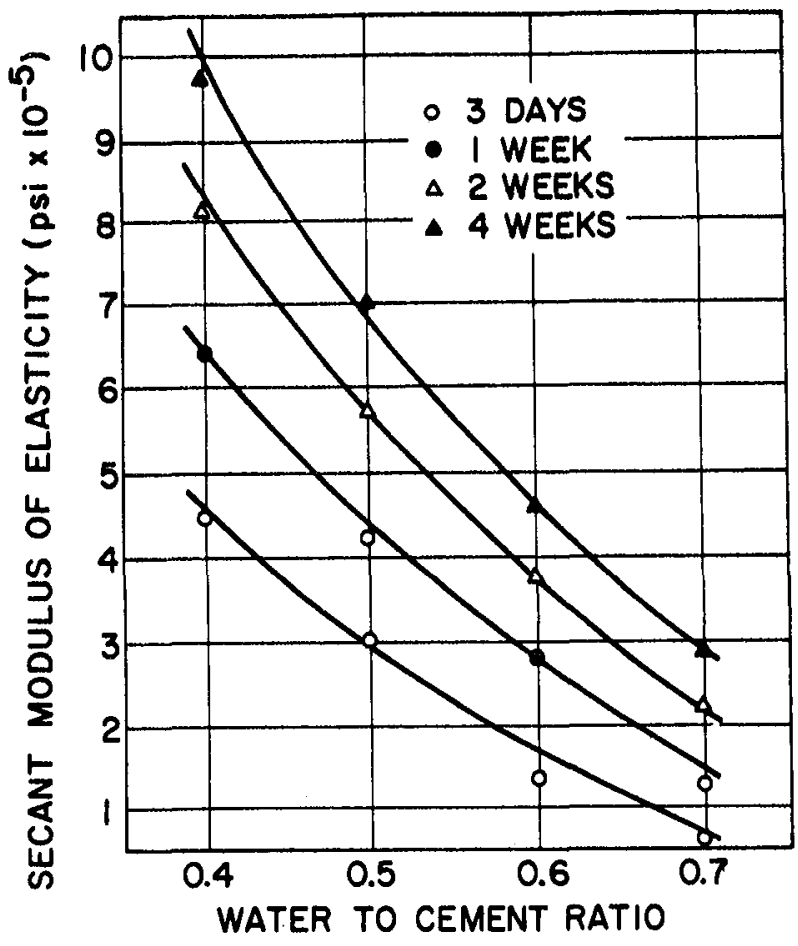

Fig 6 Concrete secant modulus vs water to cement ratio

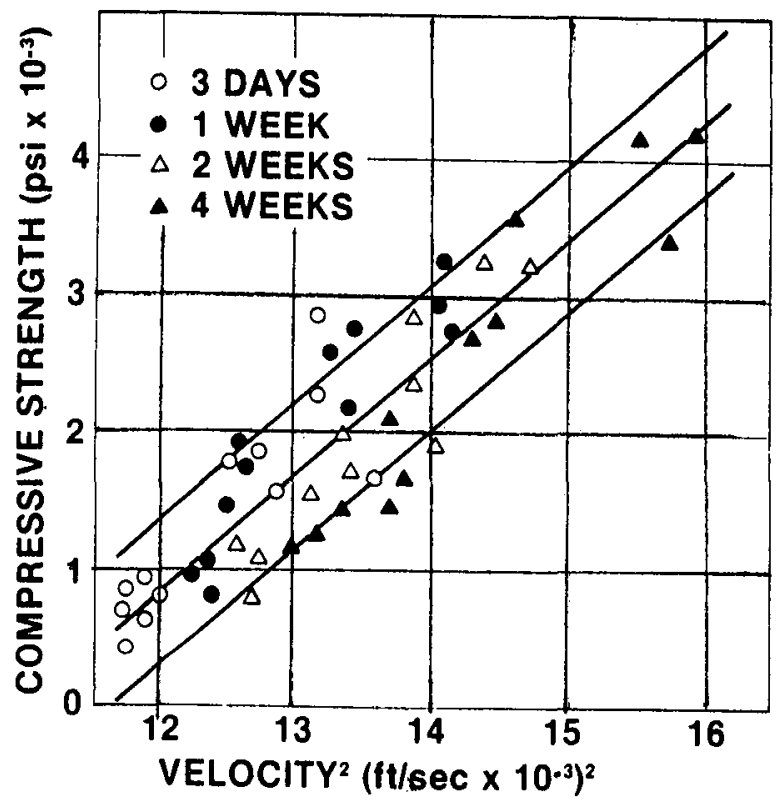

Fig 8 Secant modulus/velocity correlation in concrete. 


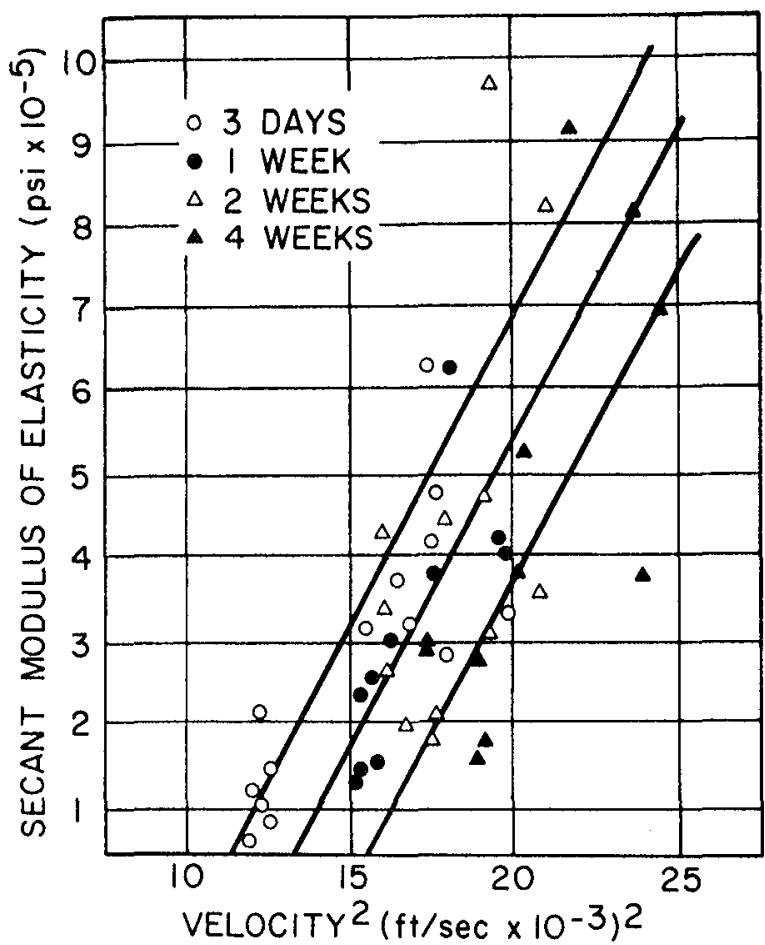

Fig 9 Secant modulus/velocity correlation in concrete.

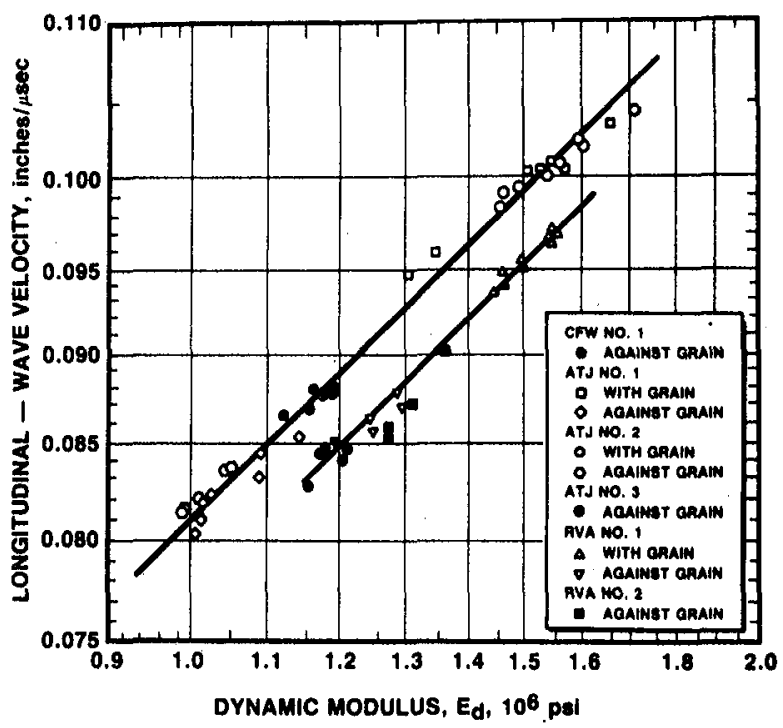

Figd Velocity/modulus correlations in graphite. Densities are $1.72 \& 1.85$ $\mathrm{gm} / \mathrm{cm}^{3}$ for the top and bottom plots, respectively,

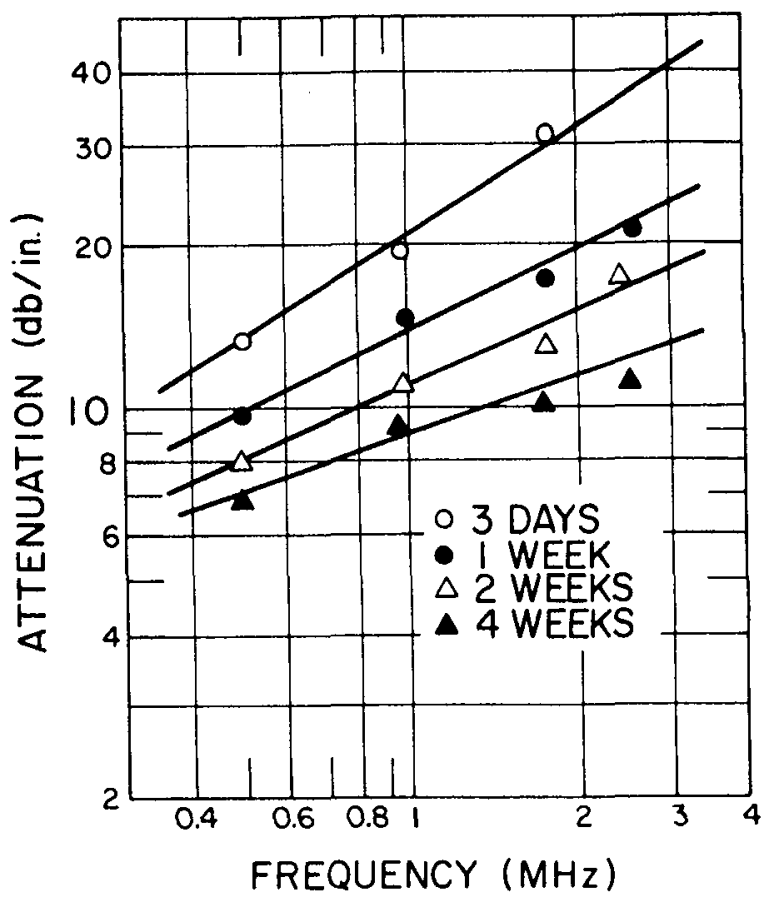

Fig 10 Attenuation monitoring of cure in concrete with a water to cement ratio of 0.5 .

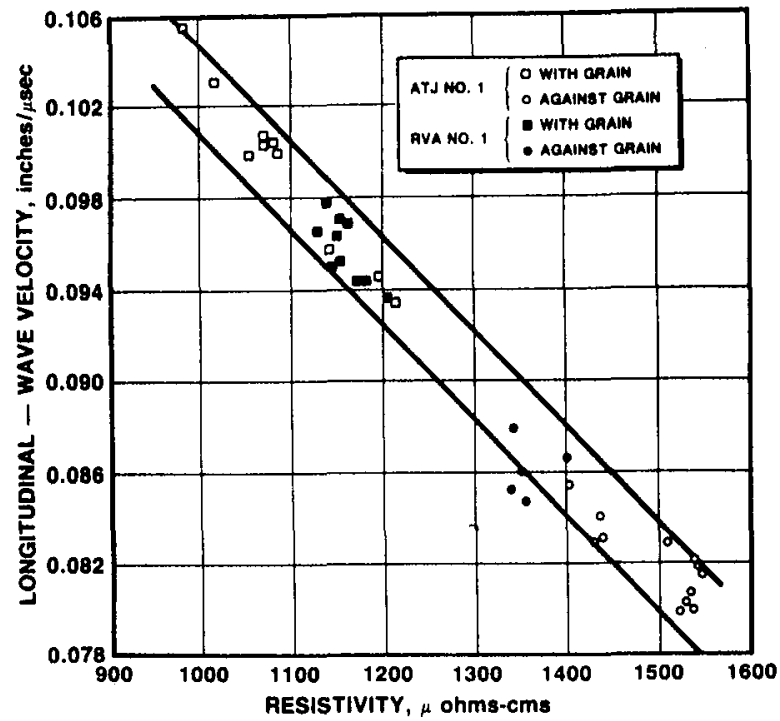

Fig 12 Velocity/resistivity correlation in graphite. 


\begin{tabular}{|c|c|c|}
\hline $\begin{array}{c}\text { WAYELENGTH TO } \\
\text { GRAIN DIAMETER } \\
\text { RANGE }\end{array}$ & MECHANISH & ATTENUATION \\
\hline$\lambda>>\bar{D}$ & RAYLEIGH & $\overline{\left(\frac{\Delta K}{K}\right)^{2}} \bar{D}^{3} \mathrm{Q}^{4}$ \\
$\lambda-\bar{D}$ & PHASE & $\overline{\left(\frac{\Delta K}{K}\right)^{2}} \bar{D}^{2}$ \\
$\lambda<<\bar{D}$ & DIFFUSION & $\overline{\left(\frac{\Delta K}{K}\right)^{2}} \frac{1}{\bar{D}}$ \\
\hline
\end{tabular}

Fig 13 Ultrasonic scattering losses.

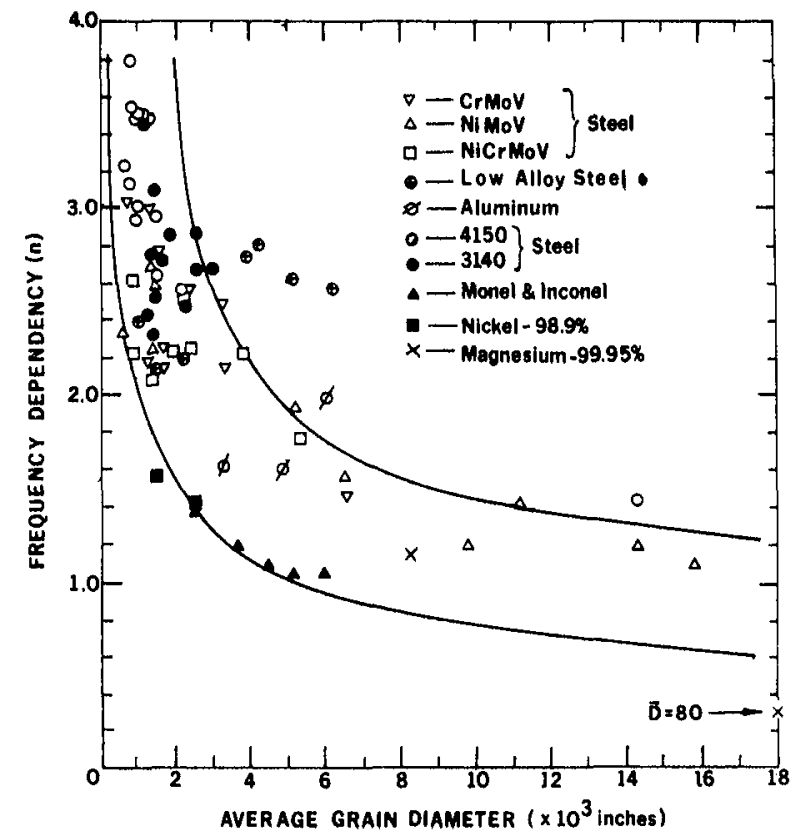

Fig 15 Frequency dependency as function of grain size.

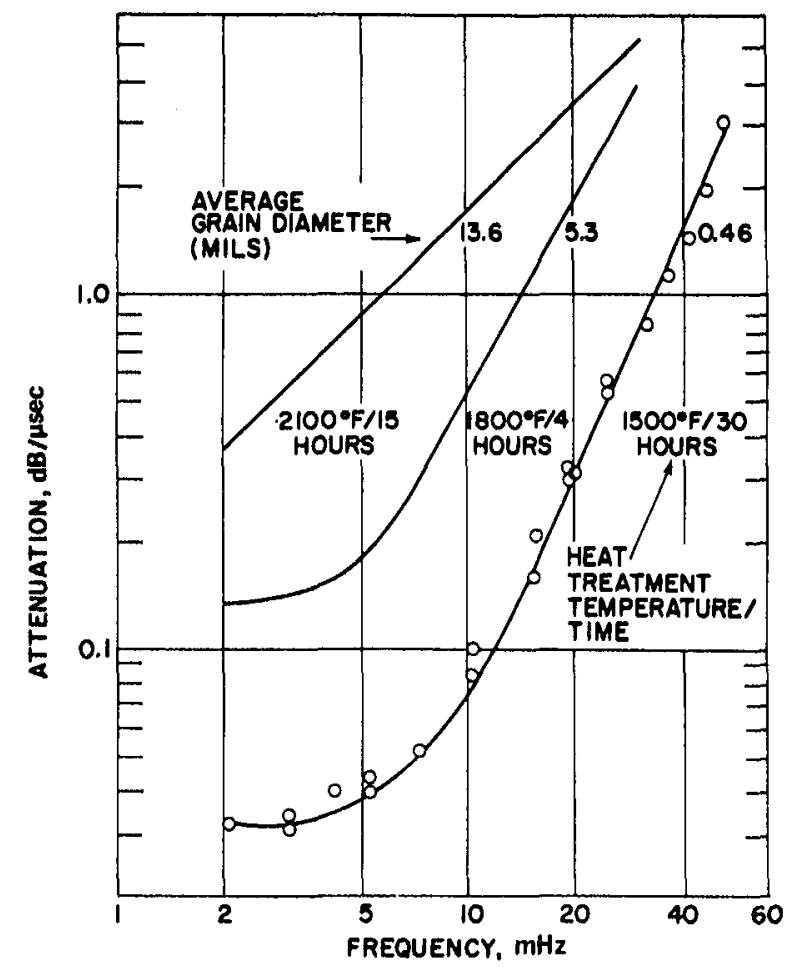

Fig 14 Attenuation/grain size effects.

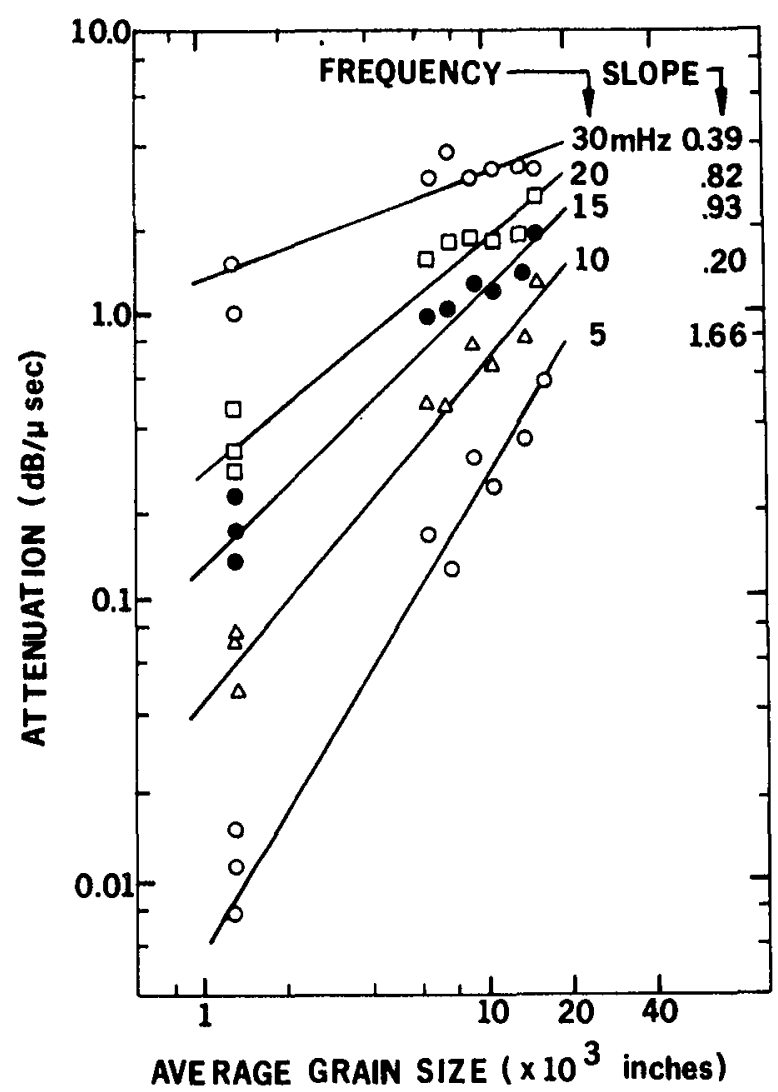

Fig 16 Cross plot of attenuation/grain size data typified by Fig 14. 


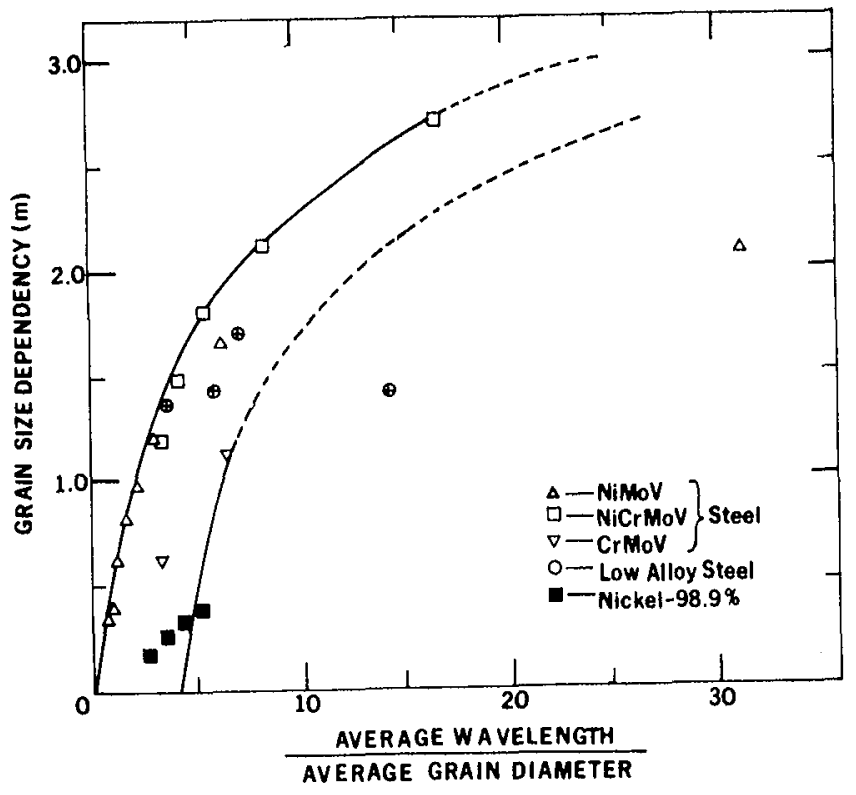

Fig 17 Grain size dependency of

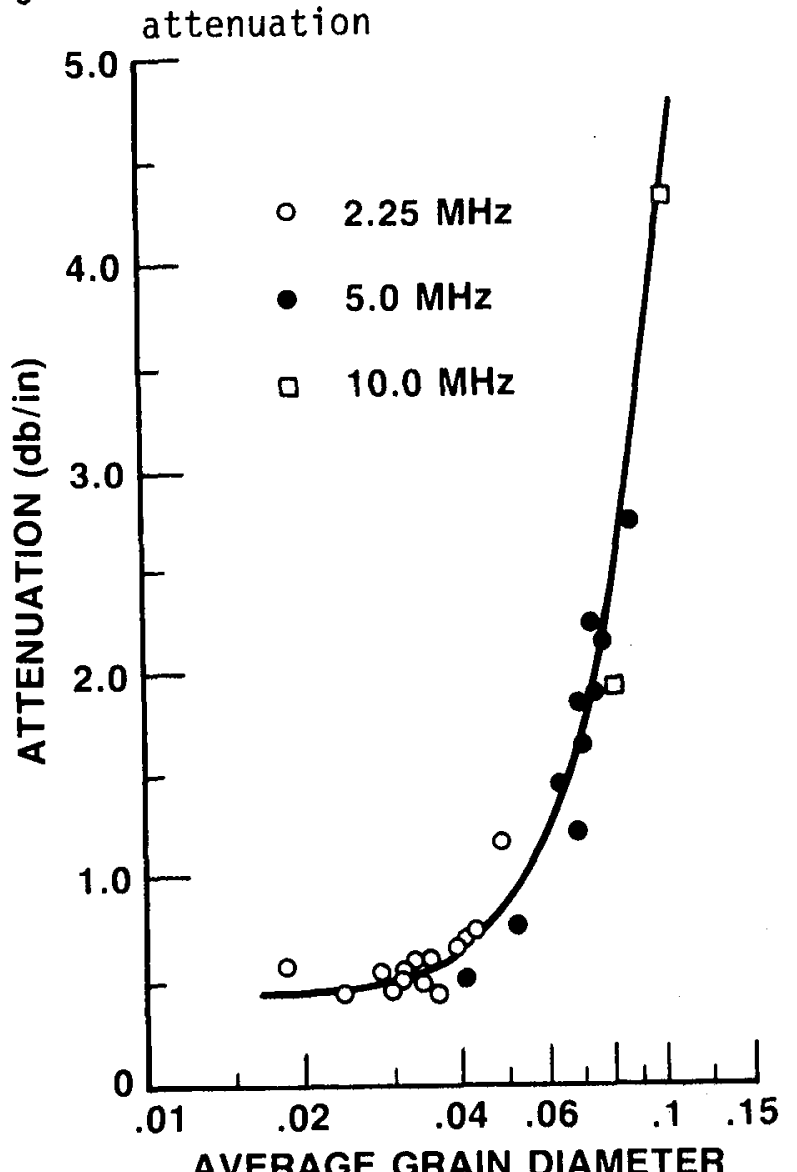

AVERAGE GRAIN DIAMETEB.

\section{WAVELENGTH}

Fig 19 Attenuation as a function of the ratio of the average grain size to wavelength.

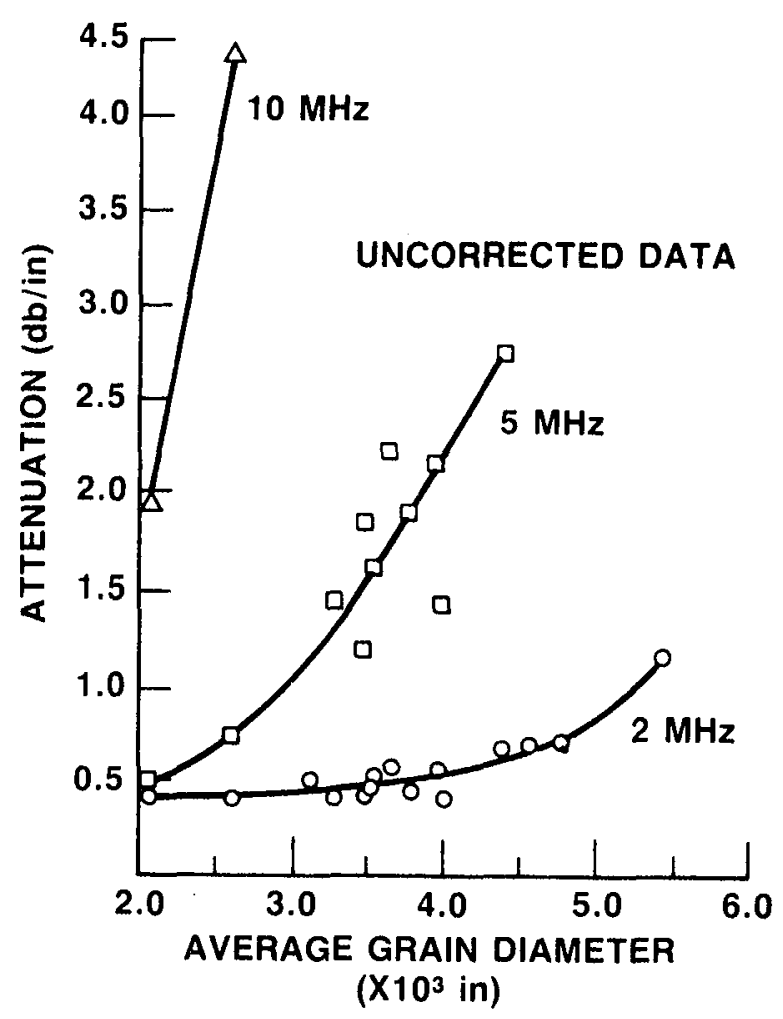

Fig 18 Attenuation/grain size for a Timkin alloy $(6-26-16)$

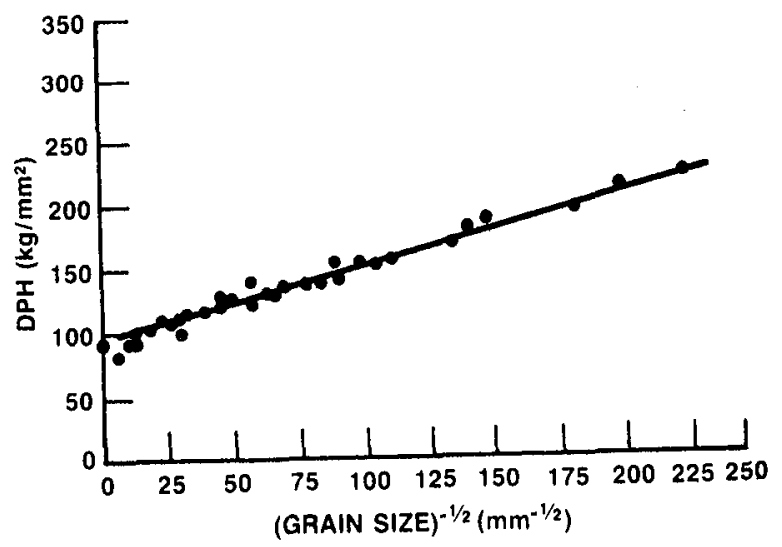

Fig 20 Hardness vs grain size in in titanium. After Armstrong (6) 


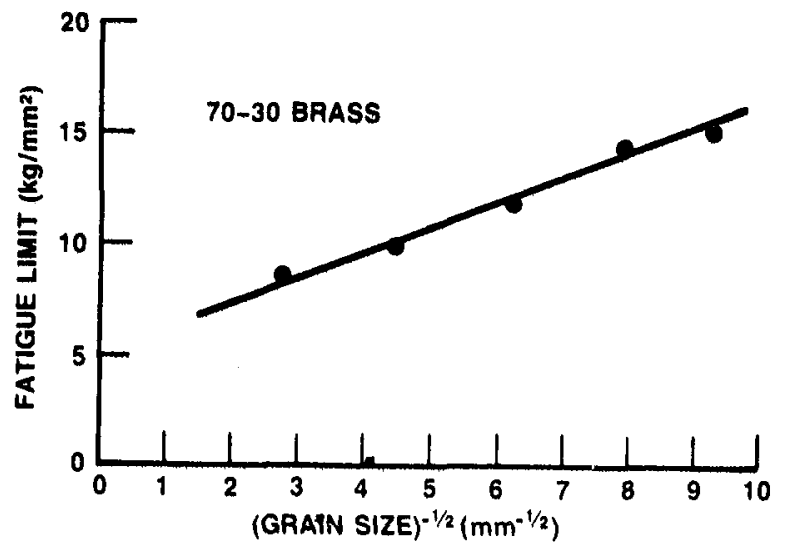

Fig 21 Fatique limit in $70-30$ brass. After Sinclair and Craig (7).

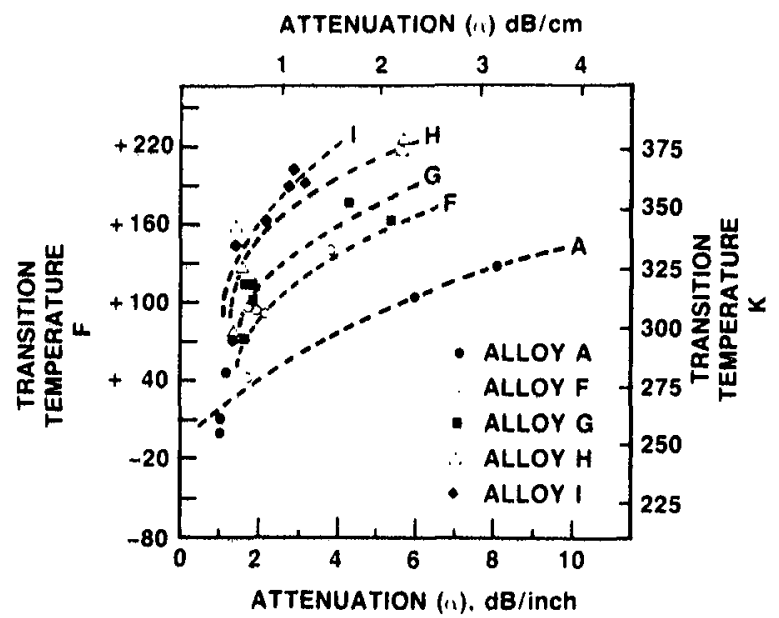

Fty 23 Attenuation monitoring of the transsition temperature of carbon steels. Carbon increases the transition temperature. After Klineman et al (9)

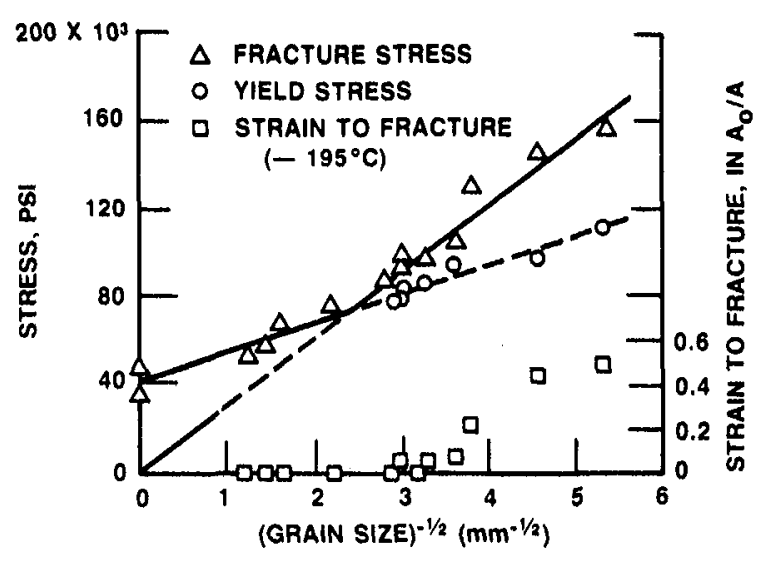

Fig 22 Influence of grain size on various properties of low carbon steels. After Low (8)

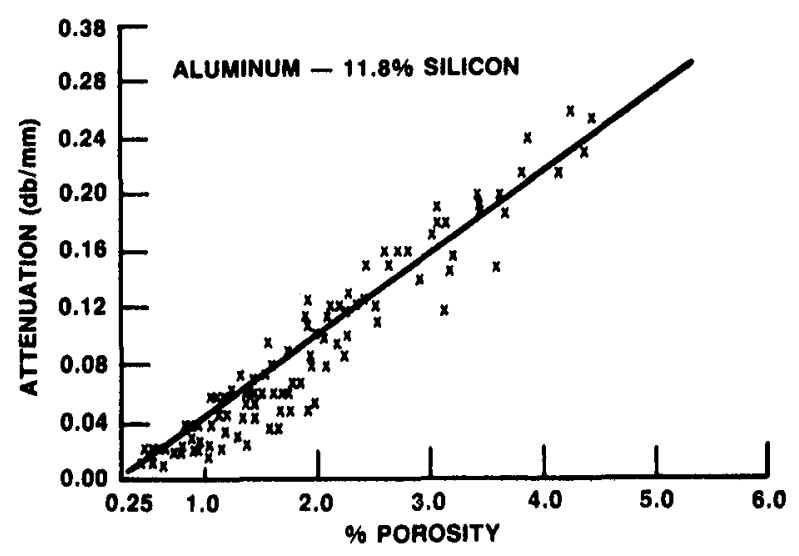

Fig 24 Attenuation/porosity correlations. After Chinnathambi and Prabhak (10). 


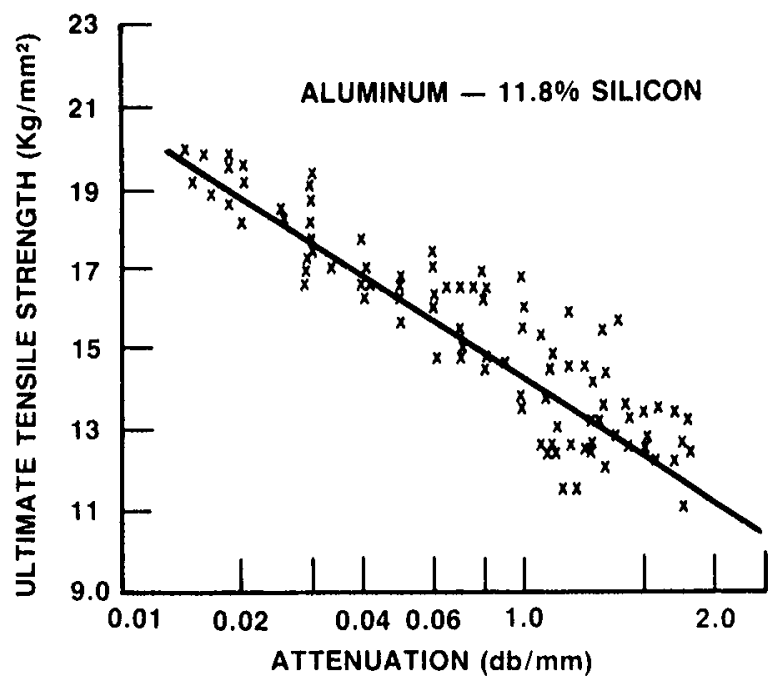

Fig 25 Attenuation monitoring of ultimate tensile strength. After Chinnathambi and Prabhak (10)

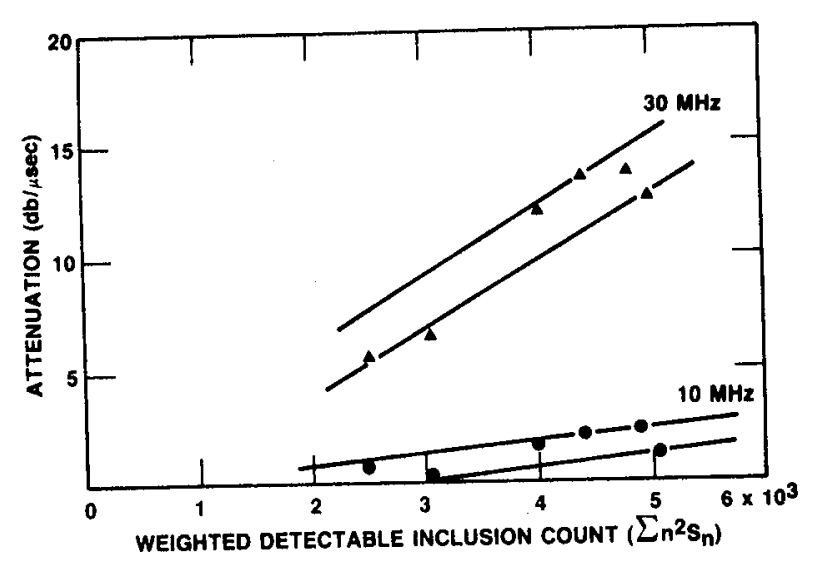

Fig 26 Attenuation monitoring of inclusion content in stainless steel.

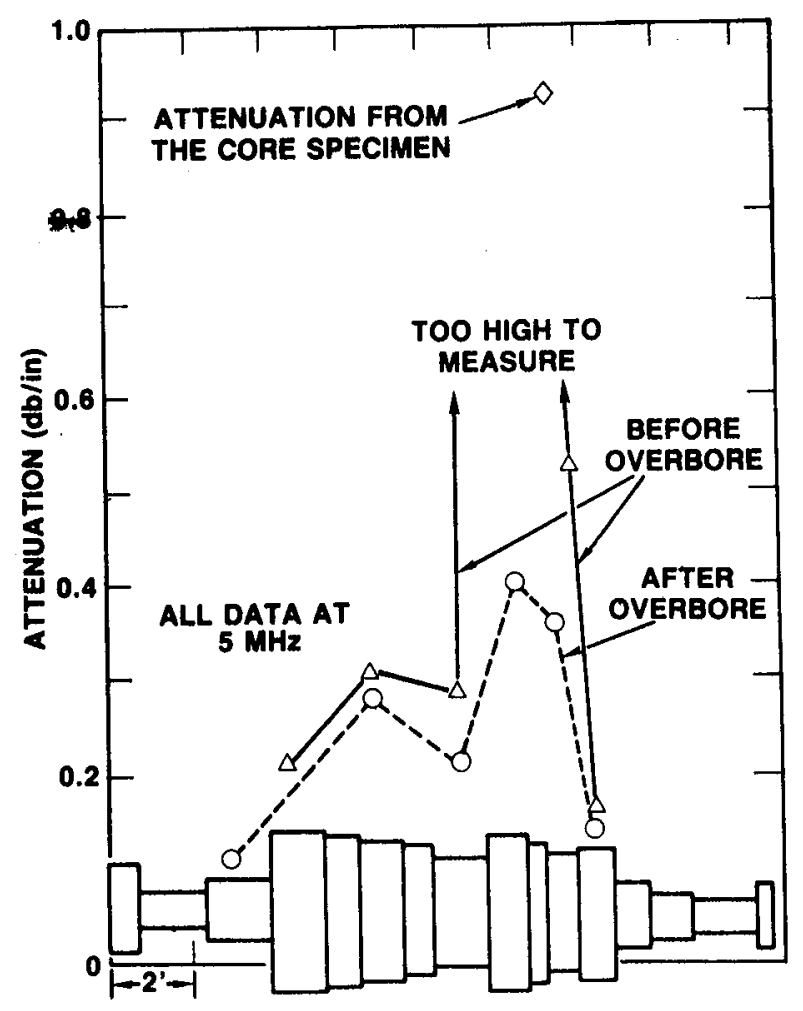

Fig 27 Monitoring of inclusion content in large rotor forgings. 


\title{
DYNAMIC MODULI AND LOCALIZED DAMAGE IN COMPOSITES
}

\author{
L.S. Fu \\ The Ohio State University \\ Columbus, Ohio 43210
}

The alm in nondestructive evaluation (NDE) by sound and ultrasound is to extract material and fracture properties from pulses sent by and recelved at transducers. The analysis is usually given either in the time domain or the frequency domain. The introduction of the size factor, the wavelength, in the model mechanics problems in NDE plays the important role of relating the far-field measurable quantities such as phase velocities and attenuation to the near-field physical situation such as local geometric dimensions and elastic properties. This aspect of the analysis thus allows "nondestructive" testing methods to be employed for "experimental" validation of the predictions by the theories (refs. 1 to 3 ).

Solution to the elastic wave scattering due to a single embedded inhomogeneity is available by different methods that are appropriate at different frequency ranges. The methods that offer a solution in an analytic form and are useful for inhomogeneous media with multiple components are the long-wave approximation, the polarization approach, and the extended method of equivalent inclusion (refs. 4 and 5 ).

Several averaging schemes or theorems exist in the literature for finding the dynamic effective moduli and mass density. Some efforts concentrate on the average stress $\mathscr{\sigma}$ and strain $\varepsilon$ fields or on the average displacement field $\underset{\sim}{u} ;$ others use a variational approach. These theories are appropriate mostly at Rayleigh or longwave limits and do not exhibit dispersive effects. Dispersiveness and attenuation are important in evaluating dynamic material properties.

This study dealt first with the scatter of elastic waves due to a thin, flat ellipsoidal inhomogeneity, either penny shaped or elliptical. An average theorem appropriate for dynamic effective mass density and effective moduli was developed via a self-consistent scheme. Effective material properties of two-component media consisting of randomly distributed spheres are given here as a special case.

\section{PRELIMINARIES}

Displacement Field due to Presence of Mismatch

The inhomogeneous media considered in this paper are assumed to consist of a homogeneous matrix of elastic moduli $C^{0}$ and mass density $\rho^{0}$ and a distribu. tion of inhomogeneities with moduli $\underset{\sim}{\sim}(r)$ and mass density ${ }_{p}(r)$ occupying regions $\Omega_{r}, r=1,2, \ldots, n$ (fig. 1). The total displacement field $\underline{u}$ can be separated into two parts.

$$
\underline{u}=\underline{u}^{(i)}+\underline{u}^{(m)}
$$

where the superscripts ( $i$ ) and ( $m$ ) denote "incident" and "mismatch, "respectively. It is clear that when no mismatch components (i.e., inhomogeneities) are present, 
the total displacement field is entirely the same as the incident displacement wave field. On the other hand, if there is no incident wave field, the only field that exists is the null field.

\section{Eigenstrains}

The eigenstrains $\varepsilon^{*}$, which are also termed transformation strains, are defined as the part of the total strain $\varepsilon$ that must be subtracted before the remaining part can be related to stresses $\underset{\sim}{\sim}$ through Hooke's law

$$
\begin{gathered}
\varepsilon_{r s}^{*}=\varepsilon_{r s}-\varepsilon_{r s}^{e} \\
\varepsilon_{r s}^{e}=c_{j k r s}^{-1} \sigma_{j k}
\end{gathered}
$$

where $\mathcal{C}^{-1}$ are the elastic compliances. The method of equivalent inclusion is a method that allows the inhomogeneous media to be replaced by media of homogeneous matrix effective moduli with distributed transformation strains in the regions originally occupied by inhomogeneities; hence

$$
\varepsilon_{r s}^{*}= \begin{cases}0 & \text { in } \Omega_{0} \\ \varepsilon_{r s}^{*} & \text { in } \Omega_{r}, r=1,2, \ldots, n\end{cases}
$$

For the two problems to be equivalent, the transformation strains must give rise to a field that is exactly the same as the "mismatch" field $\underset{\sim}{\mathrm{u}}(\mathrm{m})$. This leads to equivalence conditions that ensure that identical field quantities at any given point will be obtained in the two problemst (ref. 4):

$$
\begin{aligned}
& \Delta C_{j k r s} u_{r, s}^{(m)}(\bar{r})+C_{j k r s} \varepsilon_{r s}^{*}(1)(\bar{r})=-\Delta C_{j k r s} u_{r, s}^{(1)}(\bar{r}) \quad \text { in } \Omega \\
& \Delta \rho \omega^{2} u_{j}^{(m)}(\bar{r})+c_{j k r s} \varepsilon_{r s, k}^{*(2)}(\bar{r})=-\Delta \rho \omega^{2} u_{j}^{(1)}(\bar{r}) \quad \text { in } \Omega
\end{aligned}
$$

Two types of transformation strains, or eigenstrains, arise in elastodynamic situations due to the mismatch in elastic moduli $\Delta c$ and mass density $\Delta \rho$. It is often convenient and useful to define associated quantities such as

$$
\begin{gathered}
m_{j k}=c_{j k r s}{ }^{*}{ }_{r s}(1) \\
\pi_{j}=c_{j k r s^{\varepsilon} r s, k}{ }^{*(2)}
\end{gathered}
$$

where $m_{j k}$ and $\pi_{j}$ are moment density tensor and equivalent force or eigenforce, respectively.

the conditions (eqs. (5) and (6)) are similar to those of willis (1980) and those of Mura, Proc. Int. Conf. on Mechanical Behavior of Materials, $\underline{5}$, Socjety of Materials Science, Japan, 12-18 (1972). 
The volume average and time average of a field quantity, say $F(r, t)$, are denoted by using brackets \langle\rangle$_{V}$ and \langle\rangle$_{T}$, respectively, and are defined as

$$
\begin{aligned}
& \langle F(\underline{r}, t)\rangle=\frac{1}{V} \int F(\underline{v}, t) d V \\
& \langle F(\underline{r}, t)\rangle=\frac{1}{T} \int F(\underline{r}, t) d T
\end{aligned}
$$

where $V$ and $T$ are volume and time period, respectively, and $\underline{v}$ is velocity field.

\section{Volume Integrals of Ellipsoid Associated with Inhomogeneous Helmholtz Equation}

Volume integrals of an ellipsoid associated with the integration of the inhomogeneous Helmholtz equation are used in this work. The inhomogeneous scalar Helmholtz equation takes the form

$$
\nabla^{2} \Phi+k^{2} \Phi=-4 \pi \gamma(\underline{r})
$$

where $r(\underline{r})$ is the source distribution or density function and $\nabla^{2}$ and $k$ are the Laplacian and wave number, respectively. A particular solution to equation (11) is

$$
\Phi(\underline{r})=\int_{\Omega} Y\left(\underline{r}^{\prime}\right) R^{-1} \exp (i k R) d V^{\prime}, R=\left|\underline{r}-\underline{r}^{\prime}\right|
$$

in which $(4 \pi R)^{-1} \exp (i k R)$ is the steady-state scalar wave Green's function and $\Omega$ is the region where the source is distributed. The source distribution function $r(\underline{r})$ can be expanded in basic functions or polynomial form, depending on the geometry of the volumetric region $\Omega$. For an ellipsoidal region, the choice of using a polynomial expansion separates this work from other theories of elastic wave scattering:

$$
\gamma\left(\underline{r}^{\prime}\right)=\left(x^{\prime}\right)^{\lambda}\left(y^{\prime}\right)^{\mu}\left(z^{\prime}\right)^{v}
$$

in which $\lambda, \mu$, and $v$ are integers and $k$ is either longitudinal or transverse wave number.

For elastic wave scattering in an isotropic elastic matrix, two types of volume integrals and their derivatives must be evaluated: The $\psi$-integrals are given by

$$
\begin{aligned}
\psi(\underline{r}) & =\int_{\Omega} R^{-1} \exp (i \alpha R) d V^{\prime} \\
\psi_{k}(\underline{r}) & =\int_{\Omega} x_{k}^{\prime} R^{-1} \exp (i \alpha R) d V^{\prime} \\
\psi_{k \ell \ldots s}(\underline{r}) & =\int_{\Omega} x_{k}^{\prime} x_{\ell}^{\prime} \ldots x_{s}^{\prime} R^{-1} \exp (i \alpha R) d V^{\prime}
\end{aligned}
$$




$$
\begin{aligned}
& \psi,_{p}(\underline{r})=\frac{\partial}{\partial x_{p}} \psi(\underline{r}) \\
& \psi_{k, p}(\underline{r})=\frac{\partial}{\partial x_{p}} \psi_{k}(\underline{r})
\end{aligned}
$$

where $\alpha^{2}=\omega^{2} / V^{2}=\rho \omega^{2} /(\lambda+2 \mu)$. The other type, the $\phi$-integrals, are obtained by replacing $\alpha$ with $\beta$ in equations (12) and (13), where $\beta^{2}=\rho \omega^{2} /(\mu)$. Details of the integration are given in reference 6 . In this work, only the limiting values at $r \rightarrow 0$ and $r \rightarrow \infty$ are of interest.

\section{ISOLATED, FLAT ELLIPSOIDAL INCLUSION}

Formulation

Consider the physical problem of an isolated inhomogeneity embedded in an infinite elastic solid that is subjected to a plane time-harmonic incident wave field as depicted in figure 1. Replacing the inhomogeneity with the same material as that of the surrounding medium, with moduli $c_{j k r s}$ and mass density $\rho$, and including in this region a distribution of eigenstrains and eigenforces, the physical problem is now replaced by the equivalent inclusion problem.

The total field is now obtained as the superposition of the incident field and the fleld induced by the presence of the mismatches in moduli and in mass density written in terms of eigenstrains $\varepsilon_{i j}^{*}(1)$ and eigenforces $\pi_{j}^{*}$

$$
\underset{\sim}{F}={\underset{\sim}{F}}^{(i)}+\underset{\sim}{F}(m)
$$

where $\underset{F}{ }$ denotes either the displacement field $u_{j}$, the strain field $\varepsilon_{i j}$, or the stress field $\sigma_{i j}$.

For uniform distributions of eigenstrains and eigenforces, the fields can be obtained as

$$
\begin{gathered}
u_{m}^{(m)}\left(\underline{r}^{\prime}\right)=-\pi_{j}^{*} S_{j m^{\prime}}\left(\underline{r}^{\prime}\right)-c_{j k r s^{\varepsilon}}^{*}(1) S_{j m, k^{\prime}}\left(\underline{r}^{\prime}\right) \\
\varepsilon_{m n}^{(m)}=\left[u_{m, n}^{(m)}+u_{n, m}^{(m)}\right] / 2 \\
\sigma_{p q}^{(m)}=c_{p q m n} \varepsilon_{m n}^{(m)}
\end{gathered}
$$

where a comma denotes partial differentiation and

$$
S_{j m}(\underline{r})=\int_{\Omega} g_{j m}\left(\underline{r}-\underline{r}^{\prime}\right) d V^{\prime}
$$

in which $g_{j m}$ is the spatial part of the steady-state elastic wave Green's function and $\Omega$ is the region occupied by the inhomogeneity. The integrals $S_{j m}$ and their derivatives must be evaluated for the regions $\underline{r}>\underline{r}^{\prime}$ and $\underline{r}<\underline{r}^{\prime}$ 
(ref. 6). The solution form represented in equations (14) to (18) gives the fields inside and outside an isolated inhomogeneity of arbitrary shape.

\section{Far-Field Scattered Quantities}

Let the incident displacement fleld be longitudinal and of frequency $\omega$ and amplitude $u_{0}$ :

$$
u_{j}^{(1)}=u_{0} q_{j} \exp \left(i \alpha x_{1} k_{1}-i \omega t\right)
$$

where $i^{2}=-1$ and $q_{j}$ is the unit vector in the normal direction of the plane time-harmonic incident wave and $k_{j}$ is wave vector. For a linear isotropic medium, the spatial part of the free-space Green's function is well known. Substituting $g_{j m}\left(r-r^{\prime}\right)$ in equations (18) and (20) and using the limiting concept

$$
\begin{aligned}
& \lim _{a_{3 \rightarrow 0}} a_{3} \pi_{j}^{*}=A_{j} \quad \text { constants } \\
& \lim _{a_{3} \rightarrow 0} a_{3} \varepsilon_{i j}^{*}(1)=B_{i j} \text { constants }
\end{aligned}
$$

the scattered displacement $u_{m}(s)(r, t)$ from a thin, elliptical flat crack can easily be obtained as

$$
\begin{aligned}
\frac{u_{m}^{(s)}(\underline{r}, t)}{\left(\alpha a_{1}\right)^{3} u_{0}} & =\left.\frac{u_{m}^{(m)}(\underline{r}, t)}{\left(\alpha a_{1}\right)^{3} u_{0}}\right|_{r \rightarrow \infty} \\
& =\left[\left(C G_{m} \exp i \alpha r\right) / \alpha r+\left(D H_{m} \exp i \beta r\right)\right] \beta r \quad \exp (-i \omega t)
\end{aligned}
$$

where

$$
\begin{gathered}
G_{m}=-\left(a_{2} / a_{1}\right)\left[-l_{m} \ell_{j} A_{j}^{*}+\left(1-2 \alpha^{2} / \beta^{2}\right) l_{m} B_{j j}^{*}+2\left(\alpha^{2} / \beta^{2}\right) l_{m} l_{k} \ell_{j} B_{k j}^{*}\right] \\
H_{m}=\left(a_{2} / a_{1}\right)\left[-(\beta / \alpha)^{3}\left(l_{m} \ell_{j}-\delta_{m j}\right) A_{j}^{*}-2(\beta / \alpha)^{2} \ell_{k} B_{k m}^{*}+2(\beta / \alpha)^{2} \ell_{m} \ell_{k} \ell_{j} B_{k j}^{*}\right] \\
C=\left[j_{0}\left(\alpha r_{e}\right)+j_{2}\left(\alpha r_{e}\right)\right] / 3=\sin \alpha r_{e} /\left(\alpha r_{e}\right)^{3}-\cos \alpha r_{e} /\left(\alpha r_{e}\right)^{2} \\
D=\left[j_{0}\left(\beta r_{e}\right)+j_{2}\left(\beta r_{e}\right)\right] / 3=\sin \beta r_{e} /\left(\beta r_{e}\right)^{3}-\cos \beta r_{e} /\left(\beta r_{e}\right)^{2} \\
r_{e}^{2}=a_{i}^{2} l_{j}^{2}, \quad a_{3}=0
\end{gathered}
$$

in which $m, j, k=1,2,3$ and $\left(a_{1}, a_{2}\right), \ell_{m}, \alpha$, and $\beta$ denote the semiaxes of the flat ellipsoid, direction cosines of scattered displacements, longitudinal wave 
number, and shear wave number, respectively. Also, $A_{j}^{\star}$ and $B_{j k}^{*}$ are the reduced nondimensional forms of $A_{j}$ and $B_{j k}$, respectively, defined as follaws:

$$
\begin{gathered}
A_{j}^{*}=A_{j} /\left(\Delta \rho \omega^{2} u_{0}\right), \quad \Delta \rho=\rho^{\prime}-\rho \\
B_{j k}^{*}=-B_{j k} /\left(i \alpha u_{0}\right)
\end{gathered}
$$

Expressions for the differential cross section $\mathrm{dP}(\omega) / \mathrm{d} \Omega$ and the total cross section $P(\omega)$ can be obtained as (ref. 5):

$$
\begin{gathered}
\frac{d P(\omega)}{d \Omega}=\sigma^{L}(\theta, \phi)+(\alpha / \beta) \sigma^{\top}(\theta, \phi) \\
P(\omega)=\int\left[\sigma^{L}(\theta, \phi)+(\alpha / \beta) \sigma^{\top}(\theta, \phi)\right] d \Omega
\end{gathered}
$$

where $d \Omega$ is the differential element of solid angle and

$$
\begin{aligned}
& \alpha^{2} \sigma^{L}(\theta, \phi)=\left(\alpha a_{1}\right)^{6}\left[\mathrm{CG}_{m}\right]\left[\overline{\mathrm{CG}_{m}}\right] \\
& \beta^{2} \sigma^{T}(\theta, \phi)=\left(\alpha a_{1}\right)^{6}\left[D H_{m}\right]\left[\overline{D H_{m}}\right]
\end{aligned}
$$

in which the overbars denote complex conjugates. The constants $A_{j}^{*}$ and $B_{j k}^{*}$ must be evaluated from the equivalence conditions (eqs. (5) and (6)) with the use of the limiting concepts in equations (21) and (22) and of the integration method developed in reference 6 .

\section{Determination of $A_{j}^{*}$ and $B_{j k}^{*}$}

In equations (21) to (25) the scattered displacement field is given in terms of the "reduced' forms of the eigenforces and eigenstrains (1.e., $A_{j}^{*}$ and $B_{j k}^{*}$ ). These constants must in turn be determined from the equivalence conditions. By writing the incident wave field in a Taylor series, the governing simultaneous algebraic equations can be easily obtained. Since $f_{i j}[0]$ and $F_{m i j}[0]$ vanish automatically, these governing equations become uncoupled and lead to a three-bythree system for $A_{j}^{*}$ and a six-by-six system for $B_{j k}^{*}$. For a linear elastic medium, they are

$$
\Delta \rho \omega^{2} u_{0} f_{j s}[0] A_{j}^{*}+A_{s}^{*}=-q_{s}
$$

$\left\{\Delta \lambda \delta_{s t} D_{m m j j}[0]+2 \Delta \mu D_{s t j k}[0]\right\} B_{j k}^{*}+\left(\lambda \delta_{s t} B_{m m}^{*}+2 \mu B_{s t}^{*}\right)$

$$
=-\left(\Delta \lambda \delta_{s} q_{m} q_{m}+2 \Delta \mu q_{s} q_{t}\right)
$$

where the subscripts $s, t, m, j, k=1,2,3$ denote sum from 1 to 3 and

$$
4 \pi \rho \omega^{2} f_{j s}(\underline{r})=-\beta^{2} \phi \delta j s+\psi, m j-\phi, m j
$$


$4 \pi \rho \omega^{2} D_{s t j k}(r)=2 \mu(\psi, s t j k+\phi, s t j k)-\mu \beta^{2}(\phi, j t \delta k s+\phi, j s \delta k t)-\lambda \alpha^{2} \psi, m n \delta_{j k}$ in which

$$
\Delta \lambda=-\lambda, \quad \Delta \mu=-\mu, \quad \Delta \rho=-\rho
$$

The $\phi$ - and $\psi$-integrals and their derivatives are evaluated by the method suggested in reference 6 . Retaining terms up to $\alpha_{1}$ or $\mathrm{Ba}_{1}$ of the fourth order, the constants are obtained as

$$
\begin{gathered}
A_{j}^{*}=-q_{j} /\left\{u_{0} \omega^{2} \Delta \rho f_{j}[0]+1\right\}, \text { no sum on } j \\
f_{j}[0]=\left(f_{11}[0], f_{22}[0], f_{33}[0]\right) \\
\left\{B_{i}^{*}\right\}=\left[b_{i j}\right]^{-1}\left[c_{j}\right], 1, j=1,2,3
\end{gathered}
$$

$\left\{B_{i j}^{*}\right\}=-q_{i} q_{j} /\left[1+\xi\left(\phi_{j}[0]+\phi_{j}[0]\right)\right]$, no sum on $i, j$

$$
i \neq j ; i, j=i, 2,3 ; \xi=1 / 4 \pi
$$

where in equation (37) $B_{1}^{*}=B_{11}^{*}, B_{2}^{*}=B_{22}^{*}, B_{3}^{*}=B_{33}^{*}$, and

$$
\begin{gathered}
c_{j}=(\Delta \lambda+2 \Delta \mu)\left(1+q_{j}^{2}\right), j=1,2,3 \\
b_{11}=(\lambda+2 \mu)+\lambda \zeta \psi, j j[0]+2(\lambda+2 \mu) \xi \phi, 11[0]+2 \mu \zeta \psi, 11[0] \\
b_{12}=\lambda+\lambda \zeta \psi, j j[0]+2 \mu \zeta \psi,{ }_{11}[0]+2 \xi \lambda \phi, 22[0] \\
b_{13}=\lambda+\lambda \zeta \psi, j j[0]+2 \mu \zeta \psi,{ }_{11}[0]+2 \lambda \xi \phi,{ }_{33}[0] \\
b_{21}=\lambda+\lambda \zeta \psi, j j[0]+2 \mu \zeta \psi, 22[0]+2 \xi \lambda \phi,{ }_{11}[0] \\
b_{22}=(\lambda+2 \mu)+\lambda \zeta \psi, j j[0]+2 \mu \zeta \psi, 22[0]+2 \xi(\lambda+2 \mu) \phi, 22[0] \\
b_{23}=\lambda+\lambda \zeta \psi, j j[0]+2 \mu \zeta \psi, 22[0]+2 \xi \lambda \phi,{ }_{33}[0] \\
b_{31}=\lambda+\lambda \zeta \psi, j j[0]+2 \mu \zeta \psi, 33[0]+2 \lambda \xi \phi,{ }_{11}[0] \\
b_{32}=\lambda+\lambda \zeta \psi, j j[0]+2 \mu \zeta \psi,{ }_{33}[0]+2 \lambda \xi \phi,{ }_{22}[0] \\
b_{33}=(\lambda+2 \mu)+\lambda \zeta \psi, j j[0]+2 \mu \zeta \psi, 33[0]+2 \xi \phi,{ }_{33}(\lambda+2 \mu)[0]
\end{gathered}
$$

Note that $b_{1 j} \neq b_{j 1}$. In equation $(38), \phi_{1}=\phi_{11}[0], \phi_{2}=\phi_{22}[0]$, and $\phi_{3}=\phi_{33}[0]$. The $f-$ and $\phi-$ functions are given as

$$
\begin{gathered}
4 \pi \rho \omega^{2} f_{j s}[0]=-\beta^{2} \phi[0] \delta_{j s}+\psi, j s[0]-\phi, j s[0] \\
\beta_{\phi}^{2}[0]=\pi a_{1} a_{2} \beta^{2}\left\{I_{0}-\left[\left(\beta a_{1}\right)^{2} / 16\right] I_{1}+1(8 / 3) \beta\right\} \\
\phi,{ }_{17}[0]=-\left[\left(\pi a_{1}^{3} a_{2} \beta^{4}\right) / 12\right] I_{1}
\end{gathered}
$$




$$
\begin{gathered}
\phi, 22[0]=-\left[\left(\pi a_{1}^{3} a_{2} \beta^{4}\right) / 12\right] \quad I_{1} \\
\phi \cdot 33[0]=0 .
\end{gathered}
$$

in which

$$
\begin{gathered}
I_{0}=\int_{0}^{\infty} \frac{d \psi}{\Delta(\psi)}=F(\theta, k) \frac{2}{a_{1}} \\
I_{1}=\int_{0}^{\infty} \frac{\psi d \psi}{\left(a_{1}^{2}+\psi\right) \Delta(\psi)}=\frac{2}{a_{1}}\left[\frac{E(\theta, k)}{k^{2}}-\frac{k^{\prime 2}}{k^{2}}\right] F(\theta, k) \\
I_{2}=\int_{0}^{\infty} \frac{\psi d \psi}{\left(a_{2}^{2}+\psi\right) \Delta(\psi)}=\frac{2}{a_{1}}\left[\frac{F(\theta, k)}{k^{2}}-\frac{E(\theta, k)}{k^{2}}\right] \\
F=\int_{0}^{\theta} \frac{d \omega}{\left(1-k^{2} \sin ^{2} \omega\right)^{1 / 2}}, E=\int_{0}^{\theta}\left(1-k^{2} \sin ^{2} \omega\right)^{1 / 2} d \omega
\end{gathered}
$$

and as $a_{3} \rightarrow 0, \theta \rightarrow \pi / 2, k^{2} \rightarrow\left(1-a_{2}^{2} / a_{1}^{2}\right)$ and $k^{\prime 2}=\left(1-k^{2}\right) \rightarrow a_{2}^{2} / a_{1}^{2}$, if $a_{1}>a_{2}$. If $a_{3} \rightarrow 0$ and $a_{1}=a_{2}$, we have $I_{0}=\pi / a_{1}$ and $I_{1}=I_{2}=\pi / 2 a_{1}$. The $\psi$-functions are obtained by replacing $\beta$ with $\alpha$ in the $\phi$-functions (fig. 2).

\section{Numerical Calculations and Graphical Displays}

It is clear from equations (37) to (40) that the uniformly distributed eigenstrains and eigenforces in their reduced forms, $B_{i j}$ and $A_{j}$, respectively, depend only on the characteristics of the incident wave field and the geometric factors of the inhomogeneity for a given material system. It is observed from equations (37) to (41) that $B_{j}$ and $B_{i j}$ as functions of dimensionless wave number $\alpha a_{1}$ or $\alpha a_{2}$ would exhibit large peak values at certain incident wave frequencies when $b_{i j} \rightarrow 0$. The values of these critical frequencies depend only on the matrix elastic moduli, the crack dimensions, and the measurement direction. For a given aspect ratio $a_{2} / a$ and a measurement direction, the difference in frequencies at subsequent peak values is proportional to $a_{7}$, the largest dimension of the inclusion.

Computational data of elastic wave scattering due to a flat embedded inhomogeneity in any given isotropic material system can be obtained by employing equations (24) to (26) and (33) to (50). Scattered displacement amplitudes can easily be obtained for any given aspect ratio. In figures 3 and 4 , computational data for the back-scattered situation are displayed for a tungsten disk in an aluminum matrix for aspect ratios $a_{2} / a_{1}=0.01$ and $a_{2} / a_{1}=0.50$. In figures 5 to 8 , critical frequencies were observed in $B_{i j}^{*}$, but no critical frequencies were identified for $A_{j}^{\star}(j=1,2,3)$. The critical frequencies for $C\left|G_{m}\right|$ and $D\left|H_{m}\right|$ are, however, clearly identified. The position along the aa axis at which the first critical 
frequency occurred depended on the aspect ratio and matrix elastic moduli. A sufficientiy smali increment in al must be used in order not to miss any peak values. Since the solution form given in equations (24) to (26) and (33) to (50) is analytic in frequency, this can easily be achieved. Most of the scattered energy is carried by the transverse components of the scattered displacement ( $1 . e .$, $\left|D H_{m}\right|>\left|C G_{m}\right|$, figs. 3 and 4$)$.

\section{DYNAMIC MODULI AND DAMAGE OF COMPOSITES}

Consider the problem of an inhomogeneous medium as 117ustrated in figure 9 , under a plane time-harmontc incident wave fleld. The true composite thus occupies the whole region and possesses effective moduli $\mathcal{C}^{*}$ and mass density $\rho^{*}$. To determine the effective moduli and mass density, an average strain energy and kinetic energy were used. The effective properties were found to depend on a fourth-rank tensor $\underset{\sim}{A}$ and a second-rank tensor $\underset{\sim}{D}$. A self-consistent scheme was then developed for determining these tensors.

\section{Average Theorem}

To determine the effective moduli and mass density, the following definitions were used:

$$
\begin{gathered}
\langle\underline{q}\rangle=\mathscr{c}^{*}\langle\underline{q}\rangle \\
\langle\underline{q} \underline{\sim}\rangle=\underline{\mathcal{C}}^{*}\langle\underline{\varepsilon}\rangle\langle\underline{\varepsilon}\rangle \\
\langle\rho \underline{v}\rangle=\rho^{*}\langle\underline{v}\rangle \\
\langle\rho \underline{v} \cdot \underline{v}\rangle=\rho^{*}\langle\underline{v}\rangle\langle\underline{v}\rangle
\end{gathered}
$$

where $g, \underset{\sim}{\mathcal{E}} \underline{v}$ are the stress, strain, and velocity fields, the $\sim$ denotes a tensoriai quantity, and the angular brackets $\langle$ denote the volume average of a field quantity (eq. (9)). The left and the right sides of equations (52) and (54) can be shown to be equivalent under the so-called Hill's condition ( $r e f .7),\langle\sigma\rangle\langle\varepsilon\rangle$ $=\langle\sigma \varepsilon\rangle$. From equations (53) and (54), it is clear that the kinetic energy per unit volume of the effective medium can be made equal to that of the physical medium if, and only if, a frequency-dependent mass density is defined. This is the same as requiring, by again using the Hill's conditions $\langle p, \underline{v} \cdot \underline{v}\rangle=\langle p \underline{v}\rangle\langle\underline{v}\rangle$, the average linear momentum per unit volume to be the same as the effective unit volume (eq. (53)). These conditions (eqs. (53) and (54)) are not met if the static definition of effective mass density is used.

Let $f_{r}$ denote the volume fraction of the $r_{\text {th }}$ inclusion material. Then the volume averages of the stress and velocity fields $\sigma$ and $u$, respectively, are

$$
\begin{aligned}
& \langle\underline{\sigma}\rangle=\sum_{r=0}^{n} f_{r{ }^{\sigma}}(r) \\
& \langle\underline{\dot{u}}\rangle=\sum_{r=0}^{n} f_{r} \underline{\dot{u}}^{(r)}
\end{aligned}
$$


where $\underset{\sim}{g}(r)=\underset{\sim}{\stackrel{c}{c}}(r) \underset{\sim}{g}(r),(r)=0,1,2, \ldots, n$ and for a time-harmonic situation

$$
\underline{\dot{u}}=\underline{v}=-i \omega \underline{u}
$$

Equations (52) and (54) can be rearranged, by using equations (55) and (56), as

$$
\begin{gathered}
\langle\underline{q \varepsilon}\rangle=c^{0}\langle\underline{\varepsilon}\rangle\langle\underline{\varepsilon}\rangle+\sum_{r=1}^{n} f_{r} \mathcal{c}^{(r)_{\varepsilon}} \underset{\sim}{(r)}\langle\underline{\varepsilon}\rangle \\
\langle\rho \underline{v} \cdot \underline{v}\rangle=\rho^{0}\langle\underline{v}\rangle\langle\underline{v}\rangle+\sum_{r=1}^{n} f_{r} \Delta \rho(r) \underline{v}^{(r)}\langle\underline{v}\rangle
\end{gathered}
$$

Consider now the case where the solution form possesses a linear relation between the velocity and strain fields in the $r^{\text {th }}$ component and the average velocity and strain fields of the effective medium, that is,

$$
\begin{aligned}
& \underline{\varepsilon}^{(r)}=\stackrel{A}{\sim}^{(r)}\langle\varepsilon\rangle \\
& \underline{v}(r)=\underset{\sim}{\stackrel{D}{\sim}(r)}\langle\underline{v}\rangle
\end{aligned}
$$

where $\underset{\sim}{A}(r)$ and $\underset{\sim}{D}(r)$ are tensors of fourth and second rank that must be

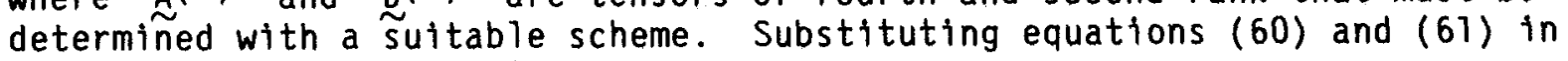
equations (58) and (59) leads to

$$
\begin{aligned}
& \langle\tilde{\sigma} \boldsymbol{\sim}\rangle=\left[\mathcal{L}^{0}+\sum_{r=1}^{n} f_{r} \Delta \mathcal{C}^{(r)_{A}}(r)\right]\langle\underline{\varepsilon}\rangle\langle\varepsilon\rangle \\
& \langle\rho \underline{v v}\rangle=\left[\rho^{0}+\sum_{r=1}^{n} f_{r} \Delta \rho(r)_{D}(r)\right]\langle\underline{v}\rangle\langle\underline{v}\rangle
\end{aligned}
$$

Comparing these equations with equations (52) and (54) gives the following expressions for the dynamic effective moduli and mass density:

$$
\begin{aligned}
& \stackrel{\mathcal{L}}{*}^{*}=\mathcal{L}^{0}+\sum_{r=1}^{n} f_{r} \Delta \mathcal{L}^{(r)} \stackrel{\mathcal{A}}{(r)}^{(r)} \\
& \left.\begin{array}{c}
\rho^{*}=\rho^{0}+\sum_{r=1}^{n} f_{r} \Delta_{\rho}(r)_{0}(r) \\
D^{(r)}=D_{j j}^{(r) / 3}
\end{array}\right\}
\end{aligned}
$$

Note that the tensors $A^{(r)}$ and $D^{(r)}$ are frequency dependent and replace the static expressions when frequency approaches zero and when proper care is taken. The assumption of a general 1inear dependence between $\underline{v}(r)$ and $\langle\underline{v}\rangle$ (eq. (61) must be specialized such that the second-rank tensor $D^{-}$will degenerate into a scalar. This specialization is automatic for randomly distributed spheres where $D_{m j}=D \delta_{m j}$, in which $D=D_{11}=D_{22}=D_{33}$. 
To determine the explicit form of the tensors $\underset{\sim}{A}(r)$ and $\underset{\sim}{\sim}(r)$, the strain and velocity fields in the $r^{\text {th }}$ component are determined by using the method of equivalent inclusion as presented in the previous section.

\section{Self-Consistent Scheme for Determining Effective Properties}

The tensor fields $\underset{\sim}{A}(r)$ and $\underset{\sim}{D}(r)$ are of ranks four and two, respectively, and are functions of wave numbers, geometric properties, and effective and inclusion material properties. Let the average strain and velocity be the same as those derived from the incident wave field. Then the governing conditions for determining $\underset{\sim}{A}(r)$ and $\underset{\sim}{(r)}$ for the $r^{\text {th }}$ inclusion are simply obtained by rewriting the equivalence conditions for an effective medium with mass density $\rho^{*}$ and moduli $C^{*}$ :

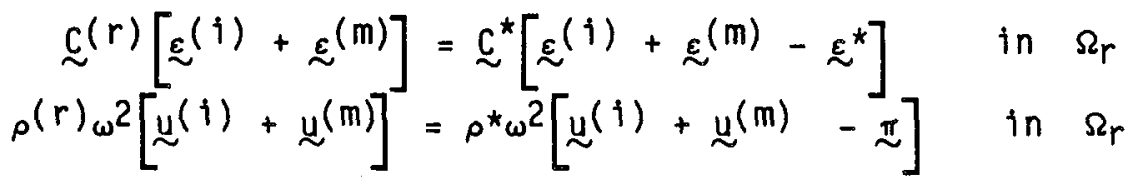

in which the superscripts (m) and (i) denote mismatch and incident, respectively. Slightly different approaches for finding effective moduli for heterogeneous materials that apply to static cases with different constituents and situations are given in references 7 and 8 . The strengths and dynamic responses of composites have been investigated (refs. 9 and 10) and reviewed (refs. 11 and 12).

\section{Effective Properties of Two-Component Media: Randomly Distributed Spheres}

Let the moduli, mass density for the matrix, inclusion material particles, and the effective medium by denoted by $C_{j k r s,}^{0} \rho^{0} ; C_{j k r s}, \rho^{\prime}$; and $C_{j k r s}^{*}$ and $\rho^{*}$, respectively. By using the elastodynamic solution for a single elipsoidal inhomogeneity, the displacement and strain fields inside an inhomogeneity are found, when the average displacements are made equal to the plane time-harmonic incident wave field:

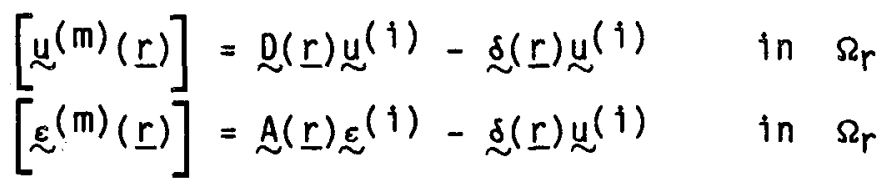

Employing the volume-averaging process as described by equation (9) and substituting in equations (58) and (59), the effective properties are easily defined as follows:

$$
\begin{gathered}
\rho^{*}=\rho+f \Delta \rho D, \quad D=\frac{1}{3} D_{j j} \\
\mathscr{C}^{*}=\underset{\sim}{C}+\stackrel{\Delta C A}{\sim}
\end{gathered}
$$

where $f$ is the volume fraction of inclusion material. The tensor fields $\underset{\sim}{D}$ and $\stackrel{A}{\sim}$ are

$$
D_{m j}=-\left\langle f_{m j}(\underline{r})\right\rangle /\left\{f_{M J}[0]+4 \pi\left(p^{\prime}-p^{\star}\right) \omega^{2}\right\} \text { no sum on } M, J
$$




$$
A_{m n p q}=\left\langle F_{m j k, n}(\underline{r})+F_{n j k, m}(\underline{r})\right\rangle S_{j k p q} / 2 p^{*} \omega^{2}
$$

in which the tensors $\underset{\sim}{f}$ and $\underset{\sim}{\mathcal{F}}$ are defined as

$$
\begin{gathered}
4 \pi \rho \omega^{2} f_{m j}(\underline{r})=-\beta_{\phi \delta_{m j}}^{2}+\psi_{m j}-\phi,_{m j} \\
4 \pi \rho \omega^{2} F_{m i j}(\underline{r})=-\left(\lambda \alpha^{2} \psi \cdot m^{\delta_{i j}}+2 \mu \beta^{2} \phi, j \delta_{m j}-2 \mu \psi \cdot m j\right.
\end{gathered}
$$

The $\psi$ - and, by implication, the $\phi$-integrals given in equations (14) and (15), etc., are the volume integrals associated with the inhomogeneous Helmholtz equation. They can be carried out for an ellipsoidal region by expanding (exp $i k R) / R$ in Taylor series expansions with respect to $r^{\prime}$ for $r>r^{\prime}$ and with respect to $r$ for $r<r^{\prime}$. Here $k$ can be either $\alpha$ or $\beta$. Detalls are given in reference 6 . This type of expansion for the integrand is particularly useful in determining the coefficients of a "polynomial" distribution of $\pi_{j}^{*}$ and $\varepsilon_{i}^{*}(1)$.

The fourth-rank tensor $S_{j k p q}$ is the connecting tensor between the eigenstrains and the applied strains, that is,

$$
\left.\begin{array}{c}
\varepsilon_{j k}^{*(1)}=S_{j k p q} \sigma_{p q}^{(a)} \\
S_{j k p q}=S_{j k p q}\left(C, a_{i}, \omega\right)
\end{array}\right\}
$$

for the case of uniform eigenstrains and eigenforces. In developing these expressions, the volume average of the $\phi$-integrals must be evaluated. Finally, note that $\rho^{*}$ and $\mathcal{C}^{*}$ are complex, where the real and imaginary parts are associated with the velocity and attenuation, respectively.

\section{Example: Spherical Inclusion Materials}

Let the spherical inclusion materials of radius a be randomly distributed over the whole volume of the matrix. If the matrix and the inhomogeneities are isotropic, the effective medium is also isotropic. It is easy to show that

$$
D_{m j}=\delta_{m j}\left\{\frac{-\left\langle f_{33}(r)\right\rangle}{f_{33}[0]+4 \pi\left(\rho^{\prime}-\rho^{*}\right) \omega^{2}} \mid=\delta_{m j} D\right.
$$

and

$$
\left.\begin{array}{l}
s_{j k p q}=s_{k j p q}=s_{j k q p}=s_{p q j k} \\
s_{1111}=s_{2222}=s_{3333}=c_{1} \\
s_{2323}=s_{1313}=s_{1212}=c_{3} \\
s_{1122}=s_{1133}=s_{2233}=c_{2}
\end{array}\right\}
$$


where

$$
\begin{gathered}
C_{1}=\left(C_{1}^{*}+C_{2}^{*}-2 G C_{2}^{*}\right) /\left[\left(C_{1}^{*}\right)^{2}+C_{1}^{*} C_{2}^{*}-2\left(C_{2}^{*}\right)^{2}\right] \\
C_{2}=\left(C_{1}^{*} G-C_{2}^{*}\right) /\left[\left(C_{1}^{*}\right)^{2}+C_{1}^{*} C_{2}^{*}-2\left(C_{2}^{*}\right)^{2}\right] \\
C_{3}=\left\{2 F_{122,1}[0]+\mu^{*} /\left(\mu^{\prime}-\mu^{*}\right)\right\} \\
C_{2}^{*}=G_{111,1}[0]+(G+1) F_{122,1}[0]+H \\
C_{1}^{*}=F_{111,1}[0]+2 G F_{122,1}[0]-F \\
F \equiv-\left(\lambda^{*}+2 \mu^{*}\right) / G \\
G=\left(\lambda^{\prime}-\lambda^{*}\right) /\left[\left(\lambda^{\prime}-\lambda^{*}\right)+2\left(\mu^{\prime}-\mu^{*}\right)\right] \\
H=\lambda^{*} / G
\end{gathered}
$$

Following the theory developed in the previous sections, the effective moduli and mass density are found to be

$$
\begin{gathered}
\rho^{*}=\rho+f \Delta \rho D \\
\lambda^{*}=\lambda+f\left[\Delta \lambda\left(A_{1111}+2 A_{1122}\right)+2 \Delta \mu A_{1122}\right] \\
\mu^{*}=\mu+f \Delta \mu\left(A_{1212}+A_{1221}\right) \\
K^{*}=K+f\left[\left(A_{1111}+2 A_{1122}\right) \Delta \lambda+(2 / 3)\left(3 A_{1122}+A_{1212}+A_{1221}\right) \Delta \mu\right]
\end{gathered}
$$

Clearly, the velocities are dispersive. At frequencies above the Rayleigh limit, this phenomenon is pronounced. From figures 10 to 12 , the bulk moduli, shear moduli, and longitudinal velocities are shown as functions of the volume concentration of spherical inclusion materials for aluminum spheres in germanium for different dimensionless wave numbers aa. For a given fixed concentration, the moduli $K^{*}$ and $\mu^{*}$ and the velocities $v_{L}$ and $v_{T}$ are increased as the dimensionless wave number a is increased. The dispersiveness of effective shear modulus is minimal and that of effective bulk modulus is more pronounced (figs. 12 and 13).

As an example of detecting localized damage by void nucleation, let all small voids be localiy nucleated within a localized small region $\Omega$ of radius $R$ (fig. 14). The effective moduli of this composite can therefore be obtained from equations (64) and (65). If void nucleation outside the region $\Omega$ can be ignored, the scattering of the composite sphere can easily be obtained. By using the computer program developed in reference 17 for that of a single spherical inclusion. the scattering cross section for a composite sphere consisting of small voids in titantum is displayed as a function of dimensionless wave number for different concentrations of voids (fig. 15). As the volume fraction of voids inside $\Omega$ is changed, the effective properties $\rho^{*}, \lambda^{*}$, and $\mu^{*}$ are also changed. Hence, the attenuation effect is pronounced as the concentration of voids is increased. The 
scattering cross section, which is essentially proportional to the attenuation (ref. 1) increases with increasing concentration $f_{r}$. It appears that these curves can be used to locate and calibrate porosity in a structural component.

\section{CONCLUDING REMARKS}

The velocity and attenuation of ultrasonic waves in two-phase media were studied by using a self-consistent averaging scheme. The effective medium had to possess the same strain and kinetic energy as the physical medium. The concept of volume averaging for physical quantities was employed. The solution depended on the scattering of a single inhomogeneity. The theory is general and can be applied to any multicomponent material system. Since the scattering of an ellipsoidal inhomogeneity is known, the average theorem presented in this report can be used to study the velocity and attenuation of distributed inhomogeneities of shapes such as disks and short fibers. The orientation of these inhomogeneities as well as their sizes, as in the spherical geometry, will necessarily induce anisotropy in the effective medium. The scheme developed herein was not compared with test data. The use of a self-consistent scheme in determining static material properties for composites has been explored (ref. 13).

Results for randomly distributed spherical inclusions of radius a are presented. Effective moduli and mass density were found to be dispersive. A simple model of localized damage was studied. It is well known that porosity is directly related to the strength of rocks and ceramics. Therefore the theoretical study of velocity and attenuation in two-phase media may be a viable means for data analysis in ultrasonic evaluation of dynamic material properties for composite bodies (ref. 14) and polycrystals (ref. 15). As in determining the static properties of materials, the problem may be more in the mechanics than in the manufacturing (ref. 16). Deformation processes such as rolling, sheet metal forming, and drawing often involve large plastic flow, moisture absorption, and thermal cycling. Residual stresses and anisotropy are introduced into the material and 1 imit the amount of deformation to fracture with a directional dependence. Theoretical development in acoustoelasticity appears to be lacking (ref. 18).

\section{REFERENCES}

1. R. True 11, C. Elbaum, and B.B. Chick, Ultrasonic Methods in Solid State Physics, Academic Press, N.Y., 1969.

2. Elastic Waves and Non-Destructive Testing of Materials, edited by Y.H. Pao, AMD-29, American Society of Mechanical Engineers, New York, 1978.

3. L.S. Fu, "Mechanics Aspects of NDE by Sound and UTtrasound," Applied Mechanics Review, vol. 35, no. 8, 1982, pp. 1047-1057.

4. L.S. Fu, "A New Micro-Mechanical Theory for Randomly Inhomogeneous Media," Symposium on Wave Propagation in Inhomogeneous Media and UTtrasonic Nondestructive Evaluation, AMD-62, June 1984, pp. 155-174.

5. L.S. Fu and T. Mura, "The Determination of Elastodynamic Fields of the Ellipsoidal Inhomogeneity," Trans. ASME J. App 1. Mech., vol. 50, 1983, pp. 390-397. 
6. L.S. Fu and T. Mura, "Volume Integrals of Ellipsoids Associated with the Inhomogeneous Helmholtz Equations," Wave Motion, vol. 4, 1982, pp. 141-149.

7. R. Hill, "The Elastic Behavior of a Crystalline Aggregate," Proc. Phys. Soc. A65, 1952, p. 319 .

8. B. Budiansky and T.T. Wu, "Theoretical Prediction of Plastic Strains of Polycrystals," Proc. 4th U.S. Nat. Cong. App1. Mech., 1962, pp. 1175-1185.

9. C.W. Bert, "Models for Fibrous Composites with Different Properties in Tension and Compression," J. Eng. Mater. Technol. ASME, vo1. 99, 1977, p. 344.

10. A.B. Schultz and S.W. Tsai, "Dynamic Moduli and Damping Ratio in FiberReinforced Composites," J. Comp. Materials, vol. 2, no. 3, 1968, pp. 368-379.

11. J. Achenbach, Wave Propagation in Elastic Solids, North Holland Pub. Co., 1975.

12. T.C.T. Ting, "Dynamic Response of Composites," Applied Mechanics Reviews, vol. 33, no. 12, Dec. 1980.

13. L.S. Fu, "On the Feasibility of Quantitative Ultrasonic Determination of Fracture Toughness - A Literature Review," International Advances in Nondestructive Testing, vol. 7, May 1981. (Also NASA CR-3356, 1980.)

14. L.S. Fu, "Scatter of Elastic Waves Due to a Thin Flat Elliptical Inhomogeneity," NASA CR-3705, 1983.

15. A. Vary, "Correlations Between U1trasonic and Fracture Toughness Factors in Metallic Materials," ASTM STP 677, pp. 563-578, 1979.

16. 2. Hashin, "Analysis of Composite Materials - A Survey," J. Appl. Mech., Trans ASME, vol. 50, 1983, p. 481 .

17. Y.C. Sheu and L.S. Fu, "The Transmission or Scattering of Elastic Waves by an inhomogeneity of Simple Geometry: A Comparison of Theories," NASA Contractor Report CR-3659, Jan. 1983.

18. H. Fukuoka, et al., "Acoustoelastic Stress Analysis of Residual Stress in the Rim of Railroad Wheels," ASME AMD-62, ed. G. Johnson, June 1983. 


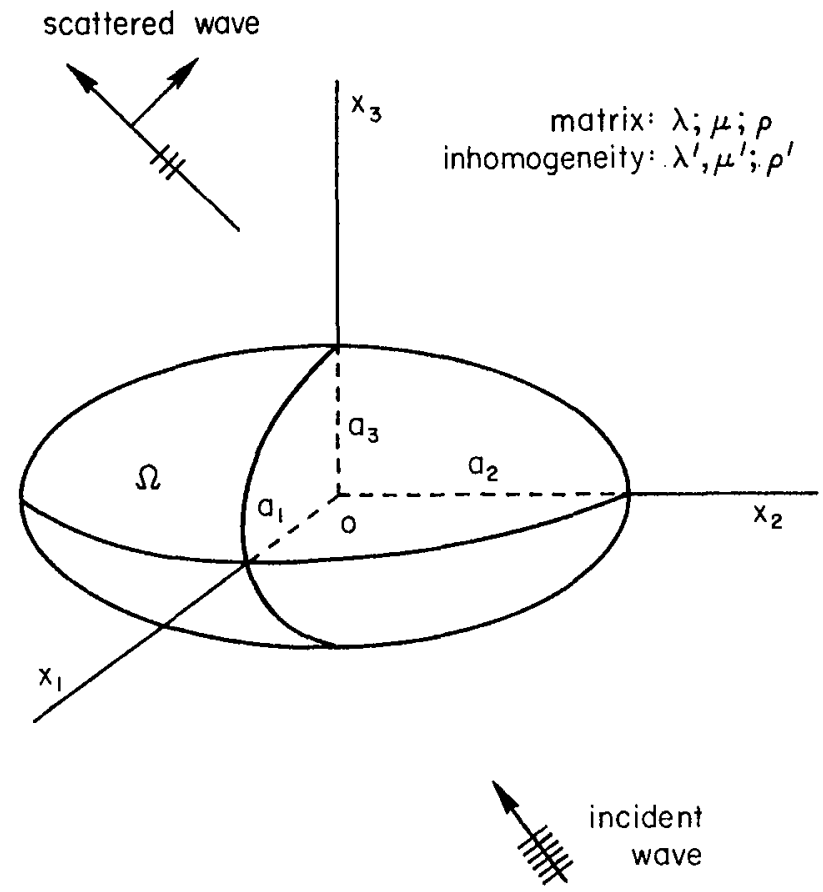

Figure 1. - Ellipsoidal inhomogeneity embedded in elastic matrix.

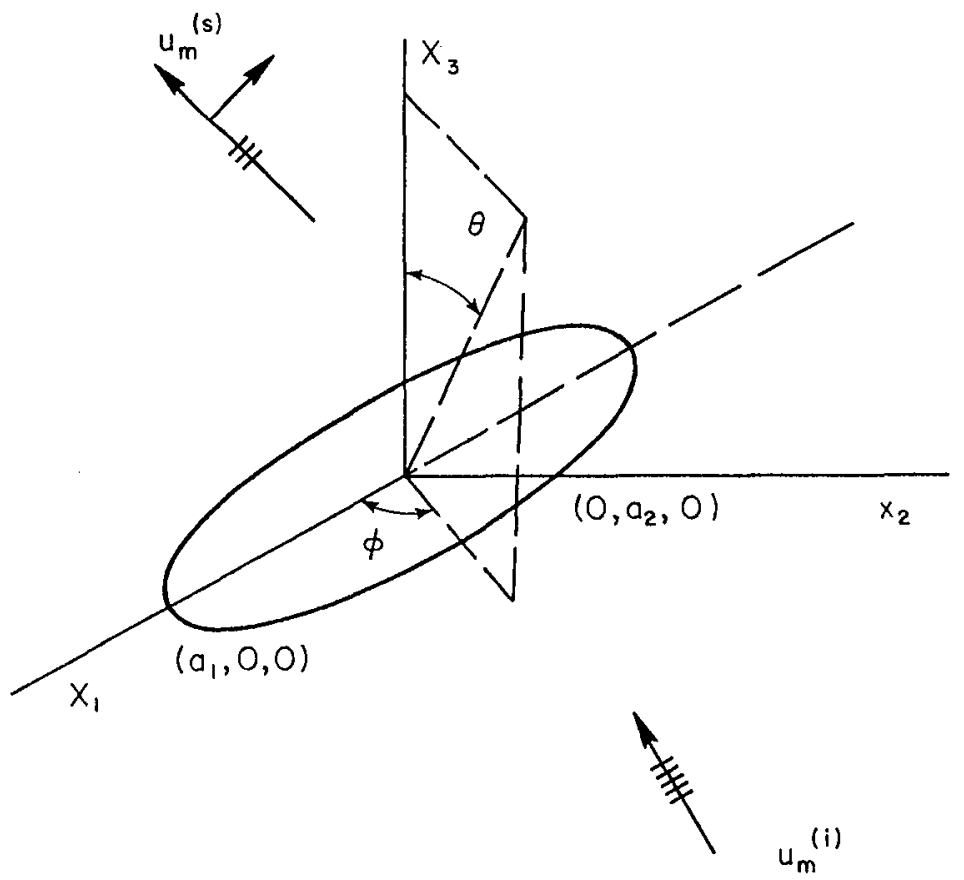

Figure 2. - Elliptical crack: $a_{3} \rightarrow 0$. 


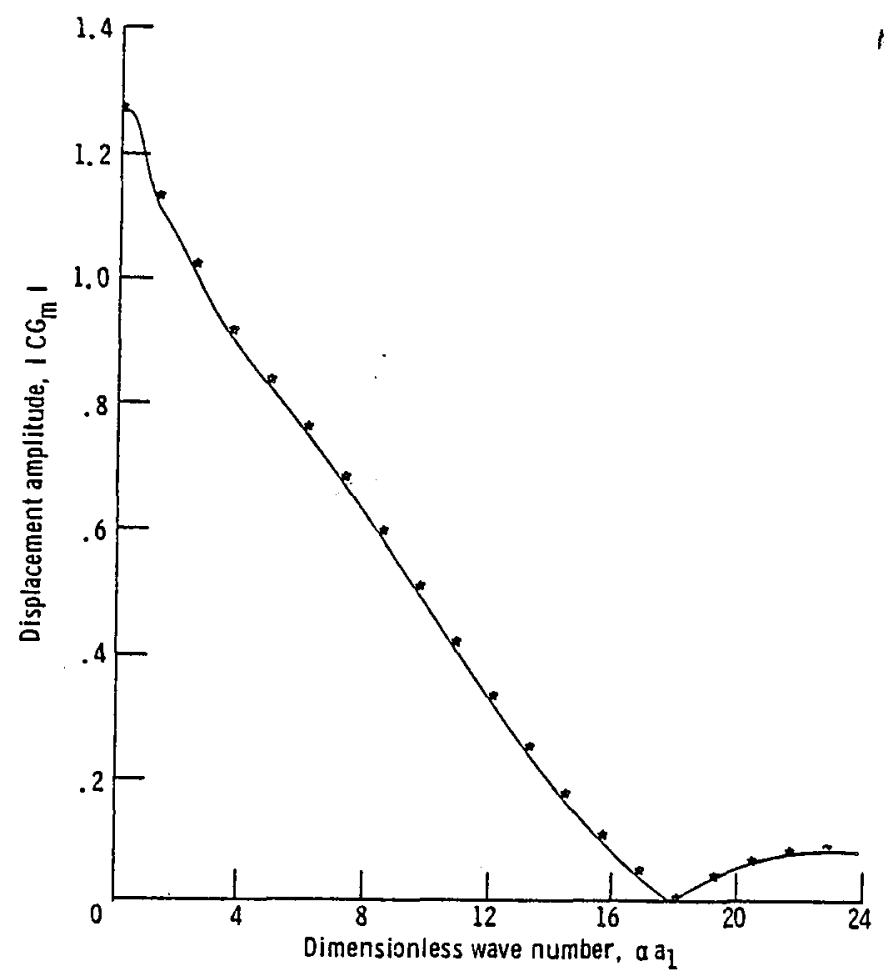

Figure 3. - $\left|C G_{m}\right|$ versus $\alpha a_{1}$ for back-scattered data for tungsten disk in aluminum matrix. $a_{2} / a_{1}=1 ; a_{1}=0.5 ; \phi=25^{\circ} ; \theta=30^{\circ} ; a_{m}=-l_{m}$.

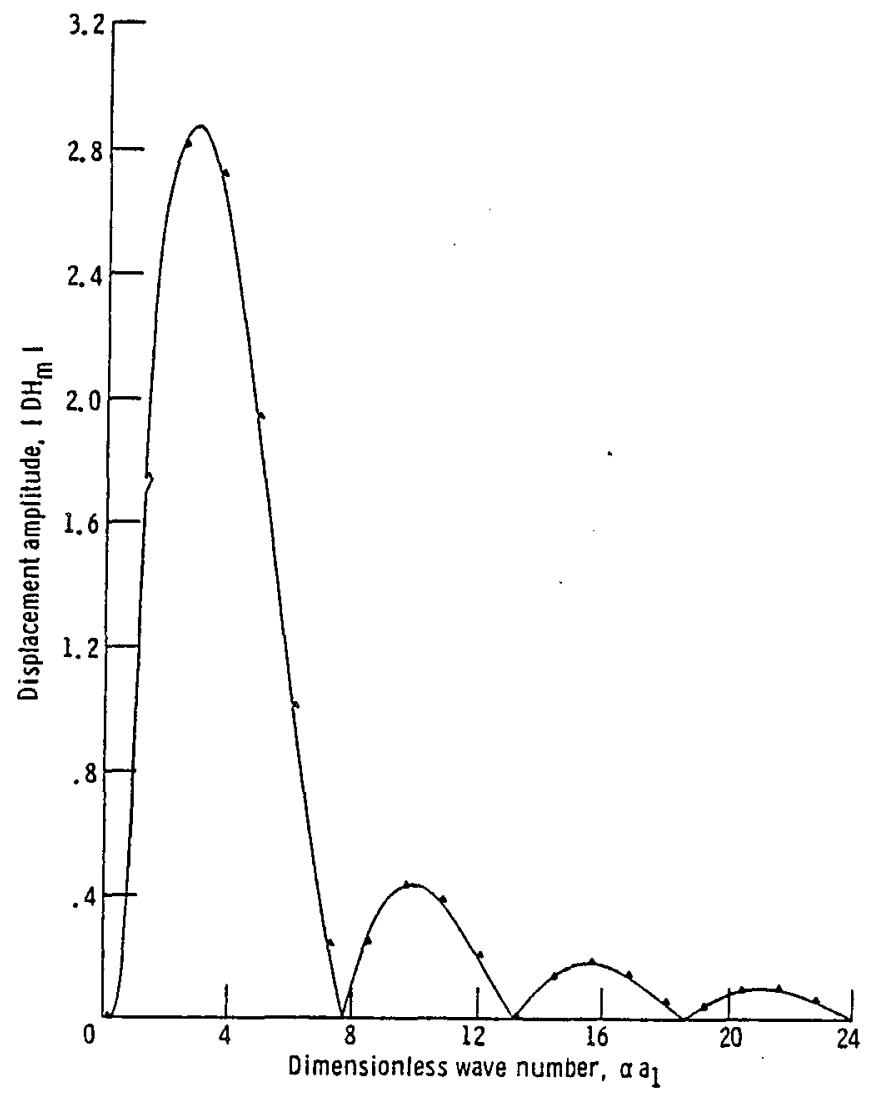

Figure 4. - $\left|\mathrm{OH}_{m}\right|$ versus $\alpha_{a}$ for back-scattered data for tungsten disk in aluminum matrix. $a_{2} / a_{1}=1 ; a_{1}=0.5 ; \phi=25^{\circ} ; \theta=30^{\circ} ; a_{m}=-l_{m}$. 


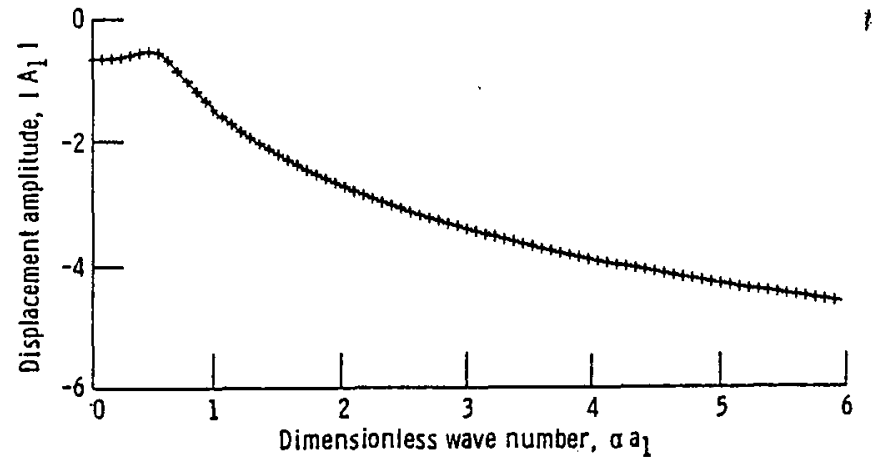

Figure 5. - $\left|A_{1}\right|$ versus $\alpha a_{1}$ for back-scattered data for tungsten disk in aluminum matrix. $a_{2} / a_{1}=1 ; a_{1}=1.0 ; \phi=25^{\circ} ; \theta=30^{\circ} ; q_{m}=-l_{m}$.

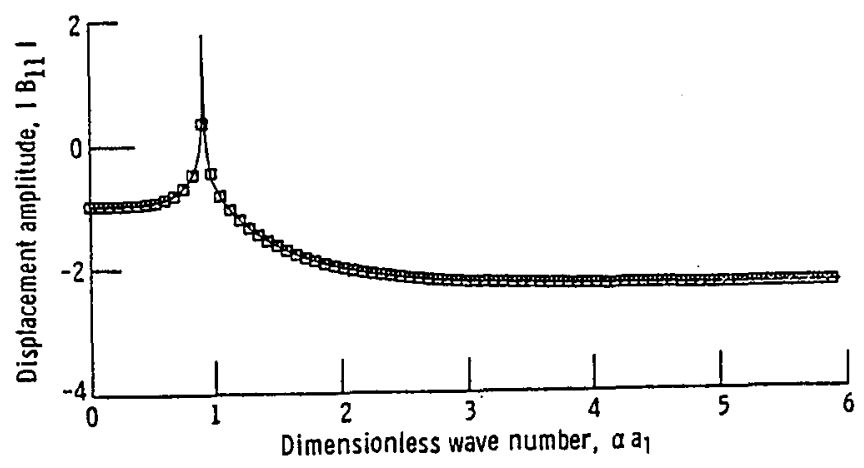

Figure 6. - $\left|B_{11}\right|$ versus $\alpha a_{1}$ for back-scattered data for tungsten disk in aluminum matrix. $a_{2} / a_{1}=1 ; a_{1}=1.0 ; \phi=25^{\circ} ; \theta=30^{\circ} ; a_{m}=-l_{m}$.

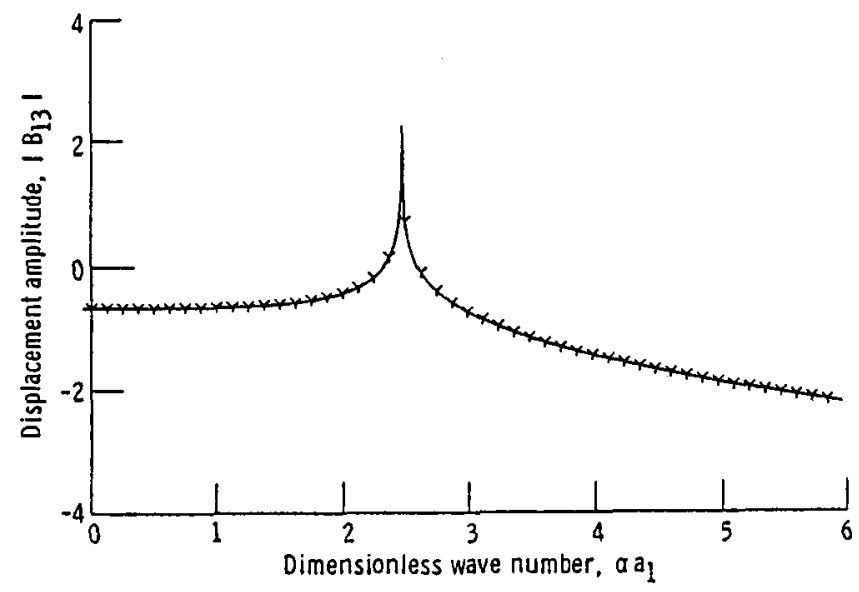

Figure 7. - $\left|B_{13}\right|$ versus $\alpha_{1} a_{1}$ for back-scattered data for tungsten disk in aluminum matrix. $a_{2} / a_{1}=1 ; a_{1}=1.0 ; \phi=25^{\circ} ; \theta=30^{\circ} ; a_{m}=-l_{m}$. 


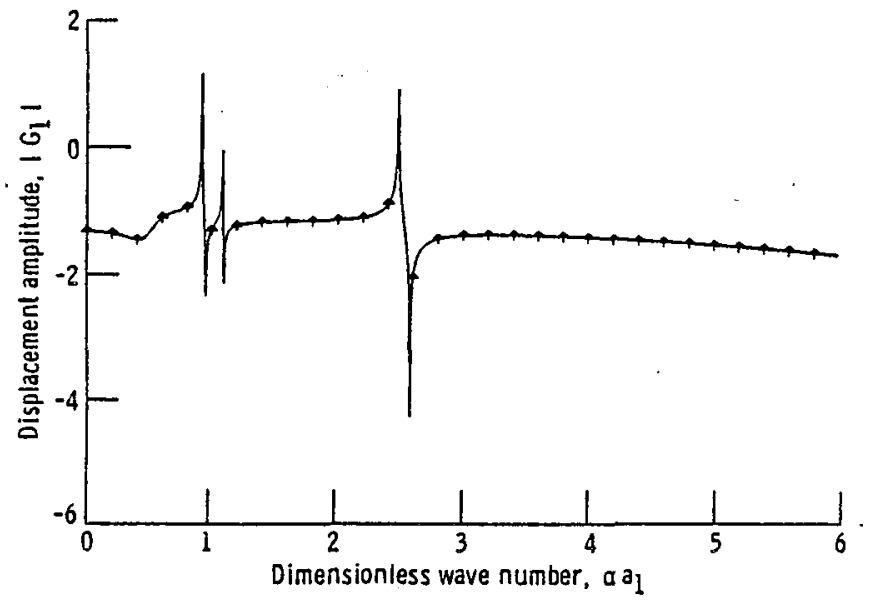

Figure 8. - $\left|G_{1}\right|$ versus $\alpha a_{1}$ for back-scattered data for tungsten disk in aluminum matrix. $a_{2} / a_{1}=1 ; a_{1}=1.0 ; \phi=25^{\circ} ; \theta=30^{\circ} ; a_{m}=-l_{m}$.

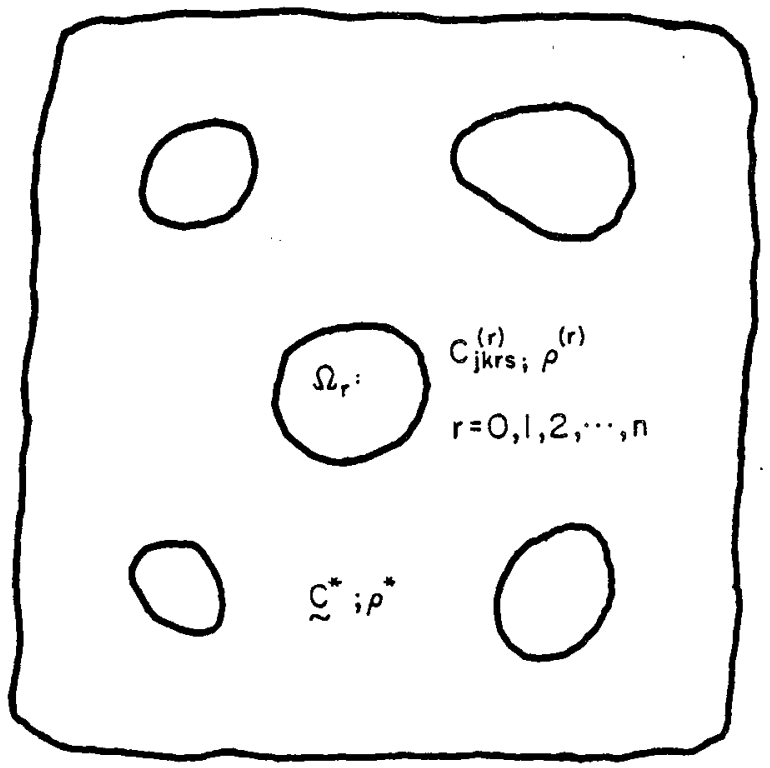

Figure 9. - Inhomogeneous medium with multicomponents. 


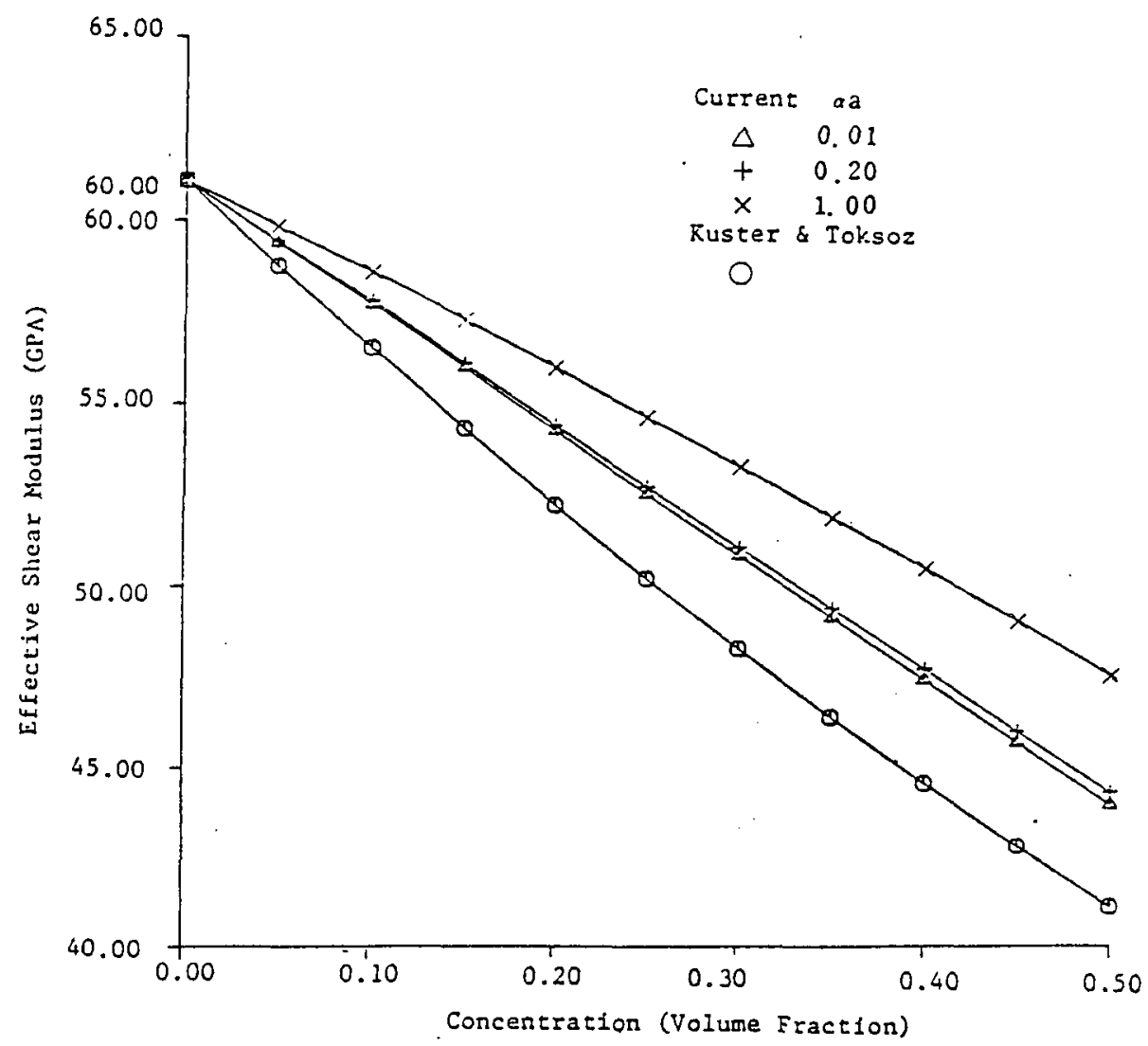

Figure 10. - Effective shear modulus versus concentration: aluminum spheres in germanium. 


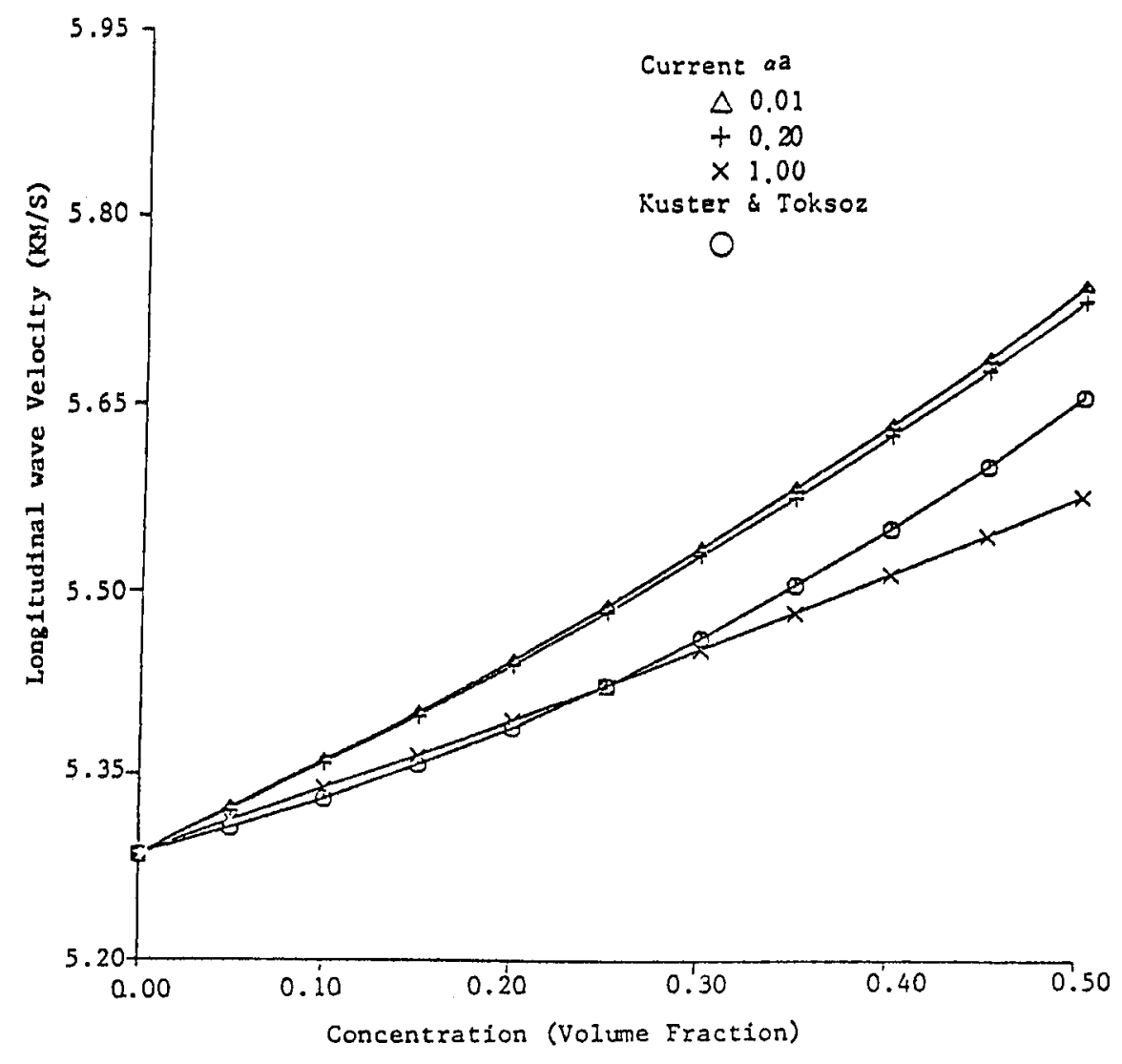

Figure 11. - Longitudinal wave velocity versus concentration: aluminum spheres in germanium.

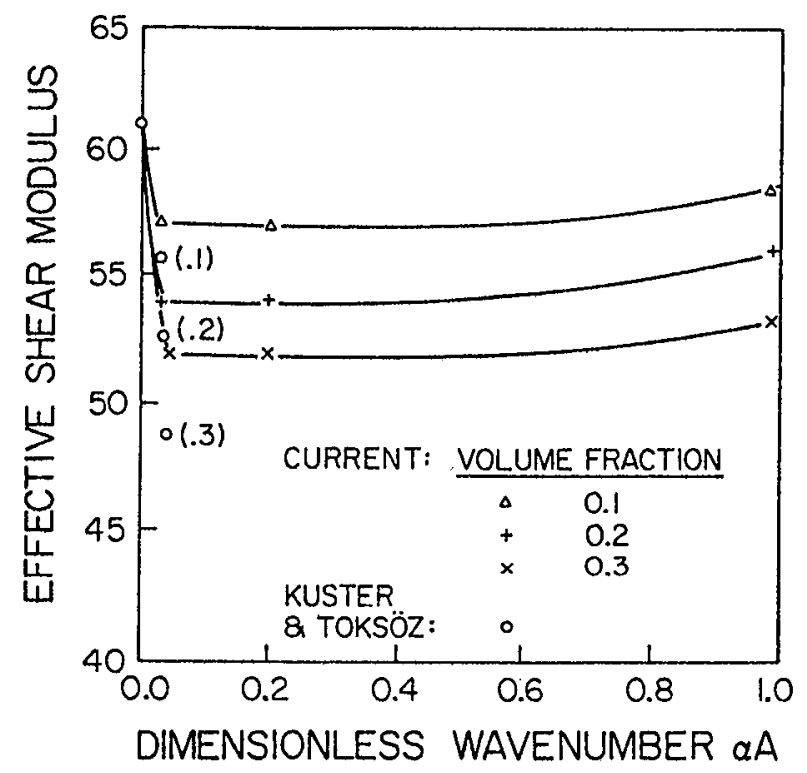

Figure 12. - Effective shear modulus versus wave number: aluminum spheres in germanium. 


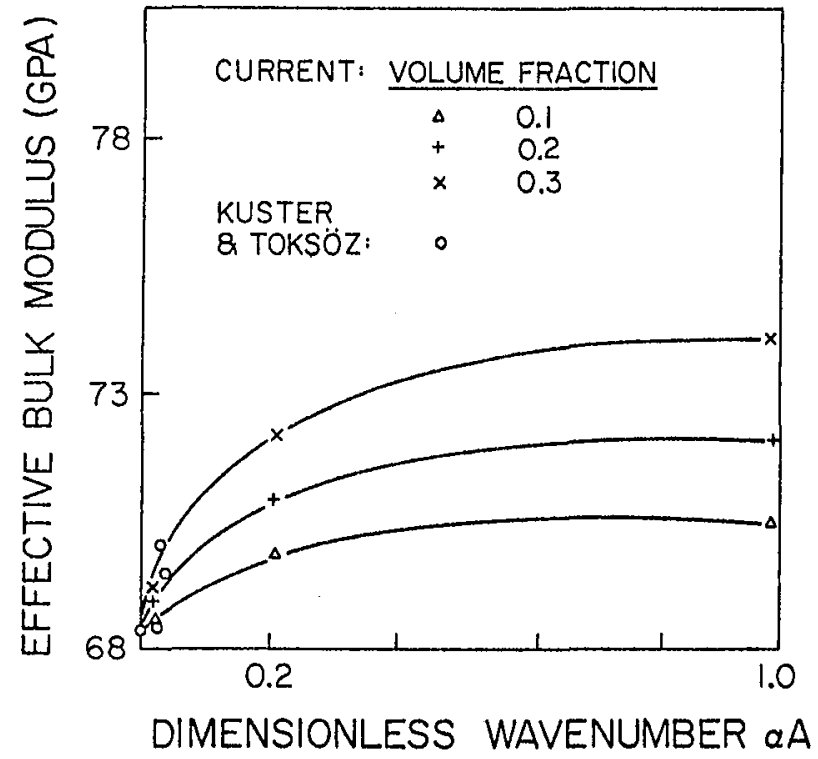

Figure 13. - Effective bulk modulus versus wave number: aluminum spheres in germanium. 


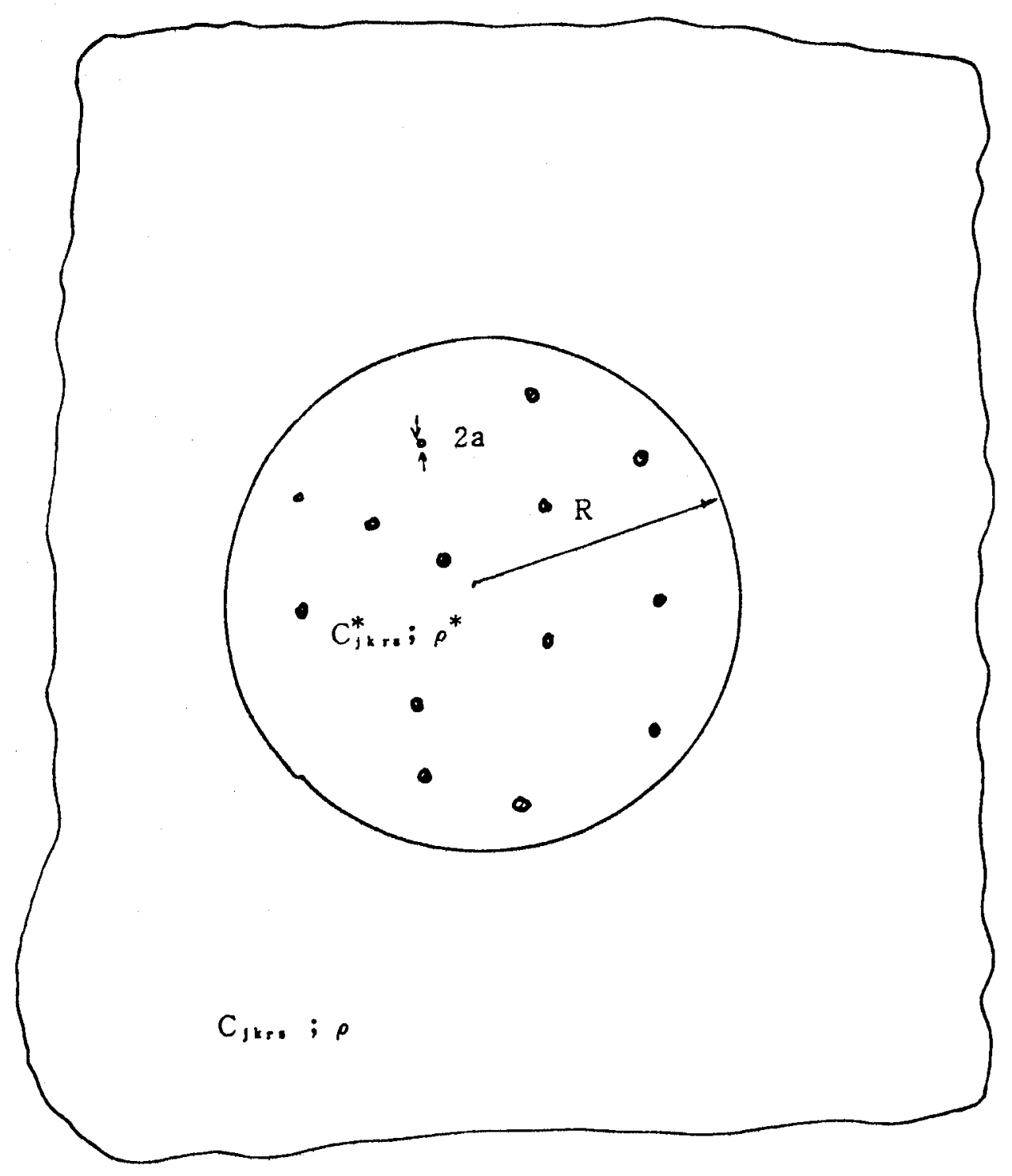

Figure 14. - Schematic diagram of localized damage in infinite solid. 


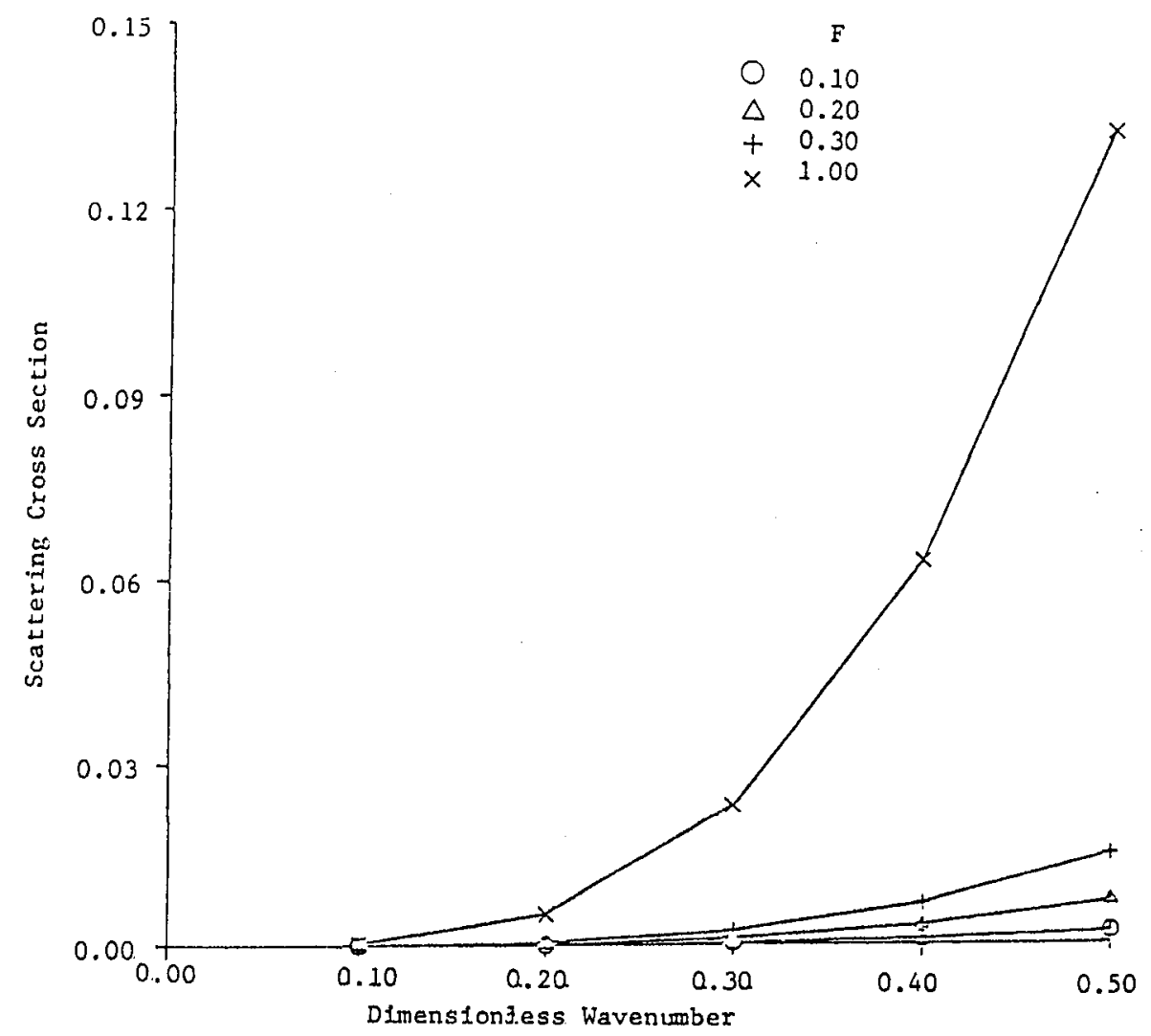

Figure 15. - Scattering cross section versus dimensionless wave number: distributed voids within a spherical domain in titanium matrix, $a / R=0.01$. 


\title{
TRANSFER FUNCTION CONCEPT FOR ULTRASONIC CHARACTERIZATION OF MATERIAL MICROSTRUCTURES
}

\author{
Alex Vary and Harold E. Kautz \\ National Aeronautics and Space Administration \\ Lewts Research Center \\ Cleveland, Oh10 44135
}

Ultrasonic attenuation measurements provide nondestructive means for characterizing material properties. In polycrystalline solids the frequency dependence of attenuation characterizes microstructure and hence the mechanical properties governed by microstructure. Microstructure, morphology, specific mechanical properties, and ultrasonic characteristics of materials are often uniquely interrelated (Green, 1973a, 1981, 1985; Vary, 1984).

Although expressions that relate ultrasonic attenuation to specific microstructural factors exist, they are fragmented and unsatisfactory because they consist of diverse piecemeal descriptions and ad hoc combinations of attenuation mechanisms over various frequency ranges of interest (Merkulov, 1956; Papadakis, 1960, 1965b; Serabian and Williams, 1978). A unified approach is needed to overcome these shortcomings and to help resolve ques... tions that arise in attempts to identify and evaluate key microstructural factors that govern mechanical properties.

The approach given herein depends on treating material microstructures as elastomechanical "filters" that have analytically definable transfer functions. These transfer functions can be defined in terms of the frequency dependence of the ultrasonic attenuation coefficient (Vary, 1980b, 1984). The transfer function concept provides a basis for synthesizing expressions that characterize polycrystalline materials relative to microstructural factors such as mean grain size, grain-size distribution functions, and grain boundary energy transmission. Although our approach is nonrigorous, it leads to a rational basis for combining the previously mentioned diverse and fragmented equations for ultrasonic attenuation coefficients.

\section{BACKGROUND AND THEORY}

\section{General}

Ultrasonic materials characterization involves both velocity and attenuation measurements. Velocity measurements are useful for measuring elastic constants, residual stresses, density, etc. This paper concentrates on attenuation measurements because they are pivotal to understanding interrelations between microstructure and mechanical properties. The characterization of mechanical properties depends on precise measurements of attenuation.

U1trasonic frequency-spectrum analysis provides a means for characterizing microstructural factors that govern mechanical properties (Papadakis, 1976; Fitting and Adler, 1981; Vary, 1984). The frequency range emphasized 
herein is that normally used for interrogating polycrystalline engineering materials (i.e., about 10 to $100 \mathrm{MHz}$ ). By using ultrasonic frequency-spectrum analysis and signal deconvolution algorithms (Bracewell, 1978; Bendat and Pierso1, 1980; Vary, 1980b; Williams, et a1., 1985a), it is possible to define transfer functions that characterize microstructures relative to their effect on wave propagation and also on mechanical properties. It will be seen that these transfer functions are definable in terms of the frequency dependence of the attenuation coefficient.

\section{Pulse-Echo Method}

F'or the purposes of this paper we assume a material sample with flat, parallel opposing surfaces. The sample is examined with a broadband, pulseecho system using digital signal acquisition and processing (fig. 1). A buf fered, piezoelectric transducer collects a set of echoes returned by the back surface of the sample. Only the first two echoes, $B_{1}$ and $B_{2}$, are selected for analysis. Signal acquisition, processing, and analysis are done as described by Papadakis (1976), Vary (1979b, 1980a,b), Generazio (1984), and Kline (1984). The echoes are transformed to the frequency domain by digital Fourier transform algorithms. Ultrasonic attenuation as a function of frequency and therefore information on material microstructure is obtained by deconvolution of echoes $B_{1}$ and $B_{2}$.

\section{Material Transfer Function}

In the following discussion the quantities $B_{1}, B_{2}, I_{1}, I_{2}, T$, and $R$ (fig. 2) are Fourier transforms of corresponding time-domain quantities. The quantities $B_{1}, B_{2}, I_{1}$, and $I_{2}$ are spectra of waveforms, and $T$ is the material transfer function, to be defined in the frequency domain. (Phase spectra and such associated topics as waveform synthesis and phase interference effects are not considered herein.) The reflection coefficient $R$ is also a function of frequency, albeit a weak function in most cases (Papadakis, 1968; Generazio, 1984). The transfer function will be defined in terms of ${ }^{\mathrm{B}_{1}}, \mathrm{~B}_{2}$, and $\mathrm{R}$.

Internal echo $I_{1}$ is the source of the "reduced" signal $B_{1}$ (i.e., reduced by the factor $(1+R)$, where $R$ is the reflection coefficient at the specimen transducer interface). The reflection coefficient is unity (i.e., 1) at the free surface. Algebraic signs carried by reflection coefficients depend on whether they are defined as power or amplitude quantities and on the input-output direction of signals across interfaces. The "power" (energy) definition as given in appendix $A$ applies to $R$ herein. A part of the energy of $I_{1}$ is reflected and appears as the second internal echo $I_{2}$ giving the reduced echo $\mathrm{B}_{2}$; thus

$$
\begin{aligned}
& \mathrm{B}_{1}=(1-\mathrm{R}) \mathrm{I}_{1} \\
& \mathrm{~B}_{2}=\operatorname{TR}(1-\mathrm{R}) \mathrm{I}_{1}
\end{aligned}
$$

where $B_{1}, B_{2}$, and $I_{1}$ are the "power" (i.e., magnitude-squared spectra of corresponding amplitude squared time-domain signals). Combining equations (1) and (2) yields 


$$
T=\frac{\mathrm{B}_{2}}{\mathrm{RB}_{1}}
$$

There are transfer functions associated with the instrumentation, signal transduction, and other aspects of the signal acquisition system. These can be ignored because in the pulse-echo configuration they vanish, just as the term $(1+R) I_{1}$ vanishes in equation ( 3$)$.

\section{Attenuation Coefficient}

Papadakis (1968; 1976) has demonstrated that the attenuation coefficient can be found by frequency spectrum analysis and that it may be written as

$$
\alpha=\left(\frac{1}{2 x}\right) \ln \left(\frac{\mathrm{RB}_{1}}{\mathrm{~B}_{2}}\right)
$$

where $x$ is the sample thickness. The attenuation coefficient $\alpha$ is a function of frequency and hence as with the quantities $T, B_{1}$, and $B_{2}$, it is appropriate to treat it as a Fourier transform of a time-domain quantity. Then because the transfer function $T$ and the attenuation coefficient $\alpha$ are both defined in terms of $B_{1}, B_{2}$, and $R$, equations ( 3 ) and (4) can be combined to get

$$
T=\exp (-2 x \alpha)
$$

Thus the transfer function is defined in terms of an attenuation coefficient. Conversely, if the material transfer function $T$ can be prescribed apriori, appropriate expressions can be found for the attenuation coefficient $\alpha$. It will be seen that a general, unified expression can be derived for $\alpha$ for the various energy loss mechanisms in polycrystalline solids by using the preceding equations and the transfer function concept.

\section{Attenuation Mechanisms}

The energy loss mechanisms that govern ultrasonic attenuation in the frequency ranges (bandwidths) of interest for polycrystalline engineering solids are known (Mason and McSkimmin, 1947; Lifsitz and Parkomovskii, 1950; Granato and Luicke, 1956; Merkulov, 1957; True11, et al., 1969; Bahtia, 1976). The two principal causes of attenuation are scattering and absorption. Diffusion, Rayleigh, and stochastic (or phase) scattering losses are extrinsic; absorption losses from dislocation damping, anelastic hysteresis, relaxation, and thermoelastic effects are intrinsic to individual grains (i.e., crystallites).

We are not concerned with geometrical losses, such as those due to diffraction effects, which are not inherent to material microstructures. These effects can be either controlled or eliminated from attenuation measurements by wel1-known experimental and data reduction procedures (Papadakis, 1975) in order to get the true attenuation coefficient as a function of frequency. 
Scattering usually accounts for the greatest portion of losses in polycrystalline aggregates (Papadakis, 1964a,b, 1965a,b, 1970; Serabian and Williams, 1976; Goebbels, 1980). For polycrystalline aggregates expressions that have been developed for the scatter attenuation coefficient $\alpha$ as a function of frequency $f$ are referred to three regimes that are defined by the ratio of mean grain size a to dominant wavelength $\lambda$.

For the Rayleigh scattering regime, where $\lambda \gg \pi a$,

$$
\alpha_{r}=c r^{a^{3} f^{4}}
$$

For the stochastic (phase) scattering regime, where $\lambda \cong \pi a$,

$$
\alpha_{p}=c_{p} a f^{2}
$$

For the diffusion scattering regime, where $\lambda \ll \pi a$,

$$
\alpha_{\mathrm{d}}=\mathrm{c}_{\mathrm{d}^{\mathrm{a}}}
$$

In equation (6) $a^{3}$ represents grain volume, where the grains are to be considered as having a variety of nonequiaxed, nonspherical shapes (Papadakis, $1965 \mathrm{~b}$ ). The constants $c$ contain other quantities (i.e., geometric factors, longitudinal and transverse velocities, density, and elastic anistropy factors)(Mason and McSkimmin, 1947, 1948; Lifsitz and Parkomovskii, 1950;

Merkulov, 1957; Truell, et al., 1969). These factors will be considered later in this paper.

\section{Intrinsic Mechanisms}

Absorption losses due to dislocation damping, hysteresis, thermoelastic effects, etc., are intrinsic to grains (crystallites) and involve direct conversion of acoustic energy to heat. These attenuation mechanisms are essen-tially independent of grain size, shape, volume, etc.

For hysteresis losses (Kolsky, 1953; Nowick and Berry, 1972)

$$
\alpha_{h}=c_{h} f
$$

For thermoelastic losses (Lucke, 1956)

$$
\alpha_{t}=c_{t} f^{2}
$$

Hysteresis losses (eq. (9)) arise when acoustic waves cause stress-strain damping. Hysteresis losses with a first-power frequency dependence are usually observed in single crystals and amorphous solids and with difficulty in polycrystalline solids (Mason, 1958). Equation (10) gives the frequency dependence of thermodynamic losses that arise when longitudinal waves produce heat flow from dilatation to compression regions. 
Viscous losses (Auld, 1973) also exhibit a second-power frequency dependence but are generally negligible in solids. Models for absorption losses caused by dislocation vibrations, relaxation effects, and internal friction predict second-power frequency dependence down to frequency independence (Granato and Lücke, 1956; Seeger and Schiller, 1962; Truel1, et al., 1969). Absorption losses due to magnetic domains, electrons, or phonons are special cases involving ferromagnetic materials, very high frequencies, or cryogenic temperatures, respectively, and are not considered here.

\section{Combined Expressions}

Total attenuation coefficients are usually written ad hoc as sums of coefficients for scattering and absorption. For example, equations (7) and (9) are combined to form an expression for the total attenuation coefficient $\alpha$, where

$$
\alpha_{h p}=c_{h} f+c_{p} a f^{2}
$$

or equations (6) and (9) are combined to get

$$
\alpha_{h r}=c_{h} f+c_{r} a^{3} f^{4}
$$

Combined expressions as in equations (11) and (12) are convenient for fitting experimental data and for analyzing the contributions of attenuation mecha-nisms and underlying microstructural factors.

An inconsistency occurs in attempting to combine the expressions for Rayleigh and stochastic attenuation in equations (6) and (7) because as ultrasonic frequency increases, the fourth-power term for Rayleigh scattering becomes dominant. This contradicts the expectation that for higher frequencies, as wavelength diminishes and approaches grain size, the second power of frequency due to stochastic scattering should dominate. Papadakis (1965b) and others have dealt with this issue by applying equations (6) and (7) piecemeal and adapting a smoothing function to form continuity between the Rayleigh and stochastic regimes. Smith (1984) has observed that the transition from Rayleigh to stochastic scattering depends on grain-size distribution and suggests introducing a probability function in scatter attenuation equations proposed by Roney (1950) and Serabian and Williams (1978).

Maisel (1984) overcomes the problem of expressing the transition from Rayleigh to stochastic scattering by using an electrical network analog to model ultrasonic scattering. Maisel's results suggest expressions for the attenuation coefficient that combine Rayleigh and stochastic frequency dependencies in a continuous function. One such expression is

$$
\alpha_{r p}=c_{r p} \frac{4 a^{3} k^{4}}{1+4 a^{2} k^{2}}
$$

where $c$ is a material constant incorporating average fractional deviations of density and compressional modulus, a is mean scatterer size, $k$ is wave number $(2 \pi f / v)$, and $v$ is velocity. The formulation of equation (13) allows 
the fourth power of frequency to dominate at low frequencies and the second power to dominate at high frequencies. A similar formulation was derived by Perkeris (1947) by assuming an autocorrelation function for polycrystalline inhomogeneity. Although both formulations allow a smooth transition from fourth- to second-power frequency dependence with increasing ultrasonic frequencies, no allowance is made for frequency independence at extreme frequencies in accordance with equation (8). Rokhlin (1972) describes a generalized formulation that encompasses Rayleigh, stochastic, and diffusion scattering. However, in Rokhlin's approach a smooth transition between scattering regimes is lost because of the periodic nature of the sine function that was used.

\section{MICROSTRUCTURE CONSIDERATIONS}

\section{Grain Size}

Before dealing with transfer function synthesis we briefly review some relevant aspects of the interplay of microstructure and the previously discussed formulations for ultrasonic attenuation.

A universally cited quantity for characterizing polycrystalline microstructures is "mean grain size." This quantity is used regardless of difficulties inherent in measuring it or assigning appropriate values to it, especially in materials that exhibit complex microstructures (subgrains, second phases, etc.). Nevertheless it is appropriate, for many single-phase polycrystallines with "uniform" microstructures, to speak of a mean grain size. This depends on discovering a definitive grain-size distribution function (DeHoff, 1968). Most polycrystallines exhibit grain-size distributions that are highly skewed and nonGaussian because of a high incidence of large grains (Papadakis, 1964b, 1965b; Schückler, 1968; Evans, et al. 1978; Smith, 1984; Tittmann, et al., 1985).

There are polycrystalline aggregates that have microstructures with readily defined mean grain sizes (Schuickler, 1968). When expressions of the form of equations (11) and (12) are fitted to experimental attenuation data for samples of these materials, a new expression for the same sample after annealing to a different mean grain size can be obtained by modifying the coefficients of frequency by a numerical scale factor (e.g., the ratio of mean grain sizes). This has been demonstrated for polycrystalline samples of nickel and copper where measurements were predominantly in the stochastic scattering regime (i.e., principal wavelengths commensurate with grain size (Generazio, 1985)). A general scaling parameter for grain size has been described by Serabian and Williams (1978).

Scaling of attenuation expressions probably applies only to homomorphic microstructures having grain-size distribution functions that are invariant with changes in mean grain size. Photomicrographs of the successive heat treatments produced by Generazio (1985) seem to scale (i.e., map) exactly in this sense. Apparently scaling can be done even with rather complex microstructures despite difficulties in producing or identifying homomorphic grainboundary patterns. 
Scattering theories usually consider ensembles of scattering centers embedded in a featureless continuum. Current theories for polycrystallines account only for scattering by equiaxed grains and neglect effects of texture, anisotropy, grain-size variations, etc. Indeed, well-formulated theories for attenuation exist only for the simple case of single-phase polycrystalline solids with virtually identical, equiaxed grains and for frequencies that satisfy the conditions $k a \ll 1$ or $k a \gg 1$, where $k$ is wave number and a is mean grain (scatterer) size.

Underlying equations (6) and (7) is the assumption of single scattering by independent scatters; the conceptual and theoretical difficulties that arise with multiple scattering where scatterers interact and velocity dispersion effects are likely to arise are ignored. For example, equation (6) is based on the "Rayleigh criterion" and ideal elastic scatterers that are sufficiently separated to ensure single scattering (Truell, et al., 1969).

Fourth- and second-power relations given in equations (6) and (7), respectively, have been experimentally confirmed for special cases (Winkler and Murphy, 1984). Papadakis (1981) and Sayers (1985) realized the fourthpower relation of equation ( 6 ) by measuring scattering due only to "minority" phases (e.g., carbon nodules and inclusions) in polycrystalline aggregates. Evans, et al., (1978) used a similar approach for materials with sparse distributions of "predominant" scatterers in a polycrystalline "continuum."

Apparently, when used with materials exhibiting discrete, widely separated, "strong" scattering centers, the Rayleigh criterion is satisfied and the difficulties due to multiple scattering are avoided. It seems remarkable that frequency dependences predicted by rather simplified scattering models can be realized even with complex polycrystallines.

\section{Empirical Correlations}

For most polycrystallines the overall experimentally determined frequency dependence of attenuation is not an integral power. Exponents on frequency even in the Rayleigh or stochastic regimes rarely equal exactly 4 or 2 , respectively (Papadakis, 1981). This is probably due partly to imprecisions in experimental measurements and to attenuation data being defined only within the bandwidth used to interrogate the sample. Within limited bandwidths the best $f$ it of experimental data simply reflects only a portion of the actual attenuation function.

Because of the aforementioned factors it is often possible to fit attenuation data with expressions that allow the exponent on frequency to be an experimentally determined variable (Vary, 1980a,b). Among the simplest expressions of this kind is

$$
>a<=\operatorname{cf}^{\mathrm{m}} \quad \mathrm{f}_{1}<\mathrm{f}<\mathrm{f}_{2}
$$

where $>\alpha<$ denotes that $\alpha$ is empirically defined only in a limited frequency range and where $m$ may assume noninteger values. The quantities $c$ and $m$ will change correspondingly with microstructural changes that affect 
attenuation (mean grain size, grain size distribution, etc.). Experimental results show that equation (14) is appropriate for fitting attenuation data over a frequency range that spans the Rayleigh scattering regime for a variety of polycrystalline materials (Papadakis, 1964a, 1976; Vary, 1978; BozorgGrayeli, 1981; Vary and Hu11, 1982, 1983). It will be seen that equation (14) gives statistically valid descriptions of data in the Rayleigh regime.

\section{Grain Interfaces}

From a purely physical standpoint grain facets and surface areas should influence mechanical properties of polycrystallines. This is certainly true of properties that depend on the surface energy of grains, properties affected by the grain-boundary thickness, properties for which grain boundaries are obstacles, and properties connected with grain- and phase-boundary migrations and obliterations. The transfer function concept, described next, provides a basis for a unified expression for attenuation that incorporates the previously discussed attenuation mechanisms and also a means for studying the effects of variations in grain-boundary energy transmission factors.

\section{TRANSFER FUNCTION SYNTHESIS}

\section{Approach}

An attenuation transfer function for polycrystalline solids is defined in terms of a lattice model that incorporates the effects of grain-boundary (interface) spacing and energy transmission across interfaces. The lattice model is generalized by the introduction of probability distribution functions to account for variations in grain and scatterer size and boundary spacing. The transfer function concept provides a convenient and rational basis for incorporating the previously described attenuation mechanisms into a unified expression for ultrasonic attenuation.

\section{Interface Transfer Function}

The transfer function of an interface between two media is defined in terms of the reflection coefficient at the interface. We assume ideal flat interfaces between dispersion-free media with unequal acoustic impedances. In this case the reflection coefficient $R$ is taken as a frequency-independent Heaviside step function in the frequency domain, corresponding to a delta (impulse) function in the time domain, see figure 3 and appendix $B$.

The reflection coefficient $R$ in equations (1) to (4) conforms with the interface transfer function notion given here. If it becomes necessary to consider velocity dispersion or nonideal (thick, strained, acoustically rough, etc.) interfaces, expressions for $R$ (and the quantity $G$, defined below) would simply assume frequency-dependent forms (Papadakis, 1976; Kinsler, et al., 1982; Generazio, 1984). 
The lattice model illustrated in figure 4 represents a volume that consists of series of plane parallel interfaces separating thin layers with unequal acoustic impedances (compare with Rokhlin (1972), where a volume is divided into equal rectangular parallepipeds). The model is one dimensional and accounts for energy losses suffered by longitudinal rays traveling at normal incidence to the interfaces. Each of the $n$ internal interfaces has a transfer function defined in terms of an internal reflection coefficient $G$ (appendix B). For the moment consider each layer as a featureless (scatterer free) medium with an intrinsic attenuation function

$$
A=|A(f)|=\exp \left(-\alpha_{i} \ell\right)
$$

Where $\ell$ is layer thickness and $A$ is the magnitude (power) spectrum of $A(f)$. As developed in appendix $C$ the transfer function $T$ for the lattice model can be reduced to the form

$$
T=(H-G)^{2 n} A^{2(n+1)}\left[\sum \ldots\right]
$$

where $H=|H(f)|=1$ is the Heaviside unit step function that will be rendered as 1 hereinafter (i.e., $(H-G)=(1-G)$ ). The multiple convolution (multiple reflection) factor $\left[\sum \ldots\right]$ in equation (16) is

$\left[\sum \ldots\right]=\left[\sum_{1}^{q}(R G)^{q-1} A^{2(q-1)}\right]^{2}\left[\sum_{1}^{q} G^{q-1} A^{2(q-1)}\right]\left[\sum_{1}^{q} G^{2(q-1)} A^{2(q-1)}\right]^{2(n-1)}$

The number of first-order multiple reflections in each lattice layer is $q-1$, and the number of layers is $n+1$. Equation (16) defines $T$ in terms of power (energy) reflection functions $R$ and $G$ (appendix $A$ ). The terms $A, G, R$, and $T$ are Fourier transforms. The various powers and products of $A, G$, and $R$ are convolutions of their time-domain counterparts. For example, RG in equation (17) is the convolution of corresponding time-domain quantities. The quantity $\left[\sum \ldots\right]$ given in equation (17) explicitly contains only first-order multiple reflections. It will be seen that this is sufficient for generalizing the model.

\section{Lattice Model with Scattering}

The lattice model may be extended to include scattering by taking $A$ in equations (16) and (17) to consist of both intrinsic and extrinsic attenuation factors,

$$
A=\left[\exp \left(-\alpha_{i} \ell\right)\right]\left[\exp \left(-\alpha_{e} \ell\right)\right]
$$

If it is postulated that each layer contains an independent scattering center in addition to intrinsic attenuation, equation (16) takes the form

$$
T=(1-G)^{2 n}\left[\exp \left(-\alpha_{i} l\right) \exp \left(-\alpha_{e} l\right)\right]^{2(n+1)}\left[\sum \ldots\right]
$$


Let the intrinsic attenuation be due to anelastic hysteresis, and in accordance with Nowick and Berry (1972) express it as

$$
\alpha_{i}=c_{h} f=\left(\frac{h}{v}\right) f
$$

where $h$ is a hysteresis constant and $v$ is velocity. For extrinsic attenuation take the Rayleigh scattering expression derived by Mason and McSkimmin (1947),

$$
\alpha_{e}=c r^{a^{3} f^{4}}=\left(\frac{16 \pi^{4} K}{3 v^{4}}\right) a^{3} f^{4}
$$

where $a$ is mean scatterer size (with $4 \pi a^{3} / 3$ replacing the mean grain volume in the Mason and McSkimmin (1947) derivation) and $k$ is elastic anisotropy ( $i . e .$, average change in elasticity encountered from one layer to the next (Mason and McSkimmin, 1948; Lifsitz and Parkomovskii, 1950; Merkulov, 1957)).

\section{Generalization for Polycrystallines}

We generalize the lattice model to resemble a polycrystalline by postulating unequal layer thicknesses and nonparallel interfaces. Thus any ray traveling at normal incidence to the front and back surfaces (still mutually parallel) encounters a sequence of interfaces similar to those in a polycrystalline aggregate.

Combining equations (4) and (19), noting that, for large values of $n$, $(n / x)=(1 / \ell)$ while $(n+1) \ell=x$ and taking $\ell$ as mean layer thickness, an expression is obtained for $\alpha$ in terms of $T$,

$$
\alpha=-\left(\frac{1}{l}\right) \ln (1-G)+\alpha_{i}+\alpha_{e}+\left(\frac{1}{2 x}\right) \ln \left[\sum \ldots\right]
$$

The quantity $G$ is now taken as average reflection coefficient for the randomly oriented interfaces. If $G$ is sufficiently small and energy is indeed scattered away in lattice layers, the contribution of higher order terms in the sumnations in equation (17) may be ignored. This is equivalent to setting $q=1$ in equation (17) so that $\ln [\Sigma \ldots]=0$ (see DISCUSSION). Although the energy transmission coefficient $I-G$ may approach unity, the interface transparency term ln $(1-G)$ is always finite (i.e., a nonzero negative).

Given the preceding argument and noting that $l$ corresponds to a mean grain size represented by the quantity a and combining equations (20) to (22),

$$
\alpha=-\left(\frac{1}{a}\right) \ln (1-G)+\left(\frac{h}{v}\right) f+\left(\frac{16 \pi^{4} K}{3 v^{4}}\right) a s^{2} f^{4}
$$

Although a spherical scatterer with volume $4 \pi \mathrm{a}^{3} / 3$ is implicit to equation (21), in equation (23) scatterer geometry is made abstract so that $s$ is an effective mean scatterer diameter while a is mean scatterer length. Thus 
we allow for nonequiaxed grains or scatterers with aspect ratios a/s of 1 or greater. Rokhlin (1972) assumes grains to be parallelepipeds having square ends but different side lengths in the direction of wave propagation.

\section{Generalization for Grain-Size Distribution}

The range of grain sizes usually contained in a polycrystalline can be described by a histogram or, for a large grain population, by an analytical probability distribution function. Grain-size distributions are usually nonGaussian and their probability distributions are typically log-normal functions (DeHoff, 1968; Schuickler, 1968; Smith, 1984). A function that describes grain-size distributions representative of most polycrystalline solids is given by

$$
p(y)=\left(\frac{4 y}{a}\right) \exp \left(\frac{-2 y}{a}\right) \quad p(y)=0 \text { for } y<0
$$

where $y$ is a spatial (length) variable and $a$ is mean grain size. We have pointed out that the quantity $\alpha$ is defined in the frequency domain and can therefore be treated as the Fourier transform of a time-domain quantity. To incorporate probability distribution functions in the expression for $\alpha$ given by equation (23), we transpose to the tine domain, take the Fourier transform, and find the results of convolution with probabalistic factors in equation (23). Therefore let

$$
p(t)=\left(\frac{4 t}{u^{2}}\right) \exp \left(\frac{-2 t}{u}\right) \quad p(t)=0 \text { for } y<0
$$

where $u$ is mean transit (grain delay) time corresponding to mean grain size $a$ and $a=u v$, where $v$ is average velocity (fig. 5). The Fourier transform of $p(t)$ is

$$
P(f)=\left[1+(\pi u f)^{2}\right]^{-1} \exp \left[-2 i \tan ^{-1}(\pi u f)\right]
$$

The phase spectrum given by the exponential term in equation (26) is ignored since we are interested only in the magnitude spectrum of $P(f)$ (i.e., $P=|P(f)|)$. The implicit assumption is that phase interference effects are insignificant. In general, the probability distribution function of the scatterer diameter $s$ is not identical to that of its axial length $a$. Therefore we admit two separate expressions for $P$,

$$
\begin{aligned}
& P_{a}=\left[1+\left(\pi u_{a} f\right)^{2}\right]^{-1} \\
& P_{s}=\left[1+\left(\pi u_{s} f\right)^{2}\right]^{-1}
\end{aligned}
$$

where subscripts $s$ and a refer to mean scatterer diameter and length, respectively. That is, $u_{a}=a / v$ and $u_{s}=s / v$. 
Now consider the first term in equation (23) as a probabilistic quantity. In the frequency domain the probabilistic weighting of the reflection function for transit time variations is GP and the probabilistic weighting for trans-mission is $G(1-P)$. Therefore the frequency dependence of the boundary energy transmission term $1-G$ in equation $(23)$ is $\ln [1-G(1-P)]$. The expression for $P$ in equation (27) will be used for this case. The middle term in equation (23) is independent of grain size and remains unaltered. In the third term of equation (23) a and $s$ are probabilistic so that

$$
\alpha=-\left(\frac{1}{v u_{a}}\right) \ln \left[1-G\left(1-P_{a}\right)\right]+\left(\frac{h}{v}\right) f+\left(\frac{16 \pi^{4} K}{3 v u_{a}}\right) u_{a}^{2} u^{2} f^{4} P_{a} P^{p}
$$

where $u_{a}=a / v, u_{s}=s / v$, and $v$ is average velocity. The corresponding probability functions $P_{2}$ and $P_{S}$ are to be defined according to the nature of the various microstructural factors that govern attenuation (grains, precipitates, etc.) in a particular solid. A further generalization would take grain-boundary spacing and scatterer spacing (and dimensions) to be independent of each other.

\section{VERIFICATION AND APPLICATIONS OF MODEL \\ Comparison with Scattering Theory}

We examine a special case of equation (29) wherein grains are equiaxed scatterers (i.e., $a=s=v u$ ). Also, take $P_{a}=P_{s}=P=|P(f)|$. For the moment ignore the first two terms in equation (29) so that consequences of the preceding derivations may be compared with classic expressions for scattering. The extrinsic attenuation (third) term of equation (29) expands to

$$
\alpha_{e}=\left(\frac{16 \pi^{4} k}{3 v u}\right)\left\{\frac{(u f)^{4}}{\left[1+(\pi u f)^{2}\right]^{2}}\right\}
$$

For $\lambda \gg \pi$ equation (30) reduces to the expression for Rayleigh scattering (Mason and McSkimmin, 1947),

$$
\alpha_{\text {er }}=\left(\frac{16 \pi^{4} K}{3 v^{4}}\right) a^{3} f^{4}
$$

For $\lambda \cong \pi a$ equation (30) reduces to the expression for stochastic (phase) scattering,

$$
\alpha_{e p}=\left(\frac{16 \pi^{2} K}{3 v^{2}}\right) a f^{2}
$$

For $\lambda \ll \pi a$ equation (30) reduces to the expression for diffusion scattering,

$$
\alpha_{\text {ed }}=\left(\frac{16 \mathrm{~K}}{3}\right) \mathrm{a}^{-1}
$$


Thus we have in equation (30) an expression for extrinsic attenuation that covers all theoretically postulated scattering mechanisms continuously over all frequencies.

Although we have adopted the scatter attenuation expression of Mason and McSkimmin (1947), the form developed by Lifsitz and Parkomovskii (1950) would be appropriate for defining extrinsic attenuation in equations (21) and (30) to account for elastic randomization and attendant effects of mode conversion. However, it is convenient and sufficient for our purposes to continue using the expression for extrinsic attenuation given in equation (21).

\section{Comparison with Experiment}

Consider a simple polycrystalline where, as in the previous section, grains and other scatterers are taken to be identical, so that $a=s=v u$, where $a$ is mean grain size, $u$ is mean grain delay, and $v$ is mean velocity. Using equation (30) for extrinsic scattering, equation (29) becomes

$$
\alpha=-\left(\frac{1}{a}\right) \ln \left(1-G\left\{1-\left[1+(\pi u f)^{2}\right]^{-1}\right\}\right)+\left(\frac{h}{v}\right) f+\left(\frac{16 K}{3 a}\right)\left\{\frac{(\pi u f)^{4}}{\left[1+(\pi u f)^{2}\right]^{2}}\right\}
$$

We assign appropriate values to $G, K$, and $h$ for comparing attenuation coefficients predicted by equation (34) with experimental data for nickel, copper, and iron polycrystallines.

Serabian and Williams (1978) estimated hysteresis constants for several metals (e.g., $\mathrm{h}=2 \times 10^{-5}$ for 97.4 percent iron alloys and $\mathrm{h}=3 \times 10^{-4}$ for 98.9 percent nickel alloys). Herein we assume $h=3 \times 10^{-4}$ for 99.99 percent copper.

Both $G$ and $K$ are estimated from equivalent equations and may be taken to be identical for cubic crystals (Mason and McSkimmin, 1947, 1948; Mason, 1958):

$$
K=\left[\frac{\left\langle c_{11}-\left\langle c_{11}\right\rangle\right\rangle}{\left\langle c_{11}\right\rangle}\right]^{2}=\left(\frac{z_{2}-z_{1}}{z_{2}+z_{1}}\right)^{2}=G
$$

where $c^{\prime}$ is the elastic tensor coefficient (compression modulus) and $\left\langle c^{\prime}\right\rangle$ is its average value. The $z=\rho v$ terms are defined in appendix $A$ and are based on principal longitudinal velocities. We can assume that for any given material $G$ is directly proportional to $\mathrm{K}$ and that for cubic crystallites $\mathrm{G}=\mathrm{K}$. According to Mason and McSkimmin (1948) $\mathrm{G}=\mathrm{K}=0.0074$ for copper, 0.0067 for iron, and 0.0072 for nickel.

Attenuation-versus-frequency curves generated with equation (34) are compared with experimental curves for the cubic materials iron, copper, and nickel in figures 6 to 8 . The estimated values for $K$ and $G$ do not account for the effects of oblique incidence or mode conversion at boundaries; these effects would increase their effective values. Therefore the values for $k$ cited above should give low predictions of attenuation since shear waves produced by mode conversions are more effectively scattered. Elastic anisotropy 
for shear waves can exceed that for longitudinal waves by perhaps a factor of 10. It is probably for this reason that the best agreement with experimental data is obtained when we double the estimated values for $K$ for use in equation (34). It will be seen that $K$ is in general a composite quantity containing other scattering factors.

Figure 6 compares original experimental data of Merkulov (1957) for iron with attenuation-coefficient-versus-frequency curves generated with equation (34). Excellent agreement between the experimental and predicted attenuation coefficients is evident in figures $6(a)$ and (b) for the particular values selected for $K, G$, and $h$ for iron, even to the extent that curve crossovers at approximately $14 \mathrm{MHz}$ are predicted. Figures $6(\mathrm{c})$ and (d) exhibit attenuation curves for iron over a broader frequency range of $100 \mathrm{MHz}$, as opposed to the $20-\mathrm{MHz}$ range in figure $6(\mathrm{~b})$. Figure $6(\mathrm{c})$ is based on $K=0.0067$ and figure $6(d)$ on $K=0.0134$ in equation (34) for grain sizes from 40 to $250 \mu \mathrm{m}$. Figures 7 and 8 compare original experimental data of Generazio (1985) for copper and nickel, respectively, with attenuation-versus-frequency curves generated with equation (34).

\section{Data simulation for Curve Fitting}

It is apparent that prior knowledge of the material parameters $h, G$, and $K$ is needed to predict the attenuation coefficient with equation ( 34 ). Estimates of these quantities were sufficient to simulate attenuation curves for iron, copper, and nickel in figures 6 to 8 . We illustrate an empiricalgraphical approach for estimating and verifying values for $h, G$, and $K$ in solids, where these quantities are not easy to determine.

Our approach is founded on the observation that over a limited frequency range attenuation data can of ten be fitted quite well with equation (14) (Papadakis, 1964a; Vary, 1978, 1982; Bozorg-Grayeli, 1981). It has been shown that for data in the Rayleigh regime extrapolations based on the empirical constants $c$ and $m$ lead to excellent correlations with mechanical properti.es (e.g., fracture toughness)(Vary, 1978; 1979; Vary and Hull, 1982, 1983).

No analytical proof is offered to show why an equation of the form of equation (14) should be expected to represent either equation (34) or (29) in any particular frequency regime. However, we will show that equation (14) satisfactorily represents experimental data in restricted frequency regimes by using equation (34) to generate raw data sets in the Rayleigh regime. The point is illustrated by simulating data sets for a range of mean grain size $a$ and values of $K, G, h$, and $v$ that represent iron.

A series of discrete data sets generated with equation (34) are exhibited in figure 9 for the frequency range 10 to $100 \mathrm{MHz}$, where the Rayleigh wavelength criterion is satisfied (i.e., $\lambda \gg \pi$ ). We ignored data below $15 \mathrm{MHz}$, where diffraction effects are most pronounced for actual data sets. Treating the simulated data as actual raw attenuation data, we determined $c$ and $m$ values by a least-squares fit to equation (14) for each "material sample." The $c$ and $m$ values and the correlation coefficients of the curve fittings were tabulated for each data set. As seen in figure 9 calculated frequency exponents $m$ were clearly nonintegral and ranged from approximately 1.8 to 2.3 , corresponding to mean grain sizes from 2 to $20 \mu \mathrm{m}$, in the case illustrated. The simulated exponents spanned a range similar to experimentally 
determined frequency exponents for a number of polycrystallines in the Rayleigh regime (Vary, 1978).

F'or the material parameters chosen for generating data for figure 9, the correlation coefficient was always greater than 0.999 . Thus by treating the simulated data precisely the way one could treat a set of actual attenuation data, we found that equation (14) renders a statistically valid representation within the frequency bounds specified.

\section{Interrelation of Parameters}

In addition to representing attenuation data for a given material sample within specified frequency bounds, the parameters $c$ and $m$ are interrelated for variously conditioned samples of the same material. As an example, exper-imentally determined values of $c$ and $m$ for two maraging steels were plotted and tabulated ( $\mathrm{fig} .10$ ). All materials for which the quantities $c$ and $m$ have been experimentally measured exhibit the strong functional relation between $c$ and $m$ evident in figure 10. The quantities $c$ and $m$ are evidently interdependent for any set of related materials or samples of the same material that apparently differ due to metamorphic changes brought about by heat treatment or other thermomechanical processing methods that retain global microstructural patterns but alter mechanical properties.

By varying the material parameters $G, K, h$, and $v$ in equation (34) and allowing the mean grain size to take representative values, the $c$ and $m$ quantities corresponding to any particular material can be duplicated. Parametric fields based on varying $G, K$, and $h$ appear in figure 11 . The fields in figure 11 were created by using the curve-fitting method illustrated in figure 9 (i.e., by simulating attenuation data with equation (34), fitting with equation (14) in the 15 - to $100-\mathrm{MHz}$ range and including in the fields only points for which the curve fit correlation exceeded 0.999). Values selected for $h, G$, and $K$ corresponded to those for maraging steel and $v$ was taken to equal $0.56 \mathrm{~cm} / \mu \mathrm{s}$, the mean value for a 250 -grade maraging steel (Vary, 1978).

Coplotted in each field in figure 11 are data for the 250-grade maraging steel from figure 10 for which the mean grain size ranged from about 8.5 to $13 \mu \mathrm{m}$. Accordingly, in figure 11(a) the maraging steel data fall near the curve for mean grain size a equal to $10 \mu \mathrm{m}$. This is not fortuitous, since the location and shape of the field, relative to experimental $c$ and $m$ values for the maraging steel, depend on judicious selection of values for $h$ and G. Figures $11(\mathrm{~b})$ and (c), where we assume that $a=10 \mu \mathrm{m}$, appear to confirm the values $G=0.007$ and $h=0.00002$ for the $250-$ grade maraging steel.

An unexpected result is that each field in figure 11 indicates that the quantity $K$, the elastic anisotropy factor, varies by approximately a half order of magnitude among the maraging steel samples represented. Similar results were obtained for the 200-grade maraging steel data in figure 10 . This supports the notion that a material will have a different value of $k$ after thermomechanical conditioning. Indeed, it has been suggested that $k$ should be viewed as a composite quantity that includes a scattering coefficient (Mason, 1958; Papadakis, 1965a; Serabian and Williams, 1978). Heat treatment, strain, deformation, etc., alter attenuation properties due to effects on 
dislocations and grain boundaries (Serabian, 1985). These effects are apparent when experimental values for $c$ and $m$ are coplotted in appropriate parametric fields as in figure 11.

\section{DISCUSSION}

General

Our goal has been to develop a unified, comprehensive expression for ultrasonic attenuation. The incentive was to $f$ ind a rational basis for identifying and evaluating key microstructural factors that govern mechanical properties. We noticed that although previous expressions for attenuation are theoretically self-consistent, they provide only piecemeal, fragmented descriptions of ultrasonic attenuation mechanisms over the broad frequency range relevant to materials characterization.

We describe a transfer function concept and a heuristic lattice model that form bases for incorporating well-known attenuation mechanisms into a unified expression for ultrasonic attenuation. Although we ignore phase interference effects, our generalized analytical expression continuously accounts for the waxing and waning of all of the attenuation mechanisms previously embodied in separate equations. The generalized equation is shown to predict salient features of experimental attenuation-versus-frequency curves obtained for typical polycrystalline solids.

We believe that our chief contribution is the transfer function approach, in which expressions for attenuation coefficients are recognized and handled as frequency-domain transforms of time-domain quantities.

\section{Microstructure Encompassed}

We are concerned with polycrystallines, where ultrasonic attenuation depends primarily on such extrinsic microstructural factors as grain-size distribution and properties of grain interfaces. Using equation (34) we concentrate on relatively simple fine-grained, isotropic, homogeneous materials with mean grain sizes in the range $1 \mu \mathrm{m}$ to $>100 \mu \mathrm{m}$. We expect that the concepts given herein apply equally well to anisotropic materials and to amorphous or single-crystal materials that exhibit microporosity, dispersoids, etc. For example, equation (29) admits additional variables to account for grain anisotropy and bimodal distribution functions for dispersed scatterers.

\section{Probability Distribution Functions}

It should be apparent that our selection of a probability distribution function for use in equation (29) and equations developed from it was quite deliberate. In addition to representing typical polycrystalline grain populations, the probability distribution function given in equation (25) has salutary mathematical properties. These are evident in the consequences exhibited by equations (31) to (33), which depend on the form of the probability function. Moreover, once the mean is specified for the distribution function in equation (25), the variance is specified also (i.e., the variance equals half the square of the mean). Alternative distribution functions, such as Gaussians 
or combinations of normal and log-normal, may provide better descriptions of particular grain populations. Our immediate purpose was to indicate a rational method for introducing probabilistic factors in attenuation equations.

\section{Interface Transfer Function}

We applied the transfer function concept to a generalized version of the lattice model to represent polycrystalline materials. One consequence of the model was the introduction of a grain-boundary (interface) energy transmission function (e.g., represented by the term (1/a) ln ( $1-\mathrm{G}$ ) in eq. (23)). This term is similar to the diffusion scattering expressions of equations (8) and (33). It is related to $\mathrm{K}$, the elastic anisotropy factor in equation (33). Unlike the extrinsic (scatter) attenuation terms that include $\mathrm{K}$, the In $(1-G)$ term does not vanish in the absence of scatterers. Our model requires (and retains) it to account for the energy lost by reflection at internal interfaces. In general, $G$ is frequency dependent (as in the case of nonideal, thick, or acoustically rough interfaces, see appendix $A$ ).

\section{Multiple Reflection Term}

In our generalization the multiple reflection term [ $\left.\sum \ldots\right]$ of equation (17) is ignored. This is justifiable if the model represents polycrystallines that exhibit no significant forward-or backscatter. The waveform shown in figure 1 is a typical of this case (i.e., where no evidence of scatter wavelets appears either between or near the main echoess). This would not be true for coarse-grained materials that can exhibit considerable backscatter. In general, the $\left[\sum \ldots ..\right]$ term needs to be considered (e.g., when first- and second-order multiple reflections are significant as in layered structures (composite laminates)).

The quantity $\left[\sum \ldots\right]$ collects all multiple reflections that are not attenuated or deflected out of the interrogating beam. Referring to equations (17) and (22), we notice that, in our model, as $G \rightarrow 0$ the lattice interfaces become transparent, and overall attenuation will be due to intrinsic absorption and extrinsic scattering, say, due to point scatterers, voids, etc. In this case equation (22) reduces to $\alpha=\alpha_{i}+\alpha_{e}$. Note that for all values of $G$ the first term of each summation in equation (17) equals 1 .

When the average internal reflection coefficient $G$ lies between 0 and 1 , the interfaces assume varying degrees of opacity. For sufficiently large values of $G$ we can no longer ignore the first and higher power terms associated with $q=2,3, \ldots$, in equation (17). In this latter case backscatter would become significant and the effects of the reflection coefficient $R$ at the transducer-specimen interface enter the expression for $\alpha$, as for example in equation (22). Under this condition as in the case of equation (4) measurement of $\alpha$ depends on apriori knowledge or independent measurement of $R$.

\section{Power Functions of Frequency}

Integral exponents on frequency, as given by equations (6) to (10), should not be expected to apply to experimentally determined relations between the attenuation coefficient and frequency. Our analysis and experimental evidence 
show that even over narrow frequency ranges attenuation is a nonintegral power function of frequency. Exponents on frequency will assume values between 1 and 4 according to the degree of influence of material parameters such as those appearing in equations (29) and (34). Indeed, figures 6 to 8 show that exponents are themselves functions of frequency.

Heretofore, we and other investigators have found it practical to use nonintegral, fixed exponents to describe empirical relations between attenuation and frequency within bounded regimes. By using the generalized attenuation equations we show that this approach is statistically valid (e.g., as in the case of $\mathrm{fig} .9$ ). However, it is now clear that considerably more information concerning microstructure may be extracted by applying the generalized expressions for attenuation in equations (29) and (34).

\section{Elastic Anisotropy and Related Factors}

Our preliminary analysis of the implications of the interrelation of the empirical quantities $c$ and $m$ from equation (14) confirmed the importance of considering variations in the effective values of $K$ (and $G$ ) in applying equation (34), especially to polycrystallines modified by thermomechanical processing. We infer that changes can occur in the effective values of $K$ that transcend changes in grain geometry (size, shape, etc.). These correspond to changes in mechanical properties (fracture toughness, yield strength, etc.) in materials in which only slight or no changes in grain size have occurred. In effect, equations (29) and (34) appear to provide entry to analysis of subtle metamorphic changes that arise from thermomechanical processing of polycrystallines.

\section{CONCLUDING REMARKS}

A unified expression for ultrasonic attenuation has been introduced to supplant currently diverse and fragmented equations. A transfer function concept and a heuristic lattice model with an interface energy transmission factor have been described as rational bases for combining a variety of attenuation mechanisms and probabilistic factors in a comprehensive equation. Although our model is essentially one dimensional and we ignore phase interference effects, the resultant generalized equation predicts salient features of experimental attenuation-versus-frequency curves obtained for typical polycrystalline solids. Some consequences of the unified expression have been explored relative to characterization of key microstructural factors that govern mechanical properties of polycrystallines. 


\section{APPENDIX A \\ REFLECTION COEFFICIENT AND ACOUSTIC IMPEDANCE}

The reflection coefficient can be defined either in terms of power (energy intensity) or amplitude (pressure) (Krautkrämer, 1969; Szilard, 1982). Taking $R$ as the reflection coefficient and $Q$ as the transmission coefficient across an interface, from medium 1 to 2 , we have for the power case,

$$
\begin{gathered}
R_{p}+Q_{p}=1 \\
R_{p}=\left(\frac{z_{2}-z_{1}}{z_{2}+z_{1}}\right)^{2} \\
Q_{p}=\frac{\left(4 z_{2} z_{1}\right)}{\left(z_{2}+z_{1}\right)^{2}}
\end{gathered}
$$

and for the amplitude case,

$$
\begin{aligned}
& R_{a}+1=Q_{a} \\
& R_{a}=\frac{z_{2}-z_{1}}{z_{2}+z_{1}} \\
& Q_{a}=\frac{2 z_{2}}{z_{2}+z_{1}}
\end{aligned}
$$

where $z_{1}$ and $z_{2}$ are acoustic impedances of media 1 and 2 ,

$$
\left.\begin{array}{l}
z_{1}=\rho_{1} v_{1} \\
z_{2}=\rho_{2} v_{2}
\end{array}\right\}
$$

$\rho$ is density, and $v$ is velocity.

In polycrystallines grain-.boundary interfaces will reflect as well as transmit ultrasonic energy because of acoustic impedance "jumps" across differently oriented contiguous crystals.

The preceding equations for reflection and transmission apply to ideal interfaces that have no thickness. If there is an intervening medium that has a finite thickness d such that d greatly exceeds the ultrasonic wavelengths transmitted, $d \gg \lambda$, the transmission and reflection coefficients are frequency dependent (Kinsler, et al., 1982). Letting $v_{3}$ be velocity in the intervening medium (medium 3 ) between media 1 and 2 and $z_{3}$ be its acoustic impedance, $R$ becomes a function of frequency $f$ with the magnitude 


$$
|R(f)|=\frac{\left(1-\frac{z_{1}}{z_{2}}\right)^{2} \cos ^{2}\left(\frac{2 \pi f d}{v_{3}}\right)+\left(\frac{z_{3}}{z_{2}}-\frac{z_{1}}{z_{3}}\right)^{2} \sin ^{2}\left(\frac{2 \pi f d}{v_{3}}\right)}{\left(1+\frac{z_{1}}{z_{2}}\right)^{2} \cos ^{2}\left(\frac{2 \pi f d}{v_{3}}\right)+\left(\frac{z_{3}}{z_{2}}+\frac{z_{1}}{z_{3}}\right)^{2} \sin ^{2}\left(\frac{2 \pi f d}{v_{3}}\right)}
$$

When $\lambda_{3}=\left(v_{3} / 2 \pi f\right) \gg d$, equation (A4) reduces to equation (AI). 


\section{APPENDIX B \\ REFLECTION COEFFICIENT TRANSFER FUNCTION}

In this paper the reflection coefficient $R$ is always taken as a frequency-domain quantity. For an ideal interface between two nondispersive media with unequal acoustic impedances, $R$ is independent of ultrasonic frequency (Papadakis, 1976; Generazio, 1984). We define $R$ as identical to the Heaviside step function $H(f)$, (Bracewell, 1978)( $f i g .3$ )

$$
|R(f)|=|H(f)|
$$

where

$$
|H(f)|=\left\{\begin{array}{lll}
1 & \text { for } & f \geq 0 \\
0 & \text { for } & f<0
\end{array}\right.
$$

The time-domain counterpart ( $i . e .$, inverse Fourier transform) of $R(f$ ) is then the delta (impulse) function $\delta$ defined by

$$
\int_{-\infty}^{+\infty} \delta(t) d t=1
$$

where $\delta=0$ for $t \neq 0$ and $t$ is time.

We adopt the additional formalism that the delta function is the inverse transform of $H(f)$ with arbitrary magnitude and phase spectra. This allows the delta function to be positioned at any point in the time domain and to have any value $w$ (Bendat and Piersol, 1980) so that

$$
\int_{t_{0}-\varepsilon}^{t_{0}+\varepsilon} w \delta\left(t-t_{0}\right) d t=w
$$

where

$$
w \delta\left(t-t_{0}\right)=\left\{\begin{array}{lll}
\infty & \text { for } & t=t_{0} \\
0 & \text { for } & t \neq t_{0}
\end{array}\right.
$$

and where $|H(0)|=w$ by definition of the Fourier transform of $\delta(t)$. In a nondispersive medium $|\mathrm{H}(\mathrm{f})|=\mathrm{w}$, a constant over all frequencies $f$ equal to or greater than 0 . 


\section{APPENDIX C}

\section{DEVELOPMENT OF LATTICE MODEL EQUATION}

A ray is traced through the lattice model as depicted in figure 4. starting with the "source" $E_{0}$, transmissions and first-order multiple reflections at each interface encountered are summed to determine $B_{1}$ and $B_{2}$.

The lattice front surface has the reflection coefficient $R$ as a result of coupling to a buffered transducer as in figure 1 . There are $n$ interfaces within the lattice, each with the identical reflection coefficient $G$. The back surface interfaced with a vacuum (or air) has a reflection coefficient of unity.

In this appendix, as through the main text, the quantities $A, B, G, E$, $F$, and $R$ are frequency-domain quantities (i.e., Fourier transforms of corresponding time-domain quantities). Reflection coefficients are based on the power definition given in appendix $A$ and therefore $B_{1}$ and $B_{2}$ must be taken as power (energy) spectra of corresponding waveforms.

Ignoring backscatter (second-order multiple reflections) caused by $E_{0}$, the first back echo is

$$
B_{1}=E_{0}(H-R)
$$

where $H$ is the Heaviside unit step function $H=|H(f)|=1$ (appendix $B$ ). The second back echo is

$$
\begin{aligned}
B_{2}=E_{0}(H-R) R(H-G)^{2 n} & {\left[\sum_{1}^{q}(R G)^{q-1} A^{2 q-1}\right]^{2} \cdot \cdot } \\
& {\left[\sum_{1}^{q} G^{q-1} A^{2 q}\right] \cdot \cdot \cdot\left[\sum_{1}^{q} G^{2(q-1)} A^{2 q-1}\right]^{2(n-1)} \cdot \cdots }
\end{aligned}
$$

where $A$ is unspecified intrinsic and/or extrinsic attenuation within the lattice layer and is identical in each layer. The echo $B_{2}$ is obtained by summing the internal transmissions and reflections $F_{1}, F_{2}, \ldots, F_{n}$ and $E_{1}$, $\mathrm{E}_{2}, \ldots, \mathrm{E}_{\mathrm{n}}$. For forward-going terms (e.g., $\mathrm{F}_{1}$ to $\mathrm{F}_{\mathrm{n}}$ )

$$
F_{1}=E_{0}(H-G) \sum_{1}^{q} R_{G}^{q_{G} q-1} A^{2 q-1}=F_{11}+F_{12}+\ldots+F_{1 q}
$$


where

$$
\begin{gathered}
F_{11}=E_{0} R(H-G) A \\
F_{12}=E_{0} R^{2} G(H-G) A^{3} \\
F_{13}=E_{0} R^{3} G^{2}(H-G) A^{5} \\
\cdot \\
\vdots \\
F_{1 q}=E_{0} R^{q_{G} q-1}(H-G) A^{2 q-1}
\end{gathered}
$$

and

$$
F_{n}=F_{(n-1)}(H-G) \sum_{1}^{q} G^{2(q-1)} A^{2 q-1}
$$

For returning echo terms (e.g., $E_{1}$ to $E_{n}$ )

$$
E_{1}=F_{n}(H-G) \sum_{1}^{q} G^{q-1} A^{2 q}=E_{11}+E_{12}+\ldots+E_{1 q}
$$

where

$$
\begin{gathered}
E_{11}=F_{n}(H-G) A^{2} \\
E_{12}=F_{n} G(H-G) A^{4} \\
E_{13}=F_{n} G^{2}(H-G) A^{6} \\
\vdots \\
E_{1 q}=F_{n} G^{q-1}(H-G) A^{2 q}
\end{gathered}
$$

and

$$
E_{n}=E_{(n-1)}(H-G) \sum_{1}^{q} G^{2(q-1)} A^{2 q-1}
$$

Then

$$
B_{2}=E_{n}(H-R) \sum_{1}^{q}(R G)^{q-1} A^{2 q-1}
$$




$$
\begin{aligned}
\frac{B_{2}}{R B_{1}}=(H-G)^{2 n_{A} 2(n+1)}\left[\sum_{I}^{q}(R G)^{q-1} A^{2(q-1)}\right]^{2} \cdot \cdot \\
\\
{\left[\sum_{I}^{q} G^{q-1} A^{2(q-1)}\right] \cdot \cdot \cdot\left[\sum_{1}^{q} G^{2(q-1)} A^{2(q-1)}\right]^{2(n-1)} \cdot \cdot \cdot }
\end{aligned}
$$

In equation (C10) ". . . indicates that the summations are to be multiplied together and that additional factors comprised of summations of second-order multiple reflections are omitted. These additional factors arise from backtransmissions and re-reflections that originate with first-order terms (e.g., $E_{1}, E_{2}, \ldots, E_{n}$ and $\left.F_{1}, F_{2}, \ldots, E_{n}\right)$.

Ignoring second-order multiple reflections within the lattice layers, the lattice transfer function $T$ is obtained by combining equation (C10) with equation ( 3 ) to obtain equation (16) in the main text. 
REFERENCES

Auld, B.A. (1973), Acoustic Fields and Waves in Solids, vol. 1. Wiley, New York.

Bhatia, A.B. (1967), Ultrasonic Absorption. Clarendon Press, Oxford, England.

Bendat, J.S, and Piersol, A.G. (1980), Engineering Applications of Correlation and Spectral Analysis. Wiley-Interscience, New York.

Bozorg-Grayeli, N. (1981), Acoustic Nondestructive Evaluation of Micro-

structure. Ph.D. Thesis, Stanford University, Stanford, CA.

Bracewell, R.N. (1978), The Fourier Transform and its Applications.

McGraw-Hill, New York.

DeHoff, R.T. (1968), The Statistical Background of Quantitative Metallography. Quantitative Microscopy, R.T. DeHoff and F.N. Rhines, eds., McGraw-Hill, New York, pp. 11-44.

Evans, A.G., et al. (1978), Ultrasonic Attenuation in Ceramics. J. Appl. Phys., vol. 49, no. 5, pp. 2669-2679.

Fitting, D.W. and Adler, L. (1981), Ultrasonic Spectral Analysis for Nondestructive Evaluation. Plenum Press, New York.

Generazio, E.R. (1985), The Role of the Reflection Coefficient in Precision Measurement of Ultrasonic Attenuation. Mater. Eval., vol. 43, no. 8, pp. 995-1004.

Generazio, E.R. (1985), Ultrasonic Verification of Microstructural Changes due to Heat Treatment. Analytical Ultrasonics in Materials Research and Testing, NASA CP-2383.

Goebbels, K. (1980), Structure Analysis by Scattered Ultrasonic Radiation. Research Techniques in Nondestructive Testing, Vol. 4, R.S. Sharpe, ed., Academic Press, London, pp. 87-157.

Granato, A. and Lücke, K. (1956), Theory of Mechanical Damping Due to Dislocations. J. Appl. Phys., vol. 27, no. 6, pp. 583-593.

Green, R.E., Jr. (1973a), Ultrasonic Investigation of Mechanical Properties. Treatise on Materials Science and Technology, Vol. 3, Academic Press, New York.

Green, R.E., Jr. (1973b), Ultrasonic Attenuation Detection of Fatigue Damage. Ultrasonics International 1973, IPC Science and Technology Press, Guilford, Surrey, England, pp. 187-193.

Green, R.E., Jr. (1981), Effect of Metallic Microstructure on Ultrasonic Attenuation. Nondestructive Evaluation: Microstructural Characterization, and Reliability Strategies, 0 . Buck and S.M. Wolf, eds., Metallurgical Society, AIME, Warrensdale, PA, pp. 115-132. 
Green, R.E., Jr. (1985), Ultrasonic and Acoustic Emmision Nondestructive Materials Characterization. Analytical Ultrasonics in Materials Research and Testing, NASA CP-2383.

Haines, N.F., Bel1, J.C., and McIntyre, P.J. (1978), The Application of Broadband U1trasonic Spectroscopy to the Study of Layered Media. J. Acoust. Soc. Am., vol. 64, no. 6, pp. 1645-1651.

Kinsler, L.E., et al. (1982), Fundamentals of Acoustics, 3rd ed., Wiley, New York, Chapter 6.

Kline, R.A. (1964), Measurement of Attenuation and Dispersion Using an Ultrasonic Spectroscopy Technique. J. Acoust. Soc. Am., vol. 76, no. 2, pp. 498-504.

Klinman, R., et al. (1980), Ultrasonic Prediction of Grain Size, Strength, and Toughness in Plain Carbon steel. Mater. Eval., vol. 38, no. 10, pp. 26-32.

Kolsky, H. (1963), Stress Waves in Solids. Dover, New York.

Krautkrämer, J. and Krautkrämer, H, (1969), U1trasonic Testing of Materials. Springer-Verlag, New York.

Lifsitz, I.M. and Parkhomovskii, G.D. (1950), Theory of Propagation of supersonic Waves in Polycrystals. Zhur. Ekspt1. Theo. Fiz., vol. 20, pp. 175-182.

Lïcke, K. (1956), U1trasonic Attenuation Caused by Thermoelastic Heat Flow. J. App 1. Phys., vol. 27, no. 12, pp. 1433-1438.

Maisel, J.E. (1.984), Preliminary Investigation of an Electrical Network Model for Ultrasonic Scattering. NASA CR-3770.

Mason, W.P. and McSkimin, H.J. (1947), Attenuation and Scattering of High Frequency Sound Waves in Metals and Glasses. J. Acoust. Soc. Am., vol. 19, no. $3, \mathrm{pp} .464-473$.

Mason, W.P. and McSkimin, H.J. (1948), Energy Losses of Sound Waves in Metals Due to Scattering and Diffusion. J. Appl. Phys., vol. 19, no. 10, pp. 940-946.

Merkulov, L.G. (1957), Investigation of Ultrasonic Scattering in Metals. Sov. Phys. Tech. Phys., vol. I, no. 1, pp. 59-69.

Nowick, A.S. and Berry, B.S. (1972), Anelastic Relaxation in Crystalline Solids. Academic Press, New York.

Papadakis, E.P. (1960), Ultrasonic Attenuation in S.A.E. 3140 and 4150 Steel. J. Acoust. Soc. Am., vol. 32, no. 12, pp. 1628-1639.

Papadakis, E.P. (1964a), Ultrasonic Attenuation and Velocity in Three Transformation Products in Steel. J. Appl. Phys, , vol. 35, no. 5, pp. 1474-1482.

Papadakis, E.P. (1964b), From Micrograph to Grain-Size Distribution With Ultrasonic Applications. J. Appl. Phys., vol. 35, no, 5, pp. 1586-1594. 
Papadakis, E.P. (1965a), Revised Grain Scattering Formulas and Tables.

J. Acoust. Soc. Am., vol, 37, no, 4, pp. 703-710.

Papadakis, E.P. (1965b), Ultrasonic Attenuation Caused by Scattering in Polycrystalline Metals. J. Acoust. Soc. Am., vol. 37, no. 4, pp. 711-717.

Papadakis, E.P. (1968), Buffer-Rod System for Ultrasonic Attenuation Measurements. J. Acoust. Soc. Am., vol. 44, no. 5, pp. 1437-1441.

Papadakis, E.P. (1970), Ultrasonic Attenuation and Velocity in SAE 52100 Steel Quenched From Various Temperatures. Metal1. Trans., vol. 1, no. 4, pp. 1053-1057.

Papadakis, E.P. (1975), Ultrasonic Diffraction From Single Apertures with Application to Pulse Measurements and Crystal Physics. Physical Acoustics: Principles and Methods, Vol. 11, W.P. Mason and R.N. Thurston, eds., Academic Press, New York, pp. 151-211.

Papadakis, E.P. (1976), Ultrasonic Velocity and Attenuation: Measurement Methods With Scientific and Industrial Applications. Physical Acoustics: Principles and Methods, Vo1. 11, W.P. Mason and R.N. Thurston, eds., Academic Press, New York, pp. 277-374.

Papadakis, E.P. (1981), Ultrasonic Attenuation Caused by Rayleigh Scattering by Graphite Nodules in Nodular Cast Iron. J. Acoust. Soc. Am., vo1. 70, no. 3, pp. 782-787.

Pekeris, C.L. (1947), Note on the Scattering of Radiation in an Inhomogeneous Medium. Phys. Rev., vol. 71, no. 4, pp. 268-269.

Reynolds, W.N. and Smith, R.L. (1984), Ultrasonic Wave Attenuation Spectra in Steels. J. Phys. D, vol. 17, no. 1, pp. 109-116.

Roklin, L.L. (1972), Scattering of Ultrasound in Polycrystalline Materials. Soviet Physics-Acoustics, vol. 18, no. 1, pp. 71-75.

Roney, R.K. (1950), The Influence of Metal Grain Structure on the Attenuation of an Ultrasonic Acoustic Wave. Ph.D. Thesis, California Institute of Technology, Pasadena, CA.

Sayers, C.M. (1985), Scattering of Ultrasound by Minority Phases in Polycrystalline Metals. Wave Motion, vol. 7, no. 1, pp. 95-104.

Schreiber, E., Anderson, O.L. and Soga, N. (1973), Elastic Constants and Their Measurement. McGraw-Hi.11, New York.

Schückler, F. (1968), [Determination of] Grain size [Distribution]. Quantitative Microscopy, R.T. DeHoff and F.N. Rhines, eds., McGraw-Hill, New York, pp. 201-265.

Scott, W.R. and Gordon, P.F. (1977), U1trasonic Spectrum Analysis for Nondestructive Testing of Layered Composite Materials. J. Acoust. Soc. Am., vol. 62, No. 1, pp. 108-116. 
Seeger, A. and Schiller, P. (1962), Bildung und Diffusion von Kinken als Grundprozess der Versetzungsbewegung bei der Messung der Inneren Reibung. Acta Metall., vol. 10, no. 4, pp. 348-357.

Serabian, S. and Williams, R.S. (1978), Experimental Determination of Ultrasonic Attenuation Characteristics Using the Roney Generalized Theory. Mater. Eval., vol. 36, no. 8, pp. 55-62.

Serabian, S. (1980), Frequency and Grain Size Dependency of Ultrasonic Attenuation in Polycrystalline Materials. Brit. J. Non-Destr. Test., vol. 22, no. 2 , pp. 69-77.

Serabian, S. (1985), Ultrasonic Material Property Determinations. Analytical Ultrasonics in Materials Research and Testing, NASA CP-2383.

Shukla, S.S. and Yun, S.S. (1981), Ultrasonic Attenuation in GaAs. J. Acoust. Soc. Am., vol. 70, no. 6, pp. 1713-1716.

Smith, R.L. (1982), The Effect of Grain Size Distribution on the Frequency Dependence of the Ultrasonic Attenuation in Polycrystalline Materials. Ultrasonics, vol. 20 , no. 5 , pp. 211-214.

Szilard, J. (1982), Ultrasonic Testing - Nonconventional Techniques, John Wiley, N.Y.

Tittman, B.R., Ahlberg, L.A., and Fertig, K. (1985), Ultrasonic Characterization of Microstructures in Powder Metal Alloy. Analytical Ultrasonics in Materials Research and Testing, NASA CP-2383.

Truel1, R., Elbaum, C., and Chick, B.B. (1969), Ultrasonic Methods in Solid state Physics. Academic Press, New York.

Vary, A. (1978), Correlations Among Ultrasonic Propagation Factors and Fracture Toughness Properties of Metallic Materials. Mater. Eval., vol. 36 , no. $7, \mathrm{pp} .55-64$.

Vary, A. (1979a), Correlations Between Ultrasonic and Fracture Toughness in Metaliic Materials. Fracture Mechanics, ASTM-STP-677, C.W. Smith, ed., ASTM, Philadelphia, PA, pp. 563-578.

Vary, A. (1979b), Computer signal Processing for Ultrasonic Attenuation and Velocity Measurements. Proceedings of the $12 \mathrm{th}$ symposium on Nondestructive Evaluation, W.W. Bradshaw, ed., Southwest Research Institute, San Antonio, TX, pp. $33-46$.

Vary, A. (1980a), Ultrasonic Measurement of Material Properties. Research Techniques in Nondestructive Testing, Vol. 4, R.S. Sharpe, ed., Academic Press, London, pp. 159-204.

Vary, A. (1980b), Concepts and Techniques for Ultrasonic Evaluation of Material Mechanical Properties. Mechanics of Nondestructive Testing, W.W. Stinchcomb, ed., Plenum, New York, pp. 123-141.

Vary, A. (1980c), Simulation of Transducer-Couplant Effects on Broadband Ultrasonic Signals. NASA TM-81489. 
Vary, A. and Hull, D.R. (1982), Interrelation of Material Microstructure, Ultrasonic Factors, and Fracture Toughness of a Two-Phase Titanium Alloy. Mater. Eval., vol. 41, no. 3, pp. 309-314.

Vary, A. and Hull, D.R. (1983), Ultrasonic Ranking of Toughness of Tungsten Carbide. Proceedings of the 14th Symposium on Nondestructuve Evaluation, D.W. Moore and G.A. Matzkanin, eds., Southwest Research Institute, San Antonio, TX, pp. 212-219.

Vary, A. (1984), Ultrasonic Nondestructive Evaluation, Microstructure, and Mechanical Property Interrelations. NASA TM-86876.

Karagulle, H., Lee, S.S., and Williams, J.H., Jr.(1985a), Input-Output Characterization of an Ultrasonic Testing System by Digital Signal Analysis. Analytical Ultrasonics in Materials Research and Testing, NASA CP-2383.

Williams, J.H., Jr., and Lee, S.S. (1985b), Pattern Recognition Characterization of Micromechnical and Morphological Materials States Via Analytical Quantitative Ultrasonics. Analytical Ultrasonics in Materials Research and Testing, NASA CP-2383.

Winkler, K.W. and Murphy, W.F., III (1984), Scattering in Glass Beads: Effects of Frame and Pore Fluid Compressibilities. J. Acoust. Soc. Am., vol. 76 , no. 3, pp. 820-825.

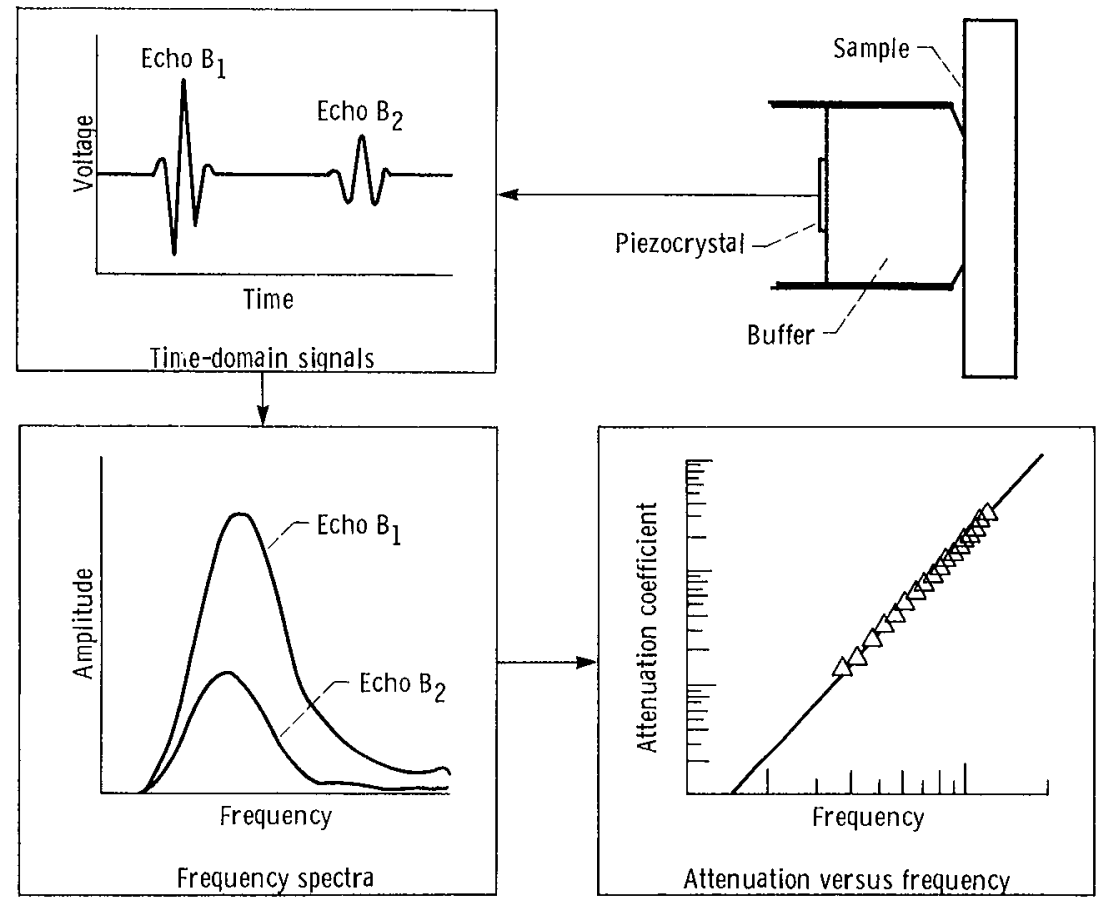

Figure 1. - Schematic of signal acquisition and data-processing stages for determining frequency dependence of attenuation coefficient by using broadband ultrasonic pulse-echo method. 


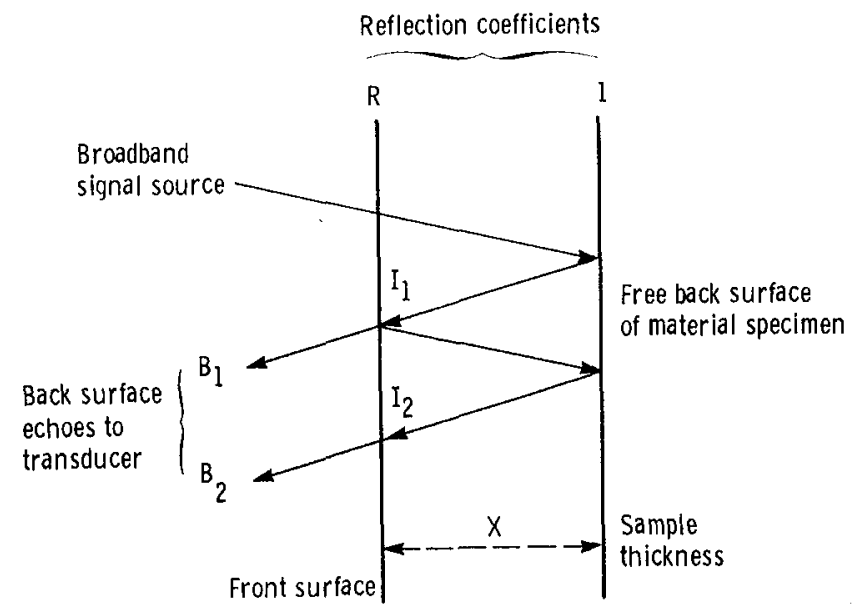

Fiqure 2. - Diagrami of broadband ultrasonic echo system for determining frequency dependence of attenuation coefficient and deriving material microstructure transfer function.

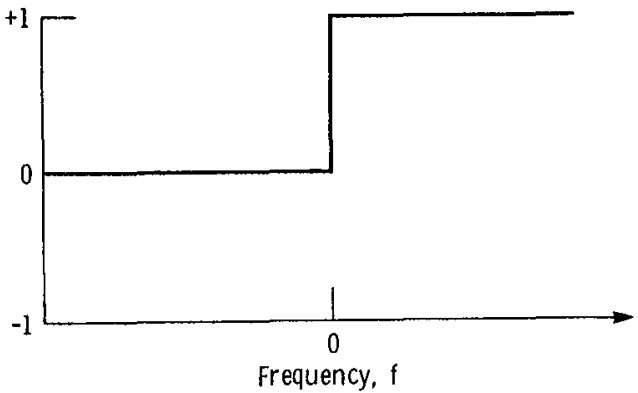

(a) Heaviside step function I $H(f) \mathrm{I}$.

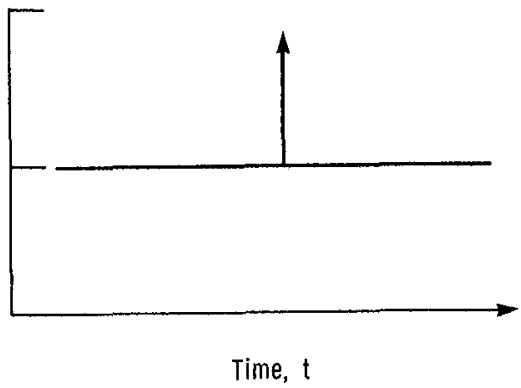

(b) Delta function $\delta(f)$.

Figure 3. - Delta function and Heaviside step function representing time- and frequency-domain counterparts, respectively, of refiection coefficient, where $|R(f)|-|H(f)|-1$ at free surface (appendix B). 


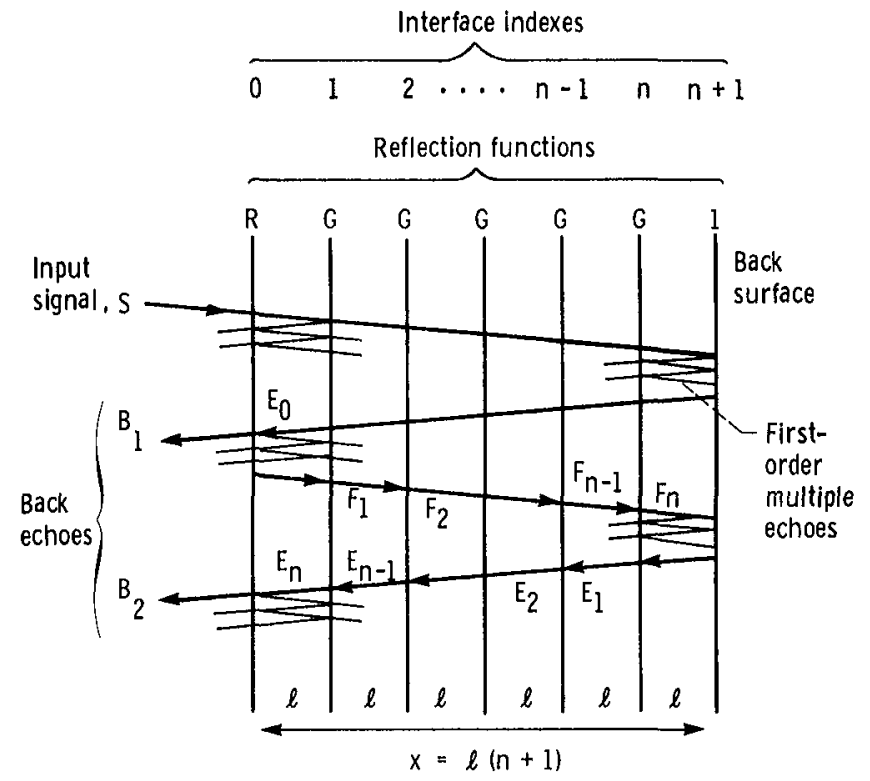

Figure 4. - Diagram of lattice model for synthesizing material structure transfer function (appendix C).

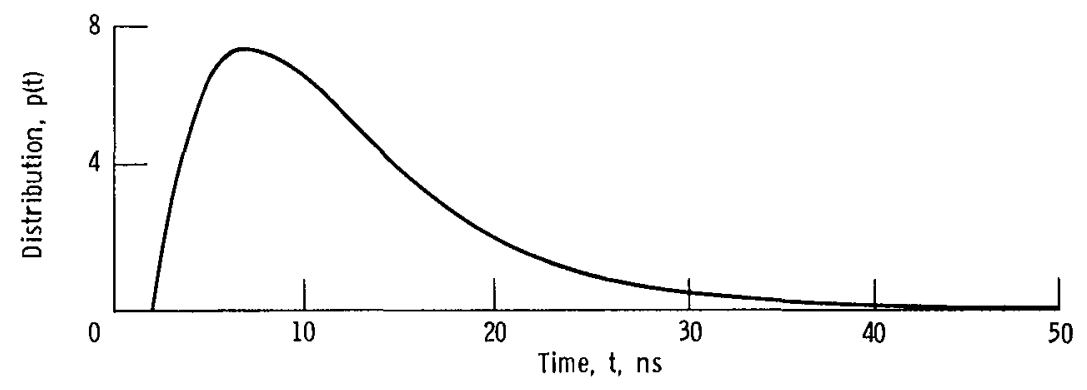

(a) Grain-delay probability distribution function.
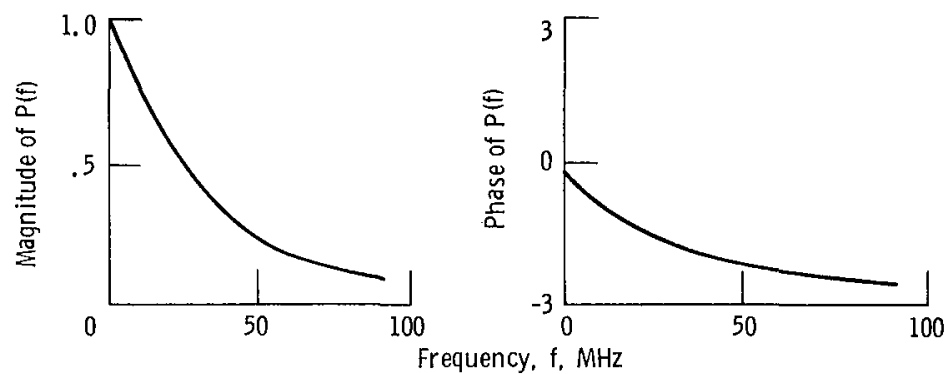

(b) Magnitude and phase spectra of grain-delay distribution function.

Figure 5. - Time-domain probability distribution function for grain-delay (transit time) and its Fourier transform, magnitude, and phase. 


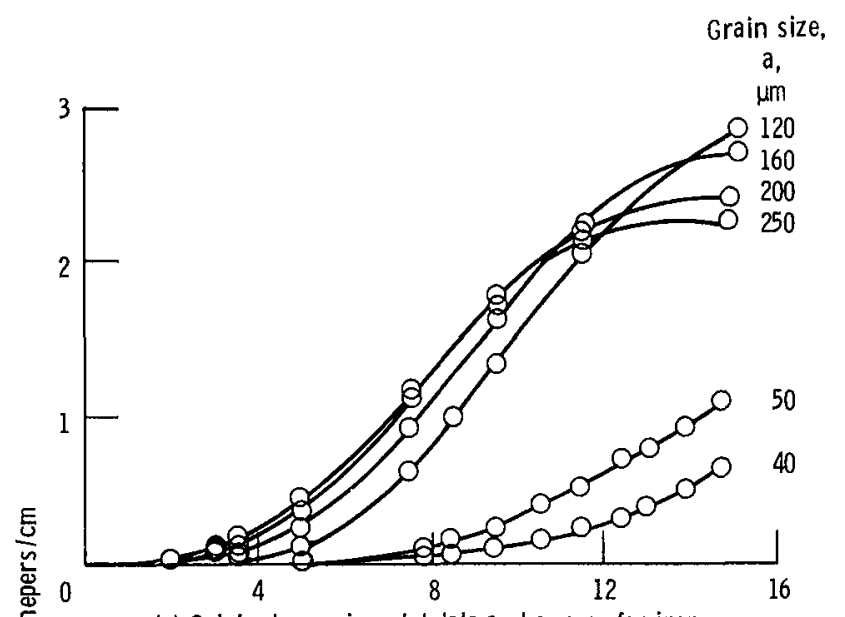

(a) Original experimiental data and curves for iron.

을

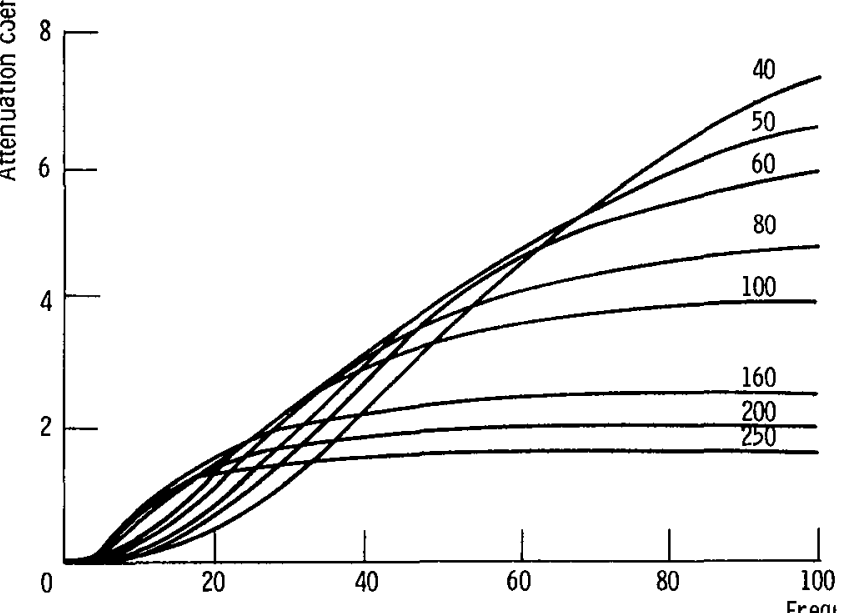

(c) Simulated curves for iron to $100 \mathrm{MHz}(\mathrm{G}=\mathrm{K}=0.0067, \mathrm{~h}=0.00002$ $v=0.59 \mathrm{~cm} / \mu \mathrm{s})$.

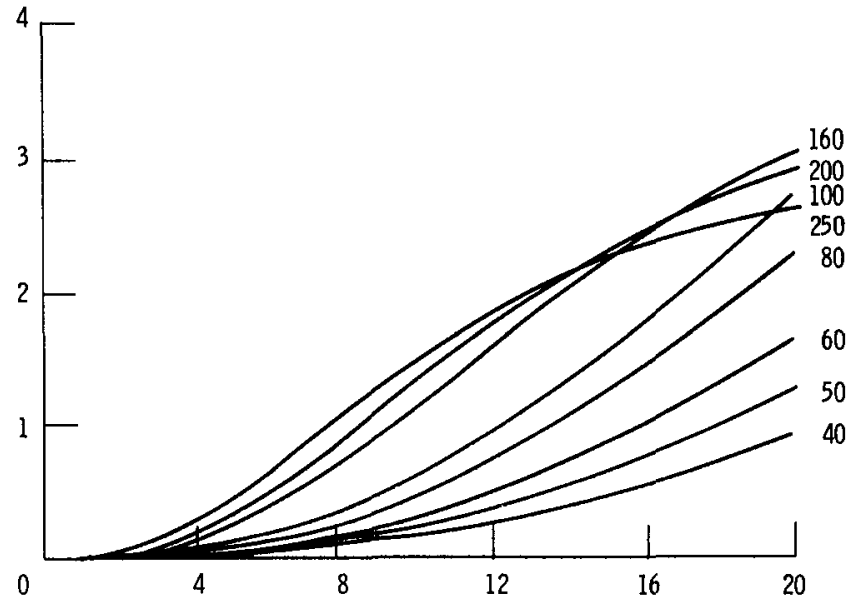

(b) Simulated attenuation data curves for iron $G=K=0.0134$, $h=0.00002, v=0.59 \mathrm{~cm} / \mu \mathrm{s}$.

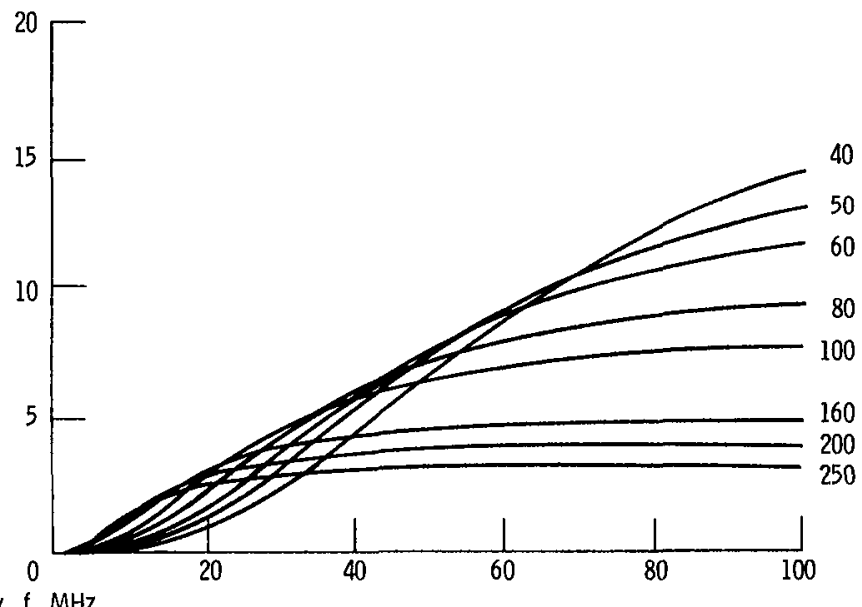

(d) Simulated curves for iron to $100 \mathrm{MHz}$ (G $=K=0.0134, h=0.00002$, $v=0.59 \mathrm{~cm} / \mu \mathrm{s})$.

Figure 6. - Comparison of experimiental attenuation-coefficient-versus-frequency curves for iron with curves generated with equation (34). Oriqinal experimental data fromi Merkulov (1957). 

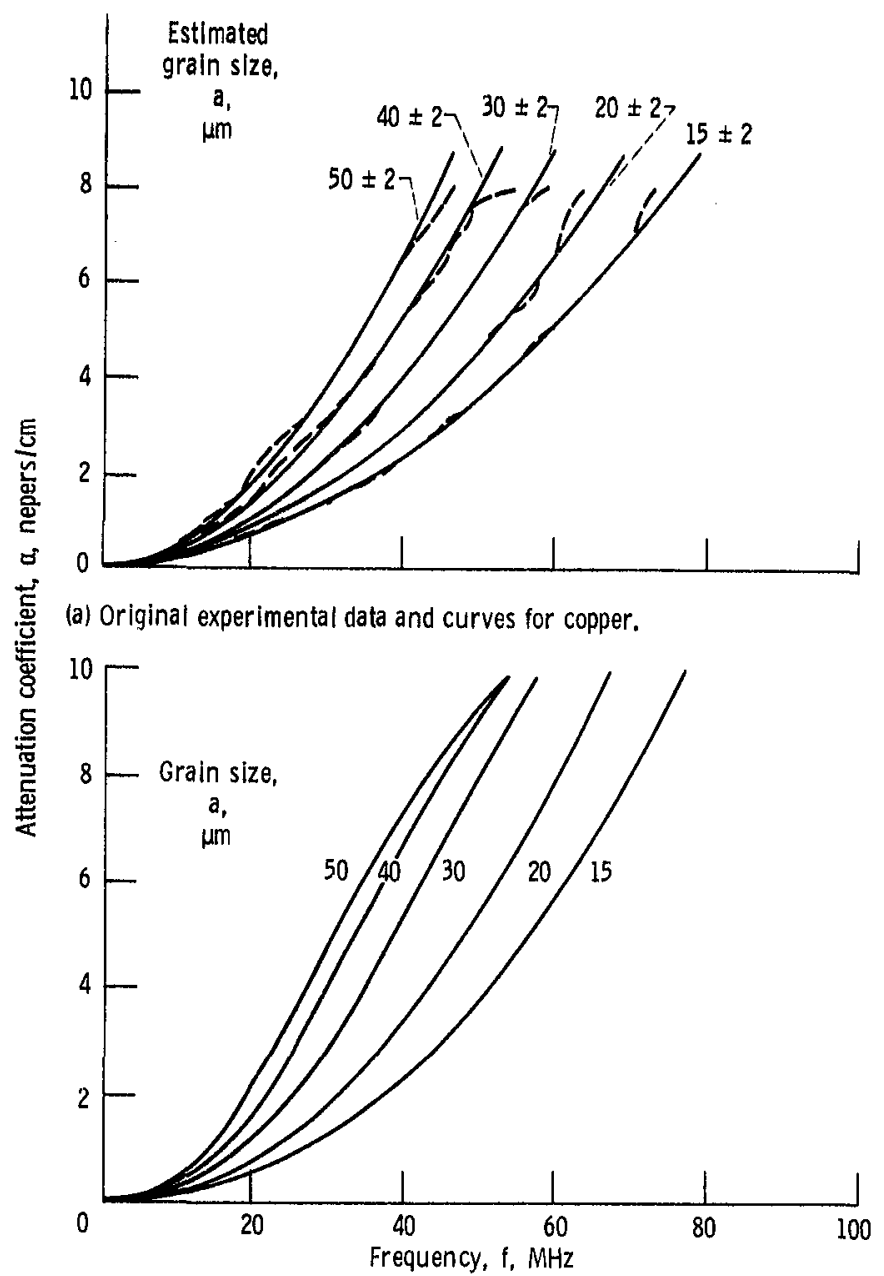

(b) Simulated attenuation data curves for copper $(G=K=0.0124$, $h=0.0003, v=0.47 \mathrm{~cm} / \mu \mathrm{s}$ ).

Figure 7. - Comparison of experimiental attenuation-coefficient-versusfrequency curves for copper with curves generated with equation (34). original experimental data and curves fromı Generazio (1985). 


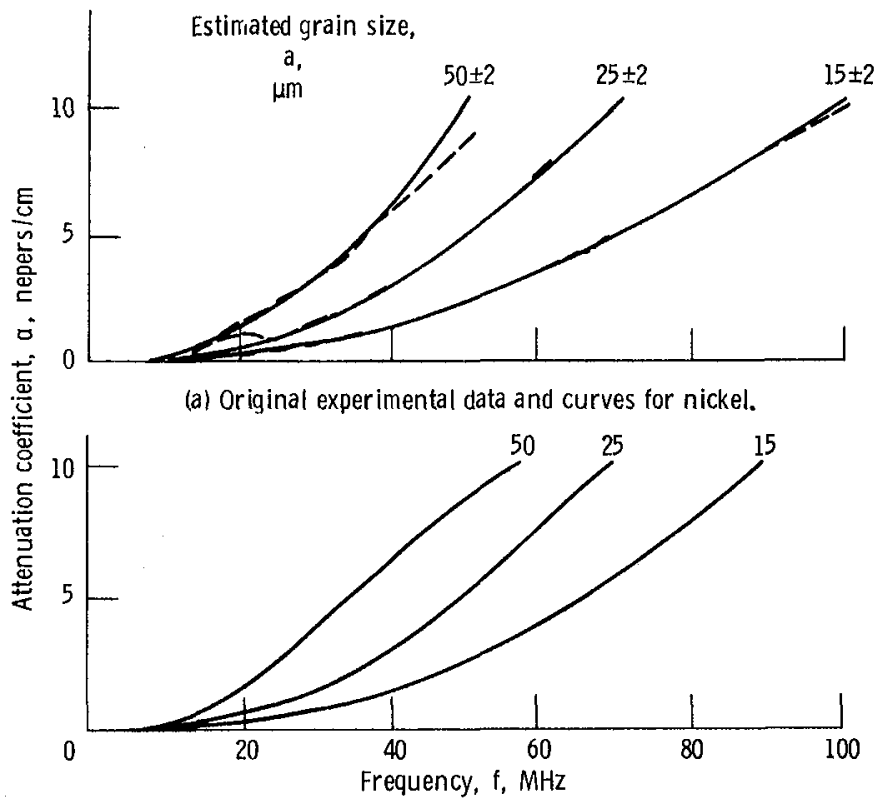

(b) Simulated attenuation data curves for nickel $G=K=0.0144$, $h=0.0003, v=0.563 \mathrm{~cm} / \mu \mathrm{s}$ ).

Figure 8. - Comparison of experimiental attenuation-coefficient-versusfrequency curves for nickel with curves generated with equation (34). Original experimental data and curves from Generazio (1985). 

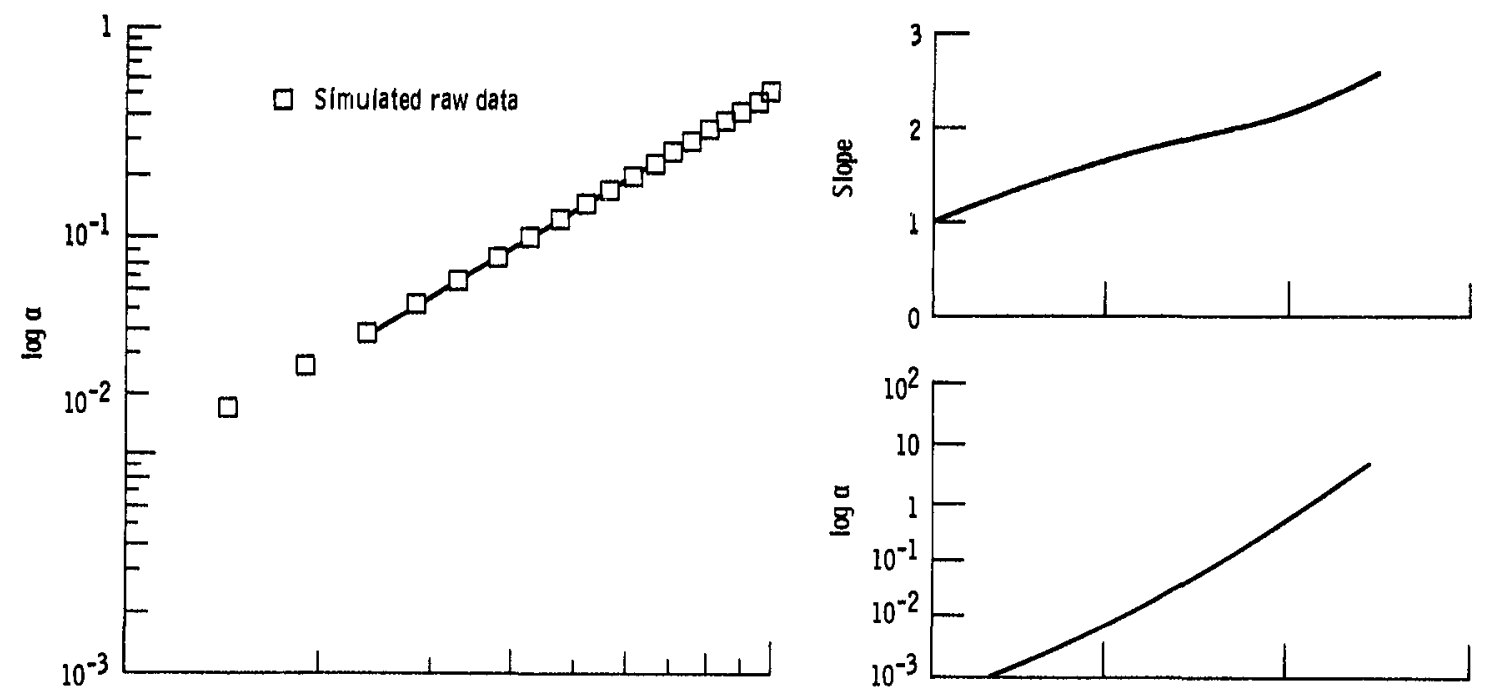

(a) Mean grain size, $2 \mu \mathrm{m} . c=1.016 \times 10^{-4} ; \mathrm{m}-1.835$; least squares coefficient $=0.99954$.
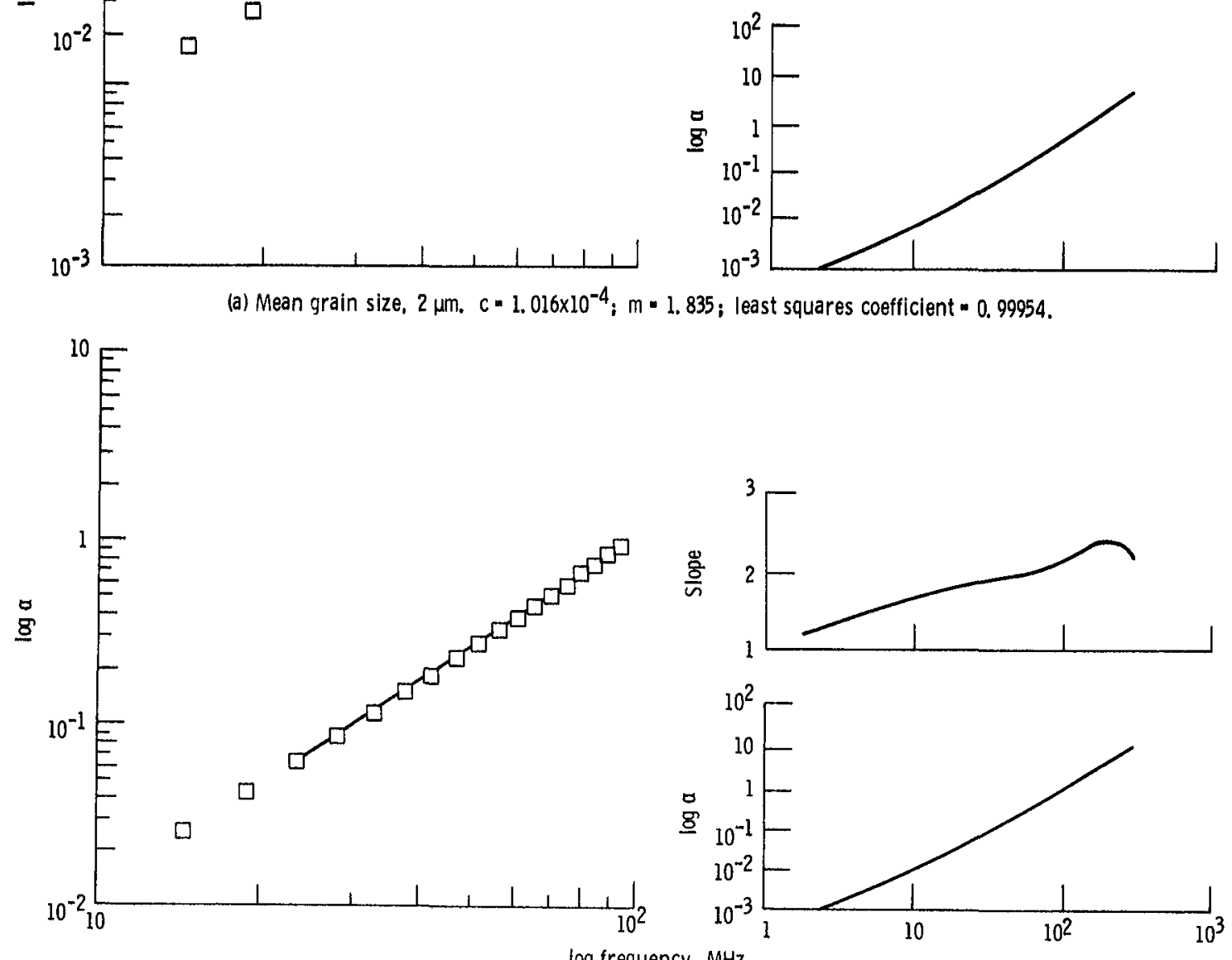

(b) Mean grain size, $4 \mu \mathrm{m}, c=1.143 \times 10^{-4} ; \mathrm{m}=1.975$; least squares coefficient $=0.99947$.

Figure 9. - Simulation of attenuation-coefficient-versus-frequency data in 10- to 100-MHz range using equation (34) and determination of $\mathrm{c}$ and $\mathrm{m}$ values by least squares fit to equation (14) from 15 to $100 \mathrm{MHz}$. $v-0.56 \mathrm{~cm} / \mu \mathrm{s} ; \mathrm{K}=0.007$; $G=0.007 ; h=2.0 \times 10^{-4}$ 

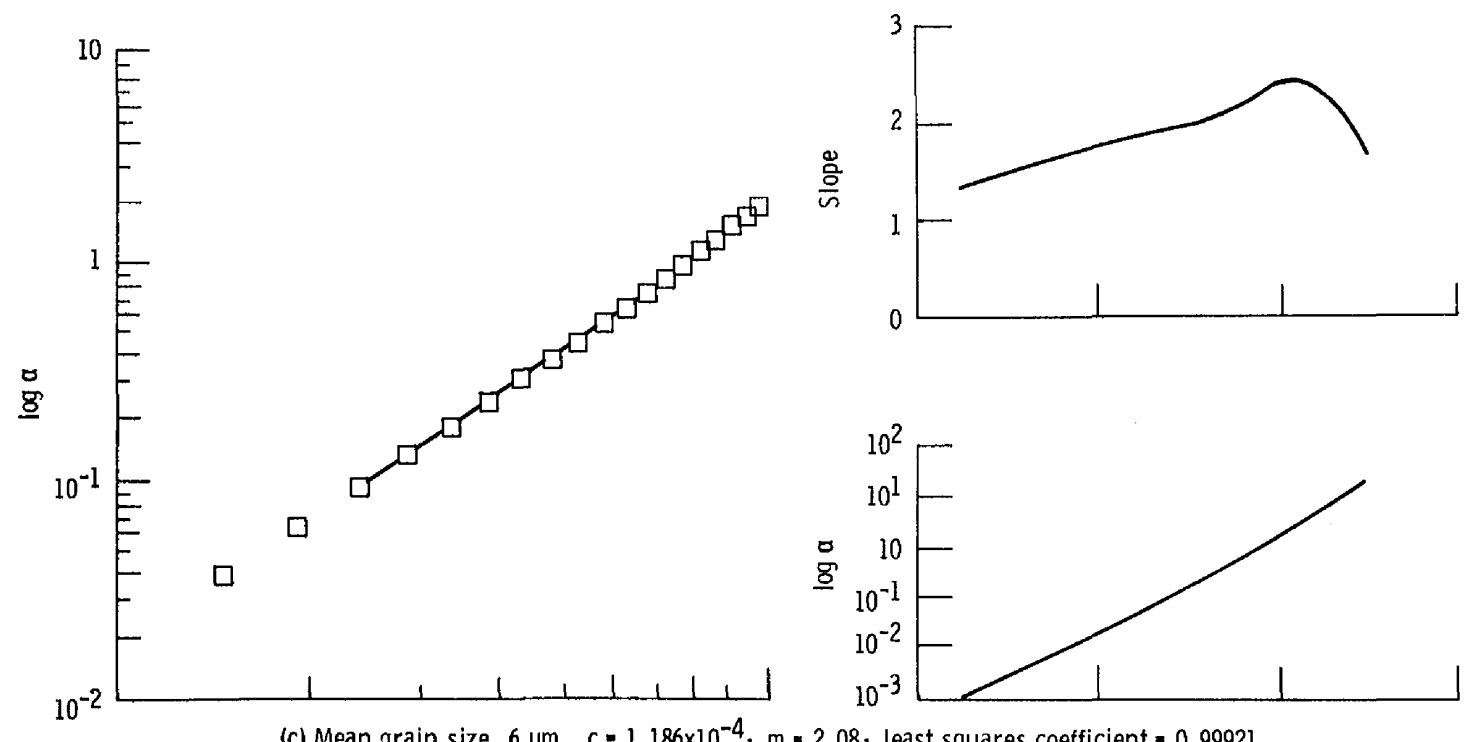

(c) Mean grain size, $6 \mu \mathrm{m}, c=1.186 \times 10^{-4} ; \mathrm{m}=2.08$; least squares coefficient $=0.99921$.
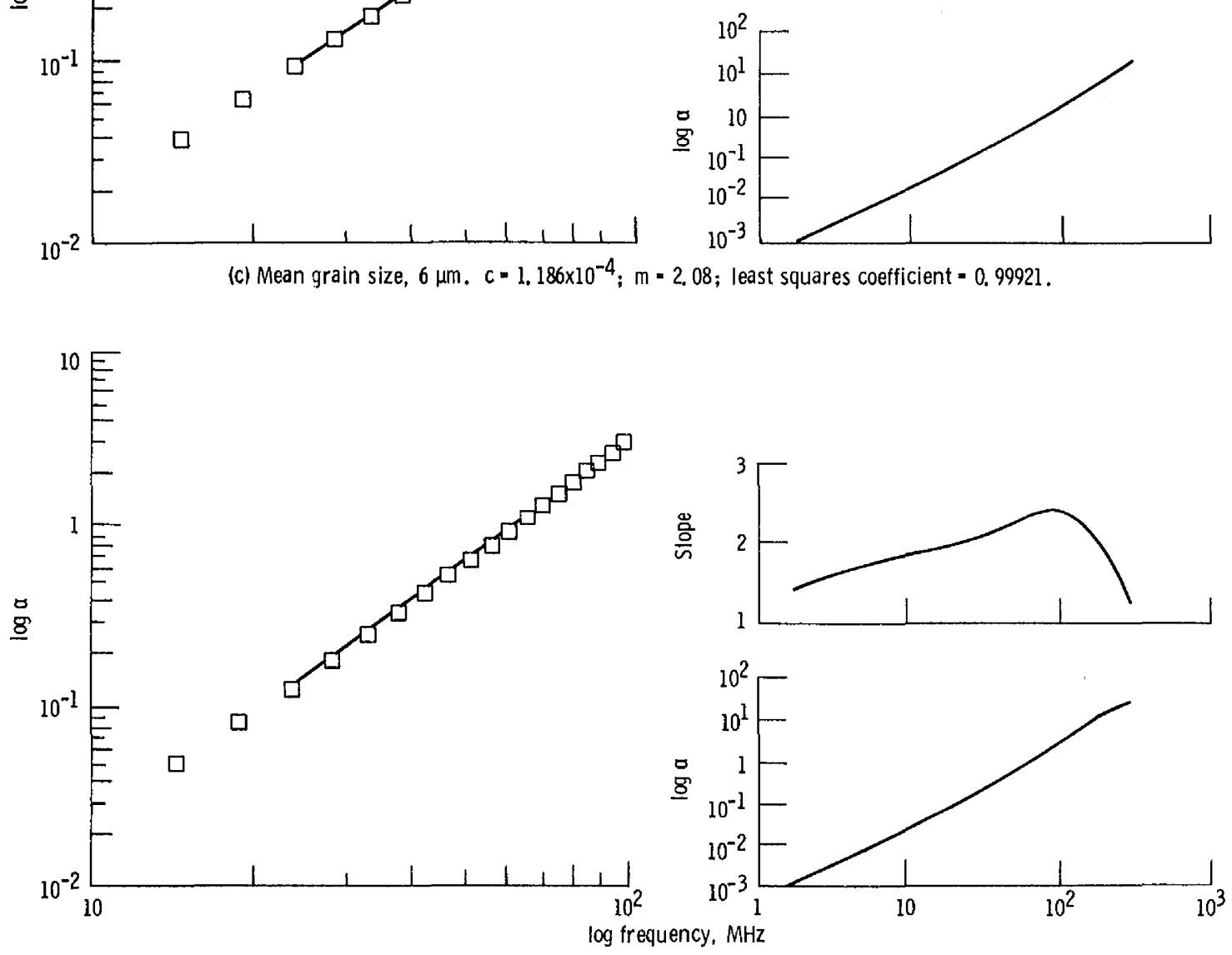

(d) Mean grain size, $8 \mu \mathrm{m}, c=1.200 \times 10^{-4} ; \mathrm{m}=2.166$; least squares coefficient $=0.99906$.

Figure 9. - Continued. 

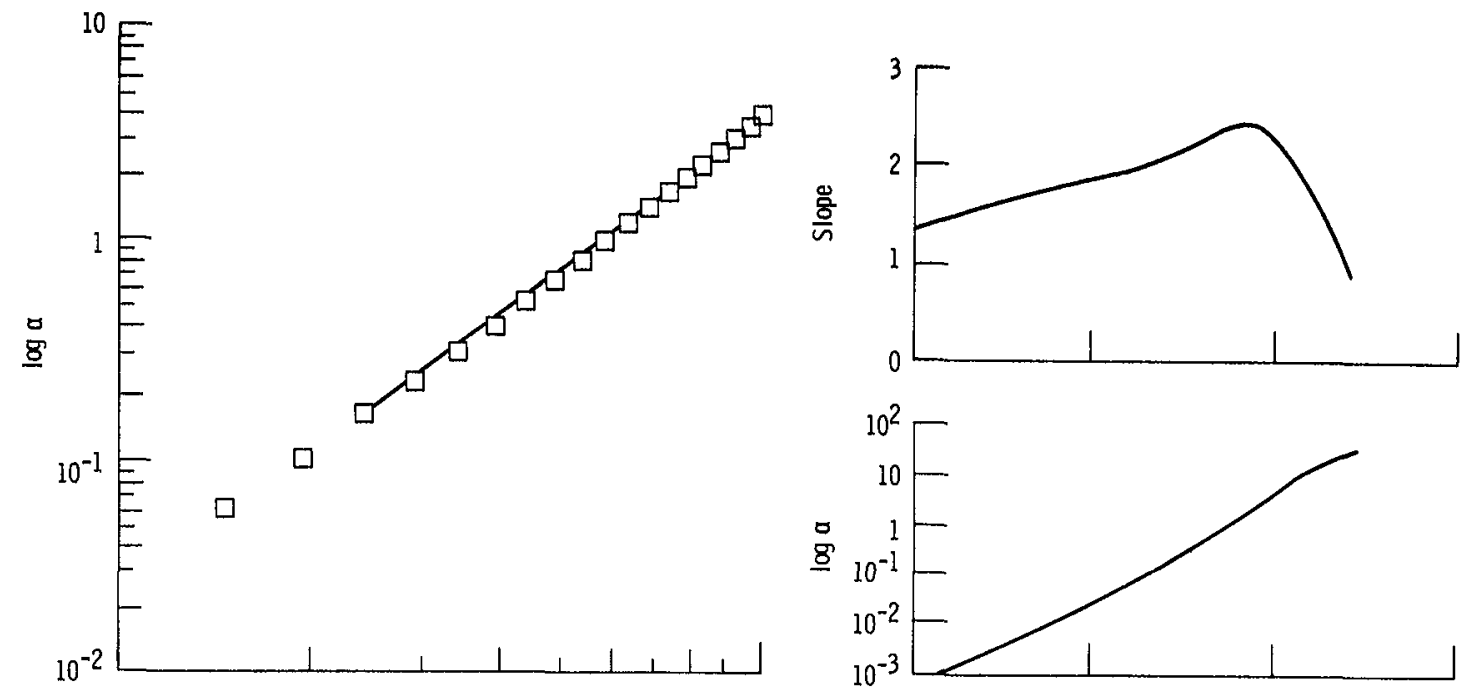

(e) Mean grain size, $10 \mu \mathrm{m}, c=1.238 \times 10^{-4} ; \mathrm{m}=2.231$; least squares coefficient $=0.99913$.
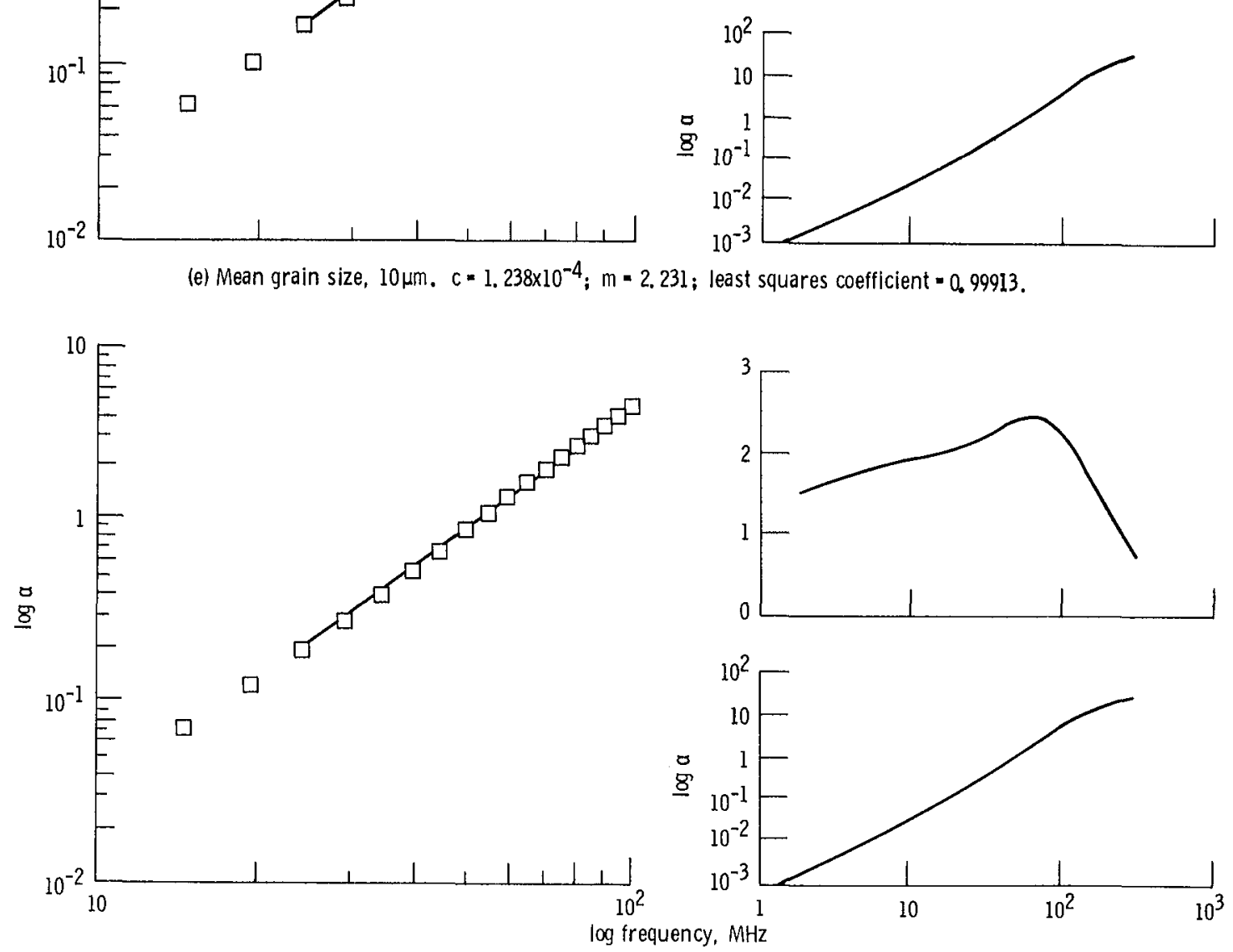

(f) Mean grain size, $12 \mu \mathrm{m} . c=1.328 \times 10^{-4} ; \mathrm{m}=2.274$; least squares coefficient $=0.99935$.

Figure 9. - Continued. 

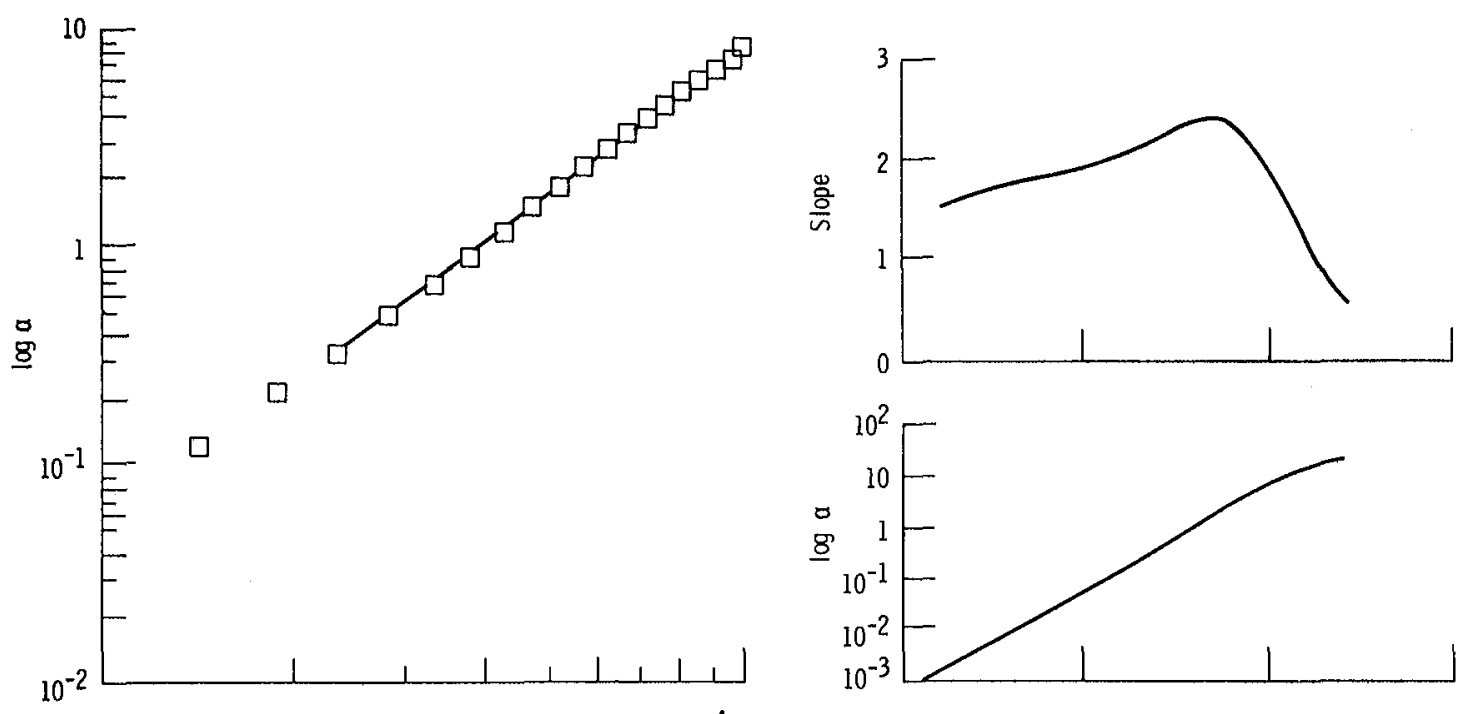

(g) Niean grain size, $14 \mu \mathrm{m} . c=1.487 \times 10^{-4} ; \mathrm{m}=2.297$; least squares coefficient $=0.99958$.
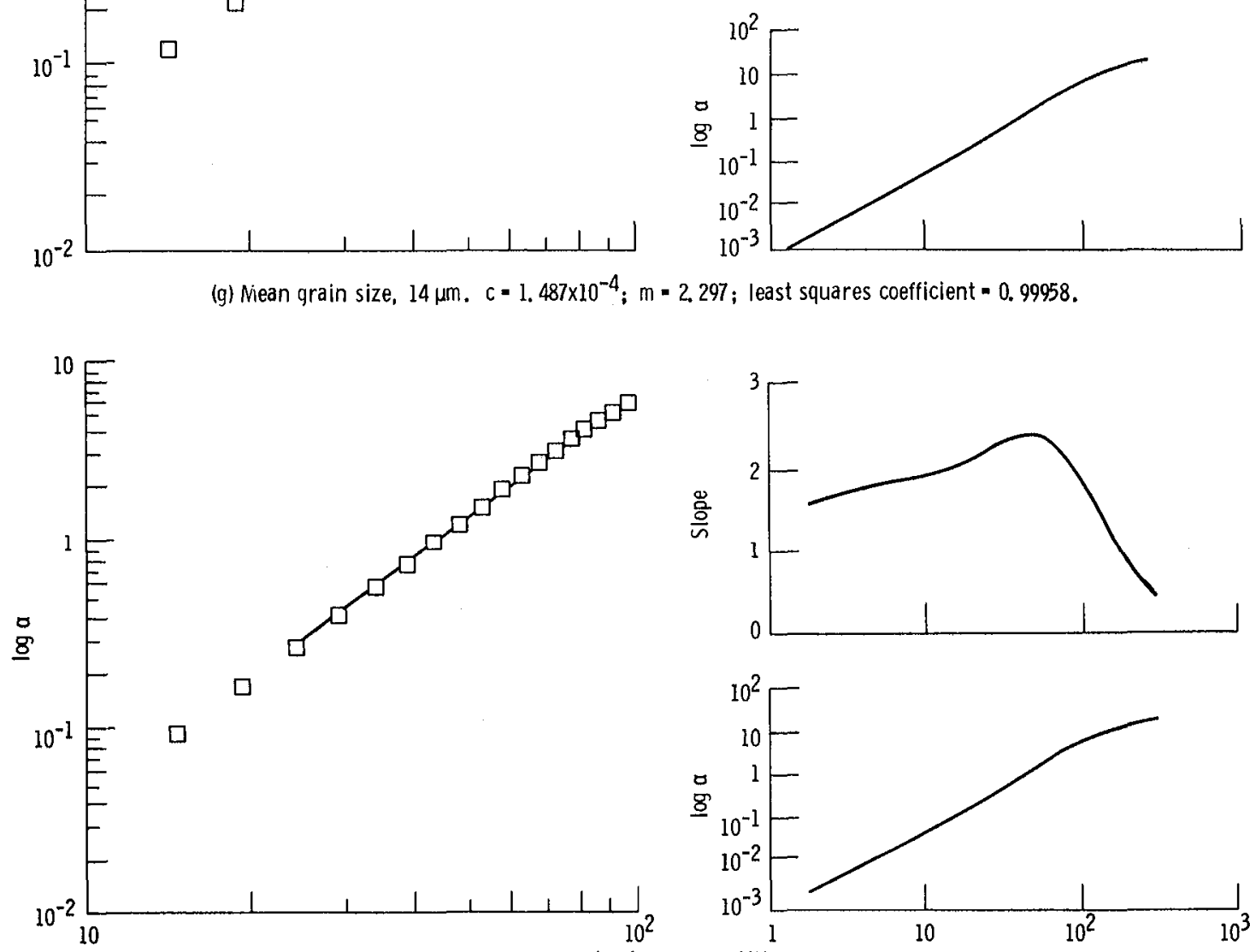

$\log$ frequency, $M H z$

(h) Mean grain size, $16 \mu \mathrm{m} . c=1,729 \times 10^{-4} ; m=2,301$; least squares coefficient $=0.99971$.

Fiqure 9. - Continued. 

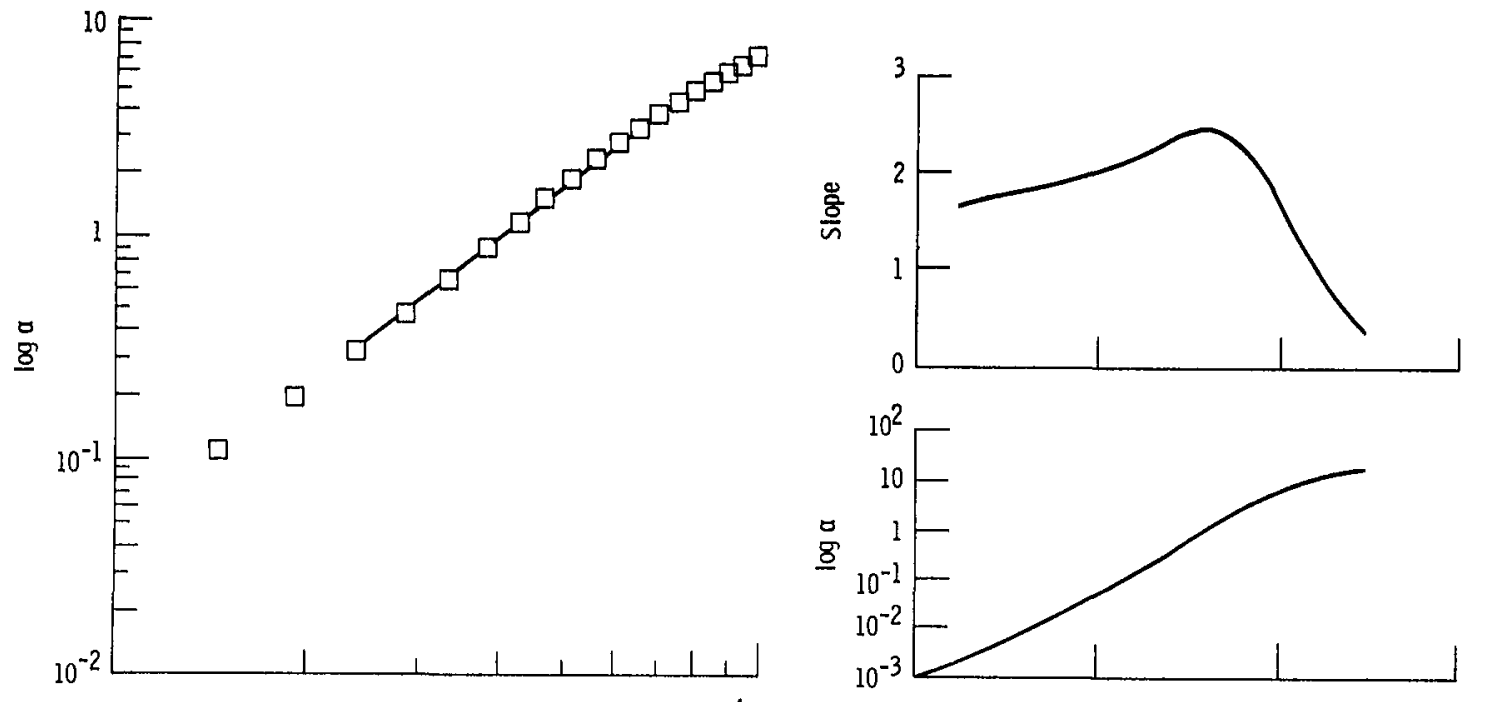

(i) Mean grain size, $18 \mu \mathrm{m} . c=2.077 \times 10^{-4} ; m=2.291$; least squares coefficient $=0.99965$.
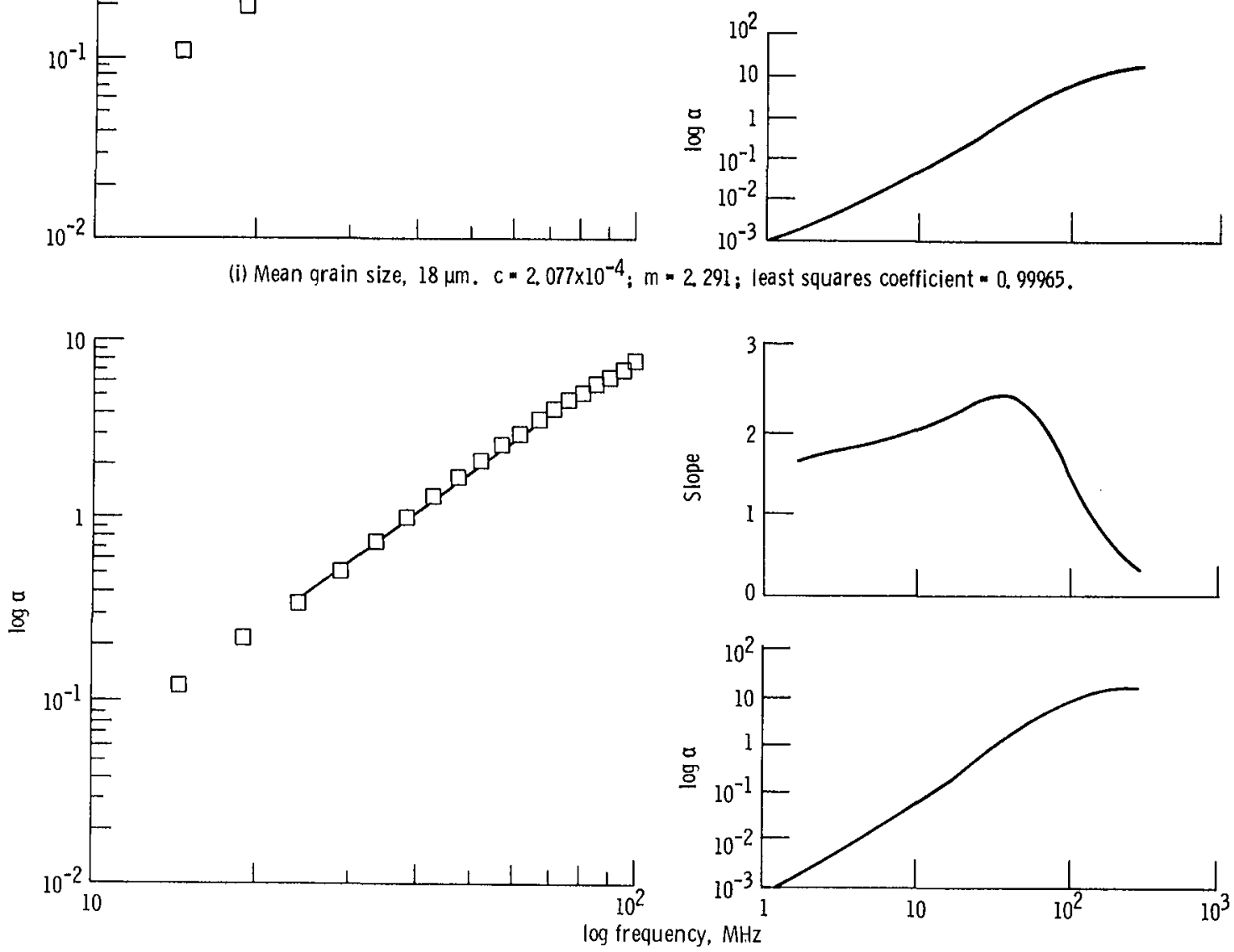

(j) Mean grain size, $20 \mu \mathrm{m} . c=2.556 \times 10^{-4} ; m=2.268$; least squares coefficient $=0.99936$.

Figure 9. - Concluded. 


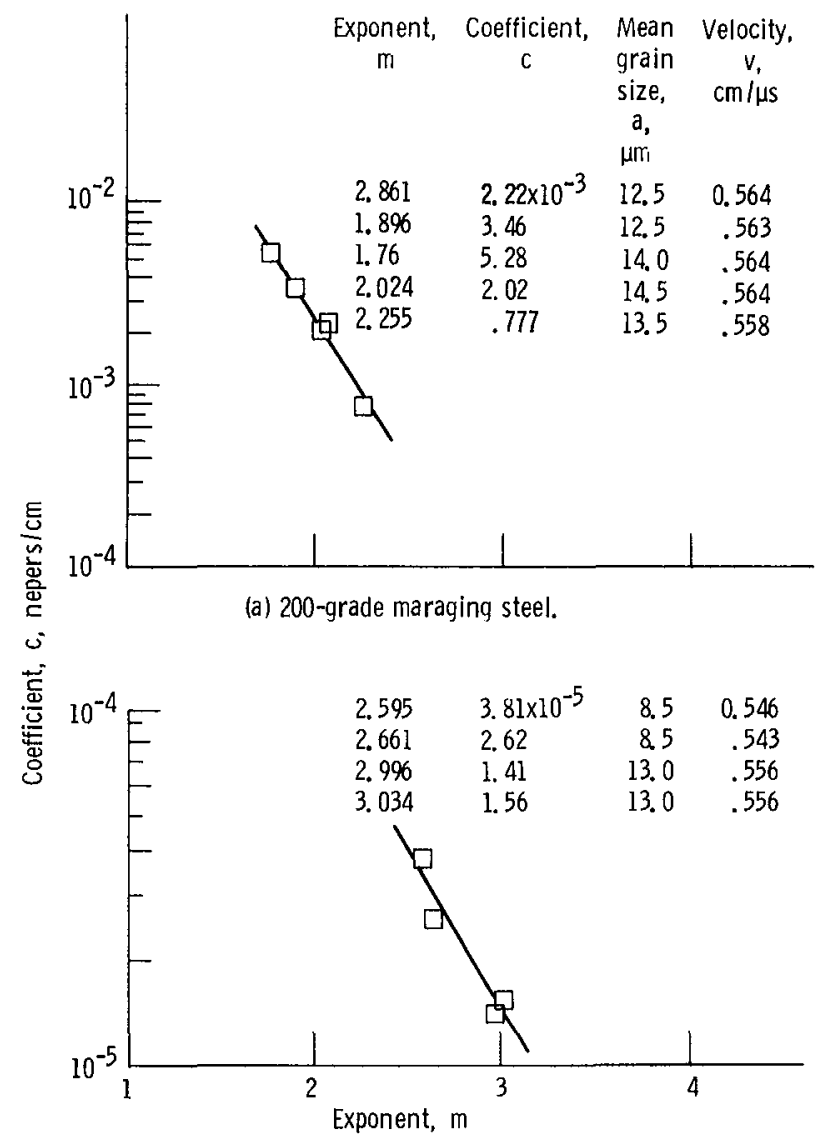

(b) 250-grade maraging steel.

Figure 10. - Inter relations between experimentally determined attenuation parameters $c$ and $m$ from equation (14) for two maraging steels. Data from Vary (1978). 


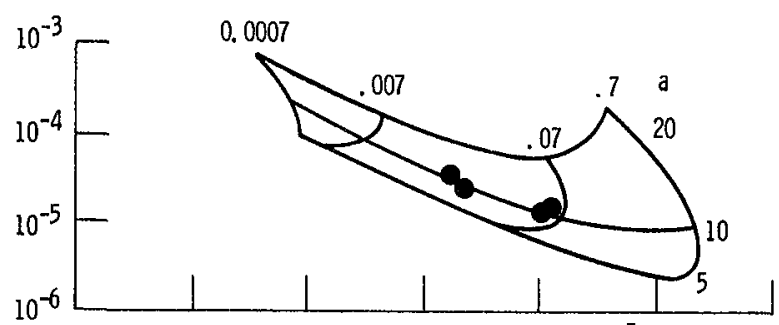

(a) Parametric field for $v=0.55 \mathrm{~cm} / \mu \mathrm{s}, \mathrm{h}=2 \times 10^{-5}, G=0.007$.

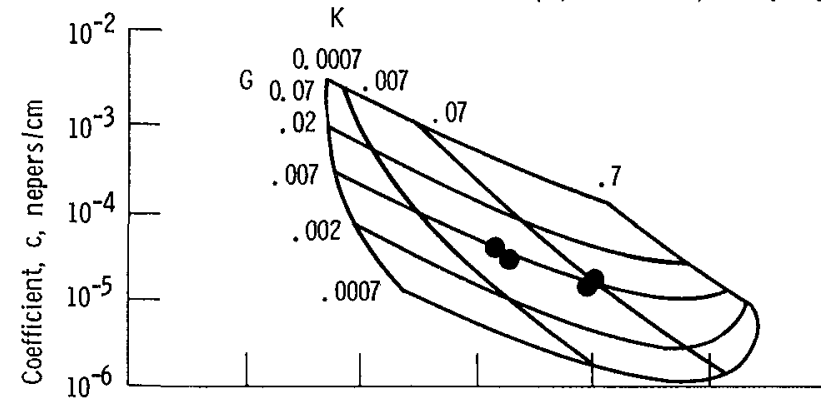

(b) Parametric field for $v=0.55 \mathrm{~cm} / \mu \mathrm{s}, a=10 \mu \mathrm{m}, h=2 \times 10^{-5}$.

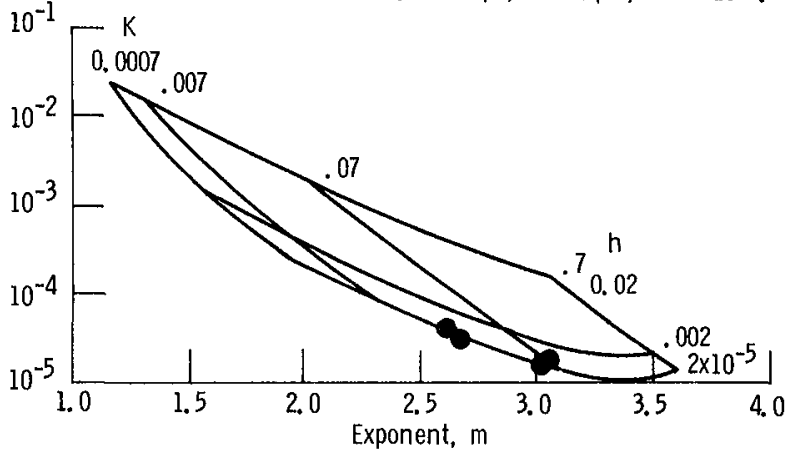

(c) Parametric field for $v=0.55 \mathrm{~cm} / \mu \mathrm{s}, a=10 \mu \mathrm{m}, G=0.007$.

Figure 11. - Parametric fields of $c$ versus $m$ based on equations (14) and (34) for assigned values of mean grain size a, velocity $v$, hysteresis constant $h$, average reflection coefficient $G$, and elastic anisotropy factor $K$. Using the curve-fitting method illustrated in figure 9 the fields contain only points for which the correlation coefficient exceeds 0.999 over the frequency range 15 to $100 \mathrm{MHz}$. Data for 250 -grade maraging steel from figure 10 are coplotted within fields. 



\title{
ADHESIVE JOINT EVALUATION BY ULTRASONIC INTERFACE
}

\author{
AND LAMB WAVES \\ S.I. Rokhlin* \\ The Ohio State University \\ Department of Welding Engineering \\ Columbus, Ohio 43210
}

The effectiveness of ultrasonic methods for the evaluation of material properties and process control has been known for a long time. It was also found that uitrasonic methods can be used for material strength prediction and verification. Application of ultrasonic methods for evaluation of adhesive bonds has a number of specific features. It involves checking a thin layer of adhesive, the thickness of which is much smaller than that of adherents. It is necessary to detect not only violation of the continuity of the adhesive, but also its effective elastic properties, which correlate with the strength of the adhesive bonds. Most of the previous techniques used longitudinal ultrasonic waves normally incident on the adhesively bonded interface. But these waves are insensitive to the adhesion properties between adhesive and adherends.

We suggest an alternative method using a guided waves. The advantage of this technique is that this wave produces shear stresses on the interface. This method was previously discussed (ref. (1) - (6)) and reviewed (ref. 7).

In this paper first, we describe application of interface waves for cure monitoring of structural adhesives; second, the Lamb technique which is applicable for study of adhesive joints of thin sheets is discussed.

\section{ELASTIC INTERFACE WAVES}

The general case of propagation of interface waves in adhesively bonded interfaces was studied by Rokhlin et al (ref. 1). The case of propagation of interface waves in a system of two half spaces separated by a viscoelastic layer was analyzed. The shear modulus $\mu_{0}$ of the layer was assumed to be smaller than the shear modulus of the half spaces, then in the case of identical half spaces there always exists a guided interface wave.

A possible method for obtaining interface waves is shown schematically in figure 1. The surface wave, excited in the lower substrate, is transformed in the interface region into an interface wave. Due to the closeness of velocities of the surface and interface waves, only a small part of the energy is transformed into bulk waves. The interface wave leaving the interface zone is retransfomred into a surface wave, and is sensed by a receiver.

*On leave from Ben-Gurion University of the Negev Beer-Sheva, Israel. 


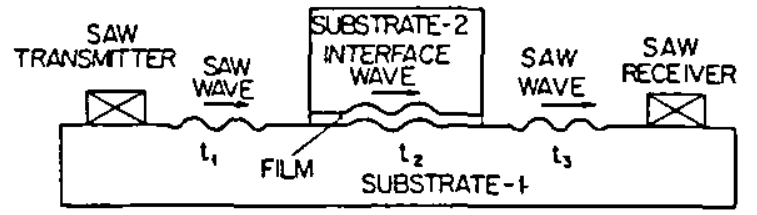

Fig. 1 Illustration of the interface wave method.

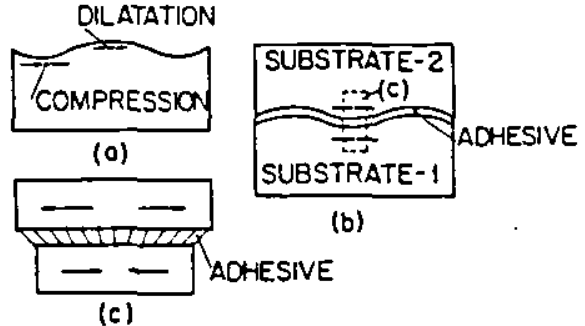

Fig. 2 Deformation of an interface film upon propagation of an interface wave. The thickness of the interface film is much smaller than the interface wavelength. The figure c corresponds, on a magnified scale, to element $c$ singled out in figure $b$.

The deformation of a thin solid interface $f i l m$ by the interface wave is illustrated schematically in figure 2. Figure $2 a$ shows the deformation produces by the surface wave in the subsurface layer. It shows tension and compression regions separated from one another by a half wavelength. Figure $2 b$ shows the deformation of an interface layer, the thickness of which is much smaller than the wavelength. Due to antisymmetry of motion, the interface layer is subjected to shear strain (fig. $2 \mathrm{c}$ ).

One can estimate the viscoelastic properties of the interface layer by measuring the velocity and attenuation of the interface wave (ref. 1). We showed that the complex shear modulus of an interface wave $\mu_{0}$ is related by a simple expression to the interface wave velocity $V_{j}$.

$$
\mu_{0} / \mu=\bar{n} \Delta_{r} /\left(\beta-\bar{h} \frac{\rho_{0}}{\rho} 1-2 \bar{h}^{2}{ }^{2} a_{2}\right)
$$

where $\alpha=V_{t} / V_{j}$ is the normalized wave number for the interface wave; $a_{1}=\alpha^{2}-\beta \gamma$; $a_{2}=2 \beta \gamma-W ; W=\alpha^{2}+\beta^{2} ; B=\left(\alpha^{2}-1\right)^{\frac{1}{2}} ; \gamma=\left(\alpha^{2}-V_{t}^{2} / V_{\ell}^{2}\right)^{\frac{1}{2}} ; \Delta_{r}=\left(W^{2}-4 \alpha^{2} B \gamma\right) ; \Delta_{r}$ is the characteristic function for the Rayleigh wave, $V_{t}$ and $V_{\ell}$ are the shear and longitudinal wave velocities in the substrate, $\rho_{0}$ is the density of the interface film material, $\rho$ and $\mu$ are the density and the shear modulus, respectively, of the substrate, $f$ is the frequency and $\bar{h}=2 \pi \mathrm{fh} / V_{t}$ is the nondimensional film thickness, $2 \mathrm{~h}$ is the thickness of the film.

Equation (1) is valid at $h \ll \lambda^{0}$, where $\lambda^{0}$ is the length of shear waves in the material of the interface film. When this condition is not satisfied, shear modulus $\mu_{0}$, determined from Equation (1) can be refined on the basis of the more exact equations (ref. 1). To determine the complex shear modulus $\mu_{0}$, one should substitute into Equation (1) the complex wave number $\alpha$, which is calculated on the basis of measured velocity and loss factor of interface waves. 
Numerical analysis shows that the sensitivity of this method is high in the range of film thicknesses which are small as compared with the length of interface waves. Hence at film thicknesses from 100 to 1 um the measurements should be performed at relatively low ultrasonic frequencies from 0.5 to $5.0 \mathrm{MHz}$.

It is possible to single out the principal features of the method, which indicate that the use of the interface waves for the evaluation of thin interface layers (in particular adhesively bonded structures) is promising.

1) The interface wave produces shear stresses at the interface; these stresses are most sensitive to variations of adhesion quality.

2) The interface wave propagates along the interface and hence is sensitive to small changes in the properties of the adhesive and of the bond between the adhesive and the adherends.

3) The interface wave can be used for evaluation of very thin layers, when $2 \mathrm{~h} / \lambda_{t}<0.01$, where $2 \mathrm{~h}$ is the thickness of the interface layer and $\lambda_{t}$ is the length of the shear wave in the substrate. Conversely, bulk longitudinal waves, usually employed for evaluation of adhesively bonded structures, are insensitive to the existence at the interface of a infinityly thin liquid layer which exhibits no shear resistance.

4) As shown below, interface waves can be used for evaluation of multilayered interface films, which simulate the adhesion properties at the adhesiveadherend interface.

As an example, reconstruction of the complex shear modulus of adhesive from experimental data is shown in figure 3 . The data were taken in the course of bonding two steel substrates with epoxy resin. The thickness of adhesive film is $12 \mu$.

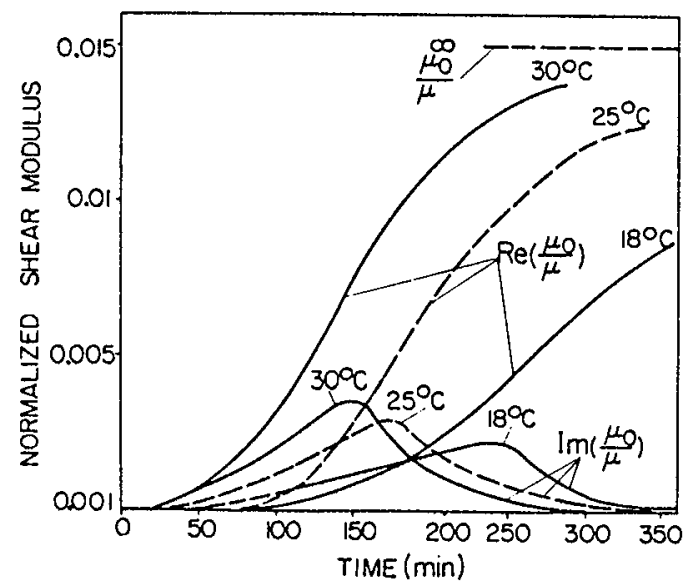

Fig. 3 The normalized complex shear modulus $\mu_{0} / \mu$ calculated from smoothed measured values of the interface wave velocity and the transmission losses. The dashed horizontal line represents direct measurements of the normalized film shear modulus $\mu_{0} / \mu$ after full polymerization. The steel shear modulus was taken as $\mu=0.809 \cdot 10^{12} \mathrm{dyn} / \mathrm{cm}^{2}$. 
An adhesive bond may fail in two ways: 1) Failure inside the adhesive, which is termed conhesive failure. The cohesive strength of a given adhesive correlates with its elastic modulus. 2) Failure along or close to the adhesive-adherend interface, which is termed interfacial or adhesion failure.

The cohesive strength of a given type of adhesive is governed by its chemical structure and by the conditions of the curing process (in particular, by the percentag of cross linking), and by the presence of various kinds of mocrodefects (e.g., microcracks or voids). These quantities also determine the shear modulus and the ultrasoni loss factor of the adhesive. By virtue of this fact, for a given type of adhesive, a change in the elastic modulus correlates with a change in its strength.

It is reasonable to consider a multilayered model of a bond line, consisting of an adhesive layer and thin interface layers between adhesive and adherends which characterize the adhesion properties.

According to this model, the shear modulus of the adhesive, calculated on the basis of the measured velocity and attenuation of interface waves is the effective shear modulus $\mu_{\text {eff. }}$. It characterizes the effective elastic properties of the multilayered adhesive system. The qualitative description of the properties of the interface wave can be given on the basis of the matrix method. We obtained a series expansion of the exact solution with respect to the thickness of interface films and compared this solution with the solution for the case of a single film (ref. 6).

According to this comparison, we obtained equation (1) for a single interface film when only linear terms are retained in the matrix expansion. A similar approximation for the multilayered system makes it possible to write the characteristic equation for the velocity of the interface wave in the form

$$
\mu_{e f f} / \mu=\bar{h} \Delta_{r} /\left(\beta-\bar{h} \frac{\rho e f f}{\rho} a_{1}-2 \bar{h}_{\alpha}{ }^{2} a_{2}\right)
$$

where

$$
\begin{aligned}
& \mu_{\text {eff }}=\mu_{0}\left(1+\frac{h_{w}}{h_{0}}\right) /\left(1+\frac{h_{w} \mu_{0}}{h_{0} \mu_{w}}\right) ; \rho_{\text {eff }}=\rho_{0}\left(1+\frac{\rho_{w} h_{w}}{\rho_{o} h_{0}} /\left(1+\frac{h_{w}}{h}\right) ;\right. \\
& h=h_{0}+h_{w}
\end{aligned}
$$

$2 h_{0}$ - is the thickness of the adhesive layer. $h_{w}, \rho_{w}, \mu_{w}$, - are the thicknesses, density and shear modulus of the weak boundary layer. The other terms have the same meanings as in Equation (1).

When contact between an adhesive and substrates is not ideal the interface wave velocity decreases. Hence, the effective shear modulus $\mu_{\text {eff found from Equation (2) }}$ on the basis of the experimentally measured velocity will be smaller than the actual modulus of the film.

For example, in the case of absence of shear bonding between the adhesive and at least one of the substrates (slip contact) the velocity of the interface wave will be close to the Rayleigh wave velocity and the calculated $\mu_{e f f}$ will be equal to zero. It is thus seen that the interface-wave velocity will characterize not only the elas- 
tic properties of the adhesive and its cohesive strength, but also the bond between the adhesive and the substrates, i.e., the interfacial (adhesion) strength. this means that the ratio $\mu_{\text {effl }} \mu_{0}$ can be used as a criterion of the bond strength. If the effective shear modulus $\mu_{\text {eff }}$ is measured for a given bond, and the shear modulus $\mu_{0}$ of the adhesive is measured on a reference specimen, then Equation (3) can be used for estimating (ref. 7) the properties of the interfacial layer between adhesive and substrates.

\section{EVALUATION OF THE CURING OF STRUCTURAL ADHESIVES BY ULTRASONIC INTERFACE WAVES}

The reliability of adhesive-bonded structures and composite materials depends on a large number of different factors. It is determined to a significant extent by the manufacturing process and the feasibility of monitoring it. In connection with this it becomes necessary to control the quality of viscoelastic properties of the adhesive in the course of curing over the required temperature and pressure ranges, and to perform real-time continuous monitoring over the entire range of variation of adhesive properties in the course of the bond's performance.

The method of ultrasonic interface waves appears to be promising in resolving this problem.

The curing of structural adhesives is performed in nonisothermal conditions. The standard cure cycle consists of a rising temperature at a constant rate for $40 \mathrm{~min}$. from room temperature to $120^{\circ} \mathrm{C}$ (for $F M-73$ ) and to $180^{\circ} \mathrm{C}$ (for $\mathrm{FM}-300 \mathrm{~K}$ ). Temperature changes modify the elastic moduli of the adhesive film and substrates (adherends). To measure the adhesive properties under nonisothermal conditions, it is necessary to eliminate the effect of temperature variations in the substrates and transducers. For this purpose a differential measuring arrangement, in the form of an acoustic bridge, was suggested and analyzed (ref. 4). A computerized ultrasonic system was further developed to measure the phase difference of the ultrasonic signals from the two arms of the bridge.

The experimental sample in the form of an ultrasonic bridge is shown schematically in Fig. 4. Locations of the heaters and thermocouples are shown in the figure. The temperature gradient in the course of measurement did not exceed $0.5^{\circ} \mathrm{C}$. As shown in the figure, pressure may be applied to the specimen. Strictly perpendicular force transmission is ensured by means of spherical contact. The comb transducers were used for excitation and reception of surface waves.

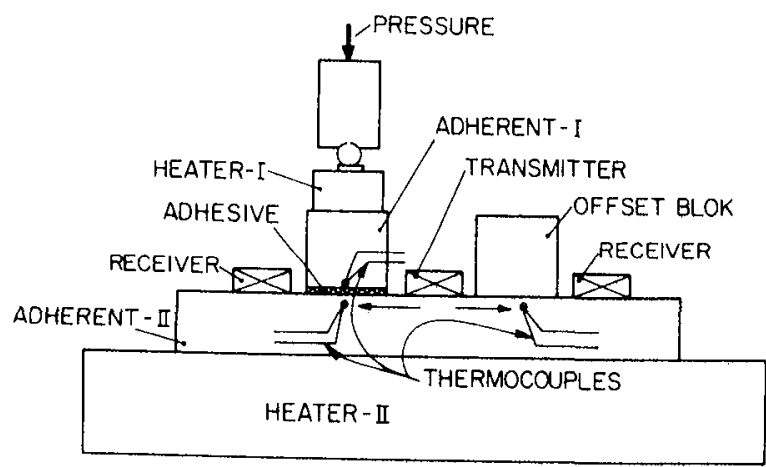

Fig. 4 Schematic of the specimen for differential measurements.

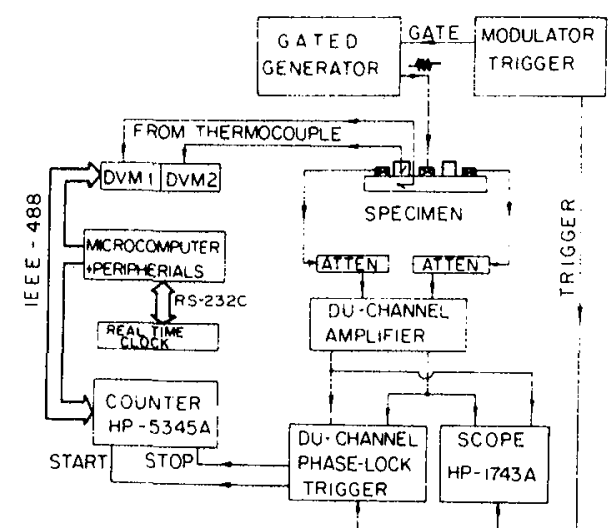

Fig. 5 The block diagram of the computerized automatic measuring measurement. 
The measuring arrangement is shown shematically in Fig. 5. A gated oscillator excites the transmitting transducer. The received signals are fed from two receiving transducers through attenuators and home-made dual-channel amplifier to a specially designed dual-channel phase-lock trigger. This unit is used for automatic following of changes in the phase of the signal between the working and reference channels. The received reference and working signals are transformed into zero-crossing rf signals. When one of the zeroes coincides with the selecting window, there is produced a short trigger/signal: trigger start, which starts up the counter. Analogously a second pulse: trigger stop, is produced in the second channel, and it stops the count. The change in the time shift between both triggers corresponds to a change in the phase between the working and reference signals. The temperature data and data from the counter operating in the time-interval averaging mode through IEEE- 488 are fed to a microcomputer.

To check the accuracy of the system, we performed an analog simulation of the measuring method. For this a reference and a working signal from a highly stable frequency synthesizer were fed to the phase-lock trigger. The synthesizer's clock signal was used as the reference. The resulting time noise was measured to the order of $30 \mathrm{psec}$, which corresponds to the synthesizer error. This means that the noise of the electronic system, namely, the phase-lock trigger and counter, does not exceed 30 ps.

Some examples of application of the method for study of the curing of structural adhesives are given below. The changes of the phase velocity of interface wave during curing of FM-73 structural adhesive are shown in Fig. 6 by black circles. The shear strength data obtained in different stages of curing process are also shown in this figure. It is seen that a rise in the velocity of the interface wave (rise in the shear modulus of the adhesive) corresponds precisely to the time interval of the bondstrength growth. Fig. 7 shows results of the measurements at different temperatures. In Fig. 8 these results are summarized in the form of an Arrhenius plot from which the activation energy of the curing reaction for the $F M-73$ adhesive was found to be equal to $9.3 \mathrm{Kcal} / \mathrm{mole}$. The data for FM - 300K structural adhesive are shown in Fig. 9. The cure temperatures for this adhesive are higher than for FM - 73 .

The effect of aging of adhesive prepregs on cure are illustrated in Fig. 10 and Fig. 11. The adhesive was aged at $40^{\circ} \mathrm{C}$ before curing. The number of days of curing ( 24 hours in a day) are shown in Fig. 10 on the right side of the experimental curves. The summary results are presented in Fig. 11. Moderate aging results in appearance of nucleation sites, with shortening of the initial incubation period. For a longer time of aging prepreg became more polymerized and not converted to the fluid in the course of curing and therefore bond formation is not effective.

The technique discussed above is applicable to evaluation of thick bonded adherends. It was further developed (ref. 9) for evaluation of thin bonded sheets using Lamb waves. 


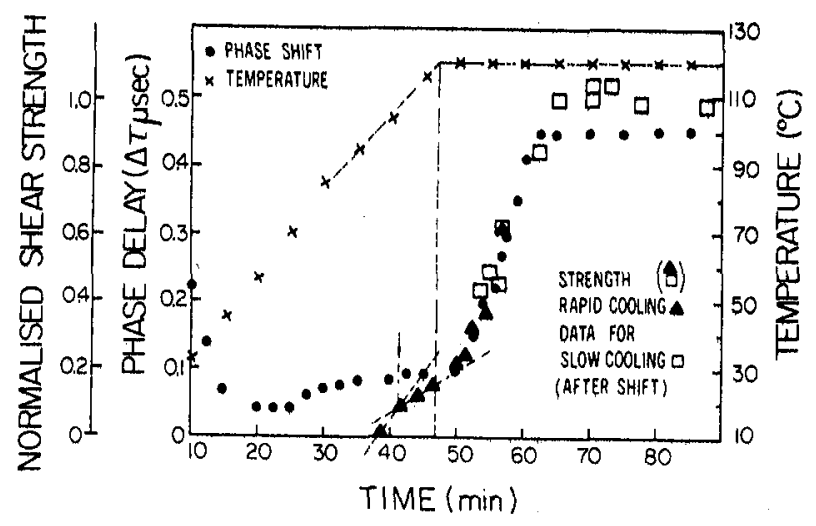

Fig. 6 Comparison of the ultrasonic data with the variation in relative bond strength in the course of the cure.

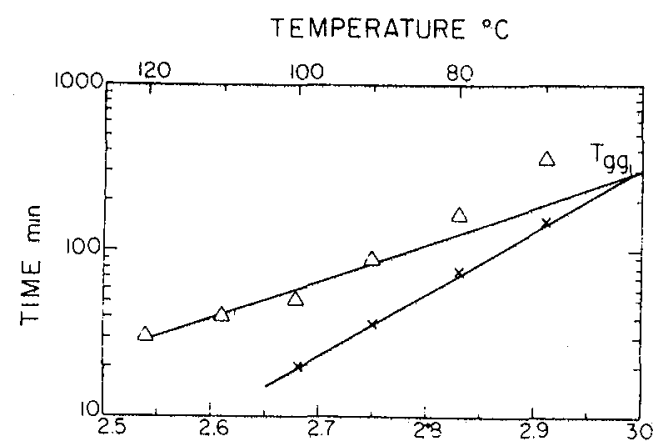

INVERSE TEMPERATURE $\left(\frac{1}{\mathrm{~T}}{ }^{\circ} \mathrm{K}\right) \times 10^{3}$

Fig. 8 An Arrhenius plot of the cure data.

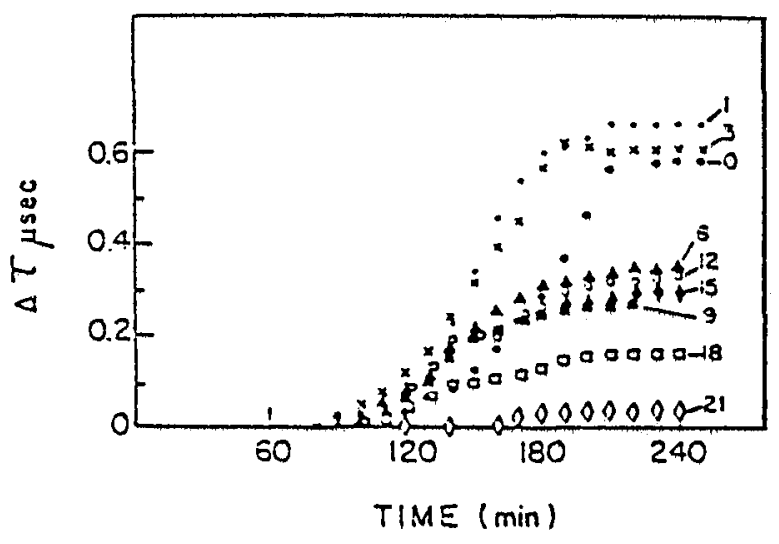

Fig. 10 The change in the interface-wave velocity as a function of curing time for aged specimens.

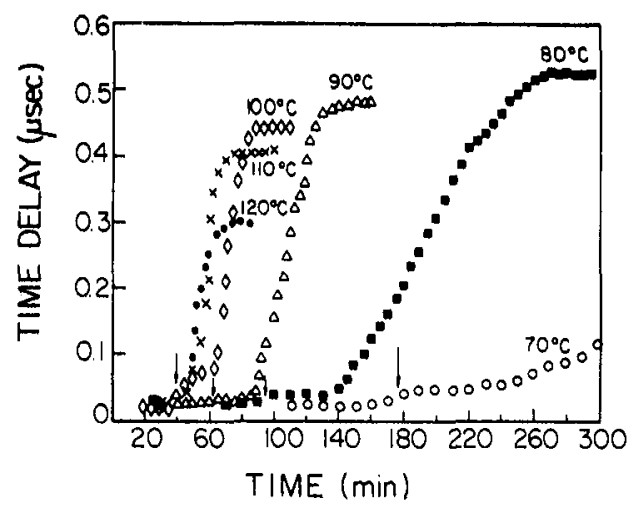

Fig. 7 Data for FM - 73 adhesive at different temperatures.

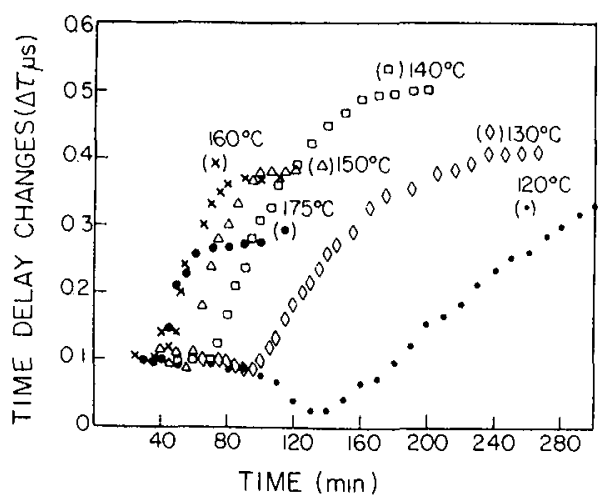

Fig. 9 Data for FM - 300K adhesive at different temperatures.

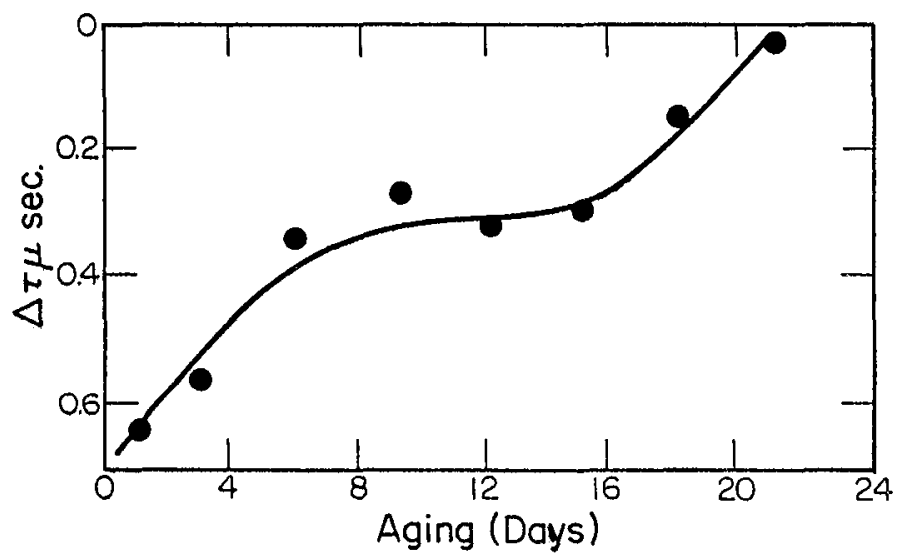

Fig. 11 The change in time delay as a function of the aging time. 
When the thicknesses of both bonded substrates are much greater than a wavelength the interface wave can propagate along the interface as was discussed in the previous section. When the thicknesses of the bonded substrates are comparable to a wavelength the problem of wave propagation becomes much more complicated: several modes with different speeds can propagate in such a structure (Lamb modes). The number of modes and their phase and group velocities depend on frequency, substrate thicknesses and boundary conditions (adhesion) between substrates.

It is possible to select Lamb modes sensitive to the changes of the bond properties. Let us for example consider the dispersion characteristic of Lamb wave for two different boundary conditions: slip and rigid. The slip boundary condition corresponds to liquid adhesive on the interface (initial stages of curing). During the curing the adhesive solidifies and the boundary condition between plates becomes closer to the rigid boundary condition. During this liquid to solid conversion the propagated mode is transformed with change of phase velocity from a mode which can propagate for the slip boundary condition to one which can propagate for the rigid boundary condition.

As an example in Fig. 12 the dispersion curves for Lamb waves in a structure with slip and rigid boundary conditions are shown (two plates of the same thickness are bounded). The mode So(h) can propagate in the case of slip bond. It is transformed to the mode $A_{1}(2 h)$ during adhesive curing. It is shown schematically for $k_{t} h=3.1$ ( $K_{t}$ is the wave number for shear waves, $h$ is the half thickness) by arrow.

UTtrasonic experiments and shear strength tests are usually performed on the lapshear specimen Fig. 13(a) (ultrasonic measurements can be also performed for the sample
shown in Fig. 13(b)). The wave behavior for such samples is much more complicated than was discussed above.

The Lamb wave is excited on the plate outside the bonded area. When it reaches the bonded area it is converted to the Lamb waves permissible in the plate region with a new value of the parameter $k_{t} h$. This transformation will be different for different contact on the bondline (slip or rigid). So it is possible that some mode which can easily be excited for the slip contact cannot be excited at all for the rigid contact. So this mode will vanish in the course of curing of the adhesive and it cannot be used for our purposes.

To clarify this matter the theoretical analysis were applied for estimation of mode conversion on the boundary between bonded and unbonded areas (ref. 8). The numerical values of the energy transmission coefficients as functions of the parameter $\mathrm{K}_{\mathrm{t}} \mathrm{h}$ are plotted in Fig. 14 ( $2 \mathrm{~h}$ is thickness of the bonded system). The first and last lower symbols shows the number of the mode in the bonded area. The middle lower symbol shows the incident and transmitted mode. The upper symbols show the type of this mode (symmetric(s) or antisymmetric(s)). The same curves can be used for slip contact: 1) antisymmetric waves may not be excited in the interface region 2) in the interface region modes with the same wave number as the incident wave can propagate in both sheets.

For the incident $S_{0}$ mode the energy is transported in the bonded region mainly by the mode $S_{0}(2 h)$ at $0<K_{t} h<2$ for both rigid and slip contacts. Additionally, for slip contact, energy is also transported by the $S_{0}(h)$ mode (the same mode as incident). 
$S_{0}(2 h)$ and $S_{0}(h)$ modes will have different phase velocities as can be seen from the dispersion caves shown in Fig. 12. For $K_{t} h 2$ the energy in the bonded region is transported by the mode $A_{1}$ and by the mode $S_{1}$ when the bond is rigid (not shown in Fig. 14). When the bond is slip the energy is transported by the $S_{0}(h)$ and $S_{1}$ modes. So with changes of the type of contact the energy will be carried by different modes.

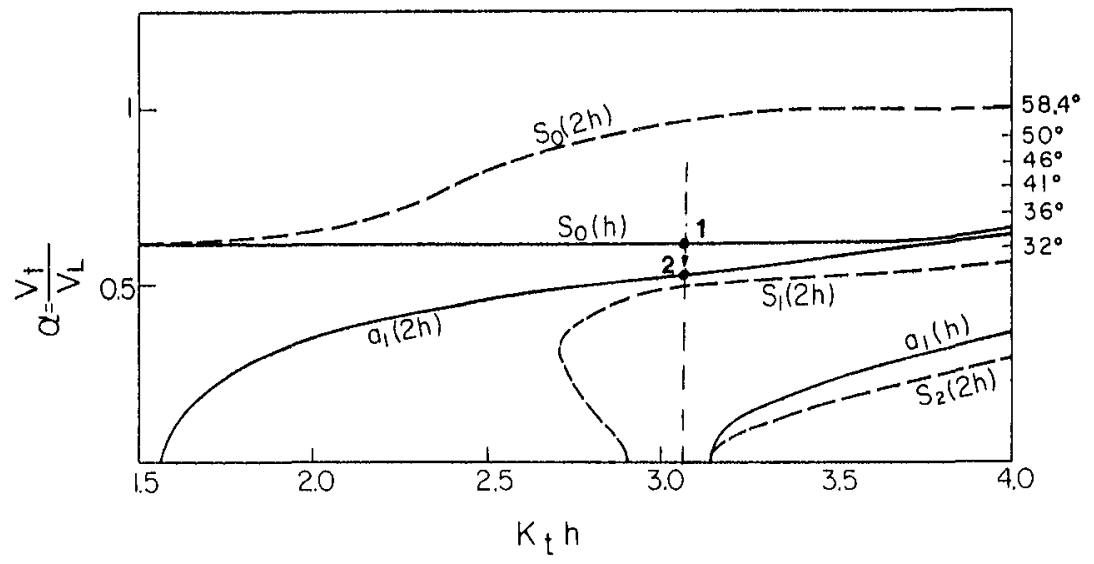

Fig. 12 The dispersion caves for the guided waves in the bonded region.

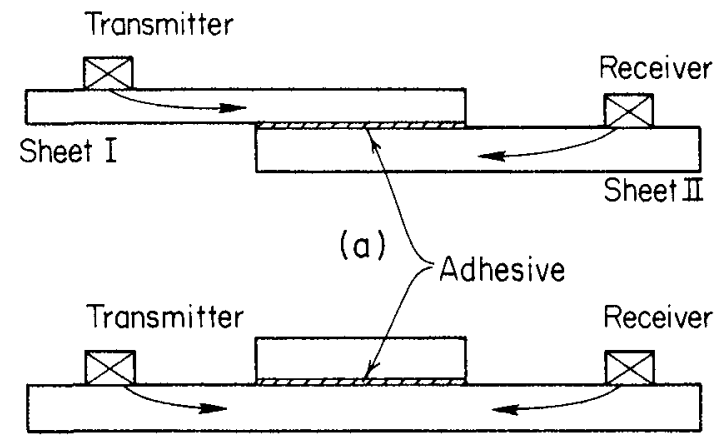

(b)

Fig. 13 Schematic illustration of the method of measurements and two sample configurations used.

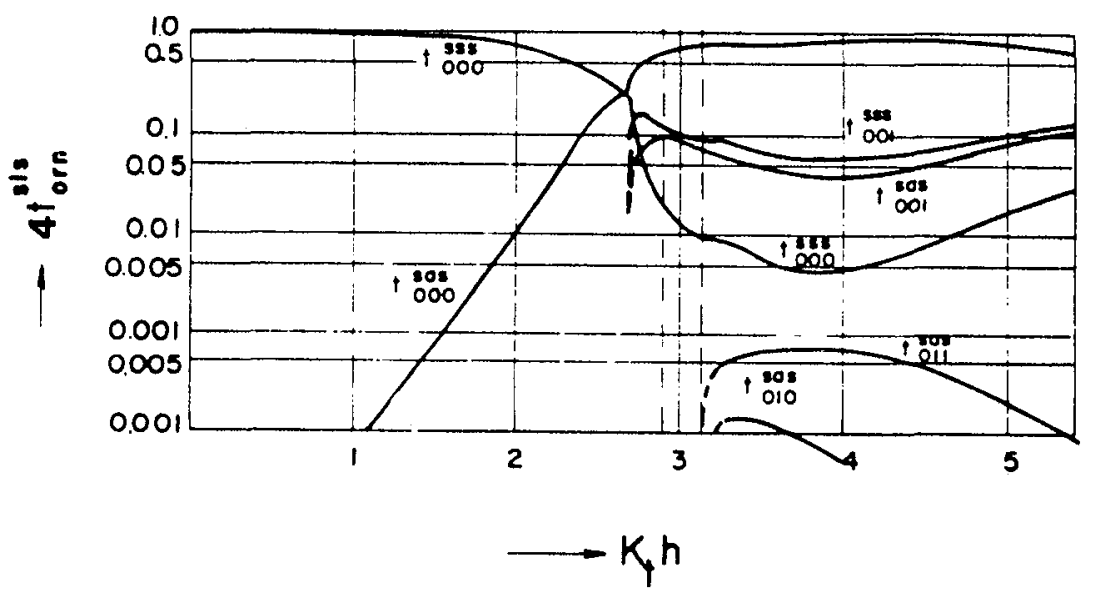

Fig. 14 Energy transmission coefficients for incident $S_{0}(h)$ mode with $S_{0}(2 h)$ mode propagates inside the bonded region. 
Now suppose that the properties of the interface change continuously from slip to rigid contact. This occurs when a thin film of liquid adhesive is cured on the interface. In which way can we attempt to select the incident mode and working frequency (parameter $K_{t} h$ so that the selected mode will be sensitive to the interface properties and in particular to monitor the state of cure? We summarize here the main directions of analysis. 1) If the mode with the same phase velocity transports energy in the bon ed region for slip and rigid contacts one can assume that the velocity changes for dif ferent bond quality will be small (this is clear because the difference between the limited values (slip and rigid) is negligible. 2) The energy transformation coefficients for the modes which are the main carriers of energy for slip and rigid contacts must be close in value. 3) The group velocities of the above modes must not be too different. Otherwise, the transmitted ultrasonic impulse for slip contact will be degraded if the contact changes and another signal will appear as the contact becomes rigid. In this case, it is impossible to make phase velocity measurements.

\section{EVALUATION OF ADHESIVE CURING USING LAMB WAVES}

The measurements were performed at frequencies $0.5,1,1.5$, and $2 \mathrm{MHz}$. The thickness of the adhesive film is typically $80-90 \mu$. We will discuss here only a few examples of the experimental data.

The experimental data for the incident $S_{0}$ mode at frequency $1 \mathrm{MHz}$ are shown in Fig. 15. The time delay changes of the ultrasonic signal during adhesive curing are plotted.

The $\mathrm{K}_{\mathrm{t}} \mathrm{h}$ in the bonded area is about 3.2. When the adhesive is liquid the $\mathrm{S}_{0}(\mathrm{~h})$ is the main energy transporter. So the shift from the point 1 to the point 2 (Fig. 12 corresponds to the phase velocity changes during the polymerization. The phase velocity decreases and the time delay correspondingly increases.

Data for the incident $S_{0}$ mode at frequency $1.5 \mathrm{MHz}$ are shown in Fig. 16. For rigid bond in the bonded area the energy transport is by the $A_{1}(2 \mathrm{~h}$ ) mode (parameter $\left.K_{t} h=4.7\right)$, and for slip contact by the mode $S_{0}(h)$. For this parameter $K_{t} h$, the phase velocity of these modes were close to one another (practially equal). Experiment shows that the velocity changes are very small. The same measurements were done on the sample shown in Fig. 13(b). The changes of the phase velocity and attenuation are very close to those shown in Fig. 15 and Fig. 16. This supports the supposition that these changes are basically due to changes of the adhesive properties and the effect of the edges of the bond does no play a significant role.

Inversely the results for frequency $0.5 \mathrm{MHz}$ (not shown here) are very different for the sample configuration shown in Fig. 13(a) and Fig. 13(b). One can assume that at this frequency the velocity changes occur not due to property changes on the bonded line but due to edge effects (ends of the bonded junction) because this is the only di ference between the specimens shown in Fig. 13(a) and Fig. 13(b) from a wave propagation point of view. This may lead to the very interesting conclusion that some of the modes are sensitive to the edge condition. This is a very important from the frac ture mechanics point of view.

Summarizing we can say that by selecting different working frequencies and incident modes for experiment, the different aspects of the bonding process can be studied. Some of the modes are sensitive to the bond line properties; some of them are more sensitive to the conditions of the edges of the bond. 


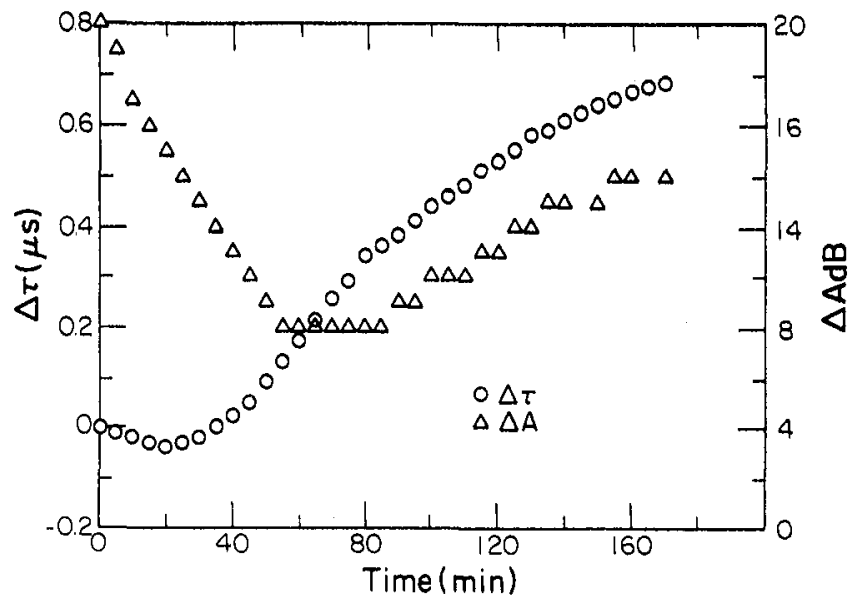

Fig. 15 The phase velocity and transmitted amplitude changes during adhesive cure for $S_{0}(h)$ incident mode at frequency $1 \mathrm{MHz}$.

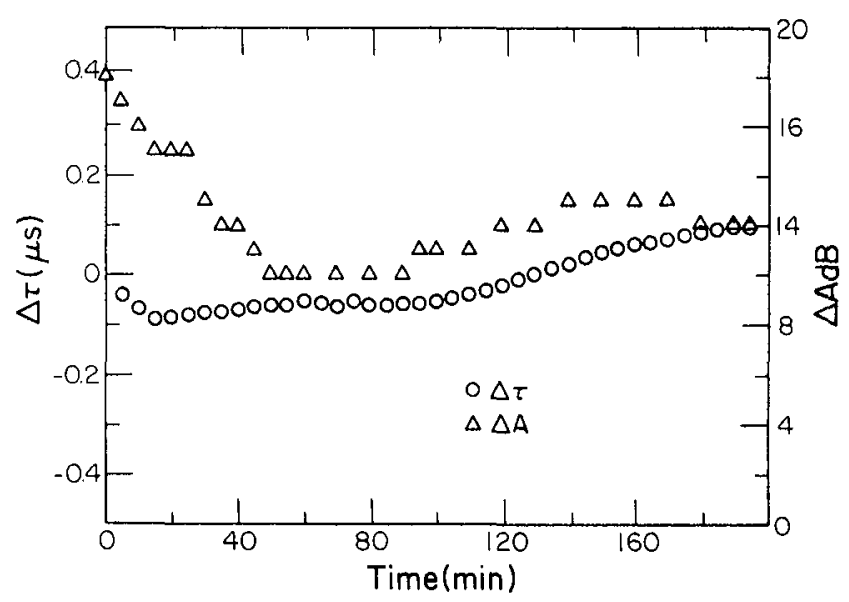

Fig. 16 The phase velocity and transmitted amplitude changes during adhesive cure for incident $\mathrm{S}_{0}(\mathrm{~h})$ mode at $1.5 \mathrm{MHz}$.

\section{CONCLUSION}

In this paper some results on application of interface and Lamb waves for study of curing of thin adhesive layers have been summarized. In the case of thick substrates (thickness much more than the wave length) the interface waves can be used. In this case the experimental data can be inverted and the shear modulus of the adhesive film may be explicitly found based on the measured interface wave velocity. It is shown that interface waves can be used for the study of curing of structural adhesives as a function of different temperatures and other experimental conditions. The kenetics of curing was studied. In the case of thin substrates the wave phenomena are much more complicated. It is shown that for successful measurements proper selection of experimental conditions is very important. This can be done based on theoretical estimations. For correctly selected experimental conditions the Lamb waves may be a sensitive probe of adhesive bond quality and may be used for cure monitoring. 


\section{REFERENCES}

1. S.I. Rokhlin, M. Hefets and M. Rosen, J. Appl. Phys., 513579 (1980).

2. S.I. Rokhlin, J. Composite Mater., 17,15 (1983).

3. S.I. Rokhlin, J. Acoust. Soc. Am., $\overline{73}, 1619$ (1983).

4. S.I. Rokhlin, M. Hafets and M. Rosen, J. Appl. Phys., 52, 2847 (1981).

5. S.I. Rokhlin, (1981), unpublished report.

6. S.I. Rokhl in and M. Rosen, Thin Solid Films, 89, 143 (1982).

7. S.I. Rokhlin in "Adhesive Joints: Their Formation Characteristics and Testing, K.L. Mittal, ed., Plenum Press, N.Y. (1984).

8. S.I. Rokhlin, (1984), unpublished report. 


\title{
INPUT-OUTPUT CHARACTERIZATION OF AN ULTRASONIC
}

\author{
TESTING SYSTEM BY DIGITAL SIGNAL ANALYSIS \\ James H. Williams, Jr., Samson S. Lee, and Hira Karagulle \\ Massachusetts Institute of Technology \\ Cambridge, Massachusetts 02139
}

The input-output characteristics of an ultrasonic testing system used for "stress wave factor" measurements were studied by coupling the transmitting and receiving transducers face to face, without a specimen in between. Some of the fundamentals of digital signal processing used are summarized.

The inputs and outputs were digitized and processed in a microcomputer by using digital signal-processing techniques. The entire ultrasonic test system, including transducers and all electronic components, was modeled as a discretetime, linear, shift-invariant system. A digital bandpass filter was introduced to reduce noise effects on the output signal. The output due to a broadband input was deconvolved with the input to obtain the unit-sample response and the frequency response of the discrete-time system. Then the impulse response and frequency response of the continuous-time uitrasonic test system were estimated by interpolating the defining points in the unit-sample response and frequency response of the discrete-time system. The ultrasonic test system was found to behave as a linear-phase bandpass filter.

The unit-sample response and frequency response of the discrete-time model of the test system were used to compute the output of the test system for a variety of inputs. The agreement between predicted and measured outputs was excellent for rectangular pulse inputs of various amplitudes and durations and for tone burst and single-cycle inputs with center frequencies within the passband of the test system. The input-output limits on the linearity of the system were determined.

\section{INTRODUCTION}

Conventional ultrasonic testing (UT) is conducted either in the throughthickness transmission or pulse-echo modes (ref. 1). In the through-thickness transmission mode, the transmitting and receiving transducers are coupled to opposite faces of the structure under inspection, and the transmitted wave field is analyzed. In the pulse-echo mode, the transmitting and receiving transducers, which may be combined into a single transducer, are coupled to the same face of the structure under inspection, and the reflected wave field is analyzed.

Recently Vary et a1. (refs. 2 and 3 ) introduced an ultrasonic nondestructive evaluation (NDE) parameter called the stress wave factor (SWF). It is similar to the pulse-echo test mode in that separate transmitting and receiving transducers are coupled to the same face of the structure. However, unlike conventional pulse-echo testing, which is generally limited to the analysis of nonoverlapping reflected wave echoes, the SWF is also valid for the analysis 
of overlapping echoes. Specifically, an input pulse having a broadband frequency spectrum is applied to the transmitting transducer and the number of oscillations of the output signal at the receiving transducer exceeding a preselected voltage threshold is defined as the SWF. The SWF has been correlated with mechanical properties of carbon-fiber-reinforced composites (refs. 2 to 4). Williams et a1. (ref. 5) theoretically and experimentally studied the ultrasonic input-output characteristics of the SWF test configuration in a thick, isotropic, elastic plate. The extension of that work to thin plates is currentiy in progress (ref.6).

An important step toward the quantitative analysis of input-output relations in any uitrasonic NDE procedure is the quantitative characterization of the experimental test system without a test specimen. The effects on the output signal of the ultrasonic transducers, the coupling of the transducers to the test specimen, and electronic components such as filters, amplifiers, attenuators, and cables in the experimental system must be characterized before the test specimen's effects on the output signal can be isolated.

This study is part of an overall effort to develop quantitative analyses of the SWF and computer-aided nondestructive evaluation (CANDE, pronounced "candy") capabilities. The experimental UT system is characterized by directly coupling the transmitting and receiving transducers face to face without a test specimen. Input and output signals are digitized with a digital oscilloscope and are processed with a microcomputer by using digital signal-processing techniques. The transfer function of the experimental UT system without any test specimen was obtained.

The results of this study should provide a useful example in the characterization of any UT system that has separate transmitting and receiving transducers. Furthermore, developments in CANDE should be facilitated by the digital signal-processing procedures summarized herein.

\section{FUNDAMENTALS OF DIGITAL SIGNAL PROCESSING}

A few results in digital signal processing are summarized in this section. Digital signal-processing techniques are discussed extensively in the literature (refs. 7 to 10). Primarily, the notations in reference 7 are followed in this outione.

Digital signal processing is concerned with the representation of signals by sequences of numbers and the processing of those sequences. Sequences correspond to discrete-time signals derived from sampling continuous-time signals. The notation $x(n)$ denotes a sequence of numbers whose entries depend on the independent parameter $n$. The sequence $x(n)$ is defined only for integer values of $n$ and represents successive samples of a continuoustime signal. For example, the sequence $x(n)$ is derived from periodic sampling of the continuous-time analog signal $x_{a}(t)$, where $t$ is time, according to

$$
x(n)=x_{a}(n T)
$$

where $T$ is the sampling period. The reciprocal of $T$ is the sampling rate or sampling frequency. The availability of high-speed digital computers and efficient signal-processing algorithms has accelerated the implementation of 
digital signal-processing techniques over that of continuous-time, analog signal-processing techniques. With the proper analysis, results from the digital signal processing of a sequence derived from sampling a continuous-time signal can accurately approximate results from continuous-time signal analyses.

A discrete-time system is defined mathematically as a unique transformation that maps an input sequence $x(n)$ into an output sequence $y(n)$. Discrete-time, linear, shift-invariant systems are discrete-time analogs of continuous-time, linear, time-invariant systems. Table 1 summarizes the inputoutput properties of discrete-time, linear and linear shift-invariant systems and their corresponding analogs in continuous-time systems. Discrete-time systems can be further characterized as stable and causal. A stable system is one for which every bounded input $x(n)$ produces a bounded output $y(n)$. A causal system is one for which the output $y(n)$ for $n$ equal to $n_{0}$ depends only on the input $x(n)$ for $n$ less than or equal to $n_{0}$. All subsequent discussions here are limited to discrete-time, linear, shift-invariant systems that are stable and causal.

The response of a linear, shift-invariant system can be characterized by the unit-sample response $h(n)$ via the convolution sum given in equation (1-1) in table 1. As a consequence, it can also be shown that the steady-state response of a linear, shift-invariant system to a sinusoidal input is sinusoidal of the same frequency as the input but with a magnitude and phase determined by the system. It is this property of linear, shift-invariant systems that makes representations of signals in terms of sinusoids or complex exponentials (i.e., Fourier representations) so useful in linear system theory. Also, it should be mentioned that the process of obtaining $y(n)$ from known $x(n)$ and $h(n)$ is called convolution, and the process of obtaining $h(n)$ from known $x(n)$ and $y(n)$ is called deconvolution.

Table 2 summarizes Fourier representations of continuous-time signals and of sequences. Fourier representations appear as equation pairs whose constituents are often referred to as the synthesis and analysis equations, as indicated in table 2. In equation $(2-1 b), x_{a}(j \Omega)$ is a continuous function in radian frequency $\Omega$ and is the Fourier transform of the continuous-time signal $x_{a}(t)$, where the symbol $j$ is defined as the square root of -1 . If $x_{a}(t)$ has units of voltage, $x_{a}(j \Omega)$ has units of voltage per radian frequency (or simply volt-second); $x_{a}(j \Omega)$ is in general complex and can be specified by its magnitude and phase.

If $x_{a}(t)$ is a real-valued function of time, the magnitude and phase of $x_{a}(j \Omega)$ wi11 be an even and odd function of frequency, respectively. Thus, it is only necessary to present the magnitude and phase of $x_{a}(j \Omega)$ for positive frequencies. If $x_{a}(t)$ is real and an even function of time, the phase of $x_{a}(j \Omega)$ is zero. If $x_{a}(t)$ is real and an odd function of time, the phase of $x_{a}(j \Omega)$ is $\pm \pi / 2$. If $x_{a}(t)$ is shifted (i.e., advanced or delayed) in time, the magnitude of $x_{a}(j \Omega)$ is not affected, but a phase linearly proportional to frequency (i.e., linear phase) is introduced in the phase of $X_{a}(j \Omega)$. More specifically, the phase (in radians) is proportional to the radian frequency with a proportionality constant having units of time. The negative of the value of the proportionality constant is the time delay (ref. 9). 
The remainder of table 2 deals with the Fourier representations of sequences. If properly computed, the fourier transform $x_{a}(j \Omega)$ can be approximated by the Fourier representation of a sequence derived from sampling $x_{a}(t)$. Examples of the Fourier representations of continuous-time signals and sequences are fllustrated in figure 1 and will be discussed shortiy.

In equation (2-2b) in table $2 x\left(e^{j \omega}\right)$ is a continuous function of radian frequency $\omega$ and is the (discrete time) Fourier transform of a sequence $x(n)$. The frequency $\omega$ is in units of radians per jncrement of $n$, which is simply radians. If $x(n)$ has units of voltage, $x\left(e^{j \omega}\right)$ has units of voltage per radian. The Fourier transform of a sequence is useful for analyzing general sequences. The discrete Fourier series representations of a periodic sequence $x(n)$ of period $N$ are also shown in table 2 . A periodic sequence $\widetilde{x}(n)$ of period $N$ is one such that $\widetilde{x}(n)$ equals $\widetilde{x}(n+N)$. In equation $(2-3 b) \widetilde{X}(k)$ is a periodic sequence of period $N$, and the numbers in this sequence are the discrete Fourier series coefficients of the periodic sequence $\widetilde{x}(n)$ having period $N$. If $\widetilde{x}(n)$ has units of voltage, $\widetilde{x}(k)$ has units of voltage also. The discrete Fourier series of a periodic sequence serves as a prelude to the discrete Fourter transform (DFT). In equation $(2-4 b) \quad X(k)$ is a sequence of length $N$ and is called the DFT of a finite-length sequence $x(n)$ of length $N$ (ref. 7). If $x(n)$ has units of voltage, $x(k)$ has units of voltage also. The DFT is useful for digital signal processing because sequences processed by a computer are of finite length and also because efficient DFT computational algorithms are available.

By comparing the entries in table 2 for the discrete Fourier series of a periodic sequence of period $N$ and the DFT of a finite-length sequence of length $N$, it is observed that the DFT representation is obtained from the discrete Fourier series representation by interpreting the finite-length sequence as one period of a periodic sequence. The properties of the DFT are similar to those of the Fourier transform, except that because of the implied periodicity, shifts of $x(n)$ in $n$ by one period $N$ and an integer multiple of one period are indistinguishable and a shift in $n$ of larger than $N$ is the same as a shorter shift.

The DFT was computed via a fast fourier transform (FFT) algorithm. The inverse DFT computed via an inverse FFT (IFFT) algorithm was used to evaluate $x(n)$ from $X(k)$. The FFT computation is particularly efficient when the length of the sequence is an integer power of 2 . A sequence of length $N$ is called an $N$-point sequence. The DFT of an $\mathrm{N}$-point sequence is called an $\mathrm{N}$-point DFT and is computed via an $\mathrm{N}$-point FFT algorithm. Figure 1 illustrates that the Fourier transform of a continuous-time signal can be reconstructed approximately from the DFT of a sequence derived from sampling the continuoustime signal. Again, note that where DFT relations are concerned, a finitelength sequence is represented as one period of a periodic sequence.

Figure 1 shows Fourier representations of continuous-time signals and of sequences derived from sampling the continuous-time signals. In part ( 1 ) of figure $1(a)$, the continuous-time signal $x_{a}(t)$ is assumed to be bandimited in frequency with Fourier representation $x_{a}(j \Omega)$ as shown. The highest frequency of $x_{a}(t)$ is assumed to be $\Omega_{0} / 2$. In part ( $\left.i j\right)$ of figure $1(a), a$ sequence $x(n)$ results from the periodic sampling of $x_{a}(t)$ with a sampling ing period of $T$. The fourier transform of the sequence $x(n)$ into a continuous function $x\left(e^{j \omega}\right)$ is also shown. It is observed that $x\left(e^{j \omega}\right)$ can be obtained from $x_{a}(j \Omega)$ by the superposition of an infinite number of $x_{a}(j \Omega)$ 
shifted in frequency $\omega$ by integer multiples of $2 \pi$. The magnitude and frequency of $x\left(e^{j \omega}\right)$ are scaled from those of $x_{a}(j \Omega)$ by $1 / T$ and $T$, respectively. The Fourier transform $x_{a}(j \Omega)$ can be recovered exactly, except for the scaling factors, from the Fourier transform $x\left(e^{j \omega} \omega\right)$ of the sequence $x(n)$ in the interval of $-\pi \leq \omega \leq \pi$ by low-pass filtering $x\left(e^{j \omega}\right)$ with a cutoff frequency of $\pi$, on one condition. The condition is that $\Omega_{0} T / 2<\pi$ so that there will be no overlapping of superposed $x_{a}(j \Omega)$ in forming $x\left(e^{j \omega} \omega\right)$. This condition is known as the "sampling theorem" and assures that if a continuoustime signal $x_{a}(t)$ is sampled at a frequency greater than twice the highest frequency of $x_{a}(j \Omega)$, then $x\left(e^{j \omega}\right)$ is identical to $x_{a}(j \Omega)$ in the interval $-\pi \leq \omega \leq \pi$, except for scaling factors. Because $x_{a}(j \Omega)$ is recovered, the continuous-time signal $x_{a}(t)$ can be recovered from the sequence $x(n)$. This minimum required sampling frequency is called the Nyquist rate or the Nyquist frequency (refs. 7, 9, and 10). The distortion due to overlapping of superposed $X_{a}(j \Omega)$ when the sampling theorem is violated is called aliasing. In part (ii) in figure 1(a), the case where there is no overlapping of superposed $X_{a}(j \Omega)$ is fllustrated; thus the signal is not aliased.

Whereas figure $1(\mathrm{a})$ deals with general continuous-time signals, figure $7(b)$ deals with finite-duration continuous-time signals. In part (i) in figure $1(b)$, a continuous-time signal $x_{a}(t)$ with nonzero values over a finite time duration and its Fourier representation $X_{a}(j \Omega)$ are shown. The duration where the signal is nonzero is assumed to be $t_{0}$, and sampling the continuous-time signal with a sampling period $T$ is assumed to result in a sequence of length $N$. It is observed that $x_{a}(j \Omega)$ for a finite-duration signal is not bandlimited in frequency. In part (ii) in figure $1(b)$, a sequence $x(n)$ results from the periodic sampling of $x_{a}(t)$ with a sampling period of T. All numbers in the sequence $x(n)$ are zero for $n<0$ and for $n>N-1$. Thus $x(n)$ is a finite-length sequence of length $N$. The Fourier transform of the sequence $x(n)$ into the continuous function $x\left(e^{j \omega}\right)$ is also shown. Similar to the results in figure $1(a), \quad x\left(e^{j \omega}\right)$ can be obtained from $X_{a}(j \Omega)$ by the superposition of an infinite number of $x_{a}(j \Omega)$ shifted in frequency $\omega$ by integer multiples of $2 \pi$. However, for this case, because $x_{a}(j \Omega)$ is not bandlimited in frequency, there is overlapping of superposed $x_{a}(j \Omega)$ in forming $x\left(e^{j \omega}\right)$. Thus there is aliasing and $x_{a}(j \Omega)$ cannot be recovered exactly from $X\left(e^{j \omega}\right)$ in the interval $-\pi \leq \omega \leq \pi$. However, if the sampling frequency is greater than twice most of the significant frequency components in $x_{a}(j \Omega)$, then $x_{a}(j \Omega)$ can still be approximated adequately by $X\left(e^{j \omega} \omega\right)$. The DFT $X(k)$ of the finite-length sequence $X(n)$ is often computed instead of $X\left(e^{j \omega}\right)$. However, in DFT evaluations, a finite-length sequence is represented as one period of a periodic sequence.

In part ( $11 i$ ) of figure $7(b)$, a periodic sequence $\widetilde{x}(n)$ of period $N$ constructed by using the $N$-point sequence $\widetilde{x}(n)$ as a period is shown. The discrete Fourier series representation $\widetilde{x}(k)$ of the periodic sequence $\tilde{x}(n)$ is also shown; $\widetilde{X}(k)$ is a periodic sequence of period $N$. The DFT $X(k)$ of the finite-length sequence $x(n)$ can be interpreted as a period of $\widetilde{x}(k)$ for $0 \leq k \leq N-1$ as shown. It is observed that $x\left(e^{j \omega}\right)$ can be obtained from $\widetilde{x}(\bar{k})$ by interpolating points in $\widetilde{x}(k)$. Because $x_{a}(j \Omega)$ can be approximated adequately by $x\left(e^{j \omega}\right)$ if the sampling frequency is greater than twice most of the significant frequency components in $x_{a}(j \Omega)$, interpolations of $\widetilde{x}(k)$ or $X(k)$ can be used to estimate $x_{a}(j \Omega)$. The numbers in $X(k)$ are scaled approximately from the values of $X_{a}(j \Omega)$ by a factor of $1 / T$, and the spacing between successive numbers in the sequence $x(k)$ can be interpreted as $2 \pi /(N T)$ 
in radian frequency, which is the frequency domain resolution. Again, the reconstruction of the Fourier transform of a finite-duration, continuous-time signal from the DFT of a finite-length sequence by interpolation is generally an approximation due to aliasing.

Table 3 lists the conditions for the special case where there is exact correspondence (to within a scaling factor) of the Fourier transform of a continuous-time signal and the DFT of a finite-length sequence derived from sampling (ref. 10). Also, the $N$-point sequence $x(n)$ can be considered as an M-point sequence if $M$ is greater than $N$ with the last $M-N$ numbers in the $M$-point sequence being zero. As discussed here next regarding convolution procedures, sometimes it is convenient to extend the length of a sequence by appending zeroes.

Because the response of a linear, shift-invariant system can be evaluated via the convolution sum in equation (i-1) in table 1 , convolution representations are discussed here. Table 4 summarizes convolution representations of continuous-time signals and of sequences. The Fourier representations of the convolutions are also given in table 4. Equation (4-1a) gives the linear convolution of continuous-time signals $x_{a 1}(t)$ and $x_{a_{2}}(t)$, resulting in $x_{a_{3}}(t)$. The Fourier representation of the convolution is given in equation (4-1b). Convolution in the time domain results in multiplication in the frequency domain. Conversely, because of the duality between time and frequency domains in Fourier representations, convolution in the frequency domain results in multiplication in the time domain, such as time windowing (ref. 9). The concepts of periodic and circular convolutions are introduced as preludes to the efficient evaluation of linear convolution of sequences using DFT procedures.

Equation (4-2a) in table 4 gives the periodic convolution of two periodic sequences $\widetilde{x}_{1}(n)$ and $\widetilde{x}_{2}(n)$, each of period $N$, resulting in a periodic sequence $\widetilde{x}_{3}(n)$ of period $N$. By restricting attention to one period of the periodic convolution, circular convolution of two $N$-point sequences $x_{1}(n)$ and $x_{2}(n)$ to obtain an N-point sequence $x_{3}(n)$ can be defined from the periodic convolution and is given in equation $(4-3 a)$. The two periodic sequences $\widetilde{x}_{1}(n)$ and $\widetilde{x}_{2}(n)$, each of period $N$, are constructed by using the $N$-point sequences $x_{7}(n)$ and $x_{2}(n)$ as periods, respectively. Equations (2-3b) and $(2-4 b)$ in table 2 have been used to obtain the simplified equation (4-3b) because $\tilde{x}_{7}(n)$ and $\widetilde{x}_{2}(n)$ are equal to $x_{1}(n)$ and $x_{2}(n)$ for $n$ ranging from zero to $N-1$. Thus $\widetilde{x}_{1}(k)$ and $\widetilde{x}_{2}(k)$ for $0 \leq k \leq N-1$ become $x_{1}(k)$ and $X_{2}(k)$, respectively. The circular convolution of $N$-point sequences is called an N-point circular convolution. The designation "circular" is derived from a graphical representation of how an $\mathrm{N}$-point sequence can be used to construct a periodic sequence of period $N$ (ref. 7 ). It can be imagined that the $N$ points from the $N$-point sequence are equally spaced in angle around a circle with a circumference of exactly $N$ points. The periodic sequence of period $N$ is obtained by traveling around the circumference of the circle a number at a time recording the $\mathrm{N}$-point sequence repeatediy. Also, a rotation of the circle corresponds to circular shifting of the $N$-point sequence (ref. 7 ).

The linear convolution of an $N_{1}$-point sequence $x_{7}(n)$ and an $N_{2}$-point sequence $x_{2}(n)$ is defined as

$$
x_{3}(n)=\sum_{m=0}^{N_{1}-1} x_{1}(m) x_{2}(n-m)
$$


Because the resulting sequence $x_{3}(n)$ is of length $N_{1}+N_{2}-1$ (ref. 7), the evaluation of linear convolution via circular convolution requires ali sequences to be of the same length, which has to be $N_{1}+N_{2}-1$. Equation $(4-4 a)$ in table 4 gives the linear convolution of finite-length sequences $x_{7}(n)$ and $x_{2}(n)$ to obtain a finite-length sequence $x_{3}(n)$ evaluated via circular convolution. Computation of circular convolution using DFT is preferred over the direct evaluation of linear convolution because of computational efficiencies. The $\left(N_{1}+N_{2}-1\right)$-point DFT's of the two convolving sequences are computed via an FFT algorithm and then multiplied according to equation (4-4b). The $\left(N_{1}+N_{2}-1\right)$-point inverse DFT of the product is computed via an IFFT algorithm, and the result is the desired linear convolution. Because the original sequences $x_{7}(n)$ and $x_{2}(n)$ are of lengths $N_{1}$ and $N_{2}$, respectively, $N_{2}-1$ and $N_{1}-1$ zeroes are appended to $x_{1}(n)$ and $x_{2}(n)$, respectively, to yield two $\left(N_{7}+N_{2}-1\right)$-point sequences for the $\left(\mathrm{N}_{1}+\mathrm{N}_{2}-1\right)$-point circular convolution. Alternatively, if linear convolution of two $\left(N_{1}+N_{2}-1\right)$-point sequences is conducted via an $\left(N_{1}+N_{2}-1\right)$ point circular convolution, the last $\mathrm{N}_{2}-1$ numbers in one sequence to be convolved must be zeroes and the last $N_{1}-1$ numbers in the other sequence to be convolved must be zeroes. In practice, the sequences are extended to a length that is greater than $N_{1}+N_{2}-1$ and is an integer power of 2 for efficient FFT computation.

Figure 2 shows convolution representation of continuous-time signals and their sequences derived by sampling. In figure 2(a), continuous-time signals $x_{a_{7}}(t)$ of duration $t_{1}$ and $x_{a_{2}}(t)$ of duration $t_{2}$ are convolved to form the continuous-time signal $x_{a 3}(t)$ of duration $t_{1}+t_{2}$. In part ( $i$ ) of figure $2(b)$, the continuous-time signal $x_{a l}(t)$ is sampled with a sampling period $T$ and is assumed to produce an $N_{7}$-point sequence. Then $N_{2}-1$ zeroes are appended to form an $\left(N_{1}+N_{2}-1\right)$-point sequence $x_{7}(n)$. Next a periodic sequence $\widetilde{x}_{1}(n)$ of period $N_{1}+N_{2}-1$ is constructed by using $x_{1}(n)$ as a period. Similarly, in part (ii) in figure $2(b)$, the periodic sequence $\tilde{x}_{2}(n)$ of period $N_{1}+N_{2}-1$ is obtained from $x_{a 2}(t)$, which is assumed to produce an $\mathrm{N}_{2}$-point sequence when sampled with a sampling period T. Periodic convolution of $\widetilde{x}_{7}(n)$ and $\widetilde{x}_{2}(n)$ results in the periodic sequence $\widetilde{x}_{3}(n)$. Then $x_{3}(n)$ can be identified from the circular convolution portion of $\widetilde{x}_{3}(n)$ by restricting attention to one period of the periodic sequence. It is observed that $x_{a_{3}}(t)$ can be approximated from the interpolation of the $\left(N_{1}+N_{2}-1\right)$-point sequence $x_{3}(n)$. The numbers in $x_{3}(n)$ are scaled approximately from the value of $x_{a 3}(t)$ by a factor of $1 / T$ and the spacing between successive numbers in the sequence $x_{3}(n)$ can be interpreted as T. Because the circular convolution is obtained via a DFT, restrictions on the DFT apply to the convolution also. Thus the sampling theorem applies to sampling the continuous-time signals if $x_{a 3}(t)$ is to be approximated adequately by the sequence $x_{3}(n)$.

In practice, if a continuous-time signal is to be sampled, the sampling frequency and the sampling length must be specified. Table 5 summarizes the significance of the sampling parameters. Equation (5-1) is based on the earlier discussion in relation to figure $1(a)$.

\section{EXPERIMENTAL SYSTEM}

A schematic of the experimental system including the specimen is shown in figure 3. This is a typical stress-wave-factor test configuration. The goal 
of this study was to characterize the experimental test system without the test specimen. Thus, all testing in this study was conducted with the transmitting and receiving transducers directly coupled face to face without any test specimen in between.

The system consisted of a pulse/function generator (Wavetek mode1 191); a 5-MHz (corresponding to -3-dB point) low-pass filter (Allen Avionics F2516); broadband $(0.1$ to $3.0 \mathrm{MHz}$ ) transmitting and receiving transducers (Parametrics VI05) having an approximately flat sensitivity of $-85 \mathrm{~dB}$ relative to $1 \mathrm{~V} / \mu \mathrm{bar}$; an uitrasonic interface couplant (Acoustic Emission Technology SC-6); an ultrasonic preamplifier (custom built by Parametrics) having a gain of $40 \mathrm{~dB}$ in the frequency bandwidth $1 \mathrm{kHz}$ to $10 \mathrm{MHz}$; a variable-frequency filter (A.P. Circuit Corporation AP 220-5), which could be used as a low-pass, high-pass, bandpass, or bandstop filter in the frequency range $10 \mathrm{~Hz}$ to $2.5 \mathrm{MHz}$; a digital oscilloscope (Nicolet model 2090 with plug-in model 204-A), which could sample and store analog signals at sampling frequencies from $0.05 \mathrm{~Hz}$ to $20 \mathrm{MHz}$ (corresponding to sampling periods from $20 \mathrm{sec}$ to $50 \mathrm{nsec}$ ) up to 4096 points; and an IBM personal computer (IBM PC), which was interfaced with the digital oscilloscope.

The variable-frequency filter was used as a 0.4 - to 2.6-MHz (corresponding to -3-dB points) bandpass filter, selected on the basis of the receiving transducer frequency response. All signals were sampled at a sampling frequency of $20 \mathrm{MHz}$ (i.e., one sample every $50 \mathrm{nsec}$ ) for 256 points. Thus each recorded signal was $12.8 \mu \mathrm{sec}$ in duration. The $5-\mathrm{MHz}$ low-pass filter was selected via antialiasing considerations. Table 6 is a BASIC language program listing for the FFT and IFFT computations used in this study. AII DFT and inverse DFT evaluations in this study were conducted by using this program.

\section{DIGITAL CHARACTERIZATION OF EXPERIMENTAL SYSTEM}

The objective of this study was to obtain the system transfer characteristics between the input to the transmitting transducer and the output from the receiving transducer after signal conditioning. A schematic of the experimental system is shown in figure 3 . Once again, note that the experiment was conducted with the transmitting and receiving transducers coupled face to face without a test specimen. Thus, the "system" under study consisted of the transmitting transducer, the coupling between transmitting and receiving transducers, the receiving transducer, the ultrasonic preamplifier, the variablefrequency filter, and all necessary cables.

A continuous-time system can be described by its impulse response $h_{a}(t)$ and its frequency response $H_{a}(j \Omega)$. In this study, the $h_{a}(t)$ and $H_{a}(j \Omega)$ of the test system were computed from the unit-sample response $h(n)$ and the DFT of $h(n)$ denoted $H(k)$, respectively, of a discrete-time model of the continuous-time system.

The experimental system was assumed to be a discrete-time, 1inear, shiftinvariant system. As shown in figure 3 , channel 1 of the digital oscilloscope sampled the continuous-time input signal to the transmitting transducer. The resulting discrete-time signal is called the input sequence and is denoted by $x(n)$. Similarly, channel 2 of the digital oscilloscope sampled the continuoustime output signal from the receiving transducer after preamplification and bandpass filtering. The resulting discrete-time signal is called the output 
sequence and is denoted by $y(n)$. Thus, the objective of this study was to obtain the discrete-time system transfer characteristics between $x(n)$ and $y(n)$. The discrete-time system besides being linear and shift-invariant was observed to be stable and causal. A schematic of the digital signal-processing procedures applied to obtain the unit-sample response and frequency response of the discrete-time system is shown in figure 4.

The pulse/function generator produced an approximately rectangular pulse $20 \mathrm{nsec}$ in duration. The rectangular pulse was passed through the $5 \mathrm{-MHz}$ lowpass filter before it reached the digital oscilloscope. Figure 5 shows the time histories of the input and output signals by linearly interpolating the defining points in the input and output sequences $x(n)$ and $y(n)$. The input and output signals were approximately 3 and $4 \mu \mathrm{sec}$ long, respectively. Thus for the sampling period $50 \mathrm{nsec}$, only approximately the first 60 and 80 numbers in the sequences $x(n)$ and $y(n)$, respectively, were nonzero. Actually, the sequences $x(n)$ and $y(n)$ were of length 256 after sampling; that is, the digital oscilloscope recorded 256 points each for the input and output signals. Then 768 zeroes were appended to the sequences to produce $x(n)$ and $y(n)$ of length 1024. Extended sequences were used to facilitate evaluation of linear convolution via the DFT, as discussed later. The DFT's $X(k)$ and $Y(k)$ for $x(n)$ and $y(n)$, respectively, were evaluated by using a 1024-point FFT algorithm based on 1024-point $x(n)$ and $y(n)$. Because the input and output signals were of finite duration, they were not bandlimited in frequency. However, because the sampling frequency (20 MHz) was greater than most of the significant frequency components in the input signal (significant up to $5 \mathrm{MHz}$ ) and the output signal (significant up to $2.6 \mathrm{MHz}$ ), the sampling theorem assured an adequate approximation of the Fourier transform of the input and output signals by the DFT of the input and output sequences. Approximations of the magnitude and phase of the Fourier transform of the input and output signals are shown in figure 5 . The magnitude and phase of the Fourier transform were obtained by linearly interpolating the defining points in the magnitude and phase of the DFT of the input and output sequences. The magnitude shown in figure 5 is presented on a decibel scale, normalized with respect to the magnitude of the largest component in the Fourier transform. The magnitudes of the largest components in the Fourier transforms of the input and output were $2.3 \times 10^{-7}$ and $9.3 \times 10^{-7} \mathrm{~V}-\mathrm{sec}$, corresponding to frequencies of 0.4 and $1.4 \mathrm{MHz}$, respectively. When the magnitude was small, both the magnitude and the phase were erratic because of sensitivity to noise contamination (fig. 5 ).

Letting the 1024-point DFT of the unit-sample response $h(n)$ of the discrete-time system be defined as $H(k)$, we can then use equation (4-4b) in table 4 to relate the DFT of the input $X(k)$ and the DFT of the output $Y(k)$ as

$$
Y(k)=H(k) X(k)
$$

where $0 \leq k \leq 1023$ for this case. Then $H(k)$ for the system can be evaluated by rearranging equation (3) as

$$
H(k)=Y(k) / X(k)
$$

The inverse DFT of $H(k)$ is the desired unit-sample response $h(n)$. The procedure for obtaining $h(n)$ from known $x(n)$ and $y(n)$ is called deconvolution. 
However, results from attempts to perform the direct division in equation (4), using $X(k)$ and $Y(k)$, as shown in figure 5 , were unsatisfactory because of noise. Thus, the output $y(n)$ was filtered digitally before division. The digital filter was selected by trial and error. Various digital filters were applied, and the resulting $H(k)$ and the unit-sample response $h(n)$ were used to evaluate output properties for various inputs and were compared with experimental results. The digital filter selected was a bandpass filter utilizing time windowing via a 701-point Blackman window (ref. 7). Specifically, the digital filter was obtained via the following steps: (1) obtain the unitsample response $h_{d}(n)$ of a desired ideal bandpass filter of 0.2 to $4.5 \mathrm{MHz}$, (2) shift $h_{d}(n)$ by 350 points to obtain the unit-sample response $h_{3}(n)$, and (3) multiply $h_{3}(n)$ by a 701-point Blackman window $w_{1}(n)$ to obtain a 701-point unit-sample response $h_{4}(n)$ of the filter. These steps are 111 ustrated in figure 4 also. The resulting digital filter was a causal, 1inearphase bandpass filter. Step 1 identified the desired bandpass filter. Because an ideal filter is noncausal, as indicated by its $h_{d}(n)$, which is nonzero for $n$ less than zero, step 2 shifted $h_{d}(n)$ by delaying it by 350 points. A causal filter is especially important for real-time signal processing.

Because the desired digital filter was a finite impulse response (FIR) filter, which by definition should have a unit-sample response of finite duration (ref. 7$), h_{3}(n)$ was truncated and only numbers between $n$ of zero and 701, inclusively, were retained. To minimize the effects of truncation, $h_{3}(n)$ was multiplied by a 701-point Blackman window $w_{1}(n)$ in step 3 to form $h_{4}(n)$. The length of 701 points minimized the effects of truncation and yet allowed a 1024-point DFT evaluation. The length of the sequence $h_{4}(n)$ of the filter affected the DFT evaluation because filtering in the frequency domain (i.e., multiplying the responses of the filter and the signal in the frequency domain) corresponded to convolution in the time domain. Because only the first 80 numbers in the sequence $y(n)$ were nonzero, proper evaluation of the linear convolution of the sequence $y(n)$ with the sequence $h_{4}(n)$, which was nonzero for the first 701 numbers, required at least a 780-point DFT, according to discussions associated with equation (4-4) in table 4. By increasing the number of points to the nearest integer power of 2 , which for this case was 1024, 1024 point sequences and 1024 DFT's resulted. Thus, sufficient zeroes were appended to any sequence to extend its length to 1024 points, unless otherwise indicated. Also, 1024-point DFT's evaluated via a 1024-point FFT were used, unless otherwise indicated.

The 1024 -point DFT of the unit-sample response of the digital bandpass filter designed by time windowing the unit-sample response of an ideal bandpass filter with a Blackman window is denoted by $\mathrm{H}_{2}(\mathrm{k})$. Using equation (4-.4b) in table 4 , the DFT of the output after digital filtering was $H_{2}(k) Y(k)$. Then equation (4) could be modified as

$$
H_{1}(k)=H_{2}(k) Y(k) / X(k)
$$

where $H_{1}(k)$ was an approximation to $H(k)$, and $0 \leq k \leq 1023$.

The unit-sample response $h_{1}(n)$ was obtained from $H_{7}(k)$ by performing an inverse DFT via an IFFT algorithm. Because $h_{j}(n)$ corresponded to $h_{a}(t)$, which is a real function of time, $h_{1}(n)$ was a sequence of real numbers and corresponded to the real part of the IFFT. Because a shift of 350 points (corresponding to $17.5 \mathrm{\mu sec}$ ) was introduced in the design of the causal, digital 
bandpass filter, the unit-sample response $h_{1}(n)$ had to be shifted back by 350 points to remove the artifact introduced by the digital filter. This was done after the rectangular windowing procedure discussed in the next paragraph.

The individual values in the sequence $h_{7}(n)$ for $n \leq 355$ and $n \geq 450$ were sma11, less than $1 / 100$ th of the maximum value in the sequence. These small values were due to noise contamination introduced during signal recording and also by the digital filtering. So, the sequence $h_{1}(n)$ was time windowed by a rectangular window $w_{2}(n)$ defined by

$$
w_{2}(n)=\left\{\begin{array}{l}
1 \text { for } 355<n<450 \\
0 \text { otherwise }
\end{array}\right.
$$

Thus, the numbers in $h_{1}(n)$ were not affected for $355<n<450$, but all others had been set to zero. The resulting sequence obtained by multiplying $h_{1}(n)$ and $w_{2}(n)$ is denoted as $h_{2}(n)$. This time windowing significantly smoothed the phase of $H_{7}(k)$ in the high-frequency region.

After the rectangular windowing, the sequence $h_{2}(n)$ was shifted back 350 points to remove the shift (i.e., time delay) introduced by the bandpass digital filter discussed earlier. The 1024-point sequence $h_{2}(n)$ was shifted to obtain $h(n)$ according to circular shifting, which in this case became

$$
h(n)= \begin{cases}h_{2}(n+350) & \text { for } 0 \leq n \leq 1023-350 \\ h_{2}(n-1024+350) & \text { for } 1024-350 \leq n \leq 1023 \\ 0 & \text { otherwise }\end{cases}
$$

The sequence $h(n)$ was the unit-sample response of the test system. The numbers in $h(n)$ were zero for $n \geq 100$ due to the windowing described in equation (6). Circular shifting was used instead of linear shifting because $h_{2}(n)$ was the result of a few DFT evaluations and, when a DFT evaluation was performed, a finite-length sequence was implied as a period of a periodic sequence. Thus, circular shifting procedures corresponding to shifting a periodic sequence were required.

The DFT of the unit-sample response $h(n)$ was $H(k)$. Because $h(n)$ was nonzero only for $<100$, the first 512 points of $h(n)$ were used to compute $H(k)$ via a 512-point FFT. Thus, $H(K)$ as obtained was a 512-point sequence; $H(k)$ was the frequency response of the discrete-time model of the test system.

Figure $6(a)$ shows the impulse response $h_{a}(t)$ of the test system as obtained by linearly interpolating the defining points in the unit-sample response $h(n)$. An impulse response has units of response per unit of excitation multiplied by time (ref. 11$)$. Thus, $h_{a}(t)$ in figure $6(a)$ is shown with units of volts per volt-second. The duration of the unit-sample response was approximately $3 \mathrm{\mu sec}$. Figure $6(\mathrm{~b})$ shows the magnitude and phase of the frequency response $\mathrm{H}_{\mathrm{a}}(j \Omega)$ of the test system as estimated by linearly interpolating the defining points in the frequency response $H(k)$. A frequency response magnitude has units of response per unit of excitation (ref. 11). Thus, the magnitude of $\mathrm{H}_{\mathrm{a}}(j \Omega)$ has units of volts per volt. The magnitude of $H_{a}(j \Omega)$ is shown in figure $6(c)$ on a decibel scale, normalized with respect to the magnitude of the largest component in $H_{a}(j \Omega)$, which is 
$2.7 \times 10^{-7} \mathrm{~V} / \mathrm{V}$ corresponding to a frequency of $1.7 \mathrm{MHz}$. The normalized magnitude had a maximum value at $1.7 \mathrm{MHz}$, and the values of the normalized magnitude decreased by $6 \mathrm{~dB}$ from the maximum value at 0.6 and $2.3 \mathrm{MHz}$. The phase of $\mathrm{H}_{\mathrm{a}}(\mathrm{j} \Omega)$ was linear from 0.3 to $2.7 \mathrm{MHz}$. The linear-phase behavior ind $1-$ cates that inputs of frequencies from 0.3 to $2.7 \mathrm{MHz}$ will result in outputs delayed in time without distortion. (However, distortion occurred because the magnitude of $\mathrm{H}_{\mathrm{a}}(j \Omega)$ was not constant from 0.3 to $2.7 \mathrm{MHz}$.) The introduced time delay corresponding to the linear phase can be computed to be $0.49 \mu \mathrm{sec}$. As shown by the magnitude plot, the system was insensitive to frequencies below $0.6 \mathrm{MHz}$ and above 2.3 $\mathrm{MHz}$; the information in these frequencies was easily contaminated by noise and also displayed the effects of digital filtering. Thus, to summarize, the system behaved as a linear-phase bandpass filter in the frequency range 0.6 to $2.3 \mathrm{MHz}$.

\section{RESULTS AND DISCUSSION}

The outputs of the test system due to different inputs can be predicted by using the unit-sample response $h(n)$ and frequency response $H(k)$ of the discrete-time model of the test system. If the input is $x(n)$ and the output is $y(n)$, the input-output relationship is given by the convolution in equation (1-1) in table 1. For finite-length sequences, equation (1-1) can be written in a similar form as equation (2). The convolution is conveniently evaluated via the DFT. If the DFT of the input is $X(k)$, the DFT of the output $Y(k)$ can be evaluated by using equation (3). So, the output $y(n)$ due to the input $x(n)$ is the inverse DFT of $Y(k)$. The frequency response $H(k)$ is shown in figure 6 . Figures 7 to 10 show the outputs of the system to various inputs. The time histories of the predicted outputs were obtained by linearly interpolating the defining points in the sequence $y(n)$ as obtained via the DFT procedure for 512-point DFT's.

Figures 7 and 8 show the outputs of the system to tone burst inputs of approximately $6 \mu \mathrm{sec}$ in duration and 1.5 and $2.5 \mathrm{MHz}$ in center frequency, respectively. The agreement between the experimentally measured and the predicted outputs was excellent. The arrival times of peaks of individual cycles were predicted to within $\pm 25 \mathrm{nsec}$ in both cases. The measured peak amplitudes of all the individual cycles were correctly predicted to within maximum errors of 5 and 9 percent for the 1.5 - and $2.5-\mathrm{MHz}$ inputs, respectively. Similar agreement was also obtained for center frequencies ranging from 0.5 to $3 \mathrm{MHz}$.

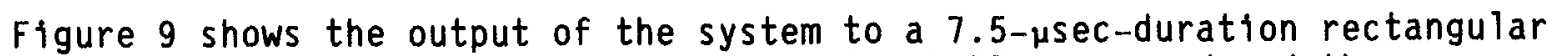
pulse input. The agreement between the experimentally measured and the predicted outputs was excellent. The arrival times of peaks of individual cycles were predicted to within $\pm 25 \mathrm{nsec}$. The measured peak amplitudes of the individual cycles were correctly predicted to within a maximum error of 5 percent. From figure 9, for the approximate time of 5 to $7 \mu \mathrm{sec}$, both the measured and the predicted outputs were constant in time. The constant values corresponded to 0 and $0.046 \mathrm{~V}$ for the measured and predicted outputs, respectively. Similar agreement was also obtained for pulse durations ranging down to $0.85 \mu \mathrm{sec}$. 
Figure 10 shows the output of the system to one cycle of $1.75-\mathrm{MHz}$ frequency input at $2.3 \mathrm{~V}$ peak to peak. The arrival times of peaks of individual cycles were predicted to within $\pm 25 \mathrm{nsec}$. The shapes of the measured and predicted outputs agreed we11, but the amplitude of the predicted output was somewhat larger than that of the measured output. As shown in figure 10, the predicted and measured peak-to-peak output amplitudes were 8.7 and $7.2 \mathrm{~V}$, respectively. The predicted output was larger than the measured output when the input and output exceeded 1.4 and $5.1 \mathrm{~V}$ peak to peak, respectively. This nonlinearity indicates that the linear model used in the prediction becomes inadequate for large-amplitude inputs and outputs. When the input and output were less than 1.4 and $5.1 \mathrm{~V}$ peak to peak, respectively, the measured output peak amplitudes were correctly predicted to within a maximum error of 6 percent. Restrictions on both input and output amplitudes were imposed because the nonlinearity may be due to the input or the output or both.

\section{CONCLUSIONS}

The SWF ultrasonic test system input-output characteristics were investigated by directiy coupling the transmitting and receiving transducers face to face without a test specimen. Some of the fundamentals of digital signal processing were summarized. Input and output signals were digitized by using a digital oscilloscope, and the digitized data were processed in a microcomputer by using digital signal-processing techniques.

The continuous-time test system was modeled as a discrete-time, linear, shift-invariant system. In estimating the unit-sample response and frequency response of the discrete-time system, it was necessary to use digital filtering to remove low-amplitude noise, which interfered with deconvolution calculations. A digital bandpass filter constructed with the assistance of a Blackman window and a rectangular time window were used. Approximations of the impulse response and the frequency response of the continuous-time test system were obtained by linearly interpolating the defining points of the unit-sample response and the frequency response of the discrete-time system. The test system behaved as a linear-phase bandpass filter in the frequency range 0.6 to $2.3 \mathrm{MHz}$. These frequencies were selected in accordance with the criterion that they were $6 \mathrm{~dB}$ below the maximum peak of the amplitude of the frequency response.

Furthermore, by using the unit-sample response and the frequency response of the discrete-time system and the assumption of a linear, shift-invariant system, the output of the system to various inputs was predicted and the results were compared with the corresponding measurements on the system. The predicted output was obtained by linearly interpolating the defining points of the discrete-time output sequence. Tone bursts of various center frequencies and durations, rectangular pulses of various durations, and angle cycle inputs at $1.75 \mathrm{MHz}$ with various amplitudes were considered. Excellent agreement between the predicted and measured results was obtained for tone bursts with center frequencies from 0.5 to $3 \mathrm{MHz}$ and rectangular pulses. Excellent agreement between the predicted and measured results was also obtained for one-cycle inputs of various amplitudes, as long as the input and output were less than $5.1 \mathrm{~V}$ peak to peak, respectively, at a $40-\mathrm{dB}$ system gain. These results are specific for the particular set of components in the test system. 
The results of this study will be useful in the quantitative analysis of the SWF when test specimens are inserted between the transducers. With known test system characteristics, the effects on the output signal due to the test specimen having a variety of flaw states can be isolated. Furthermore, using and discussing digital signal-processing methods extensively may facilitate developments in CANDE.

\section{REFERENCES}

1. J. Krautkramer and H. Krautkramer, Ultrasonic Testing of Materials, Second Edition, Springer-Verlag, N.Y., 1977.

2. A. Vary and R.F. Lark, "Correlation of Fiber Composite Tensile Strength with the Ultrasonic Stress Wave Factor", Journal of Testing and Evaluation, vo1. 7, no. 4, July 1979, pp. 185-191.

3. A. Vary and K.J. Bowles, "An Ultrasonic-Acoustic Technique for Nondestructive Evaluation of Fiber Composite Quality", Polymer Engineering and Science, vol. 19, no. 5, Apr. 1979, pp. 373-376.

4. J.H. Williams, Jr., and N.R. Lampert, "Ultrasonic Evaluation of Impact-Damaged Graphite Fiber Composite", Materials Evaluation, vol 38, no. 12, Dec. 1980, pp. 68-72.

5. J.H. Williams, Jr., H. Karagülle, and S.S. Lee, "Ultrasonic Input-Output for Transmitting and Receiving Longitudinal Transducers Coupled to Same Face of Isotropic Elastic Plate", Materials Evaluation, vol. 40, no. 6, May 1982, pp. 655-662.

6. H. Karagülle, "Ultrasonic Input-Output for Transmitting and Receiving Transducers Coupled to Same Face of Isotropic Elastic Plates", Doctoral Thesis Proposal, Submitted to the Department of Mechanical Engineering, Massachusetts Institute of Technology, Cambridge, Mass., Oct. 1982.

7. A.V. Oppenheim and R.W. Schafer, Digital Signal Processing, PrenticeHall, Inc., Englewood Cliffs, N.J., 1975.

8. S.D. Stearns, Digital Signal Analysis, Hayden Book Company, Inc., Rochelle Park, N.J., 1975.

9. A.V. Oppenheim, A.S. Willsky, with I.T. Young, Signals and Systems, Prentice-Ha11, Inc., Englewood Cliffs, N.J., 1983.

10. E.0. Brigham, The Fast Fourier Transform, Prentice-Ha11, Inc., Englewood Cliffs, N.J., 1974 .

11. S.H. Crandall and W.D. Mark, Random Vibration in Mechanical Systems, Academic Press, N.Y., 1963. 
TABLE 1 Input-Output Properties of Discrete-Time Linear, Shift-Invariant and Linear Shift-Invariant Systems and Their Corresponding Analogs in Continuous-Time Systems.

\begin{tabular}{|c|c|c|}
\hline $\begin{array}{c}\text { Discrete-Time } \\
\text { System }\end{array}$ & $\begin{array}{c}\text { System Input-Output } \\
\text { Properties }\end{array}$ & $\begin{array}{l}\text { Analog in } \\
\text { Continuous-Time } \\
\text { System }\end{array}$ \\
\hline Linear & $\begin{array}{l}\text { If } x_{1}(n) \rightarrow y_{1}(n) \\
\text { and } x_{2}(n) \rightarrow y_{2}(n), \\
\text { then } a_{1}(n)+b x_{2}(n) \\
\quad \rightarrow a_{1}(n)+b_{2}(n) \\
\text { (Principle of Superposition) }\end{array}$ & Linear \\
\hline Shift-Invariant & $\begin{array}{l}\text { If } x(n) \rightarrow y(n) \\
\text { then } x(n-k) \rightarrow y(n-k)\end{array}$ & Time-Invariant \\
\hline $\begin{array}{c}\text { Linear } \\
\text { Shift-Invariant }\end{array}$ & $\begin{array}{l}\text { If } \delta(n) \rightarrow h(n) \\
\text { then } x(n) \rightarrow y(n) \\
\text { where } y(n)=\sum_{k=-\infty}^{\infty} x(k) h(n-k) \\
\text { (Convolution Sum) }\end{array}$ & $\begin{array}{l}\text { Linear } \\
\text { Time-Invariant }\end{array}$ \\
\hline
\end{tabular}

$* \delta(n)$ is called the unit-sample sequence and is given by

$$
\delta(n)= \begin{cases}1, & n=0 \\ 0, & n \neq 0\end{cases}
$$

and $h(n)$ is called the unit-sample response. 
TABLE 2 Fourier Representations of Continuous-Time Signals and of sequences.

\begin{tabular}{|c|c|c|}
\hline Fourier Representation & Synthesis Equation & Analysis Equation \\
\hline $\begin{array}{l}\text { Fourier transform of } \\
\text { continuous-time } \\
\text { signal } x_{a}(t)\end{array}$ & $x_{a}(t)=\frac{1}{2 \pi} \int_{-\infty}^{\infty} x_{a}(j \Omega) e^{j \Omega t} d \Omega$ & $x_{a}(j \Omega)=\int_{-\infty}^{\infty} x_{a}(t) e^{-j \Omega t} d t$ \\
\hline $\begin{array}{l}\text { Fourier transform of } \\
\text { sequence } x(n) \text { into } \\
\text { the continuous } \\
\text { function } X\left(e^{j \omega}\right)\end{array}$ & $x(n)=\frac{I}{2 \pi} \int_{-\pi}^{\pi} x\left(e^{j \omega}\right) e^{j \omega n} d \omega$ & $x\left(e^{j \omega}\right)=\sum_{n=-\infty}^{\infty} x(n) e^{-j \omega n}$ \\
\hline $\begin{array}{l}\text { Discrete Fourier } \\
\text { series of periodic } \\
\text { sequence } \tilde{x}(n) \text { of } \\
\text { period N }\end{array}$ & $\tilde{x}(n)=\frac{1}{N} \sum_{k=0}^{N-1} \tilde{x}(k) e^{j k(2 \pi / N) n}$ & $\tilde{x}(k)=\sum_{n=0}^{N-1} \tilde{x}(n) e^{-j k(2 \pi / N) n}$ \\
\hline $\begin{array}{l}\text { Discrete Fourier } \\
\text { transform (DFT) } \\
\text { of finite-1ength } \\
\text { sequence } x(n) \text { of } \\
\text { length } N \text { into the } \\
\text { sequence } X(k) \text { of } \\
\text { length } N\end{array}$ & $\begin{array}{l}x(n)=\frac{1}{N} \sum_{k=0}^{N-I} x(k) e^{j k(2 \pi / N) n} \\
\quad \text { for } 0 \leq n \leq N-1 \\
x(n)=0, \text { otherwise. }\end{array}$ & $\begin{aligned} x(k)= & \sum_{n=0}^{N-1} x(n) e^{-j k(2 \pi / N) n}, \\
& \text { for } 0 \leq k \leq N-1 ; \\
x(k)= & 0, \text { otherwise. } \\
& (2-4 b)\end{aligned}$ \\
\hline
\end{tabular}

TABLE 3 Conditions for the Special Case Where There is Exact Correspondence (to within a Scaling Factor) of Fourier Transform of a Continuous-Time Signal and DFT of a Sequence Derived from Sampling [10].

\section{Conditions}

(1) The continuous-time signal $x_{a}(t)$ must be periodic.

(2) The frequency content of $x_{a}(t)$ must be bandlimited.

(3) The sampling frequency must be greater than twice the largest frequency component of $x_{a}(t)$.

(4) The sampling length must truncate $x_{a}(t)$ with a truncation interval exactly equal to one period (or integer multiple of one period) of $x_{B}(t)$. 
TABLE 4 Convolution Representations of Continuous-Time Signals and of Sequences.

\begin{tabular}{|c|c|c|}
\hline Convolution Representation & Definition & $\begin{array}{c}\text { Fourler Representation } \\
\text { of Convolution }\end{array}$ \\
\hline $\begin{array}{l}\text { Linear convolution of } \\
\text { continuous-time signals } \\
x_{a 1}(t) \text { and } x_{a 2}(t)\end{array}$ & $x_{a 3}(t)=\int_{-\infty}^{\infty} x_{a 1}(\tau) x_{a 2}(t-\tau) d \tau$ & $\begin{array}{r}x_{a 3}(j \Omega)=x_{a 1}(j \Omega) x_{a 2}(j \Omega) \\
(4-1 b)\end{array}$ \\
\hline $\begin{array}{l}\text { Periodic convolution of } \\
\text { periodic sequences } \\
\tilde{x}_{1}(n) \text { and } \tilde{x}_{2}(n) \text {, each } \\
\text { of period } N^{2}\end{array}$ & $\tilde{x}_{3}(n)=\sum_{m=0}^{N-1} \tilde{x}_{1}(m) \tilde{x}_{2}(n-m)$ & $\begin{aligned} \tilde{x}_{3}(k)=\tilde{x}_{1}(k) \tilde{x}_{2}(k) \\
(4-2 b)\end{aligned}$ \\
\hline $\begin{array}{l}\text { Circular convolution of } \\
\text { finite-length sequences } \\
x_{1}(n) \text { and } x_{2}(n) \text {, each of } \\
\text { length } N \text {. The sequences } \\
\text { are represented as } \\
\text { periods of periodic } \\
\text { sequences } \tilde{x}_{1}(n) \text { and } \\
\tilde{x}_{2}(n) \text {, each of period } N .\end{array}$ & $\begin{aligned} & x_{3}(n)= \sum_{m=0}^{N-1} \tilde{x}_{1}(m) \tilde{x}_{2}(n-m) \\
& \text { for } 0 \leq n \leq N-1 \\
& x_{3}(n)= 0, \text { otherwise. } \\
& \quad(4-3 a)\end{aligned}$ & $\begin{array}{l}x_{3}(k)=\tilde{x}_{1}(k) \tilde{x}_{2}(k) \\
\quad \text { for } 0 \leq k \leq N-1 \\
x_{3}(k)=0 \text {, otherwise. } \\
\text { This is same as: } \\
x_{3}(k)=x_{1}(k) x_{2}(k) \\
\quad(4-3 b)\end{array}$ \\
\hline $\begin{array}{l}\text { Linear convolution via } \\
\text { circular convolution of } \\
\text { finite-length sequences } \\
x_{1}(n) \text { with length } N_{1} \\
\text { and } x_{2}(n) \text { with length } N_{2} \text {. } \\
\text { The length of each } \\
\text { sequence is fncreased to } \\
\mathrm{N}_{1}+\mathrm{N}_{2}-1 \text { by appending } \\
\text { sufficient number of } \\
\text { zeroes to each sequence. } \\
\text { The resulting sequences } \\
\text { are represented as } \\
\text { periods of periodic } \\
\text { sequences } \tilde{x}_{1}(\mathrm{n}) \text { and } \\
\tilde{\mathrm{x}}_{2}(\mathrm{n}) \text { each of period } \\
\mathrm{N}_{1}+\mathrm{N}_{2}-1\end{array}$ & $\begin{array}{l}x_{3}(n)=\sum_{m=0}^{N_{I}+N_{2}-2} \tilde{x}_{1}(m) \tilde{x}_{2}(n-m) \\
\quad \text { for } 0 \leq n \leq N_{1}+N_{2}-2 \\
x_{3}(n)=0, \text { otherwise. }\end{array}$ & $\begin{array}{r}x_{3}(k)=x_{1}(k) x_{2}(k) \\
\text { for } 0 \leq k \leq N_{1}+N_{2}-2 \\
x_{3}(k)=0, \text { otherwise. } \\
(4-4 b)\end{array}$ \\
\hline
\end{tabular}


TABLE 5 Significance of Sampling Parameters for Deriving a Sequence from Sampling a Continuous-Time Signal.

\begin{tabular}{|c|c|c|}
\hline Sampling Parameter & Significance & Relationship \\
\hline $\begin{array}{l}\text { Sampling frequency } 1 / T \text {, } \\
\text { where } T \text { is sampling period } \\
\text { in sec and } 1 / T \text { is frequency } \\
\text { in } \mathrm{Hz} \text {. }\end{array}$ & $\begin{array}{l}\text { To avold distortion } \\
\text { due to aliasing, the } \\
\text { sampling frequency } \\
\text { must be larger than } \\
\text { twice the highest } \\
\text { frequency component } \\
\text { contained in the con- } \\
\text { tinuous-time signal. }\end{array}$ & $\begin{array}{l}\quad \frac{\Omega_{0}}{2}<\frac{\pi}{\mathrm{T}}(5-1) \\
\text { where } \Omega_{0} / 2 \text { is the } \\
\text { maximum radian } \\
\text { frequency of the } \\
\text { continuous-time } \\
\text { signal. }\end{array}$ \\
\hline \multirow[t]{3}{*}{$\begin{array}{l}\text { Sampling length* }{ }^{*} \text {, } \\
\text { where } \mathrm{N} \text { is length of the } \\
\text { sequence derived from } \\
\text { sampling and } \mathrm{T} \text { is } \\
\text { sampling period in sec. }\end{array}$} & $\begin{array}{l}\text { To improve DFT fre- } \\
\text { quency domain } \\
\text { resolution, the } \\
\text { sampling length must } \\
\text { be increased. }\end{array}$ & $\begin{array}{l}\text { Frequency } \\
\text { resolution }(\mathrm{Hz}) \\
=1 /(\mathrm{NT}) \\
(5-2)\end{array}$ \\
\hline & $\begin{array}{l}\text { To avoid distortion } \\
\text { due to "leakage" } \\
{[23,25] \text { resulting }} \\
\text { from truncating the } \\
\text { signal, the sampling } \\
\text { length must be } \\
\text { sufficient to totally } \\
\text { contain the signal** }\end{array}$ & $\begin{array}{r}\mathrm{NT} \geq \text { Time duration } \\
\text { of signal } \\
(5-3)\end{array}$ \\
\hline & $\begin{array}{l}\text { To utilize the best } \\
\text { efficiency of FFT } \\
\text { computation, N should } \\
\text { be an integer power } \\
\text { of } 2 \text {. }\end{array}$ & $\begin{array}{l}\quad N=2^{v} \quad(5-4) \\
\text { where } v \text { is an integer. }\end{array}$ \\
\hline
\end{tabular}

* If the sequence is to encounter a linear convolution evaluation using circular convolution via a FFT algorithm, appended zeroes may be required to extend the length of the sequence.

** If the signal is perfodic, the sampling length should be equal to the period or an integer multiple of the period. If truncation can not be avoided, the truncation effects should be minimized by smoothing the signal by proper time windowing [7-10]. 
TABLE 6 Program Listing for Microcomputer-Based N-Point FFT and IFFT Computations in BASIC when $\mathrm{N}$ is an Integer Multiple of 2 .

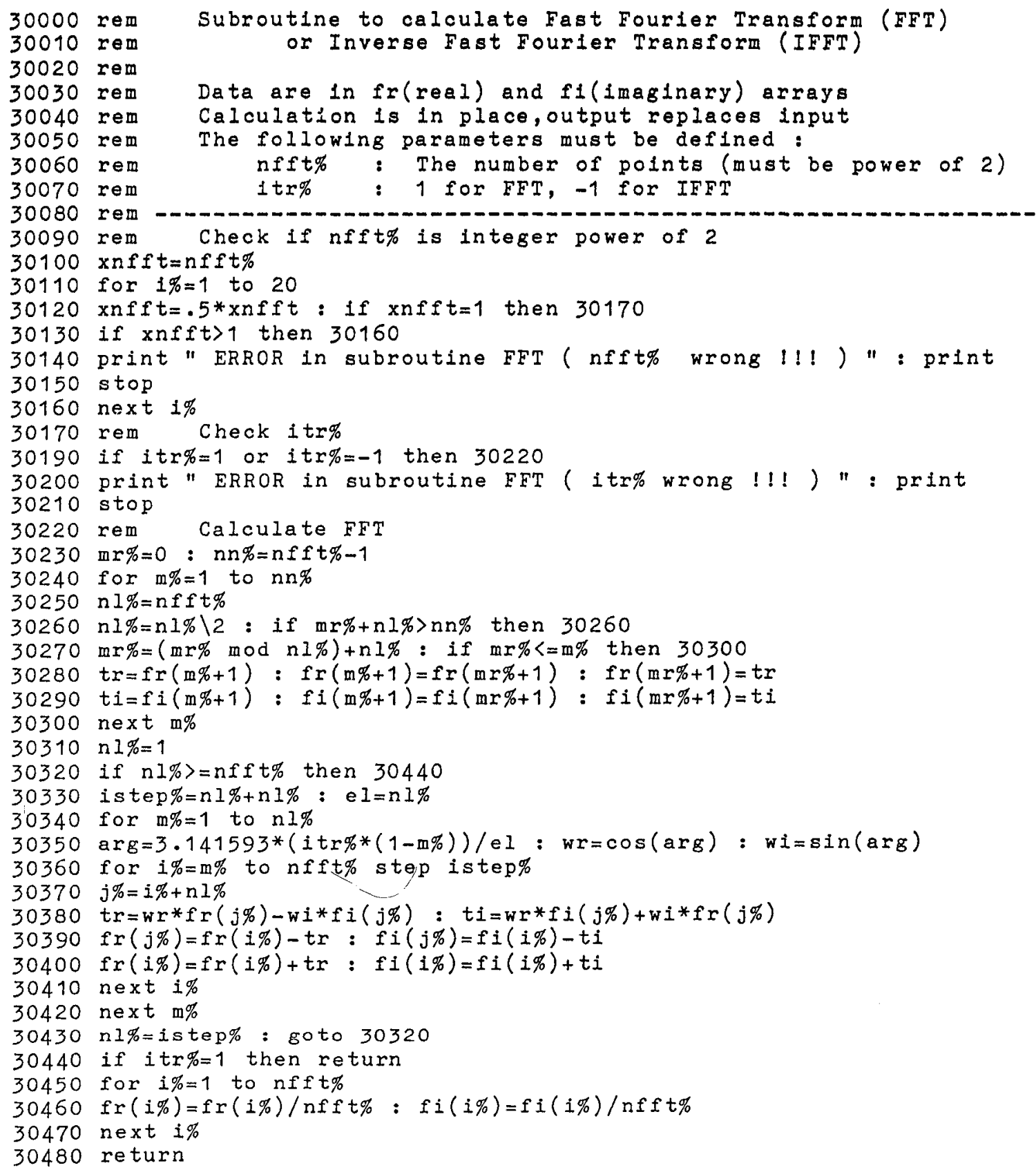


(i)
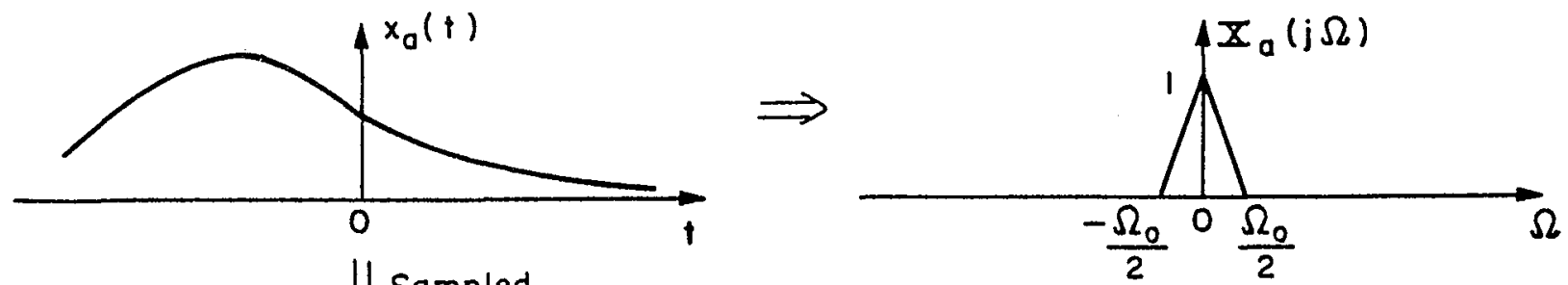

(ii)
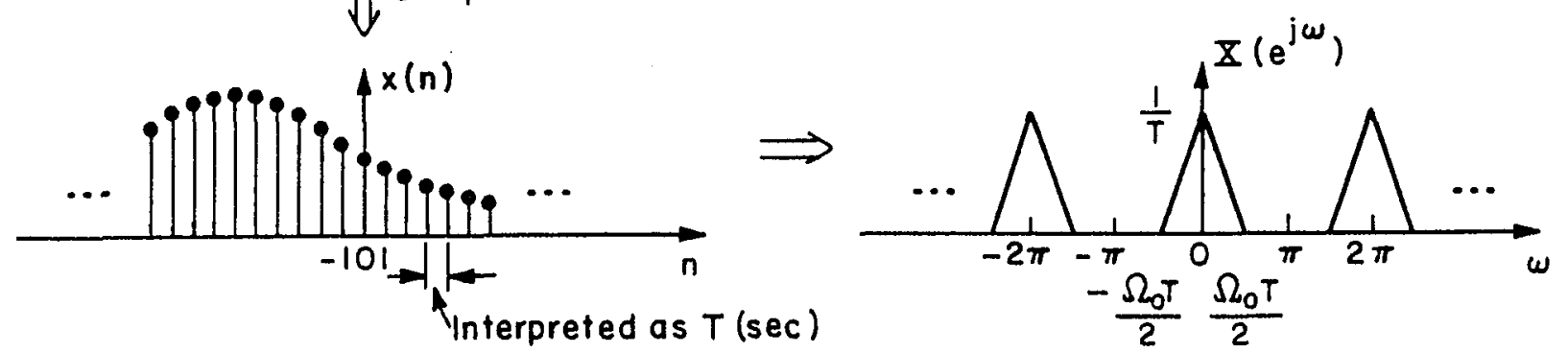

(a)

(i)
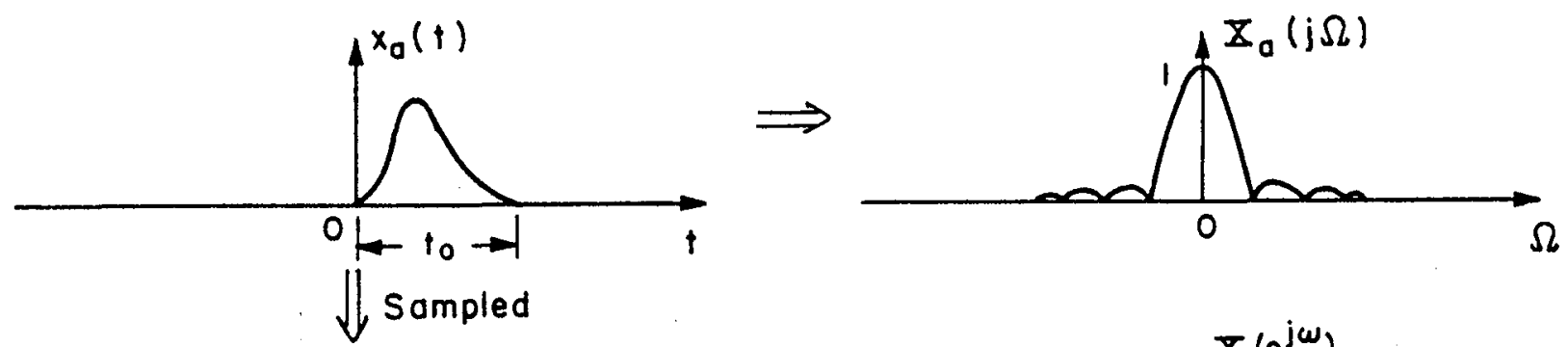

(ii)
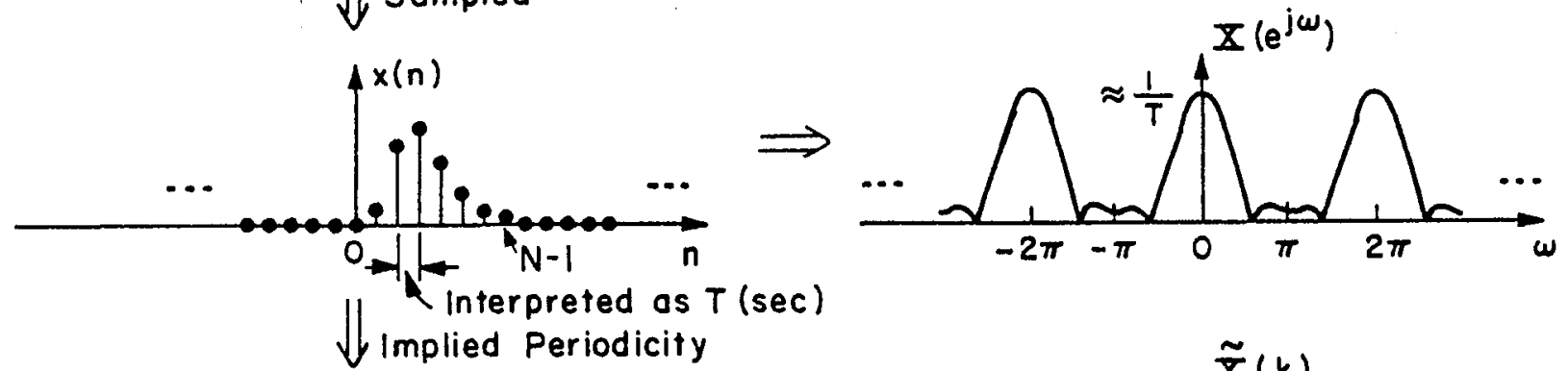

(iii)

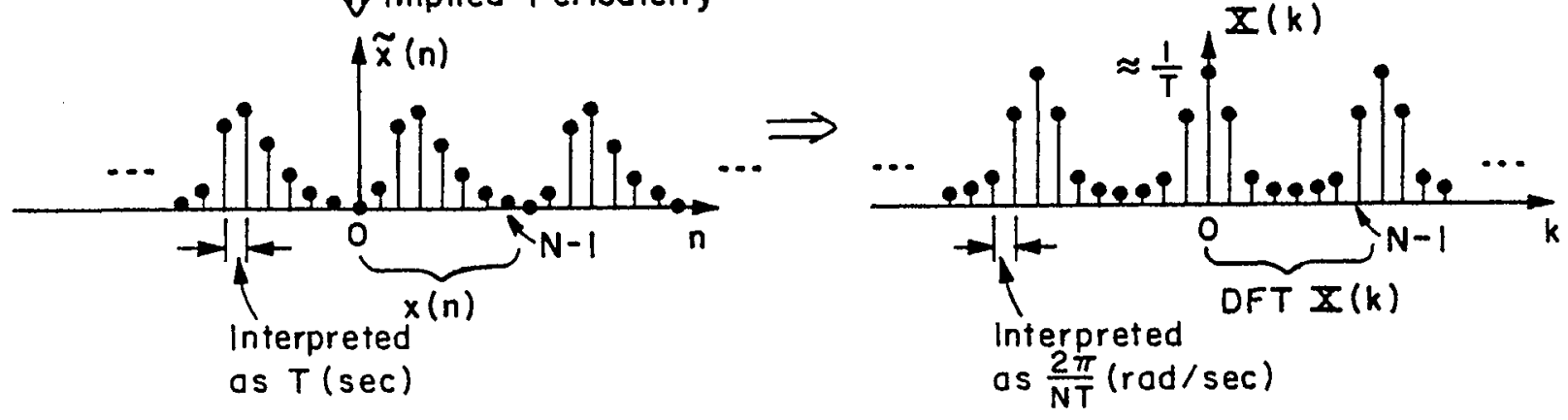

(b)

Fig. I Fourier representations of (a) continuous-time signal and sequence derived from sampling, and of (b) finite-duration continuous-time signal, sequence derived from sampling and the sequence with implied periodicity. 
(i)

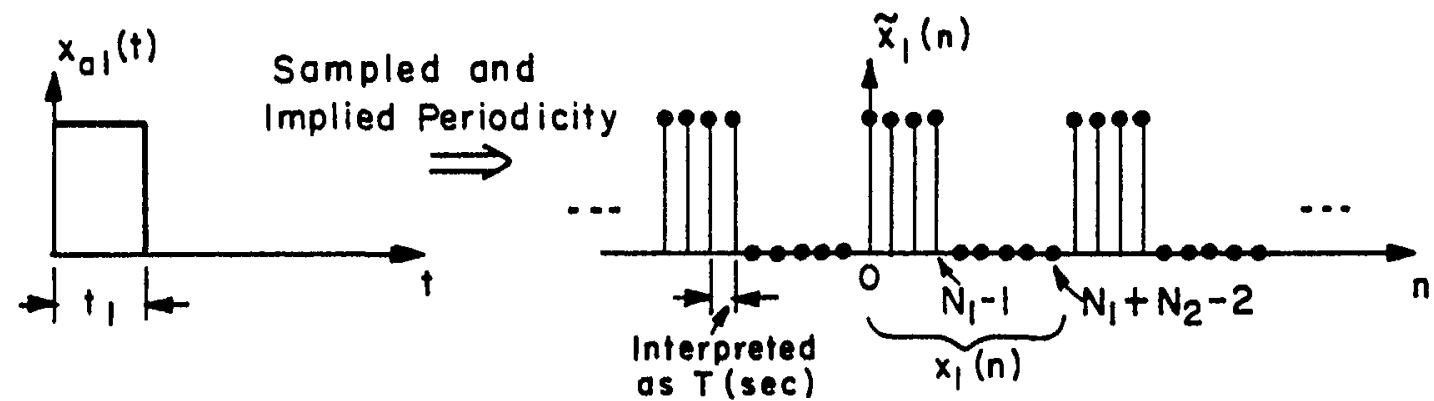

(ii)

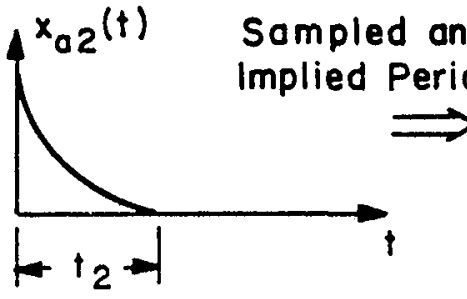

$\Downarrow \begin{aligned} & \text { Linear } \\ & \text { Convolution }\end{aligned}$

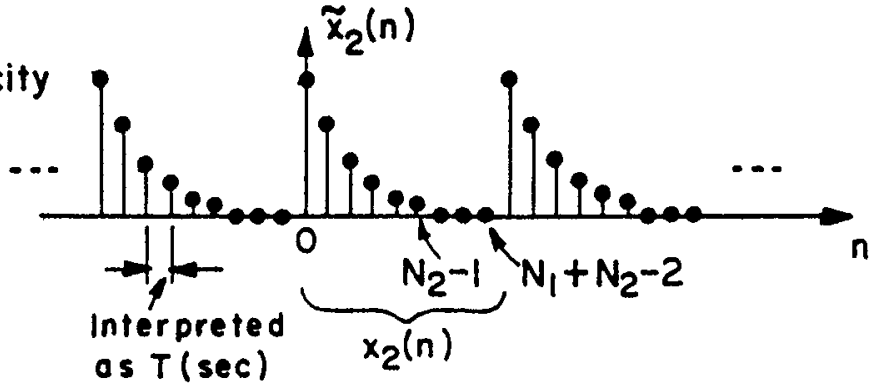

(iii)
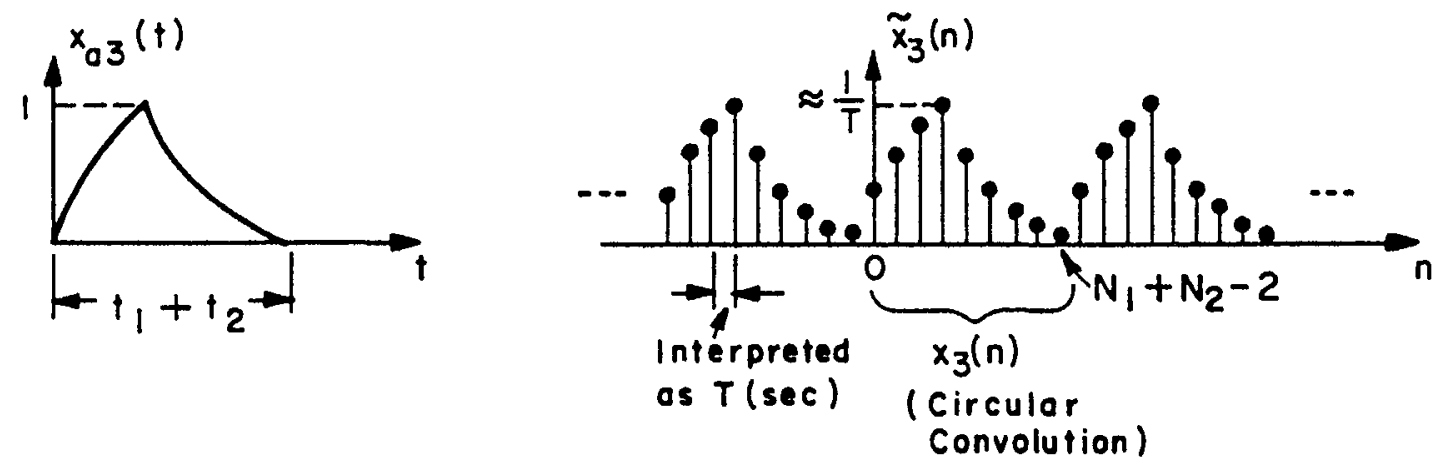

(a)

(b)

Fig. 2 (a) Linear convolution of continuous-time signals and (b) circular convolution of sequences derived from sampling the signals. 


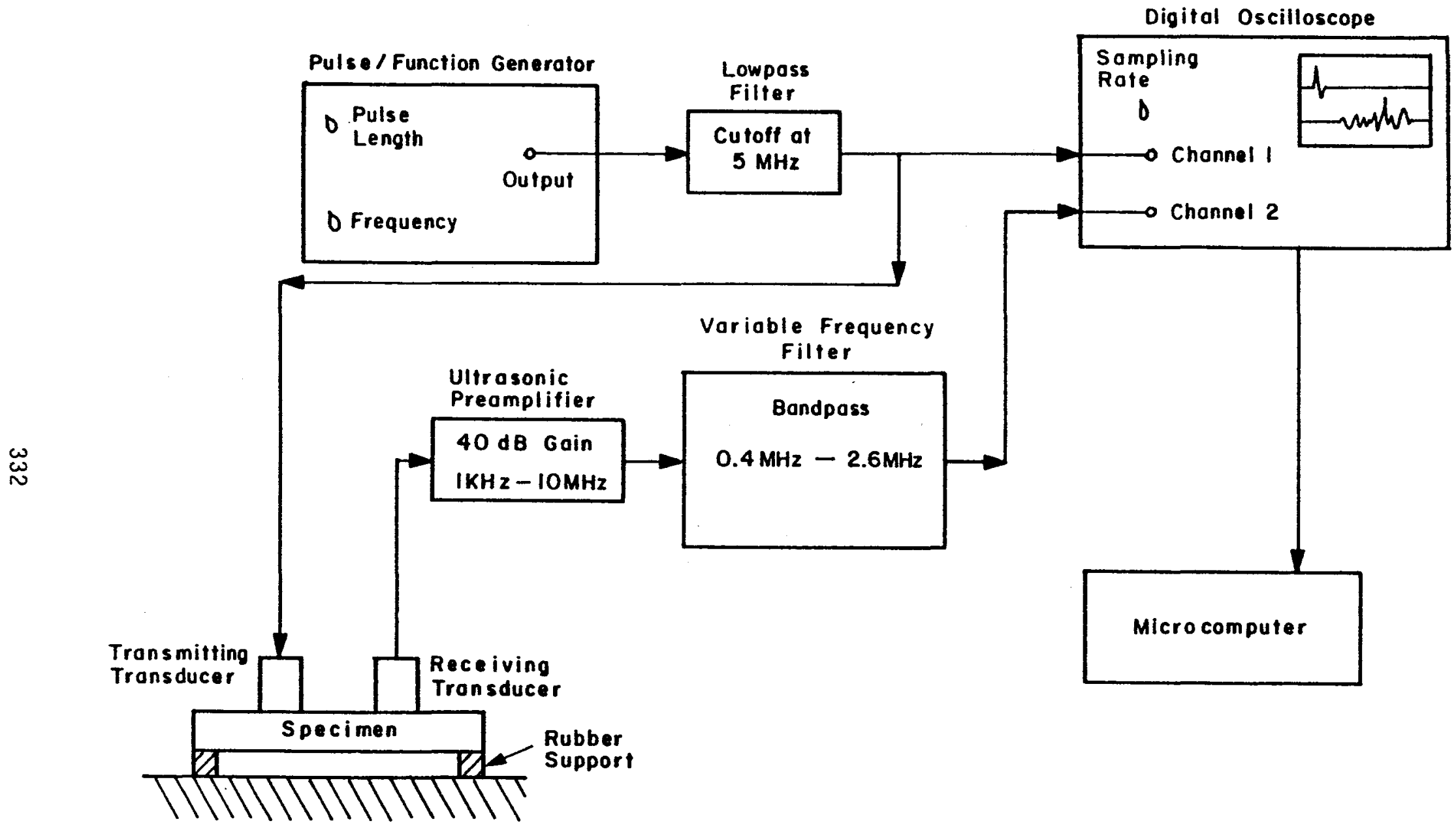

Fig. 3 Schematic of experimental components with specimen showing the "stress wave factor" test configuration. (All tests in this study were conducted with transducers coupled face to face without a test specimen.) 


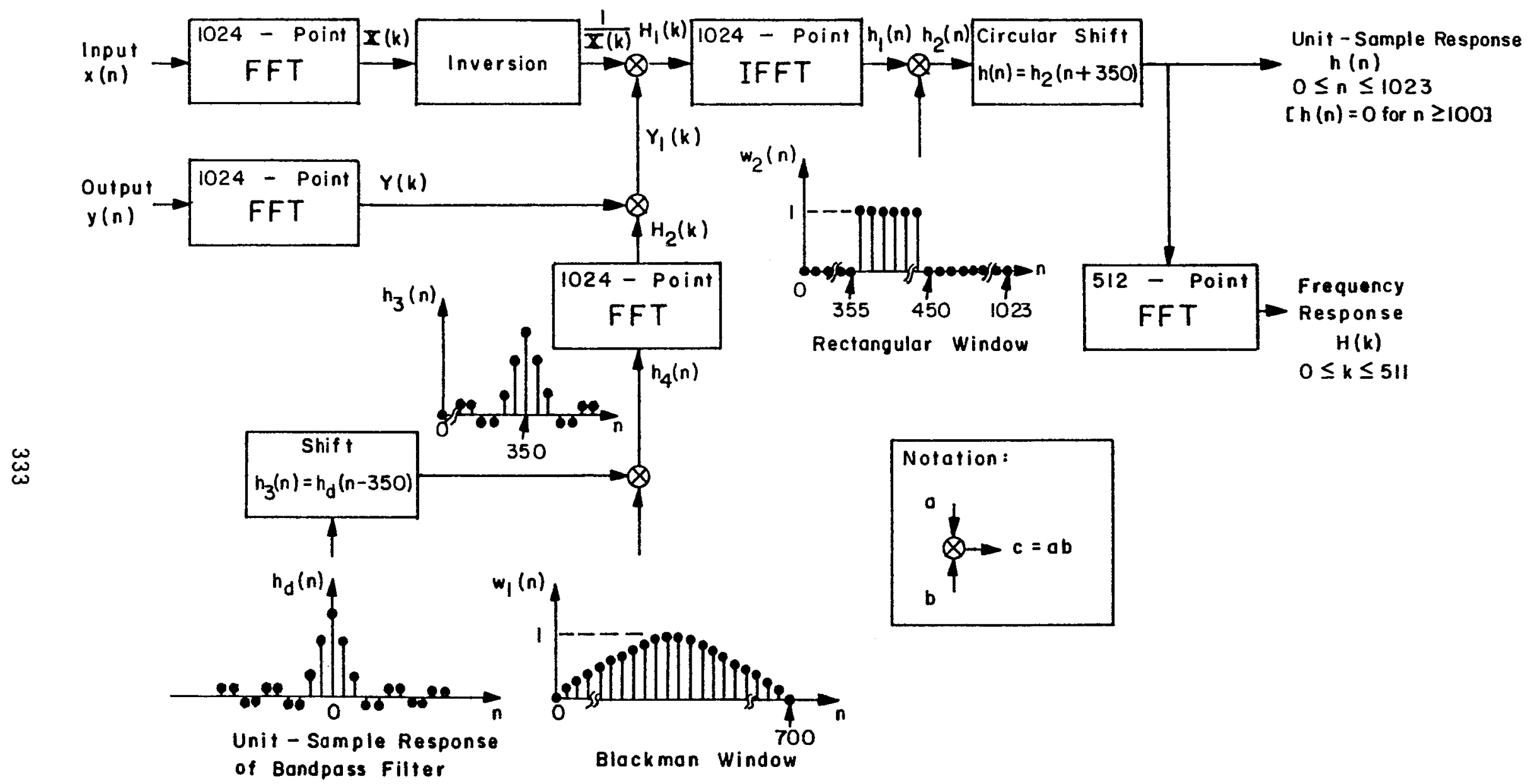

Fig. 4 Schematic of digital signal processing procedures applied to experimental input and output sequences to evaluate unit-sample response and frequency response of experimental system. 

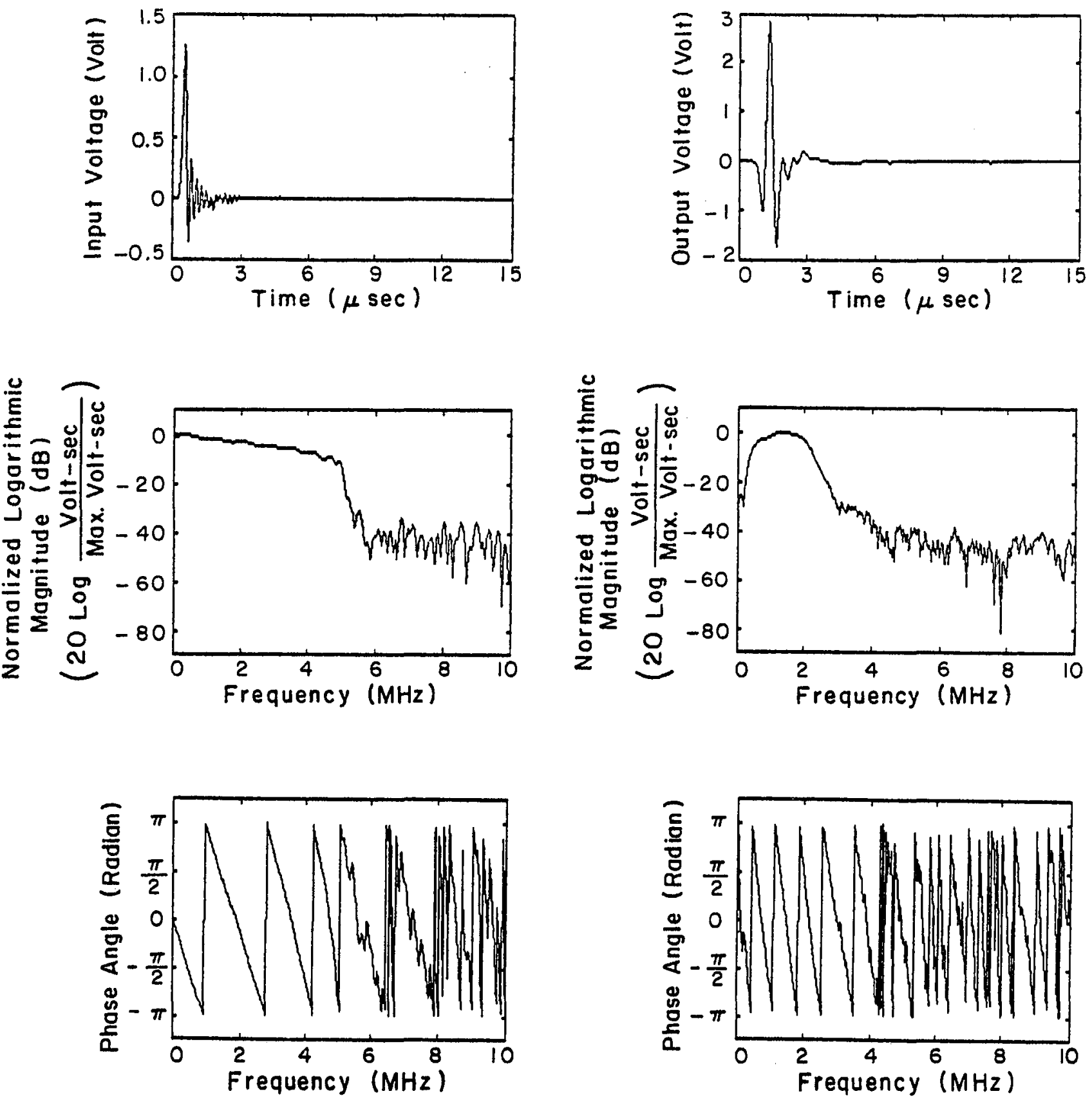

(a)

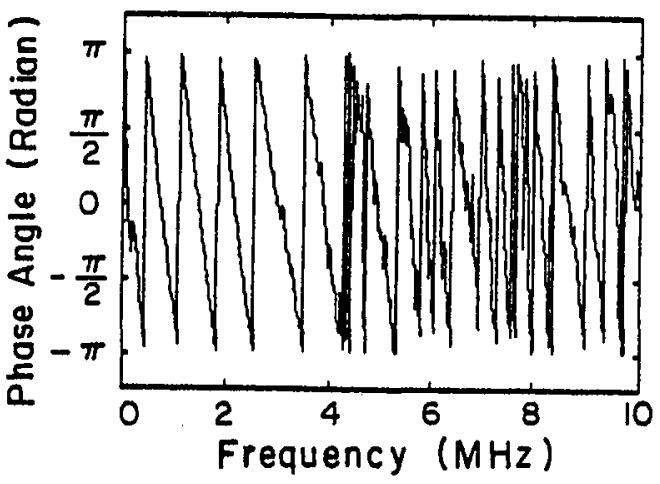

(b)

Fig. 5 Time history, magnitude and phase of (a) input signal and (b) output signal. 


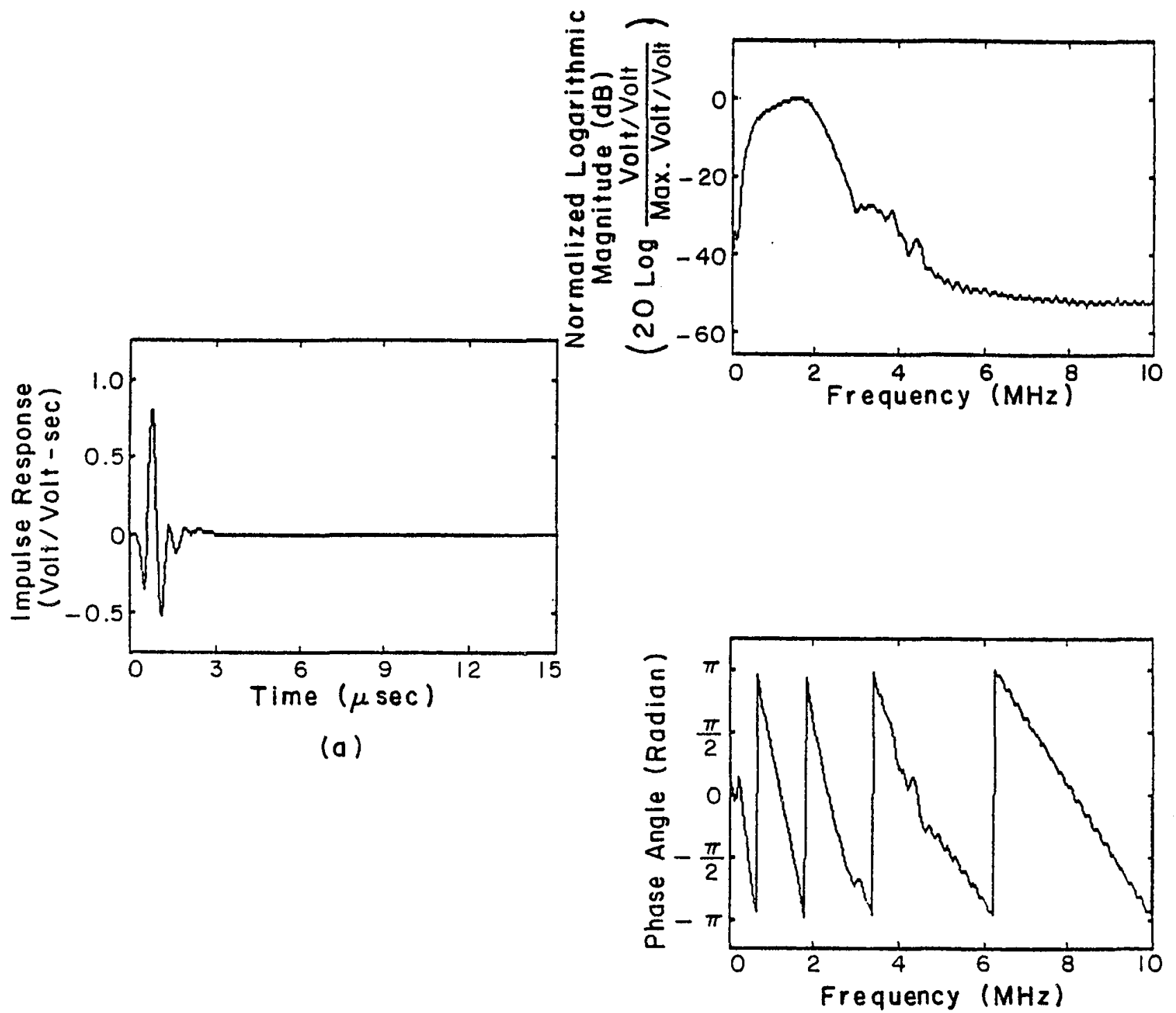

(b)

Fig. 6 Experimentally determined (a) impulse response and (b) frequency response of test system. 


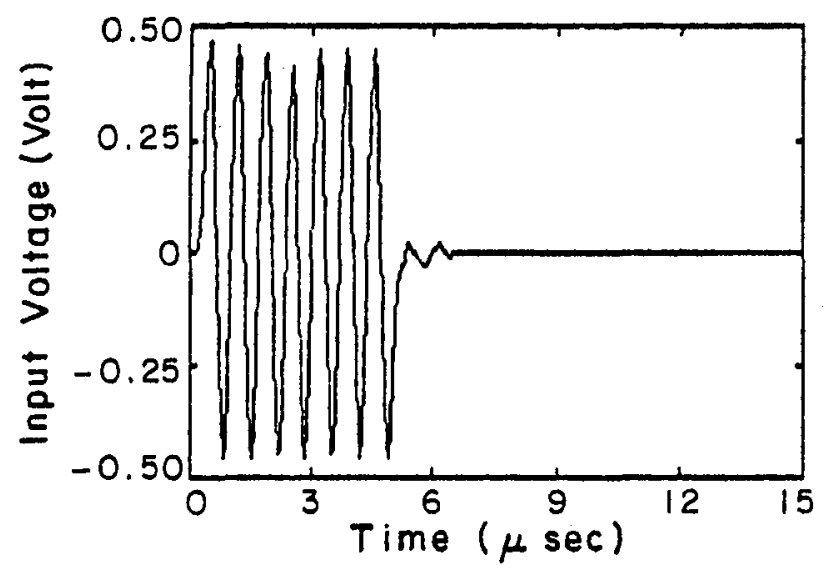

(a)

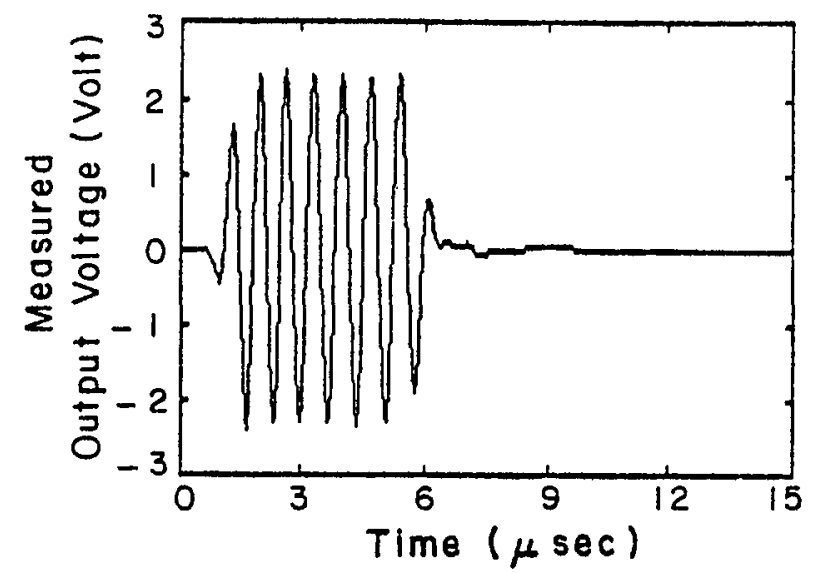

(b)

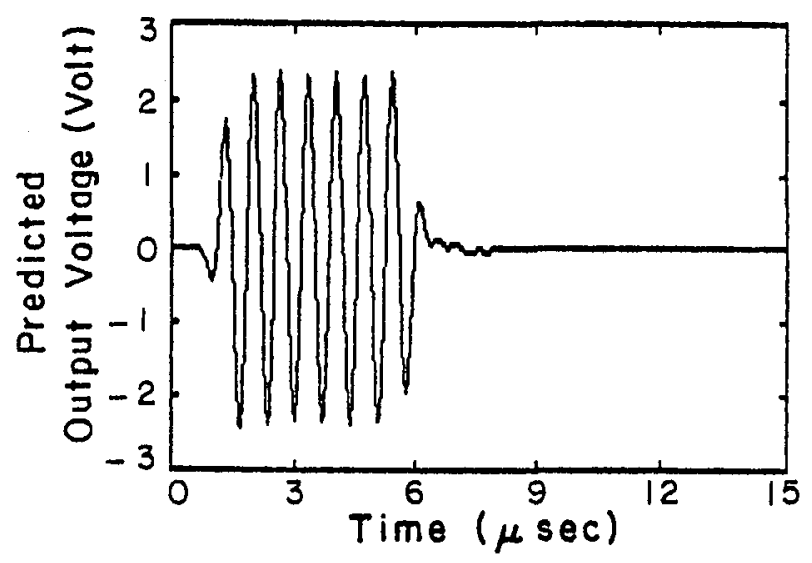

(c)

Fig. 7 Heasured and predicted system responses: (a) $1.5 \mathrm{MHz}$ center frequency tone burst input and corresponding (b) experimental output and (c) predicted output. 


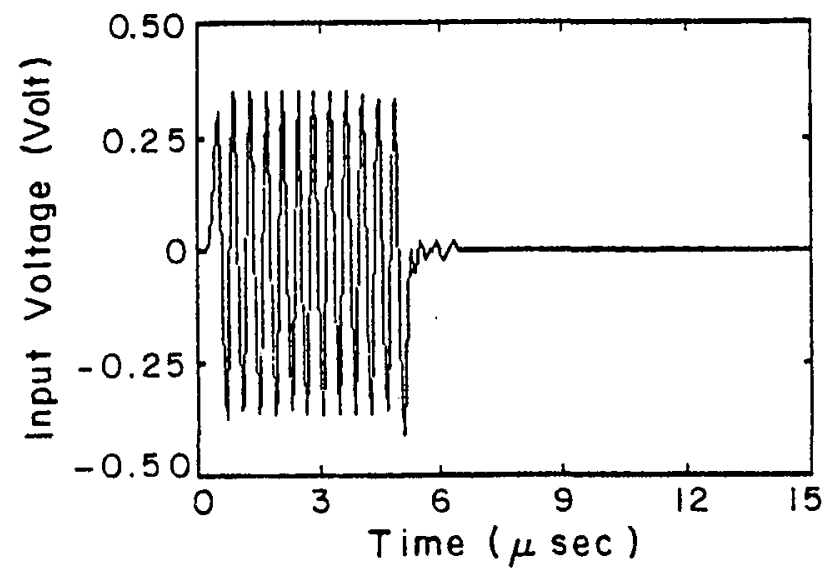

(a)

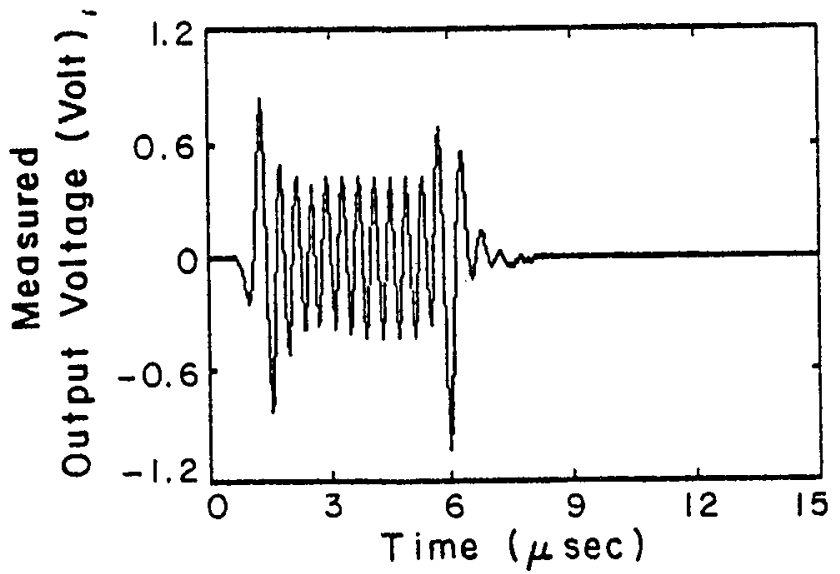

(b)

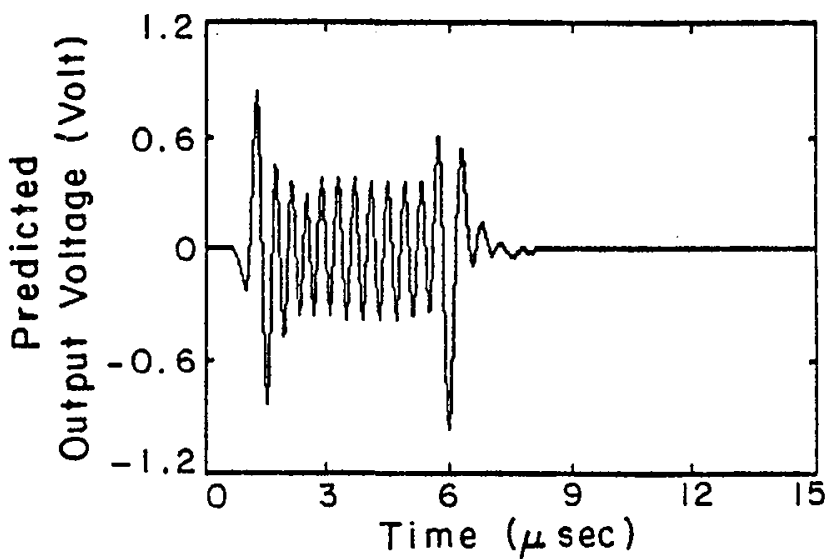

(c)

Fig. 8 Measured and predicted system responses: (a) $2.5 \mathrm{MHz}$ center frequency tone burst input and corresponding (b) experimental output and (c) predicted output. 


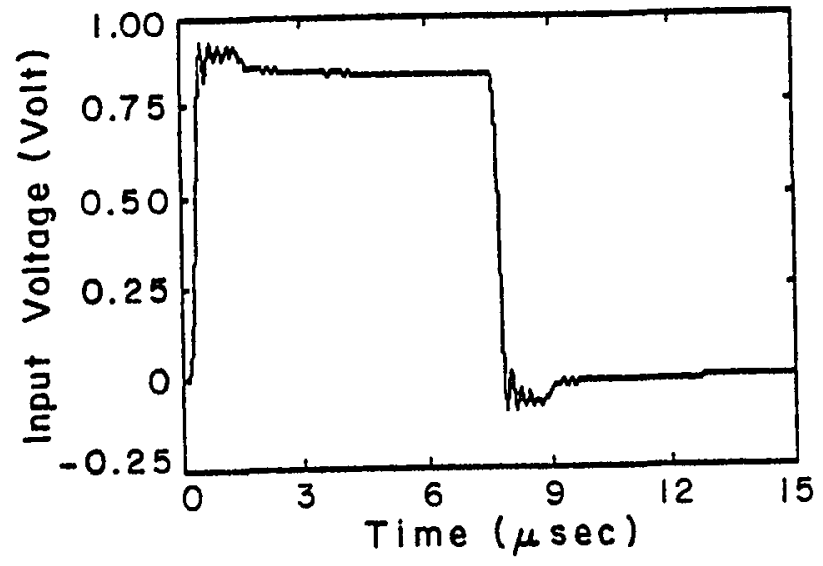

(a)

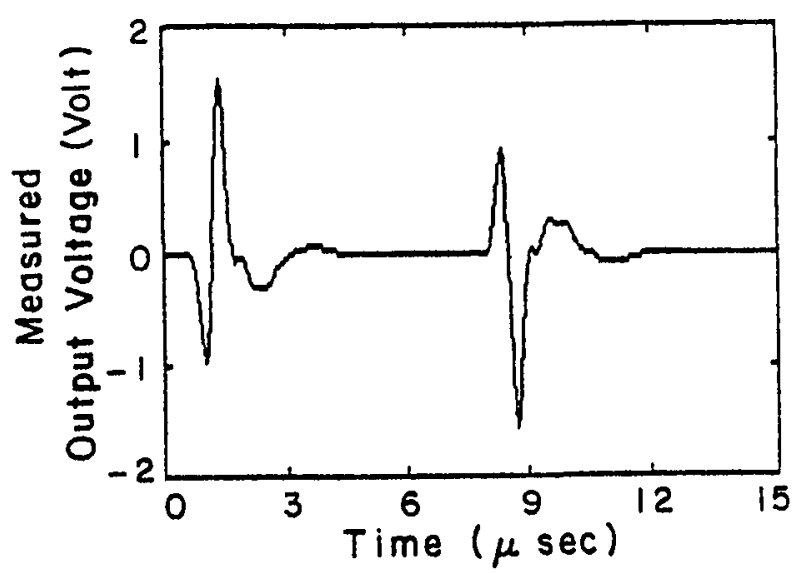

(b)

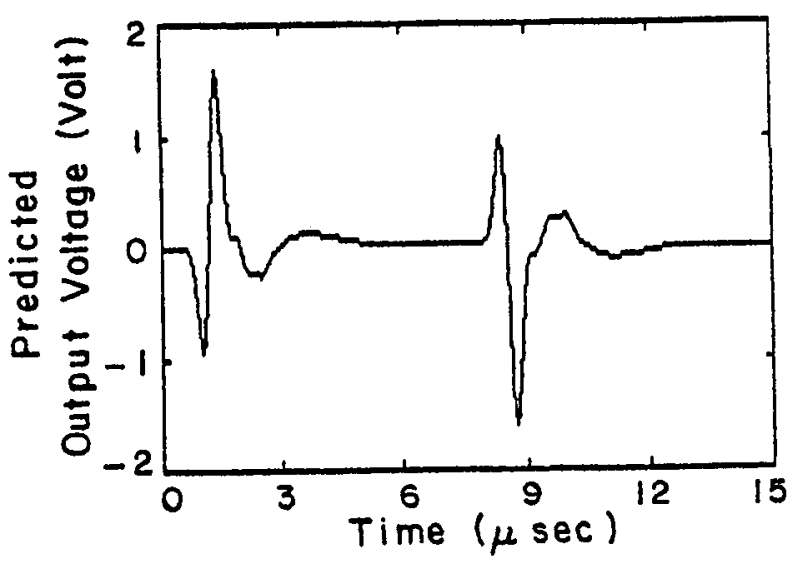

(c)

Fig. 9 Measured and predicted system responses: (a) $7.65 \mathrm{\mu sec}$ duration rectangular pulse input and corresponding (b) experimental output and (c) predicted output. 


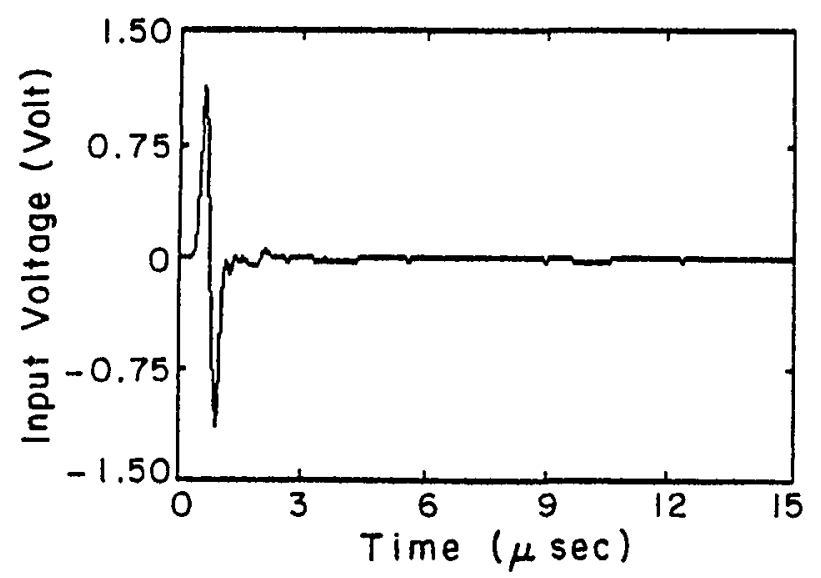

(a)

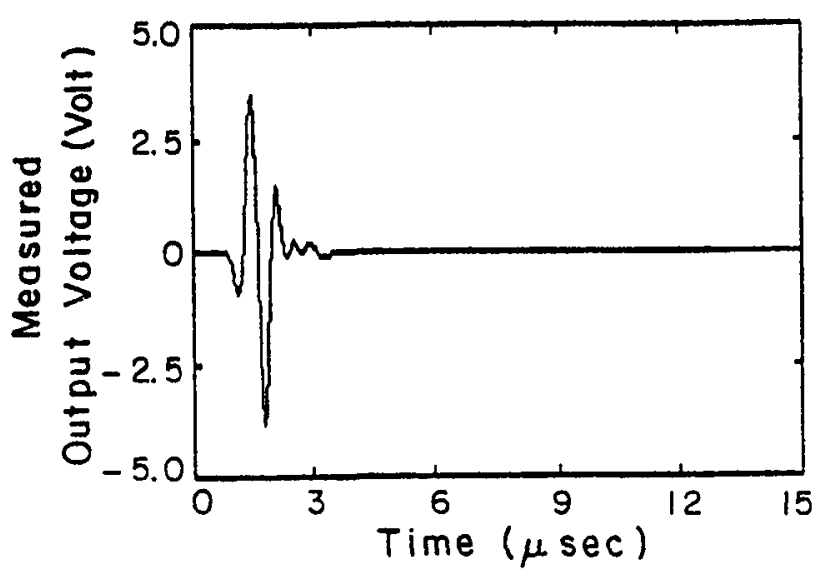

(b)

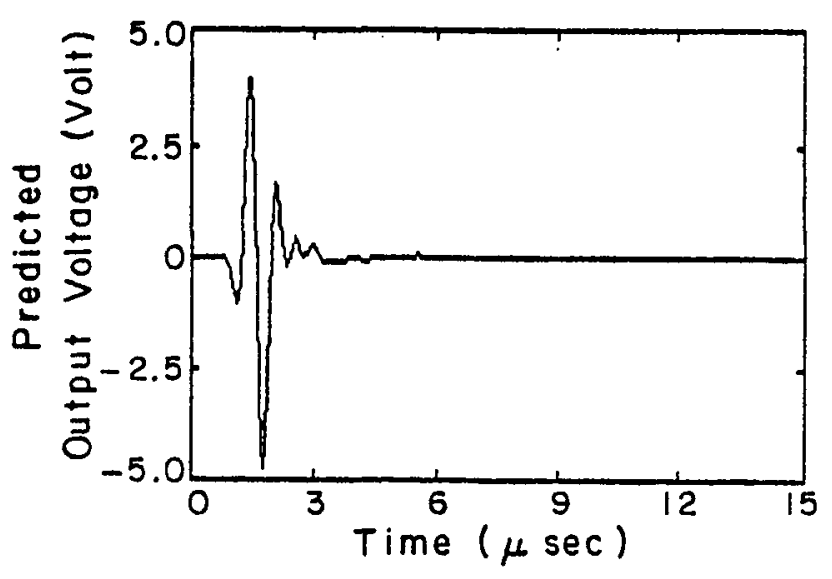

(c)

Fig. 10 Measured and predicted system responses: (a) One cycle of $1.75 \mathrm{MHz}$ frequency input at 2.3 Volts peak-to-peak and corresponding (b) experimental output and (c) predicted output. 



\title{
QUANTITATIVE FLAW CHARACTERIZATION WITH
}

\author{
SCANNING LASER ACOUSTIC MICROSCOPY \\ Edward R. Generazio and Don J. Roth \\ National Aeronautics and Space Administration \\ Lewis Research Center \\ Cleveland, Ohto 44135
}

Surface roughness and diffraction are two factors that have been observed to affect the accuracy of flaw characterization with scanning laser acoustic microscopy. Inaccuracies can arise when the surface of the test sample is acoustically rough. It is shown that, in this case, Snell's law is no longer valid for determining the direction of sound propagation within the sample. This paper investigates the relationship between the direction of sound propagation within the sample, the apparent flaw depth, and the sample's surface roughness. Diffraction effects can mask the acoustic images of minute flaws and make it difficult to establish their size, depth, and other characteristics. It is shown that for Fraunhofer diffraction conditions the acoustic image of a subsurface defect corresponds to a two-dimensional Fourier transform. Transforms based on simulated flaws are used to infer the size and shape of the actual flaw.

\section{INTRODUCTION}

With the application of fracture mechanics to the design of components under stress, the ability to accurately characterize existing material flaws by nondestructive evaluation (NDE) techniques has become extremely important. Scanning laser acoustic microscopy (SLAM) has received considerable attention in recent years as a promising NDE technique (refs. 1 to 10). SLAM is generally used for identifying the approximate position, size, shape, and depth of material flaws such as inclusions, voids, and delaminations. Materials inspected by using SLAM include electronic and structural ceramics (refs. 1 and 2).

Recent efforts have been directed toward obtaining more accurate quantitative flaw characterizations from acoustic images obtained with SLAM (refs. 1,3 , and 4). Inaccuracies can arise, however, when the surface of the test specimen is acoustically rough. The acoustic image of a flaw and therefore the characterization of the flaw depend on the surface roughness of the material in which the flaw resides. For example, voids of known dimensions in as-fired structural ceramic specimens with rough surfaces are difficult to detect and characterize from the acoustic image (ref. 5). Polishing the surface of the specimens to a mirrorlike finish improves the acoustic image, and the voids are readily detected and more easily characterized (ref. 5). The effect of surface roughness on the determination of flaw depth and the direction of sound propagation was examined in detail in this study. 
For subsurface flaws the acoustic image often consists of a diffraction pattern rather than a facsimile image of the flaw. In this case it is especially difficult to characterize the flaws (ref. 1). Nevertheless relationships have been implied between diffraction-dominated acoustic images of flaws and their actual size and depth (refs. 1 and 4 ). Since minute flaws are often detectable only by their diffraction patterns, it seems appropriate to investigate in more detail the relationship between diffraction patterns and the flaws causing them.

Some diffraction theories are applicable to typical SLAM experimental configurations. In this study we investigated methods for analyzing acoustic images and demonstrated that quantitative data can be obtained from the acoustic images when the effect of sample surface roughness and diffractive scattering are included in the analysis.

The results of two closely related investigations are presented herein. The first investigation examined the effect of sample surface roughness on the sound direction within an experimental sample. The second investigation examined the relationship between diffraction-dominated acoustic images of subsurface flaws and the characterizations of these flaws. The theoretical development and description of each experimental configuration are followed by the experimental results for each investigation. A general discussion of the interrelation of the investigations is presented.

\section{SCANNING LASER ACOUSTIC MICROSCOPY}

The development and operation of the scanning laser acoustic microscope are covered in references 7 to 10. The basic SLAM configuration is shown in figure 1(a). Longitudinal sound waves are produced by a piezoelectric transducer and transmitted through a couplant (usually distilled water) to the sample. The sound is reflected and refracted at the water-sample interface (fig. $7(b)$ ). The angle of the transmitted sound in the sample is determined by Snel1's law (ref. 11):

$$
\frac{\sin \theta_{s}}{\sin \theta_{w}}=\frac{v_{s}}{v_{w}}
$$

where $V_{W}$ and $V_{S}$ are the sound velocity in the water and sample, respectively. The angles $\theta_{W}$ and $\theta_{S}$ are the incident and refracted angles, respectively. Upon reaching the side of the sample opposite the incident-sound source, the sound displaces the surface to produce a "dynamic ripple" (ref. 8). To observe the dynamic ripple, a laser beam is scanned over the rippling side of the sample, which has been metallized with a reflective coating or covered with a metallized polymethylmethacrylate coverslip (fig. 1(a)). The laser light, angularly modulated by the surface deformations of the dynamic ripple, is reflected to a photodetector and subsequentiy demodulated to produce a real-time image on a video monitor. If the sample contains scatterers such as voids, inclusions, or density variations, the sound will be scattered or attenuated. These scattered or attenuated waves may appear as amplitude variations in the dynamic ripple and therefore may be visible as amplitude variations in the real-time video acoustic image. 


\section{EFFECT OF SURFACE ROUGHNESS ON FLAW DEPTH DETERMINATION}

\section{Background}

Two widely used methods for determining flaw depth by using SLAM are (1) the shadow method (ref. 6), which is used for surface flaws, and (2) the stereoscopic method (ref. 10), which is used for determining the depth of subsurface flaws. Both methods depend on prior knowledge of ultrasound direction in the sample. This direction can be determined by Snell's law.

The configuration for the shadowing technique is shown in figure 2, where the sound is strongly attenuated by a surface flaw to form a low-soundintensity region, or shadow. The depth of the surface flaw is estimated from the angle of sound $\theta_{s}$ in the material sample and the length $L$ of the shadow region. The depth $d$ of the surface flaw is then given by

$$
d=\frac{L}{\tan \theta_{s}}
$$

The configuration for the stereoscopic method is shown in figure 3 . Here the position $X_{R}$ of the acoustic image is noted in reference to a center position $X_{0}$. The sample is then rotated $180^{\circ}$ about the center position. The new position $X_{L}$ of the acoustic image is noted again in reference to the position $x_{0}$. If the direction of sound in the material is known, the depth $d$ of the flaw is given by

$$
d=\frac{x_{R}-x_{L}}{2 \tan \theta_{S}}
$$

These simple relationships are not generally applicable to the SLAM sample configuration. Errors in depth determination may arise when $\theta_{s}$ is inacurrately determined. Snell's law for ultrasonic waves applies to interfaces that are acoustically flat (i.e., where the sound wavelengths in both the water and the sample material are much greater than the topological variations on the surface of the sample). A typical water-ceramic interface as measured by stylus profilometry is shown in figure 4. The ceramic sample has topological variations of the order of $2 \mu \mathrm{m}$. At $100 \mathrm{MHz}$ the sound wavelengths $\lambda$ in water and in a typical ceramic sample are 14.9 and $60 \mu \mathrm{m}$, respectively. At these wavelengths and because of sample surface roughnesses, Snell's law is no longer applicable. An analogy can be drawn for Snell's law between optical and acoustical theory. An optically flat piece of glass refracts light according to Snell's law, but glass with a randomly rough surface (i.e., frosted) does not. The glass appears to be frosted because the incident light is refracted (scattered) into many angles at the air-glass interface. This analogy holds for acoustically flat and rough interfaces.

\section{Experiment}

To investigate the effect of surface roughness on the estimation of flaw depth, a macroscopic surface crack (channe1) was constructed with optically flat microscope slides (fig. 5(a)). The channel was $2.54 \mathrm{~cm}$ long, $1 \mathrm{~mm}$ deep, and $1 \mathrm{~mm}$ wide. The epoxy layer was approximately $20 \mu \mathrm{m}$ thick. The surface of the slide opposite the channel (incident-sound surface) was roughened by 
polishing in three selected regions with 1-,3-, and 15-um diamond polishing paste. The polishing direction and regions are shown schematically in figure $5(b)$. The sample was examined by SLAM with $\theta_{w}=16.8^{\circ}$.

\section{Results}

The surface roughnesses are shown in figure 6 for the optically flat area and the areas roughened with diamond paste. The peak-to-valley surface roughnesses for the optically flat region and the regions roughened with $1-, 3-$, and 15- $\mu \mathrm{m}$ diamond paste were approximately $0.04,0.2,1.0$, and $10 \mu \mathrm{m}$, respectively. These regions are cited according to their roughnesses in the remainder of this discussion.

Acoustic images of the glass slide sample near the channel are shown in figure 7. The four images shown are for incident sound on the regions of $0.04-, 0.2-, 1.0-$, and 10- $1 \mathrm{~m}$ surface roughness. The acoustic image intensity (gray level) as a function of horizontal position is also shown as a continuous white curve in each part of figure 7. The amplitude of the curve represents the amplitude of the sound. From figures $7(a)$ to (c) three distinct areas of itensity can be identified. The left-most area (solid dark area) is the inside of the channel and is unmetallized; so no sound intensity should be observed. This is also evident in the amplitude curves in the extreme left areas before the first jump in the curve. The second area is the shadow region just to the right of the solid dark area in each figure. The amplitude curves show a marked jump at the beginning of the shadow region. The noise level (image intensity) in each shadow region is relatively constant over a length $L$ (figs. $7(a)$ to $(c)$ ). This length defines the length of the shadow for each surface roughness shown. To the right of the shadow region the sound (and image) intensity increases in amplitude and fluctuates rapidiy. For this third area the sound has traveled a direct path through the sample relatively unperturbed by the presence of the channel. These three areas (channel edge, shadow, and right of shadow) are quite distinct for the regions of the glass sample having $0.04-, 0.2-$, and $1-\mu \mathrm{m}$ surface roughnesses. The region having a surface roughness of $10 \mu \mathrm{m}$, however, reveals $11 \mathrm{ttl}$ le evidence of these three areas.

If we assumed that Snell's law is valid for these configurations, the apparent depth of the channel can be determined from figures 2 and 7 (a) to (c) and equations (1) and (2). This is shown in figure 8 . The apparent channe 1 depth varied with incident-sound surface roughness. Only for the optically flat region of $0.04-\mu \mathrm{m}$ surface roughness was the true depth of the channel obtained. By assuming that Snell's law applies over the roughened regions, we obtained an error as large as 63 percent in determining the depth of the channel. Alternatively, or more correctly, we can determine the angle $\theta_{S}$ through which the sound is refracted as a function of the incident-sound surface roughness by using the known channel depth and equation (2). This is also shown in figure 8 . The refracted angle varied from $43^{\circ}$ for the optically flat region of $0.04-\mu \mathrm{m}$ surface roughness to $30^{\circ}$ for the region of $1.0-\mu \mathrm{m}$ surface roughness. The optically flat region was the only region that yielded a direction of sound (shear wave) propagation that was consistent with Snell's law (eq. (1)). 


\section{DIFFRACTION EFFECTS OBSERVED IN ACOUSTIC IMAGES OF FLAWS}

\section{Background}

The optical Fourler transform of an obstacle or aperture can be readily observed from tts diffraction pattern when the experimental configuration yields Fraunhofer diffraction conditions (refs. 12 to 14). We examined the origin of the diffraction pattern from a single slit of width a (fig. 9). plane wave, incident from the left, was diffracted at the slit opening. A minimum in the intensity of the diffraction pattern on an image plane at a distance $d$ from the slit occurred when (refs. 12 and 15)

$$
n \lambda=a \sin \alpha
$$

where $n, \lambda$, and $\theta_{s}$ are the minimum, wavelength, and angular distance between a minimum and the central maximum. For an experimental configuration the angle at which the first minimum, $n=1$, occurred is given by

$$
\alpha=\tan ^{-1}\left(\frac{x}{d}\right)
$$

where $x$ is the distance between the center of the slit and the first minimum.

A single-slit aperture will yield the two-dimensional diffraction pattern shown in figure 9 (a) if the Fraunhofer conditions are met (refs. 13 and 14). Fraunhofer diffraction patterns created by an aperture are similar to those for an obstacle with the same dimensions (refs. 3 and 12). A digital twodimensional Fourier transform of a line function simulating a single slit (fig. $9(b)$ ) is shown in figure $9(c)$. It is identical to the diffraction pattern of figure $9(a)$. The diffraction pattern for a circular aperture, first solved by Airy by 1835 (refs. 12 and 15), is more complicated than diffraction from a single slit. Airy's results indicate that for an aperture of diameter a the angle of the first minimum occurs at

$$
\alpha=\sin ^{-1}\left(1.22 \frac{\lambda}{a}\right)
$$

A digital two-dimensional Fourier transform of a circle function simulating a circular aperture (fig. 10(a)) is shown in figure 10(b). The diffraction results follow similarly for acoustic waves.

\section{Experiment}

A naturally occurring subsurface spherical void in an ordinary piece of glass (fig. 11) was used to illustrate the acoustic diffraction patterns from a spherical scatterer. The glass was ground to a thickness of approximately $4.2 \mathrm{~mm}$, and both sides were polished with 50-rm aluminum oxide. The void is $380 \mu \mathrm{m}$ in diameter and is $700 \mu \mathrm{m}$ below the surface.

To illustrate the acoustic diffraction pattern from a line scatterer, a 23-um-diameter tungsten wire was ground to have a flat surface so that it was semicylindrical in shape. The wire was $1 \mathrm{~cm}$ long and supported 300 um below the surface of a polymethylmethacrylate slab $1.5 \mathrm{~mm}$ thick (fig. 12). The slab 
was polished with 50-um aluminum oxide. Both samples were examined by using SLAM.

\section{Results}

The acoustic image for the void in glass is shown in figure 13. The diffraction pattern resembles a series of concentric circles and is similar to that of the Fourier transform of a circular obstacle shown previously ( $f$ ig. $10(\mathrm{~b})$ ). The first minimums of intensity along the vertical direction are indicated in the figure. The distance $2 x$ between these minimums is $134 \mathrm{\mu m}$. Combining the distance between the minimums with the known depth of the void and equation (5) yielded an experimental value of $6.00^{\circ}$ for the angular distance between the first minimum and the central maximum. This value agrees well with the theoretical value of $5.01^{\circ}$ obtained from equation (6).

The acoustic image for the wire embedded in polymethylmethacrylate is shown in figure 14. The image resembles the Fourier transform of a line function shown in figure $9(\mathrm{c})$. The first minimums are indicated in the figure and the distance $2 x$ between them is $232 \mu \mathrm{m}$. Combining the distance between the minimums with the depth of the wire and equation (5) yielded an experimental value of $7.02^{\circ}$ for the angular distance between the first minimum and the center of the void. This value agrees well with the theoretical value of $7.48^{\circ}$ obtained from equation (4).

Since the direction of sound propagation in samples with even moderate surface roughness varies considerably from that for an optically flat surface, an alternative method to determine this direction is needed. Both the polymethylmethacrylate and glass samples were metallized with a semitransparent gold film. The positions of the subsurface defects were simultaneousiy observed optically and acoustically (fig. 15). From the known depth $d$ and the apparent displacement $\Delta x$ of the defect, the angle of sound propagation in the sample is

$$
\theta_{s}=\tan ^{-1}\left(\frac{\Delta x}{d}\right)
$$

For the glass sample $\theta_{S}=27^{\circ}$ and corresponds to shear waves; for the polymethylmethacrylate sample $\theta_{S}=31^{\circ}$ and corresponds to longitudinal waves.

\section{DISCUSSION}

The apparent direction of sound propagation within the sample changed with the roughness of the surface on which the sound impinged. This was observed even for samples exhibiting topological variations of the order of 0.02 to $1.0 \mu \mathrm{m}$. These results indicate that Snell's law is not generally valid for determining propagation direction for samples with acoustically rough surfaces. An accurate measurement of flaw depth depends on the correct determination of the sound direction within the sample (eqs. (2) and (3)); therefore neither the shadow method nor the stereoscopic method will yield accurate flaw depths or samples with rough surfaces. 
The acoustical Fourier transforms (figs. 13 and 14) are not identical to their corresponding digital two-dimensional Fourier transforms (figs. 10 (b) and $9(c))$. The discrepancies are due to three theoretical conditions that are not satisfied by the experimental configuration. First, neither the wire nor the void is a two-dimensional object. Hence the standard theoretical development for two-dimensional apertures does not apply. The wire defect was purposely flattened on one side in an effort to achieve an experimental system that was nearly two dimensional. A similar polymethylmethacrylate sample was produced in which a cylindrical wire was embedded. The diffraction pattern on the left side of the central maximum was not observed on the acoustic image. Only by flattening one side of the wire was the full diffraction pattern observable. This result can be extended to the case of the vold. For the void the diffraction pattern on the left side of the central maximum (fig. 14) was most likely not observed because of the dimensionality of the defect and the experimental configuration. Second, the acoustic image is formed on an image plane that is not perpendicular to the direction of sound propagation. The obliqueness of this image plane must be incorporated into the diffraction theory. Third, the experimental configuration used did not result in true Fraunhofer conditions. For the sample dimensions and sound wavelengths shown herein, the diffraction patterns were observed in the transition region between Fresnel and Fraunhofer zones. As a result the phase cancellation at the minimums was incomplete and the minimums were displaced.

The acoustic image of the void shows diffraction rings that tend to be more closely packed at distances far from the central maximum. The work by Airy (refs. 12 and 15) indicates that for a circular aperture the distance between adjacent diffraction rings should decrease with distance from the central maximum. This is consistent with the experimental results. It, however, was not seen for the digital two-dimensional Fourier transform of a digitally simulated circular aperture. This discrepancy is likely to be due to the discrete nature of the digital aperture. The aperture is not a true circle but a collection of adjacent rectangular operings of various sizes. The circumference of this simulated aperture is steplike. Fourier components that would be observed if the aperture were a true circle are missing from the transform shown in figure $6(b)$. These missing Fourier components are needed to form the Airy disk pattern with varying ring spacing.

\section{CONCLUSIONS}

It was shown that accurate measurements of surface and subsurface flaw characteristics depend on determining the refracted sound propagation direction within the sample. The surface roughness of the experimental sample was found to have a substantial effect on the direction of refracted sound propagation. Therefore Snell's law is not generally applicable to even moderately rough samples. However, accurate depth measurements could be made when the direction of refracted sound propagation was determined experimentally. Acoustic diffraction patterns caused by subsurface flaws were shown to be directly related to two-dimensional digital fourier transforms of objects that simulate the size and shape of subsurface flaws. 


\section{REFERENCES}

1. Kessler, L.W., D.E. Yuhas, and C.L. Vorres, "Acoustic Microscopy of Ceramics," Nondestructive Evaluation: Microstructural Characterization and Reliability Strategies, 0. Buck and S.M. Wolf, eds., 1980, pp. 273-287, Metallurgical Soclety of AIME, Warrendale, PA.

2. Kessler, L.W., and D.E. Yuhas, "Principles and Analytical Capabilities of the Scanning Laser Acoustic Microscope (SLAM)," Scanning Electron Microscopy, Vol. 1, 1978, pp. 555-560, SEM, Inc., Chicago, IL.

3. Chou, C.H., B.T. Khuri-Yakub, and G.S. Kino, "Transmission Imaging: Foward Scattering and Scatter Reconstruction," Acoustical Imaging, Vo1. 9, K.Y. Wang, ed., 1980, pp. 357-377, Plenum, New York.

4. Yuhas, D.E., T.E. McGraw, and L.W. Kessler, "Scanning Laser Acoustic Microscope Visualization of Solid Inclusions in Silicon Nitrides," Proceedings of the DARPA/AFML Review of Progress in Quantitative Nondestructive Evaluation, AFWAL-TR-80-4078, 1980, Pp. 683-690, Rockwe11 Internationa 1, Thousand Oaks, CA.

5. Roth, D.J., J. Klima, J.D. Kiser, and G.Y. Baaklini, "Reliability of Detection of Voids in Structural Ceramics Using SLAM (Scanning Laser Acoustic Microscopy)," Paper presented at the Spring Meeting of the American Society for Nondestructive Testing in Washington, $D C$, March 11-14, 1985 .

6. Yuhas, D.E., "Characterization of Surface Flaws by Means of Acoustic Microscopy," U1trasonic Materials Characterization, NBS-SP-596, H. Berger and M. Linzer, eds., 1980, pp. 357-368, National Bureau of Standards, Washington, $D C$.

7. Whitman, R.L., M. Ahmed, and A. Korpe1, "A Progress Report on the Laser Scanned Acoustic Camera," Acoustic Holography, Vo1. 4, G. Wade, ed., 1972, pp. 11-32, Plenum, New York.

8. Kessler, L.W., P.R. Palermo, and A. Korpe1, "Practical High Resolution Microscopy," Acoustic Holography, Vol. 4, G. Wade, ed., 1972, pp. 51-71, Plenum, New York.

9. Auld, B.A., R.J. Gilbert, K. Hyllested, C.G. Roberts, and D.C. Webb, "A 1.1 Ghz Scanned Acoustic Microscope," Acoustic Holography, Vo1. 4, G. Wade, ed., 1972, pp. 73-96, Plenum, New York.

10. Kessler, L.W., and D.E. Yuhas, "Acoustic Microscopy - A Tutorial Review," Acoustical Imaging, Vo1. 9, K.Y. Wang, ed., 1980, pp. 275-299, Plenum, New York.

11. Kinsler, L.E., A.R. Frey, A.B. Coppens, and J.V. Sanders, Fundamentals of Acoustics, third edition, 1982, Chapter 5, wiley, New York.

12. Jenkins, F.A., and H.E. White, Fundamentals of Physical Optics. First edition, 1937, Chapter 5, pp. 105-127, McGraw-Hi11, New York. 
13. Cathey, W.T., Optical Information Processing and Holography, 1974, Chapter 2, Whley, New York.

14. Gonzalez, R.C., and P. Wintz, Digital Image Processing, 1973, Chapter 3, Addision-Wesley, Reading, MA.

15. Halliday, D., and R. Resnick, Fundamentals of Physics, 1974, Chapter 38, Revised Printing, Wiley, New York.

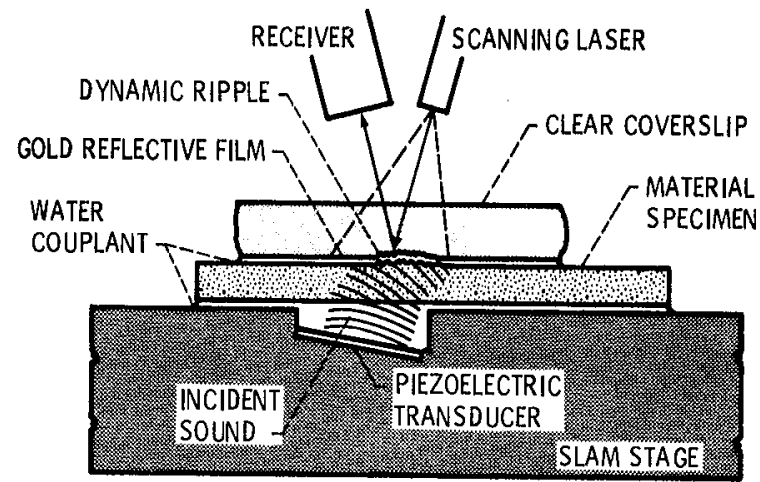

(a)

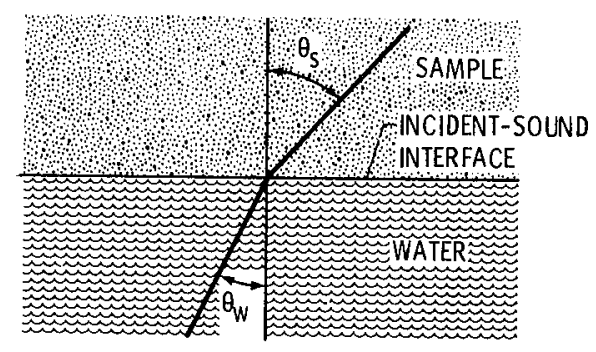

(a) General configuration used for SLAM.

(b) Schematic diagram illustrating Snell's law at incident-sound interface.

Figure 1. - Scanning laser acoustic microscopy (SLAM) technique. Ultrasound refracted at interface of water couplant and material specimen according to Snell's law.

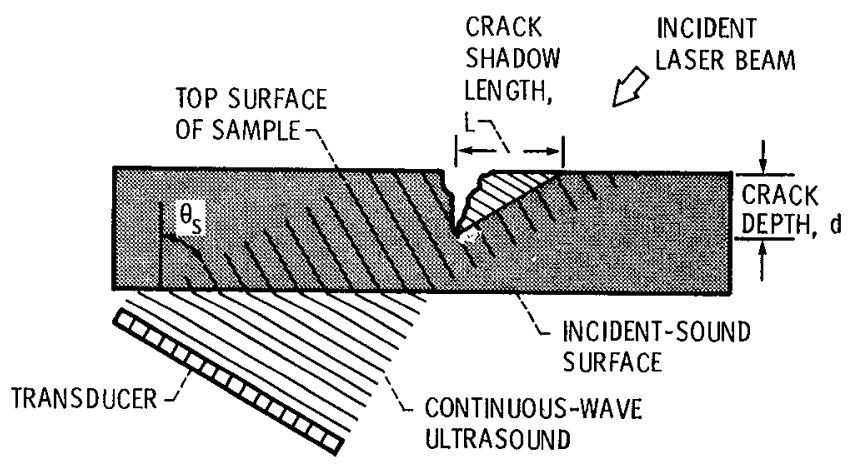

Iigure 2. - Schematic diagram showing shadow region produced when ultrasound encounters crack in top surface of sample. 


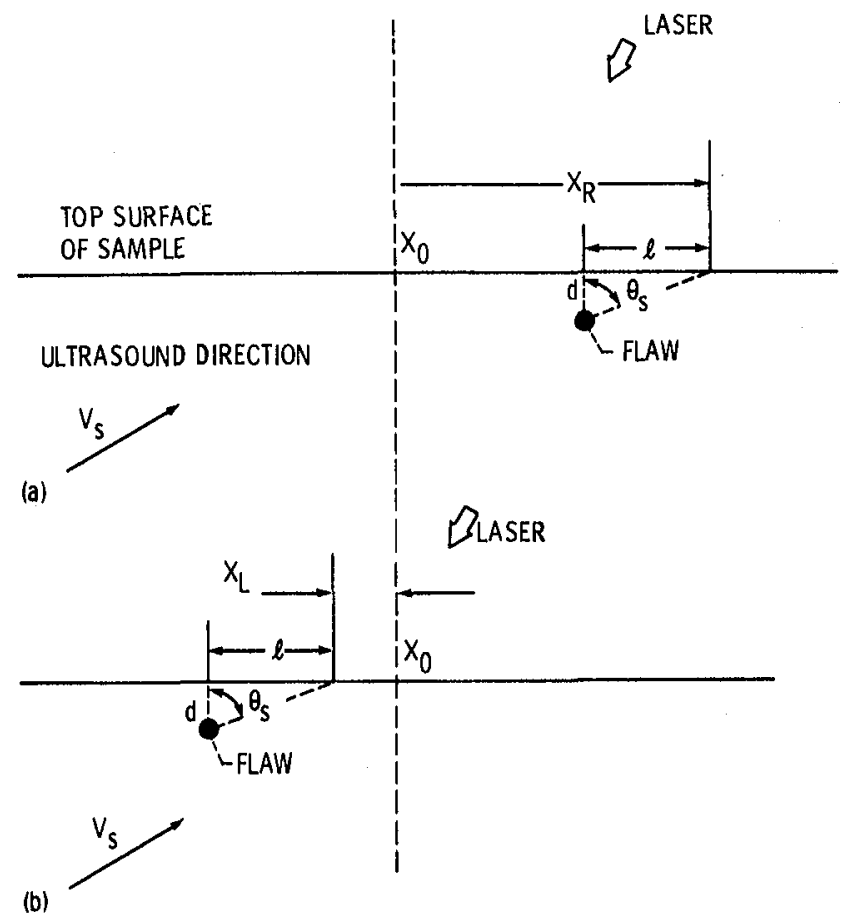

(a) Initial apparent position of flaw, $X_{R}$.

(b) Apparent position of flaw after $180^{\circ}$ rotation about position $X_{0}, X_{L}$.

Figure 3. - Schematic diagrams showing principles of stereoscopic method used to determine depth of flaw beneath sample surface.

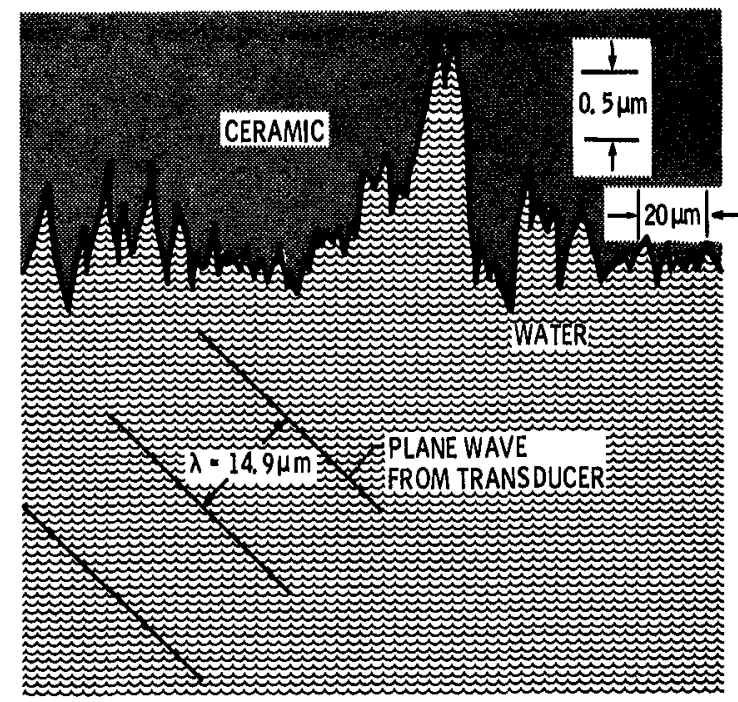

Figure 4. - Typical interface between water and ceramic sample with SLAM operating at $100 \mathrm{MHz}$. Note magnitude of wavelength of sound in water and magnitude of topological variations on surface of ceramic specimen. ) 


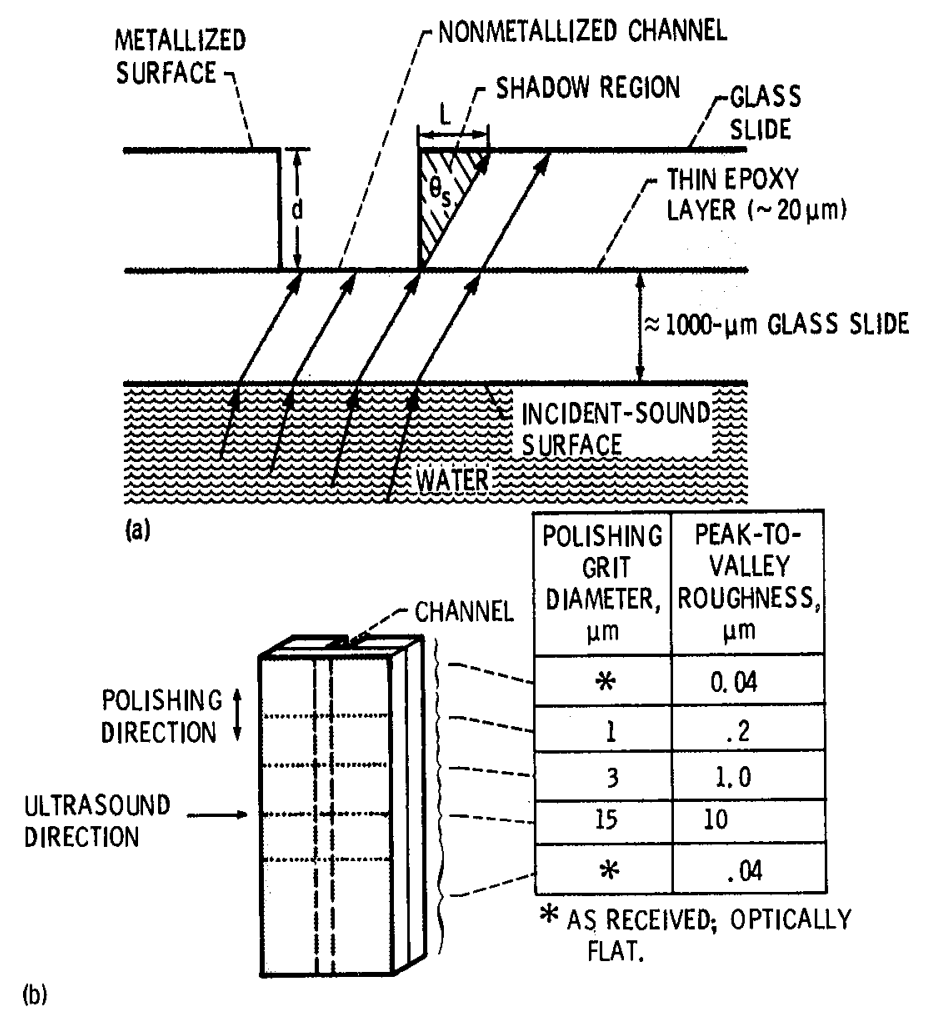

(a) Side view of specimen showing experimental setup on SLAM with resulting shadow region.

(b) Oblique bottom view of specimen showing regions polished with 1-, 3-, and 15- $\mu \mathrm{m}$ diamond polishing paste.

Figure 5. - Glass slide specimen with artificially made channel. (Channel simulates surface crack.)

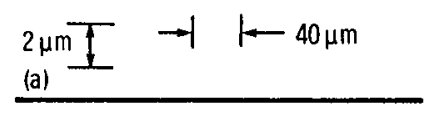

(b)
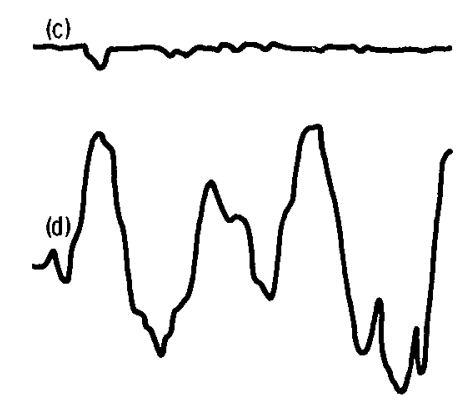

(a) Optically flat region (peak-to-valley roughness, $\leq 0.04 \mu \mathrm{m}$ ). (b) Region polished with 1- $-\mu m$ diamond paste (peak-to-valley roughness, $0.2 \mu \mathrm{m}$ ).

(c) Region polished with 3- $\mu \mathrm{m}$ diamond paste (peak-to-valley roughness, $1 \mu \mathrm{m}$ ).

(d) Region polished with $15-\mu m$ diamond paste (peak-to-valley roughness, $10 \mu \mathrm{ml}$.

Figure 6. - Surface profiles of regions of different surface roughnesses on glass slide. 

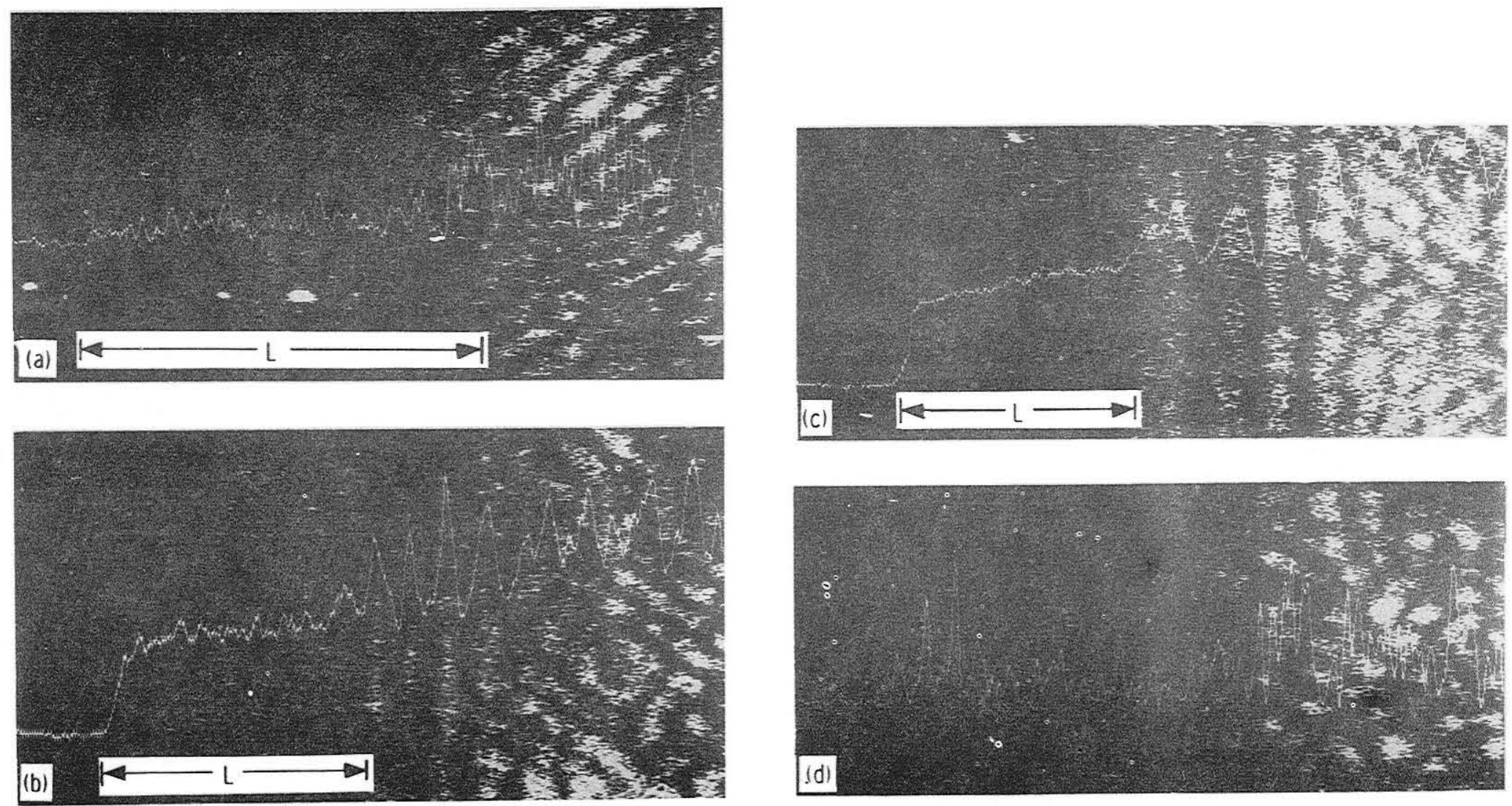

(a) Region of $0.04-\mu \mathrm{m}$ peak-to-valley roughness.

(b) Region of $0.2-\mu \mathrm{m}$ peak-to-valley roughness.

(c) Region of 1.0-um peak-to-valley roughness.

(d) Region of $10-\mu m$ peak-to-valley roughness.

Figure 7. - Acoustic images of glass slide regions of different surface roughnesses. (Images taken near channel. Continuous white curves show image intensity as function of horizontal position. Shadow length $L$ decreases with increasing surface roughness.) 


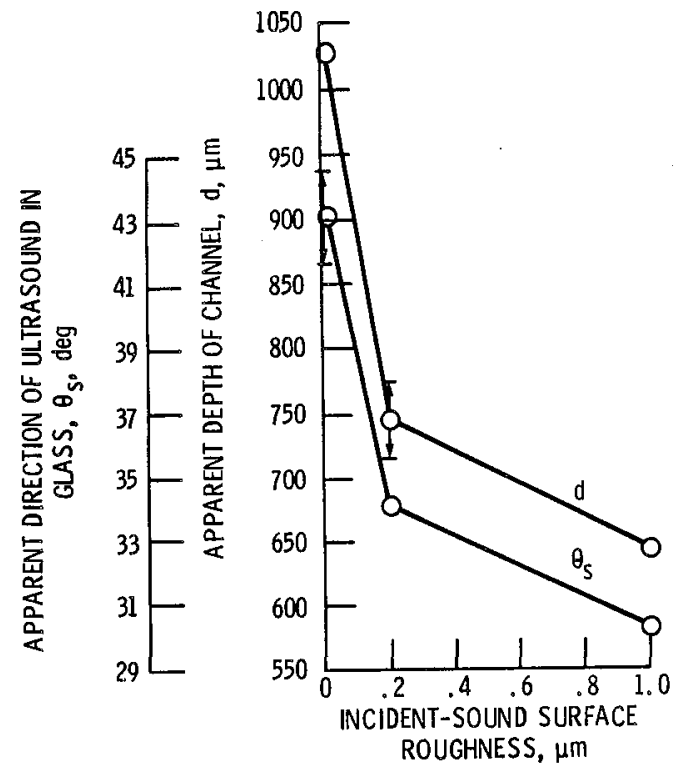

Figure 8. - Apparent depth of channel and apparent direction of ultrasound in glass as function of incident-sound surface roughness.

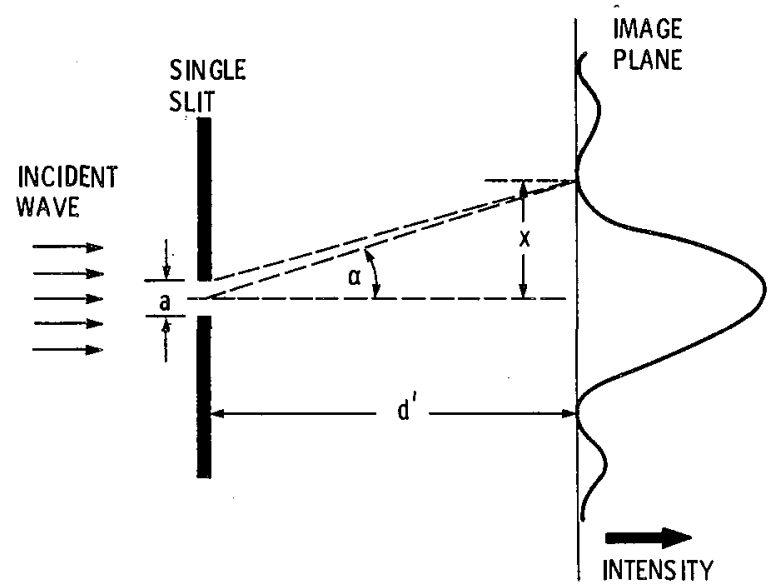

(a) Schematic diagram showing how radiation is diffracted by single slit.

Figure 9. - Diffraction of radiation from single slit under Fraunhofer conditions and Fourier transform of simulated single slit. 

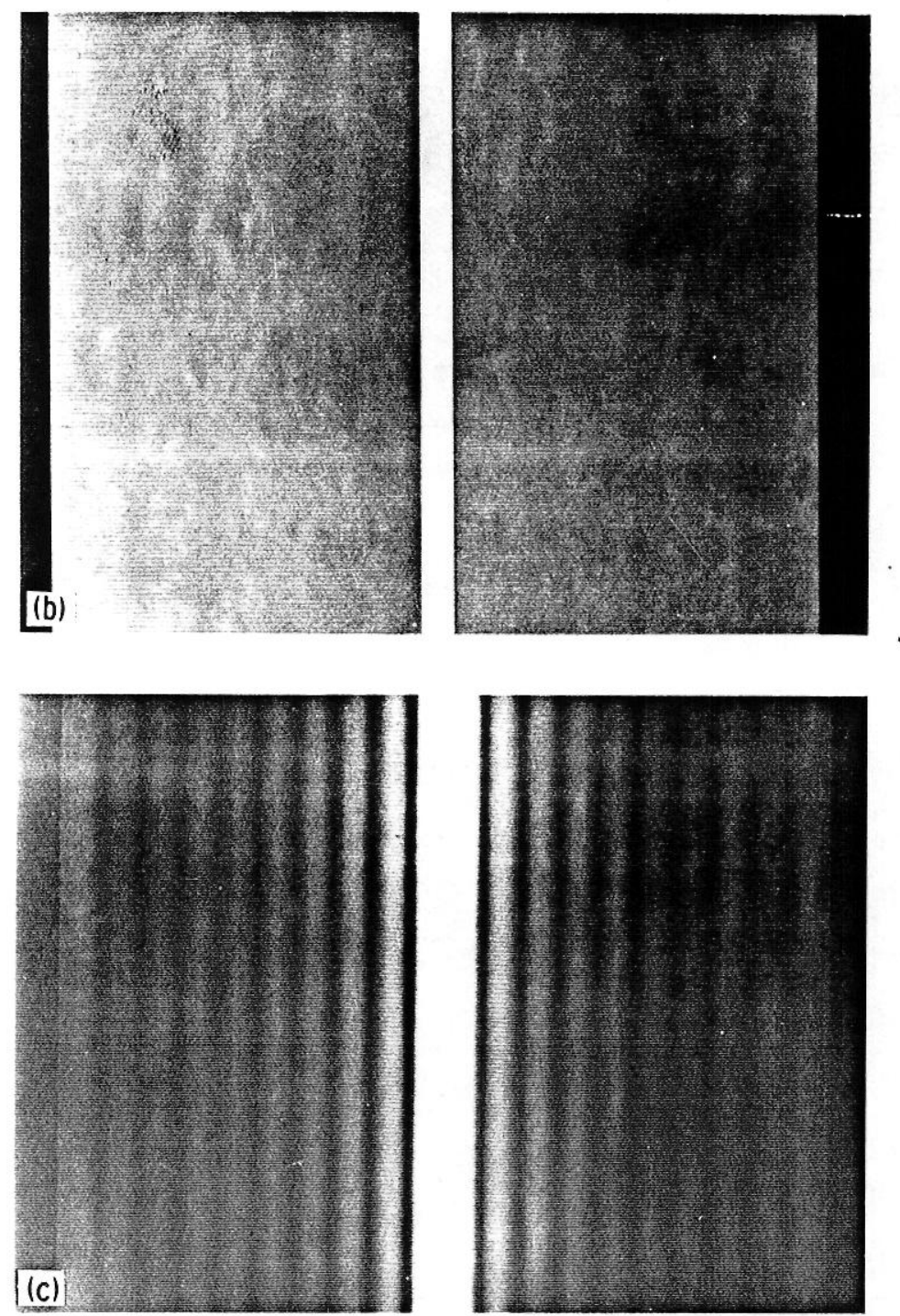

(b) Line function simulating single slit.

(c) Digital two-dimensional Fourier transform of line function.

Figure 9. - Concluded. 


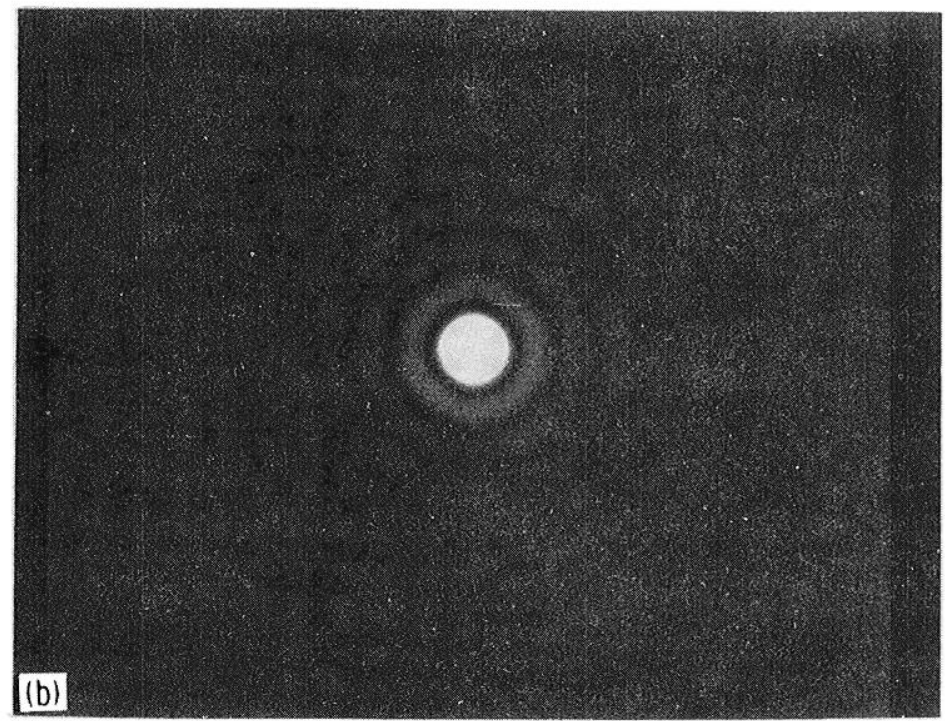

(a) Circle function simulating circular aperture.

(b) Digital two-dimensional Fourier transform of circle function.

Figure 10. - Fourier transform of simulated circular aperture. 


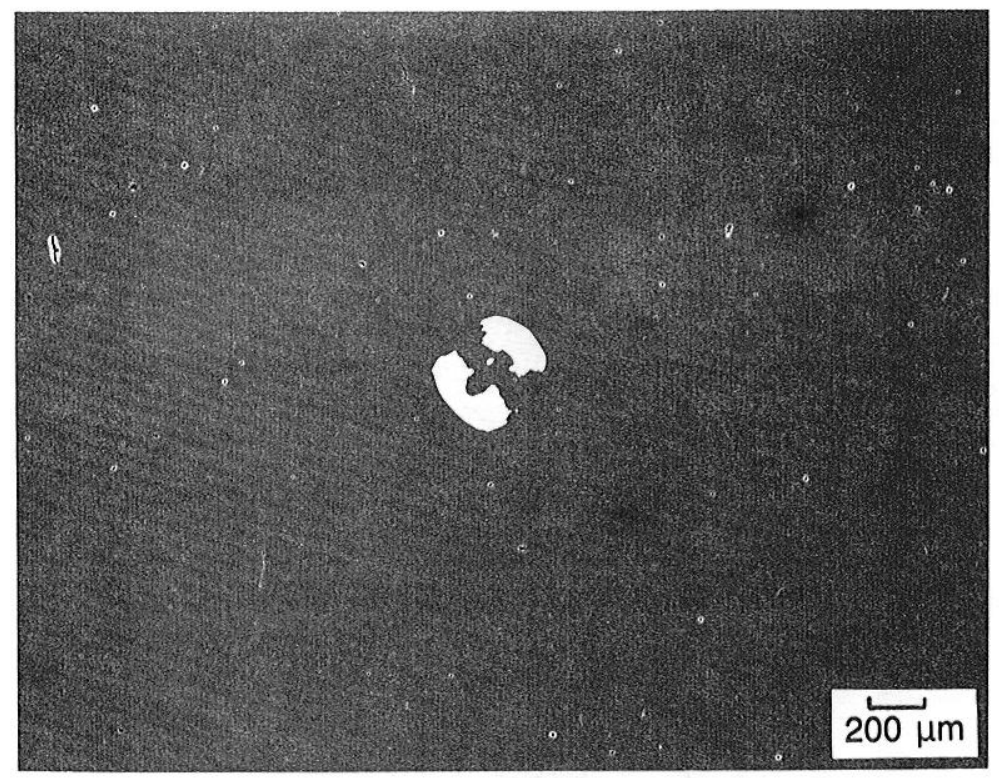

Figure 11. - Top view of subsurface spherical void occurring naturally in ordinary piece of glass, used to illustrate acoustic diffraction patterns from spherical scatterer.

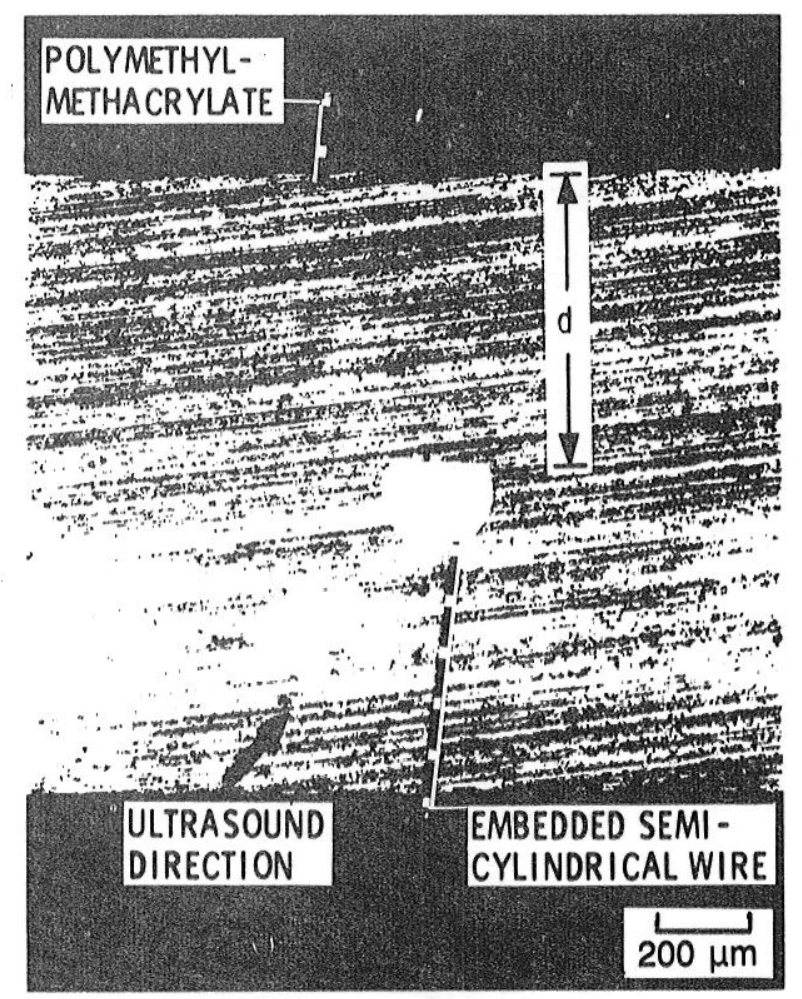

Figure 12. - Side view of tungsten wire embedded in polymethylmethacrylate slab, used to illustrate acoustic diffraction patterns from line scatterer. 


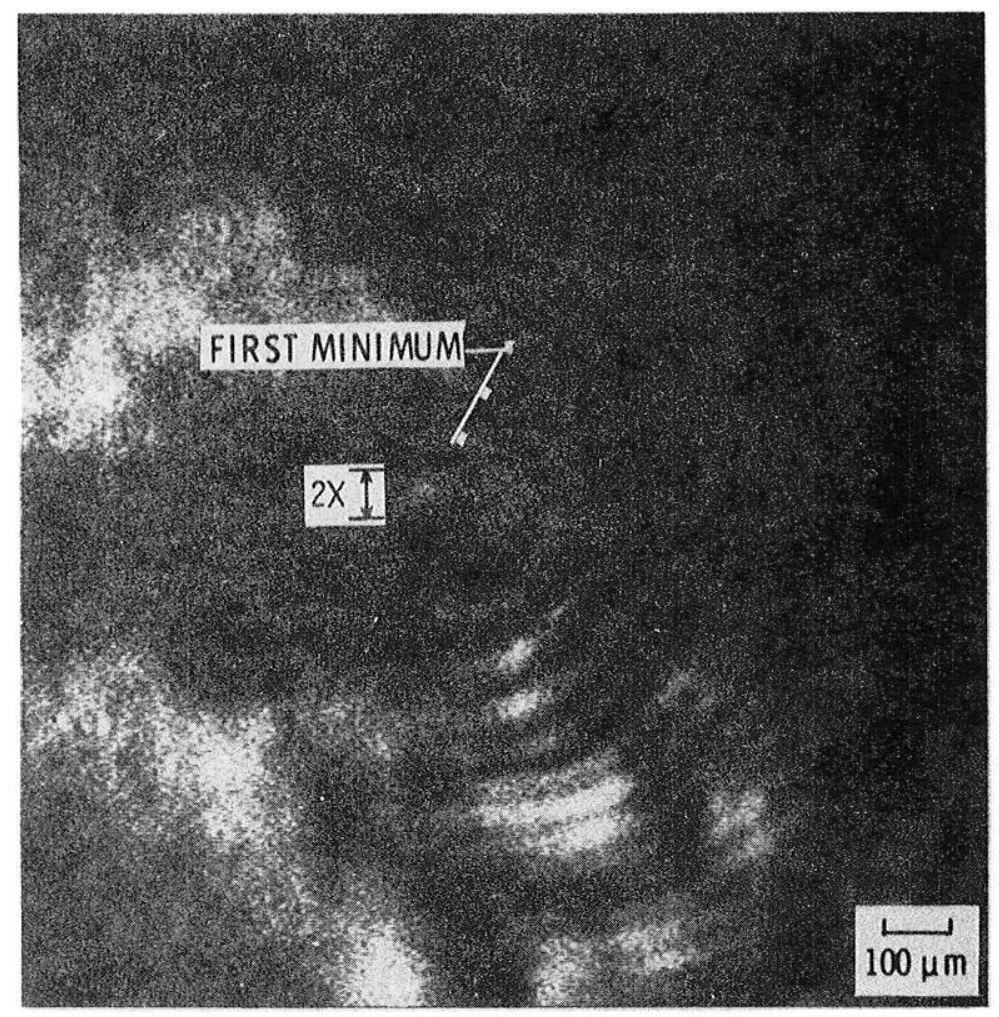

Figure 13. - Acoustic image of naturally occurring subsurface spherical void in glass (Fig. 111 .

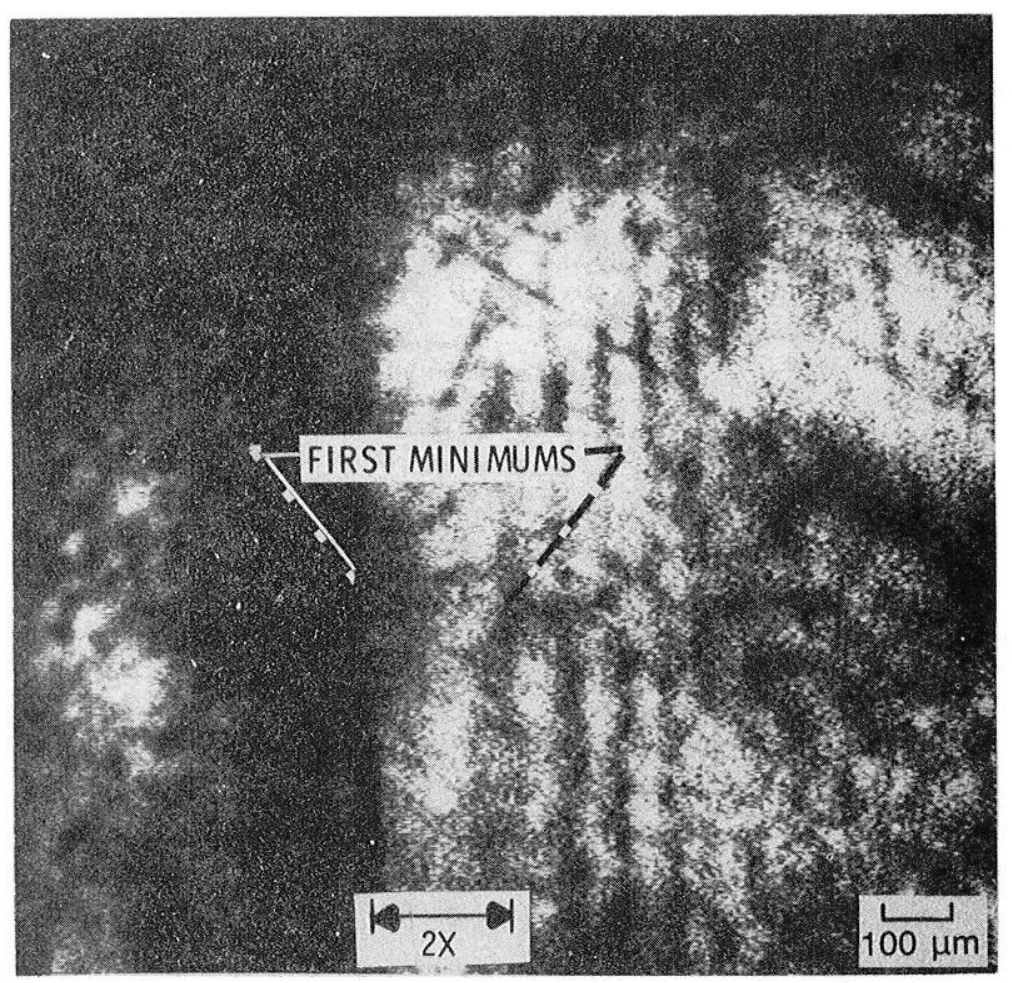

Figure 14 - Acoustic image of wire embedded in polymethylmethacrylate slab (Fig. 12). 


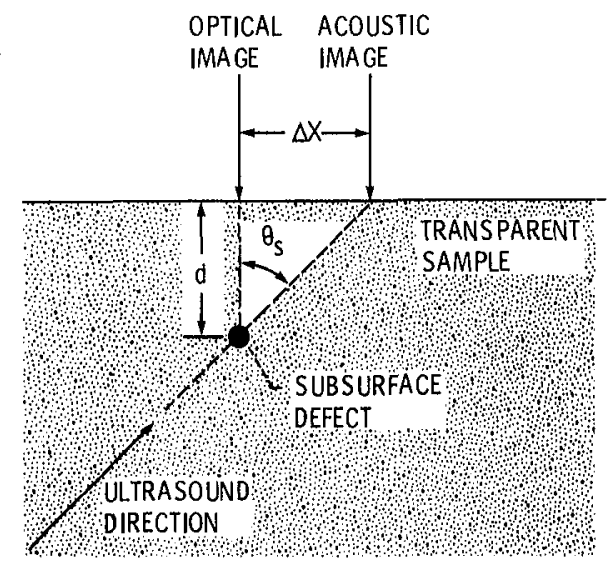

Figure 15. - Schematic diagram illustrating optical-acoustic method using SLAM for determining true ultrasound direction within material. 



\begin{tabular}{|c|c|c|}
\hline $\begin{array}{l}\text { 1. Report No. } \\
\text { NASA CP-2383 }\end{array}$ & 2. Government Accession No. & \\
\hline \multirow{2}{*}{\multicolumn{2}{|c|}{$\begin{array}{l}\text { 4. Title and Subtitle } \\
\text { Analytical Ultrasonics in Materials Research } \\
\text { and Testing }\end{array}$}} & 5 \\
\hline & & on Code \\
\hline \multicolumn{2}{|l|}{ 7. Author(s) } & on Report No. \\
\hline & 10. Work Unit No. & \\
\hline \multirow{2}{*}{\multicolumn{2}{|c|}{$\begin{array}{l}\text { 9. Performing Organization Name and Address } \\
\text { National Aeronautics and Space Administration } \\
\text { Lewis Research Center } \\
\text { Cleveland, Ohio } 44135\end{array}$}} & \\
\hline & & it \\
\hline \multicolumn{2}{|l|}{ 12. Sponsoring Agency Name and Address } & ublication \\
\hline \multicolumn{2}{|l|}{$\begin{array}{l}\text { National Aeronautics and Space Administration } \\
\text { Washington, D.C. } 20546\end{array}$} & \\
\hline \multicolumn{3}{|l|}{ 15. Supplementary Notes } \\
\hline \multicolumn{3}{|c|}{$\begin{array}{l}\text { 16. Abstract } \\
\text { The Analytical Ultrasonics in Materials Research and Testing Conference was held } \\
\text { at the NASA Lewis Research Center on November 13-14, 1984. This proceedings } \\
\text { includes } 21 \text { papers dealing with various aspects of analytical ultrasonics. } \\
\text { Analytical ultrasonics may be defined as a branch of nondestructive evaluation } \\
\text { concerned with the measurement of microstructural factors that govern mechanical } \\
\text { properties and dynamic response of structural materials. This definition goes } \\
\text { beyond flaw detection, flaw imaging, and defect characterization. Analytical } \\
\text { ultrasonics includes the assessment of material environments in which the flaws } \\
\text { reside. This proceedings presents recent results and advancements in analytical } \\
\text { ultrasonics for application to materials research and testing. }\end{array}$} \\
\hline 17. Key Words (Suggested by Author(s)) & \multicolumn{2}{|l|}{ 18. Distribution Statement } \\
\hline $\begin{array}{l}\text { Attenuation; Uitrasonic; Ultrasonic } \\
\text { attenuation; Nondestructive testing; Non- } \\
\text { destructive evaluation; Heat treatment; } \\
\text { Grain size; Grain distribution }\end{array}$ & \multicolumn{2}{|l|}{$\begin{array}{l}\text { Unclassified - unlimited } \\
\text { STAR Category } 38\end{array}$} \\
\hline $\begin{array}{l}\text { 19. Security Classif. (of this report) } \\
\text { Unc las s if led }\end{array}$ & $\begin{array}{c}\text { 21. No. of pages } \\
364\end{array}$ & $\begin{array}{l}\text { 22. Price } \\
\text { A16 }\end{array}$ \\
\hline
\end{tabular}



National Aeronautics and Space Admi sistration Code NIT-4

Washington, D.C. 20546-0001

Olficial Business

Penatty tor Private Use, $\$ 300$
SPECIAL FOURTH CLASS MAIL BOOK

$\cdot \cdot$

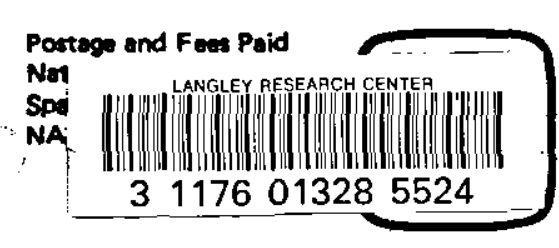

\section{N/SA}

\section{DO NOT REMOVE SLIP FROM MATERIAL}

Delete your name from this slip when returning material to the library.

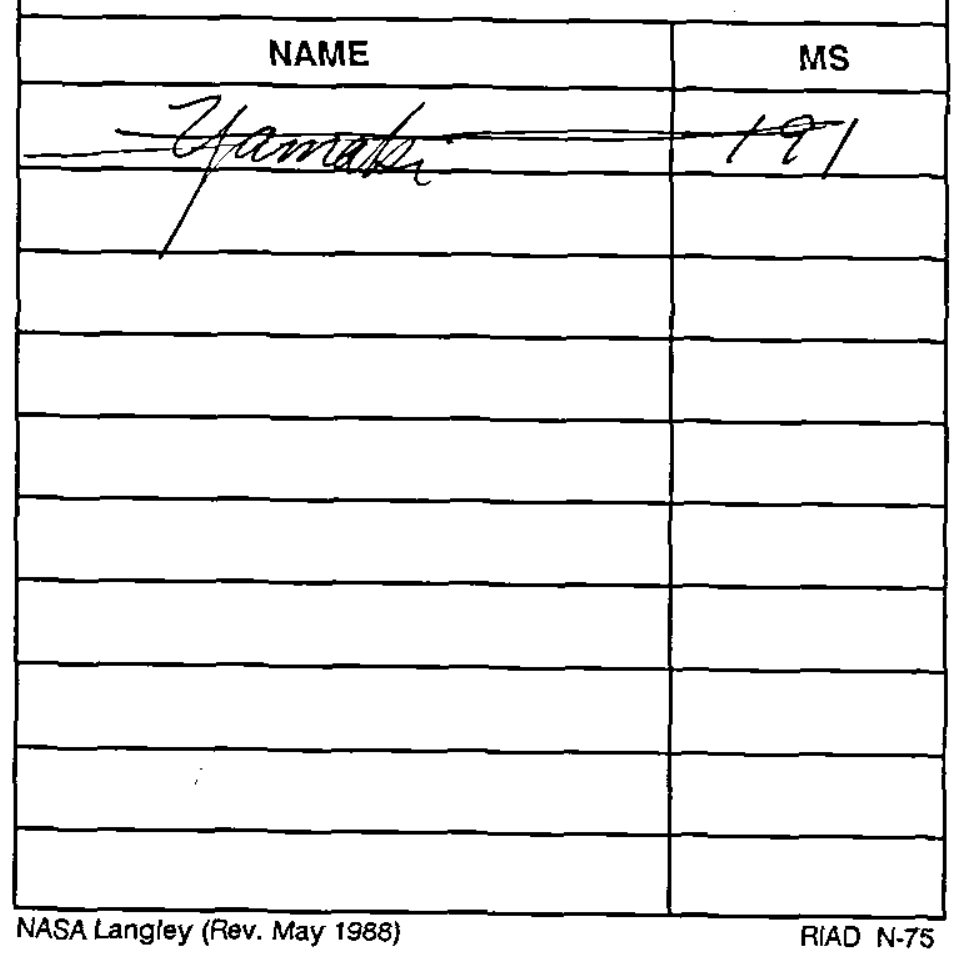

